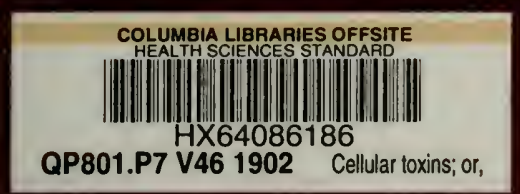

$$
\text { RECAP }
$$




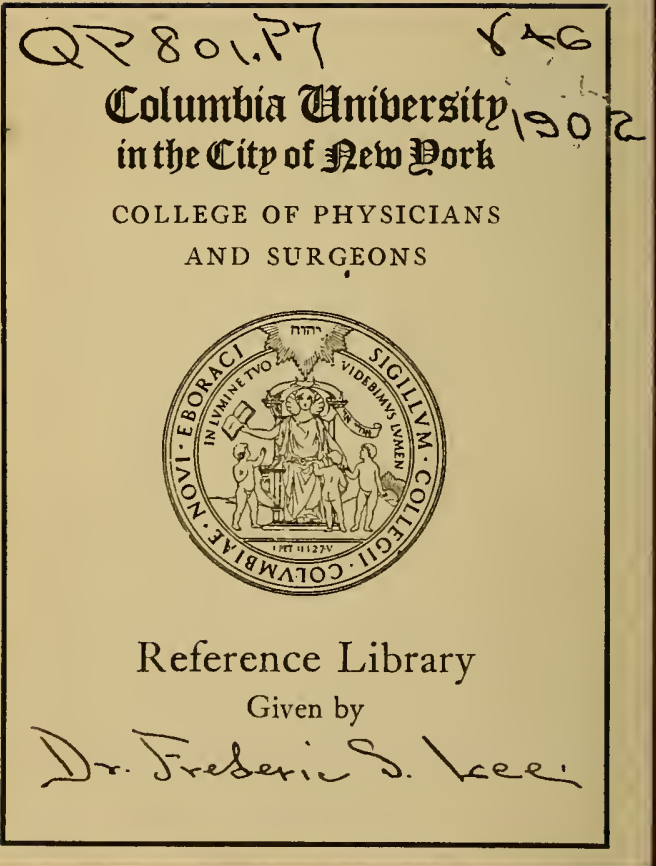






\section{CELLULAR TOXINS}

OR THE

\section{CHEMICAL FACTORS}

IN THE

\section{CAUSATION OF DISEASE}

BY

VICTOR C. VAUGHAN, M.D., LL.D.

PROPEESOR OF HYGIENE AND PHYSIOLOGICAL CHEMISTRY AND DIRECTOR OF THE HYGIENIC LABORATORY IN THE CNIVERSITY OF MICEUGAN,

AND

FREDERICK G. NOVY, M.D., Sc.D. JUNIOR PROFESOR OF HYGIENE $\triangle$ ND PHYSIOLOGICAL CHEMISTRY IN THE UNIVERSITY OF MICHIGAN.

FOURTH EDITION, REVISED AND ENLARGED

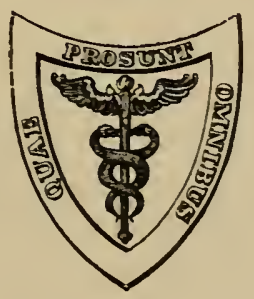

LEA BROTHERS \& COMPANY PHILADELPHIA AND NEW YORK 
Entered according to the Act of Congress in the year 1902, by LEA BROTHERS \& CO.,

In the Office of the Librarian of Congress. All rights reserved. 
ALBERT B. PRESCOTT, M.D., LL.D.

DIRECTOR OF THE OHEMICAL LABORATORY IN THE UNIVERSITY OF MICHIGAN, THIS LITTLE WORK

IS RESPECTFULLY DEDICATED

AS A SLIGHT TOKEN OF THE HIGH ESTEEM IN WHICH HE IS HELD BY HIS FORMER STUDENTS,

THE AUTHORS. 



\section{PREFACE TO FOURTH EDITION.}

During the fourteen years which have elapsed since the appearance of the first edition of this book, the subject matter of which it treats has increased in importance, has modified our conceptions of disease, and has furnished facts which are now utilized in treatment. Quite naturally, during the growth and development of the chemistry of the infectious diseases, this science has from time to time changed the relative importance of different phenomena. When the first edition of this book was written it was believed by those most competent to speak on the subject, that the basic products of bacterial growth constituted the chief factors in the causation of the infectious diseases, but it has been shown by subsequent discoveries that this conception is erroneous, and we now look for the specific bodies among the synthetic substances formed within the cells of the microörganism. This advance in knowledge has rendered the chief title selected for former editions inappropriate, and accounts for the change which we have made therein. The text has for the most part been rewritten with the intention of curtailing the space given to subjects which advanced knowledge has shown to be less important, and also for the purpose of introducing new matter. We regret exceedingly that want of space has compelled us to omit altogether the bibliography found in previous editions. References to the literature employed in the older editions have been omitted, while those bearing upon investigations which have been made since the appearance of the last edition are given in footnotes. Those to whom the third edition is accessible will be able to look up every piece of work referred to in this volume and satisfy themselves concerning the interpretation which we have placed upon the original contributions. In order to abbreviate as much as possible, we have omitted many details given in the previous editions. This curtailment has of necessity not been uniform throughout the book; certain chapters having been cut down much more than others. In some instances the desire to take up less space has possibly led us to omit statements of considerable importance. Especially is this true of the chapter devoted to poisonous foods. Several new chapters have 
been added, bringing into the volume subjects which were wholly unknown at the writing of the last edition. We have endeavored to present to our readers everything of importance done in the lines treated of down to the close of the year 1901. If this volume meets with the kind reception extended to its predecessors, its authors will feel themselves amply repaid for the labor that they have placed upon it.

UNIVERstity of Michigan, June 1, 1902. 


\title{
CONTENTS.
}

\author{
CHAPTER I.
}

The Etiology of the Bacterial Diseases: Bacterial, Protozoal, Parasitic, Toxic, Traumatic, and Autogenous Diseases. How Bacteria cause Disease.

\section{CHAPTER II.}

Classification and Definition of the Chemical Products of Bacteria: Ptomaïns; Bacterial Proteids; Bacterial Cellular Proteids;

Toxins

\section{CHAPTER III.}

A Historical Sketch of the Bacterial Poisons: Observations on Putrefactive Material ; Examination of Mixed Cultures ; The Study of Pure Cultures.

\section{CHAPTER IV.}

The Bacterial Poisons of some of the Infectious Diseases; Anthrax ; Asiatic Cholera ; Tetanus; Diphtheria ; Tuberculosis ; Suppuration; Gonorrhœa ; the Summer Diarrhœas of Infancy ; Typhoid Fever ; Hog Cholera ; Rabbit Septicæmia ; Pneumonia ; Malignant CEdema; Puerperal Fever; Glanders.

\section{CHAPTER V.}

The Germicidal Properties of Blood Serum ; Alexins; Nuclein. 102

CHAPTER VI.

The Specific Precipitins

\section{CHAPTER VII.}

The Lysins: Bacteriolysis; Hemolysis; Cytolysis; Epitheliolysis; Nephrolysis; Spermotolysis.

\section{CHAPTER VIII.}

The Agglutinins

\section{CHAPTER IX.}

Immunity: Natural Immunity; Natural Immunity to Bacteria; Natural Immunity to Toxins; Acquired Immunity; Acquired Bacterial Immunity; Acquired Immunity to Toxins ; Antitoxins.. 162 vii 
Some species of single-celled animal organisms, known as protozoa, may invade the body and there live and reproduce themselves, modifying, impairing and destroying normal tissuc. The disorders resulting from these causes are known as protozoal diseases.

Other more highly developed animals pass at least a portion of their lives as parasites, and we must recognize certain diseases as due to animal parasites.

The living cells of the animal body may be altered or destroyed by the action of poisons of mineral, vegetable or animal origin. The poisoning that results in this way may be acute or chronic; it may manifest itself in one case principally by its action on the nervous system, and in another the symptoms induced may be referred more especially to the digestive organs. Diseases due to the administration of poisons generated wholly outside the body are grouped together under the name of intoxications.

A given group of cells in the body may be so altered by mechanical violence that the continued performance of healthy function is no longer possible. A depression of the skull, as the result of a fall or blow, may induce epilepsy or insanity. Diseases induced in this manner are said to be traumatic.

Lastly, without outside interference, any group of cells in the body may, from having an excess of work thrown on it, or from other causes, many of which remain unknown, fail to do its duty, and, as a consequence, disaster may threaten the whole. These diseases may be denominated as autogenous.

This gives us a simple etiological classification of diseases into: (1) Bacterial, (2) fungous, (3) protozoal, (4) animal parasitic, (5) intoxications, (6) traumatic, (7) autogenous.

While the above given etiological classification of diseases is admissible, it must be understood that in many instances the cause is not single, but multiple, and for this reason sharp lines of classification cannot be drawn; for instance, the greatest danger in those traumatic affections in which the traumatism itself does not cause death, lies in infection. The wound has simply provided a suitable point of entrance for the infecting agent; indeed the break in the continuity of tissue may be so slight that it is of import and danger only on account of the coincident or subsequent infection, as is true in most cases of tetanus and septicemia. Furthermore, an infectious disease, whether it originates in a traumatism or not, is markedly influenced by what we are pleased to call the idiosyncrasy of the patient, and by this we mean the peculiarities of tissue metabolism taking place in the individual at the time. A dozen men may be alike exposed to the same infection, and the infecting agent may find a suitable soil for its growth and development in two of these, while in the other ten this same agent meets with such adverse influences that it dies without producing any appreciable effects; or all may be 
infected, but with differences in degree, as is evidenced by variation in symptoms, in the length of time that the infecting agent continues to grow and develop in the body, and in the ultimate result. Every physician who has had experience in the treatment of any of the infectious diseases appreciates the importance of the personal equation in his patients. It is a fact, frequently observed clinically, and capable of experimental demonstration, that privation and exhaustion not only increase susceptibility to infectious diseases but also heighten mortality from the same.

That some neurotic affections originate from traumatism, has been abundantly demonstrated; that some are largely due to malnutrition accompanied by improper metabolism or insufficient elimination, or, in other words, are to some extent autogenous, all believe. With a clear understanding that the above classification does not attempt a sharp and marked differentiation of the causes of disease, we will give our attention to a consideration of the etiology of the infectious diseases, and of the traumatic and autogenous, in so far as these are influenced by infection.

Recognizing the fact that germs do bear a causal relation to some diseases, the question arises, how do these organisms act? Inasmuch as anthrax was the first disease demonstrated to be due to bacteria, attempts to answer this question have generally been made by reference to the microörganism of this affection, or, in other words, the question is changed so as to read, "In what way does the bacillus anthracis induce the symptoms of this disease and cause death?" Of the proposed solutions of this problem the following are the most important :

1. It was suggested by Bollinger that apoplectiform anthrax is due to deoxidation of the blood by the bacilli. These germs are aërobic, and it was thought that they might act by depriving the red blood corpuscles of their oxygen. This theory was rendered more tenable by the resemblance of the symptoms of anthrax to those of carbonic acid poisoning. The most prominent of these symptoms are dyspnœa, cyanosis, convulsions, dilated pupils, subnormal temperature, and, in general, the phenomena of asphyxia. Moreover, post-mortem examination reveals conditions similar to those observed after death by deprivation of oxygen; the veins are distended, the blood is dark and thick, the parenchymatous organs are cyanotic, and the lungs are hyperemic. Apoplectiform anthrax was compared to poisoning with hydrocyanic acid, which was at one time believed to produce fatal results by robbing the blood of its oxygen.

This theory presupposed a large number of bacilli in the blood, and this accorded with the estimate of Davaine, which placed the number at from eight to ten million in a single drop; but more extended and careful observation showed that the blood of animals dead from anthrax is often very poor in bacilli. Virchow reported 
cases of this kind and Bollinger himself found the bacilli often confined to certain organs, and not abundant in the blood. Later, Siedangrotzky counted the organisms in the blood in various cases and found not ouly that the estimate made by Davaine is too large, but that in many instances the number present in the blood is small, while Joffroy observed in some of his inoculation experiments that the animals died before any bacilli appeared in the blood. These and other investigations of similar character caused workers in this field of research to doubt the truth of the theory of Bollinger, and these doubts were soon converted into positive evidence against it ; but for a while it was the subject of an interesting controversy. Pasteur, in support of the theory, reported that birds were not susceptible to anthrax, and he accounted for this by supposing that the blood corpuscles in birds do not part with their oxygen readily. However, it was shown by Oemler and Feser that the learned Frenchman had generalized from limited data, and that many birds are especially susceptible to this disease. Oemler found that the blood, even when rich in anthrax bacilli, still possesses the bright red color of oxyhemoglobin. Toepper and Roloff reported cases of apoplectiform anthrax in which there was no difficulty in respiration, and Toussaint caused animals which had been inocnlated with the anthrax bacillus to breathe air containing a large volume of oxygen and found that this did not modify the symptoms or retard death. Finally, Nencki determined the amount of physiological oxidation going on in the bodies of animals sick with anthrax by estimating the amount of phenol excreted after the administration of one gram of benzol, and found that the oxidation of the benzol was not diminished by the disease. In short, the theory that germs destroy life by depriving the blood of its oxygen has been found not to be true for anthrax, and if not true for anthrax, certainly it cannot be for any other known disease. The bacillus anthracis is, as has been stated, aërobic, while many of the pathogenic bacteria are anaërobic - that is, they live in the absence of oxygen; this element is not necessary to their existence, and indeed, when present in large amount, it is fatal to them. Moreover, in many diseases the bacteria are not found in the blood at all, and, lastly, the symptoms of these diseases are not those of asphyxia. These facts have caused a complete abandonment of this theory.

2 . If a properly stained section of a kidney taken from a guineapig, which has been inoculated with the bacillus anthracis, be examined under the microscope, the bacilli will be found to be present in such large numbers that they form emboli, which not only close, but actually distend the capillaries and even larger blood vessels, and interfere with the normal functions of the organ. A similar condition is sometimes found on microscopical examination of the liver, spleen and lungs. From these appearances it was in- 
ferred by Bollinger that the bacilli produce the diseased condition by accumulating in large numbers in these important organs, and mechanically interrupting their functions. This is known as the mechanical interference theory.

If anthrax were the only infectious disease, or if in other infections the germs were as numerous in the blood as they are in anthrax, the mechanical interference theory would still have strong support, but to the majority of germ diseases it is not at all applicable.

3. Another answer given to this question, "How do germs cause disease?" is that they do so by consuming the proteids of the body, and thus depriving it of its sustenance. The proteids are known to be necessary for the building up of cells and it is also known that microörganisms feed upon proteids. However, this theory is untenable for several reasons : in the first place, many of the infectious diseases destroy life so quickly that the fatal effects cannot be supposed to be due to the consumption of any large amount of proteid; in the second place, the distribution of the microörganisms is such that they do not come in contact with any large proportion of the proteids of the body; in the third place, the symptoms of the majority of the bacterial diseases are not those which would be produced by withdrawing from the various organs their food. The symptoms are not those of starvation.

4. Still another theory, which has been offered, is that the bacteria destroy the blood corpuscles, or lead to their rapid disintegration. But in many of the infectious diseases, as has been stated,the microörganisms, although abundant in some organs, are not present in the blood. Moreover, the disintegration of the blood corpuscles is not confirmed by microscopical examination.

5. Seeing the vital deficiencies in the above theories, and being impressed by the results obtained by the chemical study of putrefaction, bacteriologists have been led to inquire into the possibility of the symptoms of the infectious diseases being due to chemical poisons. In investigating this theory, the following possibilities suggest themselves :

(a) The microörganisms may be intimately associated with, or may produce, a soluble chemical ferment, which by its action on the body produces the symptoms of the disease and death. At one time this theory had a number of ardent supporters, among whom might be mentioned the eminent scientist De Bary; but Pasteur proved the theory false when he filtered anthrax blood through earthen cylinders, inoculated animals with the filtrate and failed to produce any effect. Nencki made a similar demonstration when he inoculated a.two per cent. gelatin preparation with the anthrax bacillus, which liquefied the gelatin, and on standing the bacilli settled to the bottom, after which the supermatant fluid which was, 
clear and alkaline in reaction, was filtered and injected into animals without producing any effect.

It must not be inferred from the above statements that bacteria do not produce ferments. Many of them do form both diastatic and peptic ferments, which may retain their activity after the bacteria have been destroyed; but there is no proof in any case that these ferments have a causal relation to the disease. After the disease process has been inaugurated some of these ferments probably play an important part in the production of morphological changes, the nature of which will be indicated when the different diseases are discussed.

(b) The microörganisms may act either directly or indirectly as ferments, splitting up complex proteids in the tissue and producing among these split products the specific poisons which induce the characteristic symptoms of the disease, and may cause death. This theory, once quite generally held, has stimulated numerous investigations, some of which have led to important discoveries; but at present it is safe to say that among the bacterial split products formed either in artificial culture media or in the body, there is not found one which, on account of its intensity of action or from the nature of the symptoms which it produces, can be regarded as the specific cause of any one of the infectious diseases. Moreover, it has been shown that some of the most virulent germs, as for instance, the bacillus of tetanus, will grow and retain their virulence in artificial cultures made up principally of inorganic substances and containing only minute quantities of organic bodies of such simple construction that it must be admitted that the specific toxins of these microörganisms cannot result from their cleavage action.

While we are forced to conclude that no specific toxin has been found among the cleavage products of bacteria, it is well established that certain powerful poisons originate in this way, and it will come within the scope of this treatise to deal with all substances formed by the cleavage action of bacteria, both upon the constituents of artificial culture media and within the animal body.

(c) Poisons may be produced by the cellular activity of bacteria much in the same way as morphin is formed in the poppy. This theory supposes that the formation of bacterial toxins is a synthetical, rather than an analytical, process. It is now generally believed that most, if not all, of the pathogenic microörganisms consist of cell walls containing cell protoplasm, and that the specific toxin is a constituent of the protoplasm, and that its formation is one of the vital phenomena manifested by the organism in its processes of growth and multiplication. In some species the cell wall is not easily permeable and the toxin is found only within the cell; while in other species the toxin formed within the cell readily passes through the wall and diffuses through the culture media in artificial growths, or 
through the tissues, when the germ is multiplying in the animal body. In at least some species the formation of a toxin is not a phenomenon which invariably accompanies growth and multiplication. This is shown to be true by the frequently observed fact that a highly virulent germ may under certain conditions wholly lose its toxicity while it continues to vegetate most luxuriantly. It seems to be evident that certain conditions of growth, as, for instance, the nature of the medium, the temperature, the supply of oxygen, and the presence or absence of certain chemical agents, determine the amount of specific toxin formed within the cell. Most pathogenic germs find the conditions suitable for the elaboration of poisons at their optimum in the animal body, and for this reason their virulence is increased by passing successively through a series of animals. However, this is not always true, and germs may decrease in virulence by being passed through certain animals; some observers have reported the finding of certain microörganisms that invariably decrease in virulence on being transferred from one animal to another. There are many interesting questions along this line which need to be investigated much more fully than has been done up to the present time before we can speak positively conceruing them. They furnish the basis of problems that are of both scientific and practical interest. In many epidemics the specific microörganism, to which the epidemic disease is due, apparently increases for a while in virulence and then gradually seems to become less dangerous. However, a discussion of these questions would take us too far away from the subject matter just now in hand.

We will now give what appears to us, in the present state of our knowledge, a correct definition of an infectious disease :

An infectious disease arises when a specific, pathogenic microörganism, having gained admittance to the body and having found conditions favorable, grows and multiplies, and in so doing elaborates a chemical poison which induces its characteristic effects.

In the systemic infectious disease, such as anthrax, typhoid fever and cholera, the specific poison is undoubtedly taken into the general circulation, and may reach and influence every part of the body. In the local infectious diseases, such as gonorrhea and infectious ophthalmia, the first action of the poison seems to be confined to the place of its formation; although even in these, when of a specially virulent type, the effects may extend to the general health, or the poison may strongly act on some distant part of the body. It is probably true that in many of the infectious diseases the chemical poison has both a local and a systemic action; thus, it is by no means certain that the nlceration of typhoid fever is due directly to the living bacillus, for it is now an established fact that this disease may exist, run a typical course, and end in death, without anatomical changes in the intestine. In diphtheria and tetanus the toxin 
formed within the bacterial cells readily diffuses through the cell walls and enters the circulation, while the organism itself is confined to relatively a small area and may not be found in the blood at all. Such diseases are properly called bacterial intoxications. In some other infectious diseases, such as anthrax and one form of the plague, the germ itself may be distributed by the blood and lymph to every part of the body ; these diseases are designated as septicemias.

With the proof established that the deleterious effects wrought by germs are due to chemical poisons elaborated by them, let us inquire what properties a microörganism must possess before it can be said to be the specific cause of a disease. The four rules of Koch have been conceded to be sufficient to show that a given germ is the sole and efficient cause of the disease with which that germ is associated. Briefly these rules are as follows:

1. The germ must be present in all cases of that disease.

2. It must be isolated from other organisms and from all other matter found with it in the diseased animal.

3. The germs thus freed from all other foreign matter must, when properly introduced, produce the disease in healthy animals.

4. The microörganism must be found properly distributed in the animal in which the disease has been induced.

We will briefly discuss the applicability of these rules. When it is stated that the germ must be present in all cases of the disease, it need not be understood that an unlimited number of cases must be examined before the causal relation of a given organism to the disease may be reasonably suspected. This would require more than a lifetime, and would demand facilities for the study of the special disease that do not and cannot exist. The number of cases in which the germ is constantly found should be reasonably large, and the larger this number the greater the probability that the organism is etiologically related to the disease. Moreover, the germ may be present in all cases, and yet it may not be found in all. To demand that it be found in all cases would be to presume that the methods of detecting. and recognizing a given organism are perfect, and there is no ground for this assumption. Again, since the results of no one man's work can be accepted in science until they have been confirmed by others, the personal equation must be considered; what one man finds, another may fail to find. Diligence, skill and accuracy are not equally developed in all men, and, moreover, the methods employed may differ. To illustrate these points: Koch, after the most painstaking study embracing twenty-nine cases of pulmonary tuberculosis, nineteen of miliary tuberculosis, twenty-one of tuberculous glands, thirteen of tuberculous joints, ten of tuberculosis of the bones, four of lupus, and seventeen of bovine tuberculosis, announced that he had discovered a bacillus which is constantly present in tubercular disease. Since this announcement thousands of physicians and bac- 
teriologists, possessing different degrees of skill, and often by different methods of staining, with microscopes of all kinds, good and bad, have sought for this bacillus, and it is not strange that now and then some man fails to find the organism in a genuine case of tuberculosis.

Another most important point in this conuection lies in the fact that the clinical and the bacteriological diagnoses do not always agree. The most skilful clinicians may differ concerning a case of membranous sore throat. One is sure that it is diphtheria; a second is in doubt and reports it as a suspicious or doubtful case; and a third is sure that it is not diphtheria. A bacteriological examination may reveal or fail to reveal the presence of the Loeffler bacillus. Again, it may be that any number of the most competent clinicians agree in saying that the case is or is not one of diphtheria, and yet a bacteriological examination may result in a contradictory diagnosis. This is exactly what has happened in the study of diphtheria. From statistics gathered by Novy, it appears that of 8,186 eases of clinical diphtheria, diagnosed as such by different men in Europe and America (to May, 1895), the Loeffler bacillus was found in 5,943, the bacteriological examinations also being made by different men. The clinical diagnosis was confirmed bacteriologically in 72.6 per cent. of these cases. On the other hand, of 333 cases diagnosed as diphtheria by Baginsky, 332 furnished the bacillus, and of 117 seen by Kossel, all were confirmed by the bacteriological examination. These figures are given to illustrate the factors of variation that may arise in the application of the first of Koch's rules.

Shall we accept the clinical or the bacteriological classification of disease? There can be no doubt that the latter is the more exact, and its adoption will lead to a more accurate and scientific study of disease. An etiological classification of the infectious diseases is one of the great desiderata of scientific medicine. Whether it will ultimately be made upon the morphological characters of the bacteria or on their poisonous products cannot yet be determined. There are certain objections to making the first of these the basis that seem well-nigh insuperable, and some of these will be discussed later.

The importance of the first of Koch's rules is self-evident; however, the invariable presence of any germ in a certain disease does not prove that the former is the cause of the latter. Indeed, so long as the investigation goes no further than this, we are justified in saying that the microörganism may be an accompaniment or a consequence of the disease; therefore, additional evidence is wanting, and is furnished by complying with the other rules of Koch.

The second rule is complied with by means of plate and other cultures, a description of which would be out of place here.

The third and fourth rules are difficult of application, because the lower animals are often immune to many of the diseases to which man is susceptible. 
The time will probably come when the true test of the specific character of a germ will be made with the chemical products. A given bacterium may not multiply in the circulating blood of a dog, and failure to do so is by no means proof that the same organism might not cause disease in man; but every germ which canses disease in man does so by its chemical products ; and if these be isolated and injected into the dog in sufficient quantity a poisonous effect will most likely follow. In the study of the bacteria of the infectious diseases, the third and fourth of Koch's rules have not been complied with in many cases, as has been stated, on account of the insusceptibility of the lower animals. The majority of investigators, meeting with this difficulty, have been inclined to rest content with the first two rules, and to conclude that when a given germ is constantly present in a given disease and not found in other diseases, that it is the cause of the disease with which it is associated. In 1889, Vaughan suggested that in those instances in which the third and fourth of Koch's rules cannot be complied with on account of the insusceptibility of the lower animals, it should be shown that the germ can produce chemical poisons which will induce in the lower animals in an acute form the characteristic symptoms of the disease, before the proof that the given germ is the cause of the disease be accepted as positive.

The science of bacteriology has of necessity been largely founded upon morphological studies. Bacteriologists have given their time and attention to the discovery of bacterial forms in the diseased organism, and to observations of characteristics in structure and growth of different species of bacterial life. This is perfectly proper, but we must also study the physiology and chemistry of germs and until this is done we must remain ignorant of the true cause of disease, and so long as we remain ignorant of the cause it cannot be expected that we shall discover scientific and successful methods of treatment. Suppose that our knowledge of the yeast plant was limited to its form and method of growth; of how little practical importance would this knowledge be. That the yeast plant requires a saccharine soil before it can grow, that given such a soil it produces carbonic acid gas and alcohol are the most important and practical facts which have been ascertained in its study. Likewise, the conditions under which pathogenic germs multiply, and the products which they elaborate in their multiplication, must be ascertained before their true and complete relationship to disease can be understood.

In saying that the morphological work upon which the science of bacteriology largely depends is inadequate, we wish that it may be plainly understood that we are not offering any hostile criticisms upon the great men who have done this work and who have formulated conclusions thercfrom. The development of bacteriology has been in accordance with the natural law governing the growth of all 
the biological sciences. The study of form naturally and necessarily precedes the study of function. The ornithologist finds a new species of bird; he first studies its shape and size, the color of its plumage, the form of its beak, the number and arrangement of the feathers of the tail and wing, the color of the eyes, etc. All this can be done with a single specimen, recognizing the fact, however, that variations more or less marked are likely to be found in other individuals. More time and wider opportunities for observation will be needed before he can tell where and when this bird is accustomed to build its nest, upon what insects, grains and berries it feeds, with what other species of birds it lives in peace, and with what it is at war. A much greater range of observation and study is necessary before the naturalist can tell how his newly discovered species would thrive if carried to a new climate where it would be compelled to live upon unaccustomed food, to build its nest of strange material, and to encounter new foes.

We repeat that it is no discredit to the science nor to those who have developed it to say that the study of bacteriology has largely been morphological. Without the morphologist, the physiologist and the physiological chemist could not exist. The science having had for its support largely morphological studies, the deductions and formulated statements arrived at by its students have been, reached in accordance with the knowledge obtained from this source; but since it has been admitted that the causal relationship between a given germ and a certain disease is dependent upon the chemical products of the growth of the germ, the fundamental lines of work must be made to correspond with this knowledge.

We may briefly enumerate some of the problems that now lie before us in our attempts to study the chemical factors in the causation of the bacterial diseases. In the first place, it will be desirable to define and classify the chemical products of bacterial activity. In doing this it will be desirable at all times to distinguish between those substances which have their origin in the cleavage action of bacteria and those which are formed by synthetical processes within the cell; but our knowledge upon this subject is not as yet sufficiently advanced, nor does it possess that degree of accuracy necessary before we can determine positively the manner of formation of these substances. In defining and classifying the chemical products of bacterial activity we will find quite naturally that there is not complete agreement among those who have worked in this field of science. Every attempt at the classification of bacterial products must at present be regarded as provisional and subject to such alterations as future discoveries may indicate. It will also be found that it is not possible in all cases to distinguish between poisonous and harmless bacterial products. This subject will be discussed more in detail later. 
In studying bacterial products it will be necessary for us to consider the conditions under which they are formed. We will be compelled to study those substances formed in artificial cultures as well as those formed in the animal body. Indeed, in some instances we will find our knowledge confined to investigations made under more or less artificial conditions, and it will be well for us to remember that caution must be exercised in drawing conclusions from knowledge thus obtained. It might be well at this place to distinguish between pathogenic and toxicogenic bacteria. A pathogenic bacterium is one which, as the name implies, induces a specific disease which is recognized by more or less well-marked and characteristic symptoms during life, or by more or less definite lesions found after death, or by both. Pathogenic bacteria are always capable of growth in the animal body, in which they multiply and elaborate their specific toxins. All pathogenic germs are toxicogenic, but it does not follow that all toxicogenic bacteria are pathogenic. A toxicogenic microörganism is one which, as this name also implies, is capable of producing a poison or poisons. A toxicogenic germ may or may not be capable of growth in the animal body. It may multiply in milk or some other article of food before its introduction into the body, and may in this menstruum elaborate more or less powerful poisons.

In the second place we will proceed to give a brief historical sketch of the discovery and study of the bacterial poisons; and, thirdly, we will pass on to a discussion of the special products of the specific pathogenic bacteria. In doing this we will not confine ourselves to the chemistry of toxins but will extend our discussion to their action on animals and the pathological changes induced by them.

After discussing the subjects mentioned above, we will proceed to the study of those conditions of the animal body which influence the growth and development of bacteria in the same. This will involve a study of the germicidal constituents of the blood, the agglutinating action of blood serum, the production and action of the toxins, the production of immunity and the treatment of the infectious diseases by serum therapy.

On the conclusion of the above-mentioned studies we will investigate questions pertaining to poisonous foods which owe their harmful properties to the presence of toxicogenic bacteria. In this connection we will present the known facts concerning food poisons and the methods of their detection. 


\section{CHAPTER II.}

\section{CLASSIFICATION AND DEFINITION OF THE CHEMICAL PRODUCTS OF BACTERIA.}

Ptomains. - While an exact classification of the chemical products of bacterial growth cannot be made at present, we know of two distinct classes, one of which contains substances which combine with acids forming salts, and which in this respect at least corresponds with the inorganic and vegetable bases. The members of this class are designated as ptomains, a name suggested by the Italian toxicologist Selmi, and derived from the Greek word $\pi \tau \omega \mu \alpha$ meaning a cadaver. A ptomaïn may be defined as an organic chemical compound, basic in character, and formed by the action of bacteria on nitrogenous matter. On account of their basic properties, in which they resemble the vegetable alkaloids, ptomains may be called putrefactive alkaloids. They have also been designated as animal alkaloids, but this is a misnomer, hecause, in the first place, some of them are formed in the putrefaction of vegetable matter; and, in the second place, the term " animal alkaloid" is more properly restricted to the leucomaïns-those basic substances which result from tissue metabolism in the body. Kobert employs the term ptomatin as etymologically preferable to ptomaïn.

While some of the ptomains are highly poisonous, this is not an essential property, and others are wholly inert. Indeed, the greater number of those which have been isolated do not, when employed in single doses, produce any apparent harmful effects. Brieger has suggested that the term ptomain be restricted to the non-poisonous basic products, and he designates the poisonous ones as "toxins"; however, such a classification would be of questionable utility. It will be necessary for us to possess more extensive and exact knowledge concerning the action of many of these substances before we can decide positively which are poisonous and which are not. The effect of a chemical compound upon the animal body depends upon the conditions under which, and the time during which it is administered. Thirty grains of quinine may be taken by a healthy man during twenty-four hours without any appreciable ill effect, yet few of us would be willing to admit that the administration of this amount daily for months would be wise or altogether free from injury. In the same manner, the administration of a given quantity of a bacterial alkaloid to a dog or a guinea-pig in a single dose may do no harm, while the daily production of the same substance in the intestine of a 
man and its absorption, continued through weeks and possibly years, may be of marked detriment to the health. We are not as yet in possession of sufficient knowledge concerning the physiological or toxicological action of the putrefactive alkaloids to render the classification proposed by Brieger worthy of general adoption; moreover, the term "toxin" is quite generally, although somewhat incorrectly, employed to designate those non-basic bacterial poisons for which Brieger suggested the name "toxalbumin."

All ptomaïns contain nitrogen as an essential part of their basic character. In this they resemble the vegetable alkaloids. Some of them contain oxygen, while others do not. The latter correspond to the volatile vegetable alkaloids, nicotin and coniin, and the former correspond to the fixed alkaloids.

The kind of ptomaïn formed in a given case will depend upon the individual bacterium engaged in its production, the nature of the material acted upon, and the conditions under which the bacterial growth proceeds, such as the temperature, the amount of oxygen present, and the duration of the process. For instance, Brieger found that although the Eberth bacillus grows well in solutions of pepton, it does not produce any ptomains; while the same bacillus growing in beef tea elaborates a poisonous alkaloid. Fitz found that while the bacillus butyricus produces by its action on carbohydrates butyric acid, in glycerin it forms propylic alcohol ; and Morin has detected amylic alcohol among the products of this germ. Brown has shown that while the mycoderma aceti converts ethylic alcohol into acetic acid, it changes propylic alcohol into propionic acid, and is without effect upon methylic alcohol, primary isobutylic alcohol, and amylic alcohol. Some bacteria will not multiply below a given temperature; thus, the bacillus butyricus will not grow below $24^{\circ} .1$

The influence of the presence or absence of certain chemical compounds upon the activity of unicellular forms of life has long been known to be great. Liebig and Knapp observed that the presence of small quantities of the chlorids of sodium and potassium intensifies the action of yeast in the decomposition of sugar, and Dubrumfaut made quantitative determinations illustrating the effects of the presence of other salts. He found that 0.5 gram of yeast in 100 c.c. of a 10 per cent. solution of sugar decomposed within a given time 0.5 gram of sugar, while in another flask to which 0.5 gram of sodium acid sulphate had been added, the same amount of yeast decomposed 0.52 gram of sugar, and the same amount of potassium sulphate in a second flask increased the activity of the yeast to 0.88 gram of sugar, and in a third flask potassium nitrate raised the quantity of sugar decomposed to 1 gram. Pasteur divided bacteria into two classes-the aërobic and the anaërobic. As these names imply, the former grow

${ }^{1}$ All temperatures given in this work are centigrade, unless otherwise specified 
and thrive in the presence of air, while the latter find their conditions of life improved by the exclusion of air; therefore, different products will be formed in decomposing matter freely exposed to the air, and in that which is buried beneath the soil or from which the air is otherwise largely excluded. Ignorance of this fact has led to some serious mistakes in toxicological work, as we shall see further on.

It is more than probable that most, if not all, ptomaïns result from the cleavage action of bacteria on the constituents of the medium in which they grow, and it follows from this that the kind of ptomain found in a given case will depend upon the stage of the bacterial disintegration of the constituents of the medium. Regarded as the results of cleavage action, ptomains are transition products in the processes of bacterial decomposition. They are temporary forms through which matter passes while it is being changed, by the activity of bacterial life, from the organic to the inorganic state. Complex organic substances, as muscle and brain, are broken up into less complex molecules, and thus the process of chemical division goes on until the simple and well-known final products, carbonic acid gas, ammonia and water, result; but the variety of combinations into which an individual atom of carbon may enter during this long series of changes is almost unlimited, and with each change in combination there is more or less alteration in nature. In one compound the atom of carbon may exist as a constituent of a highly poisonous substance, while the next combination into which it enters may be wholly inert.

It was formerly supposed that putrefaction was simply oxidation, but the researches of Pasteur and others have demonstrated the fact that countless myriads of microörganisms are engaged constantly in transforming matter from the organic to the inorganic form. If a bit of flesh be locked up so that these little workers cannot reach it, it will remain unchanged indefinitely. It may be asked if any of the changes occurring during putrefaction are to be regarded as purely chemical. Without doubt, many of the secondary products of putrefaction arise from reactions between antecedent and more complex substances, or by the action of oxygen, water, and reducing agents upon primary products. Ptomaïns formed in this way may be regarded as the indirect results of bacterial life.

While some of the basic substances formed by bacterial growth are intensely poisonous, it is not probable that any ptomain can be regarded as the most potent product of one of the pathogenic bacteria. It follows, therefore, that we must seek for the specific poisons of the infectious diseases among other chemical compounds produced by bacterial growth.

Bacterial Proteids.-Our knowledge concerning these substances still remains quite imperfect and many difficulties arise in discussing 
them. We may divide the bacterial proteids into two classes: (1) Those which constitute an integral part of the bacterial cells; and (2) those which have not been assimilated by the cells, but which have been formed by the fermentative or cleavage action of the bacteria on the proteid bodies in which they are growing. This classification, however, is of questionable value. We allow bacteria to grow for a number of days in a nutrient solution. We then separate the soluble constituents from the formed cells by filtration through porous tiles; we wash the latter and then study their proteid contents; but a considerable proportion of the contents of the living cells has already passed into solution, and the bacterial detritus left on the filter gives no exact knowledge of the constituents of the living cells. Moreover, the living cells absorb and excrete, and we are not yet able to distinguish with certainty between those substances formed within the cell and those preëxisting in the culture medium. The filtrate contains, or may contain, any one or more of the following proteid bodies : (1) Those portions of the proteid substance which were used in the preparation of the nutrient solution and which have escaped the action of bacteria; (2) proteids which have at one time been integral parts of the cells, but which have passed into solution on the death and dissolution of the bacteria ; and, (3) proteids which have been formed by the fermentative action of the bacteria, or those which are defined as constituting the second class, as given above. Attempts are now being made (1901), by growing bacteria on solid culture media extensively to distinguish with certainty the proteid constituents of the cell from those of the culture medium ; but at the present writing this work has not progressed sufficiently for us to make any positive statements concerning its results.

It is now quite certain that none of the proteids formed by the cleavage action of bacteria on the normal constituents of culture media, or on those of the animal body, are specific factors in the production of disease. It is true that many bacteria peptonize proteids and the specific poisons of certain infectious diseases have been sought for among the peptons; but there is no evidence that these are more poisonous than those formed by the gastric juice. The specific bacterial poisons are formed by synthetical rather than by analytical processes and they are constituents of the protoplasm enclosed in the cell wall.

The Bacterial Cellular Proteids.-Nencki was the first to attempt to study the chemistry of the bacterial cell. His experiments were made with putrefactive bacteria, which were obtained by decantation of liquid cultures, freed from fat with ether, dissolved in 50 parts of a potash solution of 0.5 per cent., heated for some hours at $100^{\circ}$ and filtered. The filtrate was acidified with dilute hydro- 
chloric acid and precipitated by the addition of rock salt, after which the precipitate was washed with saturated salt solution, dried at $100^{\circ}$, and freed from salt by washing with water. Nencki designates this substance as "mycoprotein," and finds that it has the formula $\mathrm{C}_{25} \mathrm{H}_{42} \mathrm{~N}_{6} \mathrm{O}_{3}$. Freshly prepared mycoprotein forms amorphous flakes, which are soluble in water, alkalis and acids. The aqueous solution is acid in reaction. After being dried at $100^{\circ}$ it is no longer wholly soluble in water. Nencki found that it is not precipitated from aqueous solution by alcohol, but by picric acid, tannic acid and mercuric chlorid; that it does not give the xanthoproteic, but does give the Millon and the biuret reactions. According to Schäffer, it is changed by acids into peptons, and on being fused with five parts of potash it breaks up into ammonia, amylamin, phenol ( 0.15 per cent. of its weight), valerianic acid (38 per cent.), leucin and traces of indol and skatol. A proteid obtained from the yeast plant has the formula $\mathrm{C}_{12} \mathrm{H}_{21} \mathrm{~N}_{3} \mathrm{O}_{3}$. It should be understood that these formulæ are of but little value inasmuch as chemically pure bodies were not secured.

The pyogenetic substance obtained from the pneumonia bacillus of Friedlander was found by Buchner to give the following reactions: It is soluble in water and the concentrated mineral acids, very soluble in dilute alkalis, from which it is precipitated on the addition of an acid. From its aqueous solution it is not precipitated by heat, nor by saturation with sodium chlorid, but is precipitated by magnesium sulphate, copper sulphate, platinum chlorid, gold chlorid, lead salts, picric acid, tannic acid, and absolute alcohol. It gives the xanthoproteic, Millon and biuret reactions.

In old bouillon cultures, the dead, disintegrated bodies of the bacteria form a sediment. It is not at all probable that an analysis of this sediment represents fairly the constituents of the living germs. During the disintegration certain constituents of the cell pass into solution, and these soluble substances are probably the most important parts of the bacterial cells, so far as they are concerned in the causation of disease. Similar processes undoubtedly occur in the body of an animal infected with a pathogenic organism and it is generally believed that these soluble substances cause the symptoms of the disease and death.

Toxins.-When it became known that some of the specific pathogenic germs elaborate both in artificial cultures and in susceptible animals poisonous basic substances or ptomaïns, it was surmised that the symptoms of the disease induced by the microörganisms were due, in all cases, to specific basic poisons, and chemists labored diligently to isolate from cultures of each germ its specific toxic products. These labors soon led to the recognition of the fact that the abovementioned surmise had been hastily drawn. It was found to be true 
that the symptoms of each and every individual disease investigated are due to the chemical products elaborated by the activity of the germ, but these chemical products are not, in the majority of these diseases, basic in character, and consequently they cannot be classed among the ptomaïns. Brieger succeeded in isolating from pure cultures of the tetanus bacillus as many as four ptomains, but the poisonous effects induced by these substances are not comparable in violence with those which follow injection of tetanus cultures from which the bacillus has been removed by filtration. The fact that the filtered culture is more poisonous than any or all of its basic contents necessitates the conclusion that the culture contains some more active constituent.

What is the nature of the powerful poisons that are formed in cultures of the bacteria of tetanus, diphtheria, tuberculosis, typhoid fever, anthrax, and other infectious diseases, and which are also formed in the bodies of animals infected with these microörganisms? At present no positive and satisfactory answer can be made to this question. At one time Roux and Yersin thought that it might be a ferment, and Brieger and Fraenkel advanced the belief that the diphtheria poison is an albuminous body; however, there are weighty objections to each of these theories, and up to the present time all attempts to isolate the specific toxins have proved unsuccessful. In 1891 Freer made an ultimate analysis of a toxin obtained by Vaughan from a toxicogenic germ found in drinking water, and obtained the following results: Carbon, 48.46 per cent.; hydrogen, 7.69 per cent.; nitrogen, 13.44 per cent.; phosphorus, 0.69 per cent. Sulphur was absent. The absence of sulphur and the very small per cent. of phosphorus were supposed at that time to indicate that the toxin was nearly pure. In 1893, Brieger and Cohn found that the tetan us toxin, in the purest form in which they could obtain it, contained no phosphorus, and only unweighable traces of sulphur, and they attributed the presence of the latter element to contamination with ammonium sulphate, which was used in precipitation. Later (1895), Brieger made an ultimate analysis of the tetanus toxin, so far purified that $0.000,000,05$ gram killed mice, with the following results : Carbon, 52.8 per cent. ; hydrogen, 8.1 per cent. ; nitrogen, 15.71 per cent. This purified toxin is not precipitated by ammonium sulphate, and it gives the biuret reaction so imperfectly that Brieger felt justified in saying that the coloration is not due to the toxin, but to proteid impurities.

Uschinsky has made an important contribution to our knowledge of the toxins, inasmuch as he has demonstrated that these substances are not split products, formed by the action of bacteria on proteids preëxisting in the culture media, but are synthetical products, and are formed when the germs are grown in culture media containing no proteids. He sueceeded in growing a number of pathogenic 
microörganisms, including those of cholera, diphtheria, tetanus and typhoid fever, in the following menstruum :
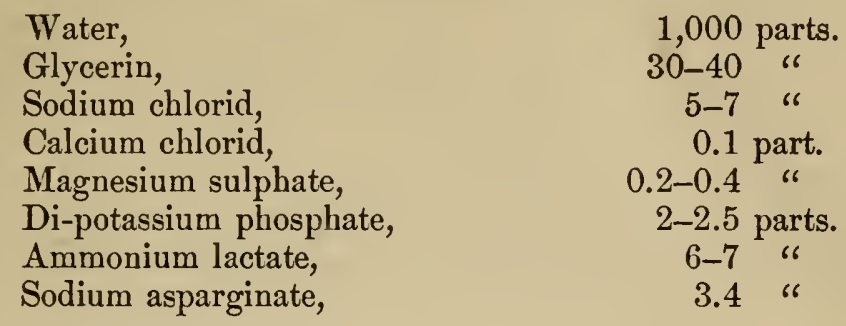

From these cultures he obtained toxins which were not less virulent than those formed in ordinary beef-tea, and thus demonstrated that their production is due to synthetical processes.

Ehrlich, ${ }^{1}$ as the result of a long series of experiments made upon animals with toxins and antitoxins, has formulated the following conclusions concerning the constitution of the diphtheria toxin :

1. The diphtheria bacillus produces two kinds of substances: $(a)$ toxins, $(b)$ toxons, both of which combine with anti-bodies. In fresh bouillon cultures toxins and toxons exist in the same proportions.

2. The toxins (and also the toxons) are not simple bodies, but easily split up into other substances which differ from one another in the avidity with which they combine with antitoxins. Among these derivatives there are found prototoxins, deuterotoxins and tritotoxins, which are here mentioned in the order of the readiness with which they combine with antitoxins, the tritotoxins combining least readily, but all of these combine with antitoxins more readily than do the toxons.

3. The complication does not stop with this division, but it is probable that every toxin cousists of equal parts of two modifications, which behave with antitoxins in the same manner, but which differ in their resistance to destructive influences; probably this difference is comparable to that existing between dextro-rotatory and levo-rotatory forms of the same compound.

4. Of these two modifications, one, which may be designated as the $\alpha$-modification, readily passes into a toxoid. Indeed, this transformation begins and frequently reaches completion in the culture medium in the incubator. The complete conversion of the $\alpha$-modification into a toxoid leaves the substance with only half of its original toxicity, and it may therefore be called a hemitoxin.

5 . The second modification, which is designated as the $\beta$-modification, varies in stability in the different kinds of poisons, the prototoxins, the deuterotoxins and the tritotoxins. The $\beta$-tritotoxin is relatively unstable and often undergoes decomposition in the incu-

1 Deutsche med. Wochenschrift, September 22, 1898. 
bator. The $\beta$-prototoxin is much more stable, but gradually decomposes and is converted into a toxoid when the culture is kept for several months. The $\beta$-modification of the deuterotoxin is under certain conditions quite stable. These facts explain the observation, previously reported, that old cultures of diphtheria gradually decrease in toxicity until a certain minimum degree is reached, after which there is no further decrease.

6. Avidity for combining with antitoxins does not suffer the least change on the conversion of a toxin into a toxoid ; for instance, the toxoid of prototoxin combines with antitoxin as readily as does the prototoxin itself.

7. Those varieties of poisons which combine less vigorously with antitoxins are neutralized by the same reagent less promptly; this explains the observation that certain poisons of the tetanus series (tetanolysin and tetanospasmin) are neutralized promptly by antitoxin only in concentrated solution.

8. The facts observed in the experiments made upon animals with toxins and antitoxins are best explained on the supposition that the toxic molecule contains two independent groups of atoms, one of which may be designated as the haptophorous and the other as the toxophorous group. It is by the action of the haptophorous group that compounds are formed with antitoxins, while the poisonous action is due to the toxophorous group. The toxons also possess these two groups and in these bodies the haptophorous groups are identical with those of the toxins, while the toxophorous groups are much more feeble in their effects.

9. The effects of the haptophorous and the toxophorous groups can in certain cases be experimentally distinguished ; for instance, Morgenroth found that when frogs were kept in the cold and successively injected with toxin and antitoxin, these animals showed no evidences of the disease, although the tetanus poison could be detected in their uerves. On the other hand, when frogs were treated at like intervals with toxin and antitoxin and kept in an incubator, they succumbed to tetanus even when all of the poison in the blood was combined with antitoxin and an excess of the latter was present. This indicates that the haptophorous group combined with the cells in the cold, while the toxophorous group manifested its effects only when the animal was kept in a warm place. The difference in time required for the action of these two groups possibly explains the incubation period which is observed in all cases of poisoning with bacterial toxins; for Dönitz has shown that the tetanus poison combines almost instantaneously with the nerve cells.

10. The toxophorous group is more complicated in structure and therefore less stable than the haptophorous. The relative lability of the toxophorous group and the stability of the haptophorous group explain the quantitative conversion of toxins into toxoids. In a 
structure so complicated it is easy to conceive of an asymmetrical atomic arrangement of the toxophorous complex, and this is more easily comprehensible on the assumption of the existence of the two modifications ( $\alpha$ and $\beta$ ) in the same amounts.

11. The haptophorous group, which under ordinary conditions is quite stable, can be broken down by the action of strong chemicals and certain physical agents (heat, iodin, etc.).

12. The anti-bodies combine exclusively with the haptophorous group and thus absorb the toxic molecule, so protecting the organism. In this way the poison may be rendered harmless without destruction of the toxophorous complex.

13. It follows from what has been said that specific antitoxins can be produced not only with toxins, but also with toxoids; indeed, highly susceptible animals (mice and guinea-pigs), can be immunized against the tetanus toxin safely and easily by means of toxoids. It is probable that in this case the degree of immunity cannot be made so great as is desirable in the preparation of a curative serum. However, it is possible that immunization with toxoids can be used directly for curative purposes, inasmuch as by this means an active immunity may be brought into existence.

14. In natural immunity, also in that form which is induced by the bacterium itself and not by the bacterial poison, the toxons probably play a very important rôle; while the toxoids do not exist in these cases since they result only from the decomposition of toxins. It is also probable that the artificial immunity which may be induced by the simultaneous employment of an antitoxic serum and living bacteria (as in rinderpest and swine erysipelas), and which is quite lasting and is produced without marked disturbances of health, is in great part due to the action of toxons.

As has already been stated, the above-mentioned theory concerning the constitution of toxins is founded upon experimental studies of the action of toxins and antitoxins in the animal body, and does not rest upon chemical experimentation; indeed, Ehrlich expresses the opinion that many decades must pass before we shall be able to isolate the toxin chemically. We shall have opportunity later on in our studies of toxins, antitoxins and immunity, to return to Ehrlich's theory. ${ }^{1}$ 


\section{CHAPTER III.}

\section{A HISTORICAL SKETCH OF THE BACTERIAL POISONS.}

THE history of man's experiments with, and his investigations of, the bacterial poisons quite naturally divides itself into three periods. During the first period man was ignorant of the existence of bacteria and of the rôle that they play in processes of putrefaction, as well as in the causation of disease, and in his experiments he did not always adopt measures which are necessary for the destruction of living microörganisms; consequently the results obtained were in some instances due wholly to infection, in others to bacterial chemical poisons, while in still others both the toxin and the bacterium probably had some influence in causing the phenomena which he observed. In the second period man recognized the existence of bacteria and made his experiments with sterilized products. But he had not at that time secured the information necessary to enable him

to isolate and classify the microörganisms ; therefore, the toxins with which he worked resulted, as a rule, at least, from the growth of mixed cultures of germs, the characteristics of which he did not know. Indeed, most of the experiments of the time were made with sterilized substances obtained from putrefying material. The third period was reached when the experimenter worked with pure cultures and for the first time was able to proceed scientifically in his investigations.

It must have been known to primitive man that the eating of putrid flesh was liable to be followed by more or less harmful effects, and when he began his endeavors to preserve his food for future use, instances of poisoning from putrefaction must have multiplied. However, the distinguished physiologist, Albert von Haller, seems to have been the first to make any scientific experiments concerning the action of putrid material upon animals. He injected aqueous extracts of decomposing flesh into the veins of various animals and found that death frequently resulted. Later in the eighteenth century, Morand gave an account of the symptoms induced in man by eating putrid meat. In the early part of the nineteenth century (1808-1814) Gaspard carried on similar experiments. His studies were made with the putrefied flesh of both carnivorous and herbivorous animals. With these he induced marked nervous disturbances, as stiffness of the limbs, opisthotonos and tetanus, and he concluded from the symptoms thus induced that the poisonous effects were not due to carbonic acid gas or hydrogen sulphid, but thought it possible that 
ammonia might have part in their production. In 1820 , Kerner published his first essay on poisonous sausage, and this was followed by a second in 1822. At that time he thought that the untoward symptoms induced by eating poisonous sansage were due to a fatty acid, similar to the sebacic acid of Thenard, and which originated during putrefaction; later, he modified these views and suggested that the poison might be a compound consisting of sebacic acid and a volatile principle. This may be regarded as the first suggestion as to the probability of a poisonous substance with basic properties in decomposing matter. In 1822 Dupré observed a peculiar disease among the soldiers under his care, who, during the warm and dry summer of that year, were compelled to drink foul water ; the disease thus induced was probably due to infection rather than to intoxication. Later, Magendie, stimulated by the investigations of Gaspard and the observations of Dupré, made many experiments, in which dogs and other animals were confined over vessels containing putrid animal matter and compelled constantly to breathe the emanations therefrom. The effects varied markedly with the species of animal and the nature of the putrid material, but in some instances symptoms closely resembling those of typhoid fever in man were induced. It might be suggested here parenthetically that a repetition of the experiments of Magendie, with such precautions as modern methods would suggest, wonld not be without value. Leuret directed his attention to the chemical changes produced in blood by putrefaction, but accomplished nothing of special value. Dupuy injected putrid material into the jugular vein of a horse and with Trousseau studied alterations produced in the blood by these injections.

During the third decade of the nineteenth century there were many investigators, in addition to those mentioned above, who endeavored to ascertain the active agent in poisonous foods. Dann, Weiss, Buchner, Schumann, Cadet de Gassicourt, and Orfila studied poisonous sausage, but made no advance beyond the work done by Kerner. Henneman, Hünnefeld, Westrumb, and Sertürner (the discoverer of morphin) made contributions concerning poisonous cheese, but all reached the conclusion that the caseic acid of Kerner is the poisonous principle.

In 1850 , Schmidt, of Dorpat, studied the decomposition products and volatile substances found in cholera stools, and two years later, Meyer, of Berlin, injected the blood and stools of cholera patients into lower animals. In 1853 , Stich made an important contribution on the effects of acute poisoning with putrid material ; he ascertained that, when given in sufficient quantity, putrid material produced an intestinal catarrh, with choleraic stools. Nervous symptoms, trembling, unsteady gait, and, finally, convulsions, were also observed, and careful post-mortem examinations were made, but no important or 
characteristic lesions were found. He concluded that the putrid material contained a ferment which induced rapid decomposition of the blood.

'The distinguished Danish physiologist, Panum, was the first to demonstrate positively the chemical character of the poison formed in putrid flesh, inasmuch as he showed that the aqueous extract of such material retained its poisonous properties after treatment which insured the destruction of all living organisms. Panum's first paper was published in 1856 and his conclusions are stated as follows:

1. "The putrid poison contained in the decomposed flesh of the $\mathrm{dog}$, and which is obtained by extraction with distilled water and repeated filtration, is not volatile, but fixed. It does not pass over on distillation, but remains in the retort.

2. "The putrid poison is not destroyed by boiling, nor by evaporation. It preserves its poisonous properties even after the boiling has been continued for eleven hours, and after the evaporation has been carried to complete desiccation at $100^{\circ}$.

3. "The putrid poison is insoluble in absolute alcohol, but is soluble in water, and is contained in the aqueous extract which is formed by treating with distilled water the putrid material which has been previously dried by heat and washed with alcohol.

4. "The albuminoid substances which frequently are found in putrid fluids are not in themselves poisonous, only so far as they contain the putrid poison fixed and condensed upon their surfaces, from which it can be removed by repeated and careful washing.

5. "The intensity of the putrid poison is comparable to that of the venom of serpents, of curare, and of certain vegetable alkaloids, inasmuch as 0.012 of a gram of the poison, obtained by extracting with distilled water putrid material which had been previously boiled for a long time, dried at $100^{\circ}$, and submitted to the action of absolute alcohol, was sufficient to almost kill a small dog."

Panum made intravenous injections with this substance, and with ammonium carbonate, ammonium butyrate, ammonium valerianate, tyrosin, and leucin, and found that the symptoms induced by the putrid poison differed from those caused by the other agents. Moreover, he found the symptoms induced by the poison to differ from those of typhoid fever, cholera, pyemia, anthrax, and sausage poisoning. He was in doubt whether the poison acted directly upon the nervous system or as a ferment upon the blood, causing decomposition, the products of which might affect the nervous centers; but he was sure that his new substance did not correspond to the ordinary ferments, inasmuch as it was not decomposed by prolonged boiling nor by treatment with absolute alcohol. Certainly, Panum's putrid poison did not consist of living organisms:

The symptoms observed by Panum varied greatly with the quantity of the poison used and the resistance of the animal experi- 
mented upon. After the intravenous injection of large doses, death followed speedily. In these cases, there were violent cramps and involuntary evacuations of the urine and feces; the respirations were labored; pallor was marked, sometimes followed by cyanosis; the pulse was feeble; the pupils were widely dilated, and the eyes projected. Autopsy did not reveal any lesion, save that the blood was dark and imperfectly coagulated, and slightly disseminated through the tissues. Post-mortem putrefaction came on with extraordinary rapidity.

When smaller doses or more vigorous animals were used, the symptoms did not appear before from a quarter of an hour to two hours, sometimes even later, and were not violent, generally terminating in recovery.

In addition to the "putrid poison," Panum obtained a narcotic substance, the two being separated by the solubility of the latter in alcohol. The alcoholic extract was evaporated to dryness, the residue dissolved in water and injected into the jugular vein of a dog. The animal fell into a deep sleep, which remained unbroken for twenty-four hours, when it awoke apparently in perfect health. This account of the finding of a narcotic substance in putrefying flesh is rendered more interesting by the later researches of Bouchard, who has shown that normal urine contains a body of like action, and this also can be extracted with alcohol from the residue obtained by evaporation.

Weber, in 1864, and Hemmer and Schwenninger, in 1866, confirmed the results obtained by Panum, and Schwenninger announced that different products are formed in the various stages of putrefaction and that these are unlike in their effects upon animals. In 1866, Bence Jones and Dupré obtained from the liver a substance which in solutions of dilute sulphuric acid gave the blue fluorescence observed in similar solutions of quinin and to which they gave the name "animal chinoidin." Subsequently, the same investigators found this substance in all organs and tissues of the body, but most abundantly in the nerves. These observations have been confirmed by others, and solutions showing this fluorescence give precipitates with the general alkaloidal reagents, but no one has as yet succeeded in isolating the basic substance supposed to be present. Indeed, it is not positively known that the body to which this reaction is due is a bacterial product, although this is the most likely assumption. It has been suggested that its presence might be due to the growth of certain fluorescing bacteria.

In 1868, Bergmann and Schmiedeberg reported that they had obtained from putrid yeast and from decomposed blood a poisonous substance in the form of a sulphate to which they gave the name "sepsin." According to these observers the sulphate of "sepsin" forms in needle-shaped crystals, and small doses (0.01 gram), dis- 
solved in water and injected intravenously into dogs, cause vomiting and bloody diarrhœa; while post-mortem examination shows ecchymoses in the stomach and intestines. It was then believed that the "putrid poison" of Panum had been isolated, and that it was identical with "sepsin." However, further investigations showed that this was not true, and it is by no means certain that the poisonous effects obtained by Bergmann and Schmiedeberg were due to the crystals seen by them in their preparation, inasmuch as these crystals were never completely isolated.

Recently Levy, working under Schmiedeberg's directions, has made further study of putrid yeast, in which he found bacilli resembling those of mouse septicemia and the proteus vulgaris. Dogs were treated with intravenous injections of liquefied gelatin cultures of the proteus, and symptoms similar to those formerly attributed to "sepsin" followed. The cultures were precipitated with absolute alcohol, and the resulting albuminous precipitate caused the same symptoms as the cultures in mice, rabbits and dogs. Levy also investigated cases of meat poisoning due to infection with the proteus. The keeper of a restaurant and some of his guests suffered from a most violent purging, which in the case of the host terminated fatally. In the vomited matter, in the stools, and in the bottom of the ice-box in which the meat was kept, the proteus was found, and cultures of it produced the symptoms of sepsin poisoning in animals. Levy concludes that the proteus is the generator of sepsin. If this conclusion be correct, it follows that the effects observed by Bergmann and Schmiedeberg were not due to the crystals obtained by them, but to other substances from which the crystals had not been separated.

In 1869 Zülzer and Sonnenschein obtained from decomposing meat extracts a nitrogenous base which, both in its chemical reactions and physiological effects, resembles atropin and hyoscyamin. When injected under the skin of animals this substance produces dilatation of the pupils, paralysis of the muscles of the intestines, and acceleration of the heart beat; but its action is uncertain and inconstant. A similar substance has been obtained from the bodies of those who have died from typhoid fever, and it is possible that the belladonna-like delirium which frequently characterizes the later stage of this disease is due to the ante-mortem generation of this or a similar poison within the body.

During the eighth decade of the nineteenth century certain toxicologists became interested in the alkaloidal reactions obtained sometimes in putrefying tissue. The most prominent of these was the Italian, Selmi, who suggested the name "ptomaïn," and whose researches furnished us with valuable information, and, what is probably of more importance, gave an impetus to the study of the chemistry of putrefactive changes, which has been productive of 
much good. Selmi showed that ptomaïns could be obtained (1) by extracting acidified solutions of putrid material with ether; (2) by extracting alkaline solutions with ether; (3) by extracting alkaline solutions with chloroform; (4) by extracting alkaline solutions with amylic alcohol; and (5), that there yet remain in the solutions of putrid matter ptomaïns that are not extracted by any of the abovementioned reagents. In this way he gave some idea of the great number of alkaloidal bodies which might be formed among the products of putrefaction, and the promising field thus discovered and outlined was soon occupied by a busy host of chemists. In the second place, he demonstrated the fact that many of the ptomains give reactions similar to those observed with the vegetable alkaloids. This led the toxicologist into investigations, the results of some of which we will ascertain further on. Selmi did not succeed in isolating any putrefactive alkaloid, and all his physiological experiments and chemical reactions were made with extracts. He remained ignorant, except in a general way, of the composition of these bodies. Nencki, in 1876, made the first ultimate analysis and determined the first formula of a ptomaïn; this was an isomer of collidin, which will be described later.

Rörsch and Fassbender, in a case of suspected poisoning, obtained a liquid which could be extracted from acid as well as alkaline solutions by ether, and which gave all the general alkaloidal reactions; but they were unable to crystallize and isolate it. They believed that this substance was derived from the liver, since fresh ox liver treated in the same manner gave them an alkaloid which could be extracted from ether as well as from acid or alkaline solution. In some of its reactions this substance resembles digitalin. Gunning found a similar body in liver sausage from which poisoning had occurred.

Schwanert, while examining the decomposing intestines, liver and spleen of a child that had died suddenly, perceived a peculiar odor and obtained by the Stas-Otto method (ether extract from alkaline solution) small quantities of a base which was distinguished from nicotin and coniïn by its greater volatility and its odor. He supposed that this substance was produced by decomposition, and in order to ascertain the truth of this supposition, he took the organs of a cadaver that had lain for sixteen days at a temperature of $30^{\circ}$ and was well decomposed. These were treated with tartaric acid and alcohol, and the acid solution was first extracted with ether but yielded no result; it was then rendered alkaline and extracted with ether. The latter extract gave, on evaporation, the same substance which had been found in the organs of the child. The residue was a yellowish oil, having an odor somewhat similar to propylamin. It was repulsive, but not bitter to the taste, and alkaline in reaction. On the addition of hydrochloric acid it crystallized in white needles, which were freely soluble in water, but soluble with difficulty in 
alcohol. On the addition of ammonium hydrate to this crystalline substance, a white vapor of unpleasant odor was given off. When dissolved in sulphuric acid the crystals formed a solution which was at first colorless, but which gradually became dirty brownish-yellow, and grayish-brown on the application of heat. On being warmed with sodium molybdate, a splendid blue color, becoming gradually gray, was produced. Potassium bichromate and sulphuric acid gave a reddish-brown, then a grass-green color. Nitric acid gave a yellow color. A tartaric acid solution of the crystals produced on the addition of platinum chlorid a dirty yellow precipitate of small six-sided stars, which contain 31.55 per cent. of platinum. Gold chlorid gave a pure yellow, amorphous precipitate; mercuric chlorid yielded white crystals ; potassio-mercuric-iodid a dirty white precipitate, and potassio-cadmic-iodid yielded no result. Tannic acid produced only a turbidity. Sodium phospho-molybdate gave a yellow flocculent precipitate which became blue on the addition of ammonium hydrate.

Liebermann, in examining the somewhat decomposed stomach and intestines of a case of suspected poisoning, found an alkaloidal body which was unlike that studied by the chemist mentioned above, inasmuch as it was not volatile. The ether extract from alkaline solution left, on evaporation, a brownish resinous mass which dissolved in water to a turbid solution. The cloudiness increased on heating. This reaction agrees with coniïn, but the odor differed from that of the vegetable alkaloid. The putrefactive alkaloid does not distil when heated on the oil-bath to $200^{\circ}$, while coniin distils at $135^{\circ}$. The former is with certainty distinguished from coniïn by its nonpoisonous properties. This substance is extracted by ether from acid as well as from alkaline solutions; on the evaporation of the ether it appears in yellow oily drops, which are soluble in alcohol.

Selmi obtained from both putrefying and fresh intestines a substance which gave the general alkaloidal reactions and had marked reducing power. When warmed with sulphuric acid it gave a violet coloration.

From human bodies from one to ten months after death Selmi removed many alkaline bases. From an ether solution of one of these, one basic substance was removed by treatment with carbonic acid gas. Another body which was insoluble in ether, but readily soluble in amylic alcohol, was found to be a violent poison, producing in rabbits tetanus, marked dilatation of the pupils, choleraic symptoms, and death.

Parts of a human body preserved in alcohol were found by Selmi to yield an easily volatile, phosphorus-containing substance, which was soluble in ether and carbon di-sulphid and gave a brown precipitate with silver nitrate. It was not the phosphid of hydrogen. With potassium hydrate it gave off ammonia and yielded a substance having an intense coniïn odor. It was volatile and reduced phosphomolybdic acid. A similar body was produced by the slow decom- 
position of the yelks of eggs. Selmi also obtained from decomposing egg albumin a body, the chlorid of which formed in needles, and possessed a curare-like action on frogs. From one arsenical body, which had been buried for fourteen days, he obtained, by extracting a solution made alkaline with baryta, with ether, a substance which formed in needles and which gave crystalline salts with acids. With sulphuric acid it gave a red color; with iodic acid and sulphuric acid it liberated free iodin and gave a violet coloration; with nitric acid it gave a beautiful yellow color which deepened on the addition of caustic potash. Platinum chlorid gave no precipitate save in highly concentrated solutions. From a second arsenical body, Selmi obtained by the same method a substance which gave with tannic acid a white precipitate; with iodin in hydriodic acid a kermesbrown; with gold chlorid a yellow, which was soon reduced; with mercuric chlorid a white; with picric acid a yellow, which gradually formed in crystalline tablets. This substance did not contain any arsenic, but was highly poisonous. From the stomach of a hog which had been preserved in a solution of arsenious acid Selmi separated an arsenical organic base. The fluid was distilled in a current of hydrogen ; the distillate, which was found to be strongly alkaline, was neutralized with hydrochloric acid and evaporated to dryness, when cross-shaped crystals, giving an odor similar to that of trimethylamin, were obtained. This substance was found by Ciaccia to be highly poisonous, producing strychnia-like symptoms. With iodin in hydriodic acid it is said to give a gray crystalline precipitate. From the liquid which remained in the retort, a non-volatile arsenical ptomain was extracted with ether. An aqueous solution of this gave with tannic acid a slowly forming, yellowish precipitate and similarly colored precipitates with iodin in hydriodic acid, platinum chlorid, auric chlorid, mercuric chlorid, potassio-mercuric iodid, potassiobismuthic iodid, picric acid, and potassium bichromate. The physiological action of this substance, as demonstrated on frogs, was unlike that of the arsines and consisted of torpor and paralysis.

Moriggia and Battistini experimented with substances obtained from decomposing bodies, upon guinea-pigs and frogs, but did not attempt their isolation because of the rapid decomposition which they undergo when exposed to the air and by which they lose their poisonous properties. These alkaloids were found to be easily soluble in amylic alcohol, less soluble in ether.

In 1871 Lombroso showed that the extract from mouldy cornmeal produced tetanic convulsions in animals. (It must not be forgotten that similar effects may be due to the cornutin of ergot.) This threw some light upon the cases of sporadic illness which had long been known to occur among the peasants of Lombardy, who eat fermented and mouldy corn-meal. In 1876 Brugnatelli and Zenoni obtained by the Stas-Otto method from this mouldy meal an 
alkaloidal substance which was white, non-crystalline, unstable and insoluble in water, but readily soluble in alcohol and ether. With sulphuric acid and bichromate of potassium it yields a color reaction very similar to that of strychnin.

The action of the ether extracts from decomposed brain resembled that of curare, but was less marked and more transitory. The beats of the frog's heart were decreased in number and strengthened in force; the nerves and muscles lost their irritability, and the animal passed into a condition of complete torpor. The pupils were dilated. Guareschi and Mosso, using the Stas-Otto method, obtained from human brains which had been allowed to decompose at a temperature of from $10^{\circ}$ to $15^{\circ}$ for from one to two months, both volatile and non-volatile bases. Among the former only ammonia and trimethylamin were in sufficient quantity for identification. With these, however, were minute traces of ptomaïns. They obtained non-volatile bases from both acid and alkaline solutions. From the former they separated a substance which gave precipitates with gold chlorid, phosphotungstic acid, phosphomolybdic acid, Mayer's reagent, palladium chlorid, picric acid, iodin in potassium iodid, and a slight one with tannic acid. This substance was not precipitated with platinum or mercury.

From the alkaline extract there was obtained a substance which in dilute hydrochloric acid solutions gave with gold chlorid a heavy yellow precipitate with reduction, also precipitates with phosphomolybdic acid, platinum chlorid, Mayer's reagent, picric acid, phosphotungstic acid, Marmé's reagent, iodin in potassium iodid, tannin, bichromate of potassium, palladium chlorid and mercuric chlorid. It reduced ferric salts. From decomposed fibrin the same iuvestigators obtained one well-defined ptomaïn. Analyses of the platinum compound of this substance gave the formula $\mathrm{C}_{10} \mathrm{H}_{15} \mathrm{~N}$. This body will be discussed in a future chapter.

From fresh brain substance they separated ammonia, trimethylamin, and an undetermined base. These, however, are not to be regarded as products of putrefaction, but as resulting from the action of reagents upon the brain substance. The trimethylamin probably arises from the splitting up of lecithin, while the undetermined base is most likely cholin, which also results from the breaking up of the lecithin molecule.

They also show that when Dragendorff's method is used basic substances can be obtained from fresh meat, and these are shown to be produced by the action of sulphuric acid on the fiesh.

In 1885, Vaughan detected in poisonous cheese an active agent to which he gave the name "tyrotoxicon," and this discovery has been confirmed by Newton, Wallace, Schaeffer, Stanton, Firth, Ladd, Wolff, Kimura, Davis, Kinnicut, and others; however, as we shall see later, this is not the substance most commonly found in harmful cheese. 
From 1882 to 1888 , Brieger succeeded in isolating and determining the composition of a number of ptomaïns. From putrid flesh he obtained neuridin, $\mathrm{C}_{5} \mathrm{H}_{14} \mathrm{~N}_{2}$, and neurin, $\mathrm{C}_{5} \mathrm{H}_{13} \mathrm{NO}$. The former is inert, while the latter is poisonous. From decomposing fish he separated a poisonous base, $\mathrm{C}_{2} \mathrm{H}_{4}\left(\mathrm{NH}_{2}\right)_{2}$, which is an isomer of ethylidenediamin, muscarin, $\mathrm{C}_{5} \mathrm{H}_{15} \mathrm{NO}_{3}$, and an inert base, $\mathrm{C}_{7} \mathrm{H}_{17} \mathrm{NO}_{2}$, gadinin. Rotten cheese yielded neuridin and trimethylamin. Decomposed glue gave neuridin, trimethylamin and a muscarin-like base. In the cadaver, he found in different stages of decomposition, cholin, neuridin, trimethylamin, cadaverin, $\mathrm{C}_{5} \mathrm{H}_{14} \mathrm{~N}_{2}$, putrescin, $\mathrm{C}_{4} \mathrm{H}_{12} \mathrm{~N}_{2}$, and saprin, $\mathrm{C}_{5} \mathrm{H}_{14} \mathrm{~N}_{2}$, all of which are inert. After fourteen days of decomposition he found a poisonous substance, mydalein, and from a cadaver which had been kept at from $-9^{\circ}$ to $+5^{\circ}$ for four months, he obtained mydin, $\mathrm{C}_{8} \mathrm{H}_{11} \mathrm{NO}$, the poisonous substance mydatoxin, $\mathrm{C}_{6} \mathrm{H}_{13} \mathrm{NO}_{2}$, also the poison methyl-guanidin. From poisonous mussel he separated mytilotoxin, $\mathrm{C}_{6} \mathrm{H}_{15} \mathrm{NO}_{2}$.

During the later years of the ninth decade of the nineteenth century, chemists began to study the products of bacterial growth in pure cultures. In this work, Brieger, following a method devised by himself, succeeded in isolating a number of basic substances, which were at that time supposed to be the specific toxins of certain diseases. He obtained typhotoxin from cultures of the typhoid bacillus, and four crystalline bodies in growths of the tetanus organism. In 1888 Roux and Yersin made an important contribution in their classical work upon the diphtheria toxin, and two years later Brieger and Fränkel confirmed these discoveries and extended this line of research. Sewall, Salmon and Smith opened up a fruitful field for investigation by showing that immunity can be secured with chemical poisons, the first-mentioned having worked with snake venom, while the others used sterilized cultures. A few years later this work was taken up and greatly advanced by Ehrlich in his now classical studies on immunity, which were first made with the poisonous principles of castor bean and jequirity, and later extended to bacterial products. In 1887 Fodor made his second contribution on the germicidal action of the blood in vita. This work, developed by the researches of Nuttall, Buchner, Metschnikoff, Pfeiffer, Ehrlich, Bordet and numerous others, has greatly advanced our knowledge of the manner in which the animal organism protects itself from bacterial invasion, and has given us some practical tests, as the Widal reaction, which are useful in the diagnosis of diseases. In 1892, blood serum therapy, which had been attempted before by Babes and Tizzoni, was brought into prominence by the researches of Behring, and confirmed by Roux in 1894. As a practical result of this line of research we have the antitoxin treatment of diphtheria, probably the most brilliant discovery yet made within the domain of curative medicine. All of these subjects will be dwelt upon in detail in subsequent chapters. 


\section{CHAPTER IV.}

\section{THE BACTERIAL POISONS OF SOME OF THE INFECTIOUS}

DISEASES.

We will now give our attention to the chemical poisons of some of the infectious diseases, and in doing this we will illustrate, substantiate and extend the statements made in preceding chapters.

Anthrax.-The definition of an infectious disease, as we have given it, is illustrated by the facts which have been learned concerning the causation of anthrax, which has probably been more thoroughly studied than any other infectious disease. Kausch taught that this disease has its origin in paralysis of the nerves of respiration, but as to the cause of this paralysis he gave no information. Delafond thought that anthrax has its origin in the influence of the chemical composition of the soil affecting the food of animals and leading to abnormal nutrition. The investigations of Gerlach, in 1845, demonstrated the contagious nature of anthrax, which was emphasized by Husinger in 1850 and accepted by Virchow in 1855. However, as early as 1849 Pollender found numerous rod-like microörganisms in the blood of animals sick with this disease, and his observation was confirmed by Brauell, who produced the disease in healthy animals by inoculations with matter taken from an anthrax pustule. Attempts were made to ridicule the idea that this organism might be the cause of the disease but, in 1863, Davaine showed that these rod-like bodies must have some causal relation to the disease, inasmuch as his experiments proved that inoculation into animals of the blood of those sick with anthrax, produced the disease only when taken at a time when the blood contained these organisms. He also demonstrated beyond any question that these rod-like bodies are bacteria, capable of growth and multiplication. The conclusions of this investigator were combated by many; but Pasteur, Koch, Bollinger, De Bary, and others studied the morphology and life history of these organisms, and then came the brilliant results of Pasteur in securing protection against inoculated anthrax by the vaccination of healthy animals with the modified germ and subsequent inoculation with the virulent form. Then the question arose, How do these bacilli produce anthrax? and in answer to this question the various theories which we have mentioned were proposed.

In 1877, Pasteur filtcred the blood of animals sick with anthrax, also anthrax cultures, through porcelain and injected the germ-free filtrate into animals without inducing the disease, and concluded 
that this bacillus does not produce any soluble poison. The first successful attempt to study the chemical poison of anthrax was made by Hoffa, who obtained from pure cultures of the bacillus small quantities of a ptomaïn which, when injected under the skin of animals, produces the symptoms of the disease and death. This substance causes at first increased respiration and action of the heart, then the respirations become deep, slow and irregular; the temperature falls below the normal, the pupils are dilated, and a bloody diarrhœa sets it. On section the heart is found contracted, the blood dark, and ecchymoses are observed on the pericardium and peritoneum. Hoffa named this poison anthracin, and later he reported that he had succeeded in isolating it from the bodies of animals dead from anthrax. It must be admitted that Hoffa's claims are not altogether satisfactory, and that they lack confirmation. Moreover, the small amount of the basic substance which he obtained renders it highly probable that in the case of a germ so virulent as that of anthrax, there must be other chemical poisons produced. In 1889 Hankin, by growing the anthrax bacillus for sone days in a nutritive solution consisting of Liebig's meat extract to which fibrin had been added, filtering and treating the filtrate with ammonium sulphate, obtained an albumose which, while not directly poisonous to animals, when injected simultaneously with an inoculation of the anthrax bacillus, causes more speedy death than when the bacillus only is used. From these observations Hankin concluded that the albumose destroys the natural resistance of the ani$\mathrm{mal}$ to the disease, after which the bacillus is able to continue the elaboration of the poison in the animal body.

Martin, by growing the anthrax bacillus for from ten to fifteen days in an alkaline albuminate from blood serum, and then by filtration through porcelain, obtains the following products :

1. Protoalbumose and deuteroalbumose, and a trace of pepton, all of which react chemically like similar substances prepared by peptic digestion.

2. An alkaloid.

3. Small quantities of leucin and tyrosin.

The most characteristic property of the albumoses is that their solutions are strongly alkaline, and the alkalinity is not removed by ether, or by dialysis.

The alkaloid is soluble in water, alcohol and amylic alcohol; and is insoluble in chloroform, ether and benzol. Its solutions are strongly alkaline and the alkaloid forms crystalline salts with acids. It is precipitated by the general alkaloidal reagents, with the exception of potassio-mercuric iodid. It is somewhat volatile and loses its poisonous properties on exposure to the air.

The mixed albumoses are poisonous only when considerable doses are taken, 0.3 gram being required to kill a mouse of 22 grams 
weight when injected subcutaneously. Smaller doses cause a local cedema and a somnolent condition, from which the animal recovers. The larger doses produce a more extensive odema, and the somnolence deepens to coma, terminating in death. In some cases the spleen is enlarged. The absence of germs was determined by plate cul ures. The alkaloid causes similar symptoms. It is, however, more poisonous, and acts more rapidly than the albumoses. The animal is affected immediately after the injection, and the gradually increasing coma terminates in death. The alkaloid also causes œedema and in many cases thrombosis of the small veins. Extravasations into the peritoneal cavity are occasionally seen, and the spleen is ordinarily enlarged and filled with blood. The fatal dose for a mouse is from 0.1 to 0.15 gram, death resulting within three hours. This alkaloid does not appear to be identical in its action with the anthracin of Hoffa. Martin also succeeded in obtaining like substances from the bodies of infected animals. He regards the albumose as an antecedent of the ptomain. It is probable that the albumoses obtained by Hankin and Martin are identical. Balp and Carbone ${ }^{1}$ succeeded in isolating from the odematous tissue of an animal dead with anthrax an albuminous body of slight toxicity, and a similar result was obtained by Landi. ${ }^{2}$ Maltzew ${ }^{3}$ found that when from 0.2 to 7 c.c. of a filtered bouillon culture of the anthrax germ was injected subcutaneously in rabbits, it induced no symptoms, but if the same animals were inoculated with anthrax within from ten to eighteen days they died much more speedily than control animals. It will thus be seen that Hankin's results have been several times confirmed. However, it should be stated that Petermann ${ }^{4}$ claims to have repeated Hankin's experiments and obtained an albumose, the only effect of which was to elevate the temperature from one to two degrees, and Klemperer ${ }^{5}$ obtained from boiled anthrax cultures an albuminous substance which caused elevation of temperature. Hankin and Wesbrook ${ }^{6}$ repeated the former's experiments, using a solution of pure pepton as a culture medium. After some weeks, the culture was filtered, treated with ammonium sulphate, centrifuged, dialyzed, and precipitated with alcohol. In this way an albumose of slight toxicity was obtained.

Brieger and Fränkel endeavored to prepare a toxalbumin from the organs of animals dead with anthrax. The finely divided tissue was thoroughly rubbed up with water, allowed to stand for twelve hours in an ice-box and filtered through porcelain. The filtrate was evaporated in vacuo at $30^{\circ}$ to one-third its volume, and, after being acidi-

1 Giornale della $R$. accademia di medicina di Torino, 1891.

${ }^{2}$ Rivista generale italiana di clinica medica, 1891.

${ }^{3}$ Russkaia Medicina, 1891.

'Annales de l'Institut Pasteur, 1892.

5 Zeitschrift f. klin. Medicin., 1892.

${ }^{6}$ Annales de $l$ Institut Pasteur, 1892. 
fied with a few drops of acetic acid, was treated with ten times its volume of absolute alcohol. This mixture was allowed to stand for twelve hours longer in an ice-box, after which the precipitate was collected on a filter, dissolved in a small volume of water, refiltered, and reprecipitated with alcohol, this being repeated until a perfectly clear aqueous solution was obtained. The albumose was further purified by dialysis, and as thus obtained, it was found to be freely soluble in water and to give the ordinary reactions for albumin. The toxicity of this albumose is much greater than that of similar substances obtained by others.

Marmier grew anthrax germs in the following medium :

$\begin{array}{lr}\text { Water, } & 1,000 \text { grams. } \\ \text { Pepton, } & 40 \text { " } \\ \text { Sodium chlorid, } & 15 \text { " } \\ \text { Sodium phosphate, } & .5 \text { " } \\ \text { Potassium phosphate, } & .2 \text { " } \\ \text { Glycerin, } & 10 \text { " }\end{array}$

The pepton used was obtained from the commercial preparation by precipitation of the other proteids with ammonium sulphate and by removing the salt by dialysis. In this menstruum the anthrax bacillus, especially the sporeless variety, grew abundantly. The toxin was obtained from the culture medium by precipitation with ammonium sulphate. When dried it is soluble in water and in a one per cent. solution of phenol; insoluble in chloroform, ether, and absolute alcohol. It is said not to give any of the reactions of albuminoids, propeptons, peptons, or alkaloids, but since there is no mention of the reactions tested, and since precipitation with ammonium sulphate is a pepton and propepton reaction, this statement must be considered as somerrhat indefinite and possibly misleading. There is no proof that the toxin obtained was pure; in fact, the report makes the reader certain that the final product was by no means chemically pure. The author was surprised in studying the poisonous effects of this toxin to find that while twenty milligrammes killed some rabbits readily, others resisted seventy milligrammes; and it does not seem to have occurred to him that this might be due to the fact that the most of his product was inert, and that these differences in effects were due to the unequal distribution of the active agent. However, we will give the author's conclusions as he states them :

1. A specific toxin may be extracted from glycerin-pepton cultures of the anthrax bacillus.

2. This toxin does not give the reactions of albuminoid substances. It does not change starch, sugar or glycogen.

3. The animals (chickens, frogs, fish) that are immune to the anthrax bacillus, are also indifferent to the toxin. Similar results 
were observed in rabbits artificially immunized with attenuated cultures.

4. This toxin is attenuated, but not destroyed, by boiling at $110^{\circ}$, thus differing from the venom of serpents, the toxins of diphtheria and tetanus, and the enzymes.

5. On the contrary, like the other bacterial toxins, it loses its effect on animals after being brought in contact with the alkaline hypochlorites. Prolonged insolation in the presence of air leads to the same result.

6. By employing carefully graduated doses of this toxin it is possible to immunize animals to the anthrax bacillus in the same way as other specific toxins give immunity to the corresponding disease.

7. Anthrax cultures in other fluids, such as blood serum, and bouillon from the flesh of the horse, ox, or calf, do not contain the toxin in appreciable quantities.

8. On the contrary, one may extract a toxin from anthrax cultures on nutritive gelatin by macerating the microörganisms in alcohol water (20 per cent. alcohol).

The toxin is contained within the bacterial cells, and in order to obtain it in the culture there must be opportunity for it to diffuse from the cells.

Heim and Geyger ${ }^{1}$ grew anthrax bacilli in eggs, after the manner of Hueppe, extracted with alcohol, precipitated with mercuric chlorid, filtered, treated the filtrate with platinum chlorid, decomposed this precipitate with hydrogen sulphid, rendered the filtrate alkaline with potassium hydrate, and extracted one portion with ether, and another with benzol. The benzol residue, when taken up with water, killed mice; the ether residue was less toxic.

Ivanow ${ }^{2}$ has shown that certain volatile fatty acids, formic, acetic and caproic, may be formed in anthrax cultures; but there is no evidence that the production of these substances has anything to do with the pathology of the disease.

Petri and Maassen ${ }^{3}$ have detected hydrogen sulphid in anthrax cultures; but spectroscopic examination of anthrax blood failed to show the presence of this substance, and there is no evidence that this gas has anything to do with the production of the disease.

Fermi has shown the presence of peptonizing and diastatic ferments in anthrax cultures; while Maumus has detected a sugarforming agent, and Roger reports the presence of a rennet. Klein ${ }^{4}$ removed the germs from agar cultures of forty-eight hours' growth, placed the same in 5 c.c. of bouillon, and after the tube had been

${ }^{1}$ Lehrbuch der Bakt. Untersuchung, 1894.

${ }^{2}$ Annales de l' Institut Pasteur, 1892.

3 Arbeiten aus dem k. Gesundheitsamte, 8, 318.

- Centralblatt $f$. Bakteriologie, 15, 598. 
held for five minutes in boiling water, injected two-thirds of its contents into the peritoneal cavity of a guinea-pig without results. From this he concludes that the anthrax bacillus does not form any intracellular poison.

Bianchi-Mariotti ${ }^{1}$ has studied the effects of the soluble constituents of anthrax cultures on the isotonia of the blood. He found that the intravenous injection of cultures filtered through porcelain, in small or medium doses, increased the isotonic properties of the blood of the rabbit, but in larger doses diminished the same; furthermore, he showed that the amount of hemoglobin is decreased after the injection, in direct proportion to the quantity of the culture used.

Conradi ${ }^{2}$ has endeavored to solve the question of the existence of an anthrax toxin by the following methods.

1. The exudates which form in the peritoneal cavities of guineapigs inoculated with anthrax, were filtered through both Kitasato and Chamberland filters and injected into susceptible animals without effect. The amount of filtered extract injected into rabbits varied from 10 to 20 c.c.

2. The livers and spleens of guinea-pigs, which had succumbed to anthrax, were immediately after death rubbed up with sterilized sand in a sterilized mortar, diluted with physiological salt solution, filtered through porcelain and injected into white rats, guinea-pigs and rabbits without effect.

3. Collodion sacs filled with virulent anthrax cultures, were placed in the abdominal cavities of susceptible animals, where they remained without apparent detriment to health. This was a repetition of similar experiments made previously by Sanarelli and Pekelharing.

4. The anthrax exudates in quantities of from 5 to 6 c.c. were placed in test-tubes, $\frac{1}{2}$ c.c. of toluol added, the tube closed with sterilized cork, thoroughly shaken, and then allowed to stand for ten days in the dark at room temperature. At the expiration of this time the contents of many tubes were placed in a separator and the toluol removed. The exudate in which the germ had thus been destroyed by toluol was injected into susceptible animals without effect.

5. Having shown that asporogenic cultures are deprived of vitality by exposure for 110 hours to $-16^{\circ}$, such cultures, after being thus sterilized and being kept for some time in the incubator to prove their sterility, were injected into susceptible animals without effect.

6. Thinking it possible that a toxin might be extracted under pressure, after the method used by Buchner in obtaining a ferment from yeast, cultures were exposed to hydraulic pressure of 500 at-

2 Zeitschrift f. Hygiene, 31, 237. 
mospheres. The resulting fluid was filtered through porcelain and injected into animals without effect.

7. The experiment of Brieger and Fränkel, in which they prepared their anthrax toxalbumin, was repeated with a negative result.

From these experiments Conradi reaches the following conclusion: "By no method known at present can it be shown that the anthrax bacillus forms either an extra-cellular or an intra-cellular poison in the animal body; indeed, these experiments increase the probability that the anthrax bacillus does not form any poisonous substance. Therefore the solution of the mamer in which anthrax infection results remains unknown. Whether improved chemical methods will lead to the detection of a poison or not cannot be predicted, but for the present the anthrax bacillus must be regarded as a purely infectious microörganism." If this be true, the mechanical interference theory is the best that can at present be offered so far as anthrax is concerned. ${ }^{1}$

Asiatic Cholera.-Bitter has shown that the comma bacillus produces in meat pepton cultures a peptonizing ferment, which remains active after the organism has been destroyed. Like similar chemical ferments, it converts an indefinite amount of coagulated albumin into pepton. It is more active in alkaline than in acid solutions, thus resembling pancreatin more than pepsin. The resemblance to pancreatin is further shown by increased activity in the presence of certain chemicals, such as sodium carbonate and salicylate. Bitter also demonstrated that this microörganism produces a diastatic ferment, inasmuch as he found that it develops an acid in nutrient solutions containing starch paste; however, all attempts to isolate this ferment were unsuccessful. A temperature of $60^{\circ}$ destroys or markedly decreases the activity of ptyalin, and this is also true of the diastatic ferment produced by the comma bacillus.

Fermi isolated the peptonizing ferment of the cholera germ in the following manner : 65 per cent. alcohol added to gelatin which has been liquefied by the bacillus, precipitates the proteid, but not the ferment; after twenty-four hours the precipitate is removed by filtration, and the ferment precipitated from the filtrate by the addition of absolute alcohol. After being collected on a filter and dried, this ferment may be dissolved in an aqueous solution of thymol and its peptonizing properties demonstrated on gelatin tubes.

Rietsch believes that the destructive changes observed in the intestines in cholera are due to the action of the peptonizing ferment.

Cantani injected sterilized cultures of the comma bacillus into the peritoneal cavities of small dogs and observed after from one-quarter to one-half hour the following symptoms : great weakness, tremor of the muscles, drooping of the head, prostration, convulsive contractions 
of the posterior extremities, repeated vomiting, and cold head and extremities. After two hours these symptoms began to abate, and after twenty-four hours recovery seemed complete. Control experiments with like amounts of uninfected beef tea gave negative results. The cultures used were three days old when sterilized. Older cultures seemed less poisonous, and a high or prolonged heat in sterilization decreased the toxicity of the fluid ; therefore, Cantani concludes that the poisonous principle is volatile, but the effect of high or prolonged heat in diminishing the toxicity was more probably due to destructive action on the toxin. The same observer found that the blood of those sick with cholera is acid; this has been confirmed by Strauss on examining the blood directly after death, and Ahrend found lactic acid in the strongly acid urine of a cholera patient.

Nicati and Rietsch killed dogs by injecting intravenously cultures from which all the germs had been removed by filtration, and they also obtained from old bouillon-pepton cultures a poisonous base. Van Ermengem also found that cultures after filtration through a Chamberland filter are poisonous.

Klebs studied the cholera toxin in the following manner: Cultures in fish preparations were acidified, filtered; the filtrate evaporated on the water-bath; the residue taken up with alcohol and precipitated with platinum chlorid. The platinum was removed with hydrogen sulphid and the crystalline residue obtained on evaporation was dissolved in water and injected into rabbits intravenously. Muscular contractions were induced, and death followed in one animal, which, in addition to the above treatment, received an injection of a non-sterilized culture. In the latter case the epithelium of the uriniferous tubules was found to be extensively calcified. Klebs believes this change in the kidney to be induced by the chemical poison, and he explains the symptoms of the disease as follows: The eyanosis is a consequence of the arterial contraction, the first effect of the poison. The muscular contractions also result from the action of the toxin. The serous exudate of the intestines follows upon epithelial necrosis. Anuria and the subsequent symptoms appear when the formation and absorption of the poison become greatest.

Hueppe states that the severe symptoms of cholera can be explained only on the supposition that the bacilli produce a chemical poison, which resembles muscarin in its action.

Villiers isolated by the Stas-Otto method from two bodies dead from cholera a poisonous base which was liquid, pungent to the taste, and possessed the odor of hawthorn. It was strongly alkaline, and gave precipitates with the general alkaloidal reagents. From one to two milligrammes of this substance injected into frogs caused decreased activity of the heart, violent trembling, and death. The heart was found in diastole and full of blood, and the brain slightly 
congested. However, the presence of this substance in the bodies of persons who have died of cholera does not prove that its production is due to the cholera bacillus.

Pouchet extracted from cholera stools, with chloroform, an oily base belonging to the pyridin series. It reduced ferric as well as platinum salts, and formed a readily decomposable hydrochlorid. It was a violent poison, irritating to the stomach, and retarding the action of the heart. Subsequently, he obtained a similar substance from cultures of the comma bacillus.

Brieger used pure cultures on beef broth, which was rendered alkaline by the addition of a three per cent. soda solution. These were kept at from $37^{\circ}$ to $38^{\circ}$, and after twenty-four hours, cadaverin was found to be present. Older cultures yielded very small quantities of putrescin, but blood serum cultures yielded much larger amounts of this base. While cadaverin and putrescin cannot be said to be poisonous, they cause necrosis of tissue into which they are injected, and their formation by the cholera germ may account for the necrotic areas observed in the intestine after death from this disease. The lecithin of the beef broth was slowly acted upon by the germ, and with age the amount of cholin increased, reaching its maximum during the fourth week. Creatin proved more resistant to the action of the bacillus; but, after six weeks, a considerable quantity of creatinin was isolated, and a similar amount of methyl-guanidin. The latter is highly poisonous, and causes muscle tremors and dyspnœa. The presence of methyl-guanidin indicates that the comma bacillus acts as an oxidizing agent, since creatin yields this substance only by oxidation. In addition to the above-mentioned ptomains, which are common products of putrefaction, Brieger found two poisons which he at that time considered as specific toxins of the comma bacillus. One of these, found in the mercuric chlorid precipitate, is a diamin, resembling trimethylendiamin. It caused muscle tremor and cramps. The other poison, found in the mercury filtrate, produced in mice a lethargic condition; the respiration and heart's action became slow, and the temperature sank so that the animals felt cold. Sometimes there was bloody diarrhœa. It is quite certain that the above-mentioned substances, neither singly nor combined, constitute the specific toxin of the cholera bacillus.

Brieger and Fränkel found in cultures of the cholera bacillus an insoluble proteid, which, when suspended in water and injected subcutaneously in guinea-pigs, caused death after from two to three days. Section showed inflammatory swelling and redness of the subcutaneous tissue, extending into the muscles, for some distance about the point of injection, but no necrosis. There was no change in the intestine and no effusion into the peritoneum. In some instances there was beginning fatty degeneration in the liver. Upon rabbits this substance, even in large doses, was without effect. 
Gamaleia employed cultures which had been sterilized at $120^{\circ}$. Subcutaneous injections of these caused transient œedema from which the animals soon recovered. When the cultures were sterilized at $60^{\circ}$, large doses (10 c.c. per kilogram body-weight) caused death. In this connection Bouchard remarks that in 1884 he obtained by the intravenous injection of the urine of a cholera patient in rabbits, muscular tremor, cyanosis, albuminuria and diarrhœa, but that he has never succeeded in inducing these symptoms with the cholera vibrio.

Petri states that the comma bacillus produces in pepton cultures large amounts of tyrosin and leucin, a small quantity of indol, fatty acids, poisonous bases, and a poisonous proteid. The proteid resembles pepton in its behavior to heat and chemical reagents and is designated by Petri as "toxopepton." In quantities of 0.36 gram per kilogram, it is fatal to guinea-pigs within eighteen hours, producing muscle tremor and paralysis. Autopsy shows an effusion into the peritoneal cavity, marked injection of the blood vessels of the intestines, and isolated hemorrhagic spots. It is possible that the substance contains the cholera toxin, but the greater part of it consists of harmless proteid bodies.

Scholl has studied the chemical products of the cholera bacillus when grown under anaërobic conditions. For this purpose he employed fresh sterilized eggs, after the method of Hueppe. The inoculated eggs, after being kept for eighteen days at $36^{\circ}$, were opened. The contents smelled intensely of hydrogen sulphid, but not of amines. The albumin was completely fluid, while the yolk was more solid and of a dark color. Five c.c. of the fluid contents injected into the abdomen of a guinea-pig caused at first paralysis of the posterior extremities, then general paralysis, and death within forty minutes. Section showed the vessels of the small intestine and stomach highly injected, a colorless effusion in the peritoneal cavity, and the heart in diastole. A like result was obtained by the use of an aqueous extract of a precipitate obtained by the addition of the albuminous content of the egg to ten times its volume of absolute alcohol. It is more than probable that the effect obtained in these experiments was due to the alcohol or hydrogen sulphid retained in the albuminous substance.

Hueppe holds that the cholera poison results from the analytic or ferment action of the germ on the proteids in which it grows, and that the proteids of the bacterial cells are not poisonous. Following the classification of bacterial proteids which we have made, Hueppe would place the cholera toxin among our bacterial proteids and the immunizing substance among the cellular proteids. At one time he claimed that these substances could be separated in the following manner: Rice-water stools from cholera patients are treated with absolute alcohol; both the toxin and the immunizing substance are 
precipitated. By collecting this precipitate and extracting it with sterilized water or physiological salt solution, the toxins only are dissolved. The belief that a separation of these two classes of proteids can be made in this way rests upon the following assumptions, neither of which can be said to be demonstrated facts: (1) The poison and the immunizing body are not one and the same thing; (2) the cellular proteids are not soluble in water or salt solution.

Hueppe claims, furthermore, that in a given case of cholera the toxin may be formed most abundantly and the immunizing substance only in small amount; in such a case the symptoms of the disease would be violent, and should recovery result, immunity to subsequent infection would be slight. With the conditions reversed, the disease might be slight and the immunity established great.

Pfeiffer and his students have held that the cholera toxin is an integral part of the bacterial cell and that it is not set free or capable of manifesting its toxic properties until the germ dies; on the other hand, Metschnikoff, Gruber, Behring, Ransom and others contend that the living cholera germ produces both in vitro and in vita a soluble, diffusible poison. It is not necessary to go into detail concerning this discussion; but we will give a brief statement of some of the experiments made by Metschnikoff and Roux. A collodion sac of about 4 c.c. capacity, filled with a 2 per cent. pepton solution, and inoculated with the cholera bacillus, was placed in the abdominal cavity of a guinea-pig. A second animal received a similar sac containing an emulsion of cholera bacilli killed by chloroform; while a third sac, containing pepton solution only, was placed in a third animal. Guinea-pig No. 3 remained unaffected; No. 2 showed during the first fow days some slight elevation of temperature and some emaciation; while No. 1 died after from three to five days, with symptoms of cholera intoxication. At autopsy the typical lesions of cholera infection were found in the peritoneum, intestines, and kidneys ; while the cholera bacillus could not be found in the peritoneal cavity, in the blood, nor in any of the organs. The collodion sac contained a large culture of highly motile cholera bacilli but no leucocytes. In order to obtain the toxin in artificial cultures, the germ was made highly virulent by passage through animals and by sac cultures. In this way they obtained a culture, $\frac{1}{16} \overline{0}$ c.c. of which sufficed to kill guinea-pigs. This highly virulent germ was grown in a culture medium consisting of 2 per cent. pepton, 1 per cent. common salt, and 2 per cent. gelatin with some serum added. Cultures in this medium, grown at $37^{\circ}$ for from three to four days and filtered through porcelain, killed guinea-pigs when administered subcutaneously in the proportion of one-third c.c. per 100-gram body weight. The toxin thus obtained was not materially changed on being boiled, but did lose its toxicity on contact with the air, especially when simultaneously exposed to light. Tubes completely filled with the germ- 
free filtrate and kept in the dark were found to lose only one-third of their toxicity after six months. Guinea-pigs were found to be most susceptible; rabbits somewhat more resistant; mice required relatively twenty times the dose fatal to guinea-pigs; while pigeons and chickens were still less susceptible. By gradually increased doses, guinea-pigs, rabbits, goats, and horses were immunized with this toxin. From a guinea-pig thus immunized an antitoxic serum was obtained, and it was found that one c.c. of this was sufficient to neutralize four c.c. of a toxin, two-thirds c.c. of which sufficed to kill a guinea-pig of 250 grams in 14 hours. By treating a horse for six months with gradually increased doses of the toxin these investigators secured an antitoxic serum, 1 c.c. of which was sufficient to neutralize four times the fatal dose of the toxin. The antitoxic serum was also found to give immunity against intra-peritoneal infection with living cultures. From these experiments it was concluded that in the treatment of cholera an antitoxic, and not an anti-bacterial, serum is needed. It should be stated that a highly active toxin is necessary for the production of a useful serum.

It is more than probable that the cholera toxin is formed within the cell by synthetical processes, and that it readily diffuses through the cell wall. On this assumption both Pfeiffer and Metschnikoff are partially right in their contentions.

Bujwid found that on the addition of from 5 to 10 per cent. hydrochloric acid to bouillon cultures of the cholera bacillus there is developed after a few minutes a rose-violet coloration, which increases during the next half hour and in a bright light shows a brownish shade. The coloration is more marked if the culture be kept at about $37^{\circ}$. In a pure culture this reaction does not occur. ${ }^{1}$

Brieger found that this color is due to an indol derivative. In cholera cultures on albumins he obtained indol by distillation with acetic acid.

Dunham finds the best medium for the "cholera reaction" to be a one per cent. alkaline pepton solution, with one-half per cent. of common salt. Bujwid prefers a two per cent., strongly alkaline, pepton solution with salt. Jadassohn finds that gelatin cultures give the reaction both before and after the liquefaction of the gelatin. The undissolved gelatin, after the addition of hydrochloric or sulphuric acid, becomes rose-violet. Cohen claims that cultures of other bacilli give a similar coloration, but Bujwid explains that the results obtained by Cohen were due to the use of impure acids, which contained nitrous acid. Salkowski agrees with Bujwid, and states that when acids wholly free from nitrous acid are used the reaction is characteristic of the comma bacillus. He explains the

1 Poehl deserves the credit of being the first to call attention to this reaction, though his work was evidently unknown to Bujwid at the time when the latter published his report. 
reaction by supposing that the germ produces nitrous acid, which exists in the culture as a nitrite; on the addition of an acid the nitrous acid is set free and acting upon the indol, which is also present, gives the coloration. After all, wider experience has shown that Cohen was right and that many germs respond to the indol reaction.

According to Hammurl, cultures of the germ newly taken from stools or intestinal content do not give the indol reaction as constantly as those germs grown on artificial media. From an exhaustive research on the importance of this test, Petri comes to the following conclusions: (1) Seven pure cultures of the cholera germ, from as many sources, gave the reaction with equal distinctness. (2) Of one hundred other bacteria tested in the same way, twenty gave a red coloration. In nineteen of these the coloration is due to the nitroso-indol reaction of Baeyer. The twentieth (anthrax) gave a color which is not due to indol. (3) In case of the cholera germ, and the others as well, the reaction is due to the reducing effect of the bacteria on nitrates. It is most marked at blood temperature and with the cholera bacillus; it is least distinct with the bacilli of Finkler and Miller. (4) None of these bacteria convert ammonia into nitrite. (5) The simple addition of sulphuric acid is sufficient to give the test, which, however, is most marked when the nutrient solution contains 0.01 per cent. nitrate. (6) The reaction is most marked if the sulphuric acid be added, after the addition of a very dilute nitrite solution.

Schuchardt calls attention to the fact that Virchow observed a red coloration on the addition of nitric acid to filtered cholera stools in 1846, and in 1885 Griesinger made mention of a similar observation.

A "cholera blue" has also been observed by Brieger in cultures in meat extract containing pepton and gelatin. This substance, which is yellow by refracted, and blue by transmitted, light, is developed by the addition of concentrated sulphuric acid to the culture. It may be separated from the "cholera red" as follows: Treat the culture with sulphuric acid, then render alkaline with sodium bydrate and extract with ether. Evaporate the ether and remove the "cholera red" with benzol, then again dissolve the "cholera blue" in ether. The characteristic absorption bands for this coloring matter begin in the first third of the spectrum between $E$ and $F$ and darken all of the zone lying beyond.

Winter and Lesage treat a bouillon culture of the cholera germ with sulphuric acid, dissolve the precipitate in an alkaline medium, reprecipitate with acid and redissolve in ether, which on evaporation leaves oily drops, and these, on cooling, form a yellow mass of the appearance of a fat. This substance is insoluble in water and acids, soluble in alkalis and ether. It melts at $50^{\circ}$, and does not lose its 
virulence on being boiled with alcohol rendered feebly alkaline. The virulence of a culture and the amount of this substance contained therein are in direct proportion to each other. Small doses (one milligram to one hundred grams of body weight) in feebly alkaline solution, introduced into the stomachs of guinea-pigs cause, as a rule, within from four to six hours, a chill and death after twenty-four hours. With larger doses the temperature falls after from one-half to one hour and death results within from twelve to twenty hours. Smaller doses cause a less marked reaction and the animal recovers within twenty-four hours. Rabbits succumb only after repeated subcutaneous injections. The substance can be extracted from the liver, muscles, kidneys, and urine of the poisoned animals. It can also be obtained from cultures of a cholera infantum germ. It is quite certain that this substance is an artificial product.

Tetanus.-Brieger has obtained from cultures of the tetanus germ four poisonous substances. The first, tetanin, which rapidly decomposes in acid, but is stable in alkaline solutions, produces tetanus in mice when injected in only a few milligrammes. The second, tetanoxin, produces first tremor, then paralysis, followed by severe convulsions. The third, to which no name has been given, causes tetanus, accompanied by free flow of the saliva and tears. The fourth, spasmotoxin, induces clonic and tonic convulsions. The same investigator isolated tetanin from the amputated arm of a man with this disease. More recent researches lead us to attach but little importance to the crystalline bodies discovered by Brieger, and it is highly probable that the crystals with which he worked were not of themselves poisonous, but were mixed with small quantities of the toxin.

In their researches on toxalbumins Brieger and Fränkel obtained from cultures of the tetanus germ in bouillon containing grape sugar, a substance soluble in water, which when injected subcutaneously in guinea-pigs caused tetanus to appear after about four days, and led to a fatal termination.

Later, Brieger and Cohn prepared tetanus poison from cultures of the bacillus in veal broth containing one per cent. of pepton, and one-half per cent. of common salt. These cultures were rendered germ-free by filtration through porcelain, and treated with ammonium sulphate to supersaturation. This throws the poison out of solution and it floats on the surface, from which it is removed by a platinum spatula. This crude poison, when dried in vacuo, is found to contain 6.5 per cent. of ammonium sulphate. Of the filtered culture 0.00005 c.c. suffices to kill mice. From one liter of the culture one gram of the dried substance was obtained, and of this $0.000,000,1$ gram killed a mouse with the typical symptoms of tetanus. This crude product contains, besides the poison, albumins, pepton, amido- 
acids, volatile substances, and ammonium sulphate, with other salts. The albumin was removed with basic lead acetate. The pepton, amido-acids and salts were removed by dialysis, and finally, evaporation in vacuo at $20-22^{\circ}$ removed the volatile substances. The toxin thus obtained is yellow, flaky, readily soluble in water, odorless, and similar in taste to gum arabic. It turns polarized light slightly to the left. It fails to give the Millon and xanthoproteic reactions, but does give with copper sulphate and caustic potash a faint, violet coloration, not identical with the rose of the biuret reaction. With the exception of ammonium sulphate, the metallic salts, as sodium chlorid and sulphate, magnesium sulphate, potassium nitrate, mercuric chlorid and potassium ferrocyanide with acetic acid, fail to precipitate the purified poison. Moreover, calcium phosphate which Roux and Yersin used for carrying down the diphtheria poison, also magnesium carbonate and aluminum hydrate, do not throw the tetanus poison out of solution. The toxin thus obtained contains no phosphorus and only unweighable traces of sulphur. Of the best preparation obtained by these investigators, $0.000,000,05$ gram killed a mouse of 15 grams weight. The authors figure from this that the fatal dose for a man of 70 kilograms would be 0.00023 gram, or 0.23 milligram, and 0.04 milligram would induce symptoms of tetanus. The smallest lethal dose of atropin for an adult is 130 milligrams, and of strychnin from 30 to 100 milligrams. "From this one can judge of the fearful weapons possessed by the bacteria in their poisons."

Fermi and Pernossi draw the following conclusions from their studies of the tetanus poison : (1) Agar cultures are the most poisonous. Next come those on gelatin, and lastly those in bouillon. Chickens, snakes, turtles and tritons are immune to the poison. In the above-mentioned animals this toxin may rom In the above-mentioned animals this toxin may remain and retain its virulence for three days, and even longer. (4) Filtrates from agar and gelatin cultures are more resistant to heat than those from bouillon. Like the enzymes, the purer the tetanus poison the less stability does it possess. (5) Dissolved in water, the tetanus poison is rendered inert by a temperature of $55^{\circ}$, but in the dry state it can be heated to $120^{\circ}$ without loss of virulence. (6) When the dry poison is mixed with ether or chloroform and heated to $80^{\circ}$, it is destroyed ; but with amylic alcohol or benzol, a temperature of $100^{\circ}$ is required to accomplish this result. (7) Dissolved in water, this poison is destroyed by direct sunlight after an exposure of eight to ten hours (with the highest temperature on a blackened thermometer at $56^{\circ}$ ) and after fifteen hours when the temperature does not exceed $37^{\circ}$. (8) In the dry state the tetanus poison can be exposed to the direct sunlight for 100 hours without loss of virulence. (9) Under the action of an electric current of 0.5 ampère, continued for two hours, the substance becomes inert. (10) Gastric juice destroys the 
poison through the activity of the hydrochloric acid and not by virtue of the pepsin. (11) Ptyalin, diastase, and emulsin have no action. The effect of trypsin has not been satisfactorily determined. (12) Putrefactive germs do not destroy the poison. (13) The living, but not the dead, intestines of guinea-pigs and cats, destroy the poison. (14) The chick does not destroy and does not absorb the poison. (15) The poison may be eliminated by the kidneys and retain its properties in the urine. (16) The poison is not a ferment.

According to Ehrlich, ${ }^{1}$ the tetanus bacillus produces two toxins, tetanospasmin and tetanolysin. To the first of these the tetanic convulsions are due, while the second has a hemolytic action. Both of these toxins are present in the precipitate formed by the addition of ammonium sulphate to bouillon cultures of the tetanus bacillus, but they do not always exist in the same proportion. One culture may have a marked tetanic effect and manifest but little hemolytic action, while a second culture may dissolve red blood corpuscles promptly but show only slight action on the central nervous system. These toxins also differ in their stability, the hemolytic poison being the less stable and undergoing complete destruction when heated to $50^{\circ}$ for twenty minutes. When a tetanus culture is placed in a menstruum containing red blood corpuscles, the greater part of the tetanolysin combines with the corpuscles while the tetanospasmin remains in solution; on the other hand, tetanospasmin combines with nerve cells, which apparently have no attraction for the other poison. Each of these substances has its specific antitoxin and in the blood of animals immunized with tetanus cultures one or the other antitoxin may predominate. It thus happens that a serum may be strongly antispastic, while it is but slightly antilytic, or the reverse may be true.

Madsen ${ }^{2}$ has made a special study of tetanolysin. He obtained a mixed toxin by precipitation of a bouillon culture of the tetanus bacillus with ammonium sulphate. The poison thus secured was of medium strength, the fatal dose for mice of 15 grams body weight, being $0.000,001$ gram. This poison dissolves the red blood corpuscles of rabbits, goats, sheep, horses and other animals. The blood of the horse and that of the rabbit was found to be especially susceptible, while that of the goat was less so. For experimental purposes the defibrinated blood of the rabbit diluted with 0.85 per cent. of common salt solution to 5 per cent., was used. For purposes of demonstration this diluted blood was placed in tall testtubes of Jena glass. This gave opportunity for the observation of the effects of the toxin on the corpuscles during the process of subsidence. To the tubes thus prepared varying quantities of the poison were added, mixed, allowed to stand for one hour at $37^{\circ}$, and then kept over night at a low temperature. The ultimate result was ob-

${ }^{1}$ Berliner klinische Wochenschrift, 1898, 273.

2 Zeitschrift für Hygiene, 32, 214. 
served twenty-four hours after the poison was added. The quantity of the poison used in the experiments of Madsen varied from 1.2 c.c. of a one per cent. solution to 0.2 c.c. of a 0.01 per cent. solution, and a control tube containing no toxin served for contrast. In this way the effects of different quantities of the poison were made plainly manifest to the eye. In those tubes containing the larger quantities of the poison all the corpuscles were dissolved and there remained a blood-red solution, uniform in tint from top to bottom. As the amount of the poison was diminished the number of undissolved corpuscles collected at the bottom of the tube was increased and the color of the supernatant fluid grew less intense towards the top. With the larger quantities of poison the destruction of corpuscles was practically instantaneous and occurred before there was time for subsidence, while in the more diluted solutions the corpuscles partially subsided before they were acted upon by the toxin. It was thus demonstrated that there is a latent period in the action of the poison and that this period increases as the quantity of the toxin is diminished. It was also found that lowering the temperature increased for a given amount of the poison the latent period. It was also rendered highly probable that the individual corpuscles vary markedly in their resistance to the hemolytic action of the poison. Furthermore, it was shown that some corpuscles are more resistant or less resistant to this toxin than to other hemolytic poisons. Thus, corpuscles that were found to be especially susceptible to tetanolysin were less resistant to another hemolytic poison, crotin.

Madsen continued his interesting work and showed that the hemolytic action of tetanolysin can be neutralized by an antitoxic serum. In these experiments he used a tetanus serum each gram of which contained fifty immunity units against tetanospasmin. This antitoxic serum was diluted to one-half per cent. with a mixture of glycerin and common salt solution. This was designated as his "stock solution" and was further diluted to one-fortieth of one per cent. with physiological salt solution for purposes of experimentation. It was found that for the complete neutralization of two c.c. of the toxin from 1.3 to 1.4 c.c. of the $\frac{1}{40}$ per cent. antitoxin solution was needed; $i$. e., when a mixture containing two c.c. of toxin solution and 1.3 c.c. of the diluted antitoxin solution was added to diluted defibrinated blood, the corpuscles remained unaffected. Following the method pursued by Ehrlich in his studies of the diphtheria toxin, Madsen tried the effect of the partial neutralization of the tetanolysin with antitoxin. As a result of these experiments he found that when 0.10 c.c. of the antitoxin solution (which is onethirteenth of the amount necessary to completely neutralize two c.c. of the poison) was added to two c.c. of the poison the hemolytic action of the toxin was decreased to one-half its value, and that when 0.25 c.c. of the antitoxin (one-fifth of the amount necessary 
for complete neutralization) was added to two c.c. of the toxin, the latter lost nine-tenths of its effect. From these and other experiments Madsen reaches the following conclusions :

1. In cultures of the tetauus bacillus, there is besides tetanospasmin another poison, tetanolysin, for which there is a specific antitoxin, which may be designated as antilysin.

2. The action of this lysin and its anti-body can be measured with great exactness by methods of color comparison.

3. Tetanolysin combines with the red blood corpuscles and the combination is preceded by a latent period which depends upon the amount of poison and the temperature.

4. Investigation shows that tetanolysin presents a complicated neutralization reaction which resembles very closely that of the diphtheria poison.

5. The substance consists of halves which differ in certain fundamental properties. One half of the poison consists of three substances, prototoxin, deuterotoxin, and tritotoxin.

6. The prototoxin makes up only one-third of the quantity of the poison but possesses one-half of the hemolytic action. Like the prototoxin of diphtheria poison, this substance is readily changed to prototoxoid, and in undergoing this transformation it loses its toxicity but retains its combining power.

7. The deuterotoxin makes up one-ninth of the poison but possesses two-fifths of the total hemolytic action. This substance resembles the deuterotoxin of the diphtheria poison and is relatively resistant to untoward influences.

8. The tritotoxin makes up one-fourth of the poison but possesses only one-tenth of the poisonous action. While the prototoxin and deuterotoxin act both under high and low temperatures, the tritotoxin has no action at a temperature below $10^{\circ}$. Special experiments show that the tritotoxin combines with the red blood corpuscles not only in smaller quantity than the other toxins, but that its toxophorous group is much less powerful than that of the proto- and deuterotoxins.

9. The second half of the toxin possesses very minute toxicity and is known as toxon.

10. The most important result of the experiments lies in the determination that in tetanolysin, as in diphtheria toxin, there are two groups, a haptophorous group by which the substance combines with antitoxin, and a toxophorous group by which the combination with the red blood corpuscles and the hemolytic action are effected. Of these groups the first one is relatively stable while the latter is easily modified and in this process of modification the toxin becomes a toxoid.

11. The probabilities are that tetanospasmin has a neutralization structure similar to that possessed by tetanolysin. 
By separating the blood corpuscles, which have been exposed to the action of varying quantities of tetanolysin for different periods of time, in a centrifuge, and subsequently treating them with tetanus antitoxin, Madsen ${ }^{1}$ has shown that the poison can be separated from the corpuscles provided a sufficient quantity of antitoxin is added before hemolysis occurs. In this way he has demonstrated that the antitoxin may act as a curative agent, as well as a preventive.

Ransom ${ }^{2}$ has shown that both the toxin and antitoxin of tetanus pass without change from the blood into the lymph. Shortly after the intravenous injection of the toxin it is found equally distributed between the blood and the lymph, while the antitoxin remains in comparative excess in the blood. From these observations it is concluded that the toxin behaves like inorganic substances in passing from the blood into the lymph, while the antitoxin resembles more closely the proteids in this respect.

Bruschettini has studied the distribution of the tetanus poison in the body and its elimination; animals poisoned by injections of the toxin were killed just before death, and bits of various organs rubbed up with sterilized water were injected into other animals. Emulsions from the liver and suprarenal capsule were invariably without effect, while those from the kidney were constantly poisonous. The blood taken from the vena cava was found to be poisonous in three out of four experiments. When injections were made under the skin the lumbar cord was active in four out of eight cases, and in all when the injections were made directly into the sciatic nerve. When the inoculations were made under the dura mater, the brain was found to be active, while the lumbar cord remained inactive; it follows from this that the toxin not only circulates in the blood, but is deposited in the central nervous system. This author has also shown that tetanus toxin is eliminated by the kidneys. Bruner in several cases of tetanus in man, Stern in two, and Brieger in one case, were not able to induce tetanus in animals by injecting even large amounts of the urine of their patients. This does not cast doubt upon the accuracy of the report of Bruschettini ; it only shows that the poison is not in all cases eliminated by the kidney in sufficient quantity to render the urine highly toxic. In a fatal case, Vulpius failed to induce tetanus with the urine voided during life, but succeeded with that found in the bladder after death.

Dönitz ${ }^{3}$ has shown that tetanus toxin combines almost instantaneously with the cells of the central nervous system. In his experiments he employed twelve times the minimum lethal dose of toxin, and injected this directly into a vein of the ear. He first determined the amount of antitoxin which, mixed with this quantity of toxin in

1 Zeitschrift für Hygiene, 32, 239.

${ }^{2}$ Zeitschrift für physiol. Chem., 29, 1900.

${ }^{3}$ Deutsche med. Wochenschrift, 1897, 428. 
vitro, was necessary for complete neutralization. When the toxin in this amount was injected intravenously and the corresponding amount of antitoxin injected into the same vein of the opposite ear within two minutes, the animals invariably died of tetanus. In order to save his animals treated with this amount of toxin it was found necessary to nearly double the dose of antitoxin when injected within two minutes; while if four minutes elapsed between the injection of the toxin and the antitoxin, six times the quantity necessary for neutralization in vitro was required, and when this time was extended to one hour, twenty-four times this amount was necessary. Of course, in the disease the toxin is generated slowly and in relatively small amounts; therefore, the proportion between toxin and antitoxin necessary to neutralize the effects of the former is not found to be so great. The same experimenter demonstrated that when tetanus is induced by inoculation with the bacillus, the antitoxin may, experimentally at least, serve as a curative agent. He has also shown that animals treated with very small quantities of tetanus toxin may die of marasmus, and thus there may be such a thing as tetanus sine tetano. This has been observed in some other infectious diseases.

Wassermann and Takaki, ${ }^{1}$ have shown that tetanotoxin combines with nervous tissue, forming a compound which, when injected into susceptible animals, is found to be inert. They prepared a solution of tetanotoxin, to which an equal part of glycerin was added for preservation purposes. This solution was of such strength that one thousandth of a cubic centimeter sufficed to kill mice in the course of three days. Quantities of this solution, containing from one to ten fatal doses were rubbed up into emulsions with the substance of the spinal cord or brain, and this mixture was injected subcutaneously into mice. As a control, equal quantities of tissue from the liver, kidney, spleen, and bone marrow were mixed in the same way with the tetanotoxin and likewise injected into mice. As a further control, corresponding amounts of tetanus poison without admixture with any tissue were injected. In the preparation of the emulsions the organs were taken from animals immediately after death and rubbed up in a mortar with physiological salt solution. For the cord from a guinea-pig, 3 c.c. of salt solution were used, and for the brain from the same animal, 10 c.c. were employed. By these experiments it was conclusively demonstrated that the normal cord and brain form an inert compound with the toxin of tetanus, while none of the other organs has any such effect. It was found from about two hundred experiments that one cubic centimeter of the brain emulsion neutralizes ten fatal doses of the tetanus toxin, and has a marked inhibitory action on several times this amount. The antitoxic action of the cord was found to be less marked than that of the brain substance. This is somewhat unexpected, inasmuch as the symptoms manifested

${ }^{1}$ Berliner klinische Wochenschrift, 35, 1898. 
in tetanus are supposed to be referable to the action of the toxin on the cord rather than on the brain. These experiments were repeated with brain and cord substance obtained from pigeons, rabbits, horses, and men with practically the same results in all instances. By means of the centrifuge, the brain emulsion with the toxin was separated into a deposit and a clear supernatant fluid, and it was demonstrated by this means that the combined toxin and brain substance was to be found in the deposit. In other words, it was shown that the supernatant fluid had no antitoxic action. The same was found to be true of fluids obtained from the ventricles of the brain. These experiments seem to demonstrate that chemical combination takes place between the toxins introduced into the body and the formed cells, and that solution of both substances entering into the compound is not essential.

Roux and Borrel, ${ }^{1}$ have demonstrated by a series of carefully conducted experiments that small doses of tetanus toxin when injected directly into the brain substance kill animals, while much larger quantities of the same poison are required when the injection is made subcutaneously or intravenously. This undoubtedly is due to the fact that when intracerebral injection is made, all of the toxin immediately combines with the side chains of the brain cells, inflicting upon this tissue an injury to which the animal speedily succumbs.

According to Ledantec, the poisonous arrows of the natives of the New Hebrides are prepared as follows : The points, which are usually made from human bones, are first covered with a vegetable resin, then smeared with the slime of swampy places.

Roncali has tested forty different germs, some of them pathogenic and others non-pathogenic, in endeavoring to find one which would neutralize, either by its growth in the body or by the action of its products, the tetanus poison. His results were wholly negative. The tetanus poison was found to act more energetically in animals inoculated with other bacteria or treated with their products, and in no case was there any evidence of antagonism in action.

Diphtheria.-In 1887, Loeffler attempted to ascertain the nature of the diphtheria toxin. A flask of bouillon containing pepton and grape sugar was, three days after it had been inoculated with the bacillus, evaporated to 10 c.c. and this was injected into an animal, but was without effect. A second flask of the same material was extracted with ether, but this extract was also found to be inert. Next, some neutral beef broth was extracted with glycerin four or five days after it had been inoculated with the bacillus. The glycerin extract, when treated with five times its volume of absolute alcohol, deposited a voluminous, flocculent precipitate, which was collected, washed with alcohol, dried and dissolved in water. Furtber precip-

${ }^{1}$ Annales de l' Institut Pasteur, 12, 1898. 
itation with alcohol and a current of carbonic acid gas, secured a white substance, and the injection of from 0.1 to 0.2 gram of this substance subcutaneously in guinea-pigs, caused marked pain, followed by a fibrinous swelling with hemorrhages into the muscles and cedema, terminating in necrosis. From these studies Loeffler concluded that the poison belongs to the enzymes.

Roux and Yersin found that bouillon cultures from which the bacillus had been removed by filtration through a Chamberland filter are poisonous, especially cultures that are four or five weeks old. The results obtained varied with the amount of the fluid, the species of animal, and the method of administration. The effects obscrved were a serous exudate into the pleural cavity, marked acute inflammation of the kidney, fatty degeneration of the liver, especially after injection into a blood vessel, and oedematous swelling in the surrounding tissue after subcutaneous inoculation. In some instances, paralysis, generally in the posterior extremities, followed. The action of the poison was slow, and death, as a rule, occurred days, and in some cases, weeks after treatment, and was preceded by marked emaciation. The cultures first employed were seven days old; older cultures (six weeks) contain more of the toxin, and the symptoms appear within a few hours. In cultures especially rich in the poison, a small amount (from 0.2 to 2 c.c.) injected under the skin in guinea-pigs, suffices to induce the symptoms. Heating to $100^{\circ}$ for twenty minutes renders the poison inert, and a temperature of $58^{\circ}$ maintained for two hours markedly lessens its virulence. The toxin is precipitated by absolute alcohol, and is carried down mechanically by the addition of calcium chlorid to the filtered cultures. The great toxicity of this substance is indicated by the statement of Roux and Yersin, that $0.4 \mathrm{mg}$. suffices to kill eight guinea-pigs or two rabbits ; and that $0.02 \mathrm{~g}$. of the calcium chlorid precipitate, containing about $0.2 \mathrm{mg}$. of the pure poison will kill a guinea-pig within four days.

Brieger and Fränkel employed cultures of bouillon and pepton containing from five to six per cent. of glycerin, and others containing two per cent. of blood serum; the latter were found to be most suitable. In all cases they confirmed the statement of Roux and Yersin, that the cultures, at first alkaline, become strongly acid, and finally again alkaline, with the exception that the glycerin cultures remain acid. For the removal of the bacteria two methods were employed. First, the bacilli were destroyed by heat; when a temperature of $100^{\circ}$ was employed the cultures became inert, but it was found that exposure for from three to four hours to $50^{\circ}$ was sufficient to destroy the germ, while the virulence of the toxin was not affected. The second method of removing the bacteria consisted in filtration through a Chamberland filter. The germ-free filtrate could be heated to $50^{\circ}$ without loss of toxicity, while a temperature of $60^{\circ}$ rendered 
it inert. In the majority of the experiments the filtration method was used, and in this way a large quantity of a poisonous fluid of uniform strength was obtained. Varying amounts of this fluid were used upon animals, mostly guinea-pigs and rabbits, and it was found that the effects depended upon the quantities employed and the methods of administration. In all cases in which death did not occur too early, paralysis appeared. The limbs were first paralyzed, and this was true whether the fluid was administered intravenously or subcutaneously. The post-mortem appearances were identical with those observed after inoculation with the bacillus, with the exception of the absence of the pseudo-membrane. After subcutaneous injection there was a gelatinous, grayish-white, sometimes reddish, cedematous fluid, formed at the point of injection, and, after large doses, necrosis. In cases in which death was delayed, there were effusions in the pleura, fatty degeneration of the liver, and inflammation of the kidneys.

Brieger and Fränkel conclude this part of their report with the following statement: "We have shown that the Loeffler diphtheria bacillus produces in its cultures a poisonous, soluble substance, separable from the bacteria, which causes in susceptible animals the same phenomena induced by inoculation with the living micro-organism. We have further shown that this substance is destroyed by a temperature over $60^{\circ}$, but that it can be heated to $50^{\circ}$, even in the presence of an excess of hydrochloric acid, without being destroyed. This last fact is contrary to the assumption that the chemical poison of the diphtheria bacillus is a ferment or an enzyme."

The filtered cultures were tested for basic products, but with negative results, excepting that small amounts of creatinin and choliu were found. They were also distilled at from $20^{\circ}$ to $35^{\circ}$ in a vacuum, but the distillate was found to be inert. The toxin is soluble in water, insoluble in alcohol, and non-dialyzable. It is precipitated by saturation with ammonium sulphate. As a result of their first work on this subject, Brieger and Fränkel concluded that the diphtheria toxin is a toxalbumin; however, they, as well as others, have since learned that while the body obtained by them contained the toxin, the bulk of it was made up of other substances.

Taugl has shown experimentally that the toxin is formed in the body as well as in culture flasks. A large piece of pseudo-membrane was macerated in an ice-chest in water for twenty-four hours, and then filtered through porcelain. The filtrate, injected into animals, produced all the symptoms that had been obtained by similar employment of artificial cultures. The same observer found that in some cases in which the animals were inoculated with the sterilized culture through the mucous membrane a pseudomembrane formed at the point of injection. Diphtheria toxin has also been found in the tissues, blood, and urine. 
While crude diphtheria toxin, in the form of sterilized cultures of the bacillus, has been used in securing immunity and in the production of antitoxin, which has proved to be of such priceless value in the treatment of the disease, no one has up to the present time been able to isolate the poison, and we remain ignorant of its chemical constitution.

From a large number of most carefully conducted experiments with the toxin and antitoxin of diphtheria, Ehrlich ${ }^{1}$ has formulated a theory concerning the constitution of the former. This theory has undergone several modifications since it was first proposed, and it is difficult to give an exact statement of it as it now stands. However, we will attempt to state in condensed form its essential points as follows : ${ }^{2}$

1. Toxins and antitoxins neutralize one another after the manner of chemical reagents. The chief reasons for this belief lie in the observed facts $(a)$ that neutralization takes place more rapidly in concentrated than in dilute solutions, and (b) that warmth hastens and cold retards neutralization. From these observations Ehrlich concludes that toxins and antitoxins act as chemical reagents do in the formation of double salts. A molecule of the poison requires an exact and constant quantity of the antitoxin in order to produce a neutral or harmless substance. This implies that a specific atomic group in the toxin molecule combines with a certain atomic group in the antitoxin molecule.

2. The antitoxin is a reaction product of the living organism and not a transformation product of the toxin introduced in securing immunity. It is thought that when the toxin is introduced into the animal body in small quantities it combines with certain side-chairs in the molecules of the living cells. These side-chains are supposed to be necessary for the proper functioning of the cells, which, finding themselves deprived in part of their function on account of combination with the toxin, produce other and similar atomic groups or side-chains, and these being formed more rapidly than they are taken up by the toxin, are cast off into the blood and constitute the antitoxin. For instance, when a small quantity of tetanus toxin is introduced into the animal body it combines with certain side-chains in the molecules of the cells of the central nervous system and renders these atomic groups useless so far as the function of the cell is concerned. The cell, in order to compensate for its loss, produces another side-chain similar to the one of which it has been deprived. Being called upon repeatedly to exercise this activity, there is not only compensation, but over-compensation, and the result is that more side-chains are formed than the cell can use, and these break off and lagen.

${ }^{1}$ Die Wertbemessung des Diphtheriaheilserums und deren theoretische Grund-

${ }^{2}$ For a more detailed statement of Ehrlich's theory see Chapter VII. 
float away in the blood, constituting the antitoxin. Moreover, the atomic group or side-chain, after being liberated from the cell, may possibly acquire greater avidity for combination with the toxin; or, in other words, the toxin will combine more readily with these sidechains when free and floating in the blood than when they constitute parts of the molecules of the cells.

3. The diphtheria toxin, as it exists in sterilized cultures, is composed of equal parts of toxin and toxon. The toxon, which according to this theory is supposed to exist in the diphtheria culture, is believed to be without any serious effect upon animals. It may cause local œdema, but never kills. Ehrlich's theory supposes that the toxon, which we may regard as inert, has quantitatively the same power of combination with antitoxin as is possessed by the toxin, but combines with the antitoxin with less avidity. In order to understand why the theory provides for the existence of toxin and toxon in equal quantities in the diphtheria culture, we will use certain formulæ proposed by Ehrlich:

$T=$ the minimum fatal dose of the toxin (a quantity sure to kill a guinea-pig of 250 grams weight within five or six days).

$I=$ one immunity unit which is capable of neutralizing the effects of $100 \mathrm{~T}$.

Now, if $I$ be mixed with $100 T$ and injected into an animal, no ill effect results. If the toxin were a simple body, $I$ plus $100 T$ plus $T$ should kill one animal. But experimentally we find that this amount has no effect, and must be greatly increased before there is enough free toxin in the mixture to kill. Ehrlich represents the quantity of poison necessary to neutralize $I$ by $L_{0}$. Then $I$ plus $L_{0}$ is a mixture which contains neither free toxin nor free antitoxin and is wholly without effect upon animals. By $L_{+}$he indicates the quantity of poison which must be added to $I$ in order to kill a guinea-pig of 250 grams weight within five or six days. Let us suppose that in a given culture $T=0.01$ c.c., then $I=1.00$ c.c. $=100 T . \quad L_{0}=$ 1.00 c.c. $=100 \mathrm{~T}$. Now, if this were a poison like strychnia we would expect that $L_{+}=1.01$ c.c. $=101 T$, would kill, but in reality, as has been stated, we find that we have to add more of the toxin to $L_{0}$ in order to produce fatal results with the mixture, and we have theoretically the following:

$$
\begin{aligned}
T & =0.01 \text { c.c. } \\
L_{+} & =2.01 \text { c.c. }=201 \mathrm{~T} . \\
L_{0} & =1.00 \text { c.c. }=100 \mathrm{~T} . \\
D & =1.01 \text { c.c. }=101 \mathrm{~T} .
\end{aligned}
$$

As here figured out, Ehrlich's theory which provides for the existence in the crude toxin of equal parts of toxin and toxon, is the only possible explanation. In $L_{0}$ both toxin and toxon are fully 
combined with antitoxin. When more toxin is added the antitoxin is dissociated from the toxon and combines with the toxin because it has greater affinity for the latter substance. This continues until all the antitoxin is separated from the toxon and combined with the toxin, and there can be no free toxin until this point is reached. Practically, however, while the difference between $L_{+}$and $L_{0}$ is al ways more than 1 , it is never as great as 101 . Therefore, we must conclude that while there is reason for beliering that there exists in diphtheria cultures substances corresponding to Ehrlich's toxin and toxon, the facts do not justify the statement that these bodies exist in equal quantities. Moreover, as has been pointed out by Park and Atkinson, the standard for $T$ is arbitrary and it might have been placed at the amount necessary to kill a guinea-pig of 250 grams within ten days, or to cause death from paralysis within two or three weeks. The same critics state: "Even if the toxin molecule be divided as he (Ehrlich) believes, still in any given bouillon there is actual destruction, as well as production, going on all the time of all substances, not only those toxic to guinea-pigs, but also of those which, though non-toxic, still neutralize antitoxin."

4. It is observed frequently that while the toxicity of a given sterilized culture decreases on standing, the quantity of antitoxin which it will neutralize remains constant. This observation has caused Ehrlich to provide in his theory for the toxoids which have already been mentioned (p. 35).

Flexner ${ }^{2}$ has studied quite minutely and exhanstively the pathological lesions induced by certain bacterial toxins. Concerning the gross appearances after death from subcutaneous or intraperitoneal injections of sterilized cultures of the diphtheria bacillus, he makes the following statement: "Much œdema, sometimes accompanied with small extravasations of blood and often of an exquisite gelatinous type, was noted. The axillary and inguinal glands were always, often greatly, enlarged and were congested or thickly dotted with hemorrhages within the substance of the capsule and the glandular tissues. The corresponding lymph glands on the opposite side were also enlarged, but usually less so than those on the side of the inoculation. This enlargement involved the superficial and deep sets. The cervical glands along the carotids and trachea to the maxillæ also showed an increase in size. The thyroids were without exception deep brownish-red in color. The thymus gland presented a rosy, and sometimes, owing to hemorrhages, a speckled appearance ; the bronchial and pericardial glands, often very difficult to find in the healthy animal, were quite prominent; the mediastinal glands were swollen and congested or ecchymotic. The mesenteric and retroperitoneal glands were also enlarged, and sometimes considerably

1 Experimental Medicine, 3, 513.

${ }^{2}$ Johns Hopkins Hospital Reports, 6, 259. 
softened. The patches of Peyer in the intestine at times showed up with a prominence sufficient to enable them to be detected through the serosa of the intestine. The peritoneal cavity often contained an excess of clear fluid; hydrothorax also occurred, and in rare instances reached such a high degree as to completely compress both lungs, the apices alone being spared and insufflated. The pericardial sac was also unduly distended with a clear serous fluid. The adrenal glands presented a reddish color externally, and on section the medulla appeared deeply congested or even hemorrhagic. The kidneys were often pale. The liver was, as a rule, congested and exhibited upon its surface and within its substance a variable number of yellowish or yellowish-white areas, some not larger than a pin-point, others 2 $\mathrm{mm}$. in extent, which were usually surrounded by a hyperemic zone. In some cases a few of such foci could be discovered with the naked eye ; in others they were almost innumerable. The spleen, as a rule, was not greatly enlarged, it was more often pale than congested, and the Malpighian bodies showed distinctly. The lungs varied in appearance, the difference depending apparently not so much upon any pathological alterations of which they were the seat, but rather upon the quantity of fluid present in the pleural cavities. The heart was pale, the chambers, especially of the right side, were distended with dark, clotted blood. Subserous hemorrhages, especially in the peritoneal cavity, were an interesting but variable feature of the disease."

The same observer made a close microscopical study of the various tissues of animals treated with crude diphtheria toxin. In the lymph glands, the earliest changes were found to consist of swelling of the nucleus and perhaps also of the protoplasm. Under larger doses of the poison the nuclei were often observed to be homogeneous and seemed converted into granular balls of variable size, staining evenly and intensely but exhibiting a metachromatic reaction. Under still larger doses, the nuclei were observed to undergo disintegration. The fragmentation was irregular, indicating the powerful effect of the toxin. In some cases the detritus was seen within the protoplasm of the original cell, while in others the fragments had become free, owing to the destruction of the cell body. Necrobiotic foci, such as have been described by Oertel after death from diphtheria, were observed in the lymph glands in certain cases. The spleen was generally hyperemic, the excess of blood being contained within the sinuses of the pulp. The earliest changes were found to consist of swelling of the cells of the Malpighian bodies. Mitotic division was observed in the cells but did not seem to progress actively. Pathological alterations were found in the pulp affecting the framework and the vascular contents. In the intestines, the most pronounced changes were encountered in the epithelium. These aiterations consisted of proliferation by mitosis and of degeneration. There was extensive fragmentation of cells within the crypts of 
Lieberkühn. In Peyer's patches the changes were similar to those observed in other lymphatic structures. There was no evidence of denudation of the surface in any part of the alimentary tract. The mucosa of the stomach showed only insignificant lesions. The surface of the liver often exhibited small yellow, opaque points and lines which upon section were found to extend down into the organ. These points and lines indicate focal degeneration and death of liver cells. In the majority of instances the liver was congested and showed more or less evidence of fatty metamorphosis. "The kidneys were not invariably the seat of fatty changes, but it was the rule to find more or less fat (1) in the convoluted tubules of the labyrinth, (2) in the straight tubules, and (3) in the glomeruli. In some instances the fatty metamorphosis of the epithelial cells was extreme. In all cases, practically, the cells were much swollen and coarsely granular, the proteid granules being combined with fat. In rare instances a deposition of lime salts was noted in the straight tubules. These deposits yielded carbonic acid gas on the addition of strong acids. Among the most interesting changes observed was a hyaline transformation of the glomerular capsules and smaller arteries." The lesions observed in the lungs were confined almost exclusively to the vessels, the endothelial cells of the pulmonary branches and the vesicular capillaries showed fragmentary degeneration. The adrenals were markedly congested with occasional hemorrhages into the tissues, and the same condition was observed in the thyroid gland. Fatty metamorphosis was the most common pathological condition found in the myocardium. The alterations observed in the muscle substance of the heart affected both the nuclei and the protoplasm, being most marked in the former. "The earliest and most common appearance consists of swelling and elongation of the nuclei and alteration in their shape, the fibers in the meantime showing little change. Certain nuclei later assume a deeper color, owing chiefly to an intense staining of three or four or more globular bodies in their interior; but soon afterwards the nuclear membrane either degenerates or becomes invisible, and the bodies become free and appear as fragments. The substance of the fibers belonging to the affected nuclei has in the meantime quite disappeared or takes on a swollen and an attenuated aspect."

Councilman, Mallory, and Pearce' have made a most exhaustive study of the gross and microscopical lesions found in man after death from diphtheria. Many of these pathological changes are evidently due to the toxin because they are found in parts of the body not reached by the bacillus. In the lymph nodes the most characteristic lesion was found to consist in the formation of discrete foci due to cell proliferation combined with necrosis. In all cases they observed

'Diphtheria, A Study of the Bacteriology and Pathology of Two Hundred and Twenty Fatal Cases, 1901. 
lesions in the kidney varying from simple degeneration to the more serious condition of acute nephritis. The more severe forms of degeneration were observed in cases which, on account of the intensity of the poison, succumbed shortly after the onset of the disease. The lesions in the human liver differ from those produced experimentally in animals chiefly in the greater frequency of the central situation of the necrosis. The changes in the intestines consist principally of hyperplasia of the lymphoid structures. "The slight extent of the lesions does not indicate the action of toxins absorbed from the alimentary canal ; they are probably due to the action of toxins from the blood principally. There is nothing in the character of the lesions to indicate the elimination of the toxin by the alimentary canal." In the heart fatty degeneration was observed in severe cases of short duration and more extensive degenerations in more prolonged cases. The pathological changes found in the heart are often sufficient to account for the impairment of this organ so greatly feared in this disease. Thrombosis occurs frequently and is due to primary necrosis of the endocardium. The changes observed in the lungs in man after death from diphtheria are largely due to other microörganisms, among which the pneumococcus is probably the most important.

Tuberculosis.-In 1865, Villemin demonstrated the infectious character of this disease by inducing it in animals by feeding them upon tuberculous sputum and tissues. In 1868, Chauveau, and, a few years later, Cohnheim experimentally confirmed the discovery of Villemin, which must be regarded as one of the most important contributions to medical knowledge made during the nineteenth century. In 1878 , Tappeiner showed that tuberculosis might be transmitted by the inhalation of infected dust. However, the nature of the infecting agent in tuberculous tissue remained unknown until 1882, when Koch, after a most exhaustive research covering different manifestations of tuberculosis in man and some of the lower animals, announced the discovery of the specific bacterium of this disease. This work was so thoroughly done that practically every statement made by Koch in his first report stands to-day unchallenged. The twenty years that have elapsed since that time have each brought its confirmations of the facts then recorded. It is not within the province of this book to discuss in any detail the bacterium of tuberculosis, and it must suffice to say that the causal relation of the bacillus tuberculosis to the disease is not now questioned by any competent authority. Every case of tuberculosis is due to infection from a preexisting case in man or beast. We will concern ourselves wholly with the chemical poisons produced by this bacterium and by virtue of which the symptoms of the disease and death are induced. We may be permitted, however, to call attention to the fact that in its later stages tuberculosis becomes a mixed infection and the chemical 
products of more than one bacterium constitute the causal factors in this form of poisoning.

Koch's tuberculin, with which he hoped to cure the disease, is the crude poison formed by his bacillus and is known as tuberculin. The methods of preparing this substance have varied somewhat and we will mention some of them. Koch prepared tuberculin in the following manner : Meat infusion containing one per cent. of pepton and from four to six per cent. of glycerin is placed in sterilized flasks with broad bottoms. The flasks are only partially filled in order that the surface of the fluid should be as great as possible. A small mass of a growth of tubercle bacilli is taken from a culture on glycerin agar or blood serum, and floated on the surface of the meat infusion in the flask, which is then placed in an incubator at $37^{\circ}$. The bacilli grow abundantly on the surface of the meat infusion, forming a thick, yellowish-white layer. After about six weeks, growth stops, the bacterial layer begins to break into pieces and these fall to the bottom of the flask. The culture is now evaporated to one-tenth its volume on the water-bath. The concentration increases the per cent. of glycerin to from forty to fifty, and this ingredient prevents the growth of extraneous bacteria and renders the fluid permanent for an indefinite time. After filtration through porcelain, this fluid constitutes the crude tuberculin of Koch. It will be seen that it must contain, in addition to the water and glycerin, any other soluble, unchanged constituent of the original meat infusion, any split products, if there be such, arising from the cleavage action of the bacilli on the components of the culture medium, and all soluble constituents of the bacterial cells. The toxin in this impure form is not destroyed by the temperature of the water-bath. Ultimate analyses of the crude tuberculin have been made, but it must be evident from what has just been said concerning the complexity of its composition that such determinations are without value. It does not contain any ptomaïn or other basic body.

Bujwid obtained tuberculin by extracting the growths of the bacillus on glycerin agar tubes, heating to $100^{\circ}$ for ten minutes, filtering through porcelain and concentrating at a low temperature. As thus prepared, the fluid resembles very much the preparation already described.

Tuberculin may also be obtained from bacilli grown on potatoes. The freshly cut surfaces of the sterilized potatoes are washed with a 1 per cent. sterilized solution of sodium bicarbonate, then moistened with sterilized water containing five or six per cent. of glycerin. On potatoes thus prepared the bacillus grows abundantly at a temperature of $37^{\circ}$. After further development has ceased, the growths are extracted with water or water and glycerin.

In $1897 \mathrm{Koch}^{1}$ reported his attempts to improve the preparation

${ }^{1}$ Deutsche med. Wochenschrift, 1897, 209. 
of tuberculin. At first he extracted the bacilli with a decinormal solution of caustic soda. This extract, when filtered through paper and injected into animals, induced a reaction similar to that which followed the use of the original preparation; but it was found that abscesses were likely to follow the employment of this material. It was also observed that it contained tubercle bacilli and for this reason it was filtered through porcelain. The filtrate was germ-free, but was found not to possess the reaction of tuberculin. It was thus evident that filtration through porcelain had removed not only the bacilli, but the active constituent of the preparation. In his further researches with the bacillus, Koch was able to extract two characteristic chemical substances, both of which belong to the unsaturated fatty acids. One of these is freely soluble in dilute alcohol and is readily saponified with alkalis, while the other is soluble only in boiling absolute alcohol and saponifies with difficulty. Both of these substances take the so-called tubercle stain, $i$. e., they are stained intensely red with carbolic fuchsin and retain this color on treatment with dilute nitric acid and with alcohol. Bacilli from which these fatty bodies have been extracted retain their form, but no longer take their characteristic stain.

Finally, Koch prepared his improved tuberculin in the following manner: The bacilli of fresh, highly virulent cultures are dried and rubbed into a very fine powder, which is then added to distilled water with which it forms a colloidal mixture. This aqueous preparation is placed in a centrifuge which makes four thousand revolutions per minute, and after half an hour it separates into an upper, opalescent but transparent layer, which contains no bacilli, and into a deposit. The deposit is dried, again rubbed up in a mortar, mixed with water and centrifuged, and this is repeated until there is obtained a perfectly clear fluid. The upper layer obtained the first time in the centrifuge Koch designated as $T O$, while the upper layers obtained by subsequent treatment in the centrifuge he distinguished as $T R$. The addition of 50 per cent. glycerin to $T O$ causes no change, while in $T R$ it produces a flocculent white precipitate. This indicates that $T R$ contains those substances which are insoluble in glycerin, while TO is made up of the constituents of the bacterial cells soluble in this reagent. In its action upon man and other animals, $T O$ resembles the original tuberculin while $T R$ is supposed to have the curative action first attributed to tuberculin without any of its ill-effects. Koch stated that with $T R$ men and animals could be so immunized that they did not react to $T O$ or crude tuberculin. $T R$ is preserved with the addition of 20 per cent. of glycerin.

De Schweinitz ${ }^{1}$ isolated from liquid cultures of the bacillus tuberculosis a crystalline substance having a melting point of $161^{\circ}$ to $164^{\circ}$, readily soluble in ether, alcohol and water. Analysis showed

1 Transactions of the Association of American Physicians, 1897. 
that this body corresponds closely to teraconic acid and its discoverer believes that the necrotic effects observed in tuberculosis are due to this agent. It is also a fever-reducing substance, while the albuminoid obtained from cultures of the tubercle bacillus causes an elevation of temperature.

Klebs ${ }^{1}$ states, as a result of his investigations, that the tubercle bacillus contains two fatty bodies. One of these may be extracted with ether; it has a reddish color, melts at $42^{\circ}$, and constitutes 20.5 per cent. of the weight of the bacillus. The other fat is insoluble in ether, but may be extracted with benzol; its melting point has not been accurately determined, but is something over $50^{\circ}$, and it constitutes 1.14 per cent. of the substance of the germ. The specific coloration of tubercle bacillus with carbolic fuchsin is due to the presence of these fatty bodies, and after their removal the bacilli, although retaining their form, fail to stain. The greater part of the tubercle bacillus, after the removal of the above-mentioned fats, consists of a nuclein, which may be purified after digestion with pepsin and hydrochloric acid, by solution in dilute alkalis and precipitation with alcohol. In this way Klebs obtained a nuclein which yielded between 8 and 9 per cent. of phosphorus. The third important constituent of the tubercle bacillus is the glycerin-water extract, which probably consists of a mixture of substances, some of which are proteid in character.

Ruppel ${ }^{2}$ thinks that there are three kinds of fatty substances in the tubercle bacillus. The first constitutes about 8 per cent. of the total weight of the organism, from which it can be removed with cold alcohol. During the process of extraction the alcohol becomes intensely red, and this is supposed to be due to a chromogen contained in the bacillus, which develops into the coloring matter on exposure to air. On evaporation of this alcoholic extract there remains a smeary mass, consisting largely of free fatty acid. When the free fatty acids are removed by the ordinary method of treatment with soda solution and ether, the mass obtained on the evaporation of the ether melts between 55 and $60^{\circ}$. This substance is easily saponified, and contains along with the free fatty acids another substance soluble in ether, which is believed to be one of the higher alcohols. The second form of fat contained in the bacillus may be extracted from the residue left after extraction with cold alcohol, by means of hot alcohol. This substance begins to liquefy at $65^{\circ}$, but does not become altogether clear until a temperature of $200^{\circ}$ is reached. The hot alcoholic extract saponifies with difficulty, and appears to consist of fatty esters of the higher alcohols. The third fatty substance may be removed by means of ether. It melts at from 65 to $70^{\circ}$, and on being heated gives off an odor similar to that of

${ }^{1}$ Centralblatt f. Bakteriologie, $20,{ }^{7} 488$.

${ }^{2}$ Zeitschrift f. physiol. Chemie, 26, 1899. 
beeswax. The total amount of fatty substances contained in the tubercle bacillus varies from 8 to 26 per cent.

According to Ruppel, the soluble albuminous substances contained in the tubercle bacillus are best obtained by the action of superheated steam on bacilli previously deprived of their fatty content. The procedure recommended is as follows : The bacilli are first extracted with a 1 per cent. soda solution or heated with a dilute solution of glycerin, thoroughly extracted with alcohol and ether, dried and rubbed into a fine powder, which is then treated with ten times its volume of 5 per cent. glycerin solution and kept in the autoclave at $150^{\circ}$ for about two hours. The extract thus obtained is filtered while still hot, and it is found that the filtrate, at first clear, forms a deposit on stauding, and complete separation may be obtained in the centrifuge. The soluble proteids obtained in this way constituted from 18 to 20 per cent. of the total weight of the bacilli, and consisted exclusively of albumoses analogous to the atmidalbumose of Neumeister. The solution of mixed atmidalbumoses is dark in color and may be precipitated by ammonium sulphate, sodium chlorid, or, best of all, with absolute alcohol after the addition of a small amount of sodium chlorid and hydrochloric acid. On drying, the alcoholic precipitate is found to consist of a light powder, which, as has been stated, constitutes about 20 per cent. of the fat-free bacilli.

Ruppel finds that when tubercle bacilli have been finely broken up, they give up about half their substance to aqueous extracts. When finely divided tubercle bacilli are shaken in water there is formed a milky emulsion, and this, when placed in the centrifuge, separates into a transparent, feebly opalescent, yellowish fluid, and a deposit. When this deposit is dried and weighed it is found to constitute about half the weight of the powdered bacilli originally taken. The reaction of the fluid portion is generally feebly alkaline, but may be neutral. It contains no coagulable proteid, and of the general color reactions, it responds only to the biuret test. Acetic acid produces a considerable precipitate, which is not soluble in excess, but which dissolves in dilute alkalis, from which it may be precipitated anew on the addition of acids. The acetic acid precipitate contains a little more than 4 per cent. of phosphorus. It responds to neither the Millon nor the xanthoproteic test, but the biuret reaction is positive. When dissolved in glacial acetic acid and warmed with sulphuric acid, it does not give a red coloration. About 25 grams of this substance was shaken with 1 per cent. sulphuric acid, and the acid extract thus obtained was precipitated by the addition of absolute alcohol. The precipitate was found to consist of a snow-white, flocculent powder, and the amount obtained was 0.7 of a gram. It is soluble in warm water, from which it partially deposits on cooling. When dissolved in warm water, the 
addition of barium hydrate precipitates barium sulphate, which may be removed by filtration and the filtrate precipitated with alcohol. The substance thus obtained does not respond to any of the color reactions for proteids with the exception of the biuret test. Ruppel believes it to be a protamin, and proposes for it the name, tuberculosamin. He believes that in the tubercle bacillus this protamin is combined with nucleinic acid, and, indeed, from the part insoluble in 1 per cent. sulphuric acid he obtained a nucleinic acid which contains 9.42 per cent. of phosphorus. He proposes that the nucleinic acid in the tubercle bacillus be known as tuberculinic acid.

According to Behring, ${ }^{1}$ tuberculinic acid, as prepared by Ruppel, contains a histon-like body, on the removal of which the tuberculinic acid is obtained chemically pure. Behring states that the nucleinic acid obtained from tubercle bacilli, or tuberculinic acid, is quite different from all other nucleinic acids. To this substance he attributes the toxic action of tubercle bacilli, and he states that one gram of tuberculinic acid is capable of destroying the life of 600 grams of normal guinea-pig when injected subcutaneously, and 90,000 grams when introduced intra-cerebrally, and that the same amount of tuberculinic acid is capable of destroying the life of 60,000 grams of guinea-pig, already infected with tuberculosis, when administered subcutaneously; and 40,000,000 grams of the same tissue when injected intra-cerebrally. In distinguishing physiologically between tuberculinic and other nucleinic acids, tuberculous animals should be selected on account of their greater susceptibility to the tuberculinic acid.

Levene, ${ }^{2}$ agrees with Ruppel that the tubercle bacillus contains both free and combined nucleinic acid, and the following is his statement concerning the method of obtaining both of these: "With the view of obtaining the free nucleic acid, neutralized, dried and pulverized bacilli were repeatedly extracted with a 5 per cent. sodium chlorid solution, and an 8 per cent. ammonium chlorid solution. The extracts obtained were then treated with picric acid and acidulated with acetic acid. To the filtrate from this precipitation alcohol was added, and the precipitate thus formed redissolved in water and reprecipitated with alcohol. The perfectly white precipitate was redissolved in water, slightly acidulated with acetic acid, and treated with a solution of cupric chlorid. The precipitate thus formed was washed with water until copper-free; then with alcohol until chlorin-free; finally with ether and then dried in vacuo over sulphuric acid, and in air-bath at $105^{\circ}$ to constant weight. ... The residue after the sodium chlorid treatment was treated for two hours with a 4 per cent. solution of sodium hydrate:

1 Berliner klinische Wochenschrift, 36.

${ }^{2}$ Journal of Medical Research, 1, 1901. 
and some sodium acetate, then neutralized with acetic acid. An excess of picric acid was then added and then rendered acid with acetic acid. To the filtrate alcohol was added and the precipitate thus formed was redissolved and reprecipitated. The precipitate was biuret-free and possessed all the properties of nucleic acids. On heating with mineral acids, it did not reduce Fehling solution. It was again redissolved in water by the aid of some alcohol, the solution was then rendered acid with acetic acid, and the copper salt of the nucleic acid obtained as above."

By this method Levene obtained from samples of tubercle bacilli grown on different culture media, nucleinic acids which varied in phosphorus content from 6.58 to 13.9 per cent. The same investigator obtained a glycogen-like substance from the tubercle bacillus. This gives an opalescent solution, which on the addition of iodin colors similarly to glycogen. On being heated with mineral acids it reduces Fehling solution. The method of obtaining this carbohydrate is given as follows: "The bacilli were treated as described above for the separation of nucleic acid. The picric acid filtrate was then treated with alcohol. The precipitate thus formed consisted of nucleic acid and glycogen. The acid can be removed on addition of a solution of cupric chlorid. The copper compound of the glycogen remains in solution and from the latter it can be precipitated by the addition of alcohol. The copper compound thus obtained is dissolved in water, the solution is acidulated with hydrochloric acid until the solution reacts acid to congo, and the glycogen is then precipitated with alcohol. Should the precipitate still contain some copper, the latter can be removed by repeating the last operations."

Koch's first announcement concerning tuberculin awakened great activity in the study of the chemistry of the bacillus of this disease. Maffucci found that cultures, when grown upon glycerin or blood serum for from one to six months, and then sterilized by being repeatedly heated to from $65^{\circ}$ to $70^{\circ}$, produced in guinea-pigs, when employed subcutaneously, a progressive marasmus; which terminated fatally within from fourteen days to six months. He also found that eggs inoculated with sterilized cultures of the chicken tuberculosis bacillus produced young which were feeble and soon died of emaciation. In neither the guinea-pigs nor chickens could he find any tubercles. Crookshank and Herroun reported the isolation of a ptomaïn and an albumose not only from artificial cultures of the bacillus, but also from bovine tuberculous tissue. Both of these bodies were said to cause an elevation of temperature in tuberculous, and a depression in healthy animals. Zuelzer reported the isolation of a poisonous ptomain from agar cultures of the bacillus tuberculosis. The injection of one cg. or less of this substance subcutaneously in rabbits or guinea-pigs caused, after from three to five minutes, 
increased frequency of respiration (to 180 per minute ?) and an elevation of temperature of from $0.5^{\circ}$ to $1^{\circ}$. Marked protrusio bulbi was a constant symptom and the pupils were dilated. From two to three cg. sufficed to kill rabbits, death occurring in from two to four days. The place of injection was found to be reddened and hemorrhagic spots were observed in the mucous membrane of the stomach and small intestines. Occasionally considerable amounts of clear fluid were found in the peritoneal cavity. As early as 1888, Hammerschlag had ascertained that cultures of the tubercle bacillus are toxic to certain animals. Subsequently he reported that almost 27 per cent. of the cellular substance of the bacillus tuberculosis is soluble in alcohol and ether. In this extract, he found, in addition to fat and lecithin, a poison which induced in rabbits and guinea-pigs convulsions followed by death. The part of the germ insoluble in alcohol and ether was found to consist of cellulose and proteids. Weyl obtained by macerating the bacillus in dilute soda solution an extract which, when injected into animals, caused local necrosis. Prudden and Hodenpyl summarized the results which they obtained by the inoculation of animals with dead tubercle bacilli, as follows: "These dead tubercle bacilli are markedly chemotactic. When introduced in considerable amount into the subcutaneous tissue or into the pleural or abdominal cavities, they are distinctly pyogenetic, causing aseptic, localized suppuration. Under these conditions they are capable, moreover, of stimulating the tissues about the suppurative foci to the development of a new tissue, closely resembling the diffuse tubercle tissue induced by the living germ. We have found that dead tubercle bacilli introduced in small numbers into the blood vessels of the rabbit largely disappear within a few hours or days, but that scattering individuals and clusters may remain here and there in the lungs and liver, clinging to the vessel walls for many days without inducing any marked changes in the latter. After a time, however-earliest in the lungs, later, as a rule, in the livera cell proliferation occurs, in the vicinity of these dead germs, which leads to the formation of new, multiple, nodular structures, bearing a striking morphological resemblance to miliary tubercles. There is in them, however, no tendency to cheesy degeneration and no evidence of proliferation of the bacilli, but rather a steady diminution in their number. It seems to us that the new structures regenerate in a proliferation of the vascular endothelium under the stimulus of the dead and disintegrating germs." Babes and Broca showed that the introduction of dead tubercle bacilli into the bodies of animals rendered them susceptible to the action of tuberculin, and the reaction was most marked when the tuberculin had not been extracted from the bacilli. They also claimed that the local changes induced by the injection of dead bacilli were improved and could be healed by injections of tuberculin; but that under the influence of the tuber- 
culin the dead bacilli were often carried from the place of deposit and distributed by means of the circulation to different parts of the body where subsequently tubercular nodules formed.

The physiological action of tuberculin is so pronounced and at first was believed to be so markedly sui generis, that its discoverer, and through him the greater part of the medical world, was for a short time led into the belief that a specific and sure cure for the greatest plague of man had been found. The grounds for this belief were founded principally upon the following effects observed in the action of tuberculin : (1) Small doses, one mg. or even less, injected subcutaneously into individuals suffering from tuberculosis caused marked elevation of temperature ; while similar doses injected in the same way into non-tubercular persons produced no appreciable effect. Moreover, persons sick with other diseases than tuberculosis were not found to be especially susceptible to the action of tuberculin. Here, then, is a substance that has a specific action, a chemical body by the effects of which one can distinguish a tubercular from a non-tubercular individual. If all the cows of a large herd be treated with tuberculin in proper amount, and a record of the temperature be made for twenty-four hours before and for the same length of time after the treatment, it will be found that in some a febrile reactionan elevation of one degree or more in temperature-occurs, while the temperature of others remains unaffected, or is but slightly changed. Now, if all these animals be killed and examined, it will be found that those that have manifested the febrile reaction are tuberculous; while those that failed to react to tuberculin are not tuberculous. A similar test to this was made by Koch on tuberculous and nontuberculous guinea-pigs. No such effects had ever before been attained by the employment of any therapeutic agent. It is small wonder then that Koch and his colaborers were surprised at the results observed, and readily accepted and too speedily announced the belief that a specific cure for tuberculosis had been found. The grounds for this belief were strengthened by their observation of additional evidence in the selective action of tuberculin.

(2) Not only does tuberculin select tubercular individuals by its action, but in the individual it selects for the demonstration of its most conspicuous effects, the exact site of the tubercular lesion. If a man who has a lupus on his face receives a tuberculin injection in the back or in any other portion of his anatomy, the tissue about the lupus soon begins to show evidence of stimulation; it becomes hyperemic, the margins of the sore begin to granulate, and if the treatment be continued the lupus often temporarily heals. However, more extended investigation has shown that the action of tuberculin is not so specific as was at first believed. The experiments of Krehl have demonstrated the fact that tubercular animals are especially prone to show elevations of temperature after the in- 
jection of various substances. For instance, paralactic acid was found to act similarly to tuberculin and on section of tubercular animals treated with this agent the diseased areas were observed to be hyperemic and to contain hemorrhagic spots. Matthes ascertained that certain albumoses and peptons affect tubercular animals in much the same way, though to a less degree, as tuberculin does.

There has been some question among pathologists concerning the manner in which tuberculin acts on tubercular tissue, and in this connection Baumgarten makes a statement which may be condensed as follows: It causes an exudative inflammation in the vascular tissue about the tubercle, and in this way the tuberculous tissue may be isolated and, when situated superficially, removed. In some cases, however, after the prolonged employment of the agent, the tuberculous tissue may, under the influence of the exudative fluid and the polynuclear leucocytes, break down and form abscesses. The bacilli themselves are in no case harmed by the use of tuberculin, and, after its constant employment for months, they retain their original form and lose none of their virulence. Some preparations seem to show that the bacilli multiply more rapidly when the injections are made, but a positive statement on this point is reserved until further studies have been made. It is certain, however, that the non-tubercular tissue of animals acquires no immunity against the disease from the injections. This is shown by the appearance of metastatic foci in animals in which from 7 to 12 grams of the original lymph (an amount which would be equivalent to from 70 to 180 grams in man) have been injected. It is further shown by the fact that in some animals treated subcutaneously tubercles have appeared at the point of injection.

Suppuration.-As early as 1879 , Leber concluded from his observations on infective keratitis, that the aspergillus must produce certain soluble products which diffuse through the cornea and set up an inflammation in the adjacent vascular tissue. In 1882 he showed that suppuration could be induced by the introduction of sterilized mercury and copper, and that the pus formed is free from microörganisms. In 1884 he induced suppuration by the injection of cultures of the staphylococcus pyogenes aureus that had been sterilized by being boiled for hours. In 1888 he reported that he had found an alcoholic extract of the dried staphylococcus to be highly pyogenetic, and from this extract he prepared a crystalline body which he calls "phlogosin." This substance is readily soluble in alcohol and ether, sparingly soluble in water, and crystallizes in needles. The crystals can be sublimed, leaving no residue, and the sublimate, which forms in rosettes, still possesses pyogenetic properties. Alkalis precipitate this substance from its solutions in amorphous granules, which dissolve in acids, forming crystalline salts. 
Buchner found that the cells of many bacteria contain pyogenetic proteids. The amount of these substances in bacterial cells varies with the kind of germ, and some species (the bacillus prodigiosus, for instance), seem to contain no such bodies. The bacillus pyocyaneus contains a large quantity of this proteid and is suitable for lecture demonstration. The germs are taken from potato cultures, rubbed up with water, and then treated with about fifty volumes of a 0.5 per cent. solution of caustic potash. This forms in the cold a mucilaginous mass which dissolves at the temperature of the waterbath, and after being heated for several hours the fluid is filtered through a number of small filters; the first portion should be refiltered. The filtrate is a greenish fluid (pyocyanin) which by the careful addition of acetic or hydrochloric acid (an excess is to be avoided) forms a voluminous precipitate (pyocyaneus proteid). This precipitate should be collected on a filter, washed with water, then suspended in water, and a few drops of a soda solution added, when a dark brown fluid, with a tendency to gelatinize, in the cold, containing about 10 per cent. of the proteid, is obtained. 13.254 grams of the moist bacteria yield 1.44 gram of dry bacterial substance, and this, after the treatment given above, furnishes 0.2739 gram of dry proteid $=19.3$ per cent. This proteid leaves 11.52 per cent. of ash, which contains phosphoric acid, but consists principally of sodium chlorid. Much smaller amounts of proteids were obtained from other organisms, but the Eberth germ, bacillus subtilis, lactic acid bacillus, red bacillus from potato, and staphylococcus pyogenes aureus furnish considerable quantities.

The chemotactic properties of these proteids were tested in the following manner : The dissolved proteid was placed in a spindleshaped glass tube, and this, after being sterilized, was introduced under the skin of the back of a rabbit with antiseptic precautions, and the ends of the tube broken off subcutaneously. After from two to three days the tubes thus prepared were removed and found to contain, in addition to some of the proteid, several cubic millimeters of fibrinous pus, which was examined microscopically and by the preparation of cultures, which remained sterile. The proteid of the Eberth bacillus was found to have specially marked pyogenetic properties. More extended investigation demonstrated that certain other proteids also have pyogenetic properties. The subcutaneous injection of sterilized preparations of wheat flour and ground peas caused suppuration. Negative results were obtained with starch and solutions of di-sodium hydric phosphate; from this it is concluded that the active agent in the flour is its gluten. Pepton was employed without effect, while gelatin was found to act energetically. Alkaline albuminates were prepared from muscle, liver, lungs and kidney, by treating finely divided portions of these organs with potash and proceeding as in the preparation of bacterial proteids; all of these 
caused the formation of pus, and the preparations from the liver were found to be specially potent. Similar preparations from blood and egg yolk were active, while those from fibrin and the white of egg had no effect. Hemi-albumose was also found to be active, and this fact is placed in contrast with the negative result obtained with pepton.

The bacterial proteids, also some of the vegetable proteids, when injected directly into the blood, cause a general leucocytosis. A very small amount of the proteid of the pyocyaneus injected under the skin of the forearm caused the following symptoms: Two hours after the injection there was marked pain along the lymphatics, especially localized in the elbow and axilla. The temperature showed no marked elevation. On the following day there was observed a distinct erysipelatous redness and swelling extending for some inches about the place of injection, and this was accompanied by severe pain. The inflamed area felt hard, and projected distinctly above the surrounding surface, and the lymphatics of the arm appeared like red cords. On the third day the swelling and redness were more marked, and extended from the wrist to the elbow; but on the fourth day the symptoms began to recede. Here we have clinically a typical erysipelas with lymphangitis, and Buchner claims that all the cardinal symptoms of inflammation-rubor, calor, dolor, tumor-could not be produced without involvement of the solid tissues. Similar, but less marked, symptoms were induced by the injection of a dilute solution of vegetable casein.

Buchner states that bacteria can not cause inflammation unless they be broken down. The pyogenetic substance contained within the bacterial cell can have no chemotactic action until the cell disintegrates. Thus, the anthrax bacillus contains a pyogenetic substance, but no pus is formed in mice with anthrax, because there is no destruction of the bacilli. The pyogenetic proteid of the anthrax bacillus, however, manifests its action in malignant pustule.

Many non-pathogenic germs may grow in wounds and by elaborating their poisons may increase and influence the general intoxication. Brunner has found the proteus vulgaris growing in a wound, and it is well known that the products of this germ are powerful poisons.

Mannotti, after treating animals with sterilized pus, states the following conclusions :

(1) Sterilized pus has substantially the same toxic properties as sterilized cultures of the staphylococcus. (2) Repeated injections of sterilized pus induce chronic intoxication and marasmus. (3) Injection under the skin causes a specially grave form of poisoning. (4) The symptoms and pathological lesions caused by these injections correspond with those observed in men suffering from chronic suppuration. 
Van de Velde ${ }^{1}$ has ascertained that the staphylococcus pyogenes produces a substance which destroys leucocytes and for which the name "leukocidin" is now used. He injected sterilized cultures of the staphylococcus into the pleural cavities of rabbits and studied the exudate which formed. He found that the leucocytes which are present in such an exudate in large numbers soon undergo characteristic changes. He next added to small quantities of the exudate living, healthy leucocytes and observed that these underwent a like transformation. Heating the exudate for ten minutes at $58^{\circ}$ prevents its action on leucocytes. The successive steps in the destruction of the white blood cells are as follows: (1) The leucocytes draw in their pseudopodia and become round. in the periphery, while the center remains granular. (3) The light zone gradually extends and the granulation completely disappears. (4) The corpuscles are apparently reduced to empty sacs with scarcely discernible nuclei which finally disappear altogether. These degenerative changes are complete in about two minutes. Similar changes are observed in leucocytes added to either avirulent or virulent cultures of the staphylococcus. These observations have been confirmed by Bail ${ }^{2}$ and Lingelsheim. ${ }^{3}$ Denys and Van de Velde ${ }^{4}$ have shown that the serum of animals rendered immune to the staphylococcus pyogenes aureus destroys the leukocidin in cultures of this germ. Neisser and Wechsberg ${ }^{5}$ have devised a method of measuring the action of leukocidin and have decided from their investigations that typical pyogenetic staphylococci, both the aureus and the albus, produce the same leukocidin, but that there are varieties of both these organisms which do not produce this poison.

Kranss $^{6}$ first observed that many red blood corpuscles are dissolved by filtered cultures of the staphylococcus. This hemolysin has been further studied by Neisser and Wechsberg, who designated it as staphylolysin. This also is a product of both the aureus and the albus. It is believed that this toxin possesses a constitution similar to that supposed to be possessed by the diphtheria toxin according to Ehrlich's theory, and that it consists of toxin and toxon. Furthermore, it is probable that the toxin is converted into toxoids; but while the toxoids of the diphtheria toxin have the same avidity for antitoxin as is possessed by the toxin, the toxoids of staphylotoxin combine with antitoxin with less avidity than do the toxins. The blood serum of healthy men contains an anti-staphylolysin, but that from different individuals varies greatly.

\footnotetext{
${ }^{1}$ La cellule, 10.

${ }^{2}$ Archiv f. Hygiene, 32.

3 Aetiologie und T'herapie der Staphylokokken-Infectionen, 1900.

- La cellule, 11.

5 Zeitschrift f. Hygiene, 36.

6 Wiener klin. Wochenschrift, 1900.
} 
Ribbert $^{1}$ in his experiments on the intravenous injection of both sterilized and unsterilized cultures of the staphylococcus in rabbits observed changes in the kidneys, heart, lungs, spleen, and bone marrow, which he attributed to the action of the toxin, because they were found after the injection of sterilized as well as after the use of living cultures. These changes were characterized by the presence of infarcts and abscesses. Neisser and Wechsberg observed certain pathological conditions in the kidneys of animals which they rendered immune to staphylotoxin by intravenous injections. Concerning these kidney lesions they make the following statement: They are located in the cortex; neither the parenchyma nor the papillæ are involved. In the cortex one readily distinguishes three zones, which, proceeding from the periphery towards the center, are as follows : (1) A circular, irregular zone in which the tubuli contorti are for the most part destroyed and the peritubular spaces are filled with fragments of broken-down leucocytes. (2) An intermediate zone in which the leucocytic reaction is less marked, but in which necrosis of the epithelium is plainly evident. Only a small part of the epithelium of the tubuli contorti possess nuclei and even these show evidence of cell disintegration. The greater part of the cells are without nuclei and are in a state of coagulation necrosis. The uriniferous tubules contain hyaline casts and fragments of broken-down leucocytes. In this zone the glomeruli are markedly altered. One observes all changes from simple hyperemia to complete necrosis in which there is no sign of a glomerulus left except a structureless capsule. (3) The third zone, like the first, is rich in broken-down leucocytes, especially in the peritubular spaces. While a part of the blood vessels are still patent, others are filled with thrombi consisting of fibrin and broken-down leucocytes. These authors were unable experimentally to demonstrate that these changes in the kidney are directly due to the staphylotoxin and they have provisionally named the poison which induces these lesions " nephrotoxin." They conclude that the hemolysin and the leukocidin of the staphylococcus are two different poisons.

The action of sterilized cultures of the gonococcus has been studied by Christmas, ${ }^{2}$ Nikolaysen, ${ }^{3}$ and Wassermann. ${ }^{4}$ It has been found that cultures in which the microörganism has been destroyed by heat are as virulent to the lower animals as are living cultures. Furthermore, it has been demonstrated that the gonotoxin is contained within the cell of the microörganism. If a culture two days old be filtered through porcelain and the filtrate be injected into mice, but little or no effect results; while if a culture from two to three weeks old be

1 Die pathologische Anatomie und die Heilung der durch den Staphylococcus pvogenes aureus hervorgerufenen Erkrankungen.

${ }^{-}$Annales de l'Institut Pasteur, 1897.

${ }^{3}$ Centralblatt $f$. Bakteriologie, 1897.

- Zeitschrift $f$. Hygiene, 1898, 27. 
treated in the same way the filtrate is found to be toxic. This shows that the toxin is contained within the cell and becomes active only after the disintegration of the organism. As is true of many other bacteria, different cultures vary markedly in their toxicity. Wassermann obtained some preparations, 0.1 c.c. of which was sufficient to kill mice, while one c.c. of other sterilized cultures was necessary in order to accomplish the same result. Gonotoxin is a fairly stable body ; it can be precipitated with absolute alcohol and can be heated to $100^{\circ}$ without marked loss of toxicity. While none of the lower animals are susceptible to infection with this microörganism, rabbits, mice and guinea-pigs are readily affected by the toxin. The subcutaneous injection of two c.c. of a sterilized culture of a virulent form of gonococcus causes an infiltration which subsequently passes into a necrosis. Treatment with 10 c.c. causes marked local inflammation, the animal loses its appetite, wastes away and dies of chronic marasmus. The pyogenic action of this toxin can easily be demonstrated by the injection of a small quantity into the anterior chamber of the eye of a rabbit. Corneal cloudiness, hypopyon and sometimes complete loss of the eye result. Wassermann injected 0.1 c.c. subcutaneously into his own arm. After about four hours the place of injection became painful, there were light chills, and in the evening the temperature reached $38^{\circ}$. During the night there was headache and pain in the joints. The next day the area around the point of injection was swollen and painful, but all symptoms disappeared after two days. The same investigator has tried this agent in the treatment of obstinate cases of chronic gonorrhea, but has found that the toxin has no curative effect and only intensifies the symptoms. Both he and Christmas have attempted to produce an antitoxin for this poison, but without any marked success. It is true that Christmas has reported the preparation of an active serum by immunizing goats, but the action of this antitoxin was very slight and Wassermann's results were wholly negative. Small quantities of gonotoxin introduced into a sound urethra cause after a few days marked suppuration, while control experiments with the toxins of other cocci were without effect. The action of gonotoxin explains the clinical course of many cases of gonorrhea. When the infection is confined to the anterior part of the urethra the germ soon dies and the toxin is thoroughly washed out with each discharge of urine; but when the infection is in the posterior urethra some of the germs find their way into the crypts of this region and pass through many generations, elaborating their toxin and causing the continued formation of pus. This also explains why this disease is so much more serious in the female than in the male.

The Summer Diarrhœas of Infancy.-In 1888, Vaughan stated that the microörganisms which produce the catarrhal or mucous diar- 
rhøea of infancy, are probably only putrefactive or saprophytic in character, and that they prove harmful by producing toxins; while those that cause the choleraic form or serous diarrhœas, are more than putrefactive, they are pathogenic. At that time it was generally believed that a specific germ would be found; but the truth of the above statement has been made more manifest with every experimental study of the subject. More recently, Booker ${ }^{1}$ makes the following statement: "No single microörganism is found to be the specific exciter of the summer diarrhœeas of infancy, but the affection is generally to be attributed to the result of the activity of a number of varieties of bacteria, some of which belong to the wellknown species and are of ordinary occurrence and wide distribution, the most important being the streptococcus and proteus vulgaris."

Vaughan has studied the chemical properties of the germs $x, a$ and $A$ of Booker's list in the following manner and with the result as stated below : Beef broth cultures were kept in the incubator at $37^{\circ}$ for ten days. They were then twice filtered through heavy Swedish filters, and the second filtrate was allowed to fall into a large volume of absolute alcohol, feebly acidified with acetic acid. A voluminous, flocculent precipitate resulted in each case, and after subsidence the supernatant fluid was decanted. The precipitates were then treated with distilled water, in which those from $x$ and $a$ were soluble, while that from $A$ proved insoluble. A large volume of absolute alcohol was again added, and the mixture allowed to stand for four days. The precipitates from $x$ and $a$ completely subsided, leaving the supernatant fluids perfectly clear; but in the case of $A$ the subsidence was not complete. The precipitates were collected, by decantation and filtration, on porous plates, and dried over sulphuric acid. These substances are proteid in composition but differ from known proteids and from one another. That from $x$ is slightly yellow, as seen deposited in the alcohol, but becomes grayish on exposure to the air. It is readily soluble in water from which it is precipitated by either heat or nitric acid, singly or combined. It gives the biuret and xanthoproteic reactions and is precipitated by saturating its aqueous solution with ammonium sulphate, and therefore cannot be classed with the peptons. Sodium sulphate and carbonic acid fail to throw it down from its aqueous solution; consequently it is not a globulin, and for the present at least it must be classified among the albumins; however, it possesses properties which do not belong to the known albumins.

The proteid prepared from cultures of the germ $a$ is, as seen under the alcohol, very light, flocculent, and perfectly white; but so soon as it is brought in contact with the air it begins to blacken, and finally dries down on the porous plate in black scales. It possesses the same general properties in regard to the action of solvents and

$$
{ }^{1} \text { Johns Hopkins Hospital Reports, 6, } 1897 .
$$


other reagents which were found to be possessed by the proteid obtained from cultures of $x$.

The proteid of $A$ is peculiar, inasmuch as it is practically insoluble in water.

All these proteids are highly poisonous, and when injected under the skin of kittens or dogs cause vomiting and purging, and, when employed in sufficient quantity, collapse and death. Post-mortem examination shows the small intestine pale throughout and constricted in places. The heart is found to be in diastole and filled with blood. The following brief notes from the record of experiments illustrate the nature of the symptoms and the post-mortem appearances :

A small amount of proteid from bacillus $x$, dissolved in water, was injected under the skin on the back of a kitten about eight weeks old. Within one-half hour the animal began to vomit and purge, and death resulted within eighteen hours. The small intestines were pale, contracted in places, and contained a frothy mucus. The stomach was distended with gas and contained yellowish mucus. The liver appeared normal, the spleen and the kidneys congested, and the heart distended.

Another kitten was treated with a proteid from bacillus $\dot{a}$ dissolved in water. The vomited and fæcal matters in this case were green. The animal died after fifteen hours, and presented appearances practically identical with those mentioned above.

A third kitten was treated with some of the proteid of bacillus $A$, suspended in water, and presented substantially the same symptoms and post-mortem appearances.

A fourth animal was treated in the same manner as above with the proteid prepared from some canned meat. This was done as a control on the above experiment, and the kitten remained unaffected, thus demonstrating the fact that the poisonous properties are peculiar to the bacterial proteids.

Concerning the amount of these proteids necessary to produce fatal results in the animals experimented upon, the following statement may be made: Under the skin on the back of a guinea-pig $10 \mathrm{mg}$. of the dry scale proteid from bacillus $a$ was injected, and caused death within twelve hours. Of two kittens treated with $15 \mathrm{mg}$. each of the $a$-albumin, one died after forty-eight hours, and the other recovered after two days of purging and vomiting. Two dogs, of about five pounds' weight, had each $40 \mathrm{mg}$., and after serious illness of two days' duration, recovered. During these two days of purging and vomiting the dogs were constantly shivering as with cold, but the rectal temperature stood at from $102.5^{\circ}$ to $103.5^{\circ} \mathrm{F}$. There was in no case any sign of inflammation at the point of injection. Plate cultures were made from the proteids themselves, and from the blood, liver, spleen, and kidneys of some of the animals killed with 
the proteid, and these remained sterile, thus demonstrating that no germ was introduced into the animals along with the chemical poison.

The following conclusions concerning the germs which cause the summer diarrhoeas of infancy may be formulated:

1. There are many microörganisms, any one of which, when introduced into the intestines of the infant, under certain favorable conditions may produce diarrhoea.

2. Many of these microörganisms are true saprophytes. A germ growing in the intestine does not necessarily feed upon living tissue. The food in the duodenum before absorption has no more vitality than the same material in a culture flask. Moreover, the excretions poured into the intestines from the body are not possessed of vitality. A bacterium which will grow upon a certain medium in a flask and produce a poison will grow on the same medium in the intestine and produce the same poison, provided it is not destroyed by some secretion of the body.

3. The only digestive secretion which is known to have any decided germicidal effect is the gastric juice; therefore, if this secretion be impaired there is at least the possibility that the living germ will pass on to the intestine, will there multiply, and will, if it be capable of so doing, elaborate a chemical poison which may be absorbed. It has been said that the gastric juice does not act as a germicidal agent, because there are other acids which are more powerfully bactericidal than hydrochloric acid, but there is no force in this argument. The question is not whether the stomach is supplied with the very best germicide, but whether it is supplied with any at all. The human eye is not by any means a perfect mechanism, but it is man's only organ of vision.

The chief reason why the breast-fed child has a better chance for life than the one fed upon cow's milk lies in the fact that the former gets its food germ-free; but a second reason is to be found in the large amount of acid required to neutralize the cow's milk, as has been pointed out by Escherich. It is also possible that some of the secretions poured into the intestines have germicidal properties, or that the cells, in absorbing the bacterial proteids, may to a limited extent so alter them that they are no longer poisonous, or that in a perfectly normal condition the liver may be able to prevent these poisons from entering the general circulation without change.

4. Any germ which is capable of growing and producing an absorbable poison in the intestines may, for all practical purposes, be considered pathogenic. It is not necessary that a bacterium be capable of growing and causing disease and death when injected under the skin or into the blood in order to establish its right to rank with the pathogenic germs. In the blood the organism is acted upon by a wholly different fluid from that by which it is surrounded 
in the intestines, and the germicidal properties of the blood have been unquestionably demonstrated.

5. The proper classification of bacteria in regard to their relation to disease can not be made from their morphology alone, but must depend largely upon the products of their growth. As has been stated, three microörganisms, differing sufficiently to be recognized as of different species, produce poisons, all of which induce vomiting and purging, and when used in sufficient quantity, death. Morphologically these bacilli may not be closely related, but physiologically they are near akin. If these deductions be true, we will avoid the introduction into the alimentary canal, not only of the specific pathogenic germs, but of all toxicogenic microörganisms.

Baginsky and Stadthagen obtained from cultures of the "white liquefying bacterium" of the former a poisonous proteid which produces in mice, after about five hours, slight dyspnœa, the coat becomes rough, the animal sits with drooping head, and when forced to move does so sluggishly but without any evidence of paralysis. The marked apathy increases, and death results after two or three days. Section shows an infiltration about the place of injection, congestion of the spleen, liver and peritoneum. The intestine is hyperemic throughout its entire length, and its upper portion contains a reddish-brown fluid. They also obtained from the same cultures a poisonous ptomain, which is probably identical with one found by Brieger in putrid horse flesh, and has the formula $\mathrm{C}_{7} \mathrm{H}_{17} \mathrm{NO}_{2}$.

That tyrotoxicon is one of the causes of the violent choleraic diarrhœa of children there can scarcely be a doubt. The symptoms induced by the poison cannot be distinguished from those induced by the disease. The post-mortem appearances are very much alike, it not identical, and the poison has been found in milk, a part of which had been given to a child not more than two hours before the first symptom of a violent attack of the disease made itself manifest; but tyrotoxicon is not so frequently found in milk as was at one time supposed.

Flügge has studied milk bacteria with special reference to their toxicogenic properties. In market milk be has frequently found four anaërobic bacilli, two of which produce poisons. The subcutaneous injection of filtered cultures of one of these in doses of from 0.3 to 0.6 c.c. in mice caused death after from three to fifteen hours, and section showed marked hyperemia of the intestines and transudates in the peritoneal and pleural cavities. The intra-abdominal injection of five c.c. of this culture killed guinea-pigs within from fifteen to twenty-four hours, and in these also the intestines were found to be engorged and the abdominal cavity filled with a serous transudate. The second toxicogenic, anaërobic bacillus develops a most disagreeable odor in milk, and consequently it is not likely 
to be taken. Summer diarrhœas can scarcely be attributed to these anaërobic germs, and yet they cannot be regarded as altogether harmless.

Flügge found and isolated twelve species of peptonizing bacteria in market milk and three of these were found to be markedly poisonous to animals. Concerning the action of cultures of these bacilli, the following statement is made : "The two days' old cultures of No. I. induce in frogs, on the injection of two c.c. into the dorsal lymph zone, first slowness of motion and reflexes, after one hour paralysis of the extremities and complete loss of reaction, and after four hours, death. Mice die after from five to six hours from the subcutaneous injection of 0.5 c.c. With the exception of lack of voluntary motion and tardiness of reaction, no symptoms are manifest. Guinea-pigs, that have received five c.c. intra-abdominally, lie on the side and have marked dyspnoa, the abdomen is retracted, and handling them causes pain. Death results after from four to seven hours. Section shows hyperemia of the kidneys, and the peritoneal and serous coat of the intestines are markedly reddened. Nothing else of interest in found. Dogs drink the milk cultures with relish and in large quantities. After one hour severe diarrhœe sets in with a movement every five minutes. When fed with normal milk recovery follows.

"Two days' old milk cultures of No. III. induce in frogs and mice no symptoms. Guinea-pigs and rabbits receiving intra-abdominal or intravenous injections remain quiet in their cages, respond quickly to irritation, but gradually recover. The cultures, when fed to puppies, induce sharp diarrhœa and apparently severe pain in the abdomen. One of the puppies showed on the second day progressive exhaustion, paralytic weakness of the extremities, and a fall of temperature. He died on the third day, and section showed hyperemia of the kidneys, nothing else worthy of note."

"Bacillus No. VII. injected in milk cultures into frogs, mice and guinea-pigs had no marked action. When the culture was filtered through a Chamberland filter and concentrated in vacuo to one-fiftl its volume, it killed mice and guinea-pigs when injected in doses of 0.6 and 5 c.c. respectively. Death, which followed in from six to twelve hours, was preceded by dyspnoea and convulsive movements. Section showed nothing characteristic. Even the unconcentrated milk cultures acted powerfully when fed to puppies. After feeding for one or two days, profuse diarrhœa set in, but disappeared the next day. The diarrhœa was accompanied by great emaciation, weakness of the extremities, and tottering gait. As soon as the use of the cultures was discontinued, and ordinary milk given, improvement began and continued to complete recovery. Two puppies, after recovery, were again fed with the cultures, and after a short time the profuse diarrhœa with its accompanying symptoms reappeared." 
Flügge discussed the question as to whether or not the poisonous properties of these peptonizing bacilli are due to the peptons formed, and came to the conclusion that this could not be the case. He found among the twelve species some that produced peptons much more energetically than do the harmful kinds. In fact, one of the most highly virulent species produced the least pepton. Furthermore, the symptoms that followed the injections were not those that are induced by pepton. It is true that different investigators, who have tested the nutritive value of peptons and albumoses on the lower animals and on healthy and sick men, are unanimous in the verdict that the long-continued employment of these preparations causes in both men and dogs severe intestinal irritation. Züntz noted that dogs fed on peptons suffered from an abundant, watery diarrhœa, and eliminated from three to six times as much nitrogen unused as those fed upon meat, and Munk obtained like results. Pfeiffer induced in himself and in another man intestinal irritation and diarrhoea by large doses of pepton and Neumeister states: "By long-continued use of these preparations, symptoms of marked irritation and injury to the intestines uniformly resulted, and consequently the prescription of albumoses in disease can scarcely be regarded as "rational." " The reason why partly digested milk is often so well borne by children is due to the fact that but little pepton is formed, and from all that we know of the nutritive value of albumoses and peptons, the long-continued employment of peptonized milk in the feeding of infants cannot be recommended; but Flügge was right in concluding that the toxic properties of cultures of peptonizing bacteria found in milk by him were not due to the peptons. This has been conclusively shown by more recent researches in Flügge's laboratory prosecuted by Lübbert. ${ }^{1}$ This investigator has extended the study of one of the most virulent species of peptonizing bacilli found by Flügge. He has shown that this microörganism rapidly digests the casein of milk, while it has no action on either the fat or the lactose. Pure milk cultures of this bacillus were readily taken by guinea-pigs and caused death within four days. Three young dogs fed with such cultures developed severe diarrhoea after two hours and died within from four to seven days; while older dogs consumed large quantities of the milk and remained unaffected. This is especially interesting, inasmuch as it is a well-known fact that after the second year of life, children seldom suffer from summer diarrhœas. Section of the animals killed with these cultures showed slight swelling and injection of the mucous membrane of the intestine. The bacteria could not be discovered outside of the intestines, either in the blood or the organs. This demonstrates that death resulted from intoxication and was not due to sepsis. Further researches demonstrated the fact that the

${ }^{1}$ Zeitschrift f. Hygiene, 22, 1. 
toxin is contained within the bacterial cells and when all these are removed by filtration, the filtrate is without harmful effect. These investigations indicate that the toxin is absorbed from the alimentary canal of young animals, while it is not taken up from the digestive tract of adults. Both the gastric and pancreatic juices were found to be without effect upon these bacilli, and the filtrates obtained after digestion of the bacterial cells in these fluids were without poisonous action. Inasmuch as no bacteria were found in the blood of any of the internal organs, we must conclude that the germ cells undergo a process of disintegration or digestion, while being absorbed through the intestinal walls. Boiling milk cultures of this toxicogenic, peptonizing bacillus destroys their virulence but it must not be inferred from this that all milk containing toxicogenic germs is rendered harmless by heat. The colon bacillus is frequently found in market milk and it is altogether likely that this germ plays an important rôle in the causation of the summer diarrhœeas of infancy and, as we shall see later, the colon toxin resists very high temperatures.

Typhoid Fever.-The specific character of the bacillus discovered by Eberth in 1880 has been abundantly demonstrated by subsequent bacteriological research, and there is no doubt that this microörganism is the cause of typhoid fever. In 1885, Brieger obtained from pure cultures of the Eberth bacillus a poisonous ptomain, which produced in guinea-pigs a slight flow of saliva, frequency of respiration, dilatation of the pupils, profuse diarrhœa, paralysis, and death within from twenty-four to forty-eight hours. Post mortem examination showed the heart in systole, the lungs hyperemic, and the intestines contracted and pale. At first Brieger was inclined to regard this as the specific poison of typhoid fever, and named it typhotoxin, but more extended investigation has shown that this substance cannot be regarded as the essential toxin of the Eberth bacillus. In their researches upon the toxalbumins, Brieger and Fränkel found in cultures of the typhoid bacillus a proteid which caused death in rabbits after from eight to ten days. This substance undoubtedly contained the typhoid toxin but was by no means a pure preparation of this substance.

Pfeiffer has demonstrated that typhotoxin is contained in the bacterial cells and he has shown that $4 \mathrm{mg}$. of the dried cells for each 100 grams of body weight suffice to kill guinea-pigs. With this poison animals can be rendered immune to the Eberth bacillus, and the blood of such immune animals contains a slightly active antitoxin, which, however, has not proved of value in the treatment of the disease.

In 1889, Vaughan isolated from mixed cultures from typhoid stools a base, forming crystalline salts and capable of inducing in cats and dogs a marked elevation of temperature accompanied by 
severe purging. The following is the record of one experiment with this substance: "An aqueous solution of the crystals was given to a dog by the mouth at $3 \mathrm{p} . \mathrm{m}$. The rectal temperature was $101^{\circ} \mathrm{F}$. before the administration. At 3.15 retching and vomiting set in and continued at intervals for more than two hours. At 3.30 the temperature was $103^{\circ} \mathrm{F}$. and at 3.55 the animal began to purge. The first discharges contained much fecal matter, but subsequently they were watery and contained mucus stained with blood. At 4, the temperature was $103.5^{\circ} \mathrm{F}$., and remained the same at 4.30. The animal was seen again at 10 a. m., the next day, when its temperature was $101.5^{\circ}$ and recovery seemed complete."

This base was not obtained in sufficient quantities for an ultimate analysis. The platino-chlorid crystallizes in fine rhombic prisms and the hydrochlorid in long, delicate, red needles. The red color seems to be inherent to the substance and not due to impurities. The mercury and platinum compounds are insoluble in alcohol, soluble in water. The hydrochlorid is soluble in both water and alcohol.

In 1890, Vaughan reported the isolation, from water supposed to cause typhoid fever, of a number of toxicogenic germs, and the chemical properties of two of these have been studied. They belong to the proteids, and an analysis of one of these by Freer shows it to belong to the nucleins. These poisons are soluble in water, the opalescent solution showing a distinctly acid reaction. They are not precipitated by heat or nitric acid singly or combined. They dissolve in nitric acid, forming a colorless solution, which becomes yellow on the addition of ammonia. They dissolve in caustic alkalis, and the solution becomes purple on the addition of copper sulphate.

On white rats these poisons produced symptoms which are identical with those following inoculations with the living germs. The rat seems to shiver with cold and gives evidence of abdominal pain. It lies with its limbs flexed and head drawn down for a few seconds, then stretches out the limbs. It lies on the side for a short time, then sits with the head drawn under the body. Dogs shiver as with cold, but at the same time the rectal temperature is from $1^{\circ}$ to $4^{\circ}$ above the normal. The following experiments seem to show that the poison accumulates in the nerve centers: Two guinea-pigs were treated with hypodermic injections of one of these poisons, the amount used being about ten times the dose which ordinarily proves fatal to one of these animals. Within twelve hours both were dead. Plate cultures made from the liver, blood, spleen, brain and spinal cord remained sterile. Small quantities of the brain and spinal cord were rubbed up in a sterilized dish with sterilized water, and two c.c. of the emulsion was injected under the skin of each of four guinea-pigs. These animals seemed to be very excitable the next day, throwing themselves about violently in their cages when slight noises were made near them. Within a period of from six- 
teen to twenty-four days all died. This experiment needs repetition and it will be necessary to prepare and inject similar emulsions made from other organs before any positive conclusions can be drawn.

Hog Cholera.-In pure cultures of this bacillus Novy has found a poisonous base, which probably has the composition $\mathrm{C}_{10} \mathrm{H}_{26} \mathrm{~N}_{2}$, and to which he has provisionally given the name susotoxin. $100 \mathrm{mg}$. of the hydrochlorid of this base causes in white rats convulsive tremors and death within one and one-half hours. Section shows the heart in diastole, lungs pale, stomach contracted, a serous effusion into the thoracic cavity, and the subcutaneous tissue pale and edematous.

Novy has also obtained a poisonous proteid from cultures of this germ. The following experiments illustrate the effects obtained from this body : 100, 50 and $25 \mathrm{mg}$., respectively, were injected into three young white rats from the same litter. The animal which received $100 \mathrm{mg}$. soon began to crawl about on its belly, being unable to rise. The eyes were soon filled with a thick secretion and the toes became red. Finally it became quiet, lying on its belly, with feet extended. The respirations became deeper, and a coma-like condition set in. The animal died, without convulsions, within about three hours. The rat which received $50 \mathrm{mg}$., went through the same course of symptoms, but these were less intense. Death resulted four hours after the injection. The one which received the $25 \mathrm{mg}$. became very sick, but finally recovered, and one week later it was given another injection of $30 \mathrm{mg}$., which produced scarcely any effect. Then it was treated at intervals of five, three, five, two and four days, respectively, to $40,50,75,100$ and $125 \mathrm{mg}$. without effect. Three days after the last injection the animal was inoculated with one c.c. of a bouillon culture of the highly virulent germ. Only a slight temporary effect was observed during the first day, after which recovery was complete and permanent. A control rat was given the same quantity of the culture, sickened the next day and died one week later. From this it will be seen that the animal was rendered immune to the bacillus.

De Schweinitz also reports the detection of a slightly poisonous base, which he designated sucholotoxin, and a poisonous proteid, and with these he has been able to secure immunity in guinea-pigs against the virulent germ. The proteid body is classed among the albumoses, and is said to crystallize in white, translucent plates when dried in vacuo over sulphuric acid and to form needle-like crystals with platinum chlorid. The same investigator has also reported the isolation of soluble ferments, or enzymes, from cultures of the hog cholera bacillus. From milk cultures both peptonizing and diastatic ferments were obtained. These are destroyed by heating above $55^{\circ}$; they contain nitrogen, and, when pure, do not give the albuminoid 
reactions. Injections of the soluble ferments conferred immunity. In 1892 Novy obtained immunity in rabbits by injection of blood serum of immunized animals, and similar results were obtained by De Schweinitz. In the same year Metschnikoff published his studies on immunization with blood serum of rabbits immune to hog cholera, but according to Smith and Moore, he did not work with the hog cholera germ, but with that of swine plague. The last-mentioned individual obtained only partial immunity with blood serum of immune guinea-pigs, and none with that of rabbits.

Rabbit Septicemia.-Hoffa has killed rabbits by inoculation with pure cultures of the bacillus of this disease, and has isolated from the bodies of these animals methylguanidin, while in the bodies of healthy rabbits this poison could not be found. He ascertained that the average fatal dose of methylguanidin for rabbits is $0.2 \mathrm{gm}$. when given subcutaneously. Hueppe thinks that the bacterium of chicken cholera is identical with that of rabbit septicæmia, and it has been ascertained that chickens also may be poisoned with methylguanidin and that the symptoms induced by this substance resemble those of the disease.

Pneumonia.-Bonardi reported the presence of certain poisonous basic substances in cultures of the diplococcus of Fränkel, but he was unable to obtain these in quantity sufficient for ultimate analysis. $\mathrm{He}$ also stated that he secured immunity against the germ by treating rabbits with a small quantity of the chemical poisons. However, temporary immunity to the diplococcus may be induced by non-specific agents, and Vaughan ${ }^{1}$ concluded a research upon this subject as follows:

1. Rabbits and guinea-pigs may be protected against virulent cultures of the diplococcus of pneumonia by previous treatment with hypodermatic injections of a solution of yeast nuclein.

2. The immunity thus secured is not due to the action of the nuclein, as a germicide, directly on the germ.

3. The process of securing this immunity is an educational one, and, most probably, depends upon the stimulant effects of the nuclein upon some organ whose function it is to protect the body against bacterial invasion.

4. The longer the nuclein injections are continued and the more frequently they are administered, the more complete is the immunity which is secured.

5. In order to obtain this immunity the inoculation with the germ must follow soon after the last treatment with the nuclein. 
Malignant Edema.-Kerry found that the bacillus of this disease decomposes albumin with the formation of fatty acids, leucin, hydroparacumaric acid, and a foul-smelling oil of the composition $\mathrm{C}_{8} \mathrm{H}_{16} \mathrm{O}_{4}$ : This oil is insoluble in water, alkalis, and acids, easily soluble in ether, benzol, bisulphid of carbon, and alcohol. It is optically inactive and on being oxidized forms valerianic acid. Nothing is said concerning its action upon animals. Among the gaseous products are carbonic acid, hydrogen, and marsh gas. The author was unable to determine whether or not free nitrogen was present.

Puerperal Fever.-Bourget concludes a research on this disease with the following statements :

1. In puerperal fever the urine contains highly poisonous bases.

2. The toxicity of the urine is most marked when the symptoms of the disease are most grave, and diminishes as the symptoms abate.

3. The ptomains obtained from the urine prove fatal when injected into frogs and guinea-pigs.

4. Toxic bases, resembling those obtained from the urine, were extracted from the viscera of a woman who had died of puerperal fever.

Glanders.-The toxin of this disease is contained in the bacterial cell, and is known as mallein or morvin. Sterilized cultures of the glanders bacillus containing the chemical poison are used in horses in the same way that tuberculin is employed to diagnose tuberculosis in cows. In glandered horses the subcutaneous injection of small quantities of mallein causes a more marked elevation of temperature than it does in healthy animals. However, Schattenfroh has shown that a like effect is produced in glandered horses by the injection of toxins from other bacteria. Commercial mallein is prepared in a number of ways, one of which is as follows: Growths of the glanders bacillus, from ten to fourteen days old, on potatoes, are removed with a sterilized spatula and rubbed up with sterilized water in the proportion of one part of the moist bacillus to nine parts of water. This emulsion is allowed to stand for twenty-four hours, and then heated to $110^{\circ}$ for fifteen minutes ; next, it is filtered through porcelain, 30 per cent. of glycerin added, and concentrated at low temperature on the water-bath to one-eighth of the original volume. This is again sterilized at $110^{\circ}$ and the preparation, now ready for use, consists of a clear, yellowish, odorless fluid of feebly acid or neutral reaction. Kressling found in mallein prepared by the above method peptons, globulins, xanthin, guanin, small quantities of tyrosin and leucin, and traces of volatile fatty acids and ammonia. 


\section{CHAPTER V.}

\section{THE GERMICIDAL PROPERTIES OF BLOOD SERUM.}

As early as 1872 Lewis and Cunningham demonstrated the fact that bacteria injected into the circulation rapidly disappear. In the blood of twelve animals that had been treated with such injections bacteria could be found after six hours in only seven. In a second series of thirty, bacteria were found after twenty-four hours in the blood of only fourteen, and in a third experiment involving seventeen animals, bacteria were found in only two, when the examination was made from two to seven days after the injection.

In 1874 Traube and Gscheidlen found that arterial blood, taken under aseptic precautions, from rabbits into the jugular vein of which one and one-half c.c. of a fluid rich in putrefactive germs had been injected forty-eight hours previously, failed to undergo decomposition for months. These investigators attributed the germicidal properties of the blood to the ozonized oxygen. Similar results were obtained by Fodor and by Wyssokowitsch. The latter accounted for the disappearance of the germs, not by supposing that they were destroyed in the blood, but that they found lodgment in the eapillaries.

The first experiments made with extra-vascular blood were conducted by Grohmann under the direction of A. Schmidt in his researches upon the cause of coagulation. It was found that anthrax bacilli, after being kept in plasma, were less virulent, as was demonstrated by their effect upon rabbits, and Grohmann supposed that in some way the bacteria were influenced by the process of coagulation.

In 1887 Fodor made a second contribution to this subject, and in this he combated the retention theory of Wyssokowitsch. One minute after the injection of one c.c. of anthrax culture into the jugular vein, in eight samples of blood, Fodor found only one colony of the bacillus. He then took the blood from the heart with a sterilized pipette and added anthrax bacilli to it. This was kept at $38^{\circ}$, and plates made from time to time showed a rapid diminution of the number of germs; after a time, when the blood had lost its germicidal properties, the number of bacteria began to increase.

In 1888, Nuttall, working under the direction of Flügge, used defibrinated blood taken from various species of animals-rabbits, mice, pigeons, and sheep-and found that this blood destroyed the bacillus anthracis, bacillus subtilis, bacillus megaterium and staphylococcus pyogenes aureus when brought in contact with them. $\mathrm{He}$ 
also confirmed the finding of Fodor that after a while the blood loses its germicidal properties and becomes a suitable culture medium in which germs grow abundantly.

Nissen continued this work under Flügge's direction and reached the following conclusions :

1. The addition of small quantities of sterilized salt solution or bouillon to the blood does not destroy its germicidal properties.

2. Cholera germs and Eberth bacilli are easily destroyed by fresh blood.

3. In a given volume of blood there is a maximum number of bacilli which can be destroyed.

4. Blood, the coagulability of which has been destroyed by the injection of pepton, is still germicidal.

5. Blood in which coagulation is prevented by the addition of 25 per cent. of magnesium sulphate has its germicidal properties decreased.

6. Filtered blood plasma from the horse is germicidal.

At one time, Behring attributed the action of the blood of the white rat on antbrax bacilli to its great alkalinity. He made a number of titrations, by which he showed that the blood serum of the rat is somewhat more alkaline than that of certain animals which are more susceptible to anthrax, such as the rabbit, the guinea-pig and the cow. This deduction is not justified, because there are many other and more important points in which these animals differ from the white rat than in slight differences in the alkalinity of the blood serum. Had he shown that the blood of the adult rat, which is not susceptible to anthrax, is more alkaline than that of the young rat, which is susceptible, his argument would have been more plausible ; but even then it would not have deserved the dignity of positive evidence.

In 1890 Buchner and his students made a valuable contribution to our knowledge of the germicidal properties of blood, and reached the following conclusions:

1. The germicidal action of the blood is not due to phagocytes, because it is not influenced by the alternate freezing and thawing of the blood, by which the leucocytes are destroyed.

2. The germicidal properties of the cell-free serum must be due to its soluble constituents.

3. Neither neutralization of the serum, nor the addition of pepsin, nor the removal of carbon dioxide gas, nor treatment with oxygen, has any effect upon the germicidal properties of the blood.

4. Dialysis of the serum against water destroys its activity, while dialysis against 0.75 per cent. salt solution does not. In the diffusate there is no germicidal substance. The loss by dialysis with water must be due to the withdrawal of the inorganic salts of the serum.

5. The same is shown to be the case when the serum is diluted 
with water and when it is diluted with salt solution; in the former instance the germicidal action being destroyed, while in the latter it is not.

6. The inorganic salts have in and of themselves no germicidal action. They are active only in so far as they affect the normal properties of the albuminates of the serum. The germicidal properties of the serum reside in its albumin constituents.

7. The difference in the effects of active serum and that which has been heated to $55^{\circ}$ is due to the altered condition of the albuminate. The difference may possibly be a chemical one (due to changes within the molecule) or it may be due to alterations in mycelial structure. The albuminous bodies act upon the bacteria only when the former are in an active state.

In the third edition of this book we pointed out an inconsistency between Buchner's experimental results and his conclusions. Experimentally he ascertained that peptic digestion of blood serum does not destroy its germicidal properties, and yet he concluded that the active principle is serum albumin. Since serum albumin is destroyed by peptic digestion, it cannot be the active germicidal agent in the serum.

Prudden found that ascitic and hydrocele fluids restrain the development of certain germs. Rovighi reported that the germicidal action of the blood is increased in febrile conditions. Pekelharing enclosed anthrax spores in bits of parchment and introduced them under the skin of rabbits. Thus treated, the spores soon lost their virulence and finally their capability of growth. The destruction of these spores could not have been due to phagocytes, which did not penetrate the parchment, but must have been caused by soluble germicides. Behring and Nissen found that the serum of the white rat and of the rabbit destroys anthrax bacilli, while serum obtained from the mouse, sheep, guinea-pig, chicken, pigeon, and frog, has no such effect. It will be observed from this that there is no constant relation between the germicidal action of the blood of animals of different species and their susceptibility to the disease caused by the germ ; thus, the rabbit is highly susceptible to anthrax, notwithstanding the fact that its blood destroys large numbers of these germs; while the chicken is immune to anthrax from the moment when it comes from the shell and yet the bacillus anthracis grows luxuriantly in the extra-vascular blood of the chick. This demonstrates that there is a great difference between the action of extra-vascular blood and that existing in the body, constantly fed, and, in case of need, altered in composition by certain glands.

Halliburton prepared from the lymphatic glands a globulin which he designated cell-globulin- $\beta$, and which agrees with fibrin ferment in inducing coagulation in plasma. Hankin tested the germicidal properties of this globulin, conducting his experiments in the follow- 
ing manner : The lymphatic glands or the spleen of a dog, or cat, are freed as much as possible from fat and connective tissue, then divided and extracted with a dilute solution of sodium sulphate (one part of a saturated solution to nine parts of water). The cell globulin passes into solution, while the other proteids are but sparingly soluble. After twenty-four hours, the fluid is filtered and mixed with an excess of alcohol. The voluminous precipitate contains the cell globulin and is collected on a filter and washed with absolute alcohol. For use, a part is dissolved in water, and a small quantity of a bouillon culture of the anthrax bacillus is added. From time to time plate cultures are made, along with control plates, and in this way the germicidal properties of the substance are demonstrated. Hankin reached the following conclusions :

1. Halliburton's cell-globulin- $\beta$ has marked germicidal properties.

2. In this respect it differs from fibrin ferment.

3. The germicidal properties of this substance seem to be identical with those of serum, as described by Buchner, Nissen and Nuttall.

4. The active properties of the serum are probably due to this or an allied body.

Bitter repeated these experiments, but failed to confirm them; however, it is certain that the spleen contains a germicidal substance, but whether it can be extracted by the method of Hankin or not we do not know.

Christmas prepared a germicidal substance from the spleen and other organs by the following method: The animal is killed with ether, opened under aseptic precautions, and the organ removed, cut into fine pieces, covered with 50 c.c. of glycerin and allowed to stand for twenty-four hours and then filtered. The filtrate is precipitated with five times its volume of alcohol and this is immediately decanted. The precipitate is washed with absolute alcohol in order to remove the glycerin, and then traces of alcohol are taken up by pressure between folds of blotting paper and the precipitate is dissolved in 25 c.c. of distilled water. Through this solution air is passed for some hours in order to remove traces of alcohol, and then the fluid is filtered and its germicidal action tested.

Bitter examined this method also, and the impartial reader may see that he did not do so with fairness. However, this fact renders the work all the more valuable because his results confirm the statements of Christmas. Bitter killed his animals by venesection and, in some cases, at least, prepared the substance in unsterilized vessels, but even when this was done the solution was germ-free and manifested marked germicidal properties. However, Bitter found a difference between this substance and the germicidal constituent of blood serum; the latter is certainly destroyed by a temperature of $65^{\circ}$, while the solution of Christmas, after having been heated to this 
temperature, is still capable of destroying from 35,000 to 40,000 typhoid bacilli within four hours.

It is possible that the more powerful action of the solution made by Christmas is due to the presence of the germicidal substance in more nearly a chemically pure condition than it exists in blood serum, and later we shall see that the temperature at which the germicidal activity of blood serum is arrested is variable, and depends upon conditions which are not thoroughly understood.

Attempts have been made to determine the nature of the germicidal constituent by the action of precipitating reagents on the proteids of blood serum. Buchner was not able to obtain a germicidal solution by precipitating all the proteids with absolute alcohol, freeing the precipitate from alcohol, drying it, and then redissolving it. Inasmuch as he failed to give the methods employed in freeing the precipitate from alcohol, the temperature or the conditions under which it was dried, and the nature of the menstruum by which re-solution was effected, his conclusion that alcohol destroys the germicidal substance must remain open to question. On the other hand, Christmas found that when the proteids are precipitated with alcohol and the precipitate dissolved in a volume of water equal to that of the original solution, the solution thus obtained has a more powerful germicidal action than the serum.

Bitter reached the conclusion that anthrax and typhoid bacilli are destroyed by "precipitated serum," but not so energetically as by normal serum. Emmerich, Tsuboi, Steinmetz and Löw studied the effect of precipitation of the proteids upon the germicidal properties of the blood serum. An active serum was dialyzed in a sterilized parchment paper tube against water for from twelve to eighteen hours. By the expiration of that time the serum globulin, becoming insoluble on account of the withdrawal of inorganic salts, was deposited. The dialyzer was dried with sterilized filter paper, and the globulin-free serum was precipitated with several volumes of alcohol. The precipitate was collected on a sterilized filter and the alcohol removed by sterilized porous plates and filter paper. The precipitate was then finely divided, dried for half an hour in vacuo at $36^{\circ}$, then rubbed up in a sterilized mortar and dissolved in sterilized water, to which salt solution had been added. In the solution thus prepared germs did not show, after from three to four hours, either a marked increase or decrease, but when the solution was heated to $100^{\circ}$, allowed to cool, and then inoculated with germs, the increase was four hundred-fold within four hours. It was next found that if instead of water a 0.05 per cent. aqueous solution of potassic hydrate was employed in dissolving the alcoholic precipitate in the globulinfree serum, this solution possessed all the germicidal strength of the original serum. The same was found to be true of dilute alkaline solutions of the alcohol precipitate in serum from which the globulin 
had not been removed. The dilute alkaline solution was shown not to have any germicidal action in and of itself. From these experiments the above-mentioned investigators concluded that the germicidal constituent of blood serum is an alkaline compound of serum albumin. They also found that heating the serum albumin alkaline solution to $65^{\circ}$, or higher destroyed its germicidal action, and they explained this effect of heat upon blood serum and other artificial solutions by supposing that the high temperature breaks up the combination of the alkali with the serum albumin. Furthermore, they found that a serum that had been rendered inactive by a temperature of $55^{\circ}$ could be regenerated, in part at least, by the addition of a small amount of alkaline menstruum.

Since Fodor and Züutz have shown that freshly drawn blood rapidly decreases in alkalinity on standing in vitro, an explanation of the fact that blood serum rapidly loses its germicidal properties naturally suggests itself. Emmerich and his co-workers confirmed their belief in this theory by demonstrating that blood serum which has been rendered feebly acid ( 0.67 part of sulphuric acid per mille) has no germicidal action, but furnishes a good culture medium. These investigators show the important rôle that the small amount of alkali plays in the germicidal action of blood serum. This had already been demonstrated by Fodor by quite a different line of investigation. The latter found that the resistance of rabbits to anthrax is markedly increased by the administration, by stomach or subcutaneously, of sodium phosphate, carbonate or bicarbonate, or of potassium carbonate. Löw concludes that the introduction of alkali into the albumin molecule increases its lability, and he cites examples from organic chemistry in support of this view. Emmerich and his assistants think it highly probable that only a comparatively small part of the albumin is active, and this small part, they suppose, originates in the albumin of the daily food which is converted into lymph cells, and by the disintegration of these it passes into solution in the blood; however, they admit that there are some reasons for believing, with Buchner, that the whole of the serum albumin is active. They state that it is possible, but highly improbable, that the germicidal substauce is not the serum albumin, but some substance that is precipitated along with this by alcohol and other agents.

In 1893 Vaughan and McClintock, after reviewing the literature, reported their work on the germicidal constituent of blood serum as follows :

1. The serum albumin is not the germicidal substance in blood serum. Either this must be true or the experiment by which Buchner demonstrated that an active pepsin does not destroy the germicidal action of blood serum must have been an error; because peptic digestion readily and completely converts serum albumin into pep- 
tons, and we know that peptons are especially favorable to bacterial growth.

2. The germicidal substance must belong to the proteids. Otherwise it would be difficult to explain the fact that a temperature of $55^{\circ}$ renders blood serum inactive.

3. The only proteid likely to be present in blood serum and which is not destroyed by peptic digestion is nuclein. Having reached these conclusions, the following questions naturally presented themselves :

1. Is there a nuclein in blood serum? 2. Has this nuclein, if there be one, germicidal properties?

The first of these questions was answered in the following manner : An active blood serum was treated with ten times its volume of a mixture of equal parts of absolute alcohol and ether. This produced a voluminous precipitate which, after repeated washings with alcohol and ether, was submitted to the action of pepsin-hydrochloric acid in the iucubator at $38^{\circ}$. Digestion was continued until the supernatant fluid failed to respond to the biuret test for peptons. Each time this test was made the fluid was decanted from the undigested part and replaced by an equal volume of fresh digestive fluid. In all cases, digestion was prompt and proceeded to a certain point, when it ceased altogether. The undigested proteid was small in amount and grayish in color. This was collected on a small sterilized filter, and washed first with a 0.2 per cent. solution of hydrochloric acid, and then with alcohol. After the washing with alcohol, the filter was allowed to stand exposed to the air for half an hour or longer in order that all of the alcohol might pass through or evaporate. The precipitate was then dissolved in a sterilized solution of potassic hydrate. The strength of this alkaline solution usually employed was 0.12 per cent. and the solution contained in addition to the alkali 0.6 per cent. of sodium chlorid. In some instances a solution containing 1.2 grams of potassic hydrate, six grams of sodium chlorid, and one gram each of sodium bicarbonate and disodium hydrogen phosphate to one liter of water, was employed as a solvent. The solution was filtered through a Chamberland tube and received in a sterilized flask. The solution thus obtained was perfectly clear, colorless, and did not respond to the biuret test. The addition of strong nitric acid produced a cloudiness, which dissolved on the further addition of the acid. This acid solution did not become yellow on being heated, but did so after the addition of ammonia. It is true that there was no ultimate analysis made of this substance, but the above experiments demonstrate the fact that blood serum contains a proteid which resists peptic digestion, does not respond to the biuret and xanthoproteic tests, and which, as we shall see later, has marked germicidal properties. Is this substance serum albumin or is it a nuclein? Moreover, the presence of nucleinic acid 
in blood serum has been confirmed by the work of Lilienfeld, whose paper was published in 1895, while that of the American investigators was published in 1893 .

The germicidal action of the nuclein, obtained as above stated, from blood serum, was abundantly demonstrated by a long series of experiments published in the original contributions on this subject and in part reproduced in the third edition of this book.

In the third edition of this book there occurred the following paragraph: "The origin of the nuclein now found for the first time in blood serum is an interesting question. Does it come from the disintegration of the polynuclear cells, or shall we regard certain white blood corpuscles as unicellular organisms whose function it is to secrete this nuclein?" It will be seen from what is to follow that practically all the investigations, which have been carried on since the above paragraph was written, have had for their object the solution of the questions there proposed.

Buchner has long used the term alexins to designate the germicidal substance or substances that exist in blood serum and whose exact nature has not yet been determined. His most potent argument against alexin being a nuclein lies in the fact that when blood serum is heated to $56^{\circ}$ or $58^{\circ}$ it loses its bactericidal properties; while it is well known that aqueous solutions of nucleinic acid are not altered by much higher temperatures. A serum whose germicidal action has been destroyed by heat is designated as inactive. It is within the range of possibility that there may be a nuclein or a nucleinic acid so labile that it loses its germicidal action at the relatively low temperature mentioned above. It is also possible that while an aqueous solution of nucleinic acid may retain its germicidal properties at $100^{\circ}$, when mixed with the constituents of blood serum it may be altered at a much lower temperature. It will be seen from this that the effect of temperature upon the alexins of the blood is a matter of importance inasmuch as it may be suggestive of the composition of these bodies. Bail ${ }^{1}$ in his studies on the action of leukocidin found that while this toxin dissolves or disintegrates the leucocytes, it does not destroy the bactericidal substance contained in them and that when this phenomenon occurs the germicidal constituent of the white blood corpuscle passes into solution, and that such a solution may be boiled without losing its germicidal properties. He also ascertained that when isolated leucocytes are extracted with alkaline sodium chlorid solution and this extract precipitated with acetic acid and then redissolved in an alkaline inactive dilute blood serum, a germicidal solution is obtained and that this may be heated for one-half hour to $85^{\circ}$ without loss of bactericidal properties. Furthermore, he slowed that the germicidal properties were due to the substance precipitated with acetic acid inasmuch as he

1 Archiv f. Hygiene, 30 and 32. 
found that on removal of this precipitate the solution became inactive. Löwit ${ }^{1}$ rubbed up isolated leucocytes with pulverized glass and extracted from the powder thus obtained a bactericidal substance which is not destroyed or even weakened in action by five minutes' boiling. The germicidal extract thus obtained is cloudy, of feebly alkaline reaction, gives the biuret test, contains but little proteid which is precipitable by heat, and yields a flocculent precipitate on the addition of acetic acid. From their investigations both Bail and Löwit conclude that the heat-resisting bactericidal substance obtained by them is nuclein or nucleinic acid or a derivative of one of these substances. Schattenfroh ${ }^{2}$ combats the conclusion reached by the above-mentioned anthors. He claims that the germicidal substance obtained by Löwit consists of sodium silicate extracted from the powdered glass, and he attempts to demonstrate this by an experiment in which the germicidal action of this compound is tested. This experiment, however, is not convincing, inasmuch as it shows that even a one per cent. solution of sodium silicate is practically without bactericidal properties; and it is not probable that water or physiological salt solution will extract from glass a stronger solution than this. In his criticism on the work of Bail, Schattenfroh admits that the results obtained by his opponent are exactly what he himself observed, but he thinks that in this case the heat-resisting germicidal substance is the alkali used in extracting the leucocytes. This criticism also is somewhat far-fetched inasmuch as Schattenfroh attempts to demonstrate the truth of his assertion by showing that Bail's extract, when neutralized or rendered feebly acid, is without germicidal properties, but everyone knows that blood serum when neutralized or rendered feebly acid also becomes inactive. Moreover, Fodor long ago demonstrated that increasing the alkalinity of the blood increases its germicidal properties. Schattenfroh's own investigations have demonstrated that a temperature of $56^{\circ}$ to $58^{\circ}$ does not destroy the alexins in aqueous extracts of isolated leucocytes. On this point he makes the following statement: "Experiment 3 and others leave no longer room for doubt that the bactericidal action of leucocytes is retained after being heated in distilled water for half an hour to from $55^{\circ}$ to $60^{\circ}$. It may be seen from what has been stated that the resistance of the alexins to heat is largely dependent upon the nature of the menstruum in which they are dissolved or held in suspension."

The evidence concerning the manner in which the leucocytes produce alexins is scarcely more satisfactory than that bearing upon the influence of temperature upon the latter. Haukin ${ }^{3}$ was probably the first to suggest that the alexins are products of the amphophil

1 Ziegler's Beiträge, 22.

${ }^{2}$ Archiv f. Hygiene, 35.

${ }^{3}$ Centralblatt f. Bakterioloqie, 12. 
leucocytes, which he proposes should be called alexocytes. According to his theory the pseudo-eosinophil granules constitute the parent substance of the alexins. He succeeded in diminishing the number of these leucocytes in the blood by the intravenous injection of leech extract and found that the blood thus obtained was not equivalent to normal blood in its germicidal properties. Denys ${ }^{1}$ and his students were among the earlier advocates of the theory that the alexins are secretion products of the leucocytes. Buchner ${ }^{2}$ obtained leucocytes in relatively large numbers by introducing, under aseptic precautions, wheat gluten or aleuron into the pleural cavities of animals; an exudate rich in white blood corpuscles forms about the foreign body after a few hours and the corpuscles thus obtained serve for experimentation. The purpose of Buchner's first experiments was to combat the phagocytic theory of Metschnikoff, and he demonstrated that the leucocytes, obtained as stated above, when destroyed by alternate freezing and thawing, markedly increased the germicidal action of the serum in which they were suspended, while at the same time they were deprived of life. In other words, these experiments demonstrated that the disintegration of white blood corpuscles liberates a germicidal substance. Leucocytes obtained after Buchner's method can be freed from serum in the centrifuge, and may be dissolved or suspended in physiological salt solution, distilled water, or in either an active or an inactive serum. By experiments of this kind Hahn ${ }^{3}$ demonstrated that the addition of leucocytes to an active serum increased the activity of the latter, while when added to an inactive serum they restored its activity. In this way it has been shown beyond controversy that blood serum owes its germicidal properties to the white corpuscles. Numerous investigators have fully demonstrated that the disintegration of the leucocyte is followed by the liberation of alexins, and the most important question still unsettled in this connection is whether or not the living white corpuscles secrete alexins. Laschtschenko ${ }^{4}$ has shown that either active or inactive serum of the horse extracts alexins from the leucocytes of the rabbit. This experiment was made in the following way: The leucocytes were obtained in a pleural exudate, prepared after the method of Buchner. This exudate was placed in a centrifuge and the corpuscles separated from the serum. The corpuscles were then repeatedly washed in the centrifuge with physiological salt solution and after they had been thoroughly separated from all the constituents of the serum they were well mixed with serum obtained from other animals and freed from their own corpuscles by means of the centrifuge. Both active and inactive sera were used in these experiments. The result, which has already been stated, is attributed to

I La cellule, 10 , et seq.

2 Münchener med. Wochenschrift, 1894.

3 Archiv $f$. Hygiene, 25.

1 Archiv f. Hygiene, 37. 
the biological stimulation of the foreign serum on the leucocytes of the rabbit. It must be admitted, however, that it is possible that the serum of the horse destroys the leucocytes of the rabbit and that the germicidal substances which pass into solution in the serum result from the disintegration of the corpuscles. From microscopical study of the leucocytes thus subjected to the action of the serum of the horse, Trommsdorff ${ }^{1}$ concludes that the leucocytes still retain their vitality while the alexins pass from them into the serum. He finds that corpuscles treated in this way still possess amœboid movement and when compared with leucocytes known to be living show no signs of disintegration. It is therefore highly probable that the living leucocyte secretes a germicidal substance.

We will close our remarks upon the germicidal constituent of the blood with the following statements: (1) The exact nature of the germicidal constituents of the blood, or alexins, is not known. (2) The alexins have their origin in the white blood corpuscles. (3) Disintegration of the white blood corpuscle liberates alexins. (4) It is probably true that alexins are also secreted by living leucocytes.

1 Archiv r. Bygiene, 40. 


\section{CHAPTER VI.}

\section{THE SPECIFIC PRECIPITINS.}

THE announcement by Widal, ${ }^{1}$ that typhoid bacilli, killed by a heat of $56^{\circ}$, are still agglutinated with the homologous serum, led Kraus ${ }^{2}$ to try the effect of an homologous serum on a filtered bacterial culture, and this experiment was the beginning of our knowledge of the specific precipitins. Cultures of the cholera bacillus were freed from germs by filtration through porcelain, and after having been proved to be sterile were treated with different quantities of a sterile cholera serum. When these two fluids were mixed the mixture became cloudy and after a while filled with fine floccules which gradually subsided, leaving a supernatant clear fluid. Further investigation showed that the germ-free bacterial culture and the serum must be homologous in order to obtain this precipitation. A serum obtained from an animal which has been rendered immune to the cholera bacillus gives a precipitate when added to filtered cultures of the cholera bacillus, but gives no cloudiness when added to like cultures of any other germ. It was also found that the serum of non-immunized animals does not give precipitates with the filtered cultures of any of the pathogenic bacteria. Kraus extended his investigations using the sera and filtered cultures not only of cholera, but also of typhoid fever and the plague, and found that the reaction observed by him was a specific one inasmuch as it was obtained only with homologous sera and bacterial filtrates, and not with heterologous fluids. Furthermore, he demonstrated that the substance in the filtered culture to which this reaction is due exists within the bacterial cell. This he did by rubbing up cholera bacilli taken from agar cultures with powdered glass and submitting this mixture to a pressure of 300 atmospheres; the compressed mass was then extracted with alkaline bouillon, diluted and filtered through porcelain, when it was found that this filtrate gave a precipitate with cholera serum. This shows that the specific reaction observed in these experiments for the first time, is due to the presence in the filtrate of a substance extracted from the bacterial cells. Cultures filtered in the ordinary way through stone give this reaction because filtrates thus obtained contain substances which originated withiu the bacterial cells.

Nicolle ${ }^{3}$ repeated, confirmed and extended the observations of

${ }^{1}$ La Semaine Med., 1897.

Wiener klin. Wochenschrift, 1897.

'Annales del' Institut Pasteur, 12. 
Kraus, and subsequent investigations by others have shown that this specific reaction holds good with all bacteria to which animals have been immunized.

The substance in the serum which enters into this reaction is known as the precipitin and the precipitate thus formed is designated as a precipitum.

Bordet found that when milk which had been partially sterilized by being heated for one hour at $65^{\circ}$ was injected intraperitoneally and this injection repeated at short intervals for some time, the blood serum of the animals thus treated gives with the milk which has been injected a precipitate. He placed three c.c. of the serum from the animal treated with the milk in one test-tube and the sera of other animals in corresponding tubes as controls, and found that on the addition of the milk which had been used in the treatment of the animal, a precipitate appears, while no cloudiness occurs on the addition of the same milk to other sera. On the addition of ten or twelve drops of milk to the homologous serum, floccules form and slowly subside, leaving a clear supernatant fluid, while in the other sera the milk diffuses, forming opalescent homogeneous fluids. Further investigations, notably those of Wassermann and Schütze, ${ }^{1}$ have shown that the serum of a rabbit treated with cow's milk gives a precipitate with cow's milk and not with the milk of any other animal, and that rabbits treated with goat's milk furnish a serum that gives precipitates with goat's milk and with no other milk; and the same holds good when the experiments are made with woman's milk. By this reaction the source of a given sample of milk can be told with certainty. The following are the details of an experiment of this kind: Several rabbits were treated subcutaneously or intraperitoneally at intervals of from three to four days with from 10 to 50 c.c. of cow's milk, sterilized with chloroform, while a like number were treated in the same way with goat's milk, and a third lot treated with woman's milk. After the rabbits had undergone this treatment for about three weeks and each had received about 100 c.c. of milk, they were bled and their sera, diluted $1: 5$, were added to milk, diluted $1: 40$, and these mixtures allowed to stand at room temperature for some hours. It was found that the serum from the rabbits treated with cow's milk precipitated the albuminous substances in cow's milk, but had no such action on the milk of either woman or goat; while the serum of the rabbit treated with goat's milk precipitated the casein of goat's milk and had no effect upon that of the woman or the cow, etc. The serum of animals that have been treated as above described with milk is designated lactoserum, and it has been found that the lactosera are in all cases specific in their reactions. Indeed, the discovery of this reaction has shown that the proteids of the milk of

${ }^{1}$ Deutsche med. Wochenschrift, 1900. 
each species of animal differ from those of every other species, and it certainly should convince us that it is impossible by any chemical process to make cow's milk a perfect substitute for the mother's milk in the feeding of infants. Fisch ${ }^{1}$ found that rabbits treated with an emulsion of the cells of the udders of animals furnish specific lactosera, and this demonstrates that milk is not a filtration product, but that some of its important constituents result from the transformation of the specific cells of the gland. Milk which has been boiled for half an hour loses its power of forming a precipitum on the addition of its specific lactoserum.

Myers ${ }^{2}$ prepared crystallized egg albumin from fresh eggs and by repeated intraperitoneal injections of this substance into rabbits, he obtained a serum that gives a dense precipitate when added to solutions of crystallized egg albumin. This precipitate forms at ordinary temperature, but its formation is accelerated at $37^{\circ}$. It is soluble in two per cent. sodium chlorid solution and such a solution gives the ordinary proteid reactions. The serum of a rabbit treated with egg albumin from the fowl forms a slight precipitate with the albumin obtained from ducks' eggs. Ovasera have no precipitating action on globulin obtained from sheep serum nor on that from bullock serum, nor on serum albumin from the sheep or the bullock, nor on pepton. Myers next prepared serum globulin from the blood of the sheep and obtained from rabbits treated with this preparation a serum which has no action on egg albumin or upon pepton, but does give a slight precipitate with globulin obtained from bullock's serum. The serum of a rabbit immunized against the globulin of the sheep's blood also agglutinates the red corpuscle of the sheep. This undoubtedly is due to the presence in the red blood corpuscles of the sheep of a substance allied to, if not identical with, the globulin obtained from sheep's blood. It was also found that the globulin serum agglutinates the washed red blood corpuscles of the fowl. From these observations Myers draws the following conclusions : "It follows from these facts that the product here called 'sheep's globulin' is a mixture of substances. The main portion of the precipitum given by its precipitin is formed from a substance which is not present in the 'serum globulin' of the bullock. One substance, present in small quantities in the sheep's globulin, is, however, also present in bullock's globulin, since the precipitin of sheep's globulin gives a small precipitum with bullock's globulin. This substance common to the two globulins is not present in the red corpuscles of the sheep or fowl, since the precipitin in bullock's globulin does not agglutinate these corpuscles. Further, we must suppose that there are two other substances in the serum globulin of the sheep, one present in fowl's red corpuscles and the other present in those of the

2 The Lancet, Vol. II., 1900. 
sheep, since, as the following experiment shows, immunization against sheep's globulin leads to the appearance in the serum of two distinct agglutinins, for sheep's and for fowl's corpuscles, respectively. It the serum of a rabbit immunized against sheep's globulin be allowed to agglutinate washed corpuscles of the sheep, the clear fluid left above the corpuscles will not now agglutinate fresh corpuscles of the sheep if sufficient corpuscles have been used in the first instance. But this fluid still has the power of agglutinating fowl's corpuscles. And, conversely, after antiglobulin has agglutinated fowl's corpuscles, it has lost the power of agglutinating fresh fowl's corpuscles, but will still agglutinate those of the sheep." Animals immunized with pepton furnished a serum which gave a precipitum with pepton and failed to react with any other proteid. The pepton precipitum, after being carefully washed with physiological salt solution and then dissolved in two per cent. saline solution, does not give the biuret test. Myers attempted to determine whether or not the precipitin is used up in the formation of the precipitum and on this point he makes the following statement: "A mixture of the precipitins of bullock's globulin and egg albumin was added to a solution of egg albumin. After standing at $37^{\circ}$ for fifteen hours the mixture was centrifugalized and the clear fluid was tested for the presence of the precipitins. It was found to give a precipitum with bullock's globulin but to give none with egg albumin. At the same time a control experiment was made with a mixture of the precipitins of egg albumin and bullock's globulin with bullock's globulin. In this case the clear fluid precipitated with egg albumin but not with bullock's globulin. Experiments of a similar kind with the precipitins of egg albumin and sheep's globulin were made, and in this case also the one or other of the precipitins disappeared. From these experiments it is concluded that the precipitins are used up in the course of their action, and bearing in mind their specificity, this is strong evidence that the action of these bodies is chemical."

Uhlenhuth $^{1}$ broke freshly laid eggs in sterilized beakers, diluted with physiological salt solution, stirred with sterilized rods, and thus prepared a solution which could be easily injected intra-peritoneally into rabbits. In this way he introduced into each animal at each time the whites of from two to three eggs, and notwithstanding the large volume of the fluid, which frequently measured 100 c.c., it was well borne. After this operation had been repeated several times the rabbits furnished ovasera which proved to be most delicate reagents in testing for egg albumin, inasmuch as a few drops of such a serum added to egg albumin diluted with one hundred thousand parts of water, give a distinct cloudiness, while nitric acid and acetic acid and ferrocyanide of potassium fail to indicate the presence of albumin when the solution is diluted only 1:1000. Moreover, this reaction

${ }^{1}$ Deutsche med. Wochenschrift, 1900. 
is specific and these ovasera, which furnish such delicate reagents for testing for egg albumin, do not give any precipitate with alkaline albuminates, peptons, casein, or blood serum. However, it was found that ovasera obtained from animals immunized to the albumin of hen's eggs do react with the albumin of pigeon's eggs. Uhlenhuth obtained feebly active ovasera by prolonged and excessive feeding of rabbits with egg albumin by the mouth. However, the serum thus obtained was only slightly active and it is probable that the small amount of precipitin in this serum was due to traces of egg albumin absorbed unchanged through the walls of the stomach or intestines. Ovasera may be heated for one hour at $60^{\circ}$ without injury to their precipitins.

Leclainche and Vallèe ${ }^{1}$ injected albuminous urine intravenously into rabbits. They used in this way 20 c.c. at a time and repeated at intervals until each animal received from 150 to 200 c.c. The serum of animals thus treated furnishes a very delicate test for albuminous urine, giving an immediate precipitation. Urinsera do not precipitate non-albuminous urine. Moreover, if the animal has been rendered immune with a urine containing serum globulin, the serum from this animal does not precipitate urine containing serum albumin, and vice versa. Urinsera also precipitate pleuritic and other exudates, thus showing that the proteids of albuminous urine and of these exudates are identical. There is some contradiction in the statements concerning the action of urinsera on the blood serum of man. This point needs more extended observation. Mertens ${ }^{2}$ immunized a rabbit with placental blood serum and found that the serum obtained from this animal produced a precipitum in human blood serum and in albuminous urine from man, but was without effect upon the blood serum of normal rabbits or upon the albuminous urine of a rabbit whose kidneys had been injured by the administration of cantharides.

Zuelzer ${ }^{3}$ treated rabbits with from 5 to 10 c.c. of albuminous urine (containing from 1 to 9 per $\mathrm{m}$. of albumin) at intervals of from one to three days for two weeks, obtaining from the animals thus treated a serum which precipitated both albuminous urine and the blood of man.

Tchistovitch ${ }^{4}$ rendered animals immune to eel serum which is toxic, and obtained from the animals thus immunized a serum which produced a precipitum in the eel serum. His conclusions are as follows :

1. Rabbits, dogs, goats, and pigeons are easily immunized against the serum of the eel; it is infinitely more difficult to immunize guinea-pigs.

${ }^{1}$ Comptes Rendus de la Société de Biologie, 1901.

2 Deutsche med. Wochenschrift, 1901.

3 Deutsche med. Wochenschrift, 1901.

'Annales de l' Institut Pasteur, 13. 
2. There appears in the blood of the immunized animals an antitoxin which in vitro neutralizes the solvent action of the toxin on the red blood corpuscles of the rabbit, and which, when injected into the blood, prevents the action of the toxin.

3. This antitoxin appears in the blood promptly, even after from two to four injections, and in the rabbit it is at this time at its maximum strength ; in general, its antitoxic value is not great.

4. In the course of a prolonged immunization, the strength of the antitoxin progressively diminishes while the resistance of the rabbit against the toxin increases.

5. The red blood corpuscles of immunized rabbits are less soluble in the serum of the eel than are the corpuscles of non-treated animals.

6. The resistance of the corpuscles is not in proportion to the amount of antitoxin present in the blood of the immunized animal ; on the contrary, one observes a certain antagonism between the resistance of the corpuscles and the strength of the antitoxin, and in instances in which the latter is of relatively great strength the solubility of the corpuscles may be slightly augmented.

7. The injection of eel serum into the blood is followed by a notable diminution in the number of leucocytes in an untreated animal and an augmentation or a slight diminution in immunized animals. In a mixture of eel serum with an antitoxin obtained from an immunized animal one observes a cloudiness and a precipitation similar to that reported by Kraus in filtered bacterial cultures. This precipitate is insoluble in water, in neutral salts, and in alkaline carbonates, but is easily soluble in alkalis and acids. Its formation resembles the coagulation of a substance dissolved in toxic or antitoxic serum.

8. The volume of this precipitate is ordinarily in proportion to the strength of the antitoxin; its formation is retarded by heating the antitoxin to $70^{\circ}$ for one-half hour; and heating the eel serum to $80^{\circ}$ renders it incoagulable by the antitoxin.

9. The coagulation is not directly due to the antitoxin for one may obtain antitoxic sera which do not give a coagulum.

10. Antitoxic sera acquire very promptly the property of agglutinating the red corpuscles of the animals whose blood or serum is injected.

11. The power of agglutinating the red blood corpuscles does not correspond with the coagulating power and the former may be pronounced in a serum which does not coagulate its homologous toxin.

12. The agglutination of red blood corpuscles is not caused by the coagulation of substances dissolved in the liquid portion.

Bordet ${ }^{1}$ injected the defibrinated blood of the chicken into rabbits and obtained from the latter a serum which produced a precipitum

${ }^{1}$ Annales de ' Institut Pasteur, 13. 
in chickens' blood, also agglutinized and dissolved the corpuscles. Uhlenhuth ${ }^{1}$ treated rabbits intraperitoneally with dilutions of chickens' blood, and obtained from the former animals a serum which gave a precipitum with chickens' blood, but had no such action on the blood of the horse, donkey, cow, sheep or pigeon; nor did it produce any precipitum in solutions of egg albumin, nor in the serum of normal rabbits.

Wolff ${ }^{2}$ allowed chickens' blood to flow into four times its volume of a one per cent. solution of salt and separated the corpuscles from the plasma and injected both of these separately into two sets of rabbits. From four to six injections at intervals of four to five days were made and only those animals treated with the blood plasma yielded a precipitum with chicken blood; those treated with the corpuscles had no such effect. Other rabbits treated in the same manner with dog's blood corpuscles and plasma gave similar results, the precipitins being found only in those animals which had been treated with the fluid portion of the blood.

Uhlenhuth ${ }^{3}$ treated rabbits intraperitoneally at intervals of from six to eight days with 10 c.c. of defibrinated blood at each injection. After the fifth injection the serum from the treated animals was obtained and used for testing blood from various sources. The blood to be tested was diluted with tap water until it was reduced to a feebly red color (1:100). This diluted blood was freed from stroma either by filtration or by decantation, and two c.c. of it was placed in test-tubes and diluted with an equal volume of double physiological salt solution (1.6 per cent.). It is important that blood should be diluted with physiological salt solution and not wholly with water, which is likely to cloud certain bloods when diluted. Blood thus prepared was obtained from the ox, horse, donkey, pig, sheep, dog, cat, deer, hare, guinea-pig, rat, mouse, rabbit, chicken, goose, turkey, pigeon and man. To tubes containing diluted blood from each of the abovementioned animals, from six to eight drops of the serum of the rabbit which had been treated with ox blood was added. The ox blood was immediately clouded, while all others remained perfectly clear. The clouding in the first mentioned grew in intensity and finally formed floccules which gradually subsided. It will be seen that by this test ox blood was easily distinguished from all others with which it was compared. Uhlenhuth was also able to distinguish ox blood from that of man or the horse, the three kinds having been obtained from stains four weeks old.

Stern ${ }^{4}$ treated animals subcutaneously with human blood, employing from five to ten c.c. at each injection with intervals of two or more days. After from two to three weeks, he obtained from the

${ }^{1}$ Deutsche med. Wochenschrift, 1900.

${ }^{2}$ Annales de $l$ Institut Pasteur, 14.

3 Deutsche med. Wochenschrift, 1901.

Deutsche med. Wochenschrift, 1901. 
rabbits a serum which precipitated the blood serum obtained from man, also albuminous urine from man, and did not precipitate the blood serum of the horse, sheep or ox. He states, however, that the reaction is not wholly a specific one, inasmuch as the serum obtained in this case gave marked cloudiness with blood sera obtained from three different species of ape.

Wassermann and Schütze ${ }^{1}$ tested serum obtained from rabbits treated with human blood on 23 kinds of blood, and found that none of these reacted except blood from man and from a baboon; however, the reaction with the blood of the baboon was not nearly so marked as that with the blood of man.

Dieudonné ${ }^{2}$ treated rabbits with human blood serum, albuminous urine, and pleuritic exudate. The rabbits treated with human blood serum furnished a serum which gave a precipitum with human blood and had no effect on the blood of the rabbit, the guinea-pig, the pigeon or the goose. The animals treated with albuminous urine gave a precipitum in the albuminous urine of man and had no effect upon the normal urine of man or that of the rabbit. The serum obtained from animals treated with human pleuritic exudate gave no reaction with a similar exudate obtained from a guinea-pig. The serum obtained from the rabbits treated with human blood serum gave a precipitum not only with human blood, but also with human albuminous urine and the pleuritic and peritoneal exudates obtained from man.

Nuttall ${ }^{3}$ obtained rabbit sera by treating these animals with the blood of man, dog, sheep, ox and horse. These sera were tested against thirty-six kinds of blood. The serum of rabbits treated with dog serum gave negative results in all cases except with the blood of the dog. The serum of rabbits treated with sheep serum produced a marked precipitum with sheep's blood, and a distinct, but less marked, reaction with the blood of the gazelle and axis deer. All the other sera and bloods remained perfectly clear, excepting that of the $o x$, squirrel and swan, in which there was very slight cloudiness. The serum of the rabbits treated with ox serum produced a marked precipitation only in ox serum dilutions or dried ox blood solutions, but gave a distinct reaction with the blood of the gazelle and axis deer. All the other bloods gave a negative reaction, a slight clouding only being produced in the blood serum of the sheep, gnu, squirrel and swan. The serum of rabbits treated with horse serum produced a precipitum only in dilutions of the horse blood or serum, not even clouding other bloods. The serum of the rabbits treated with human blood serum and pleuritic exudate produced a marked precipitum only in human blood solutions, but the blood of four spe-

1 Berliner klin. Wochenschrift, 1901.

2 Münchener med. Wochenschrift, 1901.

'Journal of Hygiene, 1. 
cies of monkeys gave a slight but distinct reaction and a very faint clouding appeared in the solutions of the bloods of the horse, ox and sheep, all other bloods remaining perfectly clear. "The test gave positive results when made with diluted human serum, pleuritic exudation, both fresh and purulent, blood and serum which had been dried on filter paper and on glass plates, with blood which had undergone putrefaction for two months, with the blood of several persons who had cut themselves (blood collected on filter paper), with the serum from a blister on the foot following upon a long walk, and with the serum from a blister following a burn on the hand. Both nasal and lachrymal secretion gave a slight but decided reaction. A faint clouding was produced in normal urine. That the precipitum formed in putrid blood dilution was specific was proved by adding the anti-sera of rabbits treated with ox, sheep and dog serum to the blood dilution, no reaction resulting."

Nuttall concludes his investigations as follows: "(1) The investigations we have made confirm and extend the observations of others with regard to the formation of specific precipitins in the blood serum of animals treated with various sera. (2) These precipitins are specific, although they may produce a slight reaction with the sera of allied animals. (3) The substance in serum which brings about the formation of a precipitin, as also the precipitin itself, are remarkably stable bodies. (4) The new test can be successfully applied to a blood which has been mixed with those of several other animals. (5) We have in this test the most delicate means hitherto discovered of detecting and testing bloods, and consequently we may hope that it will be put to forensic use." 


\section{CHAPTER VII.}

\section{THE LYSINS.}

ONE of the most important contributions to the science of bacteriology made during the last decade of the nineteenth century was the discovery of what is known as Pfeiffer's phenomenon. In his experiments, R. Pfeiffer discovered that if cholera bacteria are placed in the peritoneal cavity of a guinea-pig which has been immunized to cholera, the bacterial cells are dissolved by the peritoneal fluid. If such an injection be made and portions of the bacilli be removed with capillary glass tubes every five minutes, it can be plainly seen, under the microscope, that the bacterial cells are undergoing solution in the surrounding fluid just as lumps of salt dissolve in water. The bacilli lose their motility, swell up, and then break into small granules which gradually melt away into the fluid. Extended investigation showed that this reaction is specific and the same phenomenon may be observed in animals which have been immunized to the typhoid bacillus or other pathogenic microörganisms, and by this means the cholera bacillus may be differentiated from other vibrios. Moreover, when a mixed culture is subjected to this test, the peritoneal fluid dissolves the bacteria of the species against which the animal has been immunized and leaves all other bacteria untouched. Pfeiffer found that it was not necessary to use an immunized animal, but that the same result can be obtained by placing a small quantity of a cholera culture mixed with the serum of a guinea-pig which has been immunized to cholera, in the abdominal cavity of a normal guinea-pig. Later, Metschnikoff ${ }^{1}$ discovered that Pfeiffer's phenomenon, which is also known as bacteriolysis, takes place in vitro, when to a mixture of cholera serum and the cholera culture there be added a small quantity of the peritoneal exudate from a normal guinea-pig, and Bordet added the observation that cholera serum in and of itself suffices to induce bacteriolysis in vitro when it is perfectly fresh. On long standing it becomes inactive, but its activity even then may be restored by the addition of a small quantity of normal serum. Pfeiffer repeated Bordet's experiments, and confirmed them in a modified way, inasmuch as Pfeiffer found that not every fresh blood serum had the same effect, and that the process of bacteriolysis as observed in vitro is not comparable in intensity with that observed in the animal body.

1 Annales de $l$ Institut Pasteur, 1895. 
With these facts at his command Pfeiffer ${ }^{1}$ formulated a theory of bacteriolysis, the chief points of which may be stated as follows: The immunizing substance in cholera serum has but feeble action and is only the antecedent of a substance formed in the peritoneum of the guinea-pig which has a specific solvent action on the vibrios. The immunizing substance in the serum is a stable, relatively inactive body which bears a relation to the specific bacteriolytic substance formed in the peritoneum similar to that which glycogen bears to grape-sugar. In case of need, the inactive substance in the serum is transformed through the active agency of the cells of the body into the specific bacteriolytic form. This change can be brought about by the addition of suitable normal serum. In the added serum there is a "something" (Etwas) present in small amount which is able to transform the relatively inactive substance into the active form, but which in vitro is soon used up, while in the animal body this active principle continues to be secreted by the cells of the body as long as they are stimulated by the presence of the cholera bacteria. The active principle is a ferment and bacteriolysis is a fermentative process in which specific ferments act only on certain cells, just as certain yeasts act only on sugars of certain definite constitution.

The question of the identity of bacteriolytic and agglutinating substances in the sera of immunized animals became prominent, but Pfeiffer and Kolle ${ }^{2}$ soon discovered an immune serum which was strongly bacteriolytic but without agglutinating action; while on the other hand, Fränkel and Otto ${ }^{3}$ found that the blood serum of puppies fed upon typhoid cultures had a marked agglutinating effect, but was without bacteriolytic properties. Moreover, Widal and Sicard found that the blood serum of frogs inoculated with typhoid bacilli agglutinated these microörganisms but had no bacteriolytic action on them, and, indeed, the typhoid bacilli may retain both life and virulence after long residence in the lymph sacs of such frogs.

Bordet ${ }^{5}$ treated guinea-pigs at intervals with injections of the defibrinated blood of rabbits and obtained from the former animals a serum which in vitro dissolved the blood corpuscles of the latter with great intensity, while the serum of normal guinea-pigs was found to be without solvent action upon the corpuscles of the rabbit. In these experiments it was observed that the serum first agglutinated and then dissolved the erythrocytes; but more extended investigation showed that agglutination does not always precede solution. Moreover, Bordet demonstrated that even in this case agglutination and hemolysis are not identical or due to the same substances, inasmuch as he found that a temperature of $55^{\circ}$ deprived

${ }^{1}$ Deutsche med. Wochenschrift, 1896.

${ }^{2}$ Centralblatt $f$. Bakteriologie, 20.

S Münchener med. Wochenschrift, 1894.

4 Compt. Rend. Soc. de Biol., 11, XI.

5 Annales de l' Institut Pasteur, 12. 
the guinea-pig serum of its hemolytic action, but had no effect upon its agglutinating properties. The serum rendered inactive, so far as its hemolytic properties are concerned, by heating, was found to recover these properties on the addition of certain amounts of the serum of either normal guinea-pigs or rabbits. The active serum was found to be without effect upon the corpuscles of the guinea-pig itself and upon those of the pigeon, but with slight action on the corpuscles of the rat and the mouse. The active serum from the guinea-pig was found to be powerfully toxic when injected intravenously into the rabbit. These experiments made by Bordet demonstrated the identity of the processes of bacteriolysis and hemolysis ; and most of the subsequent investigation has been confined 'to a study of the phenomenon as observed in the solution of blood corpuscles.

Ehrlich and Morgenroth ${ }^{1}$ treated a goat at irregular intervals for eight months with subcutaneous injections of defibrinated sheep's blood diluted with 0.85 per cent. salt solution, until the mixture contained only five per cent. of blood. The serum of this goat rapidly dissolved the erythrocytes of the sheep in vitro; while the serum of a normal goat had but slight solvent action on the blood corpuscles of the sheep. In this case agglutination did not precede hemolysis. When the immune serum was heated for half an hour at $56^{\circ}$, it lost its solvent action, but on the addition of the serum of a normal animal its activity was regained. The added serum was active when obtained from either the goat or the sheep, but less so from the latter. Normal serum was found to soon lose its power of restoring the activity of heated immune serum and was effective only when freshly obtained from the animal. These investigators agreed with Pfeiffer that at least two substances must be present in the blood serum in order to induce bacteriolysis or hemolysis. For the first, which is thermo-stable, they retain the name proposed by Pfeiffer, of "immune body"; while for the second, which is thermo-labile, they substitute for the "something" of Pfeiffer the term "addiment." It will be understood that the thermo-stable or immune-body is unaltered by a heat of $56^{\circ}$, while the thermo-labile substance, or addiment, is destroyed at this temperature, but is also contained in normal serum; and this explains why the immune serum after being rendered inactive by heat is regenerated by the addition of a small amount of normal serum. It was at first supposed by Ehrlich that the immune body is a substance which is brought into existence during the process of immunizing the animal, while the addiment was supposed to be present normally in the blood. Ehrlich's theory provided for a combination between the immune body and the blood corpuscles; however, this does not lead to solution of the corpuscle until the substance addiment is brought into the compound. The following experiments furnish the basis of this theory: Tubes each containing

${ }^{1}$ Berliner klin. Wochenschrift, 1899. 
four c.c. of the five per cent. dilution of sheep's blood were treated with from one to one and three-tenths c.c. each of the serum of the goat rendered inactive by heat. The mixture was allowed to remain for fifteen minutes at $40^{\circ}$ and then centrifuged. The supernatant clear fluid was removed and treated with 0.2 c.c. of normal sheep's blood and then 0.8 c.c. of the serum from a normal goat added. This mixture was allowed to stand for two hours at $37^{\circ}$, when it was found that no hemolysis had occurred. Next, the sediment which had formed in the centrifuge was placed in four c.c. of physiological salt solution and 0.8 c.c. of the blood of a normal goat added. When this mixture was allowed to stand at $37^{\circ}$ for two hours, it was found that all the blood corpuscles were dissolved. This experiment can be explained only by supposing that the immune body in the heated serum combined with the corpuscles and subsided with them in the centrifuge, leaving the supernatant fluid without the immune body, and therefore the addition of the addiment to the supernatant fluid did not induce hemolysis. On the other hand, the presence of the immune body in the sediment was shown when hemolysis occurred after distributing the sediment in saline solution and adding a small quantity of normal blood serum.

It was shown that the combination between the corpuscles and the immune body may take place at low as well as at higher temperatures, while the addiment does not enter into the compound at low temperature. When five c.c. of the five per cent. sheep's blood dilution was treated with from 1 to 1.3 c.c. of inactive serum and 0.5 c.c. of the serum of a normal goat added, and this mixture kept at $37^{\circ}$, complete hemolysis occurred within two hours. On the other hand, when the same mixture was kept at from $0^{\circ}$ to $3^{\circ}$ there was no hemolysis, but at this temperature it was shown by a repetition of the experiment already detailed that the immune body was combined in the sediment with the corpuscles, while the addiment remained in the supernatant fluid.

It was also shown that at high temperatures the combination between the corpuscles and the immune body occurs before the addiment enters into the compound. Tubes containing blood corpuscles, inactive serum, and fresh goat serum, were placed in a water-bath at $40^{\circ}$ and the time before visible solution of the corpuscles took place was noted. It was found that no evident hemolysis occurred within ten minutes; therefore the tubes which had been kept at $40^{\circ}$ for ten minutes were centrifuged. When the sediment in the centrifuge was distributed through physiological salt solution slight hemolysis occurred, but complete hemolysis resulted only after the addition of normal serum to this mixture. This experiment demonstrated that all of the immune body had combined with the corpuscles and had subsided with them, while the greater part of the addiment remained in the supernatant fluid. The affinity of the immune body for the 
blood corpuscles is great, and leads to speedy combination between the two both at high and low temperatures; while the affinity of the immune body for the addiment is slight and leads to combination tardily and only at relatively high temperatures. The immune body is supposed to be possessed of two haptophorous groups, one of which has great chemical energy, and is that by which the immune body combines with the corpuscle; while the other haptophorous group possesses less chemical energy and is that by means of which combination with the addiment is effected. In the first statement of his theory, Ehrlich believed that the addiment is a ferment which digests and dissolves bacteria and blood corpuscles. He stated: "It is possible, yes, probable, that there are in the blood only a few, probably only a single body, which has digestive properties; while, on the other hand, there must be innumerable, different, specific immune bodies, as Gruber and others have supposed. One may presume therefore that in the different immune bodies only the group which combines with the immunizing substance differs, while the group which combines with the digestive ferment is the same in all."

In a second series of experiments, Ehrlich and Morgenroth ${ }^{1} \mathrm{im-}$ munized two goats with gradually increased quantities of defibrinated sheep's blood injected subcutaneously. From these animals there were obtained highly active sera. The serum of the first animal in quantities of from 0.2 to 0.3 c.c. completely dissolved five c.c. of the dilution of sheep's blood, while from 0.03 to 0.07 c.c. had a marked effect. The serum of the second goat in quantities of from 0.15 to 0.2 c.c. completely dissolved five c.c. of the dilution of sheep's blood. It is stated that the serurn of goat II before the process of immunizing had a feebly solvent action on sheep's blood, inasmuch as four c.c. of the serum partially dissolved five c.c. of the five per cent. dilution of sheep's blood. Heating to $57^{\circ}$ for half an hour destroyed this action, also the solvent action of the same serum on rabbit's and guinea-pig's blood. It was shown that the sera of both these animals contained the immune body which combines with the corpuscles at $0^{\circ}$. Mixtures of these sera and sheep's blood were allowed to stand ror twenty-four hours at $0^{\circ}$ and then the corpuscles were separated in the centrifuge. When the sediment was shaken up with physiological salt solution there was no hemolysis until the addiment in the form of normal goat serum was added. At a temperature of $20^{\circ}$ continued for eight minutes, both components, the immune body and the addiment, combined with the corpuscles and a sediment obtained from this mixture and distributed in physiological salt solution and kept at $37^{\circ}$ resulted in complete hemolysis. The sera obtained from these animals differed from that obtained from the goat in the first series in being more intense in action and in the fact that when heated to $56^{\circ}$ for three-quarters of an hour there was scarcely any reduction

\footnotetext{
${ }^{1}$ Berliner klin. Wochenschrift, 1899, 481.
} 
in their hemolytic action on sheep's blood, while their action on the blood corpuscles of the guinea-pig and the rabbit was completely destroyed. Indeed, heating for three hours at $56^{\circ}$, or heating the serum diluted with an equal volume of water for one-half hour at $65^{\circ}$, had no effect upon the hemolytic action on sheep's blood. This indicated that the addiment in these sera differs from that observed in the goat experimented upon in the first series, and the fact that heating to $56^{\circ}$ destroyed the action of these sera on the blood of the guineapig and of the rabbit suggested that they contain at least two addiments, one of which is necessary to the hemolytic action on the blood of the guinea-pig and the rabbit and is destroyed at $56^{\circ}$, while the other is that by virtue of which the hemolytic action on sheep's blood results and which is not destroyed at a temperature of $56^{\circ}$. In other words, there must be in these sera two addiments one of which is thermo-stable, while the other is thermo-labile.

In order to solve the problem presented by this new discovery, Ehrlich and Morgenroth determined to separate the two components in these sera. The immume body was easily obtained by combining it with erythrocytes at $0^{\circ}$ and it was found that when these sera were treated with 10 per cent. of their volume of normal hydrochloric acid and the mixture digested for from thirty to forty-five minutes at $37^{\circ}$ and neutralized, they lost their hemolytic effect on sheep's blood. The acid destroyed the specific addiment in these sera, but had no effect upon the immune body. The experiment was carried out as follows: To five c.c. of the five per cent. sheep's blood dilution there was added 0.15 c.c. of the immune serum rendered inactive with hydrochloric acid (it having been previously shown that this amount of active serum was sufficient to produce complete hemolysis in five c.c. of the blood dilution). After the mixture had stood for half an hour at room temperature, it was centrifuged and separated into sediment and supernatant fluid. To the sediment there was added two c.c. of normal goat serum and to the supernatant fluid there was added another portion of the diluted sheep's blood, and also two c.c. of normal goat serum. When thus treated the corpuscles in the sediment were completely dissolved, while the supernatant fluid had no action upon the erythrocytes which had been added, notwithstanding the presence in it of the addiment. This showed that all of the immune body was contained in the sediment. However, this experiment also showed that the addiment needed to render the immune body active is present in normal goat serum and indicates that there is in normal serum a thermo-stable addiment; but it was shown that the thermostable addiment was not present in the sera of all goats. It was concluded that in the serum of the goat used in the first experiment and in the sera of the goats used in the second experiment the same immune body was present, but that the serum of the first immunized goat contained only thermo-labile addiment, while those of the two 
goats used in the second experiment contained both thermo-labile and thermo-stable addiments.

Another experiment showed that the blood serum of a non-treated normal animal dissolved the erythrocytes of the guinea-pig and that there is in the normal serum of the goat a substance analogous to the immune body found in immunized animals, which combines with the corpuscles of the guinea-pig at $0^{\circ}$. It is evident from this that the term "immune body" is not appropriate for the substance which combines with the erythrocytes at $0^{\circ}$ and which is one of the hemolytic factors. This led Ehrlich to substitute for the term "immune body" the designation of "intermediary body," ${ }^{1}$ and inasmuch as he had demonstrated that there might be different addiments in the blood, he also dropped this term and used in its stead "complement." It should be understood that the "immune body," which we will hereafter designate as "intermediary body," has two haptophorous groups, one of which is possessed of great avidity, and it is by means of this that the intermediary body combines with the corpuscles, and its great avidity is shown by the fact that this combination takes place even at $0^{\circ}$. The other haptophorous group of the intermediary body is possessed of less avidity and it is by means of this that combination between the intermediary body and the complement takes place; and on account of the slight avidity of this haptophorous group this combination occurs only at a relatively high temperature. When an active serum is kept at $0^{\circ}$ it contains the intermediary body and the complement both in a free state; now, if susceptible erythrocytes be added to this serum still kept at $0^{\circ}$, the corpuscles and the intermediary body combine, while the complement remains free ; but when the temperature is raised to $37^{\circ}$ the complement enters into the combination by attaching itself to the intermediary body. The haptophorous group by which the intermediary body combines with the corpuscle is sometimes designated as hemotropic. Of course, there may be in certain sera intermediary bodies whose hemotropic groups have no greater avidity than their complement groups; or there may be complements which combine with intermediary bodies at a low temperature. It may happen that the intermediary body is contained in the serum of one animal while the complement may be furnished by the blood of another animal. This has been shown to be the case in the following experiment: Dog serum dissolves the blood corpuscles of the guinea-pig with great energy. If the dog serum be heated to $57^{\circ}$ it loses its hemolytic action on guinea-pig blood. But if inactive dog serum be added to five c.c. of a five per cent. dilution of guinea-pig blood and two c.c. of the serum of a normal guinea-pig be added to this mixture, complete hemolysis occurs. This is explainable only on the

"In his later papers Ehrlich has used the terms "immune body" and "intermediary body" interchangeably. 
supposition that the intermediary body exists in the dog serum, while the complement is furnished by the serum of the guinea-pig; in this case the complement is furnished by the serum of the animal whose corpuscles are dissolved. It is probable that in various sera both the intermediary bodies and the complements differ, and it is not always possible to restore the hemolytic action of a serum, which has been heated, by the addition of a complement. For instance, eel serum is hemolytic to the blood of most mammals; it loses its hemolytic action when heated for fifteen minutes to $54^{\circ}$, and so far no method of restoring its activity has been discovered.

In a third communication Ehrlich and Morgenroth ${ }^{1}$ carried out their immunization experiments in a wholly different way. 920 c.c. of the mixed blood from three goats (Nos. 1, 2 and 3), diluted with 750 c.c. of water, was injected into the abdominal cavity of a large goat $(A)$ at one time. From the second day on small quantities of the blood were taken from this animal and the hemolytic action of the serum tested. The first experiment showed that this serum possessed slight hemolytic effect on the corpuscles of other goats, and on the seventh day it reached its maximum. At this time 0.3 c.c. of the serum completely dissolved the corpuscles in one c.c. of a five per cent. dilution of goat No. 4. It was tested upon nine goats and it was found that the susceptibility of the erythrocytes of these animals to the serum varied somewhat. The serum of the treated animal was found to be without effect upon its own corpuscles. Ehrlich suggests that the hemolytic action of the blood serum of one animal upon the corpuscles of another species be designated as "heterolysis," and the active agent or agents in the hemolytic serum in this case be termed "heterolysins," while the hemolytic action of the serum of one species on the corpuscles of another individual of the same species be designated as "isolysis," and the active agents as "isolysins." If a serum should be found which dissolves the corpuscles of the individual from which the serum has been obtained, the process would be designated "autolysis," and the active agents as "autolysins." No one has as yet discovered an autolysin, although the possibility of the existence of such a substance cannot be denied. It is possible that certain diseased conditions, which we have designated as auto-intoxications, may be caused in this way.

Ehrlich designates the haptophorous group in the corpuscle, or the side-chain in the corpuscle which combines with the intermediary body, as the " receptor." He supposes that it is by the action of these receptors that hemolysins, and other toxins as well, combine with and destroy the cells of the body. He illustrates his views of the relationship between the cell, the intermediary body and the complement, also the action of other toxins, by the accompanyiug drawing.

${ }^{1}$ Berliner klin. Wochenschrift, 1900, 453. 
Frg. 1.
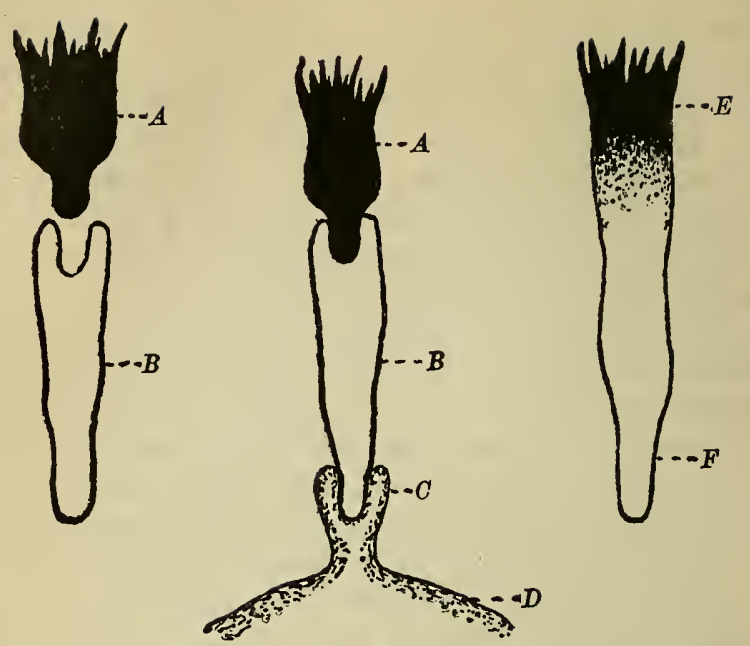

Demonstrating Ehrlich's Theory. $A$, complement; $B$, intermediary Body ; $C$, receptor; $D$, part of cell; $E$, toxophorous group of toxin ; $F$, haptophorous group.

If there be no receptors the intermediary body cannot combine with the corpuscle and consequently there is no hemolysis. Antihemolysins are supposed to be formed in the body of the animal treated with hemolytic serum in the same way that antitoxins are formed in the bodies of animals immunized to the toxins. If a hemolytic serum be injected into an animal in small but gradually increased doses at intervals, immunity to such serum is obtained and the serum of the animal thus immunized contains an anti-hemolysin. When a small amount of the hemolytic serum is injected it is taken up by the receptor in the blood cell, and provided that the amount of the hemolytic serum injected is small, the blood corpuscle is not destroyed; and needing for the performance of its function the receptor which has combined with the hemolysin, it throws out other receptors; and as in the case of the production of antitoxin, a point is reached when there is over-production of receptors and those not needed by the cell and not taken up by the hemolysin are cast off in the blood and constitute the anti-hemolysin. Ehrlich and Morgenroth prepared an anti-isolysin in the following manner: A small goat (No. 10) whose blood corpuscles had been shown to be highly susceptible to the serum of goat $A$ was treated at intervals with this serum and an anti-body obtained. When 0.4 c.c. of the serum of goat No. 10 was added to one c.c. of a five per cent. dilution of the blood of a goat which had been found susceptible to the serum of goat $A$, no hemolysis occurred. However, when the blood corpuscles of goat 
No. 10 were freed from their own serum and washed with physiological salt solution they were found to be as susceptible to the serum of goat $A$ as they were before.

Goat $B$ was treated in exactly the same manner as goat $A$. However, it was found that in this ease no isolysin appeared in the serum until the fifteenth day, when its appearance was sudden. During the first fourteen days after the injection the erythrocytes of goat $B$ remained highly susceptible to the isolysin in the serum of goat $A$; and, strange to say, this susceptibility continued even after the serum of goat $B$ manifested its hemolytic properties. It was furthermore found that the erythrocytes of certain goats were susceptible to the isolysin of goat $A$ and insusceptible to that of goat $B$. It appears from this that there are different isolysins and it was also found that anti-isolysin $A$ was wholly without action against isolysin $B$.

A third goat, $C$, received at the same time a like amount of the same blood as $B$, and first furnished a hemolysin on the seventh day. This hemolysin was also found to be an isolysin, but different from that of either $A$ or $B$. These experiments demonstrated that the exact nature of the isolysin depends upon the individual characteristics of the animal in which it is formed. A fourth goat, $D$, furnished an isolysin which dissolved the blood corpuscles of $B$ and $C$, but was without effect upon those of $A$. The sera of $A, B$ and $C$ dissolved sheep's corpuscles, while the serum of $D$ was without effect.

Ehrlich compares the intermediary body with diazo-benzaldehyde, which by means of its diazo group is capable of combining with a series of bodies, such as aromatic amins, phenols, keto-methyl bodies, etc., while by means of its aldehyde group it may combine with a different series such as the hydrazins, ammonia radicles, and hydrocyanic acid. Phenol and hydrocyanic acid will not directly combine, but with diazo-benzaldehyde acting as an intermediary body, these two substances can be brought into combination. Pushing this comparison further, we may say that the aromatic body, or the phenol, represents a constituent of the blood corpuscle. The diazobenzaldehyde is the intermediary body, while the poisonous hydrocyanic acid constitutes the complement. As has been stated, the intermediary body has two haptophorous groups. By means of one of these it combines with the receptor of the cell, while by means of the other it combines with the complement. The former may be designated as the cytophil group, while the other may be distinguished by the designation of complementophil. It should be understood that there are probably many varieties of intermediary bodies, and there may be two or more in a given blood serum. In fact, Ehrlich has demonstrated the presence of two or more kinds of intermediary bodies in the same serum. The serum of a rabbit which has been immunized to ox blood has a hemolytic action not 
only upon the corpuscles of the ox, but upon those of the goat as well. If such a blood serum be treated with a sufficient amount of the corpuscles of the ox, and the mixture separated in a centrifuge, the supernatant fluid has no solvent action upon either ox or goat corpuscles. In other words, the ox corpuscles have taken up all the intermediary bodies in the blood serum. However, if this serum be mixed with goat corpuscles and the mixture be separated in a centrifuge, the supernatant fluid has no solvent action upon goat corpuscles, but still possesses solvent action on ox corpuscles. This experiment demonstrates that there are in the blood serum of a rabbit immunized to ox blood at least two intermediary bodies, both of which are capable of combining with ox corpuscles, while only one combines with goat corpuscles. Intermediary bodies differ from one another both in their cytophil and in their complementophil groups. A given intermediary body will unite with a corpuscle only when it finds in that corpuscle its appropriate receptor. And, as has already been shown, the receptors in one and the same corpuscle, as, for instance, in the corpuscle of the ox, differ and take up different intermediary bodies. In like manner, every intermediary body will not combine with every complement; and combination between an intermediary body and a complement can occur only when their haptophorous groups are homologous. The existence of two or more kinds of intermediary bodies in a given serum has been also demonstrated by the formation of anti-intermediary bodies. If a hemolytic serum be injected in small quantities at intervals into an animal there may be obtained from that animal a serum which contains an anti-intermediary body, but its anti-action is not manifest towards all intermediary bodies and may show itself only when brought into contact with that intermediary body by means of which the anti-hemolytic serum has been obtained, and it is in this sense only that specific anti-intermediary bodies can be obtained. A serum containing an anti-intermediary body prevents the action of a hemolytic serum on the corpuscle in case that the action results from its own specific intermediary body. The action of an anti-intermediary body consists in preventing the union between the cytophil group of its specific intermediary body and the receptor of the cell. It is possible that intermediary bodies possessing different cytophil groups may have the same complementophil group, or intermediary bodies of like cytophil groups may have different complementophil groups; and it is still possible that there may be intermediary bodies possessed of only one cytophil group and having two, three or more complementophil groups. In his latest articles Ehrlich designates the intermediary body as "amboceptor," indicating that it has two haptophorous groups, but, as has just been stated, the intermediary body may be a triceptor, quadriceptor, etc.

Each complement has a haptophorous group by virtue of which it 
is able to effect a combination with the intermediary body, and a zymotoxic group by means of which it destroys the corpuscle. Ehrlich has shown the close relationship between the complement among the hemolytic factors and the bacterial toxins, inasmuch as he has demonstrated the existence of complementoids which correspond to the toxoids. As has already been stated, many of the complements are deprived of their poisonous action by a heat of $56^{\circ}$. Ehrlich has shown that this apparent destruction of the complement by heat consists only in destroying the zymotoxic group. If an animal be immunized with a hemolytic serum which has been rendered inactive by heat, there is formed in this animal not only an anti-intermediary body, but also an anti-complement. This procedure is analogous to the production of antitoxin by treating an animal with a toxoid. The anti-complement produced in this way is often quite as potent as, sometimes more so than, that obtained by immunizing an animal with an unheated or active hemolytic serum, just as a highly active antitoxin may be obtained by treating an aninal with a relatively harmless toxoid. An anti-complement prevents the action of its specific complement by rendering it impossible for the latter to combine with the intermediary body. It is possible that complements, like intermediary bodies, may vary in the number of haptophorous groups which they possess. If an intermediary body be possessed of two complementophil groups, it may take up two mono-haptophorous complements, or one di-haptophorous complement.

Bordet ${ }^{1}$ combats Ehrlich's view that the union between the intermediary body and the blood cell is a chemical one, and claims that it is a phenomenon of surface absorption. The experiment upon which this claim is based may be detailed as follows: If a guinea-pig be treated with rabbit's blood, there is produced in the former animal a hemolytic serum which will dissolve a given number of the blood corpuscles of the rabbit. Having determined the amount of blood which a given quantity of this serum will completely dissolve, provided that all the blood is added at one time to the serum, Bordet added to the determined quantity of serum half this amount of rabbit's blood and then, after allowing it to stand for a while, added the other half of the blood, when it was found that the serum dissolved only that portion of blood which was first added and had no effect upon the second portion. He compares this to the following simple experiment in surface absorption : If a small amount of methyl violet be dissolved in a given volume of water, and a piece of filter paper be immersed in this solution for a short time, the paper will absorb all the coloring matter, leaving the water colorless. Now, if the same amount of methyl violet be dissolved in the same amount of water, and a piece of filter paper half the size of that used in the first experiment be immersed in this solution, it also will absorb all

1 Annales de $C$ Institut Pasteur, 1900. 
the coloring matter and leave the solution colorless. Of course, in the latter instance, the paper, being smaller, will be more highly colored. Ehrlich has repeated Bordet's experiment with hemolytic serum and blood corpuscles and has not only confirmed his results, but has shown that even a much smaller proportion than one-half the corpuscles will combine with all the intermediary body present; but his explanation is wholly different from that suggested by Bordet. Ehrlich claims that when half the corpuscles are added to the amount of serum capable of digesting the whole number of corpuscles, each corpuscle combines with a larger number of intermediary bodies than is necessary to effect solution, but the combination having taken place, the intermediary bodies are held and are not free to act upon the corpuscles added in the second portion. Ehrlich states that reactions similar to this are well known in chemistry and he mentions the following instance: Naphthalin consists of two benzol rings linked together. If a salt-forming group, a hydroxyl or amido group, be brought in contact with naphthalin, there are formed hetero-nuclear substitution products, such as di-oxy-naphthalin, amido-naphthol, and naphthalin-diamin, whose sulpho-acids are capable of combining with either one or two molecules of a diazo-compound. If two molecules of dioxy-naphthalin be added to two molecules of a diazobenzol, there is formed exclusively a mono-azo-compound; but if two molecules of diazo-benzol be added to one molecule of dioxynaphthalin, there is formed a diazo-compound. If an additional molecule of dioxy-naphthalin be added to the already formed diazocompound, it is not capable of decomposing this substance; and the diazo-compound and the unchanged dioxy-naphthalin exist together.

We have already referred to the fact that Bordet was the first to show that the addition of a small quantity of normal serum to that of an animal which had been immunized against the cholera vibrio strengthened the bacteriolytic action of the cholera serum. This experiment opened up the way by which it has been ascertained that there are two factors present in bacteriolytic and hemolytic sera, inasmuch as the normal serum used by Bordet contained the substance which we now designate " the complement," following Ehrlich's theory. In a second series of experiments, Bordet ${ }^{1}$ treated guinea-pigs intravenously with five or six successive injections of 10 c.c. each, of the defibrinated blood of the rabbit. From the animals thus treated he obtained a serum possessed of the following characteristics: (1) This serum when mixed with the defibrinated blood of the rabbit agglutinates the corpuscles with great energy. For example, one part of the serum agglutinated all the red blood corpuscles contained in fifteen parts of the defibrinated blood of the rabbit. (2) The serum not only agglutinated but subsequently rapidly dissolved the blood corpuscles of the rabbit. When one

1 Annales de l' Institut Pasteur, 1898. 
part of the defibrinated blood of the rabbit was added to two or three parts of the active serum, the mixture became, within two or three minutes, red, clear and limpid. Microscopical examination of this fluid showed only the stroma of the globules, more or less deformed, transparent, and scarcely visible. (3) When the active serum of the guinea-pig was heated to $55^{\circ}$ for half an hour (or for less time at $60^{\circ}$ ) it lost its property of dissolving the corpuscles of the rabbit. but still agglutinated them. (4) If to a mixture of the defibrinated blood of the rabbit and the serum of the guinea-pig, the latter having been heated to $55^{\circ}$, there was added a certain quantity of fresh serum from either a normal guinea-pig or rabbit the hemolytic property of the serum was restored. (5) It follows from the above that the destruction of the hemolytic properties of the serum of the guinea-pig is only partial and the injury done this serum by heat is partially at least repaired by the addition of fresh serum from either a normal rabbit or a normal guinea-pig. (6) The serum of an untreated guinea-pig has only feeble agglutinating power on the corpuscles of the rat and has no solvent action on these bodies. (7) The active serum of the treated guinea-pig was without influence on the defibrinated blood of another guinea-pig, also without action on the corpuscles of the pigeon. It agglutinated markedly the corpuscles of the rat and the mouse. When two c.c. of the defibrinated blood of the rabbit were introduced into the peritoneal cavity of a guinea-pig which had been immunized to th e blood of the rabbit, the corpuscles thus introduced were quickly and completely destroyed. The liquid removed from the cavity after about ten minutes was found to be uniformly red and perfectly clear and limpid.

(9) If the blood of the rabbit mixed with a small quantity of the serum of the treated guineapig which had been heated to $55^{\circ}$ was injected into the peritoneal cavity of the treated guinea-pig, the phenomenon of destruction took place very rapidly. (10) The serum of the guinea-pig injected intravenously into the rabbit proved to be highly toxic.

Bordet ${ }^{1}$ has made additional valuable contributions to the subject of hemolysis. The facts ascertained in these researches agree practically in all details with those already given concerning the work of Ehrlich and Morgenroth, but Bordet's explanation of the facts differs from that given by the German investigators. Ehrlich's intermediary body is designated by Bordet as the sensitizer (substance sensibilatrice), and its function is to render impressionable globules sensitive to the action of the toxic body, which Ehrlich designates as complement and Bordet calls alexin. Ehrlich holds that the combination between the corpuscles and the intermediary body is a chemical one, while Bordet explains the action of his sensitizer on physical grounds. The term alexin, adopted by Bordet, is the same as

${ }^{1}$ Annales de l' Institut Pasteur, 1899 and 1900. 
that used by Buchner to indicate the germicidal constituent of blood serum. Bordet has also prepared anti-sensitizers and anti-alexins by immunizing animals to hemolytic sera. It will be seen from this that there is but little difference either in the experimental results obtained or in the theoretical explanation offered by the German and the French investigators. It should also be mentioned that it is generally believed that the combination between the intermediary body and the blood corpuscle, whether it be chemical or physical, is confined, so far as the corpuscle is concerned, to the stromata, and that the hemoglobin takes no part in the reaction. Müller calls the intermediary body " copula," London designates it "desmon," while Metschnikoff calls the intermediary body "philocytase" and the complement " cytase."

It has long been known that the sera of certain animals may dissolve the red blood corpuscles of animals of other species. So far as we know, the first recorded observation of this phenomenon was made by Dumas and Prevost. ${ }^{1}$ Early experiments on the transfusion of blood from one animal to another gave opportunity for repeated observation of hemolytic effects. One of the first attempts to study hemolysis by the methods of exact scientific examination was made by Ehrlich ${ }^{2}$ in 1884, when he disproved the generally held idea that cold is a causative factor in the production of hemoglobinuria. $\mathrm{He}$ closed his paper on this subject by suggesting that cold can lead to the dissolution of blood corpuscles only in specially disposed individuals in which the walls of the blood vessels produce certain agents (ferments?) which injure the " discoplasma." In 1898 Belfanti and Carbone ${ }^{3}$ ascertained that the blood serum of an animal treated with the blood of an animal of another species proved toxic when injected intravenously into the animal from which the blood had been originally obtained. Rabbit's blood was injected subcutaneously into horses and the serum of the horse injected into the rabbit caused dissolution of the corpuscles and induced death. This observation was probably the starting point of the numerous experimental studies which have been made upon this subject within the last two years. Almost simultaneously Bordet in France and Landsteiner in Austria published the results of experiments along this line. We have already referred to Bordet's work, and that of Landsteiner ${ }^{4}$ contributed nothing specially new. Von Dungern ${ }^{5}$ injected the blood of chickens and pigeons into the peritoneal cavities of guinea-pigs and observed that at first the corpuscles thus injected were slowly dissolved, but upon repeating the injections into the same animals he found that solution occurred more promptly and

1 Annales de Chimie, 1821.

${ }^{2}$ Charite-Annalen, 10.

${ }^{3}$ Giornal d. R. Acad. di Med. di Torino, 1898.

${ }^{1}$ Centralblatt $f$. Bakteriologie, 25.

5 Münchener med. Wochenschrift, 1899. 
PLATE $I$.

Ehrlich's Figures-Illustrating his Theory.

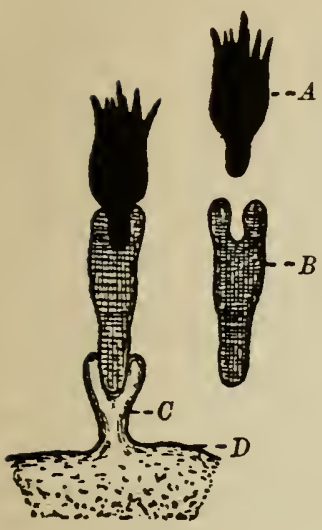

Fig. 1.

$a$. Complement ; $b$. Intermediary body ; $c$. Receptor; $d$. Part of cell.

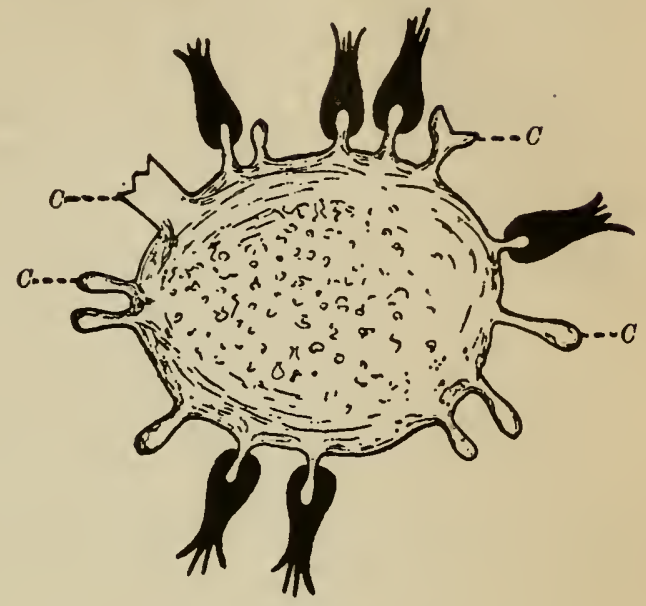

FiG. 2.

Cell with different kinds of receptors. c. Receptors.

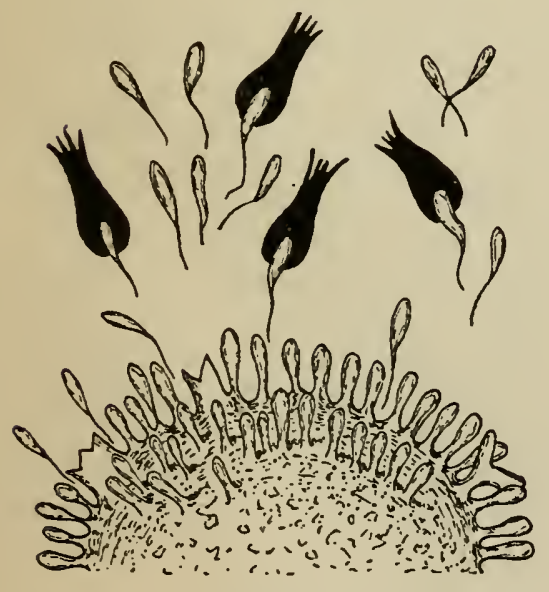

FIG. 3.

Showing separation of antitoxins, and combination of toxins with free antitoxins.

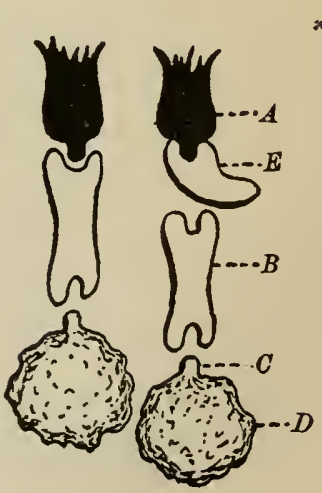

FIG. 4.

Showing the action of anti-complement. $a$. Complement ; $b$. Intermediary body ; c. Receptor; $d$. Cell. e. Anti-complement. 

that the serum of the guinea-pig agglutinated and dissolved the corpuscles of the birds both inside and outside the body. Metschnikoff $^{1}$ repeated these experiments using the blood of the goose, and Krompecher ${ }^{2}$ has done the same with the corpuscles of the frog. It should be stated that there is some advantage in the use of nucleated red cells for injection purposes, inasmuch as the nuclei are not dissolved and the number of free nuclei is an index to the degree of hemolysis. If to a drop of the serum of a rabbit which has been immunized to chicken's blood, there be added a small quantity of chicken blood and guinea-pig blood, and the mixture be examined under the microscope, it will be seen that at first the nucleated cells of the chicken blood agglutinate while the cells of the guinea-pig blood remain evenly distributed and later the chicken blood undergoes hemolysis, while that of the guinea-pig remains unaffected.

Mosso ${ }^{3}$ found that the popular belief that the blood of the eel is poisonous to mammals is true, and he demonstrated that the intravenous injection of 0.1 c.c. of eel serum per kilogram of body weight into rabbits and guinea-pigs induced death within three minutes and that post-mortem examination showed that the mammalian erythrocytes had been dissolved. Subsequent investigation showed that the hemolytic action of eel serum on the blood of mammals may be demonstrated in vitro. Kossel, ${ }^{4}$ Camus and Gley, ${ }^{5}$ and Tchistovitch ${ }^{6}$ took up this investigation and showed that rabbits, dogs and goats could be easily immunized against eel serum and that the antitoxin thus obtained prevented the hemolytic action of this serum in vitro, as well as in vivo.

Camus and Gley ${ }^{7}$ have shown that the blood corpuscles of some animals (hedgehogs and certain birds) are not susceptible to the hemolytic action of eel serum and they explain this by stating that it is due to a peculiarity of the organization of the blood cells of these animals. According to Ehrlich's theory, it might be said that the red blood corpuscles of these animals have no receptors with which the toxic substance in eel serum can combine. The venoms of the cobra and other poisonous snakes have a marked hemolytic effect upon the blood of mammals which can be arrested by the action of specifically prepared anti-hemolytic sera.

Halban $^{8}$ has made an important contribution to our knowledge of both agglutinins and hemolysins, and although the agglutinins will be discussed in a subsequent chapter, in order to save repetition we will

\footnotetext{
${ }^{1}$ Annales de l' Institut Pasteur, 1900.

${ }^{2}$ Centralblatt $f$. Bahteriologie, 28.

3 Archiv f. Experiment. Pathologie, 25.

${ }^{4}$ Berliner klin. Wochenschrift, 1898.

${ }_{5}^{5}$ Archiv Internat. de. Pharmacodynamie, 3 and 4.

6 Annales de l' Institut Pasteur, 1899.

'Compt. Rend. de l'Acad. de Sc., 1899.

8 Wiener klin. Wochenschrift, 1900.
} 
give a condensed statement here concerning Halban's work with both of these substances. He obtained sterile fetal blood drawn from the placental end of the cord cut directly after birth, while the mother's blood was secured by placing small sterile dishes in the vagina after the removal of the placenta. He prepared from each kind of blood both defibrinated blood and sera. With these preparations he tested the agglutinating and hemolytic effects of the serum of the fetus on its own mother, and vice versa; also the action of the fetal serum on the blood of other mothers. The results of these investigations may be summed up as follows: (1) The agglutinating properties of the blood of the fetus did not appear to be dependent upon those of the mother's serum, inasmuch as the mother's serum frequently agglutinated markedly, while the serum of the fetus did not agglutinate at all. Occasionally this condition was reversed, but as a rule the agglutinating action of the fetal blood was much less marked than that of the maternal. (2) A like result was obtained when the action of both kinds of serum was tested upon the blood of the mothers of other infants. (3) The same differences were observed as a rule when the sera were mixed with the blood of other individuals. (4) In no case did the serum of either fetus or mother agglutinate the corpuscles of the individual from which the serum was obtained. (5) In a considerable number of cases the serum of the mother agglutinated the blood of the fetus and the serum of the fetus agglutinated the blood of the mother. In other words, the two sera acted in this respect as did sera obtained from different individuals. When the serum of the mother agglutinated the blood of other individuals it also agglutinated that of her own child; and, likewise, when the serum of the fetus agglutinated the blood of other individuals it also agglutinated the blood of its own mother. (6) The hemolytic action of both sera as a rule paralleled their agglutinating effects. (7) The agglutinating action of both sera was tested on cholera cultures and showed the same reaction with these cells as with red blood corpuscles. (8) These investigations show that the kind and amount of agglutinins and lysins existing in the blood of the mother and the fetus differ as they would between other individuals. From these studies Halban draws conclusions which may be condensed as follows:

$A$. The fact that the maternal and fetal blood belong to two wholly distinct circulatory systems is established. It is known that the chemical properties of these two bloods differ, and Krüger has shown that the fetal blood contains only slightly more solid constituents than the maternal blood; while the fibrin of the former is markedly less than that of the latter. Scherenziss has demonstrated that the specific gravity of fetal blood is somewhat, and that of fetal serum markedly, lower than that of the mother. Fetal blood contains less hemoglobin and therefore its red corpuscles must be richer in stroma and are more easily destroyed than the corpuscles 
of the mother. On the other hand, fetal blood is richer in inorganic salts, especially the insoluble salts, than is the blood of adults. Furthermore, fetal blood is richer in sodium, poorer in potassium, and the total quantity of chlorin not combined with either potassium or sodium is markedly smaller than in the blood of adults.

$B$. It also follows from these experiments that the maternal blood often contains agglutinins while the fetal blood shows no trace of these substances; and inasmuch as the fetus must obtain albuminous substances from the maternal blood in the construction of its own tissue, and as the agglutinins are supposed to belong to the globulins, it follows that all albuminous substances are not absorbed by the fetus from the mother equally and that there must be a selective absorption manifested by the epithelium of the chorion.

C. This investigation bears upon the question of the origin of normal agglutinins in the blood; of course, it has nothing to do with specific agglutinins produced by inoculation. Since agglutinins are present in the blood of the mother, it has been supposed that those found in the blood of the fetus have simply been transferred from the former to the latter, but the above-mentioned experiments contradict this view, inasmuch as it was shown in some instances that there are agglutinins in fetal blood while they are not present in the blood of the mother. We must, therefore, suppose that these substances originate independently of the mother, and we should distinguish between inborn and hereditary agglutinins and lysins. Ehrlich and Morgenroth have proved the existence of isoagglutinins and isolysins, but it is claimed by Halban that these must be subdivided into ordinary isoagglutinins and isolysins on one hand, and idio-isoagglutinins and idio-isolysins on the other, meaning by the last given terms his inborn, non-hereditary substances. To account for the origin of these idio-iso-substances, Halban offers the following theories: (1) The idio-iso-agglutinins and idio-isolysins are due to an interchangeable immunization between mother and fetus. Blood cells in both circulatory systems are constantly disintegrating and the products of their disintegration may pass from mother into fetus and vice versa, and by this means an interchangeable immunization may be produced. The fact that the agglutinins are more frequent in the blood of the mother than in that of the fetus may be explained on the supposition that the fetus does not form agglutinins so readily. Halban supposes that this theory has some support in the demonstrated fact that children infected with typhoid fever during the first year of life as a rule show the Widal reaction less promptly than adults do; and he states that it has been shown by Schreiber that newly born infants bear without apparent effect doses of tuberculin which induce in adults marked elevation of temperature. Halban admits that the presence of agglutinins in the blood of women who have never borne children, and also in the 
blood of men, is difficult of explanation by this theory, unless we assume that every individual is possessed of the agglutinins and lysins which originated in that individual during fetal life by interchangeable immunization with the mother. This explanation provides for an existence of these substances more prolonged than seems possible. (2) The origin and continued existence of agglutinins and lysins in the body may be explained on the assumption that they are due to the frequent absorption of bacterial substances, especially from the intestines; but the fetus has been found to be germ-free, and it has been shown by Kraus and Clairmont that the serum of newlyhatched pigeons often possesses very marked bacteriolytic action. (3) There remains the possibility that mormal agglutinins and lysins may be due to self-immunization, resulting from the continued dissolution of cells in the body. It is true that Ehrlich and Morgenroth did not succeed in producing an autolysin. Halban states that there are present in normal blood not only idio-isoagglutinins and idio-isolysins, but also idio-heteroagglutinins and idio-heterolysins. The presence of the last-mentioned substances is indicated by the property of normal blood of agglutinating and dissolving certain foreign cells, also the blood corpuscles of other species of animals. These substances can hardly be supposed to have their origin in selfimmunization. So long as we are unable by experimental means to produce auto-agglutinins and auto-isolysins, it must be assumed that the agglutinins and lysins of normal serum are inborn substances.

Meltzer, ${ }^{1}$ having ascertained that the normal serum of the ox has a marked hemolytic action on the erythrocytes of the rabbit, placed this serum in the peritoneal cavities of rabbits, and, removing it after varied intervals, compared its hemolytic action on the corpuscles of the rabbit with that manifested by the same serum before it was introduced into the animal. These experiments led to the following conclusion: "The normal hemolytic power of bullock's serum for the red blood corpuscles of the rabbit disappears during a stay in the peritoneal cavity of this animal ; and the disappearance is the greater the longer the stay, and is independent of the absorption of the fluid ; disappearance takes place even during the first fifteen minutes." Further investigation showed that the disappearance of the hemolysin was not due to the formation of an anti-hemolysin, and that the hemolytic action of the ox serum was lost, but more slowly, when it was introduced into the peritoneal cavity of a dead rabbit. From this, Meltzer' concluded that the disappearance of the hemolytic power of the serum was due to the imbibition of one of the hemolytic factors, and subsequent investigation showed that the factor absorbed is the complement; however, he was not able to regenerate the serum removed from the cavity by the addition of sera containing various complements. Meltzer also ascertained that hemolytic serum ${ }^{1}$ Medical Record, 60, 1901. 
obtained by immunization becomes inactive when kept for a few hours in the peritoneal cavity; but in this case, regeneration was accomplished by the addition of fresh serum containing a complement. In these experiments rabbits were treated intravenously with guinea-pig's blood and after a serum had been obtained which had a marked hemolytic action on the blood corpuscles of the guinea-pig some of it was introduced into the peritoneal cavity of a normal rabbit, left there for three hours, and then recovered. "Such peritoneal serum, when added to guinea-pig's blood, agglutinated it, but caused no hemolysis whatsoever. Addition of immunized rabbit serum, made inactive by heating, to peritoneal serum, had no regenerating effect. But addition of fresh normal rabbit serum to the immunized peritoneal serum regenerated it completely ; the mixture of both sera dissolved guinea-pig's blood as readily as the active immunized serum alone."

In his studies of cobra lysin, Myers ${ }^{1}$ found that when this toxin is treated with anti-venomous serum it behaves similarly to the bacterial toxins. For instance, it was ascertained that, in order to nelltralize $1 \mathrm{mg}$. of cobra poison so that it would no longer manifest a hemolytic action on the blood corpuscles of man, 1.3 c.c. of antivenomous serum was required; but when 0.1 c.c. of the serum was added to $1 \mathrm{mg}$. of the venom, it deprived it of four-fifths of its toxic action, and when 0.2 c.c. of the serum was used it destroyed ninetenths of the hemolytic power of the venom. If these figures be compared with those given by Madsen in his studies of tetanolysin and already referred to on page 64 , the similarity will be evident. Myers found that the hemolytic action of a sample of dry venom remains constant for a long time, but such a venom in dilute solution rapidly decreases in hemolytic action until it reaches a minimum, when there is no further change. This is evidence that bodies similar to the toxoids described by Ehrlich are also formed in cobra lysin.

Bulloch and Hunter ${ }^{2}$ have shown that filtered cultures of the bacillus pyocyaneus dissolve the red corpuscles of the ox, the sheep, rabbit, monkey, cat, dog and rat. The more concentrated the solution of the toxin the more rapidly does its hemolytic action manifest itself, but in all the experiments there appeared to be a latent period during which there was no hemolysis. A goat was immunized with filtrates of this bacillus, and in the serum of this animal there appeared an anti-hemolytic substance which manifested its neutralizing effects in vitro. In experimenting with this substance it was observed that while small quantities of the immune serum did not prevent hemolysis and medium quantities did, excessively large amounts of the serum led to renewed hemolytic action.

1 Transactions of the Pathological Society of London, 51.

${ }^{2}$ Ibidem. 
Von Dungern ${ }^{1}$ discovered that epitheliolysins may be produced. $\mathrm{His}$ method of experimentation and the results obtained may be briefly stated as follows: He removed the trachea from an ox immediately after death, and from this he scraped the ciliated epithelial cells, taking care to avoid mixture with red blood corpuscles or connective tissue. The epithelial cells thus obtained were suspended in physiological salt solution and injected into the peritoneal cavities of guinea-pigs. From time to time some of the injected cells were removed and examined microscopically. The first change observed was one of form, in which the cells were found to be rolled up like balls, but were still capable of moving through the fluid by the activity of their cilia. Next the epithelial cells were found to be clumped in masses, with the cilia still active and many of these masses were seen moving like an individual through the fluid. Later the cells were seen to undergo a cystoid degeneration. One or more small vacuoles could be seen in the protoplasm, and these often contained leucocytes. These cysts gradually became larger, pressing the nuclei to the edges, and the cells appeared like large vacuoles surrounded by thin walls. From the cavity of an animal treated in this way for the first time visible cells can be obtained in some cases as long as six or even ten days after the injection, but when the same animal is treated after a lapse of ten or twelve days with a second injection of ciliated epithelium the cells are found to bedestroyed with much greater rapidity, and after eighteen hours no cel ls with motion can be discovered. By continuing the immunization an increased solvent action on the part of the peritoneal fluid was observed. From animals thus immunized to ciliated epithelium there was obtained a serum which in vitro dissolved similar cells. Epithelial-immune serum dissolves the red blood corpuscles as well as the tracheal epithelium of the ox. On the other hand, however, the serum of guinea-pigs immunized to ox blood, has no solvent action upon the tracheal epithelium of the ox, either in vitro or in vivo. This phenomenon can be explained in two ways. Either the epithelial immune serum contains two lysins, one of which acts upon epithelial cells, while the other exerts its effect wholly on the erythrocytes; or there may be in the epithelial immune serum only one lysin, which has a specific action on ciliated epithelium and a nonspecific action on the erythrocytes. That the latter is the correct explanation is shown by the fact that when immune serum was brought in contact with a mixture of epithelial cells and blood corpuscles, it acted only on the epithelium. Evidently the lysin in epithelial-immune serum has a greater affinity for epithelial cells than it has for blood corpuscles, and when the former of these is present in sufficient quantity in a mixture of the two, all of the lysin is used up in effecting a combination with the epithelial cells. Von Dun-

${ }^{1}$ Nünchener med. Wochenschrift, 1899. 
gern suggests the possibility of using an epitheliolysin in the destruction of the unrecognizable cancer cells that may remain in the tissue after excision by the surgeon.

In a second communication von Dungern ${ }^{1}$ has shown that rabbits and guinea-pigs immunized to cow's milk produce sera which act upon ciliated epithelial cells and also possess slight hemolytic properties.

Lindemann, ${ }^{2}$ in carrying out his investigations concerning the action of vinylamin on the kidneys recalled the fact, discovered by Claude Bernard, that the serum of an animal injected intravenously into an animal of another species induces a more or less permanent albuminuria. This led him to endeavor to strengthen the action of a heterologous serum by treating the animal with an emulsion of the kidney substance. The kidney of a rabbit was rubbed up into an emulsion and this was introduced into the peritoneal cavity of a guinea-pig. From the latter animal there was obtained a serum which when injected intravenously into rabbits caused rapid destruction of the kidneys of these animals. The following experiment illustrates the work done: A rabbit which had received without apparent effect eight c.c. of the serum of a normal guinea-pig, was seven days later given a like amount of the serum of a guinea-pig which had been treated with the renal emulsion. The latter injection provoked albuminuria within a few hours, followed within two days by complete anuria and death. The kidneys of this rabbit presented histological lesions closely resembling those found in animals killed by the administration of renal poisons. There was marked disintegration and necrosis of the epithelium of the convoluted tubules. The greater part of the tubules were transformed into granular masses containing pycnotic nuclei. The nuclei which persisted were not found to show evidences of chromatolysis, which is characteristic of coagulation necrosis, induced by metallic salts, but they resemble those found after poisoning with vinylamin and in which the alterations in the protoplasm precede changes in the nuclei. The glomeruli did not present any specific alteration. Numerous granular casts were found in the straight tubules. The serum of animals treated with emulsions of kidney is said to be nephrolytic, and the specific toxin contained in such serum is known as nephrolysin.

In a second communication Lindemann ${ }^{3}$ found that the serum of dogs which were suffering from nephritis induced by intravenous injections of chromate of potash, has marked nephrolytic effect when injected into other dogs and causes a severe nephritis terminating in death. Schütze, ${ }^{4}$ by treating rabbits with emulsions of the kidneys

\footnotetext{
1 Münchener med. Wochenschrift, 1900.

${ }^{2}$ Annales de l' Institut Pasteur, 14, 1900.

${ }^{3}$ Centralblatt $f$. Pathologie, 1900.

* Deutsche med. Wochenschrift, 1900.
} 
and liver of normal guinea-pigs, was not able to secure a kidney- or liver-serum which had a specific effect upon homologous cells. Nefédieff has gone over this work with great care and has not only confirmed all the statements made by Lindemann, but has pushed the investigation further along interesting lines. He tied the ureter of one side in each of two healthy rabbits. These animals bore the operation well, the wounds healed without suppuration, and there was marked gain in weight. Twenty-four days after the ligature had been applied he took blood from the artery of one of these animals and injected the serum obtained from it (four c.c. per kilo) into the auricular vein of a healthy rabbit. The urine of the rabbit thus treated contained on the next day a notable quantity of albumin which, however, progressively diminished and disappeared after five days. Forty-one days after the ligature was tied blood was taken from the second rabbit and its serum (five c.c. per kilo) was injected into another healthy rabbit whose urine at that time did not contain albumin, which, however, appeared in large quantity immediately after the injection, and continued for four days, rapidly diminishing. The presence of a large amount of albumin in the urine of this animal for three days after the injection led the investigator to kill the animal and make a post-mortem examination. This was done on the seventh day after the injection. The kidneys were found slightly increased in volume; section showed slight hyperemia ; and the capsule was easily removed. Microscopical examination revealed the presence of marked lesions in every part of the organ. The vessels of the glomeruli and all the capillaries of the interstitial spaces were greatly distended. The epithelium of the convoluted tubules showed necrosis, vacuolization, and modifications in the nuclei; while the tubules themselves were filled with casts. The nuclei of the epithelial cells were irregular and more or less broken. The epithelium of the straight tubules was less altered; but in these tubules there were casts consisting of epithelial cells so completely broken down as to be devoid of structure. In general, the pathological conditions found were similar to those which exist in diffuse inflammation of the kidney when accompanied by the presence of a large quantity of albumin in the urine. These experiments demonstrate that the blood of animals in which one ureter has remained tied for some time becomes laden with a nephrotoxic substance. Evidently ligature of the ureter is followed by absorption into the circulation of certain specific substances which have a destructive action upon renal cells. What the nature of the nephrotoxin is has not been determined, but the above-mentioned investigation shows that it is an isotoxin.

Landsteiner ${ }^{1}$ placed the spermatozoa of the bullock and of guineapigs in the peritoneal cavities of guinea-pigs; and removing them ${ }^{1}$ Centralblatt f. Bakteriologie, 25. 
from time to time and submitting them to examination, he ascertained that while the homologous cells were not altered and still retained their motility, the heterologous cells soon lost their motility.

Metschnikoff ${ }^{1}$ injected the spermatic fluid of one guinea-pig into the peritoneal cavity of another, and found that after some hours the spermatozoids were taken up by the leucocytes. After this, he injected into the peritoneal cavities of guinea-pigs spermatozoa of several species of animals, and observed that many of these, while still alive, are taken up by the leucocytes. The head of the spermatozoön is first absorbed by the leucocyte, and for some time thereafter the tail continues motile. Finally, however, the tail, as well as the head, is taken into the leucocyte, and is apparently digested. Twenty-four hours after the injection heads of spermatozoa within the leucocytes become more rare, and finally none can be seen. The majority of the spermatozoa are taken up and digested by the mononuclear leucocytes. Metschnikoff was not able to observe any digestion of spermatozoa outside of the leucocytes, and it may be stated here that no one has as yet prepared a fluid which dissolves spermatozoa. As we shall see later, sera have been obtained which destroy the motility of spermatozoa, but the specific substances contained in these sera are spermotoxins rather than spermotolysins. In pursuing his investigations Metschnikoff found that after all of the injected spermatozoa had been absorbed from the peritoneal cavity of the guinea-pig, that animal furnished a serum which immobilized the spermatozoa of the animal furnishing the material with which it had been treated. Later, by the subcutaneous treatment of rabbits with spermotoxic serum obtained from guinea-pigs, he produced anti-spermotoxic substances and found that spermatozoa retained their motility when placed in mixtures of spermotoxic and anti-spermotoxic sera. This anti-body may be developed in castrated as well as in normal animals, showing that other cells of the body may produce the substance. Moxter ${ }^{2}$ injected the spermatozoa of the ram, suspended in physiological salt solution, into the peritoneal cavities of guinea-pigs. After twenty-five minutes, the spermatozoa, in part still motile, were seized upon by the leucocytes, and after twentyfour hours no free spermatozoa could be found. There was observed at no time any evidence of dissolution of the spermatic cells. This phenomenon, it will be seen, is quite contrary to that observed on the introduction of erythrocytes into the peritoneal cavity of an animal of another species. The serum of the treated guinea-pig was found to possess marked spermatocidal properties. When the living spermatozoa of the ram were divided into two portions, one of which was treated with the serum of a normal guinea-pig and the other with 
the serum of the immunized animal, no marked difference in the action of these sera could be observed. In both instances the motility of the cells was arrested within from two to six minutes, when the serum was fresh. (It will be noted that this observation is not in accord with that reported by Metschnikoff.) The death of the spermatozoa in the serum is not due to the fact that the latter does not afford an adequate medium for the support of life, because the same cells retain their motility for four hours when suspended in physiological salt solution. Death is due to a toxic substance which exists in the sera of both normal and treated animals. This was shown to be the case by the following experiment: When ram's spermatozoa were mixed with the serum of either normal rabbits or immunized rabbits and the mixture allowed to stand for one hour at $37^{\circ}$ and centrifuged, the supernatant fluid was found to have lost its property of killing spermatozoa. A like result was obtained by heating either normal serum or immune serum to $58^{\circ}$. However, when immune serum was mixed with the ram's spermatozoa and the mixture placed in the peritoneal cavity of a normal guinea-pig it was found that the cells were more speedily killed than when a like experiment was made using the serum of a normal rabbit. Both immune and normal sera rendered inactive by heat were regenerated when placed in the peritoneal cavity of guinea-pigs, but after being thus regenerated, the immune serum acted much more intensely than the normal serum. Both immune and normal sera were also observed to agglutinate the spermatozoa, the former acting much more promptly than the latter. It was also found that the immune serum agglutinated the spermatozoa of the ram after these cells had been deprived of life by being kept at a temperature of $58^{\circ}$ for half an hour, while normal serum did not have this effect. The serum of the rabbit immunized against the spermatozoa of the ram did not agglutinate the spermatozoa of the horse or bull and therefore the reaction may be called a specific one. The serum of a rabbit immunized against the spermatozoa of the ram was found to have a marked hemolytic effect on the blood corpuscles of the latter animal, while it had no such effect upon the erythrocytes of the horse or ox. The question arose as to whether the spermatocidal and the hemolytic action of the immune serum were due to one or two substances. In attempting to answer this question, it was found that when a mixture of spermatozoa and erythrocytes from the ram was added to the immune serum from the rabbit, the hemolytic action of the serum was arrested. In another experiment it was ascertained that when the immune serum was treated with an excess of spermatozoa and the mixture allowed to stand for some time at $37^{\circ}$, then centrifuged, the supernatant fluid had no hemolytic action. From these observations it was concluded that the spermatocidal and the hemolytic substance in the immune serum is one and the same substance, and that 
it has greater affinity for the spermatozoön than it has for the blood corpuscle. Furthermore, it was observed that when immune serum obtained from a rabbit treated with the spermatozoa of the ram was mixed with the spermatozoa of the horse, allowed to stand, and centrifuged, the supernatant fluid still manifested its hemolytic effect on the erythrocytes of the ram. Moxter closed his paper with the following statements :

(1) The immune body kills the spermatozoa within the animal organism, but is inactive outside. There is no dissolution of the spermatozoa. (2) The immune serum possesses a specific hemolytic action on the red blood corpuscles of the ram; it combines with both the spermatozoa and the erythrocytes, but with the former with greater avidity. (3) The immune serum has a marked specific agglutinating action on the spermotozoa of the ram. (4) The erythrocytes of the ram are also agglutinated by the immune serum. This action, however, can be observed only when the hemolytic substance has been rendered inactive. (5) The serum of a normal rabbit also kills the spermatozoa of the ram, but acts less rapidly and less energetically than immune serum. The spermatocidal constituent of normal serum is identical with the hemolytic.

Metalnikoff ${ }^{1}$ has made an important contribution to our knowledge not only of spermotoxins, but of cytotoxins in general. In some particulars his investigations contradict those of Moxter. The following is a condensed statement of his results: If a rabbit be treated with a mixture of the spermatozoa and the defibrinated blood of a guinea-pig, the serum of the former animal will be found to be possessed of marked spermotoxic and hemolytic action. This serum is divided into two portions, numbers 1 and 2. To No. 1 there is added a large quantity of red corpuscles; the mixture is allowed to stand at $37^{\circ}$ for two hours and then centrifuged. The supernatant fluid does not dissolve more corpuscles when added even after the addition of more alexin in the form of the serum of a normal guinea-pig. This shows that all of the hemotoxic sensitizer (intermediary body) is fixed, but it immobilizes spermatozoa as readily as it did before it was saturated with red globules. This shows that the spermotoxic sensitizer remains intact in the serum and is not combined with the red corpuscles. To portion No. 2 of the serum there is added an excess of spermatozoa; the mixture is allowed to stand for two hours at $37^{\circ}$ and is then centrifuged. The supernatant fluid obtained in this case has neither spermotoxic nor hemolytic action. This shows that the spermatozoa have combined with not only the spermotoxic sensitizer, but also with the hemotoxic. While the red corpuscles fixed only the hemotoxic sensitizer, the spermatozoa fixed both sensitizers. These investigations show that Moxter fell into error when he concluded that the

1 Annales de l'Institut Pasteur, 1900. 
hemolytic and spermotoxic action of the immune serum was due to one and the same substance.

Both Metschnikoff and Metalnikoff have obtained spermotoxic sera which are active both in vitro and in vivo, and they have also prepared anti-spermotoxins. In Metschnikoff's experiments the spermotoxic serum of a guinea-pig was injected into a rabbit and from the latter there was obtained an anti-spermotoxic serum. After three injections, the serum of this rabbit neutralized the spermotoxic serum of the guinea-pig in the proportion of eight to one. Metalnikoff proceeded in an inverse way. He injected the spermotoxic serum of a rabbit into guinea-pigs, both subcutaneously and intraperitoneally. After three injections, he obtained a feeble anti-spermotoxic serum, from twenty to twenty-five volumes of which neutralized one volume of spermotoxic serum. He continued to give injections of the spermotoxic serum with the hope of obtaining a more active anti-spermotoxin, but instead of reënforcing his product he found that finally he obtained a serum which had absolutely no antitoxic property. He could explain this only on the supposition that while his spermotoxic serum contained two toxic factors, the sensitizer and the alexin, his anti-spermotoxic serum contained an anti-body for only one of these factors. For the neutralization of the sensitizer there must be produced an anti-sensitizer, while for the neutralization of the alexin an anti-alexin is needed. On continuing his investigations along the line suggested by this theory he ascertained that his anti-spermotoxic serum, which had been obtained from animals repeatedly treated with the spermotoxic serum and which had shown no antitoxic action, became active on the addition of fresh serum from a normal guinea-pig; for instance, one drop of the spermotoxic serum plus ten drops of the antitoxic serum heated to $56^{\circ}$ plus three drops of the serum of a normal guinea-pig killed spermatozoa within ten minutes; while one drop of the spermotoxic serum plus ten drops of the antitoxic serum heated to $56^{\circ}$ plus three drops of the serum of a normal guinea-pig heated to $56^{\circ}$, did not kill spermatozoa. This shows that while the treatment of the animals with spermotoxic serum produced an anti-sensitizer, it did not produce an anti-alexin.

Metalnikoff has shown that auto-spermotoxins may be produced in the animal body. If a guinea-pig be treated either intraperitoneally or subcutaneously with the spermatozoa of another guineapig, the serum of the treated animal is toxic for the spermatozoa of both. This auto-toxicity may be increased by repeating the injections and there may be obtained a serum ten drops of which added to one drop of the spermatic fluid of either animal causes complete immobilization of the spermatozoa within from three to four minutes. This autotoxin conforms to the general laws of the cytotoxins. It loses its toxic property on being heated to $55^{\circ}$ and is easily regen- 
erated on the addition of the serum of a normal guinea-pig. In this connection some very interesting observations are recorded. A guinea-pig whose blood contains a toxin which is markedly toxic to its own spermatozoa in vitro, has spermatozoa which are quite as motile as those of a normal animal and which when introduced into physiological salt solution live for a long time; however, if these spermatozoa be placed in the serum of a normal guinea-pig they die within from ten to twenty minutes, while the spermatozoids of a normal guinea-pig live in the serum of another normal pig for many hours. It is evident from these observations that these spermatozoa have been acted upon by the sensitizer circulating in the blood of the guinea-pig, but they are not killed by the autotoxin of the animal because the blood does not contain a free alexin, this factor existing only in the interior of the leucocytes. This explains why autotoxins are active in vitro but inactive in vivo. The cellular elements are destroyed in the serum in vitro and therefore this fluid contains both the sensitizer and the alexin in the free state; while in the living organism all the cellular elements are intact, and the alexins are held within the leucocytes, the sensitizer only circulating in the blood and fixing itself to the spermatozoa in the living animal. This view is supported by the following observation: If one injects into the peritoneal cavity of a guinea-pig, whose blood is autotoxic, living spermatozoa suspended in physiological salt solution, and examines the peritoneal exudate during the next five minutes, it will be seen that immediately after the injection there is a rapid phagolysis and that the spermatozoa do not die until the leucocytes break down, after which they are almost instantly killed. The picture is wholly different, if the same guinea-pig be treated with an injection of physiological salt solution or a small quantity of leucotoxic serum. In this case the phagolysis is very insignificant after the injection of the living spermatozoids into the cavity. Phagocytosis is established very rapidly and soon the spermatozoids while still alive are seized upon by leucocytes. Attempts were made by Metalnikoff to obtain an anti-auto-spermotoxin but without results.

Metschnikoff ${ }^{1}$ has succeeded, by inoculating guinea-pigs with emulsions of the spleen and mesenteric glands of rats, in obtaining from the former a serum which agglutinates and dissolves the leucocytes of the latter. The active agent in sera thus prepared is known as a leucolysin. Funck ${ }^{2}$ has produced leucolytic sera by treating guinea-pigs with emulsions of the spleens of rabbits. $\mathrm{He}$ began by injecting half a spleen at a time, rubbed up in physiological salt solution. After from eight to ten days the injection was repeated. From the animals thus treated he obtained sera which dissolved leucocytes both in vitro and in vivo. The alterations brought 
about can be watched under the microscope in stained specimens. The nuclei gradually lose the stain and undergo alterations in contour and apparently in location, seeming to approach the edge of the leucocyte. Finally the cell becomes more and more transparent until it wholly disappears. Serum obtained by immunizing guineapigs to emulsions of rabbit spleen dissolved the mononuclear and polynuclear cells with equal rapidity, while the serum obtained by inoculating guinea-pigs with bone marrow obtained from rabbits affected most markedly the polynuclear leucocytes.

The marked resemblance between bacterial toxins and digestive ferments has long been recognized and as early as 1893 Hildebrandt $^{1}$ immunized animals to emulsin and diastase and demonstrated the anti-fermentative action of their sera both in vitro and in vivo. $\mathrm{He}$ injected into rabbits solutions of diastase and showed that the blood taken from these animals had an anti-ferment action. The strength of the antitoxic bodies obtained by him was not great and he was able to secure only partial neutralization of the ferment in many instances. He rendered a dog immune to emulsin by frequent subcutaneous injections ; then produced in this animal a phloridzin diabetes and fed the animal upon meat and starches. $\mathrm{He}$ found that there was no marked thirst and the amount of urine eliminated was not increased above the normal and the per cent. of sugar contained in the urine was small, while companion animals in which diabetes had been induced in the same manner showed great thirst, eliminated a large amount of urine and excreted large quantities of sugar. Von Dungern ${ }^{2}$ produced anti-enzymes by treating animals with bacterial proteolytic enzymes and demonstrated the action of the anti-body in tubes of gelatin. Gheorghiewski ${ }^{3}$ immunized animals to the bacillus pyocyaneus and found that the serum of these animals added to cultures of this bacillus prevented the formation of the blue coloring matter characteristic of the germ and the formation of which is dependent upon the presence of pepton. Morgenroth ${ }^{4}$ immunized goats against rennet and obtained in the serum of these animals a fairly active anti-rennet body. The specimen of rennet which he used in inducing the immunization coagulated milk when added to it in the proportion of $1: 3,000,000$; the strongest anti-body that he could obtain when added to milk to the extent of two per cent., prevented the action of rennet when added in the proportion of $1: 20,000$, while $1: 15,000$ still induced coagulation. From his studies he concludes that rennet resembles the bacterial toxins and possesses a haptophorous and a zymophorous group, to the latter of which the specific action of the ferment is due, and it is

1 Virchow's Archiv, 131.

2 Münchener med. Wochenschrift, 1898, 1040.

3 Annales de l' Institut Pasteur, 1899.

+ Centralblatt $f$. Baliteriologie, 26. 
against this group that the anti-body manifests its action. He also showed that the milk of the immunized goat contains the anti-body and does not coagulate on the addition of rennet in quantities which are sufficient to coagulate the milk of normal animals. Buchner ${ }^{2}$ thinks that the alexin (the complement, according to Ehrlich's theory) is a ferment and that it is one and the same body in the blood of all species of animals. The intermediary body (according to Ehrlich's theory) is the only substance against which immunity can be obtained. This Buchner believes to be specific.

1 Münchener med. Wochenschrift, 1900. 


\section{CHAPTER VIII.}

\section{THE AGGLUTININS.}

IN 1889 Charrin and Roger ${ }^{1}$ in studying the action of the serum of sick and immunized animals on homologous bacteria, observed that the bacillus pyocyaneus behaved peculiarly when placed in the serum of an animal which had been immunized to this organism. In the serum of a normal rabbit, this germ was found to grow as it does in beef tea, or other liquid nutritive medium, forming an opaque culture, while in the serum of an immunized rabbit these microbes were found to form small floating floccules which soon subsided, leaving a clear, supernatant fluid. It was furthermore observed that the germ grown in its homologous serum does not produce pyocyanin, while in the serum of normal animals this coloring matter is formed by the growth of the bacillus. The sera of animals sick from inoculation with the bacillus pyocyaneus, and of those immunized against this germ, were observed to have the same effect on its growth. This early observation indicated, as has been subsequently more clearly shown, that the phenomenon of agglutination is a reaction of infection as well as of immunization.

In his studies on immunity, Metchnikoff, ${ }^{2}$ found that the vibrio which bears his own name behaves in a manner similar to that observed by Charrin and Roger with the bacillus pyocyaneus. On this point Metschnikoff makes the following statement: "In the blood and serum of non-vaccinated guinea-pigs the vibrio develops as it does in ordinary liquid media, the individual organisms retaining their motility and remaining distinct one from the other. On the other hand in the blood and serum of vaccinated animals the vibrios become immobile, and form smaller or larger floccules which float in the liquid."

In 1893 Issaeff $^{3}$ observed a like phenomenon when the pneumococcus was placed in its homologous serum, and this observation was repeated later in the studies on immunity prosecuted by the same author in conjunction with Ivanoff. ${ }^{4}$ In the last-mentioned communication there is found the following statement: "In the blood serum of healthy, non-immunized guinea-pigs the vibrio develops rapidly, and after from four to five hours at $37^{\circ}$ there is a uniform

1 Comptes Rendus de l' Academie des Sciences, 109.

2 Annales de $D$ Institut Pasteur, 5, 1891.

3 'Annales de l' Institut Pasteur, 7.

4 Zeitschrift für Hygiene, 17. 
cloudiness throughout the fluid, while the surface is covered by a scum; but in immune serum the microbes sink to the bottom of the tube, while the supernatant fluid remains clear. This condition continues for from eight to nine days, and it is not until the tenth day that the culture becomes cloudy and a scum appears on the surface."

While the above-mentioned observations were made as herewith stated, they attracted no special attention, and in all probability their significance was not appreciated by the observers themselves, and our knowledge of the phenomenon of agglutination may be said to have originated in the classical research of Gruber and Durham, ${ }^{2}$ which was reported in 1896. These investigators found that when a suspension of an agar culture of the cholera or typhoid bacillus is mixed with its homologous serum, the bacteria lose their motility and form large clumps or masses. They attributed this phenomenon to the presence of specific substances in the serum, for which they proposed the term agglutinins, and suggested that the phenomenon might be designated that of agglutination. Their researches showed that this reaction is specific, within certain limitations: For instance, a cholera serum has no agglutinating effect upon the vibrio of Finkler, that of Metschnikoff or that of Rumpel, and typhoid serum is without action upon any of the colon germs, but a cholera serum does agglutinate, v. Ivanoff, v. Berolinensis and v. Seine-Versailles. In all instances in which cholera serum was found to have an agglutinating action, it was also found to protect animals against inoculation with the germ, and these two properties vary in the same proportion, that is, the greater the agglutinating action the greater is the protective power agaiust the same germ. Typhoid serum was found to agglutinate typhoid-like bacilli, which were positively known not to be real typhoid germs. For instance, bacillus enteritidis is agglutinated in a typical manner by a highly active typhoid serum. However, there is easily observable a quantitative difference in the agglutinating action of both cholera and typhoid sera upon other than their homologous bacteria. Moreover, the agglutinating action of typhoid serum is apparently more strictly specific than is that of cholera serum, but in making a practical test with either, as we shall see later, dilution of the serum is necessary in order to avoid error. Gruber and Durham also pointed out the fact that normal sera may have a more or less marked agglutinating action upon both the vibrio of Asiatic cholera and the bacillus of typhoid fever, and they stated that in order to use this reaction for the purpose of identifying bacteria it would be found to be necessary to dilute the serum employed. It seems to have been in the mind of these investigators at the time of the publication of their first paper that the reaction described by them would find its practical application in the detection and the definite recognition of specific microörganisms

I Münchener med. Wochenschrift, 43. 
rather than in the diagnosis of disease. They recommended that large guinea-pigs should be immunized by intraperitoneal injections of dead cultures, continued for from four to six weeks, until a high degree of immunization should be secured, and that the peritoneal lymph or blood serum of these animals might be used to determine with certainty whether or not a suspected microörganism was the vibrio of Asiatic cholera or the bacillus of typhoid fever. They stated that if with such a serum from an animal immunized with the cholera germ agglutination does not occur promptly and completely, it can be said with certainty that the germ under suspicion is not the vibrio of cholera, and the same test can be applied with equal certainty in the detection of the typhoid bacillus. On the other hand, if the reaction is positive, the identity of the microörganism must still remain in doubt. We have carefully read the original paper of Gruber and Durham, and we fail to find therein any reason for believing that these investigators at that time had any idea that the phenomenon which they were investigating was soon to become one of the most certain and easily applicable methods for the diagnosis of typhoid fever. It is true that they speak of the serum test as a diagnostic measure, but from the context it appears that by the term diagnosis they mean the specific and positive identification of a suspected bacterium.

A few months after the publication of the last-mentioned contribution, Widal ${ }^{1}$ reported the successful application of the phenomenon of agglutination in the diagnosis of typhoid fever, and among clinicians it is generally known as the "Widal reaction."

Our scientific information concerning the agglutinins has been obtained principally from the researches of Widal and Sicard, ${ }^{2}$ Nicolle, ${ }^{3}$ and Winterberg. ${ }^{4}$

It is quite evident that in such a reaction as that observed in agglutination there must be two factors. One of these, which we may designate the agglutinin, is found in the serum; while the other, which may be denominated the agglutinable substance, is furnished by the bacterial culture. The product which results from the reaction between these bodies may be designated the agglutinate. We will first give our attention to the agglutinins. The normal serum of certain animals is capable of manifesting a slight agglutinating action upon certain bacteria, notably the colon bacillus and the bacillus of Eberth. The normal serum of the horse, donkey, dog, and rabbit, when added to cultures of the Eberth bacillus without dilution causes agglutination. Indeed, the serum of these animals may agglutinate the typhoid bacillus when diluted 1 to 30 . The normal serum of the guinca-pig has no agglutinative action on this or-

1 Société Medicale des Hospitaux, June 26, 1896.

${ }^{2}$ Annales de $P$ Institut Pasteur, 11.

3 Annales de l' Institut Pasteur, 8.

- Zeits. für IYygiene, 32. 
ganism. The serum of men who have never had typhoid fever may, when the dilution is not greater than one to ten, agglutinate the Eberth bacillus, and it should be remarked that the manifestation of this property by the sera of different individuals varies within wide limits. It is still a question whether the agglutinating action manifested in the serum of immunized animals, or that of persons sick with typhoid fever, is due to an exaggeration of a normal property, or is caused by the presence of a new substance.

The period in the course of typhoid fever when marked agglutination is first manifested by the serum varies widely in different cases. As a rule, agglutination in a well-marked form is not obtained until after the seventh day, but there are cases on record in which it has been obtained as early as the second day, and there are others in which it has been delayed until the second, third, and even fourth week. Likewise the disappearance of the specific agglutinating property from the serum after recovery from typhoid fever is very variable in time. The reaction has been known to fail ten days after the establishment of apyrexia, while in other instances marked agglutination has been secured many years after recovery from typhoid fever. The intensity of the reaction varies widely, and is not always in proportion to the severity of the disease. Many methods of measuring the agglutinative power of sera have been proposed. One recommended by Widal and Sicard we have found to be satisfactory. A number of tubes containing each one, two, three, four, five, etc., c.c. of bouillon are kept on hand. When a test is to be made, one adds a drop of the serum to each of these tubes and then inoculates the tube with a typhoid culture. The tubes thus prepared are placed in the incubator and kept at $37^{\circ}$ for from four to six hours. At the expiration of this time it may be seen at a glance in which tubes agglutination has occurred, inasmuch as the contents of these tubes will be unclouded and the bacilli will be found deposited on the bottom. If this reaction does not take place in tube number one, which represents a dilution of 1 to 20, the test is considered as wholly negative. If number three contains clumps, while number four is uniformly clouded, the serum is active in the proportion of one to sixty and inactive in the proportion of one to eighty. An absolute determination of the agglutinating property of a given serum is probably not within the range of possibility, and we indicate the relative activity of different sera by the dilutions in which the reactions are observable. The number of germs present certainly has some influence, and it is a well-known fact that the age of the culture is of importance in making this test. In young cultures the bacilli are more motile, and there is less tendency to manifest the phenomenon of pseudo-agglutination.

Agglutinins exist not only in the blood, but in other fluids of the body. The manifestation of agglutinating power by the urine is in 
the same individual variable from day to day and even from hour to hour, and so far we have no satisfactory explanation of this variation. Compared with the agglutinating property of the blood, that of the urine is always feeble. This, together with its variability, renders the use of this secretion in place of blood serum impracticable. Serum obtained by vesication often shows marked agglutinating power. In the few cases in which the test has been made, bile manifests this reaction in a fairly well marked manner. Often the reaction may be easily and plainly obtained with tears. However, this secretion is subject to marked variation in its agglutinating power, and it has been found that tears obtained by artificial stimulation are generally devoid of this property. In immunizing animals it has been found that pus obtained from abscesses due to the bacillus of Eberth may show a high degree of agglutinative power. Agglutinins may pass from the mother to the fetus and may be transferred from the mother to the child in the milk. The agglutinins seem to be fairly stable bodies, and some of them may be heated to $140^{\circ}$ without destruction, but that of the typhoid bacillus is destroyed at a temperature of $115^{\circ}$. Antiseptics, so far as they have been tested seem to be without effect upon the agglutinins. Typhoid serum may be treated with formalin and kept quite indefinitely without loss of its agglutinating property. However, the use of antiseptics is not necessary in order to have a serum retain its agglutinating action. A typhoid serum may be used as a culture medium for the germs of cholera, anthrax, diphtheria, or of the ordinary putrefactive bacteria without diminution of its agglutinating property. Indeed, a highly active serum will retain its agglutinative action after it has been made putrid by bacterial growth. Dilute mineral acids decrease the agglutinating property of typhoid sera, but when the acid has been added in very dilute form and has not remained long in contact with the serum, partial restoration may be induced by neutralization with alkali. Acetic acid has a like, but less marked effect. Caustic alkalis have an action similar to that manifested by acids. Apparently the agglutinins are not altered by any of the digestive ferments. Indeed, pepsin seems to protect agglutinins to some extent against the effects of dilute hydrochloric acid, and trypsin has a similar protective action against dilute alkalis.

Filtration of blood serum through porcelain removes the agglntinins, inasmuch as it has been demonstrated that the filtrate has lost largely or altogether the agglutinating property. Saturation of typhoid serum with magnesium sulphate leads to precipitation of the greater part or all of the agglutinin. If the precipitate be dissolved in water it will be found that the agglutinin has not been destroyed by the action of this reagent. Half saturation with ammonium sulphate precipitates practically all of the agglutinin; and complete saturation with chlorid of sodium has a like effect. It 
may be inferred from these reactions that agglutinin is one of the globulins or albumins existing in the blood. However, this conclusion does not necessarily follow. It is altogether within the range of possibility that the agglutinin is carried down mechanically, as happens to certain ferments and toxins when albuminous solutions are treated with neutral salts. The fact that the agglutinating action has been secured with urine in which no albumin or globulin could be detected, has led some to believe that the agglutinins belong to neither of these bodies. This conclusion also is unjustifiable. Because we cannot detect a globulin or an albumin in the urine is no positive proof that one or both of these substances may not be present. It simply means that neither is present in sufficient quantity to respond to the test employed. It must follow therefore that we are not yet in a position to determine whether or not the agglutinins belong to the proteid constituents of blood serum.

A highly active serum after having been diluted with water until no test for proteids in it can be obtained may still manifest its agglutinating action, while, on the other hand, a serum possessed of but little activity loses its agglutinating property on dilution long before positive tests for the presence of proteids fail.

Precipitation of typhoid serum with absolute alcohol destroys the agglutinin. This is probably due to the dehydrating action of alcohol, because if as soon as the precipitate is formed it be collected on a filter, and the alcohol allowed to drain away and the precipitate extracted with physiological salt solution, this extract will be found to be possessed of a certain amount of agglutinating power, while, on the other hand, if the precipitate be allowed to remain for some hours under the alcohol, the agglutinin is wholly destroyed.

The agglutinating action of a blood plasma obtained by the addition of oxalate of potassium to the blood is more marked than that of the serum of the same blood. It follows from this that the agglutinin must be in part retained in the blood clot. Indeed, this can be positively demonstrated by testing the agglutinating action of an extract of the clot made with physiological salt solution. Widal and Sicard have shown quite positively that the white blood corpuscles, outside of the body at least, do not furnish the agglutinins. The experiment by means of which this demonstration was accomplished may be briefly described as follows : A sterilized collodion tube was filled with typhoid blood, to which oxalate of potassium had been added, and allowed to stand. The red corpuscles soon formed a deposit at the bottom of the tube, and these were covered by a thin coat of white corpuscles, and above these stood the plasma. The plasma was removed with a pipette as thoroughly as possible without taking up any of the leucocytes. Next the tube was constricted by a string tied around it on a line between the layers of white and red corpuscles. After these portions had stood for twenty-four hours the rela- 
tive agglutinating property of each was tested, and it was found that the plasma containing the white corpuscles had no greater action than did that which was devoid of these elements. Of course, this experiment does not indicate that the leucocytes have nothing to do with the formation of agglutinins in the living body. However, that the agglutinins are in solution in the circulating blood is shown by their appearance in certain secretions and exudates.

Nicolle reported that the agglutinable substance is soluble in absolute alcohol and ether. If this statement had been confirmed, the separation of this body from the proteids would be comparatively easy, but the researches of Winterberg demonstrate that Nicolle had fallen into error on this point. The last-mentioned investigator made the following experiment bearing on the question : A culture of the typhoid bacillus in bouillon, which had grown for seventy days at $37^{\circ}$ and which contained a large number of germs, was treated with ten times its volume of absolute alcohol. The precipitate was extracted first with absolute alcohol and then with ether, each of which was decanted after twenty-four hours. Both of these extracts were mixed with the alcoholic filtrate obtained after precipitation, and the whole evaporated to dryness in vacuo at $30^{\circ}$. The residue was taken up with feebly alkaline bouillon and this solution again precipitated with ten times its volume of absolute alcohol, and the filtrate evaporated as before. The residue last obtained was taken up with bouillon, filtered through porcelain, and the solution failed to manifest any reaction when mixed with a highly active typhoid serum. Winterberg furthermore showed that typhoid agglutinin is produced in animals which have been treated with alcoholic precipitates obtained from both filtered and unfiltered typhoid cultures.

The fact that typhoid cultures, the germs of which have been destroyed by the use of disinfectants or the application of heat, still agglutinate homologous sera demonstrates that the agglutinable substance contained in these cultures is not destroyed when the organisms are deprived of life. Widal and sicard found that typhoid bouillon cultures exposed for half an hour to a temperature of from $70^{\circ}$ to $100^{\circ}$ still react when brought in contact with a highly potent serum, but that the reaction in this case is not so marked as that observed in living cultures. The clumps form more slowly and are less voluminous. When a typhoid culture was heated for half an hour from $57^{\circ}$ to $60^{\circ}$ and then brought in contact with an active serum the reaction was found to be apparently identical with that obtained with living cultures. It is evident from these experiments that the agglutinable substance is not destroyed by the temperatures above mentioned, and that it is present in dead as well as in living . cultures.

Widal and Sicard showed that the dried blood of typhoid patients 
can be used in making the agglutination test. They impregnated small bits of sponge with four or five drops of the blood, allowed to dry, and subsequently macerated the pieces for half an hour in from ten to fifteen drops of bouillon. The addition of this extract to bouillon cultures of the Eberth bacillus induced typical agglutination.

Johnston ${ }^{1}$ first demonstrated that a drop of blood allowed to dry upon non-absorbent paper may be transported through any distance and kept indefinitely and still be capable, after being moistened with water, of giving the agglutination test. In this way this method of making a diagnosis of typhoid fever has been popularized and placed practically within the reach of every physician. It is safe to say that at present more than 90 per cent. of the agglutination tests made are applied to dried blood stains. The only objection to this method is that exactness in dilution can not be secured, and much must depend upon the judgment and experience of the individual making the test. However, for clinical purposes, dried blood gives, in a large per cent. of cases, evidence which is of positive value. Usually the blood stain is rubbed up with either sterilized water or physiological salt solution, and this is added to a bonillon culture of the typhoid bacillus twenty-four hours old. A hanging drop of this mixture is prepared and examined under the microscope. The extent to which the blood or serum should be diluted is still a matter about which there is a difference of opinion. The general rule is that the dilution should not be less than 1 to 20 and that agglutination in this dilution should occur within thirty minutes. However, in order to be quite sure concerning the result, the dilution should not be less than 1 to 60 , and if agglutination occurs in this dilution within thirty minutes, the evidence that the blood has come from an individual with typhoid fever is quite positive. With dilutions of 1 to 30 or less, agglutination is frequently obtained with normal serum, and more frequently given in certain diseases such as tuberculosis, pneumonia, septicemia, and influenza. When agglutination does not occur in the dilution of 1 to 60 , but is observed in the dilution of 1 to 30 the weight of evidence is in favor of the existence of typhoid fever, but the proof cannot be considered as positive. In this, as in the application of many other clinical tests, much depends upon the skill, experience and good judgment of the observer. It appears that about 96 per cent. of the cases of typhoid fever furnish some time during the course of the disease a serum which will give this reaction. It will be seen from this that failure to secure the agglutination test is not absolute proof of the non-existence of typhoid fever. Experience has shown that attenuated cultures are better suited for the agglutination test than more virulent ones. However, the evidence on this point is by no means unanimous, and an explanation is not easily given. Young cultures are better than older ones,

${ }^{1}$ New York Med. Journal, 44, 1896. 
because motility of the individual organisms is greater in the former, and besides the old cultures may contain clumps consisting partly of dead and disintegrated bacterial cells, which may be mistaken for typical agglutination. A like source of error is met with when the drop to be examined is allowed to partially dry on the cover-glass. These appearances, which may be mistaken, especially by the inexperienced, for true agglutination, have been designated pseudo-reactions. There is also the possibility, especially when dried blood is used, of mistaking undissolved clumps of red cells containing threads of fibrin for the true clumping of bacilli.

When exact scientific work is to be done it is better to have the blood drawn directly from a prick on the finger or ear into a graduated pipette in which the dilution can be made with exactness. There are special tubes made for this purpose, and the pipette which accompanies the ordinary hematocytometer may be used. In hospital practice, and especially when a large amount of serum is desired, a small Spanish fly blister may be used, and if properly cared for need cause no pain. As has been already stated, cultures killed by heat or those preserved by the addition of formalin, thymol, or some other disinfectant may be used, but these are more or less unsatisfactory, inasmuch as the observer loses the opportunity of watching the gradual decrease of motility.

As has been stated, Gruber and Durham probably expected that the greatest benefit likely to come from their studies would lie in the fact that this reaction would furnish a positive means of differentiating one species of bacteria from another. It is especially desirable that we should have some positive and easily applicable method of distinguishing between the colon and typhoid bacilli, and it was thought that the agglutination test supplies this long-felt want. However, more extended observation has shown that typhoid serum occasionally agglutinates the colon bacillus, and very rarely the agglutination with this organism may be more marked than it is with the typhoid germ. It may be that in cases of this kind the typhoid patient is suffering from a mixed infection in which both the colon and the typhoid bacilli participate. When the agglutination test is used for the purpose of identifying a bacterium the serum used should be taken from an animal immunized to a pure culture of that microörganism, and reliance for the differentiation of typhoid and colon bacilli should not be placed in sera obtained from men with typhoid fever.

It must be plainly evident from what has already been said that the agglutination test has not that specificity which is possessed by the lysins and precipitins. This was plainly seen by Gruber and Durham in their original communication, and it has been insisted upon by Durham in a later paper, in which he suggests that it would be better to employ the word "special." 
There have been proposed several hypotheses concerning the phenomenon of agglutination. Gruber suggested that there must be something in the immune serum which profoundly alters its homologous bacterium. This alteration consists, in part at least, in increased viscosity of the cell membrane of the bacterium. The production of the adhesive substance is supposed to be the chief factor in the agglutination or clumping of the bacterial cells. When two or more germs are brought together, either by active or passive movement, they adhere one to the other, lose their individual motility and form small masses which gradually subside in the culture medium. According to Nicolle, the agglutinin, which is furnished by the serum, precipitates the agglutinable substance, which is formed within the bacterial cell, from which it diffuses through the culture medium to a greater or less extent. The agglutinable substance is supposed to be most abundant in the cell membrane, and by the union of the agglutinin and the agglutinable substance small coagula are formed and surround the bacterial cells. The theory proposed by Paltauf varies but slightly from that suggested by Nicolle. However, the former supposes that the combination between the agglutinin and the agglutinable substance occurs wholly outside of the bacterial cells and that the microörganisms are involved in the floccules mechanically only. The hypothesis of Dineur varies from that originally proposed by Gruber only by transferring the formation of the adhesive matter from the bacterial cell walls to the cilia. Diminished motility and subsequent agglutination are explained on the supposition that the cilia are so altered that they secrete an adhesive substance. In 1896 Bordet suggested a hypothesis in which he attempted to explain the phenomenon of agglutination wholly upon physical grounds. $\mathrm{He}$ suggested that in homologous sera there is present a substance or substances which change the relative molecular attraction between the microbes and the ambient fluid. Later Bordet developed this theory as we have already indicated in our discussion of the lysins. In accordance with the general trend of French thought along these lines, he has rejected the German idea of chemical reaction and thinks that the problem is to be solved in accordance with the laws of physics. As we have seen in a preceding chapter, Bordet supposes that both in agglutination and in bacteriolysis there is a substance in the serum which renders the blood and bacterial cells sensitive to the action of a ferment. This substance he calls the sensitizer, and he states that the phenomenon of agglutination is similar to that of coagulation, and in explaining the latter he endeavors to exclude the necessity of the presence of bodies which have other than physical action. 


\section{CHAPTER IX.}

\section{IMMUNITY.}

IT is a matter of observation as old as the history of man that all animals are not alike affected by the same diseases, and that epidemics which decimate certain species are wholly without effect upon the health and life of others. Primitive man undoubtedly recognized the fact that one attack of certain diseases gives a more or less permanent immunity to the same disorder. It has also been long recognized that certain diseases, such as diphtheria aud scarlet fever, which are so highly fatal among children, but rarely attack adults. The question of immunity has long been, is, and probably will long continue to be one of the most perplexing problems which medical science has attempted to solve. With so many chances of infection, the question is often asked how is it that the human race continues its existence, and why has it not been swept out of the world by epidemics? The answer to this question is not easy, and the time has not come when it can be given with perfect satisfaction. However, we are now in possession of numerous facts bearing on this point, and it will be our attempt in this chapter to briefly state the most important of these.

It should be plainly understood that the factors involved in securing immunity against infectious diseases are multiple in number and varied in character. A mistake has been made in endeavoring to explain immunity as being due to one, or even to a few anti-bacterial properties of the animal body. This has been admirably pointed out by Meltzer, ${ }^{1}$ from whom we make the following quotation: "I maintain in the first place that in the struggle against bacteria the defense of the body is not carried on exclusively or chiefly by a single element. It is neither the body fluids, nor the leucocytes, nor the other cells alone which can claim the exclusive merit of maintaining the health of the body, but each and every one of them has its variable share in attaining the desired end.... Let us take as an illustration the protection of the conjunctival sac. It is nearly in direct contact with the air, and we might expect to find there an extensive bacterial settlement. Nevertheless Lachowitz and Bujwid found that in 69 per cent. of cases the conjunctiva was perfectly sterile. The factors which accomplish this sterility, or at least comparative sterility, of bacteria are: The reflex which causes the closure of the lids at the approach of dust (the carrier of

1 Congress of Am. Physicians and Surgeons, 1900. 
bacteria); the blinking which occurs regularly a few times in a minute, which in conjunction with the lachrymal moisture, throws out again mechanically the already landed bacteria ; and finally, the bactericidal effect of the tears destroys the balance of the invaders. Or let us take the respiratory organ from the larynx down to the respiratory tissue, including the corresponding lymph glands. ... Through this path the outside world stands in an intimate relation with the interior of the body ; inasmuch as the air column is separated from the lymphatics and capillaries of the lungs merely by a single layer of the very thin epithelium of the air cells. Even the serous cavities are separated from the lymph spaces by thicker layers. This arrangement is, of course, indispensable for the proper exchange of the blood gases with the air. But what prevents the bacterial invasion of the interior of the body by this open and direct way? Moreover most of the writers agree that trachea, bronchi and lung tissue of healthy animals are entirely sterile. In a number of rabbits under morphine anesthesia I found all these parts to be sterile. If one vagus or a laryngeal branch was cut, then the upper part of the trachea contained bacteria, but not the lung. When both vagi were cut, then of course the lungs too were invaded. Jundell reported recently that by means of a special device he was able to test the human trachea, and found that in the majority of healthy cases the region below the glottis proved to be sterile. What protects this path? In my opinion the result is accomplished by the coöperation of the following factors: The tortuous part of the respiratory path lying above the glottis removes perhaps the greatest part of the bacteria contained in the inspired air column, and the remaining number is, under normal conditions, just small enough to be disposed of by the factors present below the larynx. Bacteria which pass the glottis are either carried back outside of the glottis from the trachea and the bronchi by the steady movements of the cilia of the epithelium, or, if the germs are carried in the center of the air column down to the air cells, they quickly penetrate the thin epithelial layer and are immediately within the reach of the lymph glauds, which take good care of them. ... In connection with the respiratory path, I would like to recall here the interesting fact that both canals which lead farthest to the innermost of the body, that is, the respiratory and the female genital canal (which latter terminates in the peritoneum), have ciliated epithelium, the movements of the cilia being outward, and are, as far as the epithelium extends, entirely or nearly sterile."

Some of the factors which lead to immunity in the infectious diseases pertain to the animal organism, while others are due to limitations of the capability of growth on the part of the infecting agents. For instance, but few if any of the pathogenic bacteria are able to penetrate the unbroken skin, and infection through this avenue can 
occur only when there is some break in the continuity of the covering of the body. Indeed some of the most powerful pathogenic microörganisms are practically harmless when introduced into the body subcutaneously, while they may speedily cause death when taken into the alimentary canal. This is true of the vibrio of Asiatic cholera. On the other hand tetanus and anthrax bacilli may do no harm when taken into a healthy stomach, while they may cause speedy death when injected subcutaneously. These examples make it evident that it is not sufficient for a pathogenic microörganism, even one to which man is highly susceptible, to come in contact with the human body in order to cause disease, but it must first find its suitable port of entry, and even after this has been reached, there are other dangers to the life of the invader with which he may have to meet before he reaches the place where he can entrench himself and begin the struggle. Such guards are furnished, as has already been seen, by the mechanical movements of epithelial ciliæ, by the tortuosity of the passage, by coming in contact with such fluids as the gastric juice, or by being hurried through the body and cast out in the excretions, as undoubtedly sometimes happens to typhoid bacilli when taken into the alimentary canal. Moreover every toxicogenic germ is not capable of maintaining even for a short time a parasitic existence. The obligate saprophytes, which are numerous, and some of which undoubtedly produce powerful toxins, are capable of harming man only under exceptional conditions which are specially favorable to their growth and multiplication. Indeed, some members of this class do not appear to be capable of growth and reproduction when introduced into any part of the animal body, and consequently they are harmful to man only when their already formed toxins are introduced into his body. These bacteria in and of themselves are not able to affect the health or endanger the life of man. Other powerful toxicogenic bacteria have their intracellular toxins locked up in cell walls which under ordinary conditions are impermeable; and it is only when some unusual condition gives opportunity for the disintegration of the bacterial cell that its toxin is liberated and may do harm. This seems to be true of the colon bacillus, which is a normal habitant of the intestines of man, and which contains a powerful intracellular poison. Under ordinary conditions this bacterium is harmless to man, because its toxin does not diffuse from the bacterial cell, and consequently cannot be absorbed by the intestinal wall ; but if the colon bacillus finds its way into the peritoneal cavity, where its cell wall is disintegrated by phagocytic or other action, its toxin is liberated to the great danger of the host.

Animals differ widely not only in their susceptibility to bacterial infection, but also to bacterial intoxication. For instance certain animals, especially birds and reptiles, are not susceptible to the toxin of 
tetanus, and mice and rats bear large doses of the diphtheria toxin without ill effect. On the other hand, many animals that are susceptible to the toxins cannot be infected with the bacteria which produce these toxins. It will be seen from this that there may be both bacterial and toxin immunity.

We may discuss the subject of immunity in detail under the following heads: (1) Natural immunity, or that possessed by certain species or races of animals at all times against certain diseases; (2) inherited immunity, which may be transmitted from the mother to the fetus through the placental circulation, or from the mother to the child through the milk; (3) acquired immunity, or that which is secured by one attack of the disease, by vaccination, or by repeated treatments with sterilized or unsterilized cultures. Acquired immunity may be either active or passive. By an active immunity we mean that form which is induced by the direct treatment of the animal with filtered or unfiltered cultures, and in which case the antitoxic or anti-bacterial substance is produced in the body of the animal thus treated. Passive immunity, on the other hand, may be secured by injecting the blood serum of an animal actively immunized into a second animal. As an illustration of active and passive immunity, we may refer to the method by which diphtheria antitoxin is prepared. The horse is treated with successive, non-fatal, gradually increased doses of diphtheria toxin until this animal furnishes a serum which contains a large amount of diphtheria antitoxin. The horse's serum when injected into the child sick with diphtheria supplies the antitoxin, which combines with the toxin, and thus protects the tissues of the child against the injury that would otherwise be inflicted on the cellular elements by the noxious agent. In this illustration an active immunity is induced in the horse, and as a result of the establishment of this condition certain cells within the animal are stimulated to a form of activity by which the antitoxin is generated. In other words, "the horse treated with successive doses of the diphtheria toxin comes to possess an active immunity. The horse's serum containing the antitoxin is injected into the child, which for the time being becomes physiologically a part of the horse, and possesses only a passive immunity.

We will now proceed to discuss in more detail the subject of natural immunity. It is a fact of common observation as well as of experimental demonstration that the lower animals are wholly immune to certain bacterial infections to which man is markedly susceptible. For instance, among men typhoid fever is one of the grave diseases, causing great morbidity, and increasing to a considerable extent the mortality lists; while among the lower animals this disease does not occur naturally, nor has anyone as yet been able to induce it by inoculation with the bacillus. It is true that many of the lower animals are susceptible to the typhoid toxin, but a true typhoidal in- 
fection with the bacterium of this disease has not been established, although frequently tried, in any of the animals upon which experiments have been made. Leprosy, scarlet fever, yellow fever and measles are other diseases which inflict themselves upon mankind, but to which the lower animals are apparently insusceptible. Some infections are prevalent among certain species of animals, while upon others they seem to be without effect. For instance, anthrax is common among cattle and sheep, while many carnivorous animals wholly escape this infection. It is an interesting fact that in nature some animals, such as the guinea-pig and rabbit, are not known to suffer from epidemics of anthrax, but this disease can be easily induced in these animals by artificial inoculation. Toxin and bacterial immunity are sometimes, but not always, possessed by the same animal. Rats and mice are insusceptible to both the bacillus and the toxin of diphtheria, but, as we have already seen, many animals succumb to the typhoid toxin, while none are susceptible to this infection.

One of the important factors in natural immunity lies in the fact that the toxicogenic germ is unable to multiply in the animal body. The bacillus pyocyaneus is frequently found upon the surface of man's body, especially in the axillary and inguinal regions, and sometimes it occurs in the intestines, but notwithstanding this almost constant proximity of this organism man is but rarely injured by it, yet, nevertheless, this bacillus produces a toxin to which man is susceptible. The micrococcus prodigiosus is not classed among the pathogenic bacteria, and yet experimentation has shown that its toxin, which has been used in the treatment of malignant growths, has a marked effect upon man. It is probable that most of the saprophytic bacteria contain intracellular substances which when repeatedly injected into animals in relatively large amounts are capable of causing death. The sarcines are usually regarded as altogether harmless, but it has been shown that the intra-peritoneal injection of the cellular elements of these organisms is followed by death. It has been found that large amounts of the spores of the tetanus bacillus, when completely freed from the toxin, may be injected into rabbits and guinea-pigs with no further injury than the formation of a pocket of pus at the place of injection, but these animals are highly susceptible to tetanus toxin, which causes in them typical symptoms of the disease and induces speedy death. The natural immunity possessed by some animals to tetanus infection may be overcome by the employment of mixed cultures, even when the accompanying microörganism is apparently wholly without effect. A like result may be secured by the simultaneous introduction into the animal body of certain chemicals, such as lactic acid. On the other hand, certain animals are quite insusceptible to the toxin, and at the same time easily infected with the microörganism. A healthy man is rela- 
tively insusceptible to tuberculin, but is easily invaded by the tubercle bacillus, and after becoming infected with the germ he loses his insusceptibility to the toxin. It may be seen from the illustrations that have already been given that inability on the part of the microorganisms to grow in the animal body is an important factor in natural immunity, but this is by no means the sole factor in the establishment of this coudition.

In the chapter on the germicidal constituents of the blood we have shown the existence of alexins not only in this fluid but in other tissues and juices of the body. It was at one time supposed that the alexins of the blood played a large part in the establishment of natural immunity, but more extended investigation has shown that this supposition is erroneous. The blood serum of the rabbit has relatively strong bactericidal effect upon anthrax bacilli, and yet this animal is most highly susceptible to this infection. Moreover, Lubarsch ${ }^{1}$ has shown that the bactericidal action of rabbit's blood on the anthrax bacillus is more marked outside than it is inside the body. The discovery of the germicidal effect of rat's blood on anthrax bacilli was at one time suggested by Behring, ${ }^{2}$ as an explanation of the relative immunity possessed by this animal to anthrax infection. However, more extended observation showed that the white rat is more susceptible to anthrax than was at one time supposed, and yet the bactericidal action of rat's blood on the anthrax bacillus can be demonstrated not only outside the animal body, but also within the organism. If anthrax bacilli be mixed with rat's blood and the mixture be injected into mice, the latter animals escape infection, but the rat from which the bactericidal blood was obtained succumbs to infection with the bacillus. On the other hand, the adult dog is immune to anthrax infection, while the blood serum of this animal has no germicidal action on this organism. Indeed, anthrax bacilli grow abundantly in the blood serum of the dog. It will thus be seen that the blood of the rabbit, an animal highly susceptible to anthrax infection, has marked germicidal action on the anthrax bacilli outside the body; while the blood of the dog, an animal immune to anthrax infection, furnishes a good culture medium for the growth of the bacillus. We must, therefore, conclude that the attempt to explain natural immunity solely by the bactericidal properties of the blood must be abandoned. Indeed, it is probable that the germicidal properties of the blood do not constitute a very important factor in the production of natural immunity. However, we do not claim that the alexins can be wholly disregarded in the study of this problem, and after bringing forward another theory concerning natural immunity, we will return to this subject. 
According to Metschnikoff, the most important factor in the production of natural immunity is to be found in the phagocytic action of certain cells within the animal body. He divides phagocytes into mobile and fixed elements. Among the former he places both the mono- and polynuclear leucocytes (with the exception of the small lymphocytes, and the mast cells of Ehrlich), and the so-called wandering cells ; while the fixed phagocytes, or macrophages, consist of endothelial cells, the elements of the spleen pulp and of bone marrow, some connective tissue cells, and possibly certain nerve and muscle cells. He states that sometimes many mononuclear phagocytes fuse together forming large plasmodia, which may be known as giant cells, and which have a phagocytic action. When bacteria are introduced into a naturally immune animal they are seized upon and devoured by either the mobile or fixed phagocytes, or by both. When a microörganism is introduced into a place where no phagocytes are present, as for instance under the skin, into the cornea, or into the anterior chamber of the eye, the mobile phagocytes collect at the point of bacterial invasion, engulf the bacterial cells with the aid of their pseudopodia and then digest them or in some other way deprive them of their capability of harming the body. This method of disposing of foreign substances introduced into the animal body is known as phagocytosis. Even those who have brought the best arguments against Metschnikoff's doctrine of phagocytosis admit that this phenomenon is especially observable in animals which are relatively immune to the invading microörganism, or in other words, they recognize the fact that in watural immunity phagocytosis is at least a frequently observed phenomenon. Pfeiffer and Kolle, ${ }^{1}$ in studying the former's reaction, which has already been referred to in our chapter on lysins, state that the greater number of bacteria destroyed in the peritoneum of normal animals are taken up by leucocytes, but that some perish in the exudative fluid. Kruse ${ }^{2}$ states that phagocytosis is frequently observed, especially in relatively insusceptible animals, and when a weak virus is used. When anthrax bacilli are injected under the skin of a $\log$ a very energetic phagocytosis takes place and indeed, according to Denys and Havet, ${ }^{3}$ this phenomenon may be observed in extra-vascular blood from the dog. Even spores are taken up by the phagocytes and sometimes they develop into bacilli within the phagocyte, thus destroying the organism which has engulfed them, but ordinarily their development is rendered impossible, and their destruction finally accomplished. The phenomenon of phagocytosis can be explained only on the assumption that the phagocyte contains some chemical substance by virtue of which it destroys the captured

\footnotetext{
1 Zeitschrift für Hygiene, 21, 1896.

${ }^{2}$ Flügge' s Mikroorganismen, 1895.

${ }^{3}$ La Cellule, 10, 1894.
} 
microörganism. What this chemical poison is, or whether it is the same in all phagocytes, we cannot at present determine. All phagocytes contain nucleic acid, and the germicidal properties of this have been abundantly demonstrated, but whether or not there may be other and more powerful bactericidal agents in certain phagocytes, we have no means at present of knowing. It is still a question whether or not the germicidal substance contained in the leucocytes is a secretion of these organisms or a result of their disintegration. As has already been stated in the chapter on the germicidal constituents of the blood, it seems quite evident that the bactericidal substances in the blood serum result from the disintegration of the white corpuscles. It is possible that a blood which furnishes a highly bactericidal serum gives off but little or no germicidal substance to the plasma in the living body. It is probable that the leucocytes in the blood of certain animals disintegrate more rapidly when removed from the body than happens to the leucocytes of other animals. In instances of the first kind we would expect to find the blood serum possessed of marked germicidal properties, while in cases of the second kind the serum may be quite devoid of bactericidal action. Again it is possible that the phagocytes in the blood of one animal may retain their germicidal constituents longer than do the leucocytes in the blood of other animals. In the serum of a blood of the first kind we would not expect to find a large amount of germicidal substance and yet within the body the phagocytes of this animal may be more effective in the destruction of germs than are those in the blood of an animal whose serum is rich in germicidal substances.

There is another chemical process in the phenomenon of phagocytosis which is of the greatest importance. The phagocytes are attracted by the introduction into the body of certain substances and repelled by others. This is known as chemotaxis, which may be either positive or negative. In other words, the foreign substance introduced into the animal body may attract the phagocytes, or it may repel them. In the former instance the phagocytes will gather in large numbers about the foreign substance introduced into the body, and phagocytic action will be most marked. It appears to us that the greatest factor in natural immunity, so far as the action of the blood of the body on the invading microörganism is concerned, lies in the fact that in natural immunity chemotaxis is positive, and the more marked the natural immunity is, the stronger is the positive chemotaxis. It should be understood that at present we have reference only to natural immunity against infection, and that we do not include in these statements our opinions concerning natural immunity against toxins.

That bacteria may retain their vitality after being taken into the phagocyte has been demonstrated by injecting phagocytes containing bacilli into susceptible animals and thus inducing infection. When 
phagocytes containing bacteria are removed from the body the phagocytes die and some of the contained bacteria demonstrate their retention of life by growth and multiplication. It sometimes happens within the body that the bacterium proves too powerful an opponent, and the phagocyte is destroyed. This condition can be brought about by the employment of a highly virulent culture or by placing the animal after inoculation under unfavorable conditions. For instance Pasteur and Joubert demonstrated that while the chicken is naturally immune to anthrax, susceptibility may be secured by keeping the inoculated animal at a low temperature, under the unfavorable influence of which normal phagocytic action is not possible.

It is probable that plasmolysis is also a factor in natural immunity. It is a well-known fact that the removal of bacteria from one medium into another containing slightly more or less of certain mineral constituents may be followed by the death of all or the greater part of the microörganisms. Bacteria consist of cell-walls with contents. Diffusion through the cell-wall is constantly taking place to a greater or less extent. By altering the mineral constituents of the medium, an osmosis, which may prove fatal to the bacterium, results. The removal of the bacterial cell from an isotonic medium to one which is either hypotonic or hypertonic leads to greater or less injury to the cell.

The subject of natural immunity to toxins is one of quite as much interest as that which we have just been discussing. Instances of insusceptibility to powerful poisons have long been known and have formed the subject-matter of much theoretical discussion. For instance, hogs can eat with impunity relatively large amounts of arsenic and antimony, and certain insects not only live, but seem to thrive in an atmosphere saturated with the vapor of formaldehyde. Metschnikoff states that there are certain invertebrates which are not affected by large doses of some of the most important bacterial toxins. The larva of a large beetle (Nashornkäfer) has been found to be wholly immune to the cholera toxin. If one of these and a small green frog of equal weight be treated with 0.5 c.c. of soluble cholera toxin, the larval beetle remains apparently unaffected, while the frog speedily dies. On the other hand, if both animals be inoculated with the cholera bacillus, the beetle succumbs to cholera sepsis, while the frog escapes infection. Metschnikoff explains these observed facts on the ground that the leucocytes of the frog consume the vibrios, but are powerless against the toxin, while the leucocytes of the larval beetle are unable to cope with the bacteria, but it must be admitted that he does not explain how it is that the beetle succumbs to the cholera toxin elaborated in its own body, while it is insusceptible to that introduced from without.

It is well known that certain vertebrates are very resistant to the most potent venoms of snakes. This is notably true of the mon- 
goose and hedgehog, and less markedly so of hogs. The crocodile is insusceptible to both the tetanus bacillus and its toxin, but is susceptible to diphtheria toxin. Many birds, including the barnyard fowl, are insusceptible to the tetanus toxin. The best explanation that has been given to natural immunity against toxins is that offered by Ehrlich's theory. This author holds that in order for a substance to be toxic to the animal organism the latter must contain cells, the molecules of which furnish side chains capable of forming chemical compounds with the toxin. When the toxin is not capable of forming a chemical combination with some constituent of the cell, it has no poisonous action on that animal. Some bacterial toxins, such as that of tetanus, form a destructive combination with the cells of the central nervous system, while others possibly form similar compounds with other tissues of the body. Therefore, according to Ehrlich, natural immunity to toxins is due to the failure to form combinations in the animal body which are destructive to some of its cellular elements.

The study of acquired immunity has furnished a rich field for research to the bacteriologist. Early in his development man must have observed that one attack of certain diseases gave to those who recovered more or less permanent immunity to that disease. The Chinese recognized this fact many centuries ago, so far as smallpox is concerned, and this led to their practice of inoculation for this disease. This custom probably spread from the Orient through Tartar tribes, and, as is well known, was introduced into England from Turkey. The next step that was made in the study of acquired immunity was the discovery of vaccination for smallpox as practiced by Jenner; but inasmuch as up to that time the specific bacteria remained unknown, the philosophy of vaccination could not be explained. The scientific study of acquired immunity may be said to date from the early investigations of Pasteur, who in 1880 discovered that inoculations with non-virulent cultures of chicken cholera gave immunity to subsequent inoculations with a virulent culture of this microörganism. Indeed, as early as 1877 Pasteur observed that susceptible animals inoculated with cultures of the anthrax bacillus mixed with other bacteria did not acquire the disease. This discovery, as we shall see later, has been amplified by others, especially by Emmerich. It may be well for us to proceed in the discussion of acquired immunity by considering the different agents employed in securing this condition. They are as follows: (1) By treatment with weakened cultures of the germ of the disease. By the employment of sterilized cultures of the specific microörganism or by the use of its toxins. (3) By treating the animal with cultures of the specific organism mixed with other bacteria, or by the employment of mixed cultures in which the specific microörganism is present. 
Pasteur ascertained that when the bacillus of chicken cholera is grown on artificial culture media through successive generations, it gradually loses its virulence and finally reaches a point when it is no longer capable of inducing the disease in susceptible animals. Then he found that animals treated with this non-virulent culture and subsequently inoculated with virulent cultures remain unaffected. With the anthrax bacillus it was found that there is no loss of virulence in successive growths on artificial media. It was therefore necessary to resort to other means of robbing this organism of its virulence. Pasteur found that this could be accomplished in several ways, the most important of which were: (1) Growth at relatively high temperatures, and (2) growth in culture media containing small amounts of certain antiseptics, such as carbolic acid. By both of these methods he was able to secure vaccines which were found to give temporary immunity. Vaccines of this kind are still employed, notably for anthrax, but the immunity secured in this way is, in most instances at least, of relatively short duration, and this method cannot be said to be altogether successful. It is true that herds of cattle and sheep may be protected from a prevailing epidemic in this way, but revaccination must be frequently practiced, and there is always danger, unless great care is exercised, of not sufficiently reducing the virulence of the germ, and epidemics have been extended by the employment of imperfectly prepared vaccines.

Attempts to secure immunity by the employment of sterilized cultures were probably suggested by the discovery of the fact that immunity to the venom of snakes may be obtained by frequent successive injections of non-fatal doses. As early as 1886, Sewall immunized pigeons to the venom of the rattlesnake, and soon thereafter Salmon and Smith immunized animals to the bacillus of hog cholera by successive treatments with sterilized cultures of the bacillus of this disease. The later researches of Ehrlich with abrin and ricin gave great impetus to study along these lines, and space cannot be given here to even an enumeration of all the work that has been done in the study of immunity by treatment with sterilized cultures. In some instances the action of the toxin has been modified by heating or by the addition of certain chemicals, such as iodin and chlorin. The production of immunity by the employment of sterilized cultures or toxins is due in most instances, if not in all, to the formation of antitoxins, and the explanation of the formation of these bodies will be discussed later.

A certain degree of immunity to some of the infectious diseases may be secured by previous treatment of the animal with substances of non-bacterial origin. The first hint that this might be accomplished was offered by Wooldridge, who reported that he had secured immunity against anthrax by previous treatment of the animals with extracts of testicles and thymus gland. Although subsequent inves- 
tigations have not fully confirmed this statement, they have led to the discovery of many interesting facts. It has been found that at least temporary immunity against some of the infections may be secured by previous treatment of the animal with normal serum, nucleinic acid, bouillon, or even physiological salt solution. For instance, Issaeff, ${ }^{1}$ found that animals can be protected against experimental cholera peritonitis by previous intra-peritoneal injections of the above-mentioned substances. This investigator found that immediately after the preventive injection there is a marked diminution in the number of leucocytes in the peritoneal lymph, but after a short period of time the leucocytes return in increased numbers. Now if at this stage the ordinarily fatal quantity of a culture of the cholera vibrio be injected into the peritoneum, death does not follow. In accordance with the phagocytic doctrine of Metschnikoff it is supposed that these substances of non-bacterial origin give temporary immunity on account of the phagocytosis which they induce. Temporary immunity against some of the most highly virulent bacteria may be secured in this manner. Thus Bordet succeeded in immunizing animals against most virulent cultures of the streptococcus by previous treatment with ordinary nutritive bouillon. This form of securing immunity is more easily demonstrated by peritoneal inoculations than in any other way. It is possible that the injection of the preventive substance leads to more or less phagolysis, and as a result of this the peritoneal fluid becomes unusually rich in bactericidal substances, which result from the disintegration of the phagocytes. Metschnikoff further suggests that the phagolysis caused by the introduction of the preventive solution is the active agent in causing the accumulation of phagocytes at the place where the phagolysis has occurred, which in this instance is in the peritoneal cavity. On account of the phagolysis, due to the introduction of such a substance as bouillon, the fluid content of the peritoneal cavity becomes rich in dissolved bactericidal substances and in this respect resembles certain blood sera. When the bacteria are subsequently injected into the peritoneal cavity many of them are destroyed by the soluble germicidal substances, and those that escape this action are seized upon by the incoming phagocytes. If a drop of the peritoneal fluid be examined at this time it will be observed that some of the bacteria have met with extra-cellular death, while others are engulfed in the phagocytes.

The most probable explanation of the good results obtained in Pasteur's treatment of hydrophobia is that by this means a toxin immunity is secured. The organism which is supposed to cause hydrophobia has not as yet been discovered, but the probabilities are that inoculation with a living organism is made by the bite of the rabid dog. In this disease a long period of incubation precedes

1 Zeitschrift für Hygiene, 16, 1894. 
the development of the first symptoms. Soon after the man has been bitten he is, according to the Pasteur method, treated successively with dried portions of spinal cords taken from animals which have died from experimental hydrophobia. These cords probably contain the toxin of the disease, or the modified microörganism, or both, and those which have been dried longest contain these in their least active form. By successive inoculations with emulsions of these cords immunity to the hydrophobia virus may be secured before the period necessary for the development of the germ introduced with the bite of the dog has passed.

For some years Emmerich has worked on a theory of immunity, which, if it should prove to be correct, gives a simple explanation of many phenomena which have puzzled bacteriologists. Recently this theory has been elaborated in two papers published by Emmerich and Löw, ${ }^{1}$ and its important points may be stated as follows: Many bacteria, and among these some of the most important pathogenic ones, produce, both in vitro and in vivo, enzymes which are capable of digesting the organism which produces them, and sometimes other bacteria as well. In proof of this statement they bring forward the following observation: If a culture of the bacillus pyocyaneus be allowed to stand at $37^{\circ}$ there forms after three days on the surface a thick scum consisting of bacilli. If the tube be thoroughly shaken some of the bacteria subside, and after three days longer a new scum forms on the surface. The process of shaking and allowing to grow is repeated six or eight times, and finally it will be observed that only a very small residue of the bacteria remains and is deposited on the bottom. If such a deposit found in a culture a few weeks old be examined microscopically there will be found a few whole bacilli which still stain well with fuchsin, but the mass of the deposit will be seen to consist of broken-dowu bacilli which do not stain readily, drops of fat, and crystals. They explain this phenomenon on the ground that the bacteria have produced an enzyme which finally manifests its bacteriolytic action on the germs. If this experiment be repeated with a culture of the bacillus of swine erysipelas, similar changes will be observed. The first effect of the enzyme is to agglutinate the bacilli, while the final result is a bacteriolytic one. It will be seen from this that both agglutination and bacteriolysis are supposed to be due to an enzyme produced by the microörganism. The difference between the change observed in artificial cultures and in immune serum is due to the fact that the latter contains a larger quantity of ready-formed enzyme, while in the former the enzyme is gradually produced. Illustrations of similar phenomena are drawn from the study of the action of enzymes on the cellulose of moulds. For instance certain parasitic moulds pierce the wood of living trees by means of enzymes produced in the innermost growths of the mould,

${ }^{1}$ Zeitschr. f. Hygiene, 31, 1899; 36, 1901. 
and by which the cellulose of the tree is digested. After a time these ferments digest not only the cellulose of the tree but also the older parts of the mould itself. The action of bacterial enzymes of this class is offered as an explanation of the degeneration forms observed in many old bacterial cultures. From such cultures there may be precipitated with alcohol an albuminous body which when dissolved in feebly alkaline solution has a bacteriolytic effect. Arrest of growth in artificial cultures occurs when the solution of the bacteriolytic enzyme is sufficient to digest newly formed bacteria. The same phenomenon is offered as an explanation of the self-limitation of the infectious diseases. When the bacteriolytic enzyme formed in the animal body has reached a sufficient degree of concentration to dissolve the bacteria which have produced it, further growth of the organism is impossible, and the disease is arrested. It is probable that the enzyme exists in the bacterial cell as a zymogen, and is able to manifest its digestive action only after liberation from the cell contents. The enzyme produced by a given bacillus may dissolve the cell membrane not only of the bacterium which has produced it, but of other bacteria as well. These enzymes are divided into two classes, which are known as conforme and heteroforme, the former being one which dissolves only the bacterium which produced it, while the latter is one which has a bacteriolytic action upon other microorganisms as well. It is proposed that bacteriolytic enzymes be given the general name of nucleases, because they digest the nucleoproteids of the bacterial cells. Special enzymes are designated by names derived from those of the bacilli which produce them. Thus the bacteriolytic enzyme of the bacillus pyocyaneus is known as pyocyanase ; that of the cholera vibrio as cholerase, etc. It is supposed that in the living body the enzymes of pathogenic bacteria combine with certain albuminous bodies, probably those derived from the leucocytes. The substances resulting from these combinations are designated immuneproteids, and one of these is distinguished from the others by prefixing the name of the special enzyme. Thus we have pyocyanase-immuneproteid, cholerase-immuneproteid, typhaseimmuneproteid, etc.

Emmerich and Löw have shown experimentally that pyocyanase readily destroys and dissolves anthrax bacilli in vitro, both under aërobic and anaërobic conditions. They have also shown that rabbits inoculated with virulent cultures of anthrax do not die if they be treated with solutions of pyocyanase. In these experiments the animals received their first treatment simultaneously with the inoculation or directly afterward, and subsequent treatments were also given. They were not able to immunize rabbits to anthrax by previous treatment with solutions of pyocyanase. However, they did succeed in preparing artificially pyocyanase-immuneproteids with which immunity to anthrax was secured. In these experiments con- 
centrated solutions of pyocyanase were obtained either by evaporation of old filtered cultures of the bacillus pyocyaneus in vacuo, or by precipitation of the enzyme and subsequent solution of the precipitate. It should be stated that Bouchard has successfully treated anthrax by injection of cultures of the bacillus pyocyaneus, and also by treatment with blood serum of sheep which had been immunized to the streptococcus of erysipelas, but inasmuch as cultures of the bacillus pyocyaneus are themselves toxic, Pettenkofer very properly said of this treatment: "One drives out the devil with Beelzebub." Emmerich and Löw hold that the pyocyanase as they have obtained it is free from toxic action. They think it probable that the poisonous constituents of filtered cultures of this bacillus are given off during the process of evaporation in vacuo or are separated from the enzyme when the latter is precipitated from old cultures. At least it appears that the animals which they treated with solutions of pyocyanase were not injuriously affected by the injections. Pyocyanase also digests typhoid, diphtheria, pest and cholera bacilli.

Pyocyanase, as precipitated by alcohol, forms a yellowish-green amorphous substance which is readily soluble in water, to which it imparts a greenish tint similar to that possessed by cultures of the bacillus. The aqueous solution is distinctly alkaline. Both Millon's reagent and the biuret reaction fail when applied to aqueous solutions of pyocyanase, while a violet coloration is produced on heating with concentrated hydrochloric acid. When pyocyanase is heated with caustic potash it becomes intensely yellow, and on the addition of lead acetate a blackish deposit is formed, thus indicating that this substance contains sulphur. It is an interesting fact that this enzyme may be heated to $90^{\circ}$ without loss to its bacteriolytic activity, and a temperature of $98.5^{\circ}$ reduces, but does not wholly rob it of this property. Notwithstanding its great resistance to heat, Emmerich and Löw conclude that this substance must be classed among the enzymes on account of its energetic action in very small amounts. They also refer to the investigation of Würtz, who has shown that the ferment papayotin retains its peptonizing action after having been exposed to a temperature of $105^{\circ}$. If a small amount of dry pyocyanase be placed in a tube of gelatin and be kept at $22^{\circ}$ the contents of the tube are completely peptonized within twentyfour hours. It also acts energetically upon fibrin.

Emmerich and Löw conclude their first paper with the following statements: (1) The fact that liquid cultures of most bacteria cease to develop after a few days or weeks is due to the elaboration by the bacteria of enzymes, which after reaching a certain degree of concentration, dissolve the bacterial cells. (2) There are bacteriolytic enzymes which digest not only their own bacteria, but others as well. (3) The curative action of both filtered and unfiltered cultures is due to the presence in these cultures of bacteriolytic enzymes.

(4) The 
production of artificial immunity with filtered or unfiltered cultures of pathogenic bacteria is due to the gradual formation within the body of a compound of the bacteriolytic enzyme with some albuminous body; the resulting immune proteid retains the bacteriolytic activity of the original enzyme. (5) This combination, which takes place very slowly in the animal body, may be speedily brought about by chemical reagents in vitro. (6) So-called agglutination is nothing more than the first stage of bacteriolytic action of enzymes. Immune sera manifest their bactericidal action more energetically under anaërobic than under aërobic conditions, but even in the presence of air, the bacterial enzymes when in highly concentrated solution show marked bactericidal action. (8) There are bacterial enzymes which in the animal body destroy not only the bacterial cells, but their toxins as well. For instance, pyocyanase protects animals which have been treated with lethal doses of diphtheria toxin. Our experiments show that pyocyanase may be used successfully in the treatment of anthrax, diphtheria, pest, etc. (10) By means of artificially prepared pyocyanase-immuneproteid a high degree of immunity against anthrax, lasting for at least fourteen days, may be secured. (11) A slight elevation of temperature (about one degree) observed in animals with anthrax treated with pyocyanase is a result of the injection of the enzyme. (12) The bactericidal action of normal blood is probably due to the presence of enzymes.

In their second paper Emmerich and Löw detail the methods which they have employed in the preparation of their nucleases and immuneproteids. The organism whose enzyme is desired is grown in a medium which is as free as possible from albuminous constituents. This is necessary in order that the enzyme employed may be obtained on precipitation with alcohol without mixture with foreign albuminous substances. As a culture medium for the bacillus pyocyaneus, the following preparation is used:

$\begin{array}{lr}\text { Distilled water, } & 1,000.0 \\ \text { Asparagin, } & 5.0 \\ \text { Sodium acetate, } & 5.0 \\ \text { Dipotassium phosphate, } & 2.0 \\ \text { Magnesium sulphate, } & 0.1 \\ \text { Sodium chlorid, } & 2.0\end{array}$

In order to prepare the nucleases in large quantities, from ten to twelve liters of the above medium is divided in portions of one-half liter each in liter flasks. These are inoculated and allowed to grow for some weeks at $25^{\circ}$, and then for some weeks longer at from $30^{\circ}$ to $37^{\circ}$. After five or six weeks the cultures are shaken thoroughly at least once in twenty-four hours, and when finally there remains only a slight deposit of germ detritus, the supernatant fluid is drained off 
by means of a vacuum pump. This fluid is filtered through porcelain and evaporated in vacuo at from $20^{\circ}$ to $36^{\circ}$ to one-tenth its volume or less. The concentrated enzyme solution is deprived of inorganic salts and a part of its toxic constituents by dialysis, continued from twelve to twenty-four hours. If after this treatment the presence of toxic substances can be shown by animal experimentation, three-tenths per cent. of trikresol is added and the solution allowed to stand for a few weeks longer, during which time apparently the bacteriolytic enzyme destroys any trace of toxic substance that may be present. The concentrated solution of enzyme thus obtained has, according to Emmerich and Löw, not only bactericidal properties, but also the capability of destroying certain toxins, notably that of diphtheria, in the animal body. As has been stated, these authors were not able to immunize animals to anthrax with the enzyme of the bacillus pyocyaneus, but this object they were able to accomplish by the employment of their pyocyanase-immuneproteid. This compound they have prepared in several ways, one of which is as follows : To each 100 c.c. of the concentrated and dialyzed pyocyaneus culture, there is added from three to five grams of fresh, fincly divided spleen pulp. This mixture is treated with three per cent. of potassium carbonate and digested at $37^{\circ}$. During this process of digestion the suspended bits of spleen substance apparently undergo a process of agglutination, resembling, on a magnified scale, the phenomenon which may be seen in bacterial cultures. It has been observed that the hay bacillus may develop in this mixture during the digestion process unless trikresol has been added. Finally the spleen pulp is apparently digested, while only small bits of capsule and fiber remained undissolved. When this method is properly carried out there is formed, according to the statements of Emmerich and Löw, a nuclease-immuneproteid which will give complete immunity against virulent anthrax, while the animal thus treated shows no elevation of temperature, and seems to remain perfectly well in every respect.

The nuclease-immuneproteid may be precipitated by the addition of ten volumes of absolute alcohol, and when dried in vacuo over sulphuric acid it may be kept for an indefinite time without loss of bacteriolytic action. Pyocyanase-immuneproteid forms a homogeneous greenish or yellowish powder, which has been found to be serviceable both in the production of immunity and in obtaining cure, after it has been kept for two years. This substance is said to keep better if about 5 per cent. of dextrin be dissolved in the bacteriolytic solution before precipitation with alcohol.

The importance of the claims of Emmerich and Löw can hardly be overestimated, provided they be confirmed by other careful workers, and be found to be generally applicable to the infectious diseases. To one who has followed the above given account of their investiga- 
tion, it must be evident, provided they have not fallen into error somewhere, that they have succeeded in preparing antitoxin artificially, and if their statements be confirmed it must be admitted that Ehrlich's theory concerning the nature of antitoxin must be considerably modified. Final decision on this matter must await future investigations.

When animals are immunized by successive treatments with a microörganism or its products, the blood serum and other fluids obtained from the body, acquire either bactericidal or antitoxic properties. In some instances the immunity secured is wholly anti-bacterial, while in others it is antitoxic. It will thus be seen that in the production of artificial immunity we may expect to find marked differences depending upon the microörganism used, and the kind of animal immunized. We will first discuss bacterial immunity. The bactericidal properties possessed by the fluids of the body of the immunized animal may manifest themselves only by an inhibitory action on the growth of the germ, or by partially depriving it of its capability of elaborating toxins. Early in his investigations of this subject, Metschnikotf found that anthrax bacilli grown on the blood serum of sheep immunized to this disease are without effect upon rabbits, but are still possessed of enough vitality to induce fatal anthrax in mice. This indicates that there is something in the blood serum of the immunized sheep which reduces the virulence of the anthrax bacillus. In some instances the inhibitory action of the immune serum manifests itself by depriving the bacterium of its ability to produce certain of its characteristic products. Thus, as has already been stated, Charrin and Roger found that when the bacillus pyocyaneus is grown in the blood serum of animals immunized to this microörganism it no longer produces its characteristic coloring matter. The inhibitory action of the body fluids may not be permanent, and when the bacillus is removed from their direct influence it may recover all of its ordinary virulence. Bordet found that when streptococci grown in the serum of immunized horses were completely freed from this medium they are as virulent as those developed in the blood serum of an unvaccinated horse, and Roger observed that the same microörganisms grow well in the serum of immunized rabbits, but that when thus grown and injected into other animals along with some of the immune serum, they induce only a temporary local disturbance. Next he inoculated rabbits with normal streptococci placed in the blood serum of immunized animals and found that the rabbits treated with this mixture remained well, while those in which the streptococci and the serum were injected into different parts of the body succumbed to the infection.

The phenomenon of agglutination is a manifestation of the inhibitory action of the sera of immunized animals on their homologous bacteria. The fact that there may be immunity without agglutina- 
tion, and vice versa, is no proof that this phenomenon is not a result of inhibitory action in those cases in which it does occur. It should be understood that agglutination is only one of various indications that the body juices of immunized animals rob their homologous bacteria in part of their virulence. Why agglutination does not take place in all instances we are not as yet able to determine, but when it does occur it is an indication that the blood of the immunized animal has some detrimental effect upon the growth and virility of the microörganism. It is true, as Metschnikoff has pointed out, that agglutination is not uniformly observed, and that there are immunized animals which furnish sera in which the homologous bacteria grow quite normally, but this observation does not overthrow the fact that agglutination is an evidence of the detrimental effect of the sera of immunized animals on the bacteria to which such animals have been immunized.

In some instances the body juices of the immunized animal manifest a bactericidal action on their homologous microörganisms, but have no effect on their toxins. This was shown to be the case by Metschnikoff in his experiment upon the coccobacillus of swine plague. The blood sera of animals immunized to this bacterium protect rabbits against infection, but furnish no protection against the toxin. In this instance we have to do with purely antiinfectious phenomena. The immunity secured is antibacterial and not antitoxic. The antiinfectious properties of the body juices are not, in all instances at least, strictly specific. The sera of certain immunized animals have a bactericidal action not only on their homologous bacteria, but sometimes on closely related microörganisms. Indeed in some instances it is not necessary that the relationship between the bacteria affected in like manner by these antiinfectious sera should be close. Thus, as has already been stated in the chapter on agglutinins, the blood serum of animals immunized to the cholera vibrio also agglutinates several other vibrios, and it has been shown by Issaeff that the blood serum of animals immunized to the vibrio of Metschnikoff has also an antiinfectious action on the pneumococcus. It has also been shown that normal serum may in some instances manifest marked antiinfectious properties. For instance, one-tenth cubic centimeter of the blood serum of a healthy man suffices to protect guinea-pigs against cholera peritonitis, and it is well known that frequently the blood serum of men, who have never had typhoid fever, agglutinates readily the bacillus of this disease. Not only is this the case, but the blood serum of such men may protect animals against the peritonitis normally induced in them by the typhoid bacillus. It follows from these observations, which might easily be multiplied, that the blood of normal animals frequently contains antiinfectious substances.

The bacteriolytic effect is the most marked form of bactericidal action that has been observed in the body juices of artificially im- 
munized animals. Of course, when the blood serum completely dissolves its homologous bacterium, it so completely destroys it that it has no longer either infectious or toxic properties. It has been shown quite positively that agglutinins and lysins are not identical, but the probabilities are that they represent the products of different stages reached in the process of immunization. A given blood serum may have an agglutinating action, and be without bacteriolytic effect, or vice versa, but it must be admitted that the possession of either one of these properties by the body juices militates against the growth of the infectious agent, and consequently each must be considered as a factor in the production of artificial immunity.

The question concerning the origin of the antiinfectious substances in the production of artificial immunity is one concerning which at present we can do but little more than theorize. The weight of evidence seems to be in favor of the view that by successive injections of the microörganism or its products the leucocytes are stimulated to increased secretion of germicidal substances. Metschnikoff claims that it is a general rule that phagocytosis is more pronounced in immunized animals than in those not immunized. He states that when a microörganism is injected into an animal which has been immunized to this germ, the phagocytes of the animal take up the invader more promptly than is done when susceptible animals are inoculated with the same germ. That phagocytosis is more marked in immunized animals is shown by introducing the microörganism in localities ordinarily free from phagocytes, such as the subcutaneous tissue and the anterior chamber of the eye. When this experiment is made, the phagocytes collect at the point of inoculation very much as they do in an animal possessed of natural immunity, and there they devour the invading organism. The probabilities certainly are that the bactericidal substances found in the serum of immunized animals originate in the phagocytes, whose capability of secreting this substance is heightened by the process of immunization. Undoubtedly it is true that phagolysis also plays a part in increasing the antiinfectious properties of the body juices of immunized animals.

We now turn to a discussion of the subject of immunity to bacterial toxins. Owing to the fact that in the chapter on lysins we have developed quite fully Ehrlich's views on this subject, a brief statement will be all that will be necessary at this place. According to Ehrlich, we are to consider that certain cells of the animal body consist of a nucleus, which he designates as a "special executive center," from which there radiate many side chains. It must be understood that this is a chemical and not a morphological conception of the cell. These side chains physiologically are concerned in processes of nutrition. Nutritive substances brought to the cells by blood or lymph can be utilized in its nutrition only after they have entered into combination with one or more of the side chains. It is con- 
ceivable that some of these side chains combine with carbohydrates, others seize upon proteids, and others still may fasten themselves to molecules of fat. In this way all serve in securing nutritive material for the growth, repair, or reproduction of the cell. It is also possible that certain pharmacological agents act upon the different tissues of the body by virtue of combinations effected with these cellular side chains. Now, in order for any substance to be poisonous to a given animal, there must be in the body of that animal cells with side chains capable of combining with the substance. A body which is unable to effect a combination with any animal tissue cannot be poisonous. It is not supposed that cells possess side chains especially provided for the purpose of combining with poisons, but it is possible that certain atomic groups which ordinarily are concerned in securing nutritive material for the cell, may combine with substances which are capable of bringing about cellular destruction. On account of the close chemical resemblance between the bacterial toxins and certain food principles, notably those proteid in character, this supposition seems quite plausible. In the first place, then, we may say that the body cells must possess toxophil side chains. By this we mean that in the molecular construction of certain constituents of the cell there are groups of atoms which may combine with bacterial toxins. In the second place, a given substance, in order to be toxic, must possess certain cytophil side chains. Both the toxophil groups of the cell, and the cytophil groups of the toxin may be designated as haptophorous bodies. This means that it is by virtue of these groups that the toxin enters into combination with certain molecules within the cell. However, the toxin is possessed not only of a haptophorous, but also of a toxophorous group, and it is by virtue of the latter that injury to the cell may be accomplished. The ordinary nutritive products must, according to this theory, possess cytophil haptophorous side chains, but they do not contain the toxophorous element. Now when a small amount of a bacterial toxin is introduced into the body of a susceptible animal, by virtue of its haptophorous group it combines with some cell in the body. Whether or not it causes a destruction of that cell depends upon the immediate injury done by this combination. If the toxin be introduced in sufficiently large quantity, it may completely destroy the cell, and if a sufficient number of cells be destroyed, death will result. If, on the other hand, the amount of toxin introduced be relatively small, and the number of toxophil groups in the cell used up in the combination be also small, the cell soon recovers from the slight injury done it, but recovers deprived of the side chain which has combined with the toxin. Feeling this loss and needing this side chain in order to supply itself with nutritive material, the cell throws out a new side chain, and when stimulated by frequent introduction of small quantities of toxin, it finally not only makes good the loss with 
which it has met, but throws out more side chains than it can possibly use. In other words, the over-stimulated cell sceretes, as it were, toxophil groups, and, as frequently happens in case of tissue stimulation, the process continnes to a point of over-compensation. Finally the excess of toxophil groups thrown out by the cell becomes so great that many of them are cast off into the blood, lymph, and other fluids of the body. These cast-off toxophil groups constitute the antitoxin. Now if a toxin be introduced into the body of an animal whose blood and lymph are filled with loose toxophil groups, the toxin is neutralized by these detached side chains, and the cell escapes injury altogether. In this way Ehrlich accounts for the formation of antitoxins and the production of toxin immunity. If the blood serum of an immunized animal be injected into a nonimmune animal and the latter be treated with the homologous toxin the poison thus introduced combines with the antitoxin dissolved in the body juices, and the cells of the animal thus treated wholly escape any injurious effects. It may be well at this point to make the following quotation from Ehrlich's own statement on the subject: "The theory above developed allows of an easy and natural explanation of the origin of antitoxins. In keeping with what has already been said, the first stage in the toxic action must be regarded as being the union of the toxin by means of its haptophorous group to certain side chains of the cell protoplasm. 'This union is, as animal experiments with a great number of toxins show, a firm and enduring one. The side chain involved, so long as the union lasts, cannot exercise its normal nutritive physiological function-the taking up of definite food-stuffs. It is, as it were, shut out from participating, in a physiological sense, in the life of the cell. We are, therefore, now concerned with a defect which, according to the principles so ably worked out by Professor Carl Weigert, is repaired by regeneration. These principles, in fact, constitute the leading conception in my theory. If, after union has taken place, new quantities of toxin are administered at suitable intervals and in suitable quantities, the side chains which have been reproduced by the regenerative process are taken up anew into union with the toxin, and so again the process of regeneration gives rise to the formation of fresh side chains. In the course of the progress of typical systemic immunization, as this is practised in the case of diphtheria and tetanus toxin especially, the cells become, so to say, educated or trained to reproduce the necessary side chains in ever-increasing quantities. As Weigert has confirmed by many examples, this, however, does not take place as a simple replacement of the defect. The compensation proceeds far beyond the necessary limit; indeed, over-compensation is the rule. Thus the lasting and ever-increasing regeneration must finally reach a stage at which such an excess of side chains is produced that, to use a trivial expression, the side chains are present in too great a 
quantity for the cell to carry, and are, after the manner of a secretion, handed over as needless ballast to the blood. Regarded in accordance with this conception, the antitoxins represent nothing more than side chains reproduced in excess during regeneration, and therefore pushed off from the protoplasm, and so coming to exist in a free state. With this explanation, the phenomena of antitoxin formation lose all their strange, one might say, miraculous characters."

It should be stated that according to Ehrlich's theory the antiinfectious substances originate in the body of the inoculated animal in a manner similar to that by which antitoxins are produced. On this point he makes the following statement: "Much more complex than in the cases hitherto discussed are the conditions when, instead of the relatively simple metabolic products of microbes, the living microörganisms themselves come to be considered; as in immunization against cholera, typhoid, anthrax, swine fever, and many other infectious diseases. There then come into existence alongside of the antitoxins produced as a result of the action of the toxins, manifold other reaction products. This is because the bacterium is a highly complicated living cell, of which the solution in the organism yields a great number of bodies of different nature, in consequence of which a multitude of antibodies are called into existence. Thus we see as a result of the injection of bacterial cultures, that there arise alongside of the specific bacteriolysins, which dissolve the bacteria, other products, as, for example, 'coagulins,' $i$. e., substances which are able to cause the precipitation of certain albuminous bodies contained in the culture fluid injected; also the so much discussed 'agglutinins,' the antiferments, and no doubt many other bodies which we have not yet recognized. It is by no means unlikely that each of these reaction products finds its origin in special cells of the body; on the other hand, it is quite likely that the formation of any single one of these bodies is not of itself sufficient to confer immunity. Thus in case of the introduction of bacteria into the body, we have to do with a many-sided production of different forms of ' antibodies,' each of which is directed only against one definite quality or metabolic product of the bacterial cell. Accordingly, in recent times, the practice of using for the production of immunization definite toxic bodies isolated from the bacterial cell has been more and more given up, and for this purpose it is now regarded as important to employ the bacterial cells as intact as possible."

According to Ehrlich's theory, antitoxins are not modified toxins, but are the products of certain cells of the animal body, and are produced by the stimulation of these cells by the toxin. If it can be shown that antitoxins have their origin in the toxin molecule, Ehrlich's theory will be overthrown. The evidence against the theory that antitoxins are modified toxins is well-nigh conclusive, and may be stated as follows: (1) The taking of large quantities of blood, 
one third or more of the contents of the body, from immunized animals, at intervals of time sufficiently long to allow the animal to recover to such an extent that its health is not seriously impaired, does not diminish the immunity of this animal as markedly as would be expected if the antitoxin was a modified toxin, and the quantity of the former could not be greater than the quantity of the latter employed in immunizing the animal. (2) If an immunized animal be bled to death, and its vascular system be washed with physiological salt solution until all the blood that can possibly be washed out is removed, yet infusions of certain tissues of this animal contain antitoxin. It must, however, be granted that the tissues may retain some of the modified toxin in the form of antitoxin after the most thorough washing out of the vascular system. (3) The quantity of antitoxin obtained is not always at least in direct proportion to the amount of toxin used in the production of the immunity, as would necessarily be the case if the antitoxin originated in the toxin. Certainly it must be admitted that if the antitoxin comes from the toxin, the amount of the former produced can never be greater than the quantity of the latter used in its production. In other words, a part can never be greater than the whole.

It has been claimed by some that toxins can be converted into antitoxins by the action of electricity. In our last edition we made the following statement concerning this claim: "Smirnow has written quite at length to show that toxins may be converted into antitoxins by the long-continued action of an electric current. We fail to find in his recorded experiments any justification of his claim. He makes quite a point of the fact that during the continuance of the electrolysis the bouillon becomes more deeply colored at one pole and nearly decolored at the other. Now, the merest tyro in physiological chemistry knows that acids deepen the color of beef tea, urine, or any other fluid, the coloring matter of which is derived from hemoglobin. So much for his chemistry. His physiology is worse. When he administers this artificial antitoxin in too large doses he kills his animals; and of what do they die?-of diphtheria. $\mathrm{He}$ has only modified and reduced the virulence of his diphtheria toxin by the acid generated by the electrolysis of the inorganic salts in the boullou. Toxins may be convertible into antitoxins, and electricity may be the agent capable of inducing this, but Smirnow has proved neither one nor the other."

Since the above was written other investigators have reported the confirmation of Smirnow's results, but after a careful study of these reports we see no reason for changing the opinion expressed above. We will investigate only one of these reports. Bolton and Pease ${ }^{1}$ passed electric currents through bouillon solutions of diphtheria toxin and found that 5 c.c. of the product from the positive pole

${ }^{1}$ Transactions of the Association of American Physicians, 11, 1896. 
mixed with 1 c.c. of diphtheria toxin, containing ten fatal doses, injected into a guinea-pig did not cause death, and from this they conclude that they have converted the toxin into antitoxin. They state that during the electrolytic progress a peculiar odor resembling that of chlorid of lime is given off, "but it was found that the fluid contained only one-tenth of one per cent. of chlorin." They made no control experiment in order to determine whether or not this amount of chlorin will destroy the quantity of diphtheria toxin employed by them, and until such an experiment has been made we will be inclined to the opinion that the 5 c.c. of electrolyzed solution contained enough chlorin not only to destroy its own toxin, but also that in the 1 c.c. of non-electrolyzed toxin with which it was mixed.

It must be admitted that it is within the range of possibility that antitoxins may contain a ferment derived from the toxin, and which in the animal body during the process of immunization has combined with the constituents of certain tissues. This view is in accord with the experimental observations of Emmerich and Löw, which we have already detailed.

Centanni endeavored to determine experimentally what organ or tissue of the body is affected in the production of immunity against rabies. His work consisted in endeavoring to induce immunity in a second series of rabbits by treating them subcutaneously with the blood serum and with emulsions made from the various organs of the members of a primary series, already rendered partially or wholly immune by the ordinary method. In doing this he used amounts of each tissue proportional to the weight of the animal; thus the central nervous system of an average rabbit is equal in weight to one three hundredth part of the total body weight of the animal, and the total blood of the same animal furnished an amount of serum equal in weight to one one hundred and fiftieth part of its body weight. Therefore he injected into a rabbit weighing 1,800 grams, six grams of nervous tissue made into an emulsion, and into another of the same weight, 12 grams of blood serum taken from rabbits already made immune, and then tested the immunity of the members of this second series. Emulsions of other tissues were employed in proportionately the same doses. By this procedure he found, as he thinks, that in the production of immunity against rabies, the immunizing substance is stored up in the central nervous system. Moreover he found that the immunizing substance remained in the nervous system long after it had disappeared from the blood and other organs. Thus it would seem that Pasteur hit upon the right thing exactly when he selected emulsions of the spinal cord as the proper material with which he could best induce immunity against hydrophobia. From these experiments, Centanni drew the general conclusion that in the production of immunity against any diseases, the immunizing substance is stored up in greatest quantity and most permanently in 
that organ or tissue most seriously affected by the disease. The experiments made by Roux show quite conclusively that the tetanus toxin combines with brain cells and it may be said that this is a partial confirmation of the general law which Centanni attempted to formulate. 


\section{CHAPTER X.}

\section{FOOD POISONING (BROMATOTOXISMUS ${ }^{1}$ ).}

UNTOWARD results frequently follow the eating of food which is ordinarily harmless. These ill effects may be due to the following causes : (1) Grains may become infected with the poisonous parasitic fungi, such as ergot. (2) Both plants and animals may feed upon substances which are not harmful to them but which may seriously affect man on account of his greater susceptibility; it is said that birds which have fed upon mountain laurel furnish food poisonous to man. (3) The flesh of some animals is poisonous during the period of physiological activity of certain glands; this is true of certain fish during the spawning season. (4) Any food may be infected with specific germs and serve as the carrier of infection ; typhoid fever is frequently disseminated in this manner. (5) The animal may be afflicted with a specific disease, and this may be transmitted to man in the meat or milk; tuberculosis may be spread in this way. (6) Foods of various kinds may become contaminated with saprophytic bacteria, which by their growth elaborate chemical poisons either

1 The following is a glossary of the new words employed in this article:

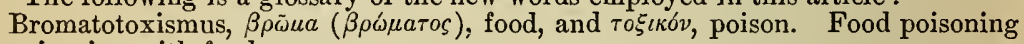
or poisoning with food.

Bromatotoxicon. A general term for the active agent in a poisonous food.

Bromatotoxin. A basic poison generated in food by the growth of bacteria or fungi.

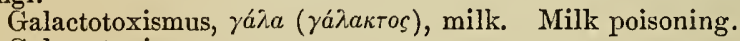

Galactotoxicon.

Galactotoxin.

Ichthyotoxismus, $₫ \theta \dot{v} \varsigma$, fish. Fish poisoning.

Ichthyotoxicon.

Ichthyotoxin.

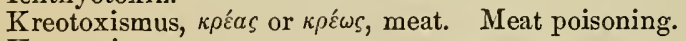

Kreotoxicon.

Kreotoxin.

Mytilotoxismus, $\mu v \tau i \lambda o s$, a sea-mussel. Mussel poisoning. Used already by Husemann.

Mytilotoxicon.

Mytilotoxin. The name given by Brieger to the ptomain discovered by him in poisonous mussel.

Sitotoxismus, oiros, cereal food. Poisoning with vegetable food.

Sitotoxicon.

Sitotoxin.

Tyrotoxismus, tvpos, cheese. Cheese poisoning. Used already by Husemann.

Tyrotoxicon.

Tyrotoxin.

Husemann uses the word zoötrophotoxismus to indicate poisoning with animal food. The same author has employed the word halichthyotoxismus to designate poisoning with fisl. 
before or after the food has been eaten; this is the most common form of food poisoning.

Mytilotoxismus.-Judging from the symptoms induced, there seem to be three kinds of poisonous mussel. In some cases the symptoms resemble those induced by a gastro-intestinal irritant. Fordere reports the case of a soldier, who, after eating a large dish of mussels, suffered from nausea, vomiting, pain in the stomach, tenesmus, and rapid pulse; after death, which occurred within two days, the stomach and intestines were found inflamed and filled with tenacious mucus. Combe and others also reported cases of the choleraic form of mussel poisoning.

However, the symptoms most frequent in man after the eating of poisonous mussels are more purely nervous. A sensation of heat and itching appears, usually in the eyelids, and soon involves the whole face, and perhaps a large portion of the body. An eruption usually called nettle rash, though it may be papular or vesicular, covers the parts. The itching is most annoying, and may be accompanied by marked swelling. Often there is asthmatic breathing, which is relieved only by ether. In some cases reported by Mohring dyspnœa preceded the eruption, the patients became insensible, the face livid, and convulsive movements of the extremities were noticed. Burrows reports similar cases with convulsive tremors, coma, and death within three days.

In a third class of cases there may be observed intoxication resembling that of alcohol, followed by paralysis, coma and death.

In 1827, Combe observed thirty persons poisoned, two of them fatally, with mussels. He described the symptoms as follows: "None, so far as I know, complained of anything peculiar in the smell or taste of the animals and none suffered immediately after taking them. In general, an hour or two elapsed, sometimes more; and the bad effects consisted rather in uneasy feelings and in debility than in any distress referable to the stomach. Some children suffered from eating only two or three; and it will be remembered that Robertson, a young and healthy man, only took five or six. In two or three hours they complained of a slight tension of the stomach. One or two had cardialgia, nausea and vomiting; but these were not general or lasting symptoms. They then complained of a prickly feeling in their hands, feet, and constriction of the mouth and throat, difficulty of swallowing, and speaking freely, numbness about the mouth, gradually extending to the arms, with great debility of the limbs. The degree of muscular debility varied a good deal, but was an invariable symptom. In some it merely prevented them from walking firmly, but in the most of them it amounted to perfect inability to stand. While in bed they could move their limbs with tolerable freedom, but on being raised to the perpendicular posture they felt their limbs sink under them. Some complained 
of a bad, coppery taste in their mouths, but in general this was in answer to what lawyers call a 'leading question.' There was slight pain in the abdomen, which increased on pressure, particularly in the region of the bladder, which organ suffered variously in its functions. In some the secretion of urine was suspended, in others it was free, but passed with pain and great effort. The action of the heart was feeble; the breathing, unaffected; the face pale, expressive of much anxiety ; the surface, rather cold ; the mental faculties, unimpaired. Unluckily the two fatal cases were not seen by any medical person, and we are, therefore, unable to state minutely the train of symptoms. We ascertained that the woman, in whose house were five sufferers, went away as in a gentle sleep, and that a few minutes before death she had spoken and swallowed."

The woman mentioned by Combe died within three hours, and the other death was that of a watchman, who was found dead in his box six or seven hours after he had eaten of the mussels. Post-mortem examination of these showed no abnormality; the stomach contained some of the food partially digested. The explorer Vancouver reports four cases similar to those observed by Combe; one of the sailors died in five and one-half hours after eating the mussels.

Schmidtmann has reported cases observed by himself in some workmen and members of their families, who had partaken of mussels taken near a newly constructed dock. The symptoms appeared, according to the amount eaten, from soon after eating to several hours later. There was a sensation of constriction in the mouth, throat and lips. The teeth were set on edge as though sour apples had been eaten. There was no headache, a sensation of flying and an intoxication similar to that produced by alcohol. The pulse was hard and rapid; no elevation of temperature ; the pupils were dilated and reactionless. Speech was difficult, broken and jerky. The limbs felt heavy; the patients grasped spasmodically at objects and missed their aim. The legs were no longer able to support the body. The knees knocked together. There was nausea, vomiting, no abdominal pain, no diarrhœa. The hands began to feel cold. The sensation of cold soon extended over the entire body, and in some the perspiration flowed freely. There was a feeling of suffocation, then a restful and dreamless sleep. One person died in one and three-quarter hours, another in three and one-half hours, and a third in five hours, after eating of the mussels. In one of these fatal cases rigor mortis was marked and remained for twenty-four hours. The vessels of all the organs were distended, only the heart was empty. There was marked hyperemia and swelling of the mucous membrane of the stomach and intestines, and the spleen was enormously enlarged, and the liver showed numerous hemorrhagic infarctions.

Many theories have been advanced to account for poisonous mus- 
sels. It was formerly believed that the effects were due to the copper which the animals obtained from the bottoms of vessels, but as Christison remarks, copper does not produce these symptoms. Moreover, Christison made analysis of the mussels which produced the symptoms observed by Combe, and was unable to detect any copper. Bouchardat found copper in some poisonous mussels, but he does not state the amount of the metal nor the source of the animals. Edwards advanced the theory that the symptoms were wholly due to idiosyncrasy of the consumer. This certainly is not a tenable hypothesis in such instances as those reported by Combe and Schmidtmann, where a large number or all those who partook of the food were affected. Coldstream stated that the livers of poisonous mussels are larger, darker, and more brittle than normal, and these changes he believes are due to a diseased condition of the animals. Many have supposed that the poisonous effects were due to a peculiar species of medusa upon which the mussels feed, and De Beume found in the vomited matter of one person some medusæ and he states that these are most abundant during the summer, when mussels are most frequently found to be poisonous. The theory of Burrows that mussels are always poisonous during the period of reproduction at one time received considerable credit; however, cases of poisoning have occurred at different seasons of the year. In 1872, Crumpe suggested that there is a species of mussel which is in and of itself poisonous, and this species is often mixed with the edible variety. It has been stated that the poisonous species has a brighter shell, a sweet, more penetrating, bouillon-like odor than the non-poisonous; also that the flesh of the former is yellow and that the water in which they are cooked is bluish. This theory, however, is opposed by the majority of zoölogists. Möbius states that the peculiarities of the supposed poisonous variety pointed out by Virchow and Schmidtmann are really due to the conditions under which the animals live, the amount of salt in the water, the temperature of the water, whether it is moving or still water, the nature of the bottom, etc. He also states that the sexual glands, which form the greater part of the mantle, are white in the male and yellow in the female. The theory of a poisonous species has been abandoned since it has been shown that edible mussels may become poisonous if left in filthy water fourteen days or longer, and, on the other hand, poisonous ones may become fit for food if kept for four weeks in good water.

Cats and dogs which have eaten voluntarily of poisonous mussels have suffered from symptoms similar to those observed in man; and rabbits have been poisoned by the administration of the water in which the food has been cooked. A rabbit treated in this manner by Schmidtmanu died within one minute. From these mussels Brieger extracted the ptomaïn mytilotoxin, which will be discussed in a subsequent chapter. Whether or not those mussels which pro- 
duce other symptoms also contain ptomaïns remains for future investigations to determine.

In 1887, three cases of mussel poisoning, one fatal case, occurred at Wilhelmshaven, the place which supplied Brieger with the mussels from which he obtained mytilotoxin. Schmidtmann found that non-poisonous mussels placed in the waters of this bay soon became poisonous, and that the poisonous mussels from the bay placed in the open sea soon lose their toxic properties. Linder has found in the water of the bay and in the mussels living in it a great variety of protozoa, amœbæ, bacteria and other organisms, which are not found in the water of the open sea nor in the non-poisonous mussels. $\mathrm{He}$ also ascertained that if the water of the bay be filtered, non-poisonous mussels placed in it do not become poisonous, and he concludes that poisonous mussels are those which are suffering from disease due to residence in filthy water. Cameron makes a somewhat similar statement about the poisonous mussels near Dublin, taken from water contaminated with sewage. He found that the livers of these animals were much enlarged, and from them he obtained a base that is probably identical with mytilotoxin. That oysters taken from beds near the outlet of sewers may be contaminated with the specific germ of typhoid fever has been well demonstrated, within the past few years, and that they may become poisonous in the same way that mussels acquire harmful properties is also well known. Pasquier reported cases of poisoning at Havre from the eating of oysters taken from an artificial bed near the outlet of a drain from a public water closet. Christison says that an unusual prevalence of colic, diarrhœea and cholera at Dunkirk was believed to have been traced to an importation of oysters from the Normandy coast. There should be police regulations against the sale of all kinds of mollusks, and all kinds of fish as well, taken from filthy water. Special attention should be given to localities that have once supplied poisonous food of this kind. Many popular rules have been formulated for the easy recognition of poisonous mussels, and to some of these credence has been given by medical authors. An unusually large mussel is regarded with suspicion, and Lohmeyer gives measurements that may guide the person in search of this article of food. Stress is placed on color by some, and one is advised to avoid the dark brown-blue, and purchase the dark-blue or dark green-blue. We may expect to see the prudent hungry man draw from his pocket a scale of colors and carefully compare it with the shell of the juicy bivalve before he consigns it to his digestive organs, if he is to observe the rules laid down in some recent medical works. Then he will take the dimensions of the whole, measure the thickness of its shell, and then its strength, for we are informed that the poisonous clam has a thin, brittle shell. Seriously, one is to avoid shellfish from impure water, and he may properly insist that they 
be washed in clean water, and certainly one should avoid eating this kind of food when it has stood for a few hours at summer heat in the form of broth.

Ichthyotoxismus.--Some fish are always poisonous; others are poisonous only during the spawning season, and still others are subject to epidemic bacterial diseases, and those affected with certain of these diseases furnish flesh that is toxic to man, or, in other words, the bacterial disease is transmitted to man with his food. Lastly, fish, like other kinds of meat, may become infected with saprophytic germs which produce toxins poisonous to man. The Spaniards use the word siguatera ${ }^{1}$ to designate the complex of symptoms induced in man by the eating of fish that are physiologically poisonous ; and Blanchard proposes the general adoption of this term, while he suggests that the word botulismus or botulism be used to designate diseased conditions which result from the eating of any kind of meat that is harmful on account of bacterial infection. His statement is substantially as follows: "There are two distinct categories of intoxication with the flesh of vertebrates :

"Botulismus is an intoxication induced by meat invaded by microbes and containing the ptomains elaborated by them; this term is applicable not only to disease caused by market meat, but also to that induced by preserved foods.

"Siguatera is an intoxication caused by fresh food, not infected by bacteria, and in which the poisonous principles are leucomaïns formed by the physiological activity of the tissues. I propose to designate this category of intoxication by the word siguatera, a name employed by the Spanish physicians of the Antilles to indicate poisoning by eating fish."

While we have not accepted Blanchard's nomenclature as applicable to all kinds of poisonous meats, the distinction made by him admirably states the differences in the two kinds of fish poisoning. It is a question whether or not we shall discuss in this connection those fish whose flesh is not harmful, but which are supplied with poisonous glands. However, as the secretions of the special glands owe their toxic properties to physiological poisons, we will include them in this category and will make brief mention of them.

Kobert makes the following classification of poisonous fish :

1. Many fish possess poisonous glands that are connected with their barbed fins with which they wound their enemies. The structure of these glands is similar to those of poisonous snakes. After the removal of the skin containing these glands the flesh is not poisonous. Such are Trachinus draco of the German lakes, and Serranus scriba of the Mediterranean sea. Stomias boa is feared on account of its bite ; many roaches have a poisonous barb in the tail. Bottard describes five classes of fish supplied with poisonous glands. 
(1) Of this class Synanceia brachio is a type and has its poison apparatus in the dorsal fin, consisting of thirteen barbs, each of which has two poison reservoirs. Each of the twenty-six reservoirs is supplied with ten or twelve tubular glands, the secretion of which is a clear, bluish, feebly acid fluid. Undiluted, this solution causes local gangrene; diluted, it induces paralysis. In Plotosus lanceatus in front of the ventral fin there is a hollow barb with closed end, connected with a poison reservoir, and the fluid flows only when the barb is broken. (2) Trachinus draco is a typical example of this class. The apparatus of Cottus scorpio and C. bubalis also is of this kind. There are three hollow barbs on the gill cover and the reservoirs connected with this secrete a poison only during the spawning season. (3) Thalassophryne reticulata has two hollow barbs, one on the gill cover and the other on the back. (4) Murena helena has on the gills an open pocket, the walls of which are lined with cells secreting a poison that moistens the teeth. (5) Scorpena scropha and S. porcus have open poison glands connected with hollow barbs set in the dorsal and ventral fins. Chemically nothing is known of the nature of these poisons ; pharmacologically it has been demonstrated that they cause severe inflammation of the subcutaneous tissue.

Trachinus draco, ordinarily known as the "dragon-weaver" or "sea-weaver," is one of the best known of the fish possessed of poisonous barbs. The varieties of this species are widely distributed in salt waters. It is a handsome fish, somewhat resembling the trout, and marked with blue and brown stripes. While bathing, men sometimes wound their feet with the barbs of this fish which lies half buried in the sand. It also happens that fishermen sometimes incautiously prick their fingers with these barbs. Almost immediately there are knife-like pains felt about the wound and these quickly extend over the body. Cardialgia may be most excruciating and there is a sensation of suffocation. The forehead is covered with profuse cold perspiration, and the heart becomes weak and beats intermittently. Pain and terror combine to render the condition agonizing to the attendant. Convulsions with mild delirium come on and finally death, occasionally from exhaustion, supervenes. This is the history of a severe case, but ordinarily the symptoms are less grave, and severe local pain accompanied by cedema and followed by gangrene are the results. Some experiments with the poison have been made on the lower animals, especially on rabbits and guinea-pigs. If the thigh of one of these animals be pierced with the barb of one of these fish there is a cry of pain and soon the limb begins to twitch. The entire body may be involved in convulsive movements, which resemble those due to strychnin, inasmuch as they are intensified by touching the animal. Respiration usually becomes difficult, and paralysis of the posterior extremities 
often results. Death may occur within one hour after the infliction of the wound.

Gressin states that there is no poison gland connected with the barbs on the gill cover of Trachinus draco, but that the pocket, in which the opercular fin lies, is lined with large epithelial cells, which probably secrete the poison. This substance kills small fish, frogs and rats, in which convulsions and fall of temperature precede death. One drop of the fluid injected subcutaneously in pigenns causes convulsive trembling and spasmodic breathing. While Gressin found that the poison of the weaver-fish at Havre induces convulsions in frogs, Pöhl found that the poison of the same fish from the Adriatic, also that of Trachinus radiatus, acts as an exquisite heart poison, retarding and finally arresting this organ in diastole. Its effect on the heart is not altered by atropin, camphor, caffein, helleborein, or hydrastin. Along with its effects on the heart, spontaneous muscular and cutaneous sensibility are impaired. A similar, though less active, poison is found in the barb of the dorsal fin. The small immovable barbs in the dorsal fin of the Scorpœna porcus (hog-fish), so much dreaded by fishermen, are supplied with an analogous but less active poison. Neither the blood serum nor the raw flesh of the Trachinus has poisonous properties.

2. The fish poisoning so well known in Japan is due to different species of the tetrodon (fugu). According to Remy, there are in Japan twelve species of fish, all belonging to the genus Tetrodon, whose ovaries are poisonous. In winter when the ovaries are atrophied they are less harmful ; however, Remy reports the following experiments made with fish,caught during the winter: Dogs fed upon the ovaries or testicles soon sickened, with salivation, severe and frequent vomiting and convulsive muscular contractions. Soon after the poison was gotten out of the stomach by vomiting recovery followed. In order to prevent this rapid elimination the organs were rubbed up in a mortar and the fluid portion administered subcutaneously. By this method, notwithstanding the fact that the experiments were made in winter, death resulted in less than two hours. The symptoms consisted chiefly of disturbances of the digestive and nervous systems. The most important were uneasiness, salivation, vomiting of much mucus, severe contractions of the abdomen, then paralytic symptoms, relaxation of the sphincters, marked dyspncea, cyanosis and dilatation of the pupils. Death was due to dyspnoa. On section the salivary glands and pancreas were found injected and hyperemic. There were small hemorrhagic spots in the stomach and intestines. The liver and kidneys were filled with dark blood as is seen in death from asphyxiation. No structural changes could be found in the nervous system.

Miura and Takesaki find that the ripe ovaries of Tetrodon rubripes contain a substance which induces in rabbits acceleration of the res- 
piratory movements, paralysis of the skeletal muscles, mydriasis, increased peristalsis of the intestines, and arrest of the heart.

Takahaschi and Inoko find the fugu poison resistant to prolonged boiling and it behaves like a basic substance in its reactions with the general alkaloidal reagents. The same observers claim that they have induced the characteristic symptoms of fugu poisoning by injecting the blood, urine, and aqueous contents of the stomach of patients into the abdominal cavity of frogs.

Tahara reports that he has isolated from the roe of the tetrodon two poisons. One of these is a crystalline base, to which he has given the name tetrodonin; while the other is a white, waxy body, and is designated as tetrodonic acid. While both are markedly poisonous, the acid is more active than the base. Of 993 cases of fugu poisoning reported in Tokio from 1885 to 1892 inclusive, 680 were fatal-a mortality of more than 68 per cent.

A disease known as kakké was at one time very prevalent in Japan and other countries along the Eastern coast of Asia. Many theories, some of which were quite naturally founded upon the superstitions of that part of the world, have been advanced to account for the etiology of this disease ; however, with the opening up of Japan to the civilized world, an investigation by scientific methods was undertaken by foreign physicians and by the observant and intelligent natives who had acquired their medical training in Europe and America. It was soon ascertained that this disease was confined to the sea coast districts, and particularly to the natives, Americans and Europeans living in Japan being almost wholly exempt. With improved transportation, kakké was found to extend towards the interior of Japan. Among the natives, the most robust seemed to be most prone to the disease. With these observations the following additional facts were recognized: (1) The inhabitants of the coast were formerly the only natives who partook largely of sea-fish; (2) improved transportation carried these food products toward the interior; (3) the foreigners did not consume these fish so largely as the natives did; (4) among the natives the most robust would quite naturally eat more food of any and all kinds than the less vigorous. The above-mentioned observations led Miura to define the disease as follows: "Kakké is a chronic or subacute, seldom an acute, intoxication due to the consumption of certain kinds of fish." He then set himself to solve the questions: (1) What fish are the bearers of the poison? (2) In what conditions are these fish poisonous? In Tokio the disease generally appears in May, reaches its greatest prevalence in August and gradually disappears in September and October. This would indicate that if the disease were due to eating fish, the poisonous species must be those that are in demand from May to September. Six species were found to be most abundantly, in fact almost exclusively, used at this time of the year; and all of 
these belong to the family of Scombridæ. This is in accordance with the observations of Gubarew, who has reported cases of poisoning from eating Scombrida saba. However, the etiological relation of these fish to kakké cannot be said to be positively established, and it is true that in some parts of the world certain species of the Scombridæ are eaten without injurious effects. Nothing definite is known about the nature of the poison of these fish, nor has it been determined whether the active agent is a physiological product of certain glands or a result of bacterial activity.

Petromyzon fluviatilis, which is not classed among fish by modern zoölogists, causes, according to Prochorow, a bloody diarrhea, frequently observed in the Jamberg district of Russia. This occurs whether the animal is eaten raw or thoroughly cooked, and it is stated that if salt be sprinkled on the animal while it is alive its skin secretes an abundant discharge of mucus, and after this the flesh is not poisonous. Böhm and others have expressed some doubt about any species of fish being per se poisonous. They have been inclined to attribute the effect so frequently observed to one or the other of the following causes: (1) Meat rapidly undergoes putrefactive changes and the ill effects are due to true botulism. (2) The observed untoward symptoms are explainable by supposing the existence of a marked idiosyncrasy in the consumer. That the first supposition is not true is shown by the following facts : (1) Poisoning with perfectly fresh fish occurs not only in the tropics, where decomposition goes on rapidly, but in the temperate zone as well, and during seasons of the year and under conditions that exclude the possibility of the ill effects being due to putrefactive changes in the meat. (2) Certain species of tetrodon and other fish are so well known to be poisonous, even when perfectly fresh, that their consumption is at times resorted to, notably in China and Japan, for suicidal purposes. That the symptoms are not due to idiosyncrasy in the consumer is demonstrated by the effects of the flesh and of the expressed juice upon the lower animals.

3. Cluppea thrissa and C. venenosa, also certain species of Scarus, have no poisonous glands, nor are their reproductive organs more poisonous than other parts of the body; still the flesh of these fish is always poisonous. According to Gunther, their harmful properties are due to the medusæ, corals, and other decomposing substances upon which they feed. In the West Indies it is a well-known fact that all the fish caught off certain coral banks are poisonous and that every part of the animal is unfit for food. The symptoms are those of a gastroenteritis, and death frequently results.

It has been suggested that ichthyotoxismus may be due to substances of vegetable origin which are employed in some countries, notably by savage and partly civilized peoples, to kill the fish. That this may be true in some instances is possible, but that this explanation 
is not generally applicable is shown by the observation that where this method of obtaining fish for food is most frequently employed, no ill results follow, and where it is not resorted to cases of fish poisoning may be very common. According to Husemann, Cocculus Indicus has been employed for the purpose of poisoning fish. The leguminous plant Piscidia, of the West Indies, owes its name to this use of its bark. In the Dutch East Indies the cortex of the root of Derris elliptica and the seed of Pachyrrhinus angulatus are employed for this purpose. Both of these, according to Greshof, contain a non-nitrogenous substance which is highly poisonous to fish, and relatively harmless to other animals. An extract of the derris root, which, in Borneo, is also used as an arrow poison, kills fish when mixed with water in the proportion of $1: 25,000$, and the active principle in a dilution of 1:5,000,000. Greshof has isolated both of these poisons and named them derrid and pachyrrhizid. A legumen, Tephrosia ichthyonecea, from West Africa, also yields a non-nitrogenous poison, but this affects other animals as well as fish. The fish poison of Java, from the seed of Milletia atropurpurea, contains saponin, and that of Ceylon, from Hydrocarpos inebrians, owes its effects to hydrocyanic acid. Robinia nicon of tropical America is used by the savage tribes for the purpose of benumbing fish and this plant has been found to contain a snow-white crystalline substance, freely soluble in alcohol, wholly insoluble in water. Water, to which an alcoholic solution of this poison has been added in the proportion of $1: 1,000,000$, killed fish. Other fish poisons of the West Indies are Jacquinia armillaris, which, on account of the fact that its dried fruit is used for bracelets is known as bois bracelet, and Serjania letalis, from which the poisonous honey of a certain wasp is prepared, the toxic action of which St. Hilaire tested upon himself. This honey, even in small quantity, is said to produce a mild intoxication. This will remind the classical student of the poisonous honey connected with the retreat of the ten thousand Greek soldiers under Xenophon, which occurred four hundred years before our era.

4. We include in this class the cases that Blanchard would describe under botulism, inasmuch as the poisoning is due to putrefactive changes. According to Anrep, there are in poisonous fish two active ptomaïns. One of these is extracted from alkaline solution with ether, chloroform and benzin. It is amorphous and insoluble in water, but forms easily soluble salts of great toxicity, so that onefourth mg. of the hydrochlorid induces poisonous effects in dogs, and one-half mg. kills rabbits. This ptomaïn may be preserved quite indefinitely in the dry state or dissolved in ether, but is speedily destroyed by strong alkalis and acids. Dissolved in phosphoric acid and evaporated it gives a red coloration, rapidly passing into a dirty green. Jakolew isolated a similar alkaloid from poisonous sturgeon 
in 1889 , but it differed from that of Anrep in the fact that the former gave precipitates with platinum chlorid and tannin, while the latter did not. Anrep's second ptomaïn is an oily substance, less poisonous than the solid. Both these substances have a paralyzing action on frogs, dogs and rabbits, arresting respiration and the action of the heart. In cats, they cause clonic convulsions. The heart's action is retarded, and just before death respiration is accelerated. The more poisonous of these alkaloids, for which the name halichthytoxin has been suggested, produces mydriasis on application to the eye.

Vaughan reports the following case of ichthyotoxismus exanthematicus : K., a vigorous man of thirty-four years, ate freely of canned salmon, while others at the table with him remarked that the taste of the fish was peculiar and refrained from eating it. Twelve hours later K. began to suffer from nausea, vomiting, and a griping pain in the abdomen; and six hours later he was found vomiting small quantities of mucus, colored with bile, at frequent intervals. The bowels had not moved and the griping pain continued, while he was covered with a scarlatinous rash from head to foot. The pulse was 140 , the temperature $102^{\circ} \mathrm{F}$., and respiration shallow and irregular. The stomach and large intestines were washed out thoroughly, and ten grains of calomel, soon followed by twelve ounces of solution of magnesium citrate, for the purpose of cleansing the small intestines, were administered. The next day the rash disappeared, but the temperature remained above the normal for four or five days, and it was not until a week later that this man was able to leave his house. The absence of inorganic poisons in the salmon was demonstrated and it was found that subcutaneous injection of twenty drops of the fluid contained in the can caused evident illness and suffering in a white rat. The only microörganism that could be found, either by direct microscopical examination or by the preparation of plate cultures, was a micrococcus, and this was present in the salmon in great numbers. This germ grew fairly well in beef-tea, but the injection of five c.c. of beef-tea cultures of different ages failed to affect white rats, kittens or rabbits. However, this micrococcus, when grown for twenty days in a sterilized egg produced a most potent poison. The white of the egg became thin," watery, markedly alkaline, and ten drops sufficed to kill white rats. It is supposed that this microörganism produced toxins only when grown under anaërobic conditions. The same observer saw two similar cases also due to eating canned salmon, and in one of these, the attending physician having arrested the vomiting and purging by the hypodermatic administration of morphin, death resulted. This illustrates the danger of interfering with nature's efforts to remove the poison which, instead of being arrested, should have been assisted by the use of the stomach tube and by irrigation of the colon. 
Potain saw a man suffering from vomiting, vertigo, ringing in the ears, and pain in the joints, due to eating lobster.

Griffiths found in sardines that had undergone putrefactive changes a base to which he has given the name sardinin; this ptomain, together with others reported by the same investigator, is described in Chapter XIV.

5. In Russia many instances of fish poisoning are due to the fact that the fish are diseased and the disease is transmitted to man in his food. Instances of this kind of fish poisoning are well known in Germany also, and here they are generally due to eating diseased barbels. The symptoms are identical with those of cholera nostras, and the disease is known as "barbencholera." The poison, the nature of which is yet unknown, evidently irritates the mucous membrane of the stomach and intestines. This form of fish poisoning is sometimes called ichthyotoxismus gastricus.

Schmidt concludes his studies on poisonous fish in Russia with the following statements: (1) Poisoning with fish is not due to putrefaction. (2) Fish poisoning (in Russia) is always due to some member of the sturgeon tribe. (3) The genesis of fish poisoning has no relation to the method of catching the fish, the use of salt, or imperfections in the method of preserving them. (4) The poisonous substance is not distributed throughout the animal, but is confined to certain parts. (5) The poisonous portion cannot be distinguished from the non-poisonous, either macroscopically or microscopically. (6) The thoroughly cooked meat is never poisonous. (7) The fish poison is an animal alkaloid, produced most probably by bacteria that cause an infectious disease in the fish.

Arustamow has studied eleven cases of fish poisoning in which five terminated fatally. In the fish, and in the liver, kidneys and spleen of the persons, germs, resembling but not identical with the typhoid bacillus, were found. The most noteworthy symptoms were general weakness, dull pain in the abdomen, dyspnœa, mydriasis, vertigo, and dryness of the mouth. This author also concludes that the ill effects are due to bacteria which are pathogenic to the fish and in the cases observed by him the meat was eaten raw.

Sieber found that the fish in an aquarium, from which some had been taken to supply a table and had proved poisonous, were sick and that as many as thirty died within the next two days. From the dead and sick fish Sieber obtained by anaërobic methods a highly toxicogenic germ to which she has given the name Bacillus piscicidus agilis. This germ consists of highly motile short rods, and old cultures show spore formation. The bacilli are easily colored with Ziehl's solution and on gelatin and agar plates the colonies are granular, gray, or yellow. This organism liquefies gelatin and produces carbonic acid gas and small quantities of methyl mercaptan. It is pathogenic to fish, frogs, mice, rabbits, dogs and guinea-pigs, and 
from the muscles of these animals the germ may be recovered in pure culture. After filtration through porcelain the sterile cultures are quite as poisonous as before sterilization. Heat does not destroy the toxin and at least one poisonous substance may be obtained by distillation. Filtered cultures give an intense red coloration with ferric chlorid. Sieber has obtained from growths of this bacillus cadaverin and other known ptomaïns, but there are at least two undetermined bases present and one of these suffices to kill frogs in doses of $3.5 \mathrm{mg}$. The symptoms induced in animals by the use of sterilized cultures consist of shortness of breath and unrest, followed by apathy and paralysis.

Kreotoxismus.-It has long been known that the flesh of animals dead from certain diseases or slaughtered while suffering from these diseases, is not a safe food for man. The Mosaic law forbade the eating of the flesh of animals dead from disease : "Ye shall not eat of anything that dieth of itself; thou shalt give it unto the stranger that is in thy gates, that he may eat it; or thou mayst sell it unto an alien, for thou art a holy people unto the Lord thy God. Thou shalt not seethe a kid in his mother's milk." (Deuteronomy, XIV, 21.) The first part of this command is certainly wise counsel, but the feeding of a visitor with such food would not be now regarded as in accord with the rules of hospitable entertainment, and the sale of it even to an alien should not be permitted by the law of any country. The most common diseases that may be transmitted from the lower animals to man by the consumption of the flesh or milk of the former as food by the latter, are tuberculosis, anthrax, symptomatic anthrax, pleuro-pneumonia, puerperal fever, glanders, various septicemias, trichinosis, mucous diarrhœa, and actinomycosis. However, it does not come within the scope of this book to discuss the transmission of these diseases from the lower animals to man, and we shall limit the subject of kreotoxismus to those untoward effects which arise from the eating of flesh infected with non-specific, toxicogenic bacteria.

Sausage poisoning, sometimes designated as botulism or botulismus, and sometimes known as allantiasis, has long been recognized as a cause of sickness and death and its causation has been a subject of theory and investigation for one hundred years or longer. It is probable that some of the earlier epidemics attributed to botulism were in fact due to trichiniasis, and it was not until the discovery of the parasite to which the latter condition is due that differentiation was possible. A large proportion of the cases of sausage poisoning have occurred in Würtemberg and the immediately adjacent portions of Baden. This fact has been correctly ascribed to the methods there practised of preparing and curing sausage. It is said to be common for people to use the blood of the sheep, ox and goat in the 
preparation of this article of diet. Moreorer, the blood is kept for days sometimes in wooden boxes and at a high temperature before it is used. In these cases it is altogether likely that putrefaction progresses to the poisonous stage before the process of curing is begun. A kind of sausage known as "blunzen" is made by filling the stomachs of hogs with the meat. In curing, the interior of this great mass is not acted upon and putrefaction sets in. The curing is usually done by hanging the sausage in the chimney and at night the fire goes out and the meat freezes. The interior of the mass is generally the most poisonous part and in many instances those who have eaten of the outer portion are often unharmed, while those who have eaten of the interior of the same sausage have been seriously affected. This method of preparing sausage in Würtemberg is not now so generally employed and poisoning from this article of food is not so common as formerly.

Many German writers state that when a poisonous sausage is cut, the putrid portion has a dirty, grayish-green color, and a soft, smeary consistency. A disagreeable odor, resembling that of putrid cheese, is perceptible, while the taste is unpleasant and sometimes a smarting of the mouth and throat is produced. Post-mortem examination shows no characteristic lesions. It is generally stated that putrefaction sets in very tardily, but Müller shows that no reliance can be placed upon this point, and states that out of forty-eight recorded autopsies, it was especially stated in eleven that putrefaction rapidly developed. In some instances there has been noticed hyperemia of the stomach and intestinal canal, but this is by no means constant. The liver and brain have been reported as congested, but this would result from failure of the heart, and would, by no means, be characteristic of poisoning with sausage.

Von Faber, in 1821, observed sixteen persons who were made sick by eating fresh unsmoked sausage made from the flesh of a pig which had suffered from an abscess on the neck. Five of the patients died. The symptoms were as follows: There was constriction of the throat, difficulty in swallowing, retching, vomiting, colic-like pains, vertigo, hoarseness, dimness of vision and headache. Later on in severer cases there was complete exhaustion, and, finally, paralysis. The eyeballs were retracted, the pupils were sometimes dilated, then contracted, they did not respond to light, there was paralysis of the upper lids. The tonsils were swollen, but not as in tonsillitis. Liquids which were not irritating could be carried as far as the esophagus, when they were ejected from the mouth and nose with coughing. Solid foods could not be swallowed. On the back of the tongue and in the pharynx there was observed a puriform exudate. Obstinate constipation existed in all, while the sphincter ani was paralyzed. Breathing was easy, but all had a croupous cough. The skin was dry and there was incontinence of urine. There was no 
delirium and the mind remained clear to the last. At autopsy the skin was found to be rongh and the abdomen retracted. The large vessels in the upper part of the stomach were filled with black blood and the content of the stomach consisted of a reddish-brown, semifluid substance, which gave off a repugnant acid odor. In one case the omentum was found greatly congested. The large intestine was pale and the right ventricle of the heart was filled with dark fluid blood.

Schüz cites thirteen cases of poisoning from liver sausage in which the symptoms differed from the foregoing in the following respects: (1) In only one out of the thirteen was there constipation. All the others had numerous typhoid-like stools. (2) Symptoms involving the sense of sight were present in only three; in all the pupils were unchanged. (3) The croupous cough was wholly wanting; though in many there was complete loss of voice. Difficulty of swallowing was complained of by only one. (4) Delirium was marked in all; and in one the disturbance of the mental faculties was prominent for several weeks. (5) There were no deaths. (6) The time between eating of the sausage and the appearance of the symptoms varied from eighteen to twenty-four hours and the duration of sickness from one to four weeks; though in one case complete recovery did not occur until after two and one-half months. The sausages were not smoked and all observed a garlic odor, though no garlic had been added to the meat.

Tripe reports sixty-four cases, in which the stools were frequent, watery, and of offensive odor; in some there was delirium. In the one fatal case the hands and face were cold and swollen; the pulse, rapid and weak ; the pupils, contracted but responsive to light; and the small intestine, inflamed.

Hedinger reports two cases with the usual symptoms, but during recovery dilatation of the pupils was followed by contraction. Birds ate of this sausage and were not affected. In Röser's cases, section showed abscess of the tonsils, a dark, bluish appearance of the mucous membrane of the pharynx, larynx, and bronchial tubes, dark redness of the fundus of the stomach, and circumscribed gray, red and black spots on the mucous membrane of the intestine; the liver was brittle and the spleen solid.

Many theories concerning the nature of the active principle of poisonous sausage have been advanced. It was once believed to consist of pyroligneous acid, supposed to be absorbed by the meat from the smoke used in curing, but it was found that unsmoked sausage also might be poisonous. Emmert believed the active agent to be liydrocyanic acid, and Jäger's theory supposed the presence of picric acid; but these acids are not found in poisonous sausage and their toxicological effects are wholly unlike those observed in sausage poisoning. Kerner believed the poison to consist of either caseic or 
sebacic acids, or both, while Buchner named it acidum botulinicum; but the acids of the former proved to be inert, and that of the latter to have no existence. Schlossberger suggested that the poisonous substance is most probably basic in character, and he found an ammoniacal base which could not be found in good sausage, and which did not correspond to any known amid, imid, or nitryl base ; however, this substance has not been obtained by anyone else, nor has it been demonstrated to be poisonous. Liebig, Duflas, Hirsch, and Simon believed in the presence of a poisonous ferment, and Van den Corput described sarcina botulina, which he believed to be the active agent. Müller, Hoppe-Seyler, and others found various microörganisms, and Virchow, Eichenberg, and others examined microscopically the blood of persons poisoned with sausage. Ehrenberg attempted to isolate the poisonous body by employing Brieger's method, but obtained only inert substances.

Gafflky and Paak made a study of sausage consisting of horseflesh and liver which poisoned a large number of people, one of whom died. In the majority, the symptoms came on with a chill within six hours, and in one instance within half an hour. The most prominent symptoms were headache, loss of appetite, pain in the bowels, vomiting and purging. From the sausage these investigators obtained a short bacillus, which, when given by the mouth, subcutaneously or intravenously, produced the above-mentioned symptoms, with a fatal termination in rabbits, guinea-pigs, mice and apes ; they were unable to isolate the chemical poison. This bacillus probably belongs to the colon group.

In June, 1880, a large number of persons were poisoned at Wellbeck, England, by eating ham in which Klein found a bacillus, inoculations with which were followed by the development of pneumonia; while the affected persons were described by Ballard, who investigated the outbreak, as suffering from "diarrhœal illness." Ballard has reported the following additional instances: In the "Chester case," the man ate of some so-called American sausage, which consisted mostly of pork. Gastroenteric symptoms with great prostration resulted, and in a few days the man died, apparently from pneumonia. No post-mortem examination was permitted, but the meat killed the animals fed with it, and in these, section showed hemorrhage in the stomach, congestion of the lungs, and hyperemia of the medullary portion of the kidneys. In the "Oldham case," members of two families partook of a newly opened can of pigs' tongues. Nausea, vomiting and diarrhœea occurred in all but one, and he fell into a comatose condition, which was not relieved until a purgative was administered and acted. In the "Bishop case," members of three families ate of ribs of beef. The meat was cooked on Saturday and it was more poisonons on Monday than on the preceding day. This meat evidently became infected after it 
was cooked, inasmuch as other portions of the same carcass caused no ill effects. In the "Whitechurch case," brawn, consisting of a gelatin made from pig's head, was eaten cold. Members of ten families residing in different parts of the town were affected. In the "Whitechurch-pork case," the meat eaten immediately after it was cooked had no ill effect, while portions eaten cold the next day killed two persons. In the "Wolverhampton-tin-salmon case," three adults ate, and two children merely tasted, the contents of a "blown" can. The one who ate the most was attacked about ten hours after eating, and died in three days; one who ate less became ill in about twelve hours, and died in five days; while the adult who partook most sparingly began to feel the ill effects in about fourteen hours, and finally recovered. In the children, the symptoms were slight and transient. Klein found in both fatal cases necrosis of the superficial layers of the mucous membrane of the stomach, fatty degeneration of the liver as in acute phosphorus poisoning, and inflammation of the kidneys. Mice fed upon the salmon died, exhibiting lesions similar to those observed in the men; no germ could be found in the blood of the mice. In the "Carlisle (A) case," twenty-four persons partook of a cold ham, which had been prepared the previous day and kept in a cellar in which milk and meat "were known to go bad." Two persons died. The only germ which could be found was a micrococcus, which was harmless, when fed by mouth to mice, cats and dogs. In the "Ironbridge case," twelve persons out of fifteen in a household ate at midday of veal pie which had been made the day before and warmed over. Mice were fed upon the pie and upon portions of the veal from which the pie had been made ; those fed on the veal showed no ill effects, while some of those fed on the pie died. In the pie several different species of microörganisms were found, and with one bacterium the following interesting observations were made: Cultures did not grow well at a temperature over $32^{\circ}$ and at above $36^{\circ}$ no growth took place. All cultures after some days growth possessed a most exquisite and delicate aroma, no trace of putridity being perceptible.

As might be expected from the fact that the bacterium did not grow at blood heat, subcutaneous inoculations into mice produced no results; but when mice were fed with the contents of a culture tube, they fell ill and died, but no bacterium could be found in any of the viscera. The obvious inference from this is that the cultures of this microörganism contained a substance which, when introduced into the stomach, produced illness and death, in the latter event severe gastro-enteritis being a conspicuous feature. Since the organism in itself is harmless when inoculated, not being capable of growth and multiplication at the temperature of the animal body, it follows that the substance which caused the poisoning was non-organized and produced by the bacterium. In the "Retford case," eighty persons 
in twenty-two families were made ill, one fatally, by eating pork pie. The meat was cooked on November 10 th and was eaten from the 11th to the 14th. With the exception of one family, none of those who ate of the pie on the 11th were made ill, and none of those who ate of it after the 14th. The harmful germ was a short bacillus, cultures of which made mice sick, killing some; but growths more than ten days old were without effect. In the "Carlisle (B) case," a pork pie was made November 1st and eaten by some twenty-five persons during the following ten days. Mice fed upon the meat developed on the second or third day a bloody diarrhoa, and died. The small intestine was filled with bloody mucus. The lungs were congested, and in the animals which lived the longest there was hepatization, chiefly in the upper lobe; the liver and spleen were also congested. In the "Portsmouth case," two bacilli -one motile, the other not-were found in the food. When first received, the meat poisoned mice fed upon it, but after standing and becoming offensive in odor, it failed to do so. Cultures of the nonmotile bacillus had a pleasant aroma, and were poisonous; while those of the motile germ were offensive and harmless. In the animals killed by the culture the lungs, kidneys and spleen were dark and the small intestines were relaxed and filled with mucus. In only one of the mice could the bacillus be found, and Klein states that this germ is not pathogenic but that its cultures contain a toxin. In the "Middlesborongh Pneumonia Epidemic," Ballard attributed 490 deaths to infected bacon, and he believed that the disease, developed in a person who had eaten of the infected food, was transmissible from the sick to those who had not partaken of the bacon. This has been observed in other epidemics of kreotoxismus and is due to infection with microörganisms first obtained in the food and then transmissible, probably through the sputum, to others. In the lungs, Klein discovered a short bacillus, which he called "bacillus pneumoniæ," differing altogether from the bacillus of Friedländer and from the diplococcus of Fränkel, neither of which was present. Of twenty samples of bacon forwarded from the infected districts, fourteen were distinctly poisonous to rodents fed with it; in two instances, there was some doubt, and only four proved not to be poisonous. In the dead animals, lesions similar to those observed in persons who died of the disease, in the infected districts, were found. Similar results followed inoculation of the juice expressed from the lungs and of pure cultures of the bacillus, and in all instances the microörganism was recoverable. During the progress of Klein's investigations an epidemic of pneumonia occurred among the animals (mice, guinea-pigs, and monkeys) kept in the building where his experiments were carried on, the bacillus pneumonix being found after death and sometimes in the heart's blood. On reëxamination after the lapse of three months, of samples of the bacon that had previously 
produced illness and death, they were found to have lost their power of infecting animals, and no growth of the bacillus was obtainable.

In meat which had poisoned a large number of persous, Gaertner found his bacillus enteritidis. The meat was from a cow that had a severe diarrhœa for two days before she was killed. The twelve persons who ate of the flesh raw, all became sick; while of those who ate of the cooked food a large per cent. were also affected. In the meat, and in the spleen of a person who died from the effects of the poison, Gaertner found the bacillus which proved fatal to animals. Good beef, inoculated with this germ and kept for some hours, killed rabbits, guinea-pigs and mice. The skin of the people who were poisoned and recovered peeled off. The period of incubation varied from two to thirty hours. Even the boiled bouillon cultures of this germ are highly poisonous, showing that the toxic properties are not destroyed by cooking the meat. Fischer reports the following: A cow, that had recently calved, had been sick for some eight days, and on account of this illness she was killed. The animal was slaughtered on Friday, and on the following Sunday at noon nineteen persons ate of the meat. The prominent symptoms were vomiting and violent purging, appearing a few hours after the meal. Vertigo, loss of consciousness, and exfoliation of the epidermis during recovery, all of which were observed by Gaertner in some of his cases, were not present in any case reported by Fischer. Notwithstanding these differences, a study of the bacillus led to the conclusion that it is identical with the bacillus enteritidis. By concentrating a filtered culture and precipitating with absolute alcohol, the crude toxin was obtained. It gave the general reactions for peptons, and boiling for one and one-half hours did not perceptibly weaken its toxic properties. Lubarsch reported the death of a child two days old from a septic pneumonia caused by the bacillus enteritidis. Section showed pleuritis and pneumonia of the left lower lobe, bilateral purulent bronchitis, atalectasis of the right lung, parenchymatous cloudiness of the kidneys, fatty infiltration and engorgement of the liver, slightly enlarged spleen, uric acid infarction of the kidneys, and icterus neonatorum. All other pathological conditions were supposed to be consequent upon the septic pneumonia. White rats and chickens proved to be wholly immune, while guinea-pigs, rabbits, and mice were susceptible to the bacillus found in the tissues of the child. These susceptible animals were killed within from sixteen to twentyfour hours by intraperitoneal inoculation and in from two to four days by subcutaneous injections. In all cases, sectiou showed marked congestion of the intestines, swelling of the follicles, and in some instances slight erosions of the mucous membrane. After intraperitoneal inoculation, sero-fibrinous or hemorrhagic peritonitis developed. After subcutaneous inoculation in rabbits, sometimes in guinea-pigs, there was a sero-fibrinous pleuritis, with compression of 
the lungs, and in one instance a circumscribed pneumonia. Sterilized cultures in larger quantities produced the same effects as the unsterilized. The symptoms and anatomical changes induced by this germ agree with those observed in Winkel's disease, in the rapidly fatal progress, cyanosis, icterus, rapid respiration, tendency to hemorrhage and fatty degeneration. The most essential difference lies in the fact that hemoglobinuria is a prominent symptom of Winkel's disease, while it does not occur after inoculation with the bacillus enteritidis.

In August, 1887, 256 soldiers and thirty-six citizens at Middleburg, Holland, were taken sick after eating meat from a cow which had been killed while suffering from puerperal fever. The symptoms were nausea, vomiting, purging, elevation of temperature, and prostration. In some, there were observed dizziness, sleeplessness, and dilatation of the pupil. After a few days these symptoms gradually disappeared, and in many an eczematous eruption of the lips gave annoyance. Pigs, cats and dogs that ate of the offal of this animal were also made sick. Thorough cooking did not destroy the poison, and those who took soup and bouillon made from the meat were affected like those who ate of the muscle fiber. In most of the cases the symptoms came on within twelve hours after eating the meat.

Basenau first found his bacillus bovis morbificans in the flesh of a cow that was killed while suffering from puerperal fever, and later ${ }^{1}$ he has detected the same microörganism in the meat of animals killed while sick with perforative peritonitis, puerperal paralysis, and chronic pyemia. It seems from the researches of this investigator that there are varieties of this germ, the toxins of some of which are destroyed by heating to $100^{\circ}$, while those of others are not. It is more than probable that Basenau's germ is a variety of the colon bacillus and that the toxin is contained within the cell, and whether or not meat infected with this organism will prove harmful depends upon the number of germs present.

In 1894, Vaughan and Perkins examined some dried beef which had quite seriously poisoned a family of four. There was nothing in the appearance or odor of the beef to cause any suspicion. In fact, it seemed to be of exceptionally good quality. Anaërobic cultures from the interior of the meat were made and developed a bacillus, from two to three times as long as broad, taking the ordinary stains, motile, with no spore formation, not liquefying gelatin, but coagulating milk, growing best at the temperature of the body, but developing its poison at ordinary temperature, producing gas abundantly, and pathogenic to white rats, rabbits, and guinea-pigs. Sterilized cultures were also poisonous. Of 200 men at a banquet at Sturgis, Mich., in April, 1894, everyone who ate of the pressed chicken served was made ill. Some who were not at the banquet but who aided in preparing it, took small bits of the chicken and these also 
were made sick. All were taken within from two to four hours after eating the chickeu, with nausea, violent griping and purging; many fainted while attempting to rise from bed. The chickens were killed Tuesday afternoon, picked and left hanging in the market room (not in cooling room) until Wednesday forenoon, when they were drawn and carried to a restaurant, and here left in a warm room until Thursday morning, when they were cooked (not very thoroughly), pressed, and served at the banquet that night. This food was examined by Vaughan and Perkins, and found to contain two microörganisms, a slender bacillus, from four to five times as long as broad, and a streptococcus. The bacillus was fatal to white rats, guinea-pigs and rabbits, when administered intra-peritoneally, intra-venously, and subcutaneously. The streptococcus was not fatal when given in pure culture, but mixed cultures of the two induced death; and in these instances, when administered subcutaneously, in addition to lesions found after the employment of pure cultures of the bacillus, there was extensive sloughing. This bacillus is motile, takes the ordinary stains readily, and is decolorized by Gram's method. It grows very slowly at ordinary temperature and rapidly at $37^{\circ}$. Of two cultures of equal age, one grown at ordinary temperature and the other at $37^{\circ}, 1$ c.c. of the former was necessary to induce death, while $\frac{1}{4}$ c.c. of the other proved fatal. The anaërobic cultures were much more powerful than the aërobic. $\frac{1}{2}$ c.c. of a beef tea culture heated to $60^{\circ}$ for thirty minutes killed, while 1 c.c. heated to $100^{\circ}$ for fifteen minutes failed to kill.

In an outbreak of bromatotoxismus at an asylum in Norway, the patients' food was veal, and in this Holst found a small bacillus similar to but not identical with that of Gärtner. Lewis found a ptomaïn, which he supposed to be neuridin, in corned beef that poisoned people in Ohio. Poels reported cases of poisoning in Rotterdam from the eating of meat supposed to be from a healthy animal. A variety of the colon bacillus was found in this meat and it was shown that sterilized cultures were sufficiently toxic to kill calves. The same bacillus has been found in other outbreaks of kreotoxismus in Holland. Zorkendorfer reported the presence of anthrax bacilli in some meat that poisoned many people, some fatally, near Teplitz, in 1894; however, his identification of the germ cannot be regarded as positive. Di Mattei has stated that the flesh of animals dead from symptomatic anthrax may retain its power of infection after having been preserved in a dry state for ten years. Siedler reported four cases of poisoning from decomposed goose grease. The symptoms consisted of giddiness, prostration and violent vomiting. Christison reported cases in which persons were seriously, some fatally, affected by eating various kinds of meat which had undergone partial putrefaction. Ollivier found six persons poisoned, four of them fatally, by eating decomposed mutton, 
and he mentions similar cases due to eating ham. Boutigmy, having failed to find any poison in the meat furnished at a festival, and to which the serious illness of many was attributed, made a meal of stuffed turkey furnished by the same dealer, but after a short time his countenance became livid, his pulse small and feeble, a cold sweat bathed his body, and violent vomiting and purging followed. Geiseler observed nausea, vomiting, purging and delirium after eating of bacon which was imperfectly cured. Schröder ${ }^{1}$ reported cases of poisoning which were due to the eating of the flesh of an animal killed while suffering from foot and mouth disease; however, both bacteriological and chemical investigations led to no results. Hermann $^{2}$ and Kænsche ${ }^{3}$ found in some meat which poisoned people at Breslau a typhoid-like bacillus whose toxin is not destroyed by boiling. Kuborn ${ }^{4}$ found a piece of poisonous meat which was infected with staphylococcus pyogenes flavus. "Johne ${ }^{5}$ reported cases of sausage poisoning in which microörganisms similar to the bacillus enteritidis were found.

The most important contribution that has recently been made to the subject of kreotoxismus consists of the discovery by Van Ermengem ${ }^{6}$ of a bacillus in poisonous meat and the subsequent investigation of this germ and its toxin. More than thirty-four persons were affected and three of these died. The period of incubation varied from nine to twenty hours, and the chief symptoms were pain in the stomach, contractions of the intestines, vomiting, obstinate constipation, pain in the head, fever and delirium, followed in those who recovered by marked prostration and muscular weakness. The man, who died on the fifth day, passed albuminous urine, and section showed acute gastro-enteritis, acute parenchymatous nephritis, and hyperemia of the lungs. Investigation of the meat in this case showed the presence of a bacillus similar to that found in other outbreaks of kreotoxismus, but the greatest interest in the work of Van Ermengem centel's about the cases which occurred at Ellezelles, in Belgium. The ham, which proved poisonous in this case, seemed to be perfectly normal. Those poisoned showed but little or no evidence of gastro-intestinal irritation, but nervous symptoms were very prominent, and these consisted of diplopia, mydriasis, ptosis, aphagia, aphonia and anuria. There were two deaths. Other portions of the carcass were eaten without harm and it was observed that the poisonous ham was the only part of the meat which was completely submerged in the brine, and it was inferred from this that the harmful germ would be found to be strictly anaërobic. This inference

1 Vierteljahrschrift f. gerichtl. Medicin, 1893.

Zeitschrift f. Fleisch-und Milchhygiene, 4.

3 Zeitschrift f. Hygiene, 22.

- Allgemeine med. Zeitung, 1894.

${ }^{5}$ Bericht über das Veterinärwesen, 1894.

${ }^{6}$ Centralblattf. Bahteriologie, 19; also Zeitschrift f. Hygiene, 26, 1. 
proved to be correct, and it was furthermore found that the bacillus produces a toxin almost as poisonous as that of the tetanus bacillus, inasmuch as it requires only $0.0005 \mathrm{mg}$. to kill a rabbit in twenty-four hours. The microörganism has been designated as bacillus botulinus, and its poisonous product as botulismustoxin. Marinesco ${ }^{1}$ found that when this toxin is administered by mouth to eats it causes mydriasis, partial paralysis, aphonia, impossibility of deglutition, and muscular weakness. He found that the lesions induced by this toxin are confined largely to the cells of the gray matter of the cord. With very small doses the lesions consist of a rarefaction of the chromatophil elements. Under the influence of larger amounts of poison the granules of Nissl undergo more or less marked disintegration or chromatolysis. When fatal doses are administered some of these cells will be found to be completely destroyed. Brieger and Boer ${ }^{2}$ have prepared this toxin by their method of precipitation with chlorid of zinc, but were unable to obtain it in a pure state. Apparently it belongs to the albumoses, and Kempner ${ }^{3}$ has prepared a specific antitoxin by immunizing animals to the toxin. The last-mentioned observer, together with Pollak, has confirmed the microscopical studies of Marinesco and furthermore has demonstrated that, under the influence of the antitoxin, cells which have been injured by the toxin show visible evidence of repair.

Tyrotoxismus.-In 1827, Hünnefeld made analyses of poisonous cheese and experimented with extracts upon the lower animals. He accepted the ideas of Kerner in regard to poisonous sausage in a somewhat modified form, and thought the active agents to be sebacic and caseic acids. About the same time, Sertürner, making analyses of poisonous cheese for Westrumb, also traced the poisonous principles, as he supposed, to these fatty acids. In 1848, Christison, after referring to the above-mentioned work, made the following statement: " His (Hünnefeld's) experiments, however, are not quite conclusive of the fact that these fatty acids are really the poisonous principles, as he has not extended his experimental researches to the caseic and sebacic acids prepared in the ordinary way. His views will probably be altered and simplified if future experiments should confirm the late inquiries of Braconnot, who has stated that Proust's caseic acid is a modification of acetic acid combined with an acrid oil." In 1852, Schlossberger made experiments with the pure fatty acids and demonstrated their freedom from poisonous properties. Since the overthrow of the fatty acid theory various conjectures have been made, but none worthy of consideration.

1 Comptes rendus de la Société de Biologie, 1896.

2 Deutsche med. Wochenschrift, 1896.

${ }^{3}$ Deutsche med. Wochenschrift, 1896 ; Zeitschrift $f$. Hygiene, 26, 481 ; 27, 213. 
In the years 1883 and 1884 there were reported to the Michigan State Board of Health about three hundred cases of cheese poisoning. As a rule, the first symptoms appeared within from two to four hours after eating the cheese. In a few, the symptoms were delayed from eight to ten hours and were very slight. The attending physicians reported that the gravity of the symptoms varied with the amount of cheese eaten, but no one who ate of the poisonous cheese wholly escaped. One physician reported the following symptoms: "Everyone who ate of the cheese was taken with vomiting, at first of a thin, watery, later of a more consistent reddish-colored substance. At the same time the patient suffered from diarrhœa with watery stools. Some complained of pain in the region of the stomach. At first the tongue was white, but later it became red and dry ; the pulse was feeble and irregular ; countenance pale with marked cyanosis. One small boy, whose condition seemed very critical, was covered all over the body with bluish spots." Notwithstanding the severity of the symptoms in many, there was no fatal termination among these cases, though several deaths from cheese poisoning in other outbreaks have occurred. Many of the physicians at first diagnosed the cases from the symptoms as arsenical poisoning, and on this supposition many of them administered ferric hydrate. Others gave alcohol and treated upon the expectant plan. Vaughan, to whom the cheese was sent for analysis, made the following report: "All of these three hundred cases were caused by the eating of twelve different cheeses. Of these, nine were made at one factory and one each at three other factories. Of each of the twelve I received smaller or larger pieces. Of each of ten I received only small amounts. Of each of the other two I received about eighteen kilograms. The cheese was in good condition, and there was nothing in the taste or odor to excite suspicion. However, from a freshly cut surface there exuded numerous drops of a slightly opalescent fluid which reddened litmus paper quickly and intensely. Although, as I have stated, I could discern nothing peculiar in the odor, if two samples, one of good, the other of poisonous cheese, were placed before a dog or cat, the animal would invariably select the good cheese; but if only poisonous cheese were offered and the animal was hungry, it would partake freely. A cat was kept for seven days and fed only poisonous cheese and water. It ate freely of the cheese and manifested no untoward symptoms. After the seven days the animal was etherized and abdominal section made. Nothing abnormal could be found. I predicted, however, in one of my first articles on poisonous cheese that the isolated poison would affect lower animals. At first I made an alcoholic extract of the cheese. After the alcohol was evaporated in vacuo at a low temperature a residue, consisting mainly of fatty acids, remained. I ate a small bit of this residue, and found that it pro- 
duced dryness of throat, nausea, vomiting and diarrhoea. The most of this extract consisted of fats and fatty acids and for some weeks I endeavored to extract the poison from these fats, but all attempts were unsuccessful. I then made an aqueous extract of the cheese, filtered this, and drinking some of it, found that it also was poisonous. But after evaporating the aqueous extract to dryness on the water-bath at $100^{\circ}$ the residue thus obtained was not poisonous. From this I ascertained that the poison was decomposed or volatilized at or below the boiling point of water. I then tried distillation at a low temperature, but by this the poison seemed to be decomposed. Finally, I made the clear, filtered aqueous extract, which was highly acid, alkaline with sodium hydrate, agitated this with ether, removed the ether, and allowed it to evaporate spontaneously. The residue was highly poisonous. By re-solution in water and extraction with ether, the poison was separated from foreign substances. As the ether took up some water, this residue consisted of an aqueous solution of the poison. After this was allowed to stand for some hours in vacuo over sulphuric acid, the poison separated in needleshaped crystals. From some samples, the poison crystallized from the first evaporation of the ether, and without standing in vacuo. This happened only when the cheese contained a comparatively large amount of the poison. Ordinarily, the microscope was necessary to detect the crystalline shape. From sixteen kilograms of one cheese I obtained about 0.5 gram of the poison, and in this case the individual crystals were plainly visible to the unaided eye. From the same amount of another cheese I obtained only about $0.1 \mathrm{gram}$, and the crystals in this case were not so large. I have no idea, however, that by the method used all the poison was separated from the cheese."

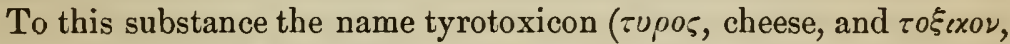
poison), which had formerly been used to designate the undiscovered active agent in poisonous cheese, was given. During 1887, Wallace found tyrotoxicon in two samples of cheese which had caused serious illness. The first of these came from Jeanesville, Pa., and the symptoms as reported to Wallace by Doolittle, who had charge of the cases, were as follows:

Some fifty persons were affected and in the majority of these the symptoms appeared within from two to four hours, and consisted of vertigo, vomiting and severe rigors, varying in their order of appearance and severity. Chills and vomiting were the most constant and marked symptoms, and were soon followed by pain in the epigastric region, cramps in the feet and lower limbs, purging and griping pain in the bowels, a sensation of numbness, especially in the limbs, and marked prostration, in some amounting almost to collapse. The vomit at first consisted of the contents of the stomach and had a strong cheesy odor; afterward, it contained mucus, bile, and, in the 
more severe cases, blood. The diarrhœeal discharges, at first fecal, later became watery and light colored. No deaths resulted, and for the most part the effects were transient, and all that remained on the following day were the prostration and numbness, which disappeared in from one to three days. Children apparently suffered less than adults. All remarked on the suddenness of the attack, feeling perfectly well until nausea and vertigo set in. Wolff detected tyrotoxicon in cheese which poisoned several persons, at Shamokin, Pa. The pores of this cheese were found filled with a grayish-green fungoid growth, though it is not supposed that this was connected in any way with the poisonous nature of the food. Tests were made for mineral and vegetable poisons with negative results, after which tyrotoxicon was recognized both by chemical and physiological tests. Ehrhart published the history of cases of poisoning from Limburger cheese. The rind was covered with a heavy mould, while the interior had become fluid from putrefaction and was of bitter taste. Three ate only of the mouldy rind, and these remained well. The next morning, five who had eaten of the other portion suffered from vertigo, nausea, vomiting and abdominal pains; no stools. The father of the family had convulsive movements of all the extremities; his pupils were dilated, and did not respond to light; there were double vision, cold sweat, cyanotic skin, distended abdomen, difficulty in swallowing, delirium, mild trismus, and a temperature of $40^{\circ}$. The temperature of the mother, on account of the great collapse, was subnormal ; she had no convulsive movements, but was unconscious for many hours. Dokkum obtained from poisonous cheese, by a modification of the method already given for the separation of tyrotoxicon, a basic substance, which when injected into frogs in doses of $5 \mathrm{mg}$. caused paralysis, and death within thirty minutes. This investigator thinks that the base thus obtained by himself is not tyrotoxicon, but a curare-like poison for which he suggests the name tyrotoxin.

For some time after the discovery of tyrotoxicon it was supposed that all cases of cheese poisoning are due to this substance, but subsequent investigations have shown that there are other toxins formed in cheese and that tyrotoxicon is a somewhat rare poison. In 1890, Vaughan, having failed to find any evidence of tyrotoxicon in numerous samples of poisonous cheese, was led to test for other toxins. He obtained an albumose, forty drops of an aqueous solution of which when injected under the skin on the back of cats produced vomiting and purging, followed by marked prostration and terminating in some instances in death. This substance belongs to the socalled poisonous albumins. From its aqueous solution it is not precipitated by heat or nitric acid, singly or combined. It is not precipitated by saturation with sodium sulphate, nor by a current of carbonic acid gas ; therefore it is not a globulin. It is precipitated by satura- 
tion with ammonium sulphate, and this fact distinguishes it from the peptons. In 1895 Vaughan and Perkins obtained from a piece of cheese which had proved fatal to one man, two bacilli, one of which elaborates an active toxin ; both filtered and heated cultures kill animals promptly. Vaughan and McClymonds ${ }^{1}$ examined sixty-five samples of cheese from as many different manufacturers. Of these, forty-nine were what is ordinarily known as American green cheese. These were made in Michigan, Wisconsin, Illinois, New York, and Canada. Eight of the forty-nine samples were sent to the laboratory because persons eating them had suffered from nausea, vomiting and purging; the other samples were not known to be poisonous. Everyone of the forty-nine samples of American green cheese furnished cultures which killed white rats, guinea-pigs and rabbits. The toxicogenic germ in all these samples belongs to the colon group, and whether or not a given sample of green cheese unpleasantly affects the consumer will depend upon the amount and virulence of the germ present in the cheese. Vaughan and Cooley ${ }^{2}$ have shown that the colon toxin is contained in the germ cell from which it does not, at least under ordinary conditions, diffuse into the culture medium. This toxin may be heated in water to a very high temperature without destruction of its toxicity, as is illustrated by the following experiment : $200 \mathrm{mg}$. of the crude toxin was placed with 10 c.c. of water in a tube which was sealed and heated to $184^{\circ}$ for thirty minutes. On opening the tube the content was found to be milky and microscopical examination showed a granular mass containing a few unbroken cells. Portions of this heated substance injected into guinea-pigs caused death, and autopsy showed the lesion usually found in these animals when killed with colon toxin. Apparatus has been devised for obtaining the colon toxin in large amount ${ }^{3}$ and it has been found that with a very virulent culture the powdered germ is often sufficiently toxic to kill guinea-pigs of 200 gram weight in doses of $\frac{1}{5} \mathrm{mg}$.

Galactotoxismus.-Tyrotoxicon has been found in milk in numerous instances, having first been detected in this fluid in 1885, soon after its discovery in cheese. In 1886 Newton and Wallace detected this poison in milk which seriously affected a large number of persons at Long Branch. The poisonous milk came solely from one dairyman and investigation showed the following condition of affairs: The cows were milked at the unusual hours of midnight and noon, and the noon's milk-that which alone was followed by illness-was placed while hot in the cans, and then, without any attempt at cooling, carted eight miles during the warmest part of the day in a very hot month. During this time the unknown germ which elaborates

1 Jacobi Festschrift, p. 108.

${ }^{2}$ Journal of the American Medical Association, 1901; also American Medicine, 1901.

${ }^{3}$ Transactions of the Association of American Physiciails, 1901. 
tyrotoxicon undoubtedly grew abundantly in the milk and its toxin was easily detected by both chemical and physiological tests. In the same year Schearer found tyrotoxicon in milk used by, and in the vomited matter of, persons made sick at a hotel at Corning, Ia. In 1887, Firth, an English army surgeon, stationed in India, reported an outbreak of milk poisoning among the soldiers of his garrison. From the milk he separated, by the method already given, tyrotoxicon and demonstrated its action upon the lower animals. In the same year Vaughan reported the Milan cases of milk poisoning, three of which terminated fatally. His report of his observation of the symptoms is as follows: I first saw these patients, Sunday, September 25th; on a sofa in the room we found the daughter, who had been vomiting during the day and seemed much exhausted. She was not inclined to talk, and seemed to be in a stupor, though when spoken to she responded rationally. Her pupils were slightly dilated, her tongue coated, her pulse 120 and weak; her face pale; and a violent throbbing could be felt over the abdomen, which was retracted. Her temperature was $96^{\circ} \mathrm{F}$. In another room were the father, mother and son, two of them dying. The father was rational and talked with some freedom, when asked concerning the kind of food they had been eating. His pupils were normal, his face could not be said to present any peculiar feature, his pulse was rapid, breathing somewhat rapid, and the throbbing in the abdominal area was plainly felt. The abdomen was retracted and there was no pain on pressure. He complained of a burning constriction of the throat, swallowed with difficulty, and said that his throat and stomach felt as though they were on fire. The mother lay with eyelids closed as if in a deep sleep. Her pulse was rapid, her face had a livid flush, her breathing was about 35 per minute, and labored. The skin was cool, but neither abnormally moist nor specially dry and harsh. She could not be aroused. In fact, she was comatose. The son rolled uneasily from one side of the bed to the other. His breathing also was labored. His eyelids were closed, and the pupils were markedly dilated-did not respond to light. He could not be aroused. In mother and son, as well as in father and daughter, the abdomen was retracted, and the throbbing in the abdominal area was easily felt.

The symptoms were not those of morphin, strychnin, digitalis, or aconite. They did have some resemblance to those of belladonna but were not identical ; the pupils were not so widely dilated as they are in belladonna poisoning; there was in none of these persons the active delirium of belladonna poisoning; there was no picking at the clothing ; no grasping of imaginary objects in the air ; no hallucinations of vision.

The family, which consisted of the four sick persons and of a daughter about twenty years of age, who was away from home at the 
time when the others were taken ill and for some months before that time, was evidently a tidy one. This was shown by their personal appearance and by the clothing and bedding; but the house in which they lived was old, and much decayed. One corner of one of the rooms had been transformed into a buttery, and in this the food was kept on shelves. On account of the more frequent scouring demanded by that part of the house the boards constituting the floor had rotted away, and a second layer had been placed over the original floor. Between these two floors there was found a great mass of moist, decomposing matter, the accumulation of years, which the broom could not reach. When this floor was taken up, a peculiar, nauseating odor was observable, and was sufficient to cause actual vomiting in one of the persons engaged in the examination. Tyrotoxicon was found in milk which had been used by the family and had stood in this room, and it was also produced in sterilized milk by inoculating it with bits of earth taken from between the floors. An autopsy held on one of the fatal cases showed as the most marked abnormality tightly constricted areas of the large intestine such as is sometimes seen in lead poisoning, and which had been quite generally observed in the lower animals experimentally killed by the administration of tyrotoxicon. Novy tested a cold-water extract of the finely-divided intestines for ptomains. The fluid, which was acid in reaction was filtered, then neutralized with sodium carbonate, and shaken with ether. The ether, after separation, was removed and allowed to evaporate spontaneously. The residue was dissolved in water and extracted again with ether. This ether residue gave the chemical reactions for tyrotoxicon and a portion of it was administered to a kitten about two months old. Within onehalf hour the kitten began to retch and soon it vomited, and within the next three hours it vomited as many as five times. There was no purging but the retching and heavy breathing, with evidences of prostration, continued more or less marked for two days, after which the animal slowly recovered. A quantity of fresh milk was divided into five portions of one quart each, placed in bottles which had been thoroughly cleansed, and treated in the following manner : No. 1 consisted of the milk only, and was employed as a control test. No. 2 was mixed with a drachm of vomited matter. No. 3 was treated with a portion of the contents of the stomach. No. 4 was treated with an aqueous extract of the intestine. No. 5 was treated with a small portion of the soil which had been taken from the floor of the buttery, stirred up with water.

These bottles were placed in an air-bath and kept at a temperature of from $25^{\circ}$ to $30^{\circ}$ for twenty-four hours, and then each was tested for tyrotoxicon, the result in No. 1 being negative, while in all of the others it was positive. These tests were both chemical and physiological. All the samples yielded a non-poisonous base 
when treated according to Brieger's method, and the same substance was obtained from perfectly fresh milk. It was most probably formed by the action of the heat and reagents employed in this method. This base was obtained in crystalline form, and several portions of it were administered to kittens without effect. Tyrotoxicon was obtained from the filtered milk by two methods: The method which has been previously described, and which consists in neutralizing the filtered milk with sodium carbonate and extracting with ether. That portion of the poison employed in the physiological tests was obtained in this way, and in order to be sure that no poison came from the ether, the extract from the milk to which nothing had been added, was given to a kitten, and was found to produce no effect. (2) The filtrate from the milk was heated to $70^{\circ}$ for some minutes and refiltered. This filtrate, which was perfectly clear, was treated with a small quantity of nitric acid in order to convert the tyrotoxicon into a nitrate. Then pure potassium hydrate in the solid form was added until the solution was strongly alkaline. This solution was concentrated so far as it could be on the water-bath. (The potassium compound of tyrotoxicon is not decomposed below $130^{\circ}$.) The dark brown residue, after cooling, was examined with the microscope and found to contain the crystalline plates of tyrotoxicon potassium hydrate, along with the prisms of potassium nitrate. The former was separated from the latter by extraction with absolute alcohol and filtration. The alcohol was evaporated to dryness on the water-bath, and the residue again extracted with alcohol. From this alcoholic solution tyrotoxicon was precipitated with ether. The precipitate was decomposed by adding acetic acid and heating, the tyrotoxicon being broken up into nitrogen and phenol. The phenol was recognized by precipitation with bromine water, and by other well-known tests.

The coroner's jury, after hearing the testimony briefly outlined above, rendered a verdict of death from poisoning with tyrotoxicon, and, so far as we know, this is the first instance in which a jury has rendered a verdict of death due to a putrefactive poison.

Camman reported twenty-three cases of milk poisoning which he attributed to tyrotoxicon, although this substance could not be found in the milk, and Kinnicutt has isolated tyrotoxicon from milk which had been kept for some hours in unclean vessels.

Gafflky has reported a case of enteritis, closely simulating typhoid fever, which was due to the presence of a virulent form of the colon bacillus in the milk consumed by the individual. The cow that gave this milk was at the time suffering from a bloody diarrhoea, and it is more than probable that some of the liquid discharged from the animal fell upon the udder or in some other manner found its way into the milk. Rehn has reported similar cases and has detected the colon bacillus in the milk taken by the sick. 
Ice cream, frozen custard, cream puffs, and other articles of food consisting largely of milk, are frequently harmful. The injurious effects of these substances have been attributed to plants eaten by the animals, and the flavoring and coloring matters used in the preparation of these foods. Even within recent years the claim has been put forward that ice cream poisoning is due to artificially prepared vanillin, but vanilla extracts used in the preparation of foods, which prove to be poisonous, have been swallowed in large quantities by chemists and have been administered to animals without the slightest effect. It has also been claimed that ice cream often owes its poisonous properties to small quantities of zinc or tin dissolved during the process of freezing. This statement is perfectly absurd when we find, as we frequently do, that a sample of ice cream will act more powerfully as an emetic than will sulphate of zinc, grain for grain. It is within the range of possibility that poisonous extracts may be used in flavoring milk preparations and it is a well-known fact that chromate of lead has been found in cream puffs. But it is certainly true that neither flavoring agent nor metals are accountable for the injurious effects observed to follow the eating of poisonous ice cream and similar milk products. Moreover, ice cream flavored with chocolate and that flavored with lemon have also been observed to be poisonous, and vanilla ice cream is more frequently poisonous for the very good reason that this flavoring is used more largely than any other, and possibly than all others combined.

Vaughan and Novy have found tyrotoxicon in numerous samples of poisonous ice cream and custard. Schearer reported the same poison in both vanilla and lemon ice cream which made many sick at Nugent, Ia. Allaben observed numerous cases poisoned with lemon ice cream, and Welford has obtained tyrotoxicon from custard flavored with lemon. It must not be inferred, however, that this is the only toxin that is found in ice cream and other milk compounds. In 1896, Vaughan and Perkins ${ }^{1}$ reported the detection of a new toxin in both ice cream and cheese. This substance differs from tyrotoxicon chemically inasmuch as it is not removed from alkaline solutions by extraction with ether. Physiologically its action on the heart closely resembles that of muscarin or neurin. Pathologically it induces a high degree of local inflammation when injected subcutaneously or intra-peritoneally; and after death the contractions of the intestines so characteristic of tyrotoxicon poisoning were never found, although more than 200 animals were experimented upon in this investigation. In the persons poisoned with this food, symptoms appeared within from three to six hours and at first consisted of nausea which in all instances was followed by vomiting. Diarrhœa was present in the majority but not in all. The vomiting was accompanied by sharp pains through the abdomen, and it is stated that

\section{'Archiv f. Hygiene, 27.}


in some the pain was partially relieved by strong pressure. The most alarming phenomenon to the physicians in attendance was feebleness of the heart's action. The hands and feet grew cold, then the entire body became cool and clammy, and in many the radial pulse was not perceptible. This condition, together with a heavy stupor in some, gave occasion for alarm, and hypodermic injections of brandy, digitalis, strychnin and nitroglycerin were employed, each physician selecting the stimulant in which he had the most confidence, or taking that which he had at hand. In one instance the patient became wildly delirious, crying out and attempting to rise from the bed. Those who vomited but little and had no diarrhœa fell into a heavy stupor, and it is highly probable that these were in greater jeopardy than the others. The early and thorough vomiting doubtless was the most potent agent in saving those who had taken the larger quantities of the infected food. The toxin formed by the germ found in this food is not destroyed by boiling. The germ which produced this toxin bears a close resemblance to the colon bacillus, but differs from a typical member of this group in the following particulars: (1) The new bacillus failed to give the indol reaction. (2) Both coagulate milk, but the new germ acts more promptly than the colon bacillus. (3) The pleasant butyric ether odor of milk cultures of the new bacillus is not developed in cultures of the colon bacillus in the same medium. (4) The new germ grows abundantly on carrots forming a creamy layer, and gives off a sour odor; while the colon bacillus grows much less vigorously and gives off no similar odor.

Undoubtediy there are many forms of the colon bacillus which frequently find their way into milk and, on account of the toxin contained within their cells, they render this and various other foods of which milk is a constituent more or less poisonous.

Sitotoxismus.-Under the heading of sitotoxismus we may include all forms of poisoning with vegetable foods infected with moulds and bacteria. All sitotoxicons are not bacterial products; however, for completeness we will briefly review the entire subject, excluding, of course, all cases of poisoning due to admixture with mineral substances. We shall also attempt to exclude as far as possible all discussion of plants that are in and of themselves poisonous.

Ergotismus, sometimes called ergotism, is due to poisoning with a fungus known as claviceps purpurea, which develops in the flowers of rye, other grains, and certain wild grasses. It is most frequently found in rye and darnel. Early in the development of the rye flower there may appear in its interior a sweet, unpleasant-smelling liquid which sometimes forms so abundantly that it overflows, runs down upon the stalk, and falls upon the ground. The sugar which it contains attracts ants and other insects, and these aid in the distribution 
of the fungus. There are certain conditions which are known to favor the development of this parasite. It is more common when there is a rainy spring followed by a hot, dry summer. Thorough cultivation of the soil kills the parasite, and for this reason ergot is more abundant in countries where the soil is not well cultivated, and ergotism has within recent years prevailed in epidemic form only in Russia and in Spain. Grains of ergot, after having been exposed to the air for a few months, lose in large part their toxicity, and consequently epidemics of ergotism follow closely upon the harvests, and especially upon poor harvests, when the parasite is most abundant and the people are compelled to feed upon what they have without close inquiry as to its quality. However, it may be pointed out here that in the present state of civilization there is but little excuse for the existence of epidemics of ergotism. In the first place, thorough cultivation of the soil would soon completely eradicate this mould, and a proper selection of seed would do much in the same direction. As early as $1858 \mathrm{Kühn}$ pointed out the benefit that would be secured by an early harvesting of fields contaminated with ergot, as by this means the spread and consequent development of this parasite would be largely prevented. Moreover, the ergot grain is much larger than that of rye, and this difference in size permits of the easy separation of the two by means of sieves especially constructed for this purpose. The commercial value of ergot is so much greater than that of rye that the time given to the separation of the two would be profitably spent, and yet so dense is the ignorance and so pronounced is the indolence of certain peasant classes that epidemics of ergotism continue and probably will continue for many years.

Kobert and his student, Grünefeld, have found three poisons in ergot; these are ergotinic acid, sphacelinic acid and cornutin. The first of these, ergotinic acid, is poisonous when injected subcutaneously or intravenously, but seems to be devoid of harmful properties when taken by the mouth, hence it can play no part in the causation of ergotism. In all cases of ergotism both the sphacelinic acid and the cornutin are contained in the ergot; therefore, a clinical picture of the disease must be a composite resulting from the combined action of the two, and it must vary with the preponderance of one or the other in the ergot taken.

It is believed that sphacelinic acid is the constituent of ergot that causes gangrene and develops the cachexia of the disease. Grünefeld fed animals with sphacelinic acid and induced gangrene in all; in cocks, the comb, then the wattles, tongue, wings and crop, respectively were affected; in hogs, the ears fell off, bit by bit; in horses and cows, the tails, ears and hoofs separated; while in dogs and cats the gangrene usually began in the skin. When locally applied in concentrated solution sphacelinic acid causes gangrene of the tissues with which it comes in contact and this explains the 
necrosis of the living tissue, the ulcerations, and the hemorrhages into the intestines.

Cornutin does not cause death of tissue, but acts directly upon the nervous system, and is believed to be the active agent in the causation of ergotismus convulsivus. It acts on the brain and cord, affecting the vagus and vasomotor centers, and acting through the lumbar cord upon the uterus. Cornutin readily undergoes decomposition, gradually losing its virulence, and is found only in fresh ergot, disappearing more quickly than sphacelinic acid. For this reason it happens that those symptoms due to cornutin are more prominent in outbreaks occurring soon after the harvest; while those due to sphacelinic acid are seen in both early and late epidemics.

There are some reasons for believing that there are bacterial products formed in ergotized bread, and to these has been attributed the septic character of certain epidemics of ergotism. However, this is a mere supposition and there has been no scientific experimentation made in its support. It is easy to see how sepsis occurs in ergotism without the necessity of supposing the presence of bacterial products in ergotized bread. In gangrene of the intestines bacterial infection through the diseased intestinal walls may easily occur; so in gangrene of the skin infection from without may take place with equal readiness.

Lathyrismus, or lathyrism, is a form of spastic spinal paralysis due to intoxication from the eating of the seeds of certain species of the genus Lathyrus of the vetch tribe. Of the more than 120 known species of lathyrus, 13 are native to the United States, and others are cultivated here on account of their showy flowers, the sweet pea of the garden being an example of the latter. In northern Africa and southern Europe lathyrism has been frequently observed, and it occasionally occurs in India and other parts of Asia. The literature of lathyrism shows that this disease was formerly much more prevalent than it is at present. As early as 1671 it was known that bread made of vetch seeds mixed with graham seriously affected those who ate of it for any length of time and the Grand Duke of Würtemberg issued an edict forbidding the use of food of this kind. It was then noticed that those who ate of this bread suffered from marked stiffness of the extremities and the disease was regarded as incurable, although death seldom resulted from it. Numerous attempts have been made to isolate the poisonous principle or principles of lathyrus, but, so far, the results obtained have been unsatisfactory and to some extent contradictory. Teilleux obtained a resinous body which, when administered to rabbits in gram doses, caused tetanic movements of the muscles and finally paralysis of the posterior extremities, death occurring within four days. From lathyrus cicera, Bourlier obtained an extract which killed frogs and 
small birds within forty-eight hours at most. An alkaloidal body was obtained by Marie from the seeds of the lathyrus sativa; however, this substance when administered subcutaneously to guineapigs does not induce any of the characteristic symptoms of lathyrism. Astier obtained an alcoholic extract which, after repeated injections, induced in dogs complete paraplegia, from which the animal slowly recovered. There are good reasons for believing that whatever the poisonous substance may be, it is destroyed at a high temperature. The Arabs of northern Africa eat vetch prepared in two ways: One preparation, known as "Kouskouson," is steamed or boiled, while the other dish, known as "Galette," is cooked at a higher temperature, and it is said to be a well authenticated fact that injurious effects more frequently follow the use of the former than of the latter. In man the first symptom of lathyrism usually manifested is a chill, which is followed by pain in the loins and legs. A girdle sensation is complained of by some, and motor lameness of the lower extremities is common. The patient walks with difficulty, and later finds locomotion wholly impossible. The knee reflex is greatly intensified, and a paresthesia with formication may be marked. It is claimed by some that gangrene of the feet and legs may occur, but it is possible that cases upon which this statement is founded were due to ergot poisoning. The old belief that recovery never occurs is not supported by more recent observation, and many of the milder cases are greatly improved by proper medicinal treatment.

Maïdismus.-Ordinarily known as pellagra, this may be defined as a progressive disease leading to paralytic and other nervous disorders, and caused by intoxication from the eating of damaged Indian corn. The geographical distribution of maïdismus is confined to that portion of Europe lying between the parallels of $42^{\circ}$ and $48^{\circ} \mathrm{N}$., with the exception of Corfu, one of the Ionian islands, but within the above-mentioned region this disease is by no means uniformly or universally distributed. It prevails in some localities to such an extent that it has become a national calamity. In 1879, one hundred thousand cases of this disease were reported in Italy, and in 1881, fifty-six thousand in Lombardy alone. Pellagra is confined to countries where the staple article of diet is maize, and yet Indian corn constitutes a most nutritious and healthful article of food in other countries, as has been abundantly demonstrated by the former well nourished condition of the large colored population of the southern United States, for there probably never has been a class of day laborers, certainly never a class of servants, better fed and nourished than were the negroes of the South before their emancipation; and corn bread, made from mature corn and properly prepared, is both healthy and nutritious. Pellagra is known only in those countries where, on account of an uncongenial 
climate, or from barrenness of the soil, or from lack of proper cultivation, maize does not mature.

While there can no longer be any doubt that pellagra is an intoxication due to poison formed in corn meal or bread, we have, as yet, no positive information concerning either the ferment which causes these harmful changes or the poisonous substance or substances that are formed. Some think that the disease is an intestinal mycosis, due to infection with a parasitic mould, which is introduced into the body with this food. Carboni found in the damaged meal used by pellagrous persons, also in their feces, a bacterium to which he has given the name bacillus maidis, and to which he ascribes the disease. Majocchi claims to have found this germ in the blood of pellagrous individuals, and according to Paltauf and Heider the grains of corn become infected during the wet season with the bacillus maidis and the bacillus mesentericus fuscus, and these decompose the moist meal producing ptomains which constitute the toxins. Others claim that the so-called bacillus maidis is nothing more than the widely distributed potato bacillus, that it is incapable of generating toxins under any conditions and that it is by no means constantly found in the intestines of pellagrous individuals. Lombroso thinks the disease is an intoxication rather than an infection, and believes that it is due to certain chemical poisons formed by bacterial activity. This investigator has obtained from powdered corn which has been allowed to ferment at from $25^{\circ}$ to $30^{\circ}$ for twenty-four to thirty-six hours, an alcoholic extract and an oily substance, and with these he thinks that he has induced the characteristic symptoms of pellagra in man and animals. The alcoholic extract of this corn contains a basic substance or substances to which Lombroso has applied the name pellagrocein, and according to his theory there are two toxins, the combined action of which gives rise to the complex symptoms of pellagra, similar to the action of sphacelinic acid and cornutin in ergotism. One of these poisons he thinks has a strychnin-like effect, while the other is narcotic in its action. Neusser believes that there is nothing directly harmful in the food when it is taken into the body, but that poisons are formed in the intestines and he makes the disease a specific form of auto-intoxication. It is claimed that sporadic cases of pellagra may be due to the use of whisky made from damaged corn ; if this be true, the poisonous substance must be volatile.

Clinicians generally state that pellagra consists of three stages, which are more or less marked and distinct. The first begins with disturbances of the digestive organs, the tongue is heavily coated, but later it loses its epithelium, there is loss of appetite as a rule, altlough in exceptional cases the desire for food may be inordinate. Usually there is diarrhea, but obstinate constipation may occur. Accompanying these digestive disturbances there is pain in the head, 
neck and back. Dizziness, muscular weakness and unsteadiness of gait are frequently observed. Mental activity becomes slow, and some complain of a numbness in the brain. In the majority of instances, not in all, there is a characteristic erythema which is most marked on the unclothed parts of the body, as the hands and face, though it may be much more widely distributed. It is to this affection of the skin that the disease owes its common name, pellagra (from pelle, skin, and agra, rough).

The appearance of certain cerebro-spinal symptoms characterizes the second stage of the disease. Chilly sensations are complained of and there is often constant ringing in the ears. The muscular weakness is increased; tremors and convulsive twitchings become frequent, and cramps and light spasms occur. The tendon reflex is more markedly exaggerated, sensibility is often diminished and the patient falls into a state of melancholia. The skin becomes pale or there is capillary injection over certain areas notably of the face, and in some instances the skin becomes hard and scaly.

Marked emaciation is one of the characteristic symptoms of the third stage. The subcutaneous fat wholly disappears, locomotion becomes impossible, incontinence of urine is persistent and uncontrollable diarrhoa makes the bed-ridden patient an object of pity. Fortunately, after this stage has been reached the individual loses all resistance to the infectious diseases, and tuberculosis or septicemia frequently closes the history. A considerable number of pellagrous individuals end their sufferings by suicide.

The most characteristic post-mortem findings may be stated as follows : The body is greatly emaciated, the intestinal walls are thin on account of the wasting a way of the muscular coat, ulceration in the intestines is frequently found, the cells of the liver and of the skin and the muscles of the heart are deeply pigmented. Atrophy seems to be most marked in those organs connected with the vagus nerve, the lungs, heart, kidneys, spleen and intestines. Although marked alterations from the normal are frequently found in the brain and cord, there seems to be no constant or characteristic lesion in these organs. 


\section{CHAPTER XI. \\ THE EXAMINATION OF POISONOUS FOODS.}

OUTBREAKs of bromatotoxismus have become common in recent years and chemists and bacteriologists are frequently asked to examine foods which are suspected of having caused untoward results. The increase in the number of cases of this kind is partly real and partly only apparent. One cause of the actual increase lies in the larger consumption of preserved foods. Meats, the appearance and odor of which would render their sale in the piece impossible, or at least doubtful, may be chopped, cooked, canned, and sold as a first-class article. We do not state that this fraud is commonly practised, but that it is a possible one cannot be denied, and that it is occasionally resorted to has been demonstrated both in this country and in Europe. This source of danger to the public health will not be removed until the necessity for scientific inspection of foods, especially of animals before slaughtering, is understood and practised. However, the greater number of cases of poisoning by prepared foods arises from imperfections in methods or from want of intelligent and conscientious attention to details. When we recognize the fact that the successful preparation of every portion of preserved food depends upon the exclusion of microörganisms, both specific and putrefactive, and when we learn that the processes are carried out for the most part by those who are ignorant of the scientific principles involved, then we can only wonder that the health of the consumer is not more frequently placed in jeopardy.

The apparent increase in the number of instances of food poisoning is due to the fact that the medical profession has only recently learned to recognize food infection as a cause of illness or has been in possession of the knowledge necessary to convert suspicion into positive demonstration. Only a few years ago we were seeking for the cause of cholera infantum in mysterious and indefinite telluric or meteorological conditions, but now we know that this disease is solely due to infected, and consequently poisonous, food. Formerly, many of these cases were believed to be due to the accidental or criminal addition of some metallic or vegetable poison to the food, and unjust accusation, possibly in some instances, unjust execution, resulted. We have also learned that typhoid and typhus fevers, scarlet fever, and other acute exanthemata, and even pneumonia, may be closely simulated by the symptoms due to infected foods.

Unfortunately, the expression "ptomaïn poisoning" has come into 
quite general use to designate illness due to infected food. While it is true that basic substances of bacterial origin constitute in some instances the actual materies morbi, this is not always, or even generally, the case. Among the poisonous bacterial products there are many that are not basic, and many others concerning the chemical nature of which we are yet very much in ignorance. In a large proportion of the instances we are ignorant not only of the chemistry of the poisons which induce the untoward effects, but of the bacteria through the activity of which these poisons are generated. Moreover, we cannot in cases of bromatotoxismus draw a sharp line of distinction between intoxication and infection. Food poisoning may originate in either of the following ways: (1) The food is infected and the poison is generated only and wholly before the food is taken. (2) The infecting organism may begin the elaboration of its poisonous products outside of and continue the same process inside the body. (3) The infection may not result in the production of poisons until the food is taken into the body. In all of these forms, infection of the food is the essential element; it is this that must be prevented, and to this especial attention must be called.

How shall we proceed in the examination of food suspected of having caused sickness or death ?

In the first place, the possibility of the ill effects having been due to metallic poisons should be considered. In cases in which this possibility exists such poisons should be sought by methods given by the best toxicologists, and of which it is not the purpose of this book to speak. In case the substance to be examined consists of canned food the tests for mineral poisons should always be made. However, when a teaspoonful of ice cream causes nausea and vomiting, the idea that these effects can be due to sulphate of zinc dissolved in the freezer is too preposterous and absurd to be worthy of serious consideration by anyone familiar with the quantity of this salt necessary to act as an emetic.

If there be a sufficient quantity of the food a portion of it should be fed to animals. As a rule, the best animals for experiments of this kind are puppies and kittens. It should be remembered that rabbits and guinea-pigs cannot vomit and we have learned by experiments that guinea-pigs fed exclusively upon pure milk die ; they are not able to digest the casein which forms hard balls in the small intestines and mushy masses in the large intestines, and the animals succumb within eight or ten days, apparently from intestinal obstruction. If they be fed upon hay or other vegetable food along with the milk, they are apparently able to digest the casein. However, under no circumstances are they fit animals for experimentation, when the purpose is to determine whether or not milk or any of its products is poisonous.

A thorough examination of foods for bacterial poisons cannot be 
made except in a properly equipped laboratory. It is our purpose to briefly point out the methods that may be followed, and in doing so we take it for granted that the one who attempts work of this kind is already familiar with the ordinary technic of bacteriological research. The line of procedure will vary somewhat with the kind of food to be examined, the form in which it has been prepared, and the quantity supplied the analyst. All samples should be examined with as little delay as possible after the article has become the object of suspicion. When delay is unavoidable, farther bacterial growth should be retarded in the meantime as far as possible; not by antiseptics, but by low temperature. Microörganisms not present at the time of the supposed poisoning may be accidentally introduced, or non-toxicogenic bacteria may multiply to such an extent that the detection of the harmful organism is rendered impossible.

As a rule, the quantity of the food supplied the analyst is not sufficient to allow of the detection or the isolation of the chemical poison directly. To try to find the toxin in a few ounces of cheese or a small bit of meat by direct extraction, is a task that would be undertaken only by one quite ignorant of the nature of these poisons. In all but exceptional instances, where many pounds of food are supplied, the portion that reaches the laboratory can only be regarded as the bearer of the germ to the activity of which the poison is due. This germ must be detected, isolated, grown in pure cultures, and its toxicogenic properties demonstrated upon the lower animals. It should be clearly understood that the most thorough study of the morphological characteristics of the germ and of the chemical properties of the poison will not suffice without an accompanying determination of the toxicological action of the culture. The infectious nature of the bacterium should also be studied.

It should always be borne in mind that the article of food has probably been through several hands, some of which may not have been germ-free. In the examination of pieces of meat and cheese, the surface should be sterilized with a broad, heated knife or other piece of iron. It has been shown that bacteria deposited on such surfaces penetrate slowly. Then with other sterilized knives, sections should be made, and one or more small bits taken from the interior should be placed in sterilized bouillon, milk or other culture medium. Not less than a dozen tubes should be inoculated in this way. Three of these should be grown aërobically at ordinary temperature; three anaërobically at the same temperature; three aërobically at $37^{\circ}$ and three anaërobically at $37^{\circ}$. Some of the toxicogenic germs grow best at relatively low temperature $\left(20^{\circ}\right.$ to $\left.25^{\circ}\right)$ and fail to develop at $37^{\circ}$. Others have their optimum growth at the last mentioned temperature. Some develop only when the air is excluded, and others only when freely supplied with air. 
In the examination of liquid and semi-liquid foods, such as milk, custard, cream, broths, and jellies, small bits or a few drops should be placed in sterilized media and grown under the conditions above mentioned.

A growth having occurred in one or more of these tubes, the bacteria should be examined in hanging-drops and in stained mounts. If more than one organism be present, plate cultures should be made and each germ should again be grown under the conditions mentioned.

The infectious character of each organism should be tested on the lower animals : (1) By feeding; (2) by subcutaneous inoculation ; (3) by intra-peritoneal inoculation, and (4) by intravenous injection. The animals generally employed in these experiments are white mice, white rats, guinea-pigs, kittens, puppies, and rabbits. A given germ may be toxicogenic to one of these animals and not to the others. The quantity of the bouillon culture-twenty-four hours old or older -employed should be relatively large-from one to ten c.c., according to the animal and the method of inoculation. If these amounts prove active, smaller quantities should be tried until the limit is reached.

Cultures sterilized both by filtration and by heat should be tested on animals. It should be borne in mind that certain toxins, notably those of the colon bacilli, are removed from cultures by filtration through porcelain, while they are not altered by a degree of heat sufficient to kill the bacteria.

If by the above mentioned experiments a toxicogenic germ has been discovered, its morphological, cultural, tinctorial and pathogenic properties may be studied as thoroughly as the investigator may desire. The study of the bacterial poison may also be carried to the same extent. The examination for ptomains and toxins can be carried out according to the methods described in subsequent chapters. 


\section{CHAPTER XII.}

\section{METHODS OF EXTRACTING PTOMAÏNS.}

From what has been given in the preceding pages, one may form an idea of the difficulties with which the chemist has to contend in endeavoring to isolate the basic products of bacterial growth. $\mathrm{He}$ has to deal with complex substances, the nature and reactions of many of which he does not know. Moreover, the bodies which he seeks to isolate are often prone to undergo decomposition and in this way escape detection. Many ptomaïns are volatile or decomposable at temperatures near that of boiling water, and in such cases solutions cannot be evaporated in the ordinary way and the poison remain in the residue. The investigator has frequently been disappointed when on the evaporation of a solution, which he has demonstrated to be poisonous, he finds that the residue is wholly inert. Again, he may destroy the substance which he attempts to isolate with the reagents which he employs. So simple a procedure as the removal of a metallic base from a solution containing a ptomaïn, by precipitation with hydrogen sulphid, has been known to wholly destroy the ptomaïn. Probably the most perplexing difficulty in the isolation of these putrefactive alkaloids lies in the great number, complexity, and diversity of the other substances present in the decomposing matters. The same ptomaïn may be present in equal quantities in two samples of milk, and yet it may be easily obtained from the one, while from the other only minute traces can be secured. The difference is due to the fact that the other constituents of the milk in the two samples are at different stages of the putrefactive process, and, consequently, differ in their reactions and in their effects upon the agents employed to isolate the poison. All chemists appreciate this difficulty.

The first thing for the chemist, who undertakes this work, to do is to ascertain whether or not his reagents are pure. We have found samples of German ether, imported on account of its supposed purity, to yield on spontaneous evaporation a residue which gave some of the alkaloidal reactions, and a few drops of which, injected under the skin of a frog, caused paralysis and death within a few minutes. We advise that 500 c.c. of the ether to be used be allowed to evaporate spontaneously, and its residue, if there be one, be examined both chemically and physiologically. The basic substance which exists in some samples of sulphuric ether is pyridin. 
Guareschi and Mosso found commercial alcohol almost invariably to contain small quantities of an alkaloidal substance, the odor of which is similar to that of nicotin and pyridin. Its solutions are precipitated by the general alkaloidal reagents, with the exception of platinum chlorid and tannic acid. It does not reduce, or reduces feebly, ferric salts. From one sample of alcohol they obtained a base which, on addition to the above reagents, did give a precipitate with platinum chlorid. Alcohol may be freed from these substances by distillation over tartaric acid.

In amylic alcohol, Haitinger found almost 0.5 per cent. of pyridin. This reagent may be purified in the manner recommended for ethylic alcohol.

Chloroform, when found to leave a residue on evaporation, should be washed first with distilled water, then with distilled water rendered alkaline with potassium carbonate, then dried over calcium chlorid and distilled.

Petroleum ether sometimes contains a base which has an odor similar to trimethylamin or pyridin, and which gives a precipitate with platinum chlorid, forming in octahedra; benzol may contain a similar substance.

The following methods have been used for the purpose of extracting putrefactive alkaloids :

The Stas-0tto Method.-This method depends upon the following facts: (1) The salts of the alkaloids are soluble in water and alcohol, and generally insoluble in ether, and (2) the free alkaloids are soluble in ether, and are removed from alkaline fluids by agitation with ether. These principles are capable of great variations in their application. The usual directions are as follows: Treat the mass under examination with about twice its weight of 90 per cent. alcohol, and from ten to thirty grains of tartaric or oxalic acid; digest the whole for some time at about $70^{\circ}$, and filter. Evaporate the filtrate at a temperature not exceeding $35^{\circ}$ either in a strong current of air or in vacuo over sulphuric acid. Take up the residue with absolute alcohol, filter, and again evaporate at a low temperature. Dissolve this residue in water, render alkaline with sodium carbonate, and agitate with ether. After separation remove the ether with a pipette, or by means of a separator, and allow it to evaporate spontaneously. The residue may be further purified by redissolving in water, and again extracting with ether.

The following modifications of this method are employed :

Instead of tartaric or oxalic acid, acetic acid may be used.

When the fluid suspected of containing a ptomain is already acid from the development of lactic or other organic acid, the addition of an acid may be dispensed with.

Ether extracts are made from both acid and alkaline solutions. 
Chloroform, amylic alcohol, and benzene are used as solvents after extraction with ether.

Dragendorff's Method.-The finely divided substance is digested for some hours with water acidulated with sulphuric acid, at from $40^{\circ}$ to $50^{\circ}$. This is repeated two or three times, and the united filtered extracts are evaporated to a syrup, which is treated with four volumes of alcohol and digested for twenty-four hours at $30^{\circ}$. After cooling, the alcoholic extract is filtered, the residue washed with 70 per cent. alcohol, and the united filtrates freed from alcohol by distillation. The aqueous residue, diluted if desirable, is filtered and submitted to the following extractions :

1. The acid liquid is shaken with freshly rectified petroleum ether, as long as this reagent leaves a residue on evaporation.

2. The acid fluid is now extracted with benzene.

3. The next solvent used is chloroform.

4. The liquid is now again extracted with petroleum ether in order to remove traces of benzene and chloroform.

5. The liquid is next made alkaline with ammonia and successively extracted with petroleum ether, benzene, chloroform, and amylic alcohol.

6. The remainder of the ammoniacal liquid is mixed with powdered glass, evaporated to dryness, the residue pulverized, and extracted with chloroform.

The residue obtained with each of the above solvents should be examined for ptomaïns.

Brieger's Method.-The substance under examination is divided as finely as possible, and then heated with water slightly acidified with hydrochloric acid. During the heating care must be taken that

FIG. 2.

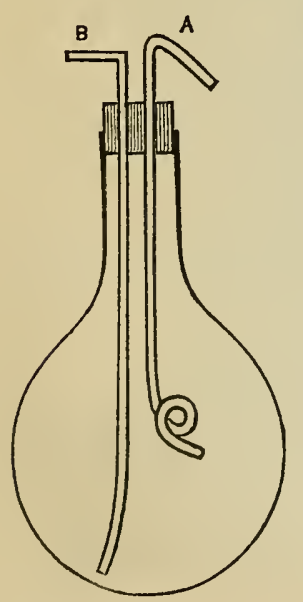
the feebly acid reaction is maintained, and the heat should continue for only a few minutes. The liquid is then filtered and concentrated, at first on a plate and then on the water-bath, to a syrup. If one has highly odorous material, a piece of apparatus devised by Bocklisch is of service. The fluid to be evaporated is placed in a globular flask, the rubber stopper of which carries two small glass tubes, one of which, $B$, extends to the bottom of the flask, while $A$ is connected with a water-pump or aspirator, which draws the vapor through the tube. In order to prevent the return of condensed fluids, the end of $A$ in the flask is curved on itself. The tube $B$ is finely drawn out and through it a current of air is constantly moving and prevents the 
formation of a deposit or a pellicle in the fluid. By regulating the amount of air entering through this tube, more or less of a vacuum will be formed in the flask. After evaporation to a syrup, an extraction is made with 96 per cent. alcohol and the filtered extract is treated with a warm alcoholic solution of lead acetate. The lead precipitate is removed by filtration, the filtrate evaporated to a syrup and again extracted with 96 per cent. alcohol. The alcohol is driven off; the residue taken up with water; traces of lead removed with hydrogen sulphid; and the filtrate, acidified with hydrochloric acid, evaporated to a syrup, which is extracted with alcohol, and the filtrate precipitated with an alcoholic solution of mercuric chlorid. The mercury precipitate is boiled with water, and on account of the differences in solubility of the double compounds with mercury, one ptomain may be separated from others at this stage of the process. (If thought best, the lead precipitate may be freed from lead and carried through the subsequent steps of the process. Brieger found small quantities of ptomains in the lead precipitate only in his work with poisonous mussels.)

The mercury filtrate is freed from mercury, evaporated, and the excess of hydrochloric acid carefully neutralized with soda (the reaction is kept feebly acid); then it is again taken up with alcohol in order to free it from inorganic salts. The alcohol is evaporated, the residue taken up with water, the remaining traces of hydrochloric acid neutralized with soda, the whole acidified with nitric acid, and treated with phosphomolybdic acid. The phosphomolybdate double compound is separated by filtration and decomposed with neutral acetate of lead. This is hastened by heating on the water-bath. The lead is removed by hydrogen sulphid, the filtrate is evaporated to a syrup and taken up with alcohol, from which many ptomains are deposited as chlorids, or double salts may be formed in the alcoholic solution. The chlorids as deposited from the alcoholic solution are seldom pure and may be isolated by precipitation with gold chlorid, platinum chlorid, or picric acid, and on account of the difference in solubility of these double salts, the process of purification is rendered more easy. The chlorid of the base is obtained by removing the metal with hydrogen sulphid, while the picrate is taken up with water, acidified with hydrochloric acid, and repeatedly extracted with ether, in order to remove the picric acid.

The Methods of Gautier and Etard.-The putrid matters, liquid and solid, are distilled at a low temperature in vacuo. The distillate $(A)$ may contain ammonium carbonate, phenol, skatol, trimethylamin, and the volatile fatty acids. The residue after filtration is treated successively by ether and alcohol.

The extraction with ether $(B)$ separates the ptomain and some fatty acids. The alcoholic extract $(C)$ removes the remainder of the 
fatty acids, as well as the acid and neutral nitrogenous bodies, almost all of which are crystallizable. The insoluble residue is boiled with dilute hydrochloric acid, with exclusion of air, finally evaporated to dryness, and the residue again extracted with alcohol. This nearly alkaline solution $(D)$ can be divided by acetate and subacetate of lead into two portions.

Gautier has also employed the following method: The putrid liquids, after the removal of fats, are feebly acidified with dilute sulphuric acid, then distilled in vacuo at a low temperature. The distillate contains ammonia, phenol, indol, and skatol. The syrupy residue, separated from any crystals which may have formed, is rendered alkaline with baryta, filtered, and extracted a number of times with chloroform, in order to dissolve the bases. The solution is distilled at a low temperature, either in vacuo or in a current of carbonic acid. The contents of the retort, on being treated with water and tartaric acid, separate into a brown resin and a liquid portion. The latter is removed and treated with a dilute solution of potash, when it gives off the odor of carbylamin, which was discovered by Gautier in 1866, and which according to Calmel, is a constituent of the venom of toads. The alkali also sets free the bases, which are removed by extraction with ether, and the ether evaporated in a current of carbonic acid under slight pressure, then under a bell-jar over caustic potash. The bases may be separated by fractional precipitation with platinum chlorid, or, if present in sufficient quantity, by distillation in vacuo.

In some instances, Gautier modified his method as follows: The alkaline putrid liquid is treated with oxalic acid to free acidulation, and as long as the fatty acids continue to separate. The liquid is then warmed and distilled until the distillate ceases to be turbid. Pyrrol, skatol, phenol, indol, volatile fatty acids, and some of the ammonia pass over. The portion remaining in the retort is rendered alkaline with lime water and the precipitate which forms and contains the greater part of the fixed acids is removed. The liquid portion, which is alkaline, is distilled to dryness, care being taken to receive the distillate in dilute sulphuric acid. The bases and ammonia pass over. The distillate is neutralized with sulphuric acid and evaporated almost to dryness, then decanted from the ammonium sulphate which crystallizes. The mother liquor is extracted with concentrated alcohol, which dissolves the sulphates of the ptomaïus. After driving off the alcohol the residue is rendered alkaline with caustic soda, and successively extracted with ether, petroleum ether, and chloroform. The lime precipitate is dried and extracted with ether which removes any fixed bases that may be present.

Remarks Upon the Methods.-Guareschi and Mosso condemn the method of Dragendorff, inasmuch as they have found that basic 
bodies are formed by the action of dilute sulphuric acid upon undecomposed albuminous substances, and they recommend the employment of the Stas-Otto method with these conditions: (1) No more acid should be added than is absolutely necessary to keep the reaction acid; (2) the heat used in evaporation should not be great, and it is better that evaporation should be made in vacuo. In this way, they say, no basic substance will be obtained from fresh tissue.

Marino-Zuco ascertained that by treating fresh eggs, brain, liver, spleen, kidney, lungs, heart and blood by either the Stas-Otto or the Dragendorff method, he could obtain a substance which gave alkaloidal reactions, and which he demonstrated to be cholin. His experiments led him to believe that cholin does not exist preformed in these fresh tissues, but that it results from the action of the dilute acids upon lecithin. Cholin was found most abundantly in those tissues which are rich in lecithin, such as the yolks of eggs, brain, liver and blood; while only traces could be obtained from the whites of eggs, lungs and the heart. The method of Dragendorff was found to furnish much larger quantities of cholin than could be obtained by the Stas-Otto process. Coppola agrees with his countrymen in condemning the method of Dragendorff as a means of extracting ptomaïns. However, the Stas-Otto method is by no means perfect, and the principal difficulties met with in its application are as follows: (1) In most instances the extraction of the base is incomplete ; (2) the degree to which the putrefactive alkaloid is removed by the solvent depends largely upon the nature of the other substances present. This fact in some cases aids and in others hinders the labors of the investigator; thus, several ptomaïns, which when pure are wholly insoluble in ether, may be removed in part, at least, from organic mixtures by this solvent by passing into the solution along with other substances; but if the attempt be made to purify one of these bases by repeated solution and extraction with ether, the result is failure, because the more completely the alkaloid is freed from impurities, the less soluble in ether it becomes. This criticism is equally applicable to the Dragendorff method, and to all others in so far as extractions are made. However, we may state that the StasOtto method is in suitable cases the best that can be employed. By it the substances are submitted to the least chemical manipulation, and the results obtained are the most reliable. Many of the more complex putrefactive products are so easily decomposed or otherwise altered that the investigator should seek to isolate them by the simplest methods possible. If it can be done without the addition of any acid or without the application of heat, so much the better.

By his method, Brieger discovered a number of basic bodies, and gave great impetus to the study of the chemistry of putrefaction ; but it is open to the objection that basic substances may be formed by the action of the reagents used, and Gram states that when the 
lactate of cholin, an inert substance which is widely distributed both in plants and animals, is heated, it is converted into a poison which has a muscarin-like action. However, Brieger obtained some of his bases by a much simplified modification of his complete method; for instance, in obtaining neuridin, he treated the aqueous extract of the putrid material, after boiling and filtration, with mercuric chlorid, collected the precipitate, decomposed it with hydrogen sulphid, evaporated the filtrate on the water-bath, and extracted the base from the residue with dilute alcohol. Brieger's method is only a modification of that employed by Bergmann and Schmiedeberg as long ago as 1868 in the preparation of sepsin from putrid yeast. 


\section{CHAPTER XIII.}

\section{THE IMPORTANCE OF BACTERIAL PRODUCTS TO THE TOXICOLOGIST.}

THE presence in the cadaver of substances which give not only the general alkaloidal reactions, but respond to some of the tests which have hitherto been considered eharacteristic of individual vegetable alkaloids, is of importance to the toxicologist. The possibility of mistaking putrefactive for vegetable alkaloids should always be borne in mind by the chemist in making any medico-legal investigations. On the other hand, cases of poisoning with bacterial products sometimes terminate fatally, and in such instances the chemist should not be satisfied with determining the absence of mineral and vegetable poison, but should strive to detect in the food or in the dead body positive evidence of the presence of the putrefactive poison.

We herewith append a brief account of some of the cases in which putrefactive substances have been found to resemble in their reactions the vegetable alkaloids.

Coniin-like Substances.-In the Brandes-Krebs trial, which took place in Braunschweig in 1874, two chemists obtained from the undecomposed parts of the body, in addition to arsenic, an alkaloid which they pronounced coniïn. This substance was referred to Otto for further examination and he reported that it was neither coniïn nor nicotin, nor any vegetable alkaloid with which he was acquainted. He converted the substance into an oxalate, dissolved it in alcohol, evaporated the alcohol, dissolved the residue in water, rendered this solution alkaline with potash, then extracted the bases with petroleum ether. On evaporation of this extract the alkaloid appeared as a bright yellow oil, which had a strong unpleasant odor, quite different, however, from that of coniïn. It was strongly alkaline, had an intensely bitter taste, and was volatile at ordinary temperature. From its aqueous solution it was precipitated by the chlorides of gold, platinum and mercury. In these reactions it resembled nicotin from which it differed in the double refractive and crystalline character of its hydrochlorid. With an ethereal solution of iodin this substance did not give the Roussin test for nicotin, but instead of the long ruby-red crystals there appeared small, dark green, needle-shaped ones. 
This substance was found to be highly poisonous, inasmuch as 7 cg. injected subcutaneously into a large frog produced instantaneous death, and $44 \mathrm{mg}$. given to a pigeon cansed a similar result. On account of its poisonous properties the jury of medical experts decided that the substance was a vegetable alkaloid, notwithstanding the fact that Otto's experiments demonstrated that this could not be true.

Brouardel and Boutmy found in the body of a woman who had died, after suffering with ten other persons, from choleraic symptoms after eating of a stuffed goose, a base which gave the odor of coniïn and the same reactions with gold chlorid and iodin in potassium iodid, etc., as coniïn. The same base was found in the remainder of the goose. It did not give a red coloration with the vapor of hydrochloric acid, and it did not form butyric acid on oxidation, and, although it was poisonous, it did not induce in frogs the symptoms of coniïn poisoning.

Selmi repeatedly found coniïn-like substances in decomposing animal tissue. By distilling an alcoholic extract from a cadaver, acidifying the distillate with hydrochloric acid, evaporating, treating the residue with barium hydrate and ether, and allowing the ether to evaporate spontaneously, he obtained a residue of volatile bases, the greater portion of which consisted of trimethylamin. After removing the trimethylamin the residue had the odor of mouse urine. Later, Selmi obtained an unmistakable coniïn odor from a chloroform extract of the viscera of a person who had been buried six months, and in another case ten months after burial. Two or three drops of an aqueous solution of the alkaline residue of the chloroform extract, allowed to evaporate on a glass plate, gave off such a penetrating odor that Selmi was compelled to withdraw from close proximity to the substance, and the odor imparted to his hands in testing with the general alkaloidal reagents remained for half an hour.

An aqueous solution of a ptomaïn obtained by Selmi by extraction with ether, according to the Stas-Otto method, from the undecomposed parts of a cadaver, had no marked odor, but after being kept for a long time in a sealed tube it not only gave off a coniïn odor, but the vapor turned red litmus paper blue. Again, the sulphate of a ptomaïn obtained from putrid egg-albumiu, on standing, formed in two layers, one of which was a golden-yellow liquid, which ou treatment with barium hydrate gave off ammonia and later the odor of coniïn. Since butyric and acetic acids were formed by the oxidation of this base, Selmi concluded that it was real coniïn or methylconiin, and that it was formed by the oxidation of certain fixed ptomaïns, or by the action of different amido bases on volatile fatty acids. The substance, which was found by Sonnenschein in a criminal trial in East Prussia and which was believed by that 
chemist to be the alkaloid of the water hemlock (Cicuta virosa), is thought by Otto, Husemann, and others, to be a cadaveric coniïn. Otto says that the symptoms reported in the case were not those of either coniin or cicuta. Sonnenschein obtained the base six weeks after the exhumation of the body, which had been buried three months. The base had the odor of coniïn, the taste of tobacco, gave with potassium bichromate and sulphuric acid the odor of butyric acid, and behaved with the reagents like coniïn.

Husemann states that it is very difficult, if not impossible, for the chemist to affirm with certainty that he has detected true couiïn in the body. The symptoms and the post-mortem appearances must couform with those induced by the vegetable alkaloid. The analysis must be made before decomposition sets in, and the amount of the base found must be sufficient for physiological experimentation.

A Nicotin-like Substance.-Wolckenhaar obtained from the decomposed intestines of a woman, who had been dead six weeks, by extraction with ether from an alkaline solution, a base bearing a close resemblance to nicotin. This base was fluid, at first yellow, but becoming brownish-yellow on exposure to the air. It was strongly alkaline and gave off an odor resembling that of nicotin, but stronger, more ethereal, benumbing, and similar to that of fresh poppy heads. It was soluble in all proportions of water, and the solutions, which did not become cloudy on the application of heat, did not taste bitter, but were slightly pungent. The peculiar odor did not disappear on saturating the base with oxalic acid. The hydrochlorid was yellow like varnish, had a strong odor and became moist on exposure to the air. Under the microscope it showed no crystals, thus differing from nicotin hydrochlorid. It also differed from nicotin in its reactions with potassio-bismuthic iodid, gold chlorid, iodin solution, mercuric chlorid, and platinum chlorid, and it did not give the Roussin test. Furthermore, it could not be identified with trimethylamin, spartein, mercurialin, lobelin, or other fluid and volatile bases.

Strychnin-like Substances.-In a criminal prosecution at Verona, Ciotta obtained from the exhumed, but only slightly decomposed, body, an alkaloid which gave a crystalline precipitate with iodin in hydriodic acid, a red coloration with hydriodic acid, and a color test similar to that of strychnin with sulphuric acid and potassium bichromate. This substance was strongly poisonous, but did not produce the tetanic convulsions characteristic of strychnin. Ciotta pronounced this substance as probably identical with strychnin, but Selmi, to whom portions of the body were subsequently submitted, found that the substance giving the color reaction was not crystalline, and that there was only the presumption of a bitter taste to it, while 
one part of strychnin in forty thousand parts of water is intensely bitter. Selmi also held that many ptomains give reactions similar to strychnin with iodin in hydriodic acid and with hydriodic acid alone. $\mathrm{He}$ also held that the physiological properties of this substance were such that it could not be strychnin. It could hardly have been aspidospermin, which reacts with sulphuric acid and potassium bichromate similarly to strychnin, because quebracho bark, in which this alkaloid is found was not at that time used as a medicine or known in Italy.

Ptomaïns giving reactions similar to those of strychnin, and also causing tetanic spasms, have been found in decomposed corn-meal, and Selmi obtained one of these substances which differed from strychnin inasmuch as it could not be extracted with ether.

Lombroso named the poisonous substance found in decomposed corn-meal "pellagrocein," but this is a mixture of ptomaïns, some of which produce narcosis and paralysis, and others induce the symptoms of nicotin poisoning instead of the spasms caused by strychnin.

Morphin-like Substances.-In the Sonzogna trial at Cremona, Italy, the experts seem to have confounded a ptomaïn with morphin. This substance was removed from either alkaline or acid solutions with ether, but could be extracted with amylic alcohol. It reduced iodic acid, but in its other reactions, also in its physiological properties, it bore no resemblance to morphin. In frogs it arrested the heart in systole, which is said never to happen in poisoning with morphin. It failed to give both the ferric chlorid and the Pellagri test for morphin.

In the same body there was found a substance which was extracted from alkaline solutions with ether, and which gave, with hydrochloric acid and a few drops of sulphuric acid, on the application of heat, a reddish residue similar to that obtained by the same reagents with codein, but in its other reactions it did not resemble this alkaloid.

Many of the tests for morphin employed by toxicologists are fallacious. In the examination of a stomach and part of a liver, sent from Lincoln, Neb., Vaughan, following the method of Dragendorff, obtained in the amylic alcohol extract from alkaline solution a residue that gave with more or less distinctuess all the principal color tests for morphin ; but failing to obtain crystals that could be identified as those of this alkaloid, the absence of morphin was reported. Haines, working with the same material, obtained similar reactions; he also was unable to secure the crystals and made a negative report. Afterward, it was quite positively shown that death had been caused in this case by a blow on the back of the head and that no morphin or other drug had been administered.

In the Buchanan case in New York, the symptoms as sworn to by the attending physician clearly were not those of morphin, and all 
the tests obtained by the experts were duplicated with putrefactive products. Many skillful chemists have carried companion portions of decomposed tissue, one portion with, and the other without morphin, through the process of extraction recommended by Dragendorff, and have obtained satisfactory results, finding that the proper residue responds to the color test in the one instance and fails to do so in the other. Tissues have been thus tested in apparently every stage of putrefaction, and the results have been satisfactory and.confirmatory of the methods generally employed. There is one point, however, which has been constantly overlooked. The putrefaction to which the tissues in these experiments are subjected has been aërobic, while that occurring in the dead body is anaërobic ; consequently, the putrefactive products are not the same in the two cases. In all experimental studies of the value of the tests for morphin in decomposing tissue, putrefaction must be allowed to proceed in the absence of oxygen. There is a second point, probably of equal importance, and this concerns the kind of tissue employed. The upper portion of the small intestines (and the adjacent tissue after death) has a bacterial flora peculiar to itself. These tissues are the ones quite universally examined in medico-legal cases, and consist of the small intestine itself, the stomach, the liver, the pancreas, the spleen, and, in some instances, the kidneys. Of course, the bacteria present in the small intestine during life may after death extend into the abdominal and thoracic viscera.

Vaughan allowed finely-chopped ox-liver to ferment for thirty days under anaërobic conditions and then divided the decomposed mass into three portions, which were marked A, B and C. To B, 130 mg. of morphin sulphate was added, and to $C$ the same amount of morphin together with 0.5 gram each of indol, skatol and phenol; no addition was made to $A$. These portions were carried simultaneously through the manipulations recommended by Dragendorff. To the residues obtained from the amylic alcohol extract of alkaline solutions, in which the chemist expects to find morphin, all the known color tests for morphin were applied and all the residues responded to these tests in the same manner. In all, nitric acid gave a lemon-brown color; sulphuric acid showed no change; sulphuric with nitric acid gave a lemon-yellow, slowly changing to a pink; ferric chlorid gave a dirty green ; iodic acid was promptly reduced; Fröhde's reagent gave a blue color without any violet; and sulphuric acid and cane sugar produced a brownish-red color, changing to a wine-red. These investigations show that the color tests for morphin, employed by toxicologists in following the scheme of Dragendorff, are wholly untrustworthy. The substances in these extracts which vitiate the tests for morphin probably consist of indol and its derivatives. Germs which produce indol and kindred substances are native and constant representatives of the bacterial flora of the 
upper portion of the small intestiue. There are many indol-forming bacteria, and while some of these may be present in any tissue, they are certainly present in health and in disease, during life and after death, in the small intestine.

Indol and its derivatives are products of anaërobic putrefaction and this accounts for the fact that the reactions obtained in the abovementioned experiments are not familiar to those toxicologists who have employed tissue allowed to putrefy in the presence of oxygen. It should be remembered that indol may be prepared synthetically and it may be obtained from putrefying tissue. Samples obtained from the two sources do not always give exactly the same reactions. Skatol is another product of anaërobic putrefaction which may interfere with the tests for morphin. While it would be comparatively easy to distinguish pure morphin from either indol or skatol, the results of the above-mentioned experiments show that the separation of morphin from tissue, decomposing in the absence of oxygen, and its identification are, by the methods now generally employed, so uncertain that the conscientious chemist will seek for methods free from these sources of error before he gives positive testimony of the presence of this alkaloid. We have spoken of indol and its derivatives as being present in decomposing tissue, and it should be stated that the number of known indol derivatives is by no means small, and how many others there may be which remain unknown no one can tell. Many of these substances give brilliant color reactions. Indoxyl is an easily decomposable substance, which gives some striking color reactions, among which may be mentioned the production of indigo blue with ferric chlorid in the presence of free hydrochloric acid.

Skatol-carbonic acid is another product of putrefaction and E. and H. Salkowski obtained 1.3 grams from $2 \mathrm{~kg}$. of moist fibrin after twenty-six days' putrefaction. Among the known color reactions of this substance Hoppe-Seyler mentions the following:

1. If a dilute solution of this acid $(1: 1000)$ be treated with a few drops of pure hydrochloric acid of 1.2 specific gravity, and then with a few drops of potassium nitrate solution ( 2 per cent.), a cherry-red coloration is produced, and later a red precipitate falls.

2 . If such a solution be mixed with an equal volume of hydrochloric acid, and then a few drops of chlorid of lime solution ( $\frac{1}{2}$ per cent.) be added, a purple-red color is produced.

3. Treated with a few drops of hydrochloric acid, then with two or three drops of a very dilute solution of ferric chlorid and heated, the mixture becomes intensely violet before boiling. Skatol-carbonic acid is non-volatile.

Skatol-acetic acid has been obtained by Nencki by the anaërobic putrefaction of serum albumin. The aqueous solutions of this substance give with ferric chlorid a white cloudiness, which on warm- 
ing becomes a brick-red, and in more concentrated solution fire red. Both indigo red and indigo blue may be formed by oxidation of indol.

Knowing that indol and its derivatives are formed in anaërobic putrefaction, and that in Dragendorff's scheme for the separation and identification of vegetable alkaloids, these substances appear in the residues which are tested for morphin, and knowing the great number and variety of color reactions given by these substances, it may be asked, How much reliance can be placed on the color tests for morphin? Besides the indol bodies, other substances are formed in the anaërobic putrefaction of proteid bodies and among these are certain aromatic products of the putrefaction of tyrosin. The following may be mentioned : (1) Hydroparacumaric acid (paraoxyphenylpropionic acid). This substance gives with ferric chlorid a distinct but evanescent blue coloration. (2) Paraoxyphenylacetic acid. This substance gives with ferric chlorid a pale grayish-violet, which soon changes to a dirty green color.

Among other products of anaërobic putrefaction of proteids, phenol and parakresol may be mentioned; with the former ferric chlorid gives a violet color and with the latter a blue.

In the case of Dr. Urbino de Freitas ${ }^{1}$ of Oporto, Portugal, who was accused of attempting the wholesale murder of his wife's family by the administration of morphin, the toxicologists relied for the detection of this alkaloid upon the color reactions applied to residues obtained by following Dragendorff's method. They also reported the presence of narcein and the probable presence of delphinin. The defense questioned the reliability of the tests used and a large number of the most prominent toxicologists in Europe became involved in the controversy. It was claimed by the prosecution that $200 \mathrm{mg}$. of morphin had been recovered. On this point Beckurts stated that it was incomprehensible to him that a toxicologist should recover $200 \mathrm{mg}$. of morphin and not be able to present a part of it in evidence, and also to furnish a portion to the experts on the other side. Brieger and Bischoff thought $200 \mathrm{mg}$. a fabulous quantity to recover and that a portion of the poison should have been presented in evidence. Husemann thought the tests relied upon insufficient, and that putrefactive substances had been mistaken for the vegetable alkaloid, and even Dragendorff himself thought it likely that this mistake had been made. For the detection of the morphin the toxicologists for the prosecution had relied chiefly upon the application to the amylic alcohol residue of the tests with iodic acid, Fröhde's reagent, and sulphoselenic acid (known as Lafon's reagent). The chemists consulted quite generally agreed that these tests are unreliable and that the amylic alcohol extract of putrefying material frequently responds to these tests when morphin is known not to be present.

${ }^{1}$ Relation medico-legale de l'affaire Urbino de Freitas. 
Atropin-like Substances.-Many investigators have found putrefactive products which in their mydriatic properties resemble atropin and hyoscyamin. To this class belongs the substance observed by Zuelzer and Sonnenschein. It was removed from alkaline solutions by ether, and formed microscopic crystals, an aqueous solution of which, when applied to the conjunctiva, produced a mydriatic effect, and, when administered internally, increased the action of the heart and arrested the movements of the intestines. Moreover, in its behavior with certain alkaloidal reagents, such as platinum chlorid, it resembled atropin; but when heated with sulphuric acid and oxidizing agents it did not give the odor of blossoms (Reuss's test). However, Selmi found ptomatropins which with sulphuric acid and oxidizing agents did give the blossom odor as distinctly as the vegetable atropin. These putrefactive bases also developed this odor spontaneously after standing for two or three days, and this does not happen with atropin. The odor was produced with the ptomatropins by nitric and sulphuric acids both in the cold and on the application of heat, while these acids in the cold did not produce the odor with atropin. Ptomatropins have been found in decomposing fish, corned beef, putrid game, and poisonous sausage. It is not known whether there is only one or more of these poisons. The symptoms often resemble those of belladonna poisoning very closely. The throat becomes dry, the muscles of deglutition seem to be paralyzed, the secretion of perspiration and saliva is arrested, mydriasis may be marked, and there may be paralysis of accommodation, ptosis, and strabismus. In some instances delirium and in others convulsions appear. The heart-beat becomes rapid and weak. The tongue is coated, and in the most dangerous cases constipation is obstinate. The general weakness may be extreme, and the voice wholly lost. Section shows the pharynx swollen, hemorrhagic spots in the œsophagus, stomach and intestines, cloudy swelling of the solitary follicles and Peyer's patches, and degeneration of the heart muscle. The brain, lungs, and kidneys are often hyperemic. Extracts of putrid material will often cause more or less dilatation of the pupil in the lower animals when applied locally. Haines and Vaughan were once appointed a commission to inquire into the tests obtained by a toxicologist who had reported four grains of atropin in the stomach of a man who had been dead for some weeks. The chief test relied upon by the chemist was that an ounce of extract from the stomach dilated a cat's pupil about as much as a solution of four grains per ounce of atropin sulphate. It is needless to comment on the validity of such evidence.

Digitalin-like Substances.-Rörsch and Fasbender discovered a putrefactive base which affected animals very much like digitalin. Trottarelli obtained a similar substance from the brain of a man in 
whose abdominal viscera he could find no poison. The sulphate of this base gave on evaporation an aromatic smelling and astringent tasting residue. It became purple with sulphuric acid only, and dark red with hydrochloric and sulphuric acids. On frogs this ptomaïn had no toxic action.

A Veratrin-like Substance.-Brouardel and Boutmy obtained from a corpse which had lain in water for eighteen months, and a large portion of which had changed into adipocere, a ptomain resembling veratrin. It was removed from alkaline solution by ether, and on being heated with sulphuric acid it became violet. With a mixture of sulphuric acid and barium peroxid it became, in the cold, brickred; and, on being heated, violet. With boiling hydrochloric acid it took on a cherry-red coloration. However, it differed from veratrin, inasmuch as it reduced ferric salts instantly, and when injected into frogs subcutaneously it did not induce the spasmodic muscular contractions characteristic of veratrin. Bechamp obtained, by the Stas-Otto method from the products of the digestion of fibrin, an alkaloidal body which gave with sulphuric acid a beautiful carminered, similar to that given with gastric juice, and again extracting, he obtained a body which behaved with sulphuric acid similarly to curarin.

A Delphinin-like Substance.-In 1870 Gibbone, an Italian of prominence, died suddenly, and his servant was accused of having poisoned him. Two chemists reported the presence of delphinin in the viscera, but it seemed improbable that the servant should know anything of so rare a substance, or that he should have been able to obtain it. However, two or more varieties of staphisagria grow in southern Italy, and it was possible that the servant had used some preparation made by himself from the plant. The supposed alkaloid was given to Selmi for further study. It was removed from alkaline solutions with ether. When heated with phosphoric acid it became red, and when brought into contact with concentrated sulphuric acid reddish-brown. In these tests the substance resembled delphinin, but with sulphuric acid and bromin water, the colorations characteristic of the vegetable poison failed to appear. Moreover, Selmi showed that delphinin gives the following reactions to which the suspected substance does not respond :

1. Delphinin dissolved in ether, and treated with a freshly prepared ethereal solution of platinic chlorid, gives a white flocculent precipitate, which is insoluble in an equal volume of absolute alcohol.

2. Delphinin gives precipitates with auro-sodium hyposulphite, and with a sulphuric acid solution of cupro-sodium-hyposulphite, the latter precipitate being soluble in an excess of the reagent. Finally Ciaccia and Vella showed that while delphinin arrests the 
heart of the frog in diastole, the suspected substance arrests it in systole.

A Colchicin-like Substance.-Baumert found, in a case of suspected poisoning, twenty-two months after death, a substance giving many of the reactions for colchicin. It was extracted from acid solutions with ether, to which it imparted a yellow color. On evaporation of the ether, a yellow, amorphous substance remained, and this dissolved in warm water with yellow coloration. It could be extracted from acid solutions also by chloroform, benzol and amylic alcohol, but not from petroleum ether. It was removed with much more difficulty from alkaline solutions. All the extracts were yellow and left on evaporation a strictly alkaline, markedly bitter, sharp tasting, amorphous yellow residue, which dissolved in water and dilute acids incompletely, forming a resin. When this resin was dissolved in dilute sodium hydrate and the solution rendered acid with sulphuric acid, the same reactions were obtained as with the original extract. With phosphomolybdic acid, phosphotungstic acid, potassio-bismuthic iodid, potassio-mercuric iodid, iodin in potassium iodid, cyanic acid, and gold chlorid, this substance gave the same reactions which were obtained by parallel experiments with colchicin. Thus, the tannic acid precipitates were both soluble in alcohol, and the precipitates with phosphomolybdic acid in both cases became blue on the addition of ammonium hydrate. Concentrated sulphuric and dilute nitric and hydrochloric acids dissolved the supposed colchicin with yellow coloration. Strong nitric acid colored the substance dirty red, scarcely to be called a violet. When the substance was purified as much as possible, this color became a beautiful carmine-red. The addition of water changed the red into yellow, and caustic soda produced a dark dirty orange. In general, in the above-mentioned reactions, the putrefactive product agreed with colchicum, but the former gave precipitates with picric acid and platinum chlorid, while the latter gave no precipitates with these reagents.

In 1886, Zeisel proposed the following test for colchicum : When a hydrochloric acid solution of the alkaloid is boiled with ferric chlorid, it becomes green, sometimes dark green, and cloudy; then, if the fluid be agitated with chloroform, the chloroform takes up the coloring matter and appears brownish-red, or dark, and the supernatant fluid clears up without becoming wholly colorless. Baumert applied this test to both colchicum and the putrefactive product. To from 2 to 5 c.c. of the suspected solution in a test-tube, he added from five to ten drops of strong hydrochloric acid, and from four to six drops of a 10 per cent. solution of ferric chlorid, then heated the mixture directly over a small flame until it was evaporated to half its volume or less. In the presence of $1 \mathrm{mg}$. of colchicin the origi- 
nally bright yellow solution became gradually green, and, on further concentration, dark green and cloudy; then, on shaking the fluid with chloroform, admitting as much air as possible, the chloroform subsided, having a red color if as much as $2 \mathrm{mg}$. of colchicin were present, and a bright yellow color if only $1 \mathrm{mg}$., and the supernatant fluid became a beautiful olive green. When either petroleum ether, benzol, carbon bisulphid, or amylic alcohol was substituted for the chloroform the coloration did not appear. From this Baumert infers that the red coloring matter is either soluble in chloroform or that it is not formed until the chloroform is added. He found that the putrefactive product did not respond to this test. Some of this substance was sent to Brieger, who decided that it was not a base, but a pepton-like substance; it was also found to be inert physiologically.

Liebermann found a similar colchicin-like substance in a cadaver. His description differed from that of Baumert only in regard to the taste of the substance, Liebermann having failed to observe any marked taste in his body, while Baumert reported a distinctly bitter taste. A colchicin-like substance has been found in beer, and it has been suggested that it was this that the above-mentioned toxicologists found in the bodies which they examined, but Liebermann states that the man whose body he analyzed had beeu a total abstainer from beer. 


\section{CHAPTER XIV.}

\section{CHEMISTRY OF THE PTOMAÏNS.}

THE basic substances described in the following pages are arranged, as far as possible, in the regular natural order. An inspection of the list of these bases will show the remarkable fact of the predominancy of the amine type. Almost two-thirds of the known ptomains contain only $\mathrm{C}, \mathrm{H}$, and $\mathrm{N}$, and represent simple ammonia substitution compounds. Of the oxygenated bases, all of those whose constitution is known possess the trimethylamin group as their basic constituent, and it is quite probable that most, if not all, of the remaining ptomains will be found to possess the same or a similar basic nucleus.

It will be seen, furthermore, that a very large number of the ptomaïns described possess little or no toxic action, and are, therefore, physiologically inert. It would seem, as Brieger has already pointed out, that a certain quantity of oxygen is necessary to the formation of poisonous bases. A free supply of oxygen, on the other hand, invariably yields non-toxic ptomaïns. The poisonous bases begin to appear about the seventh day of putrefaction, and in turn disappear if this is allowed to go on for a considerable period of time.

It has already been pointed out that the ptomaïns are to be regarded as extra-cellular products of bacterial activity. They do not originate within the bacterial cell and therefore they are not to be looked upon as direct metabolic products of the cell protoplasm but rather as secondary cleavage products. The production of amines and diamines, for example, is clearly due, as in the case of monamino acids leucin and tyrosin, to the splitting off of preëxisting groups in the substance acted upon. This fact has been made especially clear in the demonstration of the origin of putrescin and cadaverin from the proteid bases arginin and lysin; and of cholin and allied bases from lecithin.

Methylamin, $\mathrm{CH}_{3} \cdot \mathrm{NH}_{2}$; this is the simplest organic base that is formed in the process of putrefaction. It is ammonia in which one atom of hydrogen has been replaced by the methyl radical. It occurs in herring-brine (Tollens, 1866 ; Bocklisch, 1885) ; in decomposing herring, twelve days in spring (Bocklisch); in pike, six days in summer (Bocklisch); in haddock, two months at a low temperature (Bocklisch); in the fermentation of cholin chlorid (Hasebroek). Brieger found it present in cultures of comma bacillus on beef-broth 
which were kept for six weeks at $37^{\circ}-38^{\circ}$. Ehrenberg reported its possible presence in poisonous sausage, and obtained it by growing a bacillus from this source on intestines (1887). Mörner (1896) found it in fermented fish associated with di- and tri-methylamins and cholin. Emmerling (1897) found it with the latter and a base $\mathrm{C}_{8} \mathrm{H}_{11} \mathrm{~N}$ in the streptococcus decomposition of fibrin. In Brieger's method methylamin is found in both the mercuric chlorid precipitate and filtrate. The mercury double salt is readily soluble in water, and can thus be separated from any accompanying cadaverin or putrescin. Methylamin is an inflammable gas of strong ammoniacal odor, and burning with a yellow flame. It is readily soluble in water, and its solutions give reactions similar to those of ammonia. Its salts are, as a rule, also soluble in both water and alcohol.

The hydrochlorid, $\mathrm{CH}_{3} \cdot \mathrm{NH}_{2} \cdot \mathrm{HCl}$, crystallizes in large deliquescent plates. On being heated with alkali, it gives off the odor of methylamin.

The platinochlorid, $\left(\mathrm{CH}_{3} \cdot \mathrm{NH}_{2} \cdot \mathrm{HCl}\right)_{2} \mathrm{PtCl}_{4}(\mathrm{Pt}=41.31$ per cent. $){ }^{1}$ yields hexagonal plates which usually occur heaped up in several layers. It is soluble in about fifty parts of water at ordinary temperature, and can be readily recrystallized from hot water. It is insoluble in absolute alcohol and in ether.

The aurochlorid, $\mathrm{CH}_{3} \cdot \mathrm{NH}_{2} \cdot \mathrm{HCl} \cdot \mathrm{AuCl}_{3}+\mathrm{H}_{2} \mathrm{O}$, forms prisms which are readily soluble in water. There is also a readily soluble picrate.

Methylamin does not possess any toxic action, even when given in fairly large doses. This physiological indifference is shared by nearly all the monamins and diamins that have been obtained among the products of putrefaction.

Dimethylamin, $\left(\mathrm{CH}_{3}\right)_{2} \cdot \mathrm{NH}$, has been found in putrefying gelatin, ten days at $35^{\circ}$ (Brieger, 1885) ; in yeast decomposing in covered vessels for four weeks during summer (Brieger); in decomposing perch six days in summer (Bocklisch); and in herring-brine (Bocklisch, 1886); in fermented fish (Mörner, 1896). It has been found in poisonous sausage, and in cultures of a bacillus, obtained from this source, on liver and intestines (Ehrenberg, 1887). It is also formed together with trimethylamin, when neuridin hydrochlorid is distilled with sodium hydrate (Brieger, I., 23). It occurs in the mercuric chlorid precipitate as well as filtrate. From cadaverin it can be separated by platinum chlorid, since cadaverin platinochlorid is difficultly soluble in cold water, and recrystallizes from hot water, whereas the dimethylamin double salt remains in the mother-liquor. In like manner it can be separated from neuridin. From cholin it can be

${ }^{1}$ The percentages given in the following pages are calculated from $\mathrm{Au}=196.64$ (Krüss), $\mathrm{Pt}=194.46$ (Seubert), $\mathrm{Cl}=35.37, \mathrm{O}=15.96$. 
isolated by recrystallizing the mercuric chlorid precipitate from hot water.

The free base is a gas at ordinary temperature, but can be condensed to a liquid which boils at $8^{\circ}-9^{\circ}$. The hydrochlorid, $\left(\mathrm{CH}_{3}\right)_{2}$.NH.HCl, crystallizes in needles, which deliquesce on exposure to air and are soluble in absolute alcohol (Brieger, I., 56). It is insoluble in absolute alcohol (Bocklisch), but soluble in chloroform (Behrend), and can then be separated from methylamin hydrochlorid, which is insoluble in chloroform.

The platinochlorid, $\left[\left(\mathrm{CH}_{3}\right)_{2} \cdot \mathrm{NH} . \mathrm{HCl}\right]_{2} \mathrm{PtCl}_{4}(\mathrm{Pt}=39.00$ per cent.), crystallizes in long needles, which are easily soluble in hot water, less soluble in cold water. Sometimes it forms orange-yellow plates or prisms, or else small needles.

The aurochlorid, $\left(\mathrm{CH}_{3}\right)_{2}$.NH.HCl.AuCl ${ }_{3}$, forms needles (Bocklisch), or large yellow monoclinic plates (Hjortdahl), which are insoluble in absolute alcohol.

With mercuric chlorid it unites to form two double salts (Mörner).

Trimethylamin, $\mathrm{C}_{3} \mathrm{H}_{9} \mathrm{~N}=\left(\mathrm{CH}_{3}\right)_{3} \mathrm{~N}$, has been known for a long time to occur in animal and vegetable tissues. Dessaignes showed its presence in leaves of Chenopodium (1851), in the blood of calves (1857), and later in human urine. It has been obtained from ergot (Secale cornutum) by Walz (1852) and Brieger (1886) ; from herringbrine by Wertheim, Winkles, Tollens and Bocklisch. In these substances, with the exception of herring-brine, it probably does not exist pre-formed, but is rather a product of the method employed for its isolation. In fact, Brieger has shown that it does not exist in ergot, but is formed at the expense of the cholin present, which, on distillation with potash, decomposes and yields trimethylamin and glycol. Thus :

$$
\mathrm{C}_{2} \mathrm{H}_{4} \mathrm{OH} \cdot \mathrm{N}\left(\mathrm{CH}_{3}\right)_{3} \cdot \mathrm{OH}=\mathrm{N}\left(\mathrm{CH}_{3}\right)_{3}+\mathrm{C}_{2} \mathrm{H}_{4}(\mathrm{OH})_{2} \text {. }
$$

It is also formed when betain and neuridin are distilled with potash. It may have a similar origin in most of the other cases, since cholin is now known to be widely disseminated in plants and animals, either as such or as a constituent of the more complex lecithin.

Trimethylamin has been found in the putrefaction of yeast (Hesse, 1857; Müller, 1858); in cheese after six weeks in midsummer (Brieger); in human liver and spleen after from two to seven days (Brieger); in perch after six days in midsummer (Bocklisch); in mussel (Mytilus edulis) after sixteen days (Brieger); in putrefying brains after from one to two months, and in fresh braius (Guareschi and Mosso); in cultures of the streptococcus pyogenes on beef-broth, bouillon, meat-extract, and blood-serum; from cultures of the comma-bacillus (Brieger), and from cultures of proteus vulgaris (Carbone). It has also been found in cod-liver oil. Ehrenberg (1887) reports its presence in considerable quantity in poisonous sausage, 
and in cultures of a bacillus, isolated from this, grown on liver, intestines, and meat-bouillon. Stadthagen has found it in normal urine; Kulneff in the feces of a case of gastroptosis. Mörner obtained it together with cholin and the preceding amins from "fermented fish," an article of food consumed in northern Sweden (1896). Emmerling found it in putrefying gluten, also in streptococcus decomposition of fibrin together with methylamin and the base $\mathrm{C}_{8} \mathrm{H}_{11} \mathrm{~N}$ (1897).

Trimethylamin is found in both the mercuric chlorid precipitate and filtrate. It remains in the mother-liquor from which cadaverin, neuridin, and dimethylamin platinochlorids have crystallized. If an aqueous solution of mercuric chlorid is used as the precipitant, the trimethylamin will be found almost entirely in the filtrate, from which it can be obtained after removal of the mercury by evaporating the filtrate to dryness, extracting with alcohol, and treating the solution thus obtained with alcoholic platinum chlorid.

The free base is a liquid possessing a strong, fish-like odor. Its boiling-point is $9.3^{\circ}$. It is strongly alkaline in reaction and freely soluble in water.

The hydrochlorid, $\left(\mathrm{CH}_{3}\right)_{3} \mathrm{~N} . \mathrm{HCl}$, is deliquescent and freely soluble in water and alcohol. Heated to $285^{\circ}$ it decomposes. With alkalis it gives off the odor of the free base.

The platinochlorid, $\left[\left(\mathrm{CH}_{3}\right)_{3} \mathrm{~N} . \mathrm{HCl}\right]_{2} \mathrm{PtCl}_{4}(\mathrm{Pt}=36.92$ per cent. $)$, is soluble in hot water, from which, on cooling, it recrystallizes in orange-red octahedra or needles, which do not lose water when heated at $100^{\circ}-110^{\circ}$ (Bocklisch).

The aurochlorid, $\left(\mathrm{CH}_{3}\right)_{3} \mathrm{~N} . \mathrm{HCl} \cdot \mathrm{AuCl}_{3}(\mathrm{Au}=49.39$ per cent.), is easily soluble, and hence can be separated from cholin aurochlorid, which is difficultly soluble. Similarly this base can be separated from ammonia by the use of gold chlorid. The mercurochlorid contains two molecules of $\mathrm{HgCl}_{2}$ (Mörner).

Trimethylamin is not a strong poison, since very large doses of it must be given in order to bring out physiological disturbances.

Ethylamin, $\mathrm{C}_{2} \mathrm{H}_{5} \cdot \mathrm{NH}_{2}$, is formed in putrefying yeast (Hesse, 1857 ); in wheat flour (Sullivan, 1858); and also in the distillation of beat-sugar residues.

It is a strongly ammoniacal liquid boiling at $18.7^{\circ}$ and is miscible with water in every proportion. Like the other amins, it is combustible. It possesses strong basic properties, and is capable of expelling ammonia from its salts in a manner analogous to the action of the fixed alkalis.

The hydrochlorid, $\mathrm{C}_{2} \mathrm{H}_{5} \cdot \mathrm{NH}_{2} \cdot \mathrm{HCl}$, forms deliquescent plates, which melt at $76^{\circ}-80^{\circ}$. It is readily soluble in water and alcohol.

The platinochlorid, $\left(\mathrm{C}_{2} \mathrm{H}_{5} \cdot \mathrm{NH}_{2} \cdot \mathrm{HCl}\right)_{2} \mathrm{PtCl}_{4}$, forms orange-yellow rhombohedra(Weltzien), or hexagonal-rhombohedral crystals(Topsoë). 
The aurochlorid, $\mathrm{C}_{2} \mathrm{H}_{5} \cdot \mathrm{NH}_{2} \cdot \mathrm{HCl} \cdot \mathrm{AuCl}_{3}$, forms gold-yellow monoclinic prisms, readily soluble in water.

With picric acid it forms short brown prisms, not very soluble in water.

Diethylamin, $\mathrm{C}_{4} \mathrm{H}_{11} \mathrm{~N}=\left(\mathrm{C}_{2} \mathrm{H}_{5}\right)_{2} \mathrm{NH}$, has been obtained by Bocklisch from pike which were allowed to putrefy for six days in summer ; and by growing a bacillus obtained from poisonous sausages on intestines and on meat-bouillon (Ehrenberg, 1887).

It is an inflammable liquid which boils at $57.5^{\circ}$, possesses strong basic properties, and is soluble in water.

The hydrochlorid, $\left(\mathrm{C}_{2} \mathrm{H}_{5}\right)_{2} \mathrm{NH} . \mathrm{HCl}$, crystallizes in needles (Bocklisch); in long needles and prisms from absolute alcohol; in plates from ether-alcohol. These are not deliquescent and are easily soluble in water and in chloroform ; rather difficultly in absolute alcohol. Heated with sodium hydrate it gives off alkaline vapors. From an alcoholic solution it is precipitated by addition of alcoholic mercuric chlorid. The mercury double salt is difficultly soluble in hot water, from which it recrystallizes on cooling.

The platinochlorid, $\left[\left(\mathrm{C}_{2} \mathrm{H}_{5}\right)_{2} \cdot \mathrm{NH} \cdot \mathrm{HCl}\right]_{2} \mathrm{PtCl}_{4}$, crystallizes in orange-yellow monoclinic crystals which are easily soluble in water.

The aurochlorid, $\left(\mathrm{C}_{2} \mathrm{H}_{5}\right)_{2} \mathrm{NH} . \mathrm{HCl} . \mathrm{AuCl}_{3}(\mathrm{Au}=47.71$ per cent.), forms trimetric crystals (Topsoë), which are difficultly soluble (Bocklisch). It melts at about $165^{\circ}$.

With picric acid it forms an easily soluble picrate (Lea).

Triethylamin, $\mathrm{C}_{6} \mathrm{H}_{15} \mathrm{~N}=\left(\mathrm{C}_{2} \mathrm{H}_{5}\right)_{3} \mathrm{~N}$, was obtained by Brieger (1885) from haddock which were exposed for five days in an open vessel during summer. He obtained it by distilling with potash, after removal of platinum by hydrogen sulphid, the mother-liquor from which neuridin, the base $\mathrm{C}_{2} \mathrm{H}_{8} \mathrm{~N}_{2}$, muscarin, and gadinin had successively crystallized (see gadinin). It has also been found by Bocklisch (1886) in putrid pike, and by Ehrenberg (1887). The latter obtained it from cultures of a bacillus, found in poisonous sausage, and grown on meat-bouillon.

The free base is oily in character and possesses an ammoniacal odor. It is but slightly soluble in water, and boils at $89^{\circ}-89.5^{\circ}$.

The platinochlorid, $\left[\left(\mathrm{C}_{2} \mathrm{H}_{5}\right)_{3} \mathrm{~N} . \mathrm{HCl}\right]_{2} \mathrm{PtCl}_{4} \quad(\mathrm{Pt}=31.84$ per cent.), crystallizes in needles, which are readily soluble in water.

With mercuric chlorid the aqueous solution gives no precipitate.

With picric acid it yields yellow needles which are but slightly soluble in cold water.

Propylamin, $\mathrm{C}_{3} \mathrm{H}_{7} \cdot \mathrm{NH}_{2}$, is isomeric with trimethylamin, and can therefore be easily confounded with that base. There are two propylamins possible represented by the formulæ $\mathrm{CH}_{3} \cdot \mathrm{CH}_{2} \cdot \mathrm{CH}_{2} \cdot \mathrm{NH}_{2}$ 
and $\left(\mathrm{CH}_{3}\right)_{2}$. $\mathrm{CH} \cdot \mathrm{NH}_{2}$. The former, or the normal compound, boils at $47^{\circ}-48^{\circ}$, whilst the latter, or iso-propylamin, boils at $31.5^{\circ}$. Both are liquids possessing an ammoniacal, fish-like odor. They form crystalline salts ; the hydrochlorids melt respectively at $155^{\circ}-$ $158^{\circ}$ and at $139.5^{\circ}$.

Iso-propylamin (?) has been found among the distillation products of the vinasse of beet-root molasses. Propylamin has been obtained by Brieger (1887) from cultures of the bacteria of human feces on gelatin. Schwanert has isolated from the organs of a cadaver a basic substance which was said to possess an odor similar to propylamin.

Butylamin, $\mathrm{C}_{4} \mathrm{H}_{11} \mathrm{~N}$, was obtained by Gautier and Mourgues (1888) in cod-liver oil. It forms a colorless, mobile, alkaline liquid, the boiling point of which they found to be $86^{\circ}$ at $760 \mathrm{~mm}$. It absorbs carbonic acid from the air and readily forms salts. The platinochlorid forms golden-yellow plates which are quite soluble.

In animals it produces an increase in the function of the skin and kidneys, and in large doses fatigue, stupor and vomiting.

Iso-amylamin, $\mathrm{C}_{5} \mathrm{H}_{13} \mathrm{~N}=\left(\mathrm{CH}_{3}\right)_{2} \cdot \mathrm{CH} \cdot \mathrm{CH}_{2} \cdot \mathrm{CH}_{2} \cdot \mathrm{NH}_{2}$, has been obtained by Limpricht in the distillation of horn with potash; it also occurs in the putrefaction of yeast (Müller, Hesse, 1857); and in cod-liver oil (Gautier and Mourgues, 1888), where it constitutes nearly one-third of the bases present. Since leucin on heating yields amylamin and carbonic acid it may be looked upon as a source of this ptomaïn.

It is a colorless, strongly alkaline liquid, possessing an odor which is not disagreeable. At the ordinary pressure it boils at $97^{\circ}-$ $98^{\circ}$.

The hydrochlorid forms deliquescent crystals, which have a bitter, disagreeable taste. The platinochlorid crystallizes in golden-yellow slender plates, which are very soluble in boiling water. The base is, according to Gautier and Mourgues, identical with that obtained by treating iso-amylcarbimid with potash.

It is a very active poison, producing rigor, convulsions, and death. Four milligrams produce death in a greenfinch in three minutes.

Caproylamin (Hexylamin), $\mathrm{C}_{6} \mathrm{H}_{15} \mathrm{~N}$, has been found to occur by Hesse (1857) in the putrefaction of yeast. Hager isolated from some putrid material what he thought to be a mixture of amylamin and caproylamin, and named it septicin.

Hexylamin was found, in small quantity, in cod-liver oil, by Gautier and Mourgues, and according to these authors it resembles amylamin in its action, but is less toxic. 
Tetanotoxin, $\mathrm{C}_{5} \mathrm{H}_{11} \mathrm{~N}($ ?), was obtained by Brieger (1886) as one of the products of the growth of the tetanus bacillus on beef-broth or on brain-broth. It has also been obtained by Kitasato and Weyl (1890) from pure cultures of the tetanus bacillus, kept eight days at $36^{\circ}$. For its isolation see tetanin and Ber. $d$. d. chem. Ges., 19, 3120. It is tetanizing in action, produces first tremor, then paralysis and violent convulsions. It forms an easily soluble gold double salt which melts at $130^{\circ}$. The platinochlorid is difficultly soluble, and decomposes at $240^{\circ}$. The hydrochlorid is crystalline, and is readily soluble in alcohol and in water. It melts at about $205^{\circ}$. From warm alcohol it crystallizes in flat, pointed plates.

Spasmotoxin, a base of as yet unknown composition, produces in animals violent clonic and tonic convulsions. It was obtained by Brieger (1887) from cultures of the tetanus germ on beefbroth.

Another toxin was obtained by Brieger (1887), in cultures of the tetanus microbe, which produced complete tetanus, salivation and tearsecretion. In its composition it is probably a diamin. The platinochlorid forms plates which begin to decompose at $240^{\circ}$. The hydrochlorid is very deliquescent. Gold chlorid and picric acid form very soluble compounds. Besides these three bases he isolated another toxic substance, tetanin, and a base (see under tetanin).

Dihydrolutidin, $\mathrm{C}_{7} \mathrm{H}_{11} \mathrm{~N}$, was found in cod-liver oil by Gautier and Mourgues (1888). It is the first known hydrolutidin. It is a colorless, somewhat oily, very alkaline and caustic liquid, the odor of which is sharp, but somewhat agreeable when dilute. It absorbs carbonic acid from the air, darkens and thickens; is feebly soluble in water, and boils at $199^{\circ}$ at $760 \mathrm{~mm}$. pressure. The salts are bitter to the taste.

The hydrochlorid crystallizes in a confused mass of needles or in plates. The nitrate reduces silver nitrate-a property of all hydropyridin bases (Hoffmann). The sulphate forms fine stellate deliquescent needles.

The platinochlorid is readily precipitated from concentrated solutions as a canary-yellow precipitate. From warm solutions it crystallizes in lozenge-shaped plates which are often imbricated. On boiling with water it loses hydrochloric acid and forms $\left(\mathrm{C}_{7} \mathrm{H}_{11} \mathrm{NCl}\right)_{2}$. $\mathrm{PtCl}_{2}$, which possesses a lighter color, is more soluble than the normal salt, and crystallizes confusedly.

The aurochlorid crystallizes in needles which form fan- or lozengeshaped masses. It is scarcely altered even in hot water.

The iodomethylate, $\mathrm{C}_{7} \mathrm{H}_{11} \mathrm{~N} . \mathrm{CH}_{3} \mathrm{I}$, is obtained by mixing, in the cold, the base and methyl iodid. The colorless compound thus obtained is soluble in water and in alcohol, and possesses a disagree- 
able, somewhat nauseating odor. Treated with potash it yields a colorless, aromatic, very alkaline oil.

The base on oxidation with boiling potassium permanganate yields an acid, $\mathrm{C}_{7} \mathrm{H}_{7} \mathrm{NO}_{2}$, and from this fact the discoverers conclude that the base is a dihydro-dimethylpiridin, $\mathrm{C}_{5} \mathrm{H}_{4}\left(\mathrm{CH}_{3}\right)_{2} \mathrm{NH}$.

It is moderately poisonous. In small doses it diminishes the general sensibility ; in larger doses it produces trembling, especially of the head; profound depression alternating with periods of extreme excitement; paralysis of the posterior limbs, and death.

A Base, $\mathrm{C}_{8} \mathrm{H}_{11} \mathrm{~N}$, isomeric but not identical with aldehyde collidin, was obtained by Nencki as early as 1876 , by allowing a mixture of 200 grams of pancreas and 600 grams of gelatin in ten liters of water to putrefy for five days at $40^{\circ}$. The method used by Nencki for its isolation is as follows: The fluid mass was distilled with sulphuric acid, to drive off the volatile acids, then rendered alkaline with barium hydrate, and again distilled. The distillate was received in dilute hydrochloric acid, and on evaporation gave a crystalline residue of ammonium chlorid, and of a salt which formed long rhombic plates. The latter were separated from the ammonium salt by absolute alcohol. The free base was obtained from the salt by treating it with sodium hydrate, and extracting the solution with ether.

This compound, as already stated, is isomeric with collidin, and also with $\mathrm{O}$. de Coninck's base, with which it is possibly identical. The latter, however, will be described separately.

On decomposing fibrin by means of streptococci Emmerling (1897) obtained besides leucin, tyrosin and various organic acids, methylamin, tri-methylamin and a base, $\mathrm{C}_{8} \mathrm{H}_{11} \mathrm{~N}$, which apparently differed from that of Nencki and that of Gautier and Etard by the ready solubility of its platinum salt. It had no effect on guinea-pigs. It formed a syrupy liquid, and gave with gold chlorid a yellow precipitate which rapidly became reduced. Platinum chlorid gave no precipitate but the mixture on slow evaporation gave yellowish-red crystals of the platinochlorid. Concentrated solutions gave a picrate which melted at $172^{\circ}-175^{\circ}$. Owing to the slight pyridin odor of the base the latter was regarded as a propyl pyridin.

The free base is oily in character, and possesses a peculiar, not unpleasant odor. It readily absorbs carbonic acid gas from the air, forming after a time a lamellar, crystalline mass of the carbonate. The salt of this base on heating gives off an oil which burns with a smoky flame, and possesses an odor similar to that of xylol or cumol. Nencki was therefore at first of the opinion that the ptomaïn was an aromatic base, probably an isophenyl-ethylamin of the following composition: $\mathrm{C}_{6} \mathrm{H}_{5}-\mathrm{CH}\left\langle\frac{\mathrm{CH}_{3}}{\mathrm{NH}_{2}} \cdot \mathrm{He}\right.$ supposed that it was formed 
by the putrefaction of tyrosin, according to the following equation :

$$
\mathrm{C}_{9} \mathrm{H}_{11} \mathrm{NO}_{3}=\mathrm{C}_{8} \mathrm{H}_{11} \mathrm{~N}+\mathrm{CO}_{2}+\mathrm{O} \text {. }
$$

We know that tyrosin does split up, on being heated to $270^{\circ}$, into carbonic acid and oxyphenyl-ethylamin, thus :

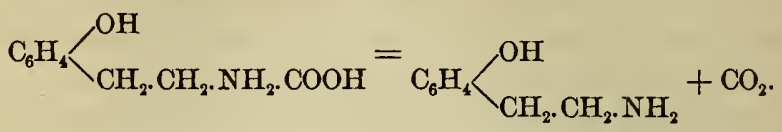

In 1883 Erlenmeyer and Lipp observed that phenyl- $\alpha$-amidopropionic acid (phenyl-alanin), on dry distillation, decomposed with the formation, among other products, of a base having the composition $\mathrm{C}_{8} \mathrm{H}_{11} \mathrm{~N}$. This base was found to be identical with phenylethylamin, $\mathrm{C}_{6} \mathrm{H}_{5} \cdot \mathrm{CH}_{2} \cdot \mathrm{CH}_{2} \cdot \mathrm{NH}_{2}$, and in its properties and composition it resembles Nencki's base. Later (1889), Nencki adopted a similar view in regard to the nature of this base, and regarded it as possessing the formula just given-that is phenyl-ethylamin. He considered phenyl amido-propionic acid-one of the three aromatic nuclei contained in the albumin molecule-as the source of this base. From the fact that phenyl- $\alpha$-amido-propionic acid is a well-known putrefactive product, it would seem that Nencki's base may arise either by the putrefactive decomposition of that acid, or by the hydrolytic cleavage of the acid as a consequence of the method employed in isolating the base. The latter would seem to be the most probable explanation of the genesis of this base, inasmuch as Brieger, by using his method for the isolation of ptomaïns, was not able to obtain it from putrid gelatin.

The platinochlorid, $\left(\mathrm{C}_{8} \mathrm{H}_{11} \mathrm{~N} . \mathrm{HCl}\right)_{2} \mathrm{PtCl}_{4}(\mathrm{Pt}=29.89$ per cent.), is readily soluble in hot, and but slightly soluble in cold water, and can be therefore recrystallized from water. It forms beautiful flat needles. On dry heating it gives off an oil which possesses an odor resembling very much that of xylol or cumol, and burns with a smoky flame. This distinguishes Nencki's base from collidin, since the platinochlorid of the latter does not show this behavior.

Nencki also obtained from putrid gelatin, under certain ill-defined conditions, especially when no glycocoll was present, a basic product which gave, with sulphuric acid, large lamellar crystals. The free base forms a thick colorless syrup, possessing a nauseous, bitter taste. It did not become crystalline even after standing some time. Unlike the base $\mathrm{C}_{8} \mathrm{H}_{11} \mathrm{~N}$, it is not volatile, and is, therefore, obtained on evaporation of the acidulated solution after previous removal of the volatile bases by distillation with baryta.

A Base, $\mathrm{C}_{8} \mathrm{H}_{11} \mathrm{~N}$, isomer of collidin and of the preceding base, with which it is possibly identical, was obtained by $\mathrm{O}$. de Coninck (1888) in the later stages of putrefaction of sea-polyps (poulpes marins). It forms a yellowish, rather mobile liquid, possessing a 
strong benumbing (vireuse) odor, and is but slightly soluble in water. It is soluble in methyl and ethyl alcohol, ether and acetone. Its density is 0.9865 . When dried over potash it boils at $202^{\circ}$ without undergoing decomposition. On exposure to the air it becomes brown, hydrates rapidly, and the boiling point is then lowered. It has not been noticed to absorb carbonic acid from the air. It resembles some of the bases obtained from Dippel's oil. The salts are in general less stable than those of the pyridin bases, and in this respect it approaches the dihydro-pyridin bases.

On treatment with hydrogen peroxid it yields an isomer of mydin, $\mathrm{C}_{8} \mathrm{H}_{11} \mathrm{NO}$. The simple and double salts of this hydroxyl derivative which de Coninck (1899) calls collidone are decomposed by boiling water.

The hydrochlorid, $\mathrm{C}_{8} \mathrm{H}_{11} \mathrm{~N}$.HCl, forms white or slightly yellowish radiate masses which are deliquescent and very soluble in water. The hydrobromid, $\mathrm{C}_{8} \mathrm{H}_{11} \mathrm{~N}$.HBr, resembles it but is less deliquescent and a trifle less soluble in cold water.

The platinochlorid, $\left(\mathrm{C}_{8} \mathrm{H}_{11} \mathrm{~N} . \mathrm{HCl}\right)_{2} \mathrm{PtCl}_{4}$, is a dark orange-colored powder which is insoluble, or almost so in cold water, and is a rather stable compound. Boiling water and water at $80^{\circ}$ decompose it into hydrochloric acid and $\left(\mathrm{C}_{8} \mathrm{H}_{11} \mathrm{NCl}\right)_{2} \mathrm{PtCl}_{2}$ which is a light-brown powder, insoluble in cold, scarcely soluble in hot water.

The aurochlorid, $\mathrm{C}_{8} \mathrm{H}_{11}$ N.HCl. $\mathrm{AuCl}_{3}$, forms a light-yellow precipitate. It is quite stable in cold, but very unstable in hot or even warm water. It cannot be modified by withdrawal of hydrochloric acid.

The base forms two compounds with mercuric chlorid. $\left(\mathrm{C}_{8} \mathrm{H}_{11} \mathrm{~N}\right.$. $\mathrm{HCl})_{2} \mathrm{HgCl}_{2}$ crystallizes in small white needles, which are slightly soluble in water and in dilute alcohol, insoluble in absolute alcohol, and on exposure to moist air undergo change. The second compound, $2\left(\mathrm{C}_{8} \mathrm{H}_{11} \mathrm{~N} . \mathrm{HCl}\right) .3 \mathrm{HgCl}$, is obtained by adding an excess of concentrated mercuric chlorid to a concentrated solution of the hydrochlorid. It forms slightly yellow, somewhat longer needles which are insoluble in the principal solvents, and are likewise changed by atmospheric humidity.

The iodomethylate, $\mathrm{C}_{8} \mathrm{H}_{11} \mathrm{~N} \cdot \mathrm{CH}_{3} \mathrm{I}$, is formed by mixing solutions of the base and methyl iodid in absolute ether. It is deposited as a network of fine white needles, which are but slowly altered in the air, and are soluble in absolute alcohol. This solution on the addition of a little potash assumes a dark-red color, which is heightened by the addition of a little hydrochloric or acetic acid, and destroyed by ammonia without any resultant fluorescence. Warmed with excess of moist solid potash it becomes garnet-red in color and gives off an odor resembling that of the dihydropyridins. It thus behaves the same as the pyridin iodomethylates.

On oxidation with potassium permanganate it yields an acid which 
melts at $229^{\circ}-230^{\circ}$, and begins to sublime at $150^{\circ}$. It presents all the characteristics of nicotinic acid, $\mathrm{C}_{6} \mathrm{H}_{5} \mathrm{NO}_{2}$, which is formed as the result of oxidation of nicotin. With hydrochloric acid it forms the compound $\mathrm{C}_{6} \mathrm{H}_{5} \mathrm{NO}_{2} \cdot \mathrm{HCl}$. With copper acetate it forms a salt; this distilled with lime, yields a substance which on boiling with platinum chlorid and water forms the compound $\left(\mathrm{C}_{5} \mathrm{H}_{5} \mathrm{NCl}\right)_{2}$. $\mathrm{PtCl}_{2}$. This same substance forms an iodomethylate, which in alcoholic solution gives, on addition of potash, the characteristic reaction of pyridin bases.

The base $\mathrm{C}_{8} \mathrm{H}_{11} \mathrm{~N}$, therefore, yields pyridin and nicotinic acid.

A Base, $\mathrm{C}_{8} \mathrm{H}_{13} \mathrm{~N}$, was obtained by Gautier and Etard (1881) from the chloroform extracts (see method, page 234) from putrefying mackerel, as well as from the decomposing flesh of the horse and ox. It is regarded by these authors as a constant and definite product of the bacterial fermentation of albuminoid substances, but this view is hardly justifiable, inasmuch as the base has not been found by other investigators. It is accompanied by the base $\mathrm{C}_{17} \mathrm{H}_{38} \mathrm{~N}_{4}$. Nencki (1882) asserted the identity of this base with the one which he had isolated in 1876 , and to which he had ascribed the formula $\mathrm{C}_{8} \mathrm{H}_{11} \mathrm{~N}$. On the other hand, Gautier and Etard consider their base to be identical with the hydrocollidin obtained by Cahours and Etard by the action of selenium on nicotin.

An isomer of this base, hemopyrrol, has been prepared from hemin and also from the chlorophyll derivative phyllocyanin. On oxidation it yields urobilin (Nencki and Marchlewski, Ber. d. d. chem. Ges., 34, 1687).

The free base is an alkaline, almost colorless, oily liquid, possessing a penetrating odor resembling that of syringa. It is volatile without decomposition, and boils at about $205^{\circ}$, while hydrocollidin boils at $210^{\circ}$. Its density at zero is 1.0296 . When exposed to the air it oxidizes slowly, becomes brown and viscous, and at the same time absorbs carbonic acid. It differs from a collidin in possessing a strong reducing action, since both the gold and platinum double salts become reduced on heating, and even in the cold.

The hydrochlorid, $\mathrm{C}_{8} \mathrm{H}_{13} \mathrm{~N} . \mathrm{HCl}$, is very soluble in water and in alcohol, and usually forms fine needles resembling snow crystals. It is neutral in reaction and possesses a bitter taste. In the presence of an excess of acid it reddens and resinifies.

The platinochlorid, $\left(\mathrm{C}_{8} \mathrm{H}_{13} \mathrm{~N} . \mathrm{HCl}\right)_{2} \mathrm{PtCl}_{4}(\mathrm{Pt}=29.7$ per cent.), is of a light-yellow, flesh color, crystalline, and but slightly soluble. It dissolves on warming, and recrystallizes in bent needles.

The aurochlorid is rather soluble, and becomes slowly reduced in the cold ; rapidly on warming.

This isomer of hydrocollidin is strongly poisonous. Even so small a dose as 0.0017 gram of the hydrochlorid produced, when in- 
jected under the skin of a bird, marked unsteadiness of gait, followed by paralysis of the extremities, and finally death. The pupils are normal and the heart stops in diastole. Larger doses (0.007 gram) cause at first vomiting and staggering, which soon give way to a condition of exaltation. Toward the end tetanic convulsions set in, followed by almost complete paralysis.

A Base, $\mathrm{C}_{9} \mathrm{H}_{13} \mathrm{~N}$, isomeric with parvolin, has been extracted by Gautier and Etard (1881) from decomposing mackerel and horseflesh. The method employed by these chemists for its isolation is given on page 233. The identity of this base with the synthetic parvolin, obtained by Waage by heating ammonia with propionic aldehyd in a sealed tube at $200^{\circ}$, cannot be considered to be definitely settled, although an apparent identity exists in regard to their boiling points. Thus, the synthetic parvolin boils at $193^{\circ}-196^{\circ}$, while Gautier and Etard assign to their base a boiling-point a little below $200^{\circ}$. Further investigation is necessary to decide upon the question of the identity of this base with parvolin, or of the ptomain $\mathrm{C}_{8} \mathrm{H}_{13} \mathrm{~N}$ with hydrocollidin.

The free base is an oily, amber-colored liquid, possessing the odor of hawthorn blossoms. It is slightly soluble in water; very soluble in alcohol, in ether, and in chloroform. Its boiling-point, as stated above, is a trifle below $200^{\circ}$. Like the bases $\mathrm{C}_{8} \mathrm{H}_{13} \mathrm{~N}$ and $\mathrm{C}_{10} \mathrm{H}_{15} \mathrm{~N}$ it becomes brown and soon resinifies on exposure to air.

The platinochlorid, $\left(\mathrm{C}_{9} \mathrm{H}_{13} \mathrm{~N} . \mathrm{HCl}\right)_{2} \mathrm{PtCl}_{4}(\mathrm{Pt}=28.65$ per cent.), is slightly soluble, crystalline, and flesh colored; exposed to the air it soon becomes pink.

The aurochlorid is quite soluble.

A Base, $\mathrm{C}_{10} \mathrm{H}_{15} \mathrm{~N}$, was isolated by Guareschi and Mosso (1883) from ox-blood fibrin which had been allowed to putrefy for five months. In 1887 it was re-obtained from putrid fibrin by Guareschi, who this time ascribed to it the formula $\mathrm{C}_{10} \mathrm{H}_{13} \mathrm{~N}$. In 1886 Oechsner de Coninck found it among the basic products formed in the putrefaction of the jelly-fish (poulpes marins, Hugounenq, page 21). The method used for its extraction was that of Gautier and Etard (see page 233). It forms a brownish oil of strong alkaline reaction, which soon resinifies. It possesses an unpleasant, weak pyridin or coniïn odor, and is but slightly soluble in water; soluble in ether and in chloroform.

In regard to the constitution of this ptomaïn we know nothing, but from its physical characters it would seem to possess a pyridin nucleus. It is isomeric with corindin, a homologue of parvolin and collidin, obtained from coal-tar.

For the behavior of the hydrochlorid to alkaloidal reagents, see Table I. 
The hydrochlorid, $\mathrm{C}_{10} \mathrm{H}_{15} \mathrm{~N} . \mathrm{HCl}$, crystallizes in colorless cholesterin-like plates which are somewhat deliquescent.

The platinochlorid, $\left(\mathrm{C}_{10} \mathrm{H}_{15} \mathrm{~N} . \mathrm{HCl}\right)_{2} \mathrm{PtCl}_{4}(\mathrm{Pt}=27.52$ per cent.), forms a light flesh-colored, crystalline precipitate, and is insoluble in water, alcohol and ether. It does not resinify, and is stable at $100^{\circ}$.

In its action this ptomaïn resembles curara, although it is by no means as strong. 0.012 gram of the free base produced in a frog dilatation of the pupil and slowing of the respiration. The nostrils were motionless, and within five hours complete paralysis of the muscles took place. The reflex excitability gradually diminished until it finally disappeared. An orange-blossom odor was observed about the frogs which were poisoned by this ptomaïn. The same amount of ptomaïn injected into a greenfinch produced vomiting, and a condition of weakness and decreased sensibility, followed soon, however, by recovery. A rat was not affected by 0.020 gram of the free base. The hydrochlorid acts much more energetically.

A Base, $\mathrm{C}_{10} \mathrm{H}_{15} \mathrm{~N}$, was isolated by $\mathrm{O}$. de Coninck, in $1886(\mathrm{Hu}-$ gounenq, page $21, C$. Rendus, 1888), from sea-polyps in an advanced stage of putrefaction, together with the base $\mathrm{C}_{8} \mathrm{H}_{11} \mathrm{~N}$. The method employed for its extraction was that of Gautier and Etard (see page 233). Nesbitt (1899) obtained a similar, if not identical, base from the intestinal contents of a dog after previous ligature of the lower part of the intestine. It was associated with cholin and neurin. It forms a slightly yellow, viscous liquid, and possesses a pleasant odor resembling that of blooming broom. Its density is about 1.18. It boils at about $230^{\circ}$ (uncorrected), with initial decomposition. In water it is but slightly soluble, readily so in ether, alcohol, acetone, and ligroïn. It is rapidly oxidized by the air, becomes brown, and resinifies but does not absorb carbonic acid.

The hydrochlorid, $\mathrm{C}_{10} \mathrm{H}_{15} \mathrm{~N} . \mathrm{HCl}$, forms fine yellowish, very deliquescent needles which in the presence of a trace of air are at once colored red; if more air is present the red changes to a brown, and in the open air a resin is formed the same as from the free base. It is very easily soluble.

The hydrobromid, $\mathrm{C}_{10} \mathrm{H}_{15} \mathrm{~N} . \mathrm{HBr}$, crystallizes in a network of fine deliquescent needles which become likewise red on exposure to air. It is very soluble in water; less so in strong alcohol, and almost insoluble in ether.

The platinochlorid, $\left(\mathrm{C}_{10} \mathrm{H}_{15} \mathrm{~N} . \mathrm{HCl}\right)_{2} \mathrm{PtCl}_{4}$, forms a dark-red powder which is insoluble in cold water; very soluble in warm water. It can be kept in dry air ; in moist air, it loses hydrochloric acid and becomes partially oxidized. Boiling water decomposes it. $\left(\mathrm{C}_{10} \mathrm{H}_{15} \mathrm{~N} . \mathrm{Cl}\right)_{2} \mathrm{PtCl}_{2}$ forms clear brown plates which are stable in moist air, and melt at $206^{\circ}$. It is insoluble in cold water, soluble in 
boiling water, but decomposes. In recrystallizing, warm, previously boiled water should be used.

The aurochlorid, $\mathrm{C}_{10} \mathrm{H}_{15} \mathrm{~N} . \mathrm{HCl} \cdot \mathrm{AuCl}_{3}$, occurs as a light yellow precipitate; insoluble in cold water, soluble in warm water. It is decomposed by boiling water; is stable when kept in a moist atmosphere.

The iodomethylate, $\mathrm{C}_{10} \mathrm{H}_{15} \mathrm{~N} . \mathrm{CH}_{3} \mathrm{I}$, in warm alcoholic solution yields, on the addition of strong potash, a bright red color, which soon becomes brown, and in about an hour the solution shows a greenish-blue fluorescence. This rapidity of change is due to the extreme oxidizability of the ptomaïn.

On careful oxidation with potassium permanganate at ordinary temperature it yields a solid acid, having a melting-point of $228^{\circ}-$ $229^{\circ}$, the same as that of the pyridin carbonic acid of Huber and Laidlin, obtained by the oxidation of nicotin. The solubility in cold and in warm water and in absolute alcohol is the same as that of nicotinic acid. It begins to sublime at $150^{\circ}$ as pearly spangles. The formula is $\mathrm{C}_{6} \mathrm{H}_{5} \mathrm{NO}_{2}$. On distillation with lime pyridin forms. It is, therefore, identical with nicotinic acid, an oxidation product of nicotin and other volatile alkaloids.

O. de Coninck considers this base, as well as $\mathrm{C}_{8} \mathrm{H}_{11} \mathrm{~N}$, as belonging to the pyridin, and not to the hydropyridin series.

A Base, $\mathrm{C}_{10} \mathrm{H}_{17} \mathrm{~N}$, was described by Griffiths (1890) as derived from cultures on pepton-agar of the bacterium allii, a germ obtained from putrid onions. The base (hydrochlorid ?) forms colorless, prismatic, microscopic, very deliquescent needles, which are soluble in warm water, alcohol, ether and chloroform. It gives a hawthornlike odor, especially when warmed. With phosphomolybdic acid it yields a white; with iodin in potassium iodid and with tannic acid a chestnut-colored precipitate. Nessler's solution produces a yellow chestnut-colored precipitate. Picric acid throws down a yellow slightly soluble deposit. The platinochlorid, $\left(\mathrm{C}_{10} \mathrm{H}_{17} \mathrm{~N} . \mathrm{HCl}\right)_{2} \mathrm{PtCl}_{4}$, is yellow, crystalline, and difficultly soluble in cold water and in alcohol; soluble in warm water. Gold chlorid produces a thick yellow precipitate soluble in water. Dilute sulphuric acid produces a violet-red color. The base is apparently a hydrocoridin.

Griffiths has also described, together with a variety of other products in physiological chemistry, the basic compounds which are given in the subjoined table. The method employed in the isolation of all but few of these compounds is that of Luff (see next chapter under urine).

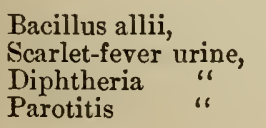

Bacillus allii,
Scarlet-fever urine,
Diphtheria "،
Parotitis

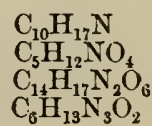

Scarlatinin,

Diphtherin,

Propyl-glycocyamin, $\mathrm{C}_{6} \mathrm{H}_{13} \mathrm{~N}_{3} \mathrm{O}_{2}$
1890.

1891. 
Glanders urine,

Pneumonia "

Measles

Whooping cough urine,

Erysipelas

Puerperal fever "،

Epilepsy

Micrococcus tetragenus,

Bacillus pluviatilis,

Eczema urine,

Influenza "

Putrid sardines,

Cancer urine,

Pleurisy

Angina pectoris urine,
Glycocyamidin,

Erysipelin, Puerperalin,

Eczemin,

Sardinin,

Cancerin,

Pleuricin,
$\mathrm{C}_{15} \mathrm{H}_{10} \mathrm{~N}_{2} \mathrm{O}_{6}$

$\mathrm{C}_{20} \mathrm{H}_{26} \mathrm{~N}_{2} \mathrm{O}_{3}$

1892.

66

1892.

$\mathrm{C}_{51} \mathrm{H}_{19} \mathrm{NO}_{2}$

$\mathrm{C}_{22} \mathrm{H}_{19} \mathrm{NO}_{2}$

$\mathrm{C}_{12} \mathrm{H}_{16} \mathrm{~N}_{5} \mathrm{O}_{7}$

$\mathrm{C}_{9} \mathrm{H}_{21} \mathrm{~N}_{2} \mathrm{O}_{5}$

$\mathrm{C}_{7} \mathrm{H}_{15} \mathrm{NO}$

$\mathrm{C}_{9} \mathrm{H}_{9} \mathrm{NO}_{4}$

$\mathrm{C}_{11} \mathrm{H}_{11} \mathrm{NO}_{2}$

$\mathrm{C}_{8} \mathrm{H}_{5} \mathrm{NO}_{5}$

$\mathrm{C}_{5} \mathrm{H}_{5} \mathrm{O}_{2}$

$\mathrm{C}_{10} \mathrm{H}_{9} \mathrm{NO}_{4}$
66

66

66

66

1893.

6

"6

1894.

1895.

It is very suspicious to find such a long array of products, isolated apparently with the greatest ease by a most simple method. This is all the more remarkable in view of the fact that other skilled workers have utterly failed in some of these cases to isolate basic substances. Thus, cultures of Loeffler's bacillus of diphtheria have been shown by Brieger and Fränkel and others not to contain ptomaïns, and yet Griffiths claims to isolate a base from the urine in the disease, and also from the pure cultures of the bacillus. The base is apparently so abundant that in 1893 he was able to sacrifice $1.5 \mathrm{~g}$. (!) in order to show that a disinfectant "izal" destroyed its poisonous properties (!). Again, from four gallons of scarlet fever urine Luff succeeded in obtaining some crystals of a base, insufficient however, for analysis. Griffiths, however, not only isolated it in sufficient quantity, but had $2.5 \mathrm{~g}$. of it to spare for testing the power of "izal." Further than that, he succeeded in isolating scarlatinin from pure cultures of the micrococcus scarlatinæ (!). We have yet to learn that scarlet fever is due to a micrococcus.

Equally interesting facts appear in connection with the base of glanders, which was isolated from the urine and also from pure cultures of the glanders bacillus. Nencki, however, had 10 liters of a bouillon culture of the glanders bacillus examined according to Griffiths's method, and failed to obtain a weighable quantity of a ptomaïn (Maly's Jahresbericht, 1894, 24, 601). The action of this basic product of glanders is remarkable, producing "an abscess at the point of inoculation, nodules in the lungs and spleen, and metastatic abscesses in various organs."

The description of these bases is so brief and unsatisfactory that the description of one applies almost equally well to all the others. Thus the bases are all white, crystalline, and soluble in water, imparting an alkaline reaction in all but two or three cases. All but four or five are characterized as poisonous, but the dose employed is never given; the method of administration is mentioned but twice, and the kind of animal employed but six times. All, or nearly all, form 
crystalline hydrochlorids, aurochlorids, and platinochlorids. Reactions are usually given with only three or four reagents. It will be noticed that one of these products has no nitrogen and yet is mentioned as a ptomain (!) ; further that the formula of five of these compounds is not in accord with the law of even numbers. Considering the amounts of the bases available for "izal" experiments, it is proper to expect accurate, exhaustive, thorough work. Chemical science is not advanced by coining names or establishing formulæ.

A Base, $\mathrm{C}_{32} \mathrm{H}_{31} \mathrm{~N}$, was obtained by Delézinier (1889) and is said to be the alkaloid isolated in 1879 by Brouardel, which in its chemical and physiological properties was described as similar to veratrin. It forms an almost colorless oily fluid, which possesses a hawthornlike odor. It is very readily oxidizable and yields the veratrin-like reactions only in the presence of air. It is soluble in alcohol, ether, toluene and benzene; and forms well-defined salts which are very deliquescent. It appears to be an amin and in its composition differs from cevadin by $9 \mathrm{H}_{2} \mathrm{O}$. Nothing is stated in regard to its source or method of preparation. The analytical results given $(\mathrm{C}=89.41, \mathrm{H}=7.3, \mathrm{~N}=3.03)$ correspond more to the formula $\mathrm{C}_{34} \mathrm{H}_{33} \mathrm{~N}$.

Ethylidenediamin (?), $\mathrm{C}_{2} \mathrm{H}_{3} \mathrm{~N}_{2}$. - This base was considered at first by Brieger to be identical with ethylenediamin, but subsequent comparison showed this to be an error. Thus the former is poisonous and does not form a gold salt, while the latter is not poisonous and does form a rather difficultly soluble aurochlorid. Again, ethylenedi- amin forms a platinochlorid which is almost insoluble in hot water, whereas the platinum double salt of the ptomaïn is much more easily soluble. Brieger, therefore, inclined to the belief that it was identical with ethylidenediamin, $\mathrm{CH}_{3} \cdot \mathrm{CH}\left(\mathrm{NH}_{2}\right)_{2}$, rather than with ethylenediamin, which has the structure $\mathrm{CH}_{2} \cdot \mathrm{NH}_{2} \cdot \mathrm{CH}_{2} \cdot \mathrm{NH}_{2}$. This ptomaïn was obtained by Brieger, 1885 (I., 44), from decomposing haddock (see gadinin). Kulneff has probably met with this base in the liquids of the stomach in gastrectasis. Carbone has reported it in cultures of the proteus vulgaris with gadinin, trimethylamin, and cholin.

The free base can be obtained without decomposition, on distilling the hydrochlorid with sodium hydrate.

The hydrochlorid, $\mathrm{C}_{2} \mathrm{H}_{8} \mathrm{~N}_{2}, 2 \mathrm{HCl}$, crystallizes in long, glistening needles which are readily soluble in water, insoluble in absolute alcohol. It gives no combination with gold chlorid. For its behavior to alkaloidal reagents see Table $\mathrm{I}$.

The platinochlorid, $\mathrm{C}_{2} \mathrm{H}_{8} \mathrm{~N}_{2} \cdot 2 \mathrm{HCl} . \mathrm{PtCl}_{4}(\mathrm{Pt}=41.49$ per cent. $)$, forms small yellow plates which are moderately difficultly soluble in water. It can be readily recrystallized from hot water. 
Frogs seem to be less susceptible to the action of this poison than mice or guinea-pigs. In the latter, it produces a short time after injection an abundant periodic flow of secretion from the nose, mouth and eyes. The pupils dilate and the eyeballs project. Violent dyspnea then comes on and predominates until the death of the animal, which does not take place for twenty-four hours or more. The heart is stopped in diastole.

Pohl (1898) has studied the effect of synthetic ethylenediamin upon animals. The subcutaneous injection of 1 gram in rabbits $\left(1 \frac{1}{2} \mathrm{k}\right.$.) produced no effect until after the lapse of a day, when a serous exudate formed at the point of inoculation. Death from parenchymatous nephritis followed on the third or fourth day. Larger doses produced clonic convulsions and paralysis of the extremities. In dogs, subcutaneous injections had no effect, but intravenously the diamin proved fatal.

Trimethylenediamin (?), $\mathrm{C}_{3} \mathrm{H}_{10} \mathrm{~N}_{2}$ (?), is a toxic base isolated by Brieger (1887) from cultures of the comma bacillus on beef-broth. It may be stated here that from the same source, cholera cultures, Kunz (1888) obtained a base which he considered to be identical with spermin or ethyleneimin (see next chapter). It is present, however, in exceedingly minute quantity, and occurs in the mercuric chlorid precipitate, from which it is obtained by the following method: The precipitate is decomposed by hydrogen sulphid, the filtrate evaporated to dryness, and the residue taken up with absolute alcohol and precipitated by an alcoholic solution of sodium picrate. The precipitate thus obtained consists of the picrates of cadaverin, creatinin, and of this new base. It is boiled with absolute alcohol to remove the insoluble cadaverin picrate; the filtrate is evaporated to expel the alcohol, and the bases then converted into the platinum double salts, whereby the easily soluble creatinin platinochlorid can be separated from the corresponding less soluble compound of the new base.

Owing to the small quantity of this substance present, a complete study of its properties has not as yet been made. It gives difficultly soluble precipitates with gold chlorid and with platinum chlorid; the compound with the latter crystallizes in long needles. With picric acid it gives a precipitate consisting of felted needles, which resemble creatinin picrate; they melt at $198^{\circ}$. Phosphomolybdic acid yields a precipitate crystallizing in plates, while potassium bismuth iodid gives dark-colored fine needles.

In its physiological action it seems to be identical with the basic substance isolated from choleraic bodies by different observers. It causes violent convulsions and muscle-tremor. The action of the synthetic base has also been studied by Pohl and apparently it is more toxic than the preceding. The action of the heart is slowed 
and irregular; the pulse is feeble, respiration is slow and deep and marked dyspnoea prevails. These symptoms soon disappear but the rabbit died in about four days with uræmic symptoms. Albuminuria was marked.

Besides trimethylenediamin another toxin was obtained by Brieger from cholera cultures, but in quantity insufficient for analysis. It was obtained from the mercuric chlorid filtrate after elimination of methylamin, trimethylamin, and traces of cholin and creatinin, as an insoluble platinum double salt. Subcutaneous injection of this base into mice produced a paralysis-like lethargic condition, slowing of respiration and heart's action, lowering of temperature, and, finally, death in twelve to twenty-four hours. In some cases bloody stools were passed.

Putrescin, $\mathrm{C}_{4} \mathrm{H}_{12} \mathrm{~N}_{2}$, is a diamin which almost invariably occurs together with cadaverin with which it is closely related. This base was also discovered by Brieger in 1885 (II., 42), who obtained it from putrefying human internal organs (for four months at a low temperature without access of much oxygen); and from the same material decomposing at the ordinary temperature of the room for from three days to three weeks. It has also been obtained from herring, twelve days in spring; from pike, six days in summer ; from haddock, two months (Bocklisch). Also from putrid mussel, sixteen days (Brieger) ; and from human as well as horseflesh. Brieger likewise obtained it from cultures of the bacteria of human feces on gelatin, and in small quantity in rather old cultures of the comma bacillus on beef-broth; in larger quantity in cultures of the same germ on blood-serum. Garcia found it in putrefying meat and pancreas, together with cadaverin and hexamethylenediamin. The diamin production at $30^{\circ}$ is considerable in twenty-four hours; reaches its maximum in three days. Putrescin appears on the first day. Roos found putrescin in stools of one case of cholera and in two cases of diarrhœa or cholerin. With cadaverin it forms in the sterile autodigestion of pig's stomach (Lawrow).

Udránszky and Baumann in 1888 demonstrated the existence of putrescin and cadaverin in the urine of cystinuria. They found the total amount of the dibenzoyl compounds in the urine in 1888 to vary from $0.2-0.4 \mathrm{~g}$. per day. Cadaverin made up about $\frac{2}{3}$ of this amount, and putrescin $\frac{1}{3}-\frac{1}{4}$. Garcia examined the same patient in 1892 and obtained, as an average of seven days, only $0.064 \mathrm{~g}$. of the dibenzoyl-compound, which contained no cadaverin (!), only putrescin. With ordinary diet the average of 11 days was $0.027 \mathrm{~g} \cdot$; with cheese-diet an average of 8 days gave $0.136 \mathrm{~g}$.; while a carbohydrate diet, an average of 7 days, gave $0.102 \mathrm{~g}$. of the dibenzoylcompound. In the feces of the same patient, on the contrary, Udránszky and Baumann found in 1888 that putrescin constituted 
by far the greater quantity, while cadaverin formed but 10 to 15 per cent. Garcia, in 1892, showed in the same patient the presence of only putrescin in the feces, no cadaverin. The feces contained on ordinary diet, an average of 11 days, $1.123 \mathrm{~g}$.; on cheese-diet, average of 8 days, $1.3978 \mathrm{~g}$; ; on carbohydrate diet, average of 7 days, $0.741 \mathrm{~g}$. of the dibenzoyl compound. Borissow, in 1894, again examined the feces of the same patient, and found as an average of four days $2.062 \mathrm{~g}$. of the dibenzoyl-compounds, which contained only traces of cadaverin. It would seem that diet and the intensity of intestinal decomposition influence the amount of excretion of diamins. The administration of salol and sulphur (Mester), or intestinal lavage (U. and B.), had no effect on diamin excretion. Normal feces, as well as the feces in various diseases, with the possible exception of cholera stools, are free from diamins. It would seem, therefore, that these bases occur in cystinuria as the result of putrefactive changes going on in the intestines; becoming partly absorbed they appear in the urine (see p. 269). In two cases of cystinuria, reported by Brieger and Stadthagen, cadaverin was found almost solely present in the urine.

According to Mester, the diamins are proportionate to the amount of cystin excreted, and therefore constitute a fixed symptom, the cause of which is the same as that of the cystinuria.

Although putrescin is recognizable on about the fourth day of the putrefaction, yet it does not occur in appreciable quantity until about the eleventh day. The amount that is formed increases as the putrefaction goes on, so that a considerable quantity may be obtained after two or three weeks. A very good source for the preparation of putrescin, cadaverin, and neuridin is gelatin which has been allowed to decompose in contact with water for some weeks. Neuridin is, apparently, formed first, but is soon replaced by the former two bases. In the process of extraction it is first obtained in the alcoholic mercuric chlorid precipitate. For its separation from cadaverin and other accompanying bases, see saprin, p. 278.

From the urine of cystinuria it is best obtained by precipitation with benzoyl chlorid (Baumanu's method). For this purpose about 1,500 c.c. of urine are treated with 200 c.c. of sodium hydrate solution ( 10 per cent.), then 20 to 25 c.c. of benzoyl chlorid are added, and the whole shaken till the odor of the latter disappears. The yellowish-white precipitate which forms may consist of insoluble phosphates, carbohydrates, polyatomic alcohols, and diamins. The cystin compound is precipitated only in concentrated solutions. The precipitate contains from a half to two-thirds of the diamins present; it is filtered off, digested with warm alcohol, and the solution filtered. The alcoholic filtrate is concentrated and then poured into about thirty times its volume of cold water. The diamin compounds then crystallize out. To separate the two diamins they are redis- 
solved in just sufficient warm alcohol to effect solution, and this is then poured into about twenty times its volume of ether. The putrescin benzoyl compound is thus thrown out of solution. The filtrate from this, on concentration, yields the cadaverin compound. To isolate that portion of the diamins which remains in the original filtrate with benzoyl-cystin, it is acidulated with sulphuric acid and extracted with ether. The residue obtained on evaporating the ethereal solution is first neutralized with a 12 per cent. sodium hydrate solution, then mixed with three to four times its volume of the same solution. The precipitate which forms consists of the sodium compounds of benzoyl-cystin and the diamins. It is washed with sodium hydrate, and the two compounds separated by their different solubilities in water-the cystin compound is readily soluble, that of the diamins insoluble. To purify the benzoyldiamins they are dissolved in warm alcohol and precipitated with excess of water.

Putrescin (from putrescere, to rot, to putrefy) is a water-clear, rather thin liquid which fumes in the air and has a peculiar semen-like odor, almost undistinguishable from that of cadaverin and reminding one somewhat of the pyridin bases. It absorbs carbonic acid energetically from the air, without losing thereby the repulsive odor. The boiling point of the free base, as ordinarily obtained, is about $135^{\circ}$. It is not decomposed by distillation with potassium hydrate, and is rather difficultly volatile with steam. With acids it forms beautiful crystalline salts. Putrescin unites with water, like ethylenediamin, to form a hydrate, and this water can only be removed by distillation over metallic sodium. The perfectly anhydrous base boils at $156^{\circ}-157^{\circ}$, and then solidifies to plates (Brieger), which melt at $24^{\circ}$ (Udránszky and Baumann). The synthetic base boils at $158^{\circ}-160^{\circ}$, and melts at $23^{\circ}-24^{\circ}$ (Ladenburg). Like cadaverin it is difficultly soluble in ether.

The constitution of putrescin has been determined by Udránszky and Baumann (1888). They showed that the dibenzoyl compound of putrescin was identical with that of the synthetic tetramethylene diamin and of the base which they found in the urine of cystinuria.

Putrescin, therefore, is tetramethylenediamin, a homologue of cadaverin, and its rational formula is :

$$
\mathrm{NH}_{2} \cdot \mathrm{CH}_{2} \cdot \mathrm{CH}_{2} \cdot \mathrm{CH}_{2} \cdot \mathrm{CH}_{2} \cdot \mathrm{NH}_{2} \text {. }
$$

The same authors (Zeitschr. f. physiol. Chem., 13, 591) pointed out that diamins might possibly occur in putrefaction as the result of oxidation of monamins. Thus, putrescin might arise from methylamin according to the equation:

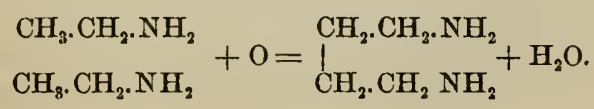


In a similar manner cadaverin might form from ethyl and propylamin. It is well known that in the decomposition of proteids in the presence of carbohydrates no aromatic compounds, as indol, phenol, tyrosin, etc., form. With reference to the formation of diamins Garcia has shown that, in the presence of cane sugar, putrefying meat and pancreas yield from one-half to less then one-tenth as much diamins as when no sugar is present. A similar decrease of diamins in cystinuria is observed (page 265) when the patient is placed on a carbohydrate diet.

The researches of Ellinger (1898), however, have definitely established the source of putrescin and of its homologue cadaverin. All proteids contain the hexon base arginin which on heating with baryta yields urea and ornithin (which see). On subjecting the latter, which is di-amido valerianic acid, to putrefaction Ellinger obtained putrescin. In like manner lysin, another hexon base, yielded cadaverin. These two ptomaïns, therefore, result from the cleavage of the di-amino acids which exist preformed in the proteid molecule. The fact that gelatin yields over 10 per cent. of these acids explains the abundant yield of putrescin and cadaverin in the putrefaction of this material.

The relation of diamins to cystinuria has until recently been scarcely understood. Baumann and Udránszky, accepting the intestinal origin of the diamins, supposed that these bases entered into combination with cystin, protecting it against oxidation. When fed to dogs, however, the diamins are in part excreted as such, but no cystin appears. Again, cystin is not present in the feces of cystinuria. Werigo considered cadaverin as a normal product of pancreatic digestion. Garcia has shown that in meat and pancreas putrefaction diamin formation begins on the first day, and reaches its maximum on the third day. Furthermore, meat and pancreas flasks inoculated with the feces of a cystinuric patient produced an increased formation of diamins, thus apparently confirming the view that diaminuria is the result of the activity of certain bacteria in the intestines.

It is possible that in diaminuria some other product is formed, which, when absorbed, combines with cystin and protects it against oxidation, so that it appears in the urine. Diaminuria and cystinuria certainly go hand in hand. Garcia endeavors to account for the presence of diamins in cystinuria by the supposition that cystin, the normal product of the body, can undergo reduction and yield putrescin under the influence of special intestinal bacteria. Cystin, however, is not present in feces in cystinuria, and when fed to dogs it merely serves to increase the amount of sulphuric acid eliminated. Moreover, the formula of cystin hardly permits of the derivation of diamins.

With reference to cystin it may be well to note that until recently it has been met with, outside of cystinuria, only in a drunkard's 
liver (Scherer); in beef kidneys (Cloetta); in decomposing pancreas (Külz); in the liver of a horse and a dolphin (Dreschel), and in a tyrosin preparation from horn(Emmerling). The studies of Baumann and of Dreschel led these investigators to regard cystin as a normal intermediate waste-product, but no definite evidence of this was brought out until Mörner (1899) succeeded for the first time in preparing cystin by the hydrolysis of horn ( 6.8 per cent.), egg-membrane ( 6 per cent.), and human hair (12.6 per cent.). Embden (1901) obtained it in like manner from serum and egg-albumin. Moreover, both confirmed Külz's observation as to the formation of cystin in tryptic digestion. Apparently all proteids which contain sulphur in a form which can easily be split off contain the cystin group. This fact, considered in connection. with the now known source of the diamins, renders it evident that cystinuria is due to a condition of abnormal tissue metabolism rather than to a peculiar intestinal decomposition. The presence of diamins in the intestine is probably due to an elimination.

Putrescin can be prepared synthetically, according to Ladenburg's method, by converting ethylene bromid into the cyanid and then reducing this by means of sodium in absolute alcohol. It is an isomer of Angeli's dimethylethylenediamin.

On heating the concentrated aqueous solution of the hydrochlorid with potassium nitrite there is produced an oil, soluble in water, from which it can be extracted with ether. This oil, on treatment with phenol and sulphuric acid, gives Liebermann's nitroso-reaction, which would seem to show that putrescin is not a primary diamin (butylenediamin), but is rather a secondary diamin (Brieger, II., 42). As a primary diamin it should take up, on repeated treatment with methyl iodid, six methyl radicals; whereas, if it is a secondary diamin, only four methyl radicals can enter the molecule. Thus, to illustrate, methy lamin, $\mathrm{CH}_{3} . \mathrm{NH}_{2}$ (a primary amin), combines with three molecules of methyl iodid to form $\left(\mathrm{CH}_{3}\right)_{4}$ N.HI. Similarly, dimethylamin, $\left(\mathrm{CH}_{3}\right)_{2} \cdot \mathrm{NH}$, requires only two molecules to form $\left(\mathrm{CH}_{3}\right)_{4}$ N.HI. In the case of diamins, double this number of methyl groups is required to effect complete saturation. As a matter of fact, Brieger (III., 101), on treating putrescin with methyl iodid, succeeded in introducing four, and only four, methyl radicals. From this, however, it does not follow that putrescin is not a primary amin, since cadaverin, an unquestioned primary diamin, yields a substitution compound containing only two methyl groups (see p. 274).

The tetra-methyl substitution product of putrescin, $\mathrm{C}_{4} \mathrm{H}_{8}\left(\mathrm{CH}_{3}\right)_{4} \mathrm{~N}_{2}$, can be distilled without decomposition. The free base crystallizes in long prisms. The hydrochlorid forms small needles which are easily soluble; with phosphotungstic acid it gives a white crystalline precipitate, with phosphomolybdic acid a yellow crystalline precipitate, with picric acid needles. Potassium bismuth iodid gives a 
brownish-red amorphous deposit, while the potassium mercuric iodid forms prisms. Gold chlorid yields difficultly, and platinum chlorid easily soluble octahedra; aqueous mercuric chlorid forms needles. The aurochlorid has the formula $\mathrm{C}_{8} \mathrm{H}_{22} \mathrm{~N}_{2} \cdot \mathrm{AuCl}_{4}$.

This tetra-methyl derivative of putrescin is enormously poisonous as compared with putrescin. The symptoms are the same as those produced by muscarin or neurin. They are : abundant salivation; dyspnœa-respiration at first increases, then decreases ; contraction of the pupils ; paralysis of the muscles of the limbs and trunk ; increased peristaltic action of the intestines, ejaculation of semen, dribbling of urine, and finally violent clonic convulsions. In the case of mice and guinea-pigs the convulsions are prominent immediately after the injection of the poison.

Putrescin hydrochlorid, $\mathrm{C}_{4} \mathrm{H}_{12} \mathrm{~N}_{2} \cdot 2 \mathrm{HCl}$, forms long colorless needles, which are very easily soluble in water; difficultly so in dilute alcohol ; entirely insoluble in absolute alcohol, and can thus be separated from cadaverin hydrochlorid. To accomplish this separation it is, perhaps, better to dissolve the mixture of the hydrochlorids in hot 96 per cent. alcohol. On cooling the solution thus obtained the putrescin salt crystallizes out, whereas that of cadaverin remains in solution. Putrescin hydrochlorid differs from cadaverin hydrochlorid in that it is not hygroscopic and can be exposed for days to the air without showing any change on the surface of the crystals.

For the behavior of the free base and the hydrochlorid to alkaloidal reagents see Table I. Putrescin is not toxic, though it possesses some marked physiological properties (see cadaverin, page 273). According to Scheurlen, putrescin, like cadaverin, produces inflammation, suppuration and necrosis. It is not poisonous to dogs (Udránszky and Baumann). It is optically inactive.

The platinochlorid, $\mathrm{C}_{4} \mathrm{H}_{12} \mathrm{~N}_{2} \cdot 2 \mathrm{HCl} . \mathrm{PtCl}_{4}(\mathrm{Pt}=39.16$ per cent.), often appears under the microscope in the form of cholesterin-like plates. In the pure condition it appears as six-sided plates, which are superposed in layers. The crystals possess a splendid silvery luster, and are rather difficultly soluble in cold water; less so in hot water.

The aurochlorid, $\mathrm{C}_{4} \mathrm{H}_{12} \mathrm{~N}_{2} \cdot 2 \mathrm{HCl} .2 \mathrm{AuCl}_{3}+2 \mathrm{H}_{2} \mathrm{O}$, crystallizes likewise in plates, which are difficultly soluble in cold water. It can, therefore, be readily separated from cadaverin aurochlorid, which is easily soluble in water. The water of erystallization can be driven off completely only at $110^{\circ}$ (Brieger). According to Bocklisch, it loses this water on standing over sulphuric acid, or on heating at $100^{\circ}$.

The picrate, $\mathrm{C}_{4} \mathrm{H}_{12} \mathrm{~N}_{2} \cdot 2 \mathrm{C}_{6} \mathrm{H}_{2}\left(\mathrm{NO}_{2}\right)_{3} \mathrm{OH}$, is difficultly soluble, and crystallizes from a hot aqueous solution in needles; from hot aqueous alcohol, on cooling, in yellow plates. It begins to brown at $230^{\circ}$, and on further heating becomes darker, till finally, at $250^{\circ}$, it decomposes with rapid evolution of gas (Bocklisch). 
The carbonate is crystalline.

The mercury double salt is easily soluble in a large quantity of water, and can thus be separated from the cadaverin salt, which is difficultly soluble. From hot concentrated aqueous solution it crystallizes in needles.

The dibenzoyl-putrescin, $\mathrm{C}_{4} \mathrm{H}_{8}\left(\mathrm{NHCOC}_{6} \mathrm{H}_{5}\right)_{2}$, forms silky plates or long needles, which are more difficultly soluble in hot alcohol than those of the cadaverin compound. From this solution it is reprecipitated by addition of water or ether. Its melting-point is $175^{\circ}$. It sublimes without decomposition.

Cadaverin, $\mathrm{C}_{5} \mathrm{H}_{14} \mathrm{~N}_{2}$, is a diamin isomeric with saprin and neuridin, and, like the latter, it occurs very frequently in decomposing animal tissues. Twelve isomers of this composition are possible. Another isomer, gerontin (see next chapter), has been described by Grandis (1890). It is a very striking fact that in ordinary putrefaction as cholin disappears the diamins appear and increase in quantity according as the time of putrefaction is extended. It is also worthy of note that cadaverin appears in putrefaction before putrescin. It has been obtained by Brieger (1885) from human lungs, hearts, livers, etc. (hence the name), which were allowed to putrefy at the ordinary temperature for three days; from the same organs, and from horseflesh, after four months in a closed vessel at $-9^{\circ}$ to $+5^{\circ}$; from horseflesh after four months at $15^{\circ}$, together with cholin, and probably muscarin (Gulewitsch); from putrid mussel after sixteen days; from putrid egg and blood-albumin. It seems to be a constant product of the growth of the comma bacillus, irrespective of the soil on which it is cultivated.

Bocklisch has isolated it from perch and pike, six days in midsummer; from herring, twelve days in spring; from haddock, two months at a low temperature; from cultivations of Finkler and Prior's vibrio proteus on beef-broth, thirty to thirty-five days at $37^{\circ}$ to $38^{\circ}$ (Ber.chem. Ges., 20, 1441). Cadaverin seems to be a constant product of the activity of the genus vibrio, and it does not usually occur in cultures in which this genus is absent. Thus, it is not present in the excrements of healthy or typhoid patients, although Jakowski did find it in the intestinal contents in a case of fistula. It has not been obtained from cultures of Emmerich's bacillus, of Eberth's bacillus, and of the pyogenic bacteria. It occurs in cultures of the bacillus of hog cholera (Schweinitz); and of the bacillus piscicidus agilis (Sieber). Oechsner de Coninck has found it in putrid jelly-fish (Hugounenq). It is present with putrescin in the urine and feces of cystinuria (Udránszky and Baumann (1888), see page 265). The odor of cholera stools and the breath of cholera patients may be possibly due to cadaverin, although Roos has not been able to obtain diamins from the rice-water discharges of cholera. In one of four 
cases a small amount of a dibenzoyl-compound, crystallizing in small white plates and needles, and melting at $175^{\circ}-177^{\circ}$, was obtained. This corresponds with the putrescin compound. No diamins were found in two cholera-urines. It would, therefore, seem that in Asiatic cholera diamins are not usually found in the feces, and since they are present in the feces and urine of cystinuria without bad results, it is evident that they cannot exercise any great action as intestinal poisons in cholera.

In a diarrhoea, where a coliform bacillus was present, Roos found both cadaverin and putrescin in the discharges, but not in the urine of one case. In another case cadaverin was likewise probably present. Werigo has reported cadaverin from the intestinal contents of a woman with intestinal fistula. He would consider cadaverin as a normal product of pancreatic digestion.

Brieger was the first to show that diamins were absent from normal feces. Baumann and Udránszky confirmed this observation with reference to man and the dog. The discharges of various diseases gave negative results except in typhoid stools, where a very small amount of dibenzoyl compounds, melting at $140^{\circ}$, was found. Roos, in 1891, was able to find but two cases with diamins in the feces. In one case of dysentery and malaria of tropical origin cadaverin was found, and in a case of cholerine putrescin was detected. It has also been obtained from caviar. Löbisch and Rokitansky have reported it in bronchiectasic sputum. Werigo has obtained it from pancreas extracts before putrefaction has set in, while Garcia isolated it from putrefying meat and pancreas together with putrescin and hexamethylenediamin.

At least one definite source of cadaverin is known and that is the hexon base lysin (which see). By allowing lysin or di-amido caproic acid to putrefy Ellinger (1900) obtained cadaverin. The presence of the lysin group in the proteid molecule is therefore necessary to the formation of this diamin. Thus, while lysin forms in sterile auto-digestion of the pancreas, in putrefaction of the latter cadaverin results.

Cadaverin occurs in the mercuric chlorid precipitate, from which it is isolated according to the methods given on pages 266 and 278 . For its isolation and separation from putrescin by the use of benzoyl chlorid, see page 266.

This base was at first ascribed the formula $\mathrm{C}_{5} \mathrm{H}_{16} \mathrm{~N}_{2}$, but subsequent researches led Brieger and Bocklisch to the adoption of the formula $\mathrm{C}_{5} \mathrm{H}_{14} \mathrm{~N}_{2}$. In 1883, Ladenburg prepared, as the first step in the synthesis of piperidin, a base, pentamethylenediamin, possessing the same empirical formula as cadaverin, and later (Ber. chem. Ges., 18, 2956) he showed the possibility of the identity of these two bases. This led to their direct comparison and the successful establishment of their identity. In fact, Ladenburg, as a crucial test of the identity, con- 
verted cadaverin into piperidin, and found the latter base to agree entirely in its chemical and physical properties with those of the natural alkaloid (Ber., 19, 2586). Ladenburg, however, observed one apparent difference between cadaverin and pentamethylenediamin, and that was in the composition of the mercury double salts. That of the former base, whether obtained from alcoholic or aqueous solution (Bocklisch, Ber., 20, 1441), was found to combine with four molecules of mercuric chlorid; whereas the double salt of pentamethylenediamin was found by Ladenburg to contain only three molecules of mercuric chlorid. Subsequently he found that he had prepared this salt by mixing the aqueous solutions of the hydrochlorid of the base and of the mercuric chlorid in the molecular ratio of 1 to 4 , and on using a larger excess of mercuric chlorid he obtained a salt containing four molecules of mercuric chlorid (Ber., 20, 2216). The complete identity of these two bases has, therefore, been established. The constitutional formula of cadaverin is, therefore :

$$
\mathrm{NH}_{2}-\mathrm{CH}_{2}-\mathrm{CH}_{2}-\mathrm{CH}_{2}-\mathrm{CH}_{2}-\mathrm{CH}_{2}-\mathrm{NH}_{2} \text {. }
$$

Cadaverin can be prepared synthetically according to Ladenburg's method. For this purpose trimethylene bromid is converted into the cyanid, and this is then reduced by sodium in absolute alcohol.

Cadaverin forms a somewhat thick, water-clear, syrupy liquid, which possesses an exceedingly unpleasant odor, resembling somewhat that of coniïn (piperidin) and of semen. When dehydrated with potassium hydrate it boils at $115^{\circ}-120^{\circ}$ (Brieger). It boils at $175^{\circ}$ (Brieger, III., 98), and fumes in the air. The base eagerly absorbs carbonic acid from the air, and solidifies into a crystalline mass, the carbonate. It is volatile with steam, and can be distilled, without decomposition, even in presence of sodium or barium hydrate or soda-lime. Neuridin, its isomer, decomposes under these circumstances. When heated with alcoholic potash and chloroform it does not give the isonitril reaction, nor does it give the characteristic odor of oil of mustard on treatment with carbon disulphid and mercuric chlorid. The absence of these reactions at first induced Brieger to conclude that cadaverin and putrescin were not primary amins, but Ladenburg (1885) showed that this conclusion was not justifiable. These two reactions are given by primary monamins, but in this case they are not given by cadaverin, a primary diamin. It is probable that this behavior holds true for all diamins.

Cadaverin is, undoubtedly, identical with the so-called "animal coniïn," which has been isolated at various times from cadavers.

Cadaverin and putrescin were at first regarded as physiologically indifferent, but more recent investigations by Scheurlen, Grawitz, and others, show that both these bases are capable of producing strong inflammation and necrosis. According to Behring, in large doses it is poisonous to mice, rabbits, and guinea-pigs ; it is not poisonous to 
dogs (Udránszky and Baumann). The synthetic putrescin according to $\mathrm{Pohl}$ produces marked dyspnœa in rabbits ; larger doses $(0.4 \mathrm{~g}$.) cause persistent tonic convulsions and paralyses of the extremities. Cadaverin is one of those substances which can set up suppuration in the absence of bacteria. In cholera Asiatica the necrosis of the intestinal epithelium is quite common, and it would seem that this pathological change, as well as the muscular spasms and algidity, are due to the presence of these bases. It should be noted, however, that Udránszky and Baumann failed to obtain any sign of intestinal irritation on feeding dogs enormous doses of cadaverin ; and, moreover, Roos (page 271) failed to find these bases in the feces of cholera. Besides these local effects, they prevent, even in small quantity, the coagulation of blood, and render it "laky." According to Grawitz, cadaverin seems to hinder the growth of bacteria. The cystitis observed in cystinuria may possibly be due to the presence of cadaverin and putrescin in the urine. Both bases are optically inactive.

When cadaverin is treated with methyl iodid, a base is obtained the hydrochlorid of which gives with platinum chlorid a double salt, having the composition, $\mathrm{C}_{5} \mathrm{H}_{12}\left(\mathrm{CH}_{3}\right)_{2} \mathrm{~N}_{2} \cdot 2 \mathrm{HCl} \cdot \mathrm{PtCl}_{4}$. This new base, therefore, is cadaverin in which two atoms of hydrogen have been replaced by two methyl radicals. The platinochlorid of this derivative forms long, clear red needles, which, unlike those of cadaverin, do not change their shape on repeated recrystallization. It is moderately difficultly soluble in water (Brieger, II., 41). Since cadaverin is a primary diamin it shonld combine with six molecules of methyl iodid to form a saturated compound. This, however, has not been obtained.

The hydrochlorid, $\mathrm{C}_{5} \mathrm{H}_{14} \mathrm{~N}_{2} \cdot 2 \mathrm{HCl}$, crystallizes in beautiful, long deliquescent needles (Brieger). According to Bocklisch it forms long, colorless needles or prisms ; crystallizes from alcohol in plates, and is not deliquescent except on long standing. From 95 per cent. alcohol it crystallizes in short, pointed stellate prisms, which are not deliquescent (Gulewitsch). On evaporation of an aqueous solution it forms very long prismatic crystals. It shows no circumpolarization. It possesses a slight bitter taste (Gulewitsch). It is soluble in water, alcohol, alcohol-ether; but is insoluble in absolute alcohol, ether, etc. It can readily be separated from putrescin hydrochlorid by its solubility in 96 per cent. alcohol (Bocklisch). The strictly pure base, as well as the hydrochlorid, does not give a blue color with ferric chlorid and potassium ferricyanid. For reactions of the hydrochlorid and of the free base, see Table I.

Cadaverin hydrochlorid on dry distillation decomposes into $\mathrm{NH}_{3}$, $\mathrm{HCl}$, and piperidin, $\mathrm{C}_{5} \mathrm{H}_{11} \mathrm{~N}$. The latter is a well-known poisonous alkaloid which exists in the combined state in black pepper. It is not known whether this change, whereby the non-poisonous cadav- 
erin is converted into a toxic base, can take place under the influence of bacteria during the process of putrefaction, or not. However, it does not seem improbable that this simple chemical change should be effected through the action of living organisms ; for Schmidt has already shown that the almost physiologically indifferent cholin, when subjected to the action of the bacteria of hay-infusion, decomposes into a neurin-like base possessing a muscarin-like action, and under certain conditions it yields a base which in its action resembles pilocarpin.

The sulphate likewise forms beautiful, well-formed needles, and in its solubility corresponds to the hydrochlorid.

The platinochlorid, $\mathrm{C}_{5} \mathrm{H}_{14} \mathrm{~N}_{2} \cdot 2 \mathrm{HCl} \cdot \mathrm{PtCl}_{4}(\mathrm{Pt}=38.08$ per cent.), crystallizes after some time, on the addition of platinum chlorid, to a not too concentrated solution of the hydrochlorid, in the form of long, beautiful orange-red needles (Bocklisch). Ordinarily it is obtained at first in long, dirty-red needles, which on repeated recrystallization become clearer and assume a form similar to that of ammonium platinochlorid. It forms chrome-yellow rhombic prisms which are short and octahedral-like. Variation in the crystalline form is observed here as in the case of the mercury compounds. In polarized light they are strongly double refracting. It is very slightly soluble in cold water; can be recrystallized from hot water (Bocklisch). Its solubility in water at $12^{\circ}$ is 1 to $113-114$; at $21^{\circ}$ it is 1 to 70.8 (Gulewitsch). It is soluble in alcohol. It decomposes at $235^{\circ}-236^{\circ}$. It does not lose weight at $125^{\circ}-135^{\circ}$; at $195^{\circ}$ it begins to darken and melts with decomposition at $215^{\circ}$ (Gulewitsch).

The aurochlorid, $\mathrm{C}_{5} \mathrm{H}_{14} \mathrm{~N}_{2} \cdot 2 \mathrm{HCl} .2 \mathrm{AuCl}_{3}(\mathrm{Au}=50.41$ per cent.), crystallizes partly in cubes, aud partly in long needles which at first possess a bright lustre, but under the desiccator soon effloresce and become opaque. It crystallizes from water acidulated with hydrochloric acid, in plates or in large, long, very pretty orange-yellow flat prisms. On rapid crystallization bright platelets form (Gulewitsch). The water of crystallization is completely removed on standing over sulphuric acid. It is very easily soluble, and melts at $188^{\circ}$ (Bocklisch); $186^{\circ}-188^{\circ}$ (Gulewitsch).

The picrate, $\mathrm{C}_{5} \mathrm{H}_{14} \mathrm{~N}_{2} \cdot 2 \mathrm{C}_{6} \mathrm{H}_{2}\left(\mathrm{NO}_{2}\right)_{3} \mathrm{OH}$, forms yellow plates which are difficultly soluble in cold water. From hot water it crystallizes in long prisms, which melt at $221^{\circ}$ with decomposition. When crystallized from 95 per cent. alcohol it forms long yellow needles, which are difficultly soluble in cold, more easily in hot 95 per cent. alcohol. It is insoluble, or very difficultly so, in absolute alcohol, and can be recrystallized from hot dilute alcohol.

Cadaverin hydrochlorid combines with mercuric chlorid, when the aqueous solutions of these two salts are mixed in the molecular ratio of 1 to 4 , to form $\mathrm{C}_{5} \mathrm{H}_{14} \mathrm{~N}_{2} \cdot 2 \mathrm{HCl} .3 \mathrm{HgCl}_{2}$. This salt can be recrys- 
tallized from hot water (Ladenburg). When an excess of mercuric chlorid is used the double salt has the composition $\mathrm{C}_{5} \mathrm{H}_{14} \mathrm{~N}_{2}, 2 \mathrm{HCl}$. $4 \mathrm{HgCl}_{2}$. This last salt melts at $216^{\circ}$ (Ladenburg); at $214^{\circ}$ (Bocklisch). It is difficultly soluble in cold water ; easily in hot water at $21^{\circ}(1-32.5$, Gulewitsch) ; from hot water it crystallizes in needles or plates (Bocklisch). On heating even on the water-bath it loses weight. At $125^{\circ}-135^{\circ}$ it loses 18.33 per cent. of its weight, due to volatilization of mercuric chlorid (Gulewitsch). As pointed out by Brieger, it is quite probable that other mercuric compounds exist than those mentioned. Gulewitsch (1894) showed interesting polymorphism of the mercury salts of cadaverin. He inclined to the belief that it may form compounds with more than three or four molecules of mercuric chlorid. On heating mercuric chlorid is given off, and hence the varieties in form. This variation in form is, therefore, not necessarily due to impurities. When first obtained the cadaverin mercurochlorid forms warty aggregates of dark-brown prisms with pointed ends. By repeated recrystallization from water it eventually forms single or stellate rhombic plates, and on further crystallization very thin, elongated, six-sided, or triangular plates result.

The neutral oxalate, $\mathrm{C}_{5} \mathrm{H}_{14} \mathrm{~N}_{2} \cdot \mathrm{H}_{2} \mathrm{C}_{2} \mathrm{O}_{4}+2 \mathrm{H}_{2} \mathrm{O}$, was prepared by Bocklisch by adding a little less than the calculated quantity of alcoholic oxalic acid to the cadaverin. The precipitate may be recrystallized from hot dilute alcohol, when it is obtained in the form of needles, which melt at about $160^{\circ}$, and at the same time give off gas.

The acid oxalate, $\mathrm{C}_{5} \mathrm{H}_{14} \mathrm{~N}_{2} \cdot 2 \mathrm{H}_{2} \mathrm{C}_{2} \mathrm{O}_{4}+\mathrm{H}_{2} \mathrm{O}$, is made by bringing the neutral salt into alcoholic oxalic acid. It is soluble in hot dilute alcohol, and recrystallizes from it in quadratic plates, sometimes in glistening needles. It melts at $143^{\circ}$ with decomposition. After it has been dried over sulphuric acid it loses, on being heated to $105^{\circ}-$ $110^{\circ}$, one molecule of water (Bocklisch, Ber., 20, 1441). The insolubility of these oxalates in absolute alcohol shows the fallacy of Tamba's distinction between ptomaïns and vegetable alkaloids.

The dibenzoyl-derivative, $\mathrm{C}_{5} \mathrm{H}_{10}\left(\mathrm{NHCOC}_{6} \mathrm{H}_{5}\right)_{2}$, crystallizes in long or small needles and plates, readily soluble in alcohol, difficultly so in ether, and insoluble in water; hence the alcoholic solution can be precipitated by addition of water or ether (separation from the putrescin compound, see page 266). It melts at $129^{\circ}-130^{\circ}$; at $130.5^{\circ}-131.5^{\circ}$ (Gulewitsch). It is not changed by boiling with dilute acids and alkalis; but boiling with concentrated hydrochloric or sulphuric acid for a long time finally breaks it up.

Neuridin, $\mathrm{C}_{5} \mathrm{H}_{14} \mathrm{~N}_{2}$, was the first diamin isolated from animal tissues (Brieger, 1883). It is one of the most common products of putrefaction, and as such has been obtained by Brieger from putrid 
horseflesh, beef, human muscle, five to six days; from haddock, five days in summer; from cheese, six weeks in summer; from gelatin, ten days at $53^{\circ}$; from decomposing human internal organs, three to eleven days; from cultures of the Eberth bacillus, with mydin. Bocklisch has obtained it from perch, six days in summer; from barbel after three days in summer.

It has also been obtained from fresh eggs in the preparation of cholin by heating with baryta; and also from fresh brain by heating with 2 per cent. hydrochloric acid (Brieger, I., 57-61). Ehrenberg (1887) found it in poisonous sausage and obtained it by growing a bacillus from this source on liver and meat bouillon.

Neuridin is almost invariably accompanied by cholin, and as the duration of putrefaction increases the latter gradually decreases in amount and yields a corresponding increase in trimethylamin, whereas the yield of neuridin increases from day to day. The amount of neuridin formed depends upon the nature of the organ employed in putrefaction. The greatest yield is obtained from gelatinous tissues such as intestines; and especially from pure gelatin. On the other hand such tissues as the spleen and liver yield but little.

Neuridin comes down in the mercuric chlorid precipitate (sometimes it occurs in the filtrate), and can then be isolated from the other bases present in a number of ways. One method is given under gadinin. Another convenient method of separation is to precipitate it from alcoholic solution by alcoholic picric acid. The picrate thus obtained is, for the purpose of further purification, recrystallized from absolute alcohol, then decomposed by extracting its acid solution with ether (to remove the picric acid) and evaporating the aqueous solution to dryness. The residue is now extracted with alcohol and the alcoholic solution precipitated by alcoholic platinum chlorid. The platinochlorid can now be recrystallized from hot water.

The free base, as obtained by the treatment of the hydrochlorid with moist, freshly precipitated silver oxid, possesses an extremely repulsive odor, similar to that of human semen.

On evaporation of its aqueous solution it yields a gelatinous-like mass, and at the same time slowly decomposes. It does not crystallize when evaporated in a vacuum, and decomposes even under these conditions. The same disagreeable odor is obtained when the hydrochlorid is warmed with potassium hydrate. Brieger (I., 24) regards this decomposition product of neuridin as an oxidation product of the original substance.

The free base is very readily soluble in water, but is insoluble in ether and absolute alcohol; difficultly soluble in amyl alcohol. It gives white precipitates with mercuric chlorid, neutral and basic lead acetates. When distilled with fixed alkali it yields di- and tri- 
methylamin, thus probably showing some relation to neurin, hence the name neuridin. It does not give Hofmann's isonitril reaction, but it does not follow from this, as shown under cadaverin, that it may not be a primary diamin. It is isomeric with cadaverin, saprin, and gerontin.

The hydrochlorid, $\mathrm{C}_{5} \mathrm{H}_{14} \mathrm{~N}_{2} .2 \mathrm{HCl}$, crystallizes in long needles which are extremely soluble in water and in dilute alcohol, but are insoluble in absolute alcohol, ether, chloroform, petroleum ether, benzene, amyl alcohol, etc. Its insolubility in absolute alcohol may be used to effect a separation from cholin hydrochlorid. It can be recrystallized from slightly warm dilute alcohol. Although the pure salt is insoluble in the reagents just given, nevertheless, in the presence of other animal matter, it is dissolved in greater or less quantity, and hence can be obtained by the Stas-Otto as well as by the Dragendorff method. The crystals resemble urea in form. On heating very cautiously the salt sublimes, and at the same time appears to undergo a partial internal decomposition, inasmuch as many of the groups of needles in the sublimate are colored red or blue. For the behavior of the hydrochlorid with the alkaloidal reagents, see Table $I$.

Pure neuridin is not poisonous, but as long as it is contaminated with other putrefaction products it possesses a toxic action similar to that of peptotoxin. This holds true for the other non-poisonous bases.

The platinochlorid, $\mathrm{C}_{5} \mathrm{H}_{14} \mathrm{~N}_{2} \cdot 2 \mathrm{HCl} . \mathrm{PtCl}_{4}$, crystallizes in beautiful flat needles. Recrystallized from hot water, it forms aggregations of small, clear, yellow needles. It is readily soluble in water, from which it is precipitated on the addition of alcohol.

The aurochlorid, $\mathrm{C}_{5} \mathrm{H}_{14} \mathrm{~N}_{2} \cdot 2 \mathrm{HCl} .2 \mathrm{AuCl}_{3}$, is rather difficultly soluble in cold water (Bocklisch), and crystallizes on cooling of the hot, saturated solution in bunches of clear, yellow, short needles.

The picrate, $\mathrm{C}_{5} \mathrm{H}_{14} \mathrm{~N}_{2} \cdot 2 \mathrm{C}_{6} \mathrm{H}_{2}\left(\mathrm{NO}_{2}\right)_{3} \mathrm{OH}$, can be recrystallized from boiling water, in which it is very difficultly soluble, in the form of needles united in plumose groups. It is almost insoluble in cold water; less difficultly soluble in alcohol. It is not fusible, but begins to brown and give off yellow vapors at $230^{\circ}$, and carbonizes completely at $250^{\circ}$.

Saprin, $\mathrm{C}_{5} \mathrm{H}_{14} \mathrm{~N}_{2}$, was found in human livers and spleens after three weeks putrefaction (Brieger, II., 30, 46, 58). It occurs together with cadaverin, putrescin, and mydalein in the mercuric chlorid precipitate. To separate these bases Brieger (1885) used the following process: The mercury salts were decomposed with hydrogen sulphid, the filtrate evaporated to dryness, and the residue then extracted with alcohol. The putrescin hydrochlorid is insoluble in alcohol, and is thus removed. The alcoholic solution was treated 
with platinum chlorid, which precipitated the greater part of the cadaverin. The mother-liquor, on concentration, yielded a mixture of the platinochlorids of cadaverin and saprin. Each successive crop contained more of the saprin double salt. The two kinds of crystals were now separated by means of a magnifying glass. The saprin platinochlorid thus obtained was finally purified by repeated recrystallization from water. The mother-liquor, after the removal of the sapriu platinochlorid, contains the mydalein salt, which on account of its solubility in water, crystallizes only on concentration, or on standing under a desiccator. The mercuric chlorid filtrate contains some mydalein and the ptomain, which yields a platinochlorid containing 28.40 per cent. platinum.

The free base is a diamin, and was first ascribed the formula $\mathrm{C}_{5} \mathrm{H}_{16} \mathrm{~N}_{2}$. It appears, however, to be isomeric with cadaverin and neuridin. The term saprin is derived from the Greek $\sigma \alpha \pi \rho o$, signifying putrid. It possesses a weak pyridin-like odor, and can be distilled with steam or with potassium hydrate without undergoing decomposition. In its reactions it behaves the same as cadaverin, except that it gives an amorphous precipitate with potassium bismuth iodid, whereas cadaverin gives a crystalline precipitate. The free base gives an immediate intense blue color with ferric chlorid and potassium ferricyanid.

The hydrochlorid, $\mathrm{C}_{5} \mathrm{H}_{14} \mathrm{~N}_{2} \cdot 2 \mathrm{HCl}$, forms flat needles which are not hygroscopic (distinction from cadaverin hydrochlorid). Its reactions are the same as those of cadaverin hydrochlorid. (See Table I.) It is, however, tinged slightly blue by a mixture of ferric chlorid and potassium ferricyanid, whereas the free base gives an intense blue. It differs from cadaverin in that it does not give the reddish-brown color with potassium bichromate and sulphuric acid. Again, it forms no aurochlorid; while, on the other hand, cadaverin hydrochlorid yields an easily soluble salt, crystallizing in splendid needles.

The platinochlorid, $\mathrm{C}_{5} \mathrm{H}_{14} \mathrm{~N}_{2} \cdot 2 \mathrm{HCl} . \mathrm{PtCl}_{4}$, forms parallel, aggregated, pointed crystals, which are somewhat soluble in water, and are thus distinguished from cadaverin platinochlorid, which crystallizes in rhombs, and is difficultly soluble in water.

Physiologically, it is indifferent.

Hexamethylenediamin, $\mathrm{C}_{6} \mathrm{H}_{16} \mathrm{~N}_{2}$ - -This compound was found by Garcia in decomposing meat and pancreas mixture, seven days at $30^{\circ}$, by the benzoyl chlorid method, together with cadaverin and putrescin. The dibenzoyl-putrescin is removed in the usual way by precipitating the alcoholic solution of the mixed beuzoyl diamins with ether. The separation of cadareriu from the new compound is more difficult owing to the great similarity in the solubilities of the two compounds. Garcia succeeded in effecting a separation by dissolving the mixture in alcohol, and raising the temperature on a 
water-bath to $70^{\circ}$; by gradual addition of water (50 volumes), taking care not to allow the temperature to rise above $70^{\circ}$, at which temperature the solution is left for twenty or thirty minutes; may be raised to $90^{\circ}$ and kept there for one hour. The solution is then rapidly filtered through an asbestos plug with the aid of a pump. On cooling, bright, long crystalline plates and needles separate from the filtrate. The residue, dissolved in alcohol and again treated by this process, finally yields a residue of pure benzoyl-cadaverin. The crystals formed in the filtrate melt at $124.5^{\circ}-125^{\circ}$; cadaverin compound at $129^{\circ}-130^{\circ}$.

The dibenzoyl compound on heating with equal parts of alcohol and concentrated hydrochloric acid on a water-bath for forty-eight hours is decomposed. The hydrochlorid is not deliquescent.

The platinochlorid, $\mathrm{C}_{6} \mathrm{H}_{16} \mathrm{~N}_{2} \cdot 2 \mathrm{HCl} . \mathrm{PtCl}_{4}(\mathrm{Pt}=37.01)$, crystallizes from hot water on cooling in elongated, well-formed needles (rhombic system) of dark-orange color. Some were more than 1 $\mathrm{cm}$. long. The crystallographic characters of the salt agree in all respects with those of the cadaverin compound with which it is therefore isomorphous. It is easily soluble in water, difficultly in strong alcohol.

Gold chlorid produces no precipitate in aqueous or alcoholic solution of the base. Picric acid gives a compound easily soluble in water and in absolute alcohol. It crystallizes in needles and plates ; at $200^{\circ}$ becomes brown, and at $210^{\circ}$ it decomposes. In its behavior to gold chlorid it resembles saprin.

This base is not present in cystinuria.

A Base, $\mathrm{C}_{7} \mathrm{H}_{10} \mathrm{~N}_{2}$. - Until very recently the nature of the basic substances which are formed as products of the alcoholic fermentation of sugar or molasses has been but little understood. Krämer and Pinner, in 1869 , found in crude fusel oil a small quantity of a volatile base which they apparently identified with a collidin. This observation was confirmed by Ordonneau and others; and in 1888 Morin contributed an elaborate paper upon the bases formed during alcoholic fermentation. The portion of crude fusel oil which boils above $130.5^{\circ}$ was extracted with slightly acidulated water, the acid aqueous solution thus obtained was made alkaline, and the oily bases which were thus set free were then distilled with vapor of water. The free bases were then dried over potassium lydrate and subjected to fractional distillation. Three fractions were thus obtained, boiling respectively at $155^{\circ}-160^{\circ}, 171^{\circ}-172^{\circ}$, and $185^{\circ}-190^{\circ}$. Only the second fraction, which boils at $171^{\circ}-172^{\circ}$, was studied, and was found to possess the formula $\mathrm{C}_{7} \mathrm{H}_{10} \mathrm{~N}_{2}$. Heated with concentrated hydrochloric acid, it is decomposed in part with the formation of ammonia. It combines with ethyl iodid to form a yellow crystalline compound which is soluble in water and alcohol, insoluble 
in ether. The hydrochlorid crystallizes in fine white needles, soluble in water and alcohol, and but very slightly soluble in absolute ether. The free base, as stated above, boils at $171^{\circ}-172^{\circ}$, is very soluble in water, alcohol, ether, etc. When pure it forms a colorless, strongly refracting, very mobile oil, which possesses a characteristic, nauseating odor, but slightly resembling that of the pyridin bases. Its density at $12^{\circ}$ is 0.9826 ; toward litmus paper the base shows no decided reaction. The platinochlorid is crystalline and is very soluble in water and alcohol, slightly soluble in ether. Potassic mercuric iodid does not precipitate the aqueous solution of the free base, but in solutions of the hydrochlorid it gives a yellow floceulent precipitate, which soon crystallizes in long brilliant yellow needles. This reaction takes place readily in solutions of 1 to 1,000 , and only after some hours in solutions of 1 to 10,000; and is not given by the bases of the pyridic and quinolinic series. Mercuric chlorid produces an immediate flocculent precipitate in solutions of the base having a concentration of 1 to 1,000 , but requires some time to appear in 1 to 10,000 . Phosphotungstic acid gives an immediate white precipitate even in a dilution of 1 to 10,000. Phosphomolybdic acid in solutions of the same strength yields a yellow precipitate.

The physiological action of this basc has been examined by $R$. Wurtz who found the lethal dose for rabbits, etc., to be about one gram per kilogram of body-weight. It produces stupor and paralysis which at first appears in the rear extremities; the sensibility becomes diminished and the pupils are dilated and unresponsive to light; the rate of heart-beat is lowered, and the rectal temperature falls as low as $35^{\circ}$; death follows a more or less prolonged coma.

Tanret obtained by the action of ammonia on glucose a number of bases, to which he applied the generic name of glucosins. One of these, having the formula $\mathrm{C}_{14} \mathrm{H}_{10} \mathrm{~N}_{2}(\mathrm{C}=6)$, corresponds in its formula and its general properties to Morin's base, $\mathrm{C}_{7} \mathrm{H}_{10} \mathrm{~N}_{2}(\mathrm{C}=12)$, and, in fact, the two bases are considered by Tanret to be identical.

It is interesting to note in this connection that alkaloidal bases have been found in petroleum by Bandrowski, and that similar basic substances have been detected by Weller in paraffin oil.

Most of the solvents in common use, such as alcohol, ether, chloroform, benzol, petroleum ether, amyl alcohol, etc., have been shown at different times to contain basic pyridin compounds, though ordinarily in very minute quantity. On the other hand, Haitinger has found in some specimens of amyl alcohol as much as 0.5 per cent. of pyridin.

Susotoxin, $\mathrm{C}_{10} \mathrm{H}_{26} \mathrm{~N}_{2}$ (?), is a base isolated by Novy in 1890 from cultures of the log cholera bacillus of Salmon (swine plague of Billings). It is probably identical with the base obtained by v. Schweinitz from the same germ, although the formula ascribed to it by him 
is $\mathrm{C}_{14} \mathrm{H}_{32} \mathrm{~N}_{2}$. The free base has not been obtained. The hydrochlorid forms a light-yellow syrup which shows no tendency to crystallize. It is soluble in water and in absolute alcohol, and is somewhat hygroscopic.

When heated with fixed alkali it gives off a strong amin odor, such as is perceived on evaporating the original culture fluid, if it happens to be alkaline in reaction.

The platinochlorid is obtained by precipitation as a light, fleshcolored, granular precipitate. It is readily soluble in water, from which it can be reprecipitated by addition of absolute alcohol. From aqueous solution, when allowed to evaporate slowly, it crystallizes in long, thick needles.

The mercurochlorid is thrown down from solutions of the hydrochlorid in absolute alcohol, by alcoholic mercuric chlorid, as a heavy, white, granular precipitate. This readily dissolves on the addition of a small quantity of water, and can be perfectly reprecipitated by addition of absolute alcohol. On treatment with hydrogen sulphid it is readily decomposed, yielding the pure hydrochlorid.

The aurochlorid is very soluble in water and alcohol. From the alcoholic solution it may be partially precipitated by ether as a lightyellow, oily precipitate, which is adherent to the sides and bottom of the tube.

The base is toxic only in relatively large doses, as seen from the following experiment. About 100 milligrams, dissolved in a little water, were injected subcutaneously into a young rat. The animal was at first quiet, apparently unwilling to move. After some ineffectual attempts at jumping it settled down to a recumbent position, and when placed on its side was unable to rise. Respiration was at first retarded, later increased, but toward the end was again very slow. Convulsive tremors shook the body at frequent intervals. The animal kicked vigorously. Reflexes were present almost to the end. As death approached the red eyes whitened and took on a glazed, opaque appearance. Death resulted in one and a half hours. The animal was on its side, the feet extended. Post-mortem examination showed the heart arrested in diastole, lungs rather pale, stomach contracted, serum in thoracic cavity, subcuta pale and œedematous. Repeated doses of smaller quantities seem to confer a partial immunity to the action of the germ.

Methyl guanidin, $\mathrm{C}_{2} \mathrm{H}_{7} \mathrm{~N}_{3}, \mathrm{NH}=\mathrm{C}<{ }_{\mathrm{NH}_{2}}^{\mathrm{NH} \cdot \mathrm{CH}_{3}}$. This base has long been known as a product of the oxidation of creatin and creatinin, but has never been met with in animal tissues. Brieger in 1886 (III., 33) obtained it from horseflesh which was allowed to decompose in a closed vessel at a low temperature $\left(-9^{\circ}\right.$ to $+5^{\circ}$ ) for four months. Bocklisch (Ber., 20, 1441) isolated it 
from impure cultures on beef-broth of Finkler and Prior's vibrio proteus, coutaining ordinary putrefaction bacteria, for twenty to thirty days at $37^{\circ}-38^{\circ}$. Vibrio proteus alone seems incapable of forming this base. The comma bacillus, after some time (six weeks), partially decomposes creatinin with formation of a small quantity of methyl guanidin (Brieger). The bacillus of anthrax likewise is capable of transforming creatin into methyl guanidin. It has been found in rabbits that died of rabbit septicemia.

It occurs in the mercuric chlorid filtrate (Brieger), from which it is obtained, after the removal of the mercury by hydrogen sulphid, on precipitation with phosphomolybdic acid. The precipitate is decomposed with neutral lead acetate, and the filtrate from this, after removal of the lead by hydrogen sulphid, is concentrated and then sodium picrate added. The resinous picrate precipitate is purified by boiling with much water, and, finally, it is recrystallized from boiling absolute alcohol. According to Bocklisch, it occurs in the mercuric chlorid precipitate (not in the filtrate), from which it is isolated, after removal of the mercury and concentration of the clear filtrate, by precipitation with sodium picrate. The precipitate, containing cadaverin, methyl guanidin, and creatinin, is boiled with absolute alcohol (cadaverin picrate is insoluble) and the alcoholic solution is then evaporated to drive off the alcohol and taken up with water. From this aqueous solution, after removal of picric acid, methyl guanidin is precipitated by gold chlorid whereas creatinin remains in solution.

This ptomaïn is identical with the synthetic methyl guanidin (methyluramin), which can be readily obtained by boiling a creatin solution with mercuric oxid or with lead dioxid and dilute sulphuric acid (Dessaignes). The parent substance of methyl guanidin as it occurs in putrefaction is undoubtedly the creatin which exists preformed in the muscular tissue. If such is the case, the bacteria engaged in its production must be considered as possessing an oxidizing action, since this base is prepared synthetically from creatin by oxidation. The change would correspond to that observed in the putrefaction of ornithin and lysin whereby putrescin and cadaverin result (p. 268). The methyl guanidin in turn may be converted into methyl urea and this into ammonia and methylamin. That creatin does not offer much resistance to the action of bacteria is shown in the fact that Friedländer's pneumococcus, which possesses but small chemical powers, is capable of slowly but steadily decomposing creatin, yielding as one of the products acetic acid. Strecker and Erlenmeyer, as well as Baumann, have shown that creatin, although a substituted guanidin, is not poisonous, but is readily converted into creatinin which is a relatively toxic substance. On the other hand, guanidin and methyl guanidin are quite violent poisons. This is, therefore, another instance in which a toxic 
substance is formed by the action of bacteria upon a previously nonpoisonous base (see page 290). According to Lossen, guanidin is formed, though in small quantity, in the oxidation of albumin.

Fischer (1897) obtained this base by oxidizing 1-7 di-methyl guanin whereas 7 methyl guanin gave only guanidin. It would appear from this that the purin antecedent may constitute a source of methyl guanidin and even of creatinin. The hexon base arginin (which see) contains a guanidin group and either it or a related body may likewise share in the production of these bodies.

The formulas of these closely related substances are here given for comparison :

$$
\begin{aligned}
& \mathrm{NH}=\mathrm{C}\left\langle\begin{array}{l}
\mathrm{NH}_{2} \\
\mathrm{NH}_{2}
\end{array}\right. \\
& \mathrm{NH}=\mathrm{C} \sum_{\mathrm{NH}_{2}}^{\text {Methyl GUanidin. }} \\
& \mathrm{NH}=\mathrm{C}\left\langle\begin{array}{l}
\text { Creatinin. } \\
\mathrm{N}\left(\mathrm{CH}_{3}\right) \cdot \mathrm{CH}_{2} \\
\mathrm{NH}-\mathrm{CO}
\end{array}\right.
\end{aligned}
$$

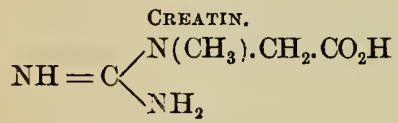
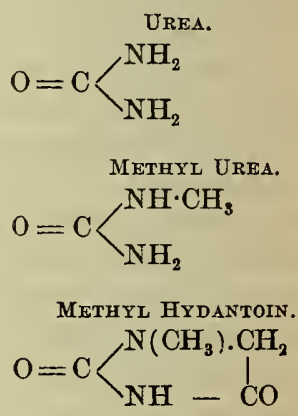

Methyl guanidin forms a colorless, easily deliquescent mass possessing a strong alkaline reaction. On heating with potassium hydrate it decomposes and yields ammonia and methylamin. It is a highly poisonous base.

The hydrochlorid, $\mathrm{C}_{2} \mathrm{H}_{7} \mathrm{~N}_{3} \cdot \mathrm{HCl}$, can be obtained from the picrate by dissolving the latter in water acidulated with hydrochloric acid, and extracting the solution with ether to remove the picric acid. The colorless aqueous solution now on evaporation yields a thin syrup which crystallizes in vacuum to compact prisms. These are insoluble in alcohol, and give with platinum chlorid a double salt of monoclinic needles (Haushofer) which are very easily soluble (1 part in about 7 parts of water, Tatarinow).

The aurochlorid, $\mathrm{C}_{2} \mathrm{H}_{7} \mathrm{~N}_{3} \cdot \mathrm{HCl} . \mathrm{AuCl}_{3}(\mathrm{Au}=47.71$ per cent.), forms rhombic crystals (Haushofer) which are easily soluble in ether, more difficultly in water or alcohol; readily soluble (Brieger). It readily decomposes on heating in pure water, but may be crystallized from water acidulated with hydrochloric acid. It melts at $198^{\circ}$.

The picrate, $\mathrm{C}_{2} \mathrm{H}_{7} \mathrm{~N}_{3} \cdot \mathrm{C}_{6} \mathrm{H}_{2}\left(\mathrm{NO}_{2}\right)_{3} \mathrm{OH}$, comes down at first as a resinous precipitate, which when boiled with much water solidifies in the form of felted needles. It is very difficultly soluble in water, 
and can be purified by repeated recrystallization from boiling absolute alcohol-distinction from cadaverin. It melts at $192^{\circ}$. According to Fischer it forms long yellow plates which melt at $200^{\circ}$ and decompose with evolution of gas at $250^{\circ}$.

The oxalate, $\left(\mathrm{C}_{2} \mathrm{H}_{7} \mathrm{~N}_{3}\right)_{2} \cdot \mathrm{H}_{2} \mathrm{C}_{2} \mathrm{O}_{4}+2 \mathrm{H}_{2} \mathrm{O}$, forms crystals which are easily soluble in water.

Methyl guauidin as obtained from putrefying flesh is identical in its physiological action with the synthetic base. It has already been stated that the non-poisonous creatin is readily converted into the relatively energetic poison creatinin. The latter substance possesses a paralyzing action differing very much from its decomposition product methyl guanidin. This base is very poisonous, and the symptoms are marked by dyspnœa, muscle-tremor, and general clowic convulsions. Brieger has observed the following symptoms on injection of about 0.2 gram of methyl guanidin into a guinea-pig: The respiration at once becomes more rapid, and in a few minutes abundant passage of urine and stool takes place; the pupils dilate rapidly to the maximum and cease to react. The animal is uneasy but motionless, though not exactly paralyzed. Respiration becomes deeper and more labored, the head moves from side to side, the extremities become gradually paralyzed; dyspnœa sets in, the animal falls on its side, and dies (twenty minutes) amid general clonic convulsions of short duration. Fibrillary twitchings of the trunkmuscles are observed only in the beginning. Post-mortem showed the heart to be stopped in diastole, the intestines filled with fluid, the bladder contracted, the cortex of the kidney hyperæmic, but the papillæ of the kidneys surprisingly pale.

Morrhuin, $\mathrm{C}_{19} \mathrm{H}_{27} \mathrm{~N}_{3}$, was obtained by Gautier and Mourgues (1888) from the mother-liquors of asellin on concentration of the platinum-containing liquid. This substance constitutes about onethird ( 0.07 per cent.) of all the bases found in cod-liver oil, and is named after Gadus morrhua, the ordinary codfish. The free base is an oily, very thick, amber-yellow liquid, the odor of which resembles somewhat that of syringa. It floats on water and partially dissolves; is more soluble in ether and in alcohol. The base is very alkaline and is caustic to the tongue. It absorbs carbonic acid and is non-volatile. The salts of copper are precipitated by it but the hydrate formed is not redissolved.

The hydrochlorid is very deliquescent. The gold salt forms a yellow precipitate which readily dissolves on warming. The platinum salt, $\mathrm{C}_{19} \mathrm{H}_{27} \mathrm{~N}_{3} .2 \mathrm{HCl} . \mathrm{PtCl}_{4}(\mathrm{Pt}=27.56$ per cent.), crystallizes in barbed needles, which are quite soluble. (Separation from asellin, p. 286.)

The base possesses the property of exciting the appetite; it acts as a diaphoretic, and, above all, as a diuretic. 0.029 gram given 
subcutaneously to a guinea-pig produced in two and a half hours a loss of 13.5 grams in the weight of the animal. The same effect is produced in birds. Strong doses (0.1 gram per kilogram) produce fatigue and hebetude.

A Base, $\mathrm{C}_{13} \mathrm{H}_{20} \mathrm{~N}_{4}$, was obtained as .early as 1868 by Oser, who observed its formation during the fermentation of pure cane-sugar by means of yeast. The hydrochlorid when dried in vacuo is said to form a white, very hygroscopic foliaceous mass which soon becomes brown on exposure to air. At first it imparts a burning taste which is soon replaced by a very bitter sensation.

A Base corresponding to the formula $\mathrm{C}_{17} \mathrm{H}_{38} \mathrm{~N}_{4}$ was obtained by Gautier and Etard from the mother-liquors of the platinochlorid of the base $\mathrm{C}_{8} \mathrm{H}_{13} \mathrm{~N}$. Very little is known, however, in regard to the general properties of this base owing to the small quantity which could be isolated. This base and the one obtained by Oser from the yeast fermentation of sugar, $\mathrm{C}_{13} \mathrm{H}_{20} \mathrm{~N}_{4}$, and asellin, $\mathrm{C}_{25} \mathrm{H}_{32} \mathrm{~N}_{4}$, are the only ptomaïns thus far isolated which are known to contain four atoms of nitrogen.

The platinochlorid, $\mathrm{C}_{17} \mathrm{H}_{38} \mathrm{~N}_{4} \cdot 2 \mathrm{HCl} . \mathrm{PtCl}_{4}(\mathrm{Pt}=27.52$ per cent.), is readily soluble and crystallizes in needles which possess a lightyellow flesh color. When heated to $100^{\circ}$, it slowly decomposes giving off a syringa-like odor.

Asellin, $\mathrm{C}_{25} \mathrm{H}_{32} \mathrm{~N}_{4}$, was isolated by Gautier and Mourgues (1888), together with five other bases, from cod-liver oil. It is present only in small quantity in the oil. The name is derived from Asellus major, the great codfish. The free base is thrown down from the solutions of the hydrochlorid by the addition of alkali, in amorphous white floccules which are almost insoluble in water. It is almost colorless, but on exposure to the air becomes slightly green. It is not. hygroscopic, and possesses a density of about 1.05. On heating it melts to a viscid yellowish fluid, possessing an aromatic odor; it is non-volatile. Although almost insoluble in water, it imparts to this an alkaline reaction and a bitter taste. It is soluble in ether, more so in alcohol.

The salts are crystallizable, but are partially dissociated by the action of warm water. The hydrochlorid forms crossed or entangled needles which are quite bitter. The gold salt is very reducible. The platinochlorid, $\mathrm{C}_{25} \mathrm{H}_{32} \mathrm{~N}_{4} .2 \mathrm{HCl} \mathrm{PtCl}_{4}(\mathrm{Pt}=24.41)$, is orangeyellow in color; soluble in warm water, insoluble in cold water (separation from morrhuin, p. 285), and is rapidly changed by boiling water. The mercury salt is precipitated in the cold, redissolves on heating, and then, on cooling, recrystallizes.

In large doses it produces fatigue, short and rapid respiration, and stupor. Three milligrams of the hydrochlorid killed a greenfinch in fourteen minutes. 
MIydin, $\mathrm{C}_{8} \mathrm{H}_{11} \mathrm{NO}$, is a non-poisonous base which was obtained by Brieger in 1886 (III., 25) from the putrefaction of about two hundred pounds of human internal organs; and also in cultures of the Eberth bacillus on peptonized blood-serum. It occurs in the mercuric chlorid filtrate and is isolated, after the removal of the mercury by hydrogen sulphid, by precipitation with phosphomolybdic acid. The gummy precipitate which is produced is decomposed on the water-bath with a solution of neutral lead acetate, and the filtrate on evaporation yields a colorless hydrochlorid, crystallizing in plates. It is purified by recrystallization of the picrate.

The free base is strongly alkaline, and possesses an ammoniacal odor. It is characterized by its strong reducing properties. The name mydin is derived from $\mu \nu \delta \alpha \alpha \omega$, to putrefy. With platinum chlorid it gives, after a time, an extremely soluble salt ; with gold chlorid, a precipitate of metallic gold. On distillation it is decomposed.

The hydrochlorid, $\mathrm{C}_{8} \mathrm{H}_{11} \mathrm{NO} . \mathrm{HCl}$, crystallizes in colorless plates. It gives a blue color with ferric chlorid and potassium ferrocyanid.

The picrate, $\mathrm{C}_{8} \mathrm{H}_{11} \mathrm{NO} . \mathrm{C}_{6} \mathrm{H}_{2}\left(\mathrm{NO}_{2}\right)_{3} \mathrm{OH}$, is obtained in broad prisms, which melt at $195^{\circ}$. It is the only salt suitable for manipulations.

In describing Nencki's collidin (page 256) it was stated that tyrosin might be looked 'upon as the source of that base. It would seem, however, to be more appropriately the parent substance of mydin, inasmuch as it decomposes on being heated to $270^{\circ}$ into carbonic acid and oxyphenyl-ethylamin, $\mathrm{C}_{8} \mathrm{H}_{11} \mathrm{NO}$. The change that takes place can be represented by the equation :

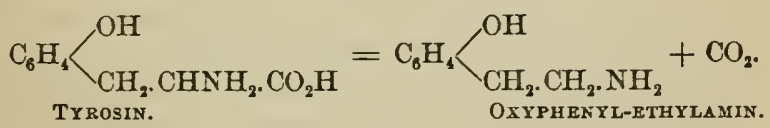

A Base, $\mathrm{C}_{5} \mathrm{H}_{11} \mathrm{NO}_{2}$, was isolated by $\mathrm{E}$. and $\mathrm{H}$. Salkowski (1883) from decomposing fibrin and meat. An amino-valerianic acid is formed in the hydrolytic cleavage by means of acids of the protamin clupein (Kossel); and of casein (Fischer, 1901). The $\delta$-acid also results in the oxidation of piperidin (Ber., 25, 2778). For diaminovalerianic acid, see ornithin.

It is extremely soluble in water, very difficultly so in alcohol, insoluble in ether, and possesses a semen-like odor and saline taste. The aqueous solution, which is not alkaline in reaction, yields on evaporation a stellate crystalline mass which on standing over sulphuric acid becomes a white powder melting at $156^{\circ}$. It dissolves silver oxid but not cupric hydrate, thus apparently indicating that it is not an amido acid. Moreover, it does not give a precipitate or blue coloration with copper acetate or ammoniacal silver nitrate. It thus differed from the then known amido-valerianic acids, its isomers. Later, however (1891), Gabriel and Aschan showed 
that $\delta$-amido-valerianic acid agrees with this base in its reactions to copper nitrate. The gold salt of the synthetic base possessed the same composition as that of Salkowski, and melted at $86^{\circ}-87^{\circ}$.

The identity of this base with $\delta$-amido-valerianic acid (homopiperidinic acid) would seem to be established, and as such it is regarded. Its structure, then, is represented by:

$$
\mathrm{NH}_{2} \cdot \mathrm{CH}_{2} \cdot \mathrm{CH}_{2} \cdot \mathrm{CH}_{2} \cdot \mathrm{CH}_{2} \cdot \mathrm{CO}_{2} \mathrm{H} \text {. }
$$

For its synthetic preparation, see Ber., 24, 1365 (1891). The base does not seem to possess toxic action.

The hydrochlorid, $\mathrm{C}_{5} \mathrm{H}_{11} \mathrm{NO}_{2} \cdot \mathrm{HCl}$, forms colorless, stellate crystals, which are permanent in the air, and are extremely soluble in water, even in absolute alcohol.

The aurochlorid, $\mathrm{C}_{5} \mathrm{H}_{11} \mathrm{NO}_{2} \cdot \mathrm{HCl} \cdot \mathrm{AuCl}_{3}+\mathrm{H}_{2} \mathrm{O}$, is obtained on slow evaporation, as large, well formed, beautiful dark yellow crystals. They are probably monoclinic, contain water of crystallization and melt at below $100^{\circ}$.

The platinochlorid gave on analysis results corresponding to the formula $\left(\mathrm{C}_{7} \mathrm{H}_{15} \mathrm{NO}_{2} \cdot \mathrm{HCl}\right)_{2} \mathrm{PtCl}_{4}$. This possibly may have been due to the presence of some higher homologues of the base $\mathrm{C}_{5} \mathrm{H}_{11} \mathrm{NO}_{2}$. It forms fine orange-yellow crystals, which are very difficultly soluble in alcohol, easily so in hot water, from which, on cooling, it crystallizes in beautiful plates.

Cholin Group.-The following four bases are closely related, and, indeed, starting from cholin, the oldest and best known individual, the remaining bases can be readily prepared from it. Moreover, they can all be prepared synthetically according to methods that will be subsequently indicated. As cholin is the most prominent member, we have thought best to class these substances together as constituting the cholin group. It is very probable that mydatoxin and mytilotoxin, when their constitution becomes known, will be found to be homologues of certain members of this group.

Neurin, $\mathrm{C}_{5} \mathrm{H}_{13} \mathrm{NO}=\mathrm{C}_{2} \mathrm{H}_{3} \cdot \mathrm{N}\left(\mathrm{CH}_{3}\right)_{3} \cdot \mathrm{OH}$. - This substance was obtained and named thus by Liebreich (1865), who prepared it by boiling protagon for twenty-four hours with concentrated baryta. Previous to its discovery as a decomposition product of protagon from the brain it was prepared synthetically by Hoffmann (1858) by treating trimethylamin and ethylene bromid with potassium hydrate or silver oxid. Baeyer (1866), by boiling an alcoholic extract of the brain with baryta water, obtained, on separation by three different methods, a base or rather a mixture of bases, which on analysis gave results corresponding to the three formulæ:

$$
\begin{aligned}
& 12 \quad 2 \quad 3 \\
& \left(\mathrm{C}_{5} \mathrm{H}_{14} \mathrm{NOCl}\right)_{2} \mathrm{PtCl}_{4} \quad\left(\mathrm{C}_{5} \mathrm{H}_{12} \mathrm{NCl}\right)_{2} \mathrm{PtCl}_{4} \quad\left(\mathrm{C}_{5} \mathrm{H}_{14} \mathrm{NCl}\right)_{2} \mathrm{PtCl}_{4}
\end{aligned}
$$


Formula No. 3 was the one accepted by Liebreich for neurin, but, according to Baeyer, Liebreich's neurin salt was not simple but a mixture of Nos. 1 and 2. He himself accepted formula No. 1 as the platinochlorid of neurin, and distinctly states (Annal. d. Chem. u. Pharm., 142, 323, 1867) that neurin is in composition trimethyl oxyethyl-ammonium hydroxid. And according to him, cholin from bile and sinkalin from white mustard appear to be identical with neurin.

This nomenclature of Baeyer's was at first adopted by Wurtz and others, who showed that the oxyethyl base was identical with cholin and sinkalin. On that account Strecker, in 1868 (Annal., $148,79)$, suggested the restriction of the name cholin to the oxyethyl base, and to reserve the name neurin for the base whose platinochlorid is represented in No. 3, as originally was done by Liebreich. In 1869 Liebreich showed that pure protagon, when heated with baryta for twenty-four hours, yields a substance having the composition of the vinyl base :

$$
\mathrm{N}\left\{\begin{array}{l}
\left(\mathrm{CH}_{3}\right)_{3} \\
\mathrm{CH}=\mathrm{CH}_{2} \\
\mathrm{OH} .
\end{array}\right.
$$

The platinochlorid of this base crystallized in five-sided yellow plates, which after a time, on exposure to the air, became cloudy ; on treatment now with water a portion dissolved and the solution was found to contain the oxyethyl base. Furthermore, he observed that when the alcoholic extract of the brain, from which all the protagon had been removed, is treated with baryta, only the latter, the oxyethyl base, is obtained. Finally, in 1870 , Wurtz abandoned the use of the term neurin to designate the oxyethyl base, and returned to the name cholin, originally applied to the oxyethyl base by its discoverer, Strecker. Nevertheless, the confusion in the use of these two terms continued to exist even until very recent years, causing no little misunderstanding. Thus, Marino-Zuco (1885), in his excellent researches on the genesis of ptomains, applies the term neurin, following Baeyer's precedent, to the oxyethyl base, $\mathrm{C}_{5} \mathrm{H}_{15} \mathrm{NO}_{2}$, which is really cholin, according to the proper nomenclature.

We have gone somewhat at this point in detail into the history and proper use of the terms neurin and cholin because of the confusion which is sure to arise if the distinction is not thoroughly borne in mind. The name neuriu, then, should be used only to denote the vinyl base $\mathrm{C}_{5} \mathrm{H}_{13} \mathrm{NO}$. It is trimethyl-vinyl-ammonium hydrate. On the other hand, cholin is the oxyethyl base $\mathrm{C}_{5} \mathrm{H}_{15} \mathrm{NO}_{2}$, which is trimethyl-oxyethyl-ammonium hydrate.

Neurin has been obtained by Brieger (1883) in the putrefaction of horse, beef and human flesh for five or six days in summer. It also occurs in the commercial, so-called "neurin," together with cholin (Brieger, I., 34); in commercial 25 per cent. cholin (Schmidt). 
Liebreich obtained it in the decomposition of protagon by baryta. Gulewitsch (1899), however, was unable to confirm this change, nor was he able to detect neurin in fresh brain matter. Liebreich's result may possibly be due to the use of partly decomposed organs, in which neurin would be present as a result of the bacterial cleavage of cholin. Brieger (I., 60) also has isolated it along with cholin from fresh human brains by boiling with baryta, but did not obtain it by digesting the brains on the water-bath with 2 per cent. hydrochloric acid. It has been found in putrid, and as a result of this change, poisonous mushrooms (Berlinerblau, 1888). For its synthetic preparation, see Gulewitsch (Zeitsch. $f$. physiol. Chem., 26, 175).

The genesis of neurin is still rather obscure, and it is to be hoped that future investigations may shed more light upon the mysterious production of this highly poisonous base. Its occurrence in the brain together with cholin would seem to indicate that it is either derived from cholin by the removal of water, or that it exists together with cholin, partly replacing the latter in the molecule of protagon (lecithin), according to the hypothesis put forward by Lippmann (page 297).

The question of its derivation from cholin by withdrawal of a molecule of water was subjected early to an interesting experimental discussion. Ch. Gram attempted to explain the production of neurin and other muscarin-like ptomaïns as due to the dehydrating action of the acids employed in the methods of extraction, and, indeed, he claimed to have converted cholin platinochlorid, by heating with hydrochloric acid, into neurin. This statement was disputed by Brieger, and by others, who showed that the platinochlorid of cholin, as well as the hydrochlorid, may be heated with fifteen or thirty per cent., or even concentrated hydrochloric acid, for six to eight hours on a water-bath without any conversion whatever (III., 15).

That neurin may be obtained from cholin, at least by chemical processes, was shown by Baeyer, in 1866, who found that cholin chlorid, when heated with several times its volume of concentrated hydriodic acid and some red phosphorus, gave a compound $\mathrm{C}_{5} \mathrm{H}_{13} \mathrm{NI}_{2}$ which, on digestion with fresh, moist silver oxid, yielded a vinyl base identical with that previously obtained synthetically by Hofmann and now known as neurin. In Hofmann's method for the synthesis of neurin the trimethylamin ethylene bromid (see synthesis of cholin, p. 296) is treated with fresh moist silver oxid. Schmidt and Bode have shown that the iodin compound resulting from the action of hydriodic acid on cholin is the same as that formed by the action of the acid on neurin ; and that on treatment with silver oxid it yields neurin. Cholin, therefore, may be readily changed into neurin. On the other hand, since neurin with hydriodic acid forms the same compound, by heating with silver nitrate cholin is formed. Hence neurin can be easily re-converted into cholin (Schmidt). 
On distilling cholin with water, or on dry distillation, a little neurin forms (Nothnagel). Brieger has tried, unsuccessfully, to bring about this dehydration by the putrefaction of pure cholin (I., 59). However, Schmidt and Weiss (1887) were more successful, and they found that cholin, as well as the hydrochlorid and lactate, is changed by the action of microörganisms into the strongly poisonous neurin. Their results are given in full under cholin (see page 299). More recently (1899) Nesbitt in his study upon auto-intoxications endeavored to show that the toxic neurin may form in the intestines by the dehydration of the cholin in lecithin. After feeding dogs on yolk of eggs for several days, the intestines were ligated and eventually the contents were examined. Cholin was found, likewise good evidence of neurin, and a ptomaïn mentioned on p. 260 . From what has been said it is evident that neurin can only arise from cholin, and this, as will be seen later, is derived from lecithin. Cholin may be kept dry or in aqueous solution for four months without change into neurin (Schmidt).

Neurin is almost invariably accompanied by cholin, from which, however, it can be readily separated by the difference in the solubilities of the platinochlorids. It occurs in the mercuric chlorid precipitate (and in the filtrate), and from this it can be obtained, after removal of the mercury, by precipitating the solution of the mixed hydrochlorids in absolute alcohol by platinum chlorid. The platinochlorids are then separated by recrystallization from water, since the neurin is difficultly soluble, while the cholin salt is readily soluble.

The free base possesses a strong alkaline reaction, and on contact with the fumes of hydrochloric acid it yields a cloud. It liberates ammonia from its salts even in the cold. It behaves toward the heavy metals like a strong base, and prevents the coagulation of albumin by heat. On boiling a concentrated solution of neurin it yields trimethylamin. It is not affected by prolonged boiling with baryta or with sodium alcoholate. According to Liebreich, the alkaline solution cannot be neutralized by passing through it carbonic acid.

With hydriodic acid neurin at $140^{\circ}-150^{\circ}$ forms trimethylamin ethylene iodid, which, on treatment with silver nitrate, as stated above, forms cholin, or on treatment with silver oxid regenerates neurin (Schmidt). This compound is the same as that prepared by Baeyer. It melts at $231^{\circ}$.

Fuming hydrobromic acid has no action on neurin at ordinary temperature or at $100^{\circ}$, but at $160^{\circ}-165^{\circ}$ it yields trimethylaminethylene bromid, which behaves with silver nitrate or oxid as above (Bode and Schmidt). Thus :

(1) $\mathrm{Br} \cdot \mathrm{N}\left(\mathrm{CH}_{3}\right)_{3} \cdot \mathrm{C}_{2} \mathrm{H}_{4} \mathrm{Br}+2 \mathrm{AgNO}_{3}+\mathrm{H}_{2} \mathrm{O}=\mathrm{NO}_{3} \cdot \mathrm{N}\left(\mathrm{CH}_{3}\right)_{3} \cdot \mathrm{C}_{2} \mathrm{H}_{4} \mathrm{OH}+2 \mathrm{AgBr}+$ $\mathrm{HNO}_{3}$.

$$
\mathrm{BrN}\left(\mathrm{CH}_{3}\right)_{3} \cdot \mathrm{C}_{2} \mathrm{H}_{4} \mathrm{Br}+\mathrm{Ag}_{2} \mathrm{O}=\mathrm{OH} \cdot \mathrm{N}\left(\mathrm{CH}_{3}\right)_{3} \cdot \mathrm{CH} \cdot \mathrm{CH}_{2}+2 \mathrm{AgBr} \text {. }
$$


This bromin compound is formed in the synthesis of neurin out of trimethylamin and ethylene bromid. It melts at $230^{\circ}$, is soluble in water and in hot alcohol and forms stellate masses of small plates or prisms. With freshly precipitated silver oxid, according to equation 2 , it yields neurin.

Hypochlorous acid, likewise, breaks up the vinyl group in neurin to form a derivative of cholin, which with silver oxid yields isomuscarin (Bode).

It decomposes readily on standing, more rapidly on heating into trimethylamin.

The chlorid, $\mathrm{C}_{5} \mathrm{H}_{12} \mathrm{~N}$.Cl, is extremely poisonous and crystallizes in fine hygroscopic needles. It is easily soluble in water and alcohol. By the action of hypochlorous acid it is changed to iso-muscarin. For behavior to alkaloidal reagents, see Table I.; also Gulewitsch.

The bromid, Br.N $\left(\mathrm{CH}_{3}\right)_{3} \cdot \mathrm{C}_{2} \mathrm{H}_{3}$, is colorless, wart-like in form, hygroscopic and easily soluble in water and in alcohol, insoluble in ether. It melts at $193^{\circ}$ (Bode).

The iodid, $\mathrm{IN}\left(\mathrm{CH}_{3}\right)_{3} \cdot \mathrm{C}_{2} \mathrm{H}_{3}$, forms colorless, permanent needles ; easily soluble in water; slightly in cold alcohol, easily in hot alcohol. When heated to $180^{\circ}$ it becomes yellow, and at $196^{\circ}$ it melts (Bode).

The picrate forms feathery, gold-yellow, long needles which when heated rapidly melt with decomposition at $263^{\circ}-264^{\circ}$. At $23^{\circ}$ the solubility in water is $1: 91.6$. On heating it dissolves more readily in water and in alcohol (Gulewitsch).

The platinochlorid, $\left(\mathrm{C}_{5} \mathrm{H}_{12} \mathrm{~N} . \mathrm{Cl}\right)_{2} \mathrm{PtCl}_{4}(\mathrm{Pt}=33.60$ per cent.), is difficultly soluble in hot water, and crystallizes in beautiful, well formed, small octahedra belonging to the regular system. The crystals are always single. No twin crystals are observed. Sometimes the crystals contain water of crystallization, at other times they do not (Brieger, I., 33). It melts at $211^{\circ}-213^{\circ}$ (Schmidt); $213^{\circ}-214^{\circ}$ (Bode); with decomposition at $195.5-198^{\circ}$ (Gulewitsch). According to Liebreich, it forms from an aqueous solution in fiveor six-sided, heaped-up plates resembling urea nitrate, while from an alcoholic solution it forms needles which on exposure to air become opaque and are partially converted into the oxyethyl basecholin. The six-sided plates are rarely met with and readily show an octahedral relation (Gulewitsch). The difficult solubility (at $20.5^{\circ}$ it is $1: 37.6$ part of water), octahedral form, always single, and the melting-point distinguish it perfectly from the cholin salt.

The aurochlorid, $\mathrm{C}_{5} \mathrm{H}_{12} \mathrm{~N}$. Cl. $\mathrm{AuCl}_{3}(\mathrm{Au}=46.37$ per cent. $)$, forms a yellow cheesy precipitate which on recrystallization yields flat prisms which are difficultly soluble in hot water (Brieger). At $21.5^{\circ}$ it dissolves in 336.5 parts of water (Gulewitsch). Dissolves easily, and can be purified by crystallization (Liebreich). It melts at $228^{\circ}-232^{\circ}$ (Gulewitsch); $238^{\circ}-239^{\circ}$ (Klein). 
The mercurochlorid may exist in two forms : $\mathrm{C}_{5} \mathrm{H}_{12} \mathrm{NCl}+6 \mathrm{HgCl}_{2}$ and $\mathrm{C}_{5} \mathrm{H}_{12} \mathrm{NCl}+\mathrm{HgCl}_{2}$. The former yields colorless, dull, very fragile plate-like crystals which are difficultly soluble and melt at $230^{\circ}-$ $234^{\circ}$. The latter forms aggregates of very narrow, rather long fragile prisms, which are more soluble in water than the preceding and melt at $198.5^{\circ}-199.5^{\circ}$ (Gulewitsch).

Physiological Action.-Neurin is exceedingly poisonous, even in small doses, and in its action it strongly partakes of the characteristic stamp of poisoning by muscarin. The injection of a few milligrams into frogs produces in a short time a complete paralysis of the extremities, with deadening of reflex excitability. Respiration stops first, while the rate of heart-beat gradually decreases till, finally, stoppage in diastole takes place. The injection of atropin at this point does away with the effect of neurin, so that the heart begins to beat again. Previously atropinized frogs, as a rule, withstand the action of the poison. Immediately after the introduction of this substance there can be observed a distinct period of exaltation, which, however, soon gives way to the characteristic stage of depression seen in the progressive slowing of the rate of heart-beat. Of the warm-blooded animals, cats seem to be much more sensitive to its action than mice, rabbits or guinea-pigs. The symptoms seen in rabbits are profuse moistening of the nasal cavities and upper lip, which is succeeded by an intensely profuse salivation; later on there is noticeable an abundant secretion from the nasal mucous membrane and from the eyes; the latter, however, ceases in a short time. The movements of the heart and of respiration are at first quickened and strengthened, but before long the paralytic effects produce a constant slowing and weakening till finally complete cessation of both movements results. The decided dyspnoea observed gradually alters its character, and just before death the respiration is irregular and superficial. The heart, as in frogs, continues to beat after the respiratory movements have ceased and finally it stops in diastole. Direct application of concentrated solutions of the poison to the eyes produces almost always a contraction of the pupil, while a similar but less constant contraction is seen when it is injected. The peristaltic action of the intestines is heightened to such an extent that continual evacuation takes place. Just before death, violent clonic convulsions occur. Atropin possesses a strong antagonistic action toward neurin and the injection of even a small quantity is sufficient to dispel the symptoms just described.

Cholin, $\mathrm{C}_{5} \mathrm{H}_{15} \mathrm{NO}_{2}=\mathrm{C}_{2} \mathrm{H}_{4} \mathrm{OH} \cdot \mathrm{N}\left(\mathrm{CH}_{3}\right)_{3} . \mathrm{OH}$. -This base is identical with the sinkalin of von Babo, the bilineurin of Liebreich, and the neurin of Baeyer, Marino-Zuco, and others. According to Schmiedeberg and Harnack, it is identical with Letellier's amanitin 
(agaricin), to which they assign, however, the formula $\left(\mathrm{CH}_{3}\right)_{3} \mathrm{~N}$. $\left(\mathrm{CHOH} . \mathrm{CH}_{3}\right) \mathrm{OH}$.

Cholin was first prepared and so named by Strecker, 1862, by treating hog-bile with hydrochloric acid. It was prepared synthetically by Wurtz (1868) by direct union of ethylene chlorhydrin and trimethylamin. 'The reaction that takes place can be represented by the equation :

$$
\left.\left.\mathrm{C}_{2} \mathrm{H}_{4}\left\{\begin{array}{c}
\mathrm{OH} \\
\mathrm{Cl}
\end{array}+\underset{\mathrm{CH}_{3 i}}{\mathrm{CH}_{3}}\right\}\right\} N=\underset{\mathrm{C}_{2} \mathrm{H}_{4} \cdot \mathrm{OH}_{3}}{\mathrm{CH}_{3}}\right\} \text { NCl. }
$$

Baeyer (1866) obtained it by boiling an alcoholic extract of the brain with baryta water; and Liebreich, in 1869, showed that if the alcoholic extract from which all the protagon had been removed be thus treated only cholin is formed, whereas pure protagon on heating with baryta yields neurin (not confirmed by Gulewitsch). It has been obtained from the yolk of eggs; from bile; from fresh brains (Brieger); from fresh eggs, blood, lungs, and hearts, and from lecithin (Marino-Zuco); from human placenta (Böhm); from the eye; from commercial neurin (Brieger); neurin was found in a specimen of commercial cholin (Schmidt); in commercial muscarin sulphate (Nothnagel); from fresh as well as decomposing internal organs of the cadaver (Brieger, 1885); in fresh blood (neurin of Marino-Zuco and Martini); from herring-brine and decomposing pike, three days in midsummer (Bocklisch). It has also been isolated from cultures of vibrio proteus (Bocklisch, Carbone) and of comma bacillus (Brieger). Ehrenberg (1887) found it in poisonous sausages, and by growing a bacillus obtained from this on liver. Gulewitsch has isolated cholin from horseflesh, putrefying at $15^{\circ}$ for four months, together with cadaverin and probably muscarin. It has been found in the intestines of dogs together with neurin and another ptomaïn (p. 260) (Nesbitt); and in fermented fish together with various amines (Mörner).

According to Halliburton (1901) cholin can be extracted from fresh brains by means of physiological salt solution. The fact that this base readily forms in the disintegration of nerve tissue has led to the belief that it may be a most important factor in auto-intoxications. Halliburton has found cholin in traces in the cerebro-spinal fluid and in the blood in nerve degenerations and during the convulsive seizures in general paralysis of the insane, and hence he regards this condition as probably due to cholin poisoning although other products may be also present. In regard to the possible conversion of cholin into neurin and the presence of the latter in auto-intoxications, see pages 291 and 299. See also Florence's crystals.

Not only has cholin been met with in the animal tissues; but it has also been observed within the last few years to be very widely 
distributed in the vegetable kingdom, especially so in fatty seeds. Thus it has been found (Harnack, 1876) accompanying muscarin in toadstool (Agaricus muscarius); in hops, and hence in beer (Griess and Harrow); in the seeds of Trigonella, in Indian hemp, arecaand earth-nuts, hemp seeds and lentils (Jahns); in the seeds of white mustard, as a glycosid (von Babo); in ergot (Brieger); in the germs of pumpkins and lupines (Schulze, Zeitschr. f. physiol. Chem., 11, $365)$; in beech-nuts and morels (Helvella esculenta, Boletus luridus, Amanita pantherina, Böhm); in flores sambuci (elder), and extracts of belladonna, hyoscyamus, ipecacuanha root and Acorus calamus (Kunz), ipecacuanha root (Arndt), and Scopolia Japonica (Schmidt and Henschke); in the sprouts and cotyledons of Soja beans (Schulze, 1888), in the fat from hog's bean, vetch, peas and lupines (Jacobson, 1889); from the lecithin of lupine seeds (Schulze and Steiger); and in Cheken leaves (Myrtus cheken, Weiss). According to Lippmann (Ber., 20,3206), it is present together with betain in the molasses from beet-root sugar. Cholin (Ritthausen) and betain (Böhm) exist together in cotton-seeds; hence, cholin occurs in the press-cakes from cotton-seeds (Böhm). Maxwell by extraction of cotton-seed cake with alcohol obtained about five times as much betain as cholin. With betain it occurs in worm-seed (Artemisia Cina, Jahns); in sprouts of malt and wheat (Schulze and Frankfurt). According to Schulze, and also Ritthausen, cholin occurs with betain and another base in the seed of the vetch, and in peas with a base resembling betain. The two bases have also been found together in the roots and leaves of Scopolia atropoïdes by Siebert.

Partheil found cholin, but not betain, in the seeds of Cytisus laburnum. Kresling obtained it from the pollen of the fir, Pinus sylvestris.

Schulze and his pupils have shown that arginin, cholin, and xanthin bases occur in lupine sprouts. The same compounds with vernin occur in gourd sprouts, whereas in the sprouts of Vicia sativa, betain, cholin, and guanidin are present. The latter is undoubtedly derived from arginin, which see.

Cholin may readily be prepared, after the method of Diakonow, from the yolk of eggs. These are extracted with ether, then with alcohol, and the extracts thus obtained evaporated, when the resulting residues are boiled with baryta for one hour. The filtrate, after the removal of the barium by carbonic acid, is evaporated and the residue is extracted with absolute alcohol. The alcoholic solution is now precipitated with platinum chlorid. Brieger (II., 55) has presented a method which is much simpler in its details and obviates the use of the expensive platinum chlorid. The tissues rich in lecithin, as yolk of eggs, brain, etc., are heated with concentrated hydrochloric acid for some hours on the water-bath. The insoluble residue is filtered off, and the filtrate, after neutralization of the ex- 
cess of free acid with carbonate of sodium, is evaporated. The residue is extracted with alcohol, and the alcoholic solution is precipitated with alcoholic mercuric chlorid. The precipitate thus obtained, on recrystallization several times from a large quantity of boiling water, yields the pure double salt of cholin.

If desirable, it can be made from pure lecithin, best prepared according to Gilson's method. Yolk of eggs is repeatedly shaken up with ether until the latter is colored only a faint yellow; the ether solution then distilled, the residue taken up in petroleum ether and filtered. The filtrate, in a separatory funnel, is well shaken with 75 per cent. alcohol, and this is repeated several times with fresh alcohol. The alcoholic extracts are combined, allowed to stand for some time, then filtered and subjected to distillation to remove traces of petroleum ether. The solution is now set aside in a cool place for several days ; the precipitate which forms consists of cholesterin, etc., and a little lecithin. The alcoholic solution is filtered by decantation, then decolored by boiling with bone-black and rapidly evaporated at $50^{\circ}-60^{\circ}$ to a syrupy consistency. This residue is extracted with ether, the solution filtered and evaporated. The lecithin thus obtained is almost perfectly pure but contains traces of cholesterin. To purify it completely, it can be dissolved in as little absolute alcohol as possible and set aside to precipitate in the cold $\left(5^{\circ}-15^{\circ}\right)$.

Cholin may be prepared synthetically according to the method of Wurtz (see Gulewitsch, Zeitschr. f. physiol. Chem., 24, 514) or of Hofmann and Bode. In the latter ethylene bromid is heated with excess of alcoholic trimethylamin. The resulting bromin compound is treated with silver nitrate, filtered, and the filtrate heated on the water-bath for about eight days yields cholin nitrate. This is the easiest and cheapest method of preparation. It may be prepared from neurin (p. 290).

In regard to the genesis of cholin the preponderance of testimony goes to show that it is derived from the decomposition of lecithin which, according to the researches of Diakonow and others, is one of the most widely distributed compounds occurring in greater or less quantity in all of the animal tissues. Lecithin which is a complex ester, decomposes under the action of acids and alkalis into a base (cholin), glycerin, phosphoric acid and fatty acids (stearic, oleic, palmitic, etc.). Gilson has shown that dilute sulphuric acid slowly decomposes lecithin, forming cholin which, after a few days, disappears; on the other hand sodium hydrate, in even 1 per cent. solution, rapidly decomposes it. This change is undoubtedly accomplished in a similar manner through the agency of bacteria. Brieger (II., 17) is inclined to believe that cholin exists preformed in the various tissues inasmuch as he has been unable to obtain it from the brain, which is rich in lecithin, by boiling with 2 per cent. hydrochloric acid. (See Schulze, page 298.) Prolonged heating with 
concentrated hydrochloric acid was necessary in order to obtain any cholin from the brain. This result of Brieger's is somewhat at variance with that of Marino-Zuco (see Relazione, etc., pages 29, 30 and 38 ) who obtained from 25 grams of lecithin, by the method of Stas, a small quantity of the aurochlorid of a base, while from a similar amount he obtained more relevant quantities by the method of Dragendorff.

The occurrence of cholin in the vegetable kingdom would be inexplicable to us at present were it not that we now know of the existence of lecithin-like bodies in plants, from the decomposition of which substantially the same products are obtained as from the lecithin obtained from the animal tissues. The existence of such a body in plants was first predicted by Scheibler in 1870, who was led to this conclusion in his celebrated study of beet-root sugar because of the presence of oleic acid, glycerin, phosphoric acid, and betain, as well as cholesterin, in the beet-root extracts. This hypothesis was confirmed by Hoppe-Seyler, who, in 1879, found a lecithin-substance in yeast. Schulze found a similar compound in the cotyledons of lupine, while Jacobson observed its presence in mustard-seeds, in fenugreek-seeds, in maize and wheat, in the fat from beans, peas, vetch, and lupines. Heckel showed its presence in globularia, and Lippmann has found it in beet-root. According to Hoppe-Seyler, this lecithin-like substance exists in all vegetable cells undergoing development. Schulze and Likiernik (1891) were the first to prepare lecithin in a pure condition from plants. It was found to possess the same properties and yield the same decomposition products as lecithin from animal tissues. Up to the present time lecithin has always been supposed to contain, as an essential component, a radical which gives rise to cholin on saponification, while on the other hand the fatty acids entering its molecule are well known to be replaceable by one another. Thus we may have a di-stearin lecithin as well as di-olein lecithin. The existence of several lecithins in the yolk of eggs has been recognized for some time, and according to Schulze and Likiernik this is also true of the lecithins in plants. Recent observations of Lippmann (Ber., 20, $3206)$ show that the above basic radical, hitherto regarded as constant in lecithin, may possibly be capable of replacement by other similar radicals. He found on saponifying with baryta two different specimens of lecithin, both obtained from beet-root, that while one of them yielded oleic acid, glycerin, phosphoric acid, and betain, the other lecithin gave oleic acid (and some other fatty acids), glycerin, phosphoric acid, and cholin, with no betain-at least not in isolable quantity. This remarkable difference has led Lippmann to suggest an explanation which, while it may not be the correct one, nevertheless possesses a high degree of probability. According to him, the lecithin molecule may contain interchangeable basic radicals in the 
same manner that it contains interchangeable acid radicals. This view is supported not only in the case of beet-root, where cholin and betain exist together, but the same two bases have been observed in cotton-seed. $A^{3}$ similar coexistence was observed in the toad-stool (Agaricus muscarius), in which cholin and muscarin were found. And, lastly, the same condition holds true probably for mytilotoxin and betain, which were shown to be present together in poisonous mussels.

Lecithin cannot always be regarded as the source of cholin in plants, since this base is known to occur as a glucosid in the seeds of white mustard. The sinapin decomposes according to the equation :

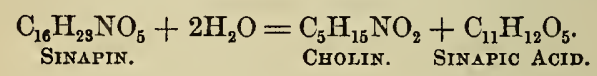

According to Schulze (1891), the cholin which is isolated from pea- and vetch-seeds exists preformed in the seeds and does not result from lecithin by the process of extraction. This is also probably true with reference to cotton-seed cake. The condition in which betain exists is not determined.

Decompositions of Cholin.-Baeyer (1866) succeeded in converting cholin into neurin by a purely chemical process. This was accomplished by heating cholin chlorid with concentrated hydriodic acid and red phosphorus in a sealed tube at $120^{\circ}-150^{\circ}$, whereby the compound $\mathrm{C}_{5} \mathrm{H}_{13} \mathrm{NI}_{2}$ was formed. Fuming hydrobromic acid heated to $160^{\circ}-170^{\circ}$ may also be employed. The iod-iodid of cholin thus obtained, on treatment with moist silver oxid (page 290), gave a base, the platinochlorid of which corresponded to the formula $\left(\mathrm{C}_{5} \mathrm{H}_{12} \mathrm{NCl}\right)_{2} \mathrm{PtCl}_{4}+\mathrm{H}_{2} \mathrm{O}$. This double salt, according to Baeyer, is readily soluble in water and gives reactions similar to cholin. Although Baeyer is emphatic in his assertion that this is the vinyl compound (neurin) formed from the oxyethyl base (cholin), yet it seems that there is room for doubt in regard to the interpretation of his results. Thus neurin platinochlorid is difficultly soluble in water, contrary to the behavior of the platinochlorid obtained by him. On the other hand, cholin platinochlorid is easily soluble in water, and it would seem, therefore, that Baeyer has not converted cholin into neurin, but rather has regenerated cholin from its iodiodid. If such were the case, we would expect that the iod-iodid of neurin, $\mathrm{C}_{5} \mathrm{H}_{13} \mathrm{NI}_{2}$, which has the same composition as the corresponding derivative of cholin, would yield, on treatment with silver oxid, the oxyethyl base. Baeyer was apparently not able to effect this change, since he held that the vinyl base may be prepared from the oxyethyl, but that the reverse, the preparation of the oxyethyl base from the vinyl compound, could not be brought about. This, however, has been successfully accomplished by 
Schmidt. Neurin can be changed into cholin, and vice versa cholin can be changed into neurin (page 290).

Whether the change described by Baeyer takes place or not, it is nevertheless certain that cholin does not readily give up a molecule of water, and thus become converted into neurin. Ch. Gram announced, in 1886, that cholin chlorid and lactate, on heating on the water-bath with dilute hydrochloric acid, decompose and that this conversion into the vinyl base was easy and complete when the aqueous hydrochloric acid solution of cholin platinochlorid was heated for five or six hours on the water-bath. In this way Gram endeavored to explain the formation of neurin as due to the action of acids upon cholin, but Brieger has shown that the platinum salt of cholin, as well as its hydrochlorid, can be heated with fifteen or thirty per cent., or even concentrated hydrochloric acid for six or eight hours without undergoing any change into neurin, thus disproving the results obtained by Gram. E. Sclimidt and Weiss have independently confirmed Brieger's observations in regard to the resistance of cholin to decomposition by acids. Schmidt has gone further, and has shown by an examination of Gram's original preparations that it was cholin and not neurin. Gulewitsch (1894) was likewise unable to split up cholin by acids into neurin. What the action of acids has failed to do is probably accomplished through the agency of bacteria. Schmidt found that cholin chlorid, when allowed to stand with hay infusion, or with dilute blood, for fourteen days at $20^{\circ}-30^{\circ}$, decomposed almost entirely, yielding large quantities of trimethylamin and a base, the platinochlorid of which resembles in form and solubility the double salt of neurin and possesses a similar physiological action. When allowed to decompose for ten days at $30^{\circ}-33^{\circ}$ neither cholin nor neurin was present. Cholin lactate in hay infusion developed an odor of trimethylamin in twelve hours, but at the end of fourteen days a good deal of cholin was still present. In this case no neurin was present, but instead a homologous base was found which can be obtained synthetically by the action of trimethylamin on allyl bromid. According to Meyer, of Marburg, this base does not possess the muscarin-like action of neurin, but resembles more closely pilocarpin.

The decomposition of cholin by putrefaction into neurin, and possibly muscarin, highly poisonous bases, may explain the production of poisons in foods. Nesbitt has in like manner endeavored to show that intestinal auto-intoxication may be due to the formation of neurin out of the cholin derived from the food lecithin (p. 291). The similar view that mental disorders might be due to neurin formation in the brain led Gulewitsch (1899) to examine the leucomains of perfectly fresh brain. He obtained cholin, two bases (possibly diamins) and urea, but no neurin. Neither was he able to confirm Liebreich's view that protagon gave neurin (p. 290). 
Brieger (I., 59) had unsuccessfully tried to transform cholin into neurin by putrefaction. He observed that the cholin decomposed with extreme slowness, even when the putrefaction was carried on at a higher temperature, yielding only trimethylamin. Wurtz (1868) showed that dilute solutions of free cholin can be heated to boiling without any perceptible decomposition. Concentrated solutions, however, decompose with the formation of trimethylamin and glycol, $\mathrm{C}_{2} \mathrm{H}_{4}(\mathrm{OH})_{2}$ (see page 250). The decomposition of cholin was studied somewhat by Mauthner (1873) who confirmed Wurtz's observation that cholin was scarcely decomposed by boiling water, and he showed that when exposed to the action of decomposing blood it yielded trimethylamin. The results obtained by K. Hasebroek (Zeitschrift f. physiol. Chem., 12, 151, 1888) deserve special mention at this place. He carried on the putrefaction of very dilute solutions of the chlorid of cholin in the presence of little or no oxygen in HoppeSeyler fermentation flasks. Sewer slime, because of its strong fermentative properties, was used to induce the putrefaction, and calcium carbonate was added to neutralize any acidity that might develop during the fermentation.

The fermentation, as shown by the evolution of gases, lasted for about three months. The total quantity of gas given off was about one liter from 1.17 grams cholin chlorid. The gases consisted almost entirely of carbonic acid and marsh gas. No hydrogen was evolved. When the fermentation ceased the flask was opened and several cubic centimeters of the almost neutral clear liquid were injected under the skin of a rabbit without producing the least effect.

This liquid distilled with alkali gave methylamin and ammonia. What is remarkable about this experiment was the total absence of the higher amins-as, for instance, trimethylamin, which has been observed so many times as a decomposition product of cholin. The absence of any poisonous base, as neurin, was probably largely connected with the absence of oxygen.

Free cholin ordinarily forms a strongly alkaline syrup which combines readily with acids to form salts, most of which are deliquescent. By oxidation it is converted into betain (see page 305), and on treatment with concentrated nitric acid it gives rise to a muscarin (see page 309). These reactions can be represented by the equations :

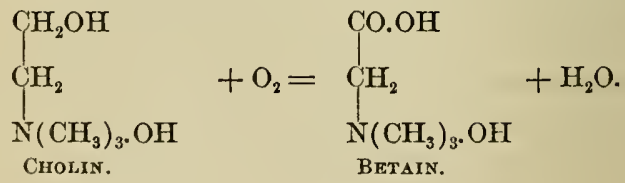

$$
\begin{aligned}
& \underbrace{}_{\substack{\mathrm{CH}_{2} \mathrm{OH} \\
\mathrm{CH}_{2}\left(\mathrm{CH}_{3}\right)_{3} . \mathrm{OH}}}+\underset{\substack{\mathrm{CHOH} \\
\text { MUSCARIN. }}}{\mathrm{CH}_{2} \mathrm{OH}}
\end{aligned}
$$


By the action of dilute nitric acid cholin is converted almost wholly into a base the platinochlorid of which is efflorescent, and forms large, bright crystals grouped in bunches. It corresponds to the formula $\left(\mathrm{C}_{4} \mathrm{H}_{10} \mathrm{~N}_{2} \mathrm{O}_{3} \mathrm{Cl}\right) \mathrm{PtCl}_{4}+2 \mathrm{H}_{2} \mathrm{O}$ (Schmiedeberg and Harnack).

Nothnagel isolated the same substance, but on attempting to convert the platinum salt into the gold compound, he obtained the aurochlorid of trimethylamin. The nature of this base, which is formed also in small quantity on oxidation with concentrated nitric acid, is uncertain.

According to Mauthner, cholin resembles the caustic alkalis in its action. Although putrefying blood decomposes it into trimethylamin, yet, when present in the proportion of 1.4 per cent., it is said to arrest putrefaction. A 1 to 2 per cent. solution like strong alkali dissolves fibrin or coagnlated albumin on boiling.

Nothnagel has shown, contrary to Arndt, that cholin cannot be distilled unchanged with baryta water. It is decomposed into trimethylamin. On distillation with water it yields a few drops of an aldehyde body, a little neurin (?), and trimethylamin. On dry distillation of cholin it yields also a little of the aldehyde body, a little neurin (?), and chiefly cholin. The latter probably results from recombination in the distillate of the trimethylamin, ethylene oxid, and water. In general it may be said that concentrated, but not dilute, solutions are decomposed by boiling.

The free base forms a syrupy liquid which eagerly takes up carbonic acid from the air and is converted into the carbonate which forms elongated six-sided plates. The base is easily soluble in water and in absolute alcohol but is insoluble in chloroform or in ether (Gulewitsch).

The chlorid, $\mathrm{C}_{5} \mathrm{H}_{14} \mathrm{NO} . \mathrm{Cl}$, is easily soluble in water and in absolute alcohol (separation from neuridin hydrochlorid and from betain). It is insoluble in ether, chloroform and benzol. From alkaline solution traces are dissolved by amyl alcohol. It crystallizes over sulphuric acid to needles which readily deliquesce in the air. Potassium mercuric iodid produces in solution of the chlorid a crystalline precipitate. For the behavior to other reagents see Table I (also Gulewitsch). The nitrate possesses the formula:

$$
\left(\mathrm{CH}_{3}\right)_{3} \cdot \mathrm{N}\left(\mathrm{NO}_{3}\right) \cdot \mathrm{CH}_{2} \cdot \mathrm{CH}_{2} \mathrm{OH} \text {. (Schmidt). }
$$

The bromid, $\mathrm{Br} . \mathrm{N}\left(\mathrm{CH}_{3}\right)_{3} \cdot \mathrm{C}_{2} \mathrm{H}_{4} \mathrm{OH}$, forms rather long, colorless rhombic plates when ether is added to an absolute alcohol solution of the salt. It deliquesces very rapidly in the air and is decomposed by sunlight, changing color to violet and brown (Nothnagel).

The iodid, I.N $\left(\mathrm{CH}_{3}\right)_{3} \cdot \mathrm{C}_{2} \mathrm{H}_{4} \mathrm{OH}$, can be crystallized in the same way as the bromid. It is less deliquescent and is turned yellow by sunlight (Nothnagel).

The platinochlorid, $\left(\mathrm{C}_{5} \mathrm{H}_{14} \mathrm{NO} . \mathrm{Cl}\right)_{2} \mathrm{PtCl}_{4}$ ( $\mathrm{Pt}=31.64$ per cent.), 
presents an interestiug case of trimorphism. It crystallizes in monoclinic plates (Rinne) which are easily soluble in water (1 to 5.82 parts at $21^{\circ}, \mathrm{G}$.) insoluble in alcohol, ether, chloroform, benzol ; also in characteristic superposed plates, sometimes in the form of orangered flat prisms (Brieger). From a warm saturated solution containing 15 per cent. alcohol it crystallizes in yellow regular octahedra containing one molecule of water of crystallization, from aqueous solutions in six-sided plates (Jahns); from aqueous solution on slow evaporation it forms plates, clinorhombic plates, or needles (HoppeSeyler) which are anhydrous. When rapidly crystallized it forms prisms (Hundeshagen, Jahns, Schulze); and if the solution is concentrated the prisms are very thin, almost needles. According to Schulze, it sometimes forms beautiful orange-red, chiefly six-sided plates. It is easily soluble, forming splendid, very large, red, superposed monoclinic plates; may crystallize from cold saturated aqueous solution in large, prismatic, or needle-shaped crystals (Schmidt). Jahns maintained that the plates and prisms belong to the same system; while Hundeshagen held that they are distinct. Instead of the salt presenting an instance of trimorphism as first stated by Hundeshagen, it would seem that but two forms occur-anhydrous monoclinic and octahedra with one molecule of water of crystallization. It contains always more or less water of crystallization, which it does not give up completely over sulphuric acid, but only at $110^{\circ}$ (Brieger). The natural platinochlorid becomes strongly electric on rubbing, whereas the synthetic cholin double salt does not become electric. It melts at $225^{\circ}$ with effervescence (Jahns) ; at about $238^{\circ}$ (Partheil); at $233^{\circ}-234^{\circ}$ (Bode); at $232^{\circ}-233^{\circ}$, and more often at $240^{\circ}-241^{\circ}$ (Schmidt), with marked effervescence. The syuthetic salt melts at $213^{\circ}-216^{\circ}$ (Gulewitsch). The solubility, crystalline form and melting-point render it wholly different from neurin. The properties of this and other salts of cholin have been carefully reëxamined by Gulewitsch (1898).

The aurochlorid, $\mathrm{C}_{5} \mathrm{H}_{14} \mathrm{NO} . \mathrm{Cl} . \mathrm{AuCl}_{3}(\mathrm{Au}=44.48$ per cent. $)$, is crystalline and is difficultly soluble in cold water, but can be recrystallized from hot water or from boiling alcohol. It forms prisms, or gold-yellow long needles which are very easily soluble in hot water and alcohol (Lippmann). It may form cubés and at $23.5^{\circ}$ it dissolves in 67.2 parts of water (G.). It can be separated from neuridin aurochlorid by its solubility in water (Brieger). On heating the gold salt melts to a brown liquid (Schulze), and decomposes at $264^{\circ}$. It melts at $244^{\circ}-245^{\circ}$ (Siebert, Jahns); at $245^{\circ}-246^{\circ}$ (Schmidt); at $241^{\circ}-243^{\circ}$ (G.).

The picrate, $\mathrm{C}_{5} \mathrm{H}_{14} \mathrm{NO} . \mathrm{OC}_{6} \mathrm{H}_{2}\left(\mathrm{NO}_{2}\right)_{3}$, forms long, broad needles which are more easily soluble than neuridin picrate, and hence can be separated by recrystallization. It is more easily soluble in alcohol than in water. 
The mercurochlorid, $\mathrm{C}_{5} \mathrm{H}_{14} \mathrm{NO} . \mathrm{Cl}+6 \mathrm{HgCl}_{2}$, is difficultly soluble even in hot water. On this account the mercury salt is very convenient for the separation of cholin from accompanying bases. It is very difficult to separate cholin from cadaverin mercurochlorid on account of the similarity in solubilities. Gulewitsch secured a separation by fractional precipitation and fractional solution. It forms small, short, cross-shaped prisms. It melts at $249^{\circ}-251^{\circ}$ (G.); at $242^{\circ}$ (Mörner). Its solubility in water is $1: 56.6$ at $24.5^{\circ}$ (Gulewitsch); at $1: 66.6,19.5^{\circ}$ (Mörner). Like creatinin it yields a crystalline precipitate with alcoholic zinc chlorid (G.).

Lactocholin, $\mathrm{Cl} . \mathrm{N}\left(\mathrm{CH}_{3}\right)_{3} \cdot \mathrm{CH}_{2} \cdot \mathrm{CH}_{2} \mathrm{O} \mathrm{CH}_{3} \cdot \mathrm{CH}_{2} \cdot \mathrm{CH}_{2} \cdot \mathrm{CH}_{2} \mathrm{O} . \mathrm{OC}>\mathrm{CH} . \mathrm{CH}$. This compound

was obtained by Schmidt by heating an aqueous solution of cholin lactate on a water-bath for six days. The platinochlorid forms long prismatic crystals with roof-shaped ends. It is easily soluble in water; difficultly in alcohol. It has two molecules of water of crystallization, and melts at $220^{\circ}-221^{\circ}$. The platinum compound on decomposition with hydrogen sulphid or potassium chlorid and precipitation with gold chlorid yields, instead of a gold salt, the aurochlorid of cholin. All attempts to obtain the aurochlorid of lactocholin failed. Lactocholin is formed from ethylidene, and not from ethylene lactic acid (Nothnagel).

Oxy-iso-butyro-cholin is produced in the same way as lactocholin. The platinochlorid has the same form and melting-point-221 . The two molecules of water of crystallization are difficultly driven off. The gold salt is not obtainable on account of reversion to cholin. The formula corresponds to that of lactocholin (Nothnagel).

Oxy-valero-cholin is also prepared the same as lactocholin. The platinum salt crystallizes with two molecules of water of crystallization, in long compact needles, which are rather easily soluble in water, and melt at $223^{\circ}-224^{\circ}$ (Nothnagel). Its composition also corresponds to that of lactocholin.

Oxy-acetic, ethylene lactic, and salicylic acids do not form anhydrid compounds. The above bodies result from the union of two molecules of cholin and one molecule of acid with elimination of two molecules of water. The platinum salts of all three anhydrides belong to the same system; all have two molecules of water of crystallization, difficultly expelled at $100^{\circ}$. The melting-point is about the same in all. They do not yield gold salts. The free anhydrids are not permanent.

Acetyl cholin. The gold salt of this compound was studied first by Baeyer, later by Nothnagel. Baeyer obtained the acetyl compound by the action of acetyl chlorid on cholin chlorid in the cold, but Nothnagel did not succeed in introducing the acetyl group short 
of $100^{\circ}$. The gold salt is anhydrous, dendritic in form, and melts at $154^{\circ}-155^{\circ}$. On decomposition with hydrogen sulphid it yields cholin. The platinochlorid crystallizes in small anhydrous needles which melt at $223^{\circ}-224^{\circ}$.

Benzoyl cholin is formed by heating dry cholin chlorid on a waterbath with benzoyl chlorid. It forms a platinum salt crystallizing in fine thread-like needles which melt at $206^{\circ}$. The gold salt forms light-yellow flat needles which are permanent in the air and melt at $183^{\circ}$. The hydrogen in the hydroxyl group of the oxyethyl is, therefore, easily replaced by acid radicals (Nothnagel).

Physiological Action of Cholin.-Cholin was regarded for a long time as physiologically inert, but this belief was set aside by Gaehtgens $(1870)$, who showed that, when given in large quantity, it possessed a marked toxic action; 0.59 gram producing almost instantaneous death in a cat. This observation of Gaehtgens has since been confirmed by Glause and Luchsinger, Brieger, and Boehm. The chlorid of cholin produces in animals the same muscarin-like symptoms of poisoning as are developed by the vinyl base neurin, the only difference lies in the intensity of the action. In order to bring about a physiological disturbance, cholin must be given in relatively large doses. Thus Brieger found it necessary to give about 0.1 gram of cholin chlorid hypodermically to a one kilogram rabbit in order to bring out the same effects as are obtained by the injection of 0.005 gram of the neurin salt. He also found that the fatal dose for a one kilogram rabbit was about 0.5 gram, which is about ten times as large as the fatal dose of neurin chlorid. Boehm observed that doses of $0.025-0.1$ gram produced in frogs general paralysis, which, in a short time, led to death or recovery; and that in its curara-like paralyzing astion cholin resembled artificial muscarin, although the latter is about five hundred times stronger. Atropin, as in the case of neurin and muscarin, antagonizes the action of cholin. Thus, 0.05 gram of the chlorid produced in a frog in one hour diastolic stoppage of the heart. This condition was removed by the injection of 0.001 gram of atropin, the heart-beat rising to the normal in about fourteen minutes; 0.05 gram of cholin chlorid, given subcutaneously to a rabbit (1,250 grams), produced salivation, which lasted but a short time and did not affect the heart-beat and respiration; 0.10 gram was necessary to bring out all the symptoms; 0.05 gram, given to guinea-pigs, had no effect whatever. According to Halliburton (1901) it causes a dilatation of the peripheral blood vessels, especially those of the intestines, and hence a fall of blood pressure.

Betain (oxyneurin), $\mathrm{C}_{5} \mathrm{H}_{13} \mathrm{NO}_{3}$. - This base has been well known for some time, because of its occurrence in the vegetable kingdom. 
Thus, it is present in cotton-seed (Boehm, Ritthausen and Weger, Maxwell), where it is about five times as abundant as cholin ; in beetroot juice (Beta vulgaris), and hence in beet-root molasses (Scheibler, 1866). It occurs also in cattle-turnip and Lycium barbarum (Husemann and Marmé, Schütte, Siebert), and is found with cholin and another base in vetch-seeds; in peas a base similar to betain exists (Schulze). With cholin it occurs in the roots and leaves of Scopolia atropoïles (Siebert). It occurs in the leaves of the potato plant, Solanum tuberosum, but not with cholin (Schütte); in worm-seed (Artemisia Cina) in about 0.5 per cent.; with cholin about 0.1 per cent. (Jahns). The two bases are also present in the sprouts of wheat and malt, betain more abundantly (Schulze and Frankfurt). It does not exist in these substances as such, but is formed from a more complex substance by the action of hydrochloric acid or baryta (Liebreich). In this respect it resembles cholin, neurin and probably muscarin. Quite recently, Lippmann (1887) has obtained a lecithin-like body from sugar-beet, which, on heating with baryta, gave oleic acid, glycerin, and phosphoric acid (glycerin-phosphoric acid), and betain. Betain, however, does not seem to be a constant constituent, inasmuch as on one occasion he obtained chiefly cholin, and little or no betain. These two bases also occur together in cotton-seed, and this fact has led Scheibler to the conclusion that it is no mere chance. Lecithin, as is well known, may contain variable acid constituents (oleic, stearic, palmitic, etc.), and reasoning on this fact, and on the results of his own experiments, Lippmann was led to suppose that it may also contain different bases in variable proportions.

Betain was first discovered by Husemann and Marmé in 1863 and 1864 and named lycin. Scheibler found it in 1866 in beets and gave it the present name. The identity of the two compounds was shown in 1875 by Husemann.

A methyl betain, trigonellin, exists in trigonella (Jahns, Hantzch).

It has been obtained from human urine (Liebreich, 1869), and from poisonous and non-poisonous mussel, but not from putrid mussel (Brieger, 1885, III., 76). Emmerling (1896) obtained it with trimethylamin by decomposing gluten with proteus vulgaris. The method for its separation from mussel is described on page 313.

Betain may be obtained synthetically in several ways: (1) By oxidation of cholin with potassium permanganate; (2) by heating sarkosin (methyl glycocoll) with methyl iodid and methyl alcohol, or with methyl iodid alone, when betain-methyl ether also forms (Paulmann); (3) by the action of silver oxid on betain aldehyde; (4) by the action of methyl iodid on glycocoll (Kraut); (5) by treating monochloracetic acid with trimethylamin. The last two methods are of value as indicating the constitution of betain, and the changes which take place can be represented by the equations : 


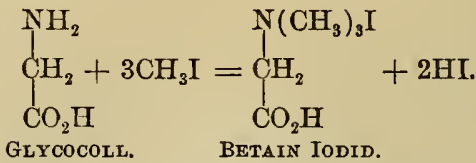

$$
\begin{aligned}
& \underset{\mathrm{CO}_{2} \mathrm{H}}{\mathrm{CH}_{2} \mathrm{Cl}}+\mathrm{N}\left(\mathrm{CH}_{3}\right)_{3}=\underset{\mathrm{CO}_{2} \mathrm{H} .}{\mathrm{CH}_{2}}
\end{aligned}
$$

MONOCHLOR-

Another method of synthesizing anhydrous betain consists in heating di-methyl amido-acetic methyl ester. The reaction is reversible (Wildstätter, Ber., 35, 585, 597).

From the formulæ of the salts of betain it is evident that betain has properly the composition $\mathrm{C}_{5} \mathrm{H}_{13} \mathrm{NO}_{3}$ which is expressed by the structural formula:

$$
\begin{aligned}
& \mathrm{N}\left(\mathrm{CH}_{3}\right)_{3} \mathrm{OH} \\
& \mathrm{CH}_{2} \\
& \mathrm{CO}_{2} \mathrm{H} .
\end{aligned}
$$

The free base is, however, readily converted into the anhydrid, $\mathrm{C}_{5} \mathrm{H}_{11} \mathrm{NO}_{2}$, trimethyl glycocoll, the structural formula of which is:

$$
\begin{aligned}
& \mathrm{CH}_{2}-\mathrm{N}\left(\mathrm{CH}_{3}\right)_{3} \\
& \mathrm{CO}-\mathrm{O} .
\end{aligned}
$$

Betain aldehyde was prepared first by Berlinerblau and later by Fischer. On oxidation with silver oxid it yields betain.

Betain is ordinarily regarded as crystallizing with one molecule of water, and the composition is expressed by the formula : $\mathrm{C}_{5} \mathrm{H}_{11} \mathrm{NO}_{2}$ $+\mathrm{H}_{2} \mathrm{O}\left(=\mathrm{OH} \cdot \mathrm{N}\left(\mathrm{CH}_{3}\right)_{3} \cdot \mathrm{CH}_{2} \cdot \mathrm{CO}_{2} \mathrm{H}\right)$. It loses this water of crystallization by heating at $100^{\circ}$, or on standing over sulphuric acid, forming an anhydrid of the formula already given. The anhydrid is very hygroscopic, and melts at $293^{\circ}$ (Wildstätter). Liebreich claimed that free betain possessed the formula $\mathrm{C}_{5} \mathrm{H}_{11} \mathrm{NO}_{2}$ because it yielded a compound having the composition $\left(\mathrm{C}_{5} \mathrm{H}_{11} \mathrm{NO}\right) \mathrm{ZnCl}_{2}$. The free base separates from alcohol in large crystals which deliquesce on exposure to the air. As obtained by Brieger from the hydrochlorid, by treatment with moist silver oxid, it possessed a sweetish taste and neutral reaction. When distilled with potassium hydrate, it yielded trimethylamin and other bases, among which a base of the formula $\mathrm{C}_{8} \mathrm{H}_{17} \mathrm{NO}_{5}$ occurred in the largest quantity. In 1893 Scheibler again studied the action of sodium hydrate on betain and found only trimethylamin; no new base, but unchanged betain.

The chlorid, $\mathrm{C}_{5} \mathrm{H}_{12} \mathrm{NO}_{2} \cdot \mathrm{Cl}$, forms beautiful monoclinic plates which are permanent in the air, and this fact can be made use of to effect a separation from the cholin salt, which is deliquescent. 
It crystallizes from aqueous solution in monoclinic plates, from hot saturated 80 per cent. alcoholic solution in beautiful prisms, often several $\mathrm{cm}$. long. It melts at $227^{\circ}-228^{\circ}$ (Jahns). It is insoluble in absolute alcohol. This fact can be made use of in their separation (Lippmann, Maxwell). It can, moreover, be easily separated from other bases by its aurochlorid which is easily soluble. If a little potassio-mercuric iodid is added to a solution of the chlorid, there forms a light yellow or whitish oily precipitate, which is soluble in excess, but on rubbing the sides of the tube with a glass rod it reappears as yellow needles. This is said to be a characteristic test (Brieger, Schulze, 1891). By the action of sodium amalgam on aqueous solutions of the chlorid a base is formed, the platinochlorid of which in form, solubility, and composition agrees with muscarin (Schmidt, Nothnagel). An iodid and a potassium iodid compound are known (Wildstätter).

The aurochlorid, $\mathrm{C}_{5} \mathrm{H}_{12} \mathrm{NO}_{2} \cdot \mathrm{Cl} \cdot \mathrm{AuCl}_{3}(\mathrm{Au}=43.12$ per cent.), forms magnificent cholesterin-like four-sided plates (or gold yellow needles, Paulmann), and is easily soluble (Brieger). The aurochlorid from sugar-beet is said to crystallize in needles and plates, and to be difficultly soluble in cold water (Scheibler, Lippmann). The double salt of the ptomain melts at $209^{\circ}$ and in this it coincides with that obtained from beet-sugar, as well as with that of the synthetically prepared base (Brieger). According to Schütte, it melts at $218^{\circ}, 220^{\circ}-222^{\circ}$; at $223^{\circ}-225^{\circ}$ (Siebert); at $220^{\circ}-221^{\circ}$, decomposing at $222.5^{\circ}$ (Paulmann) at $230^{\circ}-235^{\circ}$ (Fischer); at $227^{\circ}$ (Emmerling). The platinochlorid, $\left(\mathrm{C}_{5} \mathrm{H}_{\mathrm{II}} \mathrm{NO}_{2} \cdot \mathrm{HCl}\right)_{2} \cdot \mathrm{PtCl}_{4}$, is yellow, crystallizes in prisms and is easily soluble (E.). On rapid cooling of hot saturated solution or on precipitation with alcohol it forms more or less anhydrous fine needles; from cold saturated solution over sulphuric acid it crystallizes in plates which effloresce in the air (Jahns). It may crystallize with or without water. Liebreich and Wildstätter obtained crystals with four molecules, while Paulmann obtained crystals with one molecule of water. Jahns obtained the salt with three molecules of water.

Betain is not poisonous. It is precipitated with mercuric chlorid together with cholin. Schulze and Frankfurt separate the mercury salts of betain and cholin by partial crystallization; betain is more soluble. The two bases can be separated as chlorids by the solubility in absolute alcohol (Maxwell, Schulze and Frankfurt, Jahns).

Muscarin, $\mathrm{C}_{5} \mathrm{H}_{15} \mathrm{NO}_{3}=\mathrm{C}_{5} \mathrm{H}_{13} \mathrm{NO}_{2}+\mathrm{H}_{2} \mathrm{O}$, is the well-known toxic principle which Schmiedeberg and Koppe obtained from poisonous mushroom (Agaricus muscarius), in which it is present accompanied by cholin (Harnack). Böhm found cholin and muscarin together in Boletus luridus and Amanita pantherina. Later, Schmiedeberg isolated from a commercial specimen of muscarin 
a base possessing an antagonistic action to muscarin. Kobert believed that this "fungus-atropin" existed in the fresh mushrooms, and showed that Russula emetica contained this compound as well as cholin and muscarin.

This base is especially interesting because of the relation it bears to cholin, for Schmiedeberg and Harnack showed that it is formed when cholin, or, better still, the platinochlorid, is oxidized by concentrated nitric acid. Nothnagel by the action of concentrated nitric acid on cholin obtained muscarin, also a nitroso derivative (nitric acid and cholin ether), and a substance which is the chief product, besides a little muscarin and the nitroso compound, when the oxidation is carried out with dilute nitric acid. The muscarin from cholin does not combine with phenyl-hydrazin; betain aldehyde does. The chlorid on treatment with acetic anhydrid or benzoyl chlorid yields an anhydrid of muscarin (Nothnagel), the exact composition of which is yet undetermined; the group $-\mathrm{CH}<\mathrm{OH}$ in muscarin is probably changed into an aldehyde group - $\mathrm{COH}$.

In the preparations of muscarin from cholin a small quantity of a nitroso compound forms, the platinum salt of which resembles that of muscarin in solubility, but never in form, which is always plumose. These crystals are permanent in the air and contain two molecules of water which are not driven off at $100^{\circ}$. They melt at $223^{\circ}-224^{\circ}$ with decomposition. It possesses the formula :

$$
\text { (Cl. N } \left.\left(\mathrm{CH}_{3}\right)_{3} \cdot \mathrm{CH}_{2} \cdot \mathrm{CH}_{2} \mathrm{O} \cdot \mathrm{NO}\right)_{2} \cdot \mathrm{PtCl}_{4}+2 \mathrm{H}_{2} \mathrm{O} \text {. }
$$

The gold salt forms fine, light-yellow needles which are anhydrous and melt at $240^{\circ}$. It gives Liebermann's nitroso reaction-blue color with phenol and sulphuric acid.

In addition to the natural and synthetic bases a third "muscarin," OH.N $\left(\mathrm{CH}_{3}\right)_{3} \cdot \mathrm{CH}_{2} \cdot \mathrm{COH}$, was prepared by Berlinerblau by the action of baryta on trimethylamin and chloracetal. Fischer prepared the same compound, by the action of concentrated hydrochloric acid on acetal-trimethyl ammonium hydroxid. This base, however, differs from muscarin by the elements of water-anhydro-muscarin. In reality it is betain aldehyde, since on oxidation with moist silver oxid it yields betain (Fischer). Unlike real muscarin, it has no action on the heart of frogs or on the pupils of birds (Meyer). Like most ammonium bases it induces strong salivation and perspiration. Schmiedeberg found it to resemble cholin in its action, whereas Luchsinger found it to agree in this respect with muscarin.

A fourth base, oxycholin-OH.N $\left(\mathrm{CH}_{3}\right)_{3} \cdot \mathrm{CH} \cdot \mathrm{OH} \cdot \mathrm{CH}_{2} \mathrm{OH}$, was prepared by Bode by the action of silver oxid on hypochlorous acid and neurin chlorid. Its platinum salt melts at $254^{\circ}$ (Nothnagel). Its physiological action is different from that of muscarin. Thus, $\mathrm{n}$ frogs it slows the heart, but does not cause stoppage. Atropin 
counteracts its action. In mammals the pulse is lowered as a result of stimulation of the central vagus ganglia. The blood pressure is not lowered as is the case in muscarin, but is somewhat raised. Neither the intestines nor the iris of mammals is affected. The iris of birds is contracted as with muscarin, and the glands are affected. In cats and guinea-pigs salivation and flow of tears result. Like all ammonium bases it has a marked curara action.

A fifth base, resembling fungus- and cholin-muscarin in the form, solubility and composition of the platinochlorid, was obtained by the action of sodium amalgam on aqueous solutions of betain chlorid (Schmidt).

Lastly, Brieger in 1885 (I., 48) isolated a muscarin base from haddock which had been allowed to decompose for five days. The process by which its isolation was effected is described on page 315 . Gulewitsch isolated a small amount of a substance resembling muscarin, together with cholin and cadaverin, from horse-flesh kept at $15^{\circ}$ for four months.

It is barely possible that Brieger's base is distinct from Schmiedeberg's; nevertheless, it closely resembles it and apparently is identical.

The chlorid, $\mathrm{C}_{5} \mathrm{H}_{14} \mathrm{NO}_{2} \cdot \mathrm{Cl}$, is obtained on the decomposition of the platinochlorid with hydrogen sulphid, as a syrupy residue which, under the desiccator, slows a tendency to crystallize gradually (Brieger). It is deliquescent (Harnack). A commercial muscarin sulphate was found to be chiefly cholin (Nothnagel).

The platinochlorid, $\left(\mathrm{C}_{5} \mathrm{H}_{14} \mathrm{NO}_{2} \cdot \mathrm{Cl}\right)_{2} \mathrm{PtCl}_{4}(\mathrm{Pt}=30.08$ per cent., Brieger), forms a crystalline deposit of more or less well-formed octahedra, pinhead in size, which are difficultly soluble in water. They lose their water of crystallization $\left(2 \mathrm{H}_{2} \mathrm{O}\right)$ only on strong heating (Brieger, Nothnagel). It melts at about $240^{\circ}$ with decomposition.

The aurochlorid, $\mathrm{C}_{5} \mathrm{H}_{14} \mathrm{NO}_{2} \cdot \mathrm{Cl} \cdot \mathrm{AuCl}_{3}(\mathrm{Au}=42.82$ per cent.), crystallizes in needles, and is difficultly soluble in water (Brieger); more difficultly soluble than the cholin double salt (Harnack). From hot hydrochloric acid water it crystallizes as light-yellow, glistening platelets (Nothnagel). It is scarcely to be distinguished from the corresponding salt of cholin (Nothnagel). It begins to run together at $174^{\circ}$, gradually melts and decomposes at $232^{\circ}$. It has no water of crystallization. The separation of muscarin and cholin is very difficult. Harnack separated the two by spreading the mixed chlorids on a filter paper which absorbed the muscarin salt. Nothnagel separated the two bases as platinochlorids by repeated recrystallization from hot water and washing the crystals with cold water. The platinochlorid of cholin is easily soluble in water.

Physiological Action.-Small doses of this ptomain induce in frogs total paralysis, with stoppage of the heart in diastole, and this 
action is antagonized by subsequent injection of atropin. In the case of previously atropinized frogs it fails to antagonize. Very small doses produced in rabbits profuse salivation and lachrymation, contraction of the pupil, profuse diarrhea, and passage of urine and semen ; finally, the animal died in convulsions, which, however, were only of short duration (Brieger). Although the natural and artificial muscarin and their salts are chemically and physically alike, they are not however identical, although so considered by Schmiedeberg and Harnack. This is seen in their physiological action. Thus Böhm found that the artificial muscarin paralyzed intramuscular nerve endings. According to Meyer, $\frac{1}{10}-\frac{1}{20} \mathrm{mg}$. will do this, whereas the natural base will not have this effect. Again, 1-2 drops of a 1 per cent. solution of the artificial base will produce maximal myosis in birds in a few minutes; while the natural base has no effect on birds' pupils. The action of betain aldehyde and iso-muscarin has already been stated. Brieger's ptomaïn would seem to be nearly identical with artificial muscarin.

Constitution of the Members of the Cholin Group.-The structure of cholin was clearly demonstrated by Wurtz, who accomplished the synthesis of this base by treatment of ethylene chlorhydrin with trimethylamin. This same method can be applied to the synthesis of betain and neurin by using monochloracetic acid and vinylbromid instead of ethylene chlorhydrin. The structural formulæ which can be deduced from these reactions are as follows :
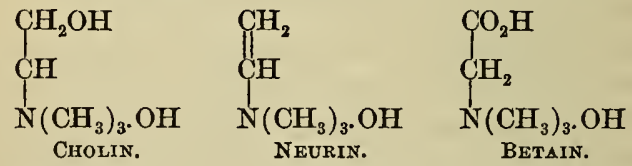

All these bases, since they can be prepared from cholin, may also be considered as oxidation products of trimethyl-ethyl-ammonium hydrate:

$$
\begin{aligned}
& \mathrm{CH}_{8} \\
& \mathrm{CH}_{2} \\
& \mathrm{~N}\left(\mathrm{CH}_{3}\right)_{3} \cdot \mathrm{OH} .
\end{aligned}
$$

The constitution of muscarin is still unsettled. Schmiedeberg and Harnack believed that it resulted from cholin by the oxidation of hydrogen connected with the same carbon as the hydroxyl group. Its formula would be either (1) or (2), as given below. The former, analogous to chloral would have the somewhat rare condition of two hydroxyl groups attached to one carbon atom. The presence of hydroxyl groups in muscarin cannot, however, be demonstrated with acetic anhydrid, or benzoyl chlorid (Nothnagel). The second formula is that of betain aldehyd. 
The formulæ of several muscarin compounds are herewith presented :

(1)

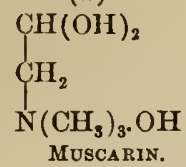

(2)

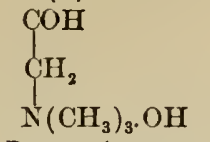

Betain Aldehyd.
(3)

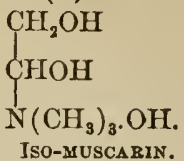

It will be observed that very slight differences in the chemical constitution of cholin, muscarin, betain and neurin are accompanied by very great differences in the physiological action of these bases. Thus, as pointed out by Schmidt, cholin may be considered as a primary alcohol and betain as a monobasic acid. Between these two relatively non-poisonous bases is the intermediate oxidation product, the aldehyde muscarin, which is highly poisonous.

Again, it will be remembered that the artificial muscarin, formed by the oxidation of cholin, had a markedly different physiological effect on the intramuscular nerve endings and on the pupils of birds than the natural muscarin. This difference must undoubtedly be ascribed to difference in stereochemical structure, as in the case of active and inactive lactic acids, and of atropin. Furthermore, Bode's isomuscarin possesses likewise an entirely different action, differing only in the position of the hydroxyl group. The same is true of betain aldehyd, which differs from muscarin by the elements of water.

Mydatoxin, $\mathrm{C}_{6} \mathrm{H}_{13} \mathrm{NO}_{2}$.-This base was obtained by Brieger in 1886 (III., 25, 32) from several hundred pounds of human internal organs which were allowed to stand in closed but spacious wooden barrels for four months, at a temperature varying from $-9^{\circ}$ to $+5^{\circ}$. $\mathrm{He}$ obtained much larger quantities of it, however, from horse-flesh which had putrefied under the same conditions. In the process of extraction it is found in the mercuric chlorid precipitate together with cadaverin, putrescin and another base, $\mathrm{C}_{7} \mathrm{H}_{17} \mathrm{NO}_{2}$. It can be isolated from this mixture by recrystallizing the mercury salts, which removes the cadaverin because of its difficult solubility in water, and decomposing the soluble mercury salts by hydrogen sulphid. The filtrate freed from mercury is now evaporated to dryness and the residue repeatedly extracted with absolute alcohol, in order to remove the putrescin hydrochlorid which is insoluble. The alcoholic solution, after standing some time to permit complete separation of any dissolved putrescin, is then evaporated to dryness and taken up with water. This solution gives, on the addition of gold chlorid, a precipitate of the aurochlorid of the base $\mathrm{C}_{7} \mathrm{H}_{17} \mathrm{NO}_{2}$. The filtrate from this precipitate, containing the mydatoxin, is treated with hydrogen sulphid to remove the gold, and then evaporated to dryness. The colorless, syrupy hydrochlorid thus obtained forms with platinum chlorid a double salt which is readily soluble in water, and 
can be purified by repeated recrystallization from absolute alcohol containing some hydrochloric acid.

The name mydatoxin is derived from $\mu \nu \delta a \omega$, to putrefy. The free base is obtained from the hydrochlorid, by treatment with moist freshly precipitated silver oxid, as a strongly alkaline syrup which solidifies in vacuo to plates. It is insoluble in alcohol, ether, etc. It does not distil without decomposition. It is isomeric with leucin and also with the base $\mathrm{C}_{6} \mathrm{H}_{13} \mathrm{NO}_{2}$, obtained by Brieger in 1888 from tetanus cultures. It is isomeric with di-methyl ethyl betain and also with tri-methyl propionyl betain (Wildstätter, Ber., 35, 606, 610). The latter is represented by formula (1). Both, however form gold salts.

The hydrochlorid, $\mathrm{C}_{6} \mathrm{H}_{13} \mathrm{NO}_{2} . \mathrm{HCl}$, is a colorless deliquescent syrup which does not form any double salt with gold chlorid. With platinum chlorid it gives an easily soluble salt. Otherwise it combines only with phosphomolybdic acid, with which it forms cubes. Ferric chlorid and potassium ferricyanid yield, after a time, Berlin blue. It is readily soluble in alcohol.

The platinochlorid, $\left(\mathrm{C}_{6} \mathrm{H}_{13} \mathrm{NO}_{2} \cdot \mathrm{HCl}\right)_{2} \mathrm{PtCl}_{4}(\mathrm{Pt}=29.00$ per cent.), melts at $193^{\circ}$, with decomposition. It crystallizes in plates which are extremely soluble in water. It can be readily recrystallized from absolute alcohol acidulated with hydrochloric acid. The mercury salt is readily soluble in water.

The exact formula of this base, of mytilotoxin, and some other bases, cannot be considered to be permanently settled, inasmuch as the formula of the hydrochlorid, $\mathrm{C}_{6} \mathrm{H}_{13} \mathrm{NO}_{2} . \mathrm{HCl}$, as deduced from the analysis of the platinum double salt, may equally apply to the base $\mathrm{C}_{6} \mathrm{H}_{14} \mathrm{NO}_{2} . \mathrm{OH}$ as to the base $\mathrm{C}_{6} \mathrm{H}_{13} \mathrm{NO}_{2}$. If the first formula is correct, then mydatoxin may be considered a homologue of betain, and its structure would be expressed by (1). Or, it may have the structure shown in formula (2).
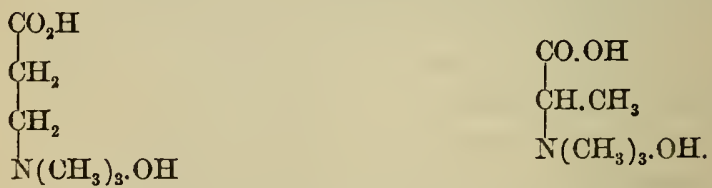

By loss of water in either case (propyl or iso-propyl betain) the formula $\mathrm{C}_{6} \mathrm{H}_{13} \mathrm{NO}_{2}$ results. In the latter instance the anhydrid would be that which, combined with pyridin, is met with in pilocarpin.

This ptomain, although it possesses toxic properties, is not, however, a strong poison. Its action is the same as that of the base $\mathrm{C}_{7} \mathrm{H}_{17} \mathrm{NO}_{2}$ (see page 318 ), with which it is associated, except that the symptoms of poisoning develop slower, so that the death of a guinea-pig does not take place for about twelve hours. White mice are very susceptible to the action of these two poisons. A short time after the 
injection of even small doses they are taken with convulsions which come on in paroxysms. The eyeballs roll upward. Lachrymation, diarrhœa, and dyspnœa develop and the mice die within a short time.

A Base (?), $\mathrm{C}_{6} \mathrm{H}_{13} \mathrm{NO}_{2}$, an isomer of the preceding and also of leucin, was obtained by Brieger in 1888 from tetanus cultures. It is not poisonous-distinction from mydatoxin. It probably is an amido acid. The platinochlorid crystallizes in plates, is easily soluble in water and in alcohol, and melts at $197^{\circ}$ with decomposition (see page 322 ).

Mytilotoxin, $\mathrm{C}_{6} \mathrm{H}_{15} \mathrm{NO}_{2}$, is the specific poison of toxic mussel (Mytilus edulis), from which it was obtained by Brieger in 1885 (III., 76 ). This poison is formed during the life of the animal under certain conditions which have been thoroughly studied by Schmidtmann, Virchow, and others (see p. 192). Brieger obtained the poison by extracting the toxic mussel with acidulated water, and evaporating this solution to a syrupy consistency. The residue was thoroughly extracted with alcohol, and this solution was treated with lead acetate in order to remove mucilaginous substances. The filtrate was then evaporated and the residue extracted with alcohol. Any lead that had dissolved was removed by hydrogen sulphid. The alcohol was expelled, and the resulting syrup was taken up with water and decolored by boiling with animal charcoal. The clear solution was now neutralized with sodium carbonate, acidulated with nitric acid, and precipitated with phosphomolybdic acid. The precipitate was decomposed by warming with neutral lead acetate, and the resulting filtrate, after the removal of the lead by hydrogen sulphid, was acidulated with hydrochloric acid and evaporated to dryness. The residue was extracted with absolute alcohol, whereby betain, on account of its insolubility, is removed, and the alcoholic solution was precipitated by alcoholic mercuric chlorid. The mercury precipitate was repeatedly recrystallized from water and the poison thus obtained as an easily soluble double salt.

The free base as obtained by the addition of alkali to the hydrochlorid possesses a disagreeable odor which disappears on exposure to air, and the substance ceases to possess poisonous properties. Brieger has proposed the application of this test for the recognition of poisonous mussel; on treatment of these with alkali the characteristic odor is developed. Mytilotosin is also destroyed on distillation with potassium hydrate, and in the distillate there is found an aromatic non-poisonous product and trimethylamin. The free base, therefore, does not exist by itself for any length of time but soon becomes converted into an inert substance. H. Salkowski has also shown that it is destroyed on boiling with potassium carbonate, whereas its hydrochloric acid solution can be evaporated to dryness and heated to $110^{\circ}$ without destroying its poisonous property. 
The hydrochlorid, $\mathrm{C}_{6} \mathrm{H}_{15} \mathrm{NO}_{2} \cdot \mathrm{HCl}$, prepared from the aurochlorid, crystallizes in tetrahedra. It is extremely poisonous, and according to Brieger produces exactly the same symptoms which have been observed by Schmidtmann in persons who have partaken of poisonous mussels (see page 190). On standing, however, the pure hydrochlorid gradually becomes dark and decomposes with loss of its poisonous property-a change corresponding to that which tetanin undergoes (p. 322). The gold salt is better adapted for preservation. The ordinary alkaloidal reagents produce in its solutions, if at all, only oily precipitates.

The aurochlorid, $\mathrm{C}_{6} \mathrm{H}_{15} \mathrm{NO}_{2} \cdot \mathrm{HCl} \cdot \mathrm{AuCl}_{3}(\mathrm{Au}=41.66$ per cent.), crystallizes in cubes. Its melting point is $182^{\circ}$.

It is well to observe that Brieger was unable to obtain this base from mussels that were allowed to putrefy for sixteen days.

As stated under mydatoxin, the formula of the hydrochlorid, $\mathrm{C}_{6} \mathrm{H}_{15} \mathrm{NO}_{2} . \mathrm{HCl}$, is applicable to either one of the two bases, $\mathrm{C}_{6} \mathrm{H}_{16}$ $\mathrm{NO}_{2} . \mathrm{OH}$ or $\mathrm{C}_{6} \mathrm{H}_{15} \mathrm{NO}_{2}$. The base corresponding to the first formula is evidently a homologue of muscarin, and should possess a similar physiological action. As a matter of fact; mytilotoxin does resemble muscarin somewhat in this respect, and its occurrence together with betain would seem to make it a decomposition product of lecithin, in which case this base must be looked upon as a member of the cholin group. A compound corresponding to the formula $\mathrm{C}_{6} \mathrm{H}_{16} \mathrm{NO}_{2} \mathrm{OH}$ was prepared by Hanriot in a manner analogous to Wurtz's synthesis of cholin, by treating glycerin monochlorhydrin with trimethylamin. This base, trimethyl-glyceryl ammonium hydrate, has the structure :

$$
\begin{aligned}
& \mathrm{CH}_{2} \cdot \mathrm{CHOH} \cdot \mathrm{CH}_{2} \mathrm{OH} \\
& \mathrm{N}\left(\mathrm{CH}_{3}\right)_{3} \mathrm{OH} .
\end{aligned}
$$

It was suggested that Hanriot's base might possibly be identical with mytilotoxin, but a careful comparison made by Brieger showed that it possesses no physiological action and that its chemical properties are entirely different.

Mytilotoxin would, therefore, seem to possess the formula $\mathrm{C}_{6} \mathrm{H}_{15}$ $\mathrm{NO}_{2}$, as originally given by Brieger. From the fact that on distillation with potassium hydrate it yields trimethylamin, it follows that mytilotoxin is a quaternary base. He is inclined to regard it as a methyl derivative of betain, which is so common in mussels, and represents it by formula No. 1 .

(1)

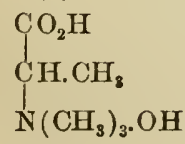

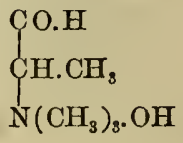


No. 1, however, is $\mathrm{C}_{6} \mathrm{H}_{15} \mathrm{NO}_{3}$, instead of $\mathrm{C}_{6} \mathrm{H}_{15} \mathrm{NO}_{2}$, as above, and has been referred to under mydatoxin. 'The formula No. 2, $\mathrm{C}_{6} \mathrm{H}_{15} \mathrm{NO}_{2}$, may be taken to represent more correctly this base. On comparing these formulæ it would appear that mydatoxin (1) is an oxidation product of mytilotoxin (2). The latter in turn would be a homologne of betain aldehyde (p. 311), and hence a derivative of cholin or muscarin.

According to Brieger, mytilotoxin produces all the characteristic effects seen in mussel poisoning. In its paralyzing action it resembles curara. This action is explainable now that Glause and Luchsinger have shown that all trimethyl ammonium bases have a muscarin-like action. For the symptoms induced by poisonous mussel see page 190.

Gadinin, $\mathrm{C}_{7} \mathrm{H}_{17} \mathrm{NO}_{2}$, was found in haddock (1885) which were allowed to decompose in open iron vessels for five days during summer. Brieger also obtained it from cultures of the bacteria of human feces on gelatin. Carbone found it in cultures of the Proteus vulgaris. The decomposing mass was thoroughly stirred every day in order to bring it into contact with atmospheric oxygen (Brieger, I., 49). It was then treated with water, and hydrochloric acid was added to acid reaction. After being warmed the mixture was filtered and the filtrate concentrated on the water-bath to a syrupy consistency. This syrupy residue was extracted with water, and the aqueous solution was precipitated with mercuric chlorid. This mercuric chlorid precipitate contained a base, the quantity of which, however, was insufficient for a complete analysis (see page 325). The mercuric chlorid filtrate, after the removal of the mercury by hydrogen sulphid, was evaporated to a syrup, and this was then repeatedly extracted with alcohol. The alcoholic solution thus obtained contained neuridin, a base of the same composition as ethylendiamin, muscarin, gadinin, and triethylamin. These bases were separated in the following manner: The alcoholic solution gave with platinum chlorid a precipitate of neuridin. The filtrate from this platinum precipitate was heated on the waterbath to expel the alcohol after which the platinum was removed by hydrogen sulphid. The aqueous filtrate was concentrated to a small volume which, on addition of platinum chlorid, gave a precipitate of the isomer of ethylendiamin. The mother-liquor from this precipitate was concentrated on a water-bath, and on cooling the platinochlorid of muscarin crystallized out. From the mother-liquor of this precipitate on standing in a desiccator, the gadinin double salt crystallized. The mother-liquor from the gadinin platinochlorid was treated with hydrogen sulphid to remove the platinum, and the aqueous filtrate on distillation with potassium hydrate gave triethylamin. 
Gadinin (from Gadus callarias, haddock) in small doses does not appear to be poisonous; large doses (0.5-1 gram) are decidedly toxic and may kill guinea-pigs. The formula of the free base as deduced from the analysis of the platinochlorid may be either $\mathrm{C}_{7} \mathrm{H}_{17} \mathrm{NO}_{2}$ or $\mathrm{C}_{7} \mathrm{H}_{18} \mathrm{NO}_{2}$. OH.

The hydrochlorid, $\mathrm{C}_{7} \mathrm{H}_{17} \mathrm{NO}_{2} . \mathrm{HCl}$, as obtained by the decomposition of the platinochlorid with hydrogen sulphid, crystallizes under the desiccator in thick, colorless needles, which are easily soluble in water; insoluble in alcohol. It forms no combination with gold chlorid, but does give crystalline precipitates with phosphomolybdic, phosphotungstic and picric acids.

The platinochlorid, $\left(\mathrm{C}_{7} \mathrm{H}_{17} \mathrm{NO}_{2} \cdot \mathrm{HCl}\right)_{2} \mathrm{PtCl}_{4}(\mathrm{Pt}=27.68$ per cent. $)$, is at first quite soluble, and on standing in a desiccator it crystallizes in golden-yellow plates which, when once formed, are again difficultly soluble in water. It can be recrystallized from hot water. It melts at $214^{\circ}$.

Typhotoxin, $\mathrm{C}_{7} \mathrm{H}_{17} \mathrm{NO}_{2}$. - This base was named thus by Brieger in 1885 (III., 86), and was regarded by him as the specific toxic product of the activity of Koch-Eberth's typhoid bacillus. It is however, certain, as in the case of tetanus, that the real poison of this germ is not this ptomaïn, but rather a toxin. He obtained the base by cultivating the bacillus on beef-hash for eight to fourteen days at $37.5^{\circ}-38^{\circ}$. The nature of the soil on which the bacillus grows has a great deal to do with the formation of the poison. An especially important factor is the temperature, for Brieger observed that no poison was produced in one case where the temperature remained by accident at $39^{\circ}$ for twenty-four hours. Under such conditions creatin is present in quantity, whereas otherwise the reverse is the rule.

In the process of extraction (p. 232) it occurs in the mercuric chlorid precipitate, and from this it is obtained, after the removal of the mercury by hydrogen sulphid, as an easily deliquescent hydrochlorid. This for the purpose of purification is converted into the difficultly soluble aurochlorid.

Typhotoxin is isomeric with gadinin and the compound $\mathrm{C}_{7} \mathrm{H}_{17} \mathrm{NO}_{2}$ which Brieger obtained from putrefying horse-flesh. In its properties it is, however, very different. Thus, the free base is strongly alkaline, and its hydrochlorid yields a difficultly soluble picrate. On the other hand, the isomer from horse-flesh possesses a slightly acid reaction, and does not form a picrate. Again, typhotoxin gives with Ehrlich's reagent (sulphodiazobenzol) an immediate yellow color, which disappears upon the addition of alkali, whereas the isomer does not yield this reaction. Furthermore, the two bases differ in their physiological action and in their behavior to alkaloidal reagents (see Table I.). Their aurochlorids, however, possess the same meltingpoint. 
The hydrochlorid is readily deliquescent, and unites with platinum chlorid to form an easily soluble double salt crystallizing in needles.

The aurochlorid, $\mathrm{C}_{7} \mathrm{H}_{17} \mathrm{NO}_{2} \cdot \mathrm{HCl} \cdot \mathrm{AuCl}_{3}$ ( $\mathrm{Au}=40.46$ per cent.), is difficultly soluble, and crystallizes in prisms, which melt at $176^{\circ}$. In its melting-point and solubility $\left(197^{\circ}\right.$, Brieger, Arch. $f$. pathol. Anat., $115,489)$ it agrees with its isomer from horse-flesh. In his first experiments with the typhoid bacillus, Brieger (II., 69) obtained a basic product differing in some of its characters from typhotoxin. Its aurochlorid, on analysis, gave 41.91 and 41.97 per cent. of $\mathrm{Au} ; 16.06$ per cent. of $\mathrm{C}$; and 3.66 per cent. of $\mathrm{H}$; while typhotoxin aurochlorid gave 40.78 per cent. Au; 17.38 per cent. $\mathrm{C}$; and 3.85 per cent. $\mathrm{H}$. For a comparison of the reaction of these two substances, see Table I.

In its physiological action typhotoxin differs from its isomer (page 318) in that the latter produces symptoms with well marked convulsions, whilst the former throws the animal into more of a paralytic or lethargic condition. The action of this base has been studied only on mice and guinea-pigs. It produces at first slight salivation with increased respiration; the animals lose control over the muscles of the trunk and extremities and fall down helpless upon their sides. The pupils become strongly dilated and cease to react to light; the salivation becomes more profuse ; the rate of heartbeat and of respiration gradually decreases and death follows in from one to two days. Throughout the course of these symptoms the animals have frequent diarrhœic evacuations, but at no time are convulsions present. On post mortem the heart is found to be in systole, the lungs are strongly hyperæmic, the other internal organs pale, the intestines firmly contracted and their walls pale.

A Base (?), $\mathrm{C}_{7} \mathrm{H}_{17} \mathrm{NO}_{2}$, was obtained by Brieger in 1886 (III., 28) on working over about one hundred pounds of horse-flesh which had been allowed to undergo slow putrefaction with limited access of air and at a low temperature $\left(-9^{\circ}\right.$ to $\left.+5^{\circ}\right)$ for four months. It occurred in the mercuric chlorid precipitate together with cadaverin, putrescin and mydatoxin, and from these bases it was separated and isolated according to the method given on page 311.

A similar, if not identical, substance having the composition $\mathrm{C}_{7} \mathrm{H}_{17} \mathrm{NO}_{2}$, was obtained by Baginsky and Stadthagen (1890) from cultures on horse-flesh, ten days at $35^{\circ}$, of a bacillus closely allied to Finkler-Prior's and isolated from stools of cholera infantum. The gold salt in its crystalline form and properties was the same as Brieger's except that it possessed a somewhat higher melting-point. It is possible that one or more of these isomers are homologues of leucin.

The free substance possesses, even after most careful purification, a slightly acid reaction. This acidity is removed from even a large quantity of the substance by the addition of a drop of alkali. On account of the acid character of the free substance Brieger does not 
consider it to be a base (a ptomaïn). It differs, however, from the amido acids in its poisonous character; in the fact that, unlike an acid, it does not unite with bases to form salts; and in not giving the characteristic red coloration (Hofmeister's reaction for the amido acids) with ferric chlorid. Whatever the true nature of this substance may be, it nevertheless in its other properties behaves like a base. Thus, it forms simple as well as double salts. On boiling with copper acetate it gives amorphous floccules. Under the desiccator it solidifies into plates which deliquesce on exposure to the air. It does not oombine either with silver oxid or with cupric hydrate. On dry distillation it yields a distillate possessing a strong acid reaction and a peculiar odor. The distillate does not give any precipitate with platinum chlorid, or with gold chlorid, nor does it react with copper acetate. With phosphomolybdic acid, however, it forms an amorphous mass; with ferric chlorid and potassium ferricyanid it yields an immediate precipitate of Berlin blue, whereas the original substance does not give any blue coloration.

The hydrochlorid, $\mathrm{C}_{7} \mathrm{H}_{17} \mathrm{NO}_{2}$. $\mathrm{HCl}$, crystallizes in fine needles which are insoluble in absolute alcohol. When the aqueous solution is treated with freshly precipitated silver oxid the resulting filtrate contains in solution some silver oxid, which can be removed by hydrogen sulphid; thus differing from an ammoniacal silver solution, which gives no precipitate on treatment with hydrogen sulphid. In this respect it resembles Salkowski's base, page 287. For reactions of the hydrochlorid, see Table I.

The aurochlorid, $\mathrm{C}_{7} \mathrm{H}_{17} \mathrm{NO}_{2} \cdot \mathrm{HCl} . \mathrm{AuCl}_{3}$, forms plates which are difficultly soluble in water and melt at $176^{\circ}$-the melting-point of the gold salt of typhotoxin. It is dimorphous, since sometimes it is also obtained in needles which can be changed into plates.

It does not form a picrate, nor does it give a reaction with sulphodiazobenzol.

This substance, when injected into frogs, produces a curara-like action. A few minutes after the injection the animal falls into a condition of paralysis and, although it can still react toward reflexes, it cannot move from its place. At times fibrillary twitchings pass over the body. The pupils dilate, the heart-action becomes gradually weaker, and finally, after several hours, the animal dies with the heart in diastole. Doses of 0.05 to 0.3 gram of the hydrochlorid, injected into guinea-pigs, produce in a short time a slight tremor, gradual increase in respiration, and slight moistening of the lower lip. The pupils at first contract, then dilate ad maximum and become reactionless. The temperature remains at first normal ; chills of short duration follow in rapid succession. The animal squats on the ground, with its snout pressing against the floor in exactly the same way as in the case of mussel-poison. Violent clonic convulsions follow in continually shorter intervals, and at the same time 
lachrymation and salivation become profuse, but not so excessive as in the case of the muscarin-like ptomaïns. The temperature sinks with the decrease in the rate of respiration, the ears previously gorged become pale and cold, and the heart-action becomes irregular and less frequent than before. General paralysis sets in, but the head still moves upward and backward. External stimuli induce violent clonic convulsions, the animal repeats frequently choking movements, and at the same time yields large quantities of saliva; finally, it falls upon its side completely paralyzed and dies. The heart stops in diastole, the intestines are pale and strongly contracted, and the bladder is empty and contracted.

Morrhuic Acid, $\mathrm{C}_{9} \mathrm{H}_{13} \mathrm{NO}_{3}$, was obtained by Gautier and Mourgues (1888) from brown cod-liver oil, together with six bases already described - namely, butylamin, amylamin, hexylamin, dihydrolutidin, asellin, and morrhuin. These bases constitute about 0.2 per cent. of the oil. The discoverers regard them as true leucomaïns, dissolved from the hepatic cells by the oil. Bouillot found that the mixed bases, or total basic product, in a dose of $0.25-0.15 \mathrm{~g}$. in man increased the volume of urine and the quantity of urea. By a microchemical reaction, exposing sections of liver to the fumes of hydrochloric or hydrofluoric acid, he detects the bases in the liver, especially in the bile-ducts. The bases, therefore, exist preformed in the cod's liver, and are derived from the bile. It is more probable, however, that these compounds are the products of initial decomposition, and for that reason they are described under the head of ptomaïns.

This compound is relatively abundant, and is basic as well as acid in character. It is resinous in appearance, and can be crystallized in flattened prisms, or large lance-shaped plates. When freshly precipitated it is oleaginous, viscous, then gradually hardens. It possesses a disagreeable aromatic odor resembling that of the seaweeds upon which the fish feed. According to the discoverers, its probable source is the lecithin derived thus from these weeds. It is soluble in alcohol, and but slightly in ether. It reddens turmeric, decomposes carbonates, and with acids forms salts which precipitate lead acetate and silver nitrate, but not copper acetate, even on warming.

The hydrochlorid is crystalline and is partially dissociated by excess of water. The platinum salt is soluble and crystallizes in very small cross-shaped prismatic needles. The gold salt is amorphous and is readily altered on heating.

The properties of this compound show that it is of a pyridin nature, and inasmuch as it does not give a precipitate with copper acetate, it would appear that the carboxyl is not directly united to the pyridin nucleus. This does not necessarily follow, now that we know that some amido acids exist which do not give a reaction with copper acetate (see page 287). Its pyridin nature is furthermore 
shown on distillation with lime. An oily alkaline base is thus obtained which forms an iodomethylate, and this with potassium hydrate yields quite an intense red color, resembling lees (de Coninck's reaction). On oxidation with permanganate of potassium it yields a monobasic acid. According to Gautier and Mourgues, the compound is probably identical with de Jungh's gaduin, and they ascribe to it the following constitution which, it should be said, lacks confirmation.<smiles>CCCC(=O)OC1CCCCC1O</smiles>

A Base, $\mathrm{C}_{5} \mathrm{H}_{12} \mathrm{~N}_{2} \mathrm{O}_{4}$, was obtained by Pouchet (1884) from the residual liquors resulting from an industrial treatment of débris of bones, flesh, and waste of all kinds, with dilute sulphuric acid. It is accompanied by another base, $\mathrm{C}_{7} \mathrm{H}_{18} \mathrm{~N}_{2} \mathrm{O}_{6}$, from which it can be separated by treatment with alcohol. The base itself forms tufts of delicate needles which alter or decompose less easily than the accompanying base. The platinochlorid, $\left(\mathrm{C}_{5} \mathrm{H}_{12} \mathrm{~N}_{2} \mathrm{O}_{4} \cdot \mathrm{HCl}\right)_{2} \mathrm{PtCl}_{4}$, forms a dull yellow powder somewhat soluble in strong alcohol, but insoluble in ether. The platinochlorid, $\left(\mathrm{C}_{7} \mathrm{H}_{18} \mathrm{~N}_{2} \mathrm{O}_{6} \cdot \mathrm{HCl}\right)_{2} \mathrm{PtCl}_{4}$, is insoluble in ether.

The hydrochlorids of these bases form silky needles, which are altered by excess of hydrochloric acid and by exposure to air. Pouchet considers them to be closely allied to the oxybetains. The general alkaloidal reagents precipitate these bases; the phosphomolybdic precipitate, on addition of ammonia, gives a blue tint. Both bases are toxic and exert a paralyzing action upon the reflex movements.

The method employed by Pouchet for their isolation was to precipitate them as tannates. The precipitate was decomposed by lead hydrate in the presence of strong alcohol, the excess of lead removed from the solution by hydrogen sulphid, and the clear liquid thus obtained was submitted to dialysis. The above bases occurred in the dialysate. In the non-dialyzable portion volatile bases were found probably identical with those described by Gautier and Etard.

Tetanin, $\mathrm{C}_{13} \mathrm{H}_{30} \mathrm{~N}_{2} \mathrm{O}_{4}$, was obtained in 1886 by Brieger (III., 94) by cultivating impure tetanus microbes of Rosenbach, in an atmosphere of hydrogen on beef-broth for eight days at $37^{\circ}-38^{\circ}$. It likewise occurs in cultures on brain-broth. Later (April, 1888), Brieger succeeded in obtaining tetanin from the amputated arm of a tetanus 
patient, identical in its physiological and chemical reactions with that isolated from cultures of Rosenbach's germs on beef-broth. The presence of tetanin during life in tetanus patients has thus been demonstrated. It has not been found in the brain and nerve tissue of persons dead from tetanus. A portion of the jelly-like mass taken from the amputated arm was found to contain tetanus bacilli as well as staphylococci and streptococci, and when planted on beef-broth tetanin was formed, but no tetanotoxin or spasmotoxin.

Kitasato and Weyl (1890), employing pure cultures of the tetanus bacillus, obtained from $1 \frac{1}{4}$ kilograms of beef used as culture medium 1.7118 gram of tetanin hydrochlorid (0.137 per cent.). Tetauotoxin was also present.

For its isolation Brieger employed the following method: The cultures were slightly acidulated with hydrochloric acid, heated and filtered; the filtrate was then treated with lead acetate and with alcoholic mercuric chlorid in the manner described under mytilotoxin (page 313). Kitasato and Weyl digest the cultures with 0.25 per cent. hydrochloric acid for some hours at $60^{\circ}$, then render slightly alkaline, filter, and distil in vacuo at $60^{\circ}$. The residue in the retort is worked for tetanin by Brieger's method, while the distillate contains tetanotoxin, ammonia, indol, hydrogen sulphid, phenol, and butyric acid. The filtrate from the above mercuric chlorid precipitate contains the greater part of the active principle, provided the precipitate has been thoroughly washed. After the removal of the mercury by hydrogen sulphid the liquid is evaporated, and the residue is repeatedly extracted with absolute alcohol, in which the tetanus poison readily dissolves and can thus be separated from the insoluble ammonium chlorid. The alcoholic solution is treated with alcoholic platinum chlorid which precipitates the ammonium and creatinin platinochlorids, whilst the platinochlorid of the poison remains in solution. The filtrate from this precipitate gives, on the addition of ether, a flocculent precipitate possessing exceedingly deliquescent properties. The precipitate is, therefore, rapidly filtered off by means of a pump, and dried in vacuo. It can then be recrystallized from hot 96 per cent. alcohol, and the beautiful clear-yellow plates thus obtained, if dried again in vacuo, become rather difficultly soluble in water, from which it can then be recrystallized and obtained in a perfectly pure condition. If boiled with boneblack, it decomposes yielding a non-poisonous crystalline compound.

Phosphomolybdic acid cannot be used in the separation of tetanin inasmuch as it destroys the poison (Brieger). Bocklisch has also observed that this reagent destroys the poison formed in the putrefaction of fish.

Tetanin obtained by treating the hydrochlorid with freshly precipitated moist silver oxid forms a strongly alkaline yellow syrup. With alkaloidal reagents it gives the same reactions as the hydro- 
chlorid, except that it does not give a blue color with ferric chlorid and potassium ferricyanid. It is easily decomposed in acid but is permanent in alkaline solution.

The hydrochlorid, $\mathrm{C}_{13} \mathrm{H}_{30} \mathrm{~N}_{2} \mathrm{O}_{4} \cdot 2 \mathrm{HCl}$, is very deliquescent, and is easily soluble in absolute alcohol. Besides with platinum it combines only with phosphomolybdic acid to form an easily soluble crystalline precipitate, which on the addition of ammonium hydrate becomes white. If, however, the hydrochlorid is impure, phosphomolybdic acid produces a precipitate which is colored an intense blue by ammonia. Potassium bismuth iodid yields a precipitate which is at first amorphous, but soon becomes crystalline. Ferric chlorid and potassium ferricyanid produce a slowly developing blue color, which probably is due to impurities.

When kept for some months the highly poisonous hydrochlorid becomes syrupy, brownish, and wholly inert. Examined at this stage, the syrup was found, by means of platinum chlorid, to contain a substance the hydrochlorid of which crystallized in plates. This is readily soluble in water and alcohol and melts at $197^{\circ}$ with total decomposition, the same as tetanin. It combines only with phosphomolybdic acid to form an easily soluble compound. The platinum salt has the composition $\mathrm{C}_{6} \mathrm{H}_{13} \mathrm{NO}_{2} \cdot 2 \mathrm{HCl}$. $\mathrm{PtCl}_{4}$. This substance is non-poisonous, and probably is an amido acid. It is different, however, from leucin and Nencki's isomers of leucin, although possessing the same composition. It is also isomeric with mydatoxin, $\mathrm{C}_{6} \mathrm{H}_{13} \mathrm{NO}_{2}$, but this is highly poisonous to mice, while the former is inert (see p. 312). Tetanin resembles mytilotoxin with respect to this loss of toxicity on standing.

The platinochlorid, $\mathrm{C}_{13} \mathrm{H}_{30} \mathrm{~N}_{2} \mathrm{O}_{4} \cdot 2 \mathrm{HCl} . \mathrm{PtCl}_{4}(\mathrm{Pt}=28.33$ per cent.), is easily soluble in absolute alcohol, from which it is precipitated on the addition of ether. From ninety-six per cent. alcohol it crystallizes in clear yellow plates. After repeated recrystallization from alcohol and drying in vacuo it becomes difficultly soluble in water so that it can be recrystallized from the latter. It decomposes at $197^{\circ}$.

This base produces the characteristic, though by no means all the symptoms of tetanus, since we know of at least three other toxins (pp. 61, 254) which occur with tetanin in cultures of the tetanus microbe. The symptoms induced by relatively large doses in warm-blooded animals, as mice, guinea-pigs, and rabbits, exhibit two distinct phases. In the first, the animal is thrown into a lethargic, paralytic condition, then suddenly becomes uneasy, and the respiration becomes more frequent. This is followed by the second phase, in which tonic and clonic convulsions, especially the former, predominate till death results. 0.5 gram has but slight action on guinea-pigs. Small doses do not seem to affect guinea-pigs, while frogs appear to be much less sensitive than mice. The characteristic convulsions and opisthotonus seen in tetanus in man are also pro- 
duced in guinea-pigs on injection of large doses of this base. Dogs and horses seem to be but slightly sensitive to the action of this poison.

A Base, $\mathrm{C}_{14} \mathrm{~N}_{20} \mathrm{~N}_{2} \mathrm{O}_{4}$, was isolated by Guareschi in 1887 from putrid fibrin. It occurs in the chloroform or ether extracts along with the base $\mathrm{C}_{10} \mathrm{H}_{13} \mathrm{~N}$, and is probably an amido acid (see page 259).

A Base, $\mathrm{C}_{7} \mathrm{H}_{15} \mathrm{~N}_{2} \mathrm{O}_{6}$, was isolated by Pouchet in 1884 . It is said to form short, thick prisms which become brown when exposed to light.

The platinochlorid, $\left(\mathrm{C}_{7} \mathrm{H}_{18} \mathrm{~N}_{2} \mathrm{O}_{6} \cdot \mathrm{HCl}\right)_{2} \mathrm{PtCl}_{4}$, crystallizes in prismatic needles which are insoluble in strong alcohol. For further details in regard to this base, see page 320 .

A Base, $\mathrm{C}_{16} \mathrm{H}_{23} \mathrm{~N}_{2} \mathrm{O}_{4}$, was obtained by Lepierre (1894) in small quantity from poisonous cheese by precipitating, in the cold, with acetate of copper. It is crystalline, bitter, inodorous, and shows a slight acid reaction to phenol-phthalein; is but slightly soluble in water, soluble in alcohol. It is dextro-rotatory, is precipitated by phosphomolybdic and picric acids, iodin in potassium iodid; not by tannin. When fed to guinea-pigs it produced diarrhœa. $0.05 \mathrm{~g}$. injected intravenously into a rabbit had no effect. The hydrochlorid is very soluble and forms large needles. The platinum and gold salts are crystalline.

Tyrotoxicon has been found in poisonous cheese (Vaughan, Wallace, Wolff, Wesener 1898), in poisonous ice cream (Vaughan, Novy, Schearer, Ladd), in poisonous milk (Vaughan, Novy, Newton, Wallace, Firth, Schearer), and in cream puffs (Stanton). The method of separating this poison and its effect upon animals have already been given with sufficient detail. Chemically, it is very instable. When warmed with water to about $90^{\circ}$, it decomposes. Hydrogen sulphid also decomposes it, therefore all attempts to isolate it by precipitation with some base, such as mercury or lead, and then removing the base with hydrogen sulphid have failed. Its unstable character is illustrated by the fact that it may disappear altogether within twenty-four hours from milk, rich in the poison, which is allowed to stand in an open beaker.

With potassium hydrate it forms a compound which agrees in crystalline form, chemical reactions, and the per cent. of potassium which it contains, with the compound of diazobenzol and potassium hydrate. This substance is best obtained from milk containing tyrotoxicon as follows: The filtered milk, which is acid in reaction, is neutralized with sodium carbonate, agitated with an equal volume of ether, allowed to stand in a stoppered glass cylinder for twentyfour hours, the ether removed, and allowed to evaporate spontaneously in an open dish. The aqueous residue is acidified with 
nitric acid, then treated with an equal volume of a saturated solution of potassium hydrate, and the whole concentrated on a water-bath (this compound is not decomposed below $130^{\circ}$ ). On being heated the mixture becomes yellowish-brown, and emits a peculiar aromatic odor. On cooling the tyrotoxicon compound forms in beautiful, sixsided plates along with the prisms of potassium nitrate.

With equal parts of sulphuric and carbolic acids, pure tyrotoxicon gives a green coloration, but in whey the color varies from yellow to orange-red. This color reaction may be used as a preliminary test in examining milk for tyrotoxicon. It is best carried out as follows: Place on a clean porcelain surface two or three drops each of pure carbolic and sulphuric acids. Then add a few drops of the aqueous solution of the residue left after the spontaneous evaporation of the ether. If tyrotoxicon be present, a yellow to orange-red coloration will be produced. This test is to be regarded only as a preliminary one, for the coloration may be due to the presence of a nitrate or nitrite, or, as Huston and Weber have shown, to butyric acid. The tyrotoxicon must be converted into the potassium compound and purified before the absence of nitrate or nitrite can be positively demonstrated. Moreover, the physiological test should always be made in testing for this poison.

With platinum chlorid in alcoholic solution tyrotoxicon forms a compound which explodes with great violence at the temperature of the water-bath. This also corresponds with the compound of platinum chlorid and diazobenzol. It should be borne in mind, however, that organic peroxids may behave in a similar manner.

Pure tyrotoxicon is insoluble in ether, and its extraction from alkaline solutions by this solvent is due to the presence of foreign matter with which the poison is taken up by the ether.

The physiological action of this ptomain has been discussed in a preceding chapter.

Mydalein ( $\mu \nu \delta \alpha \lambda \hat{\varepsilon}_{0} \varsigma$, putrid) is a poisonous base obtained in 1885 from putrefying cadaveric organs, liver, spleen, etc. (Brieger, II., 31, 48). Though it is apparently present on about the seventh day, it is unobtainable until about the third or fourth week. The method for its separation from the accompanying bases is given under saprin (page 278). It is liable to occur in the mercuric chlorid filtrate, as well as in the precipitate, inasmuch as the double salt is insoluble only in perfectly absolute alcohol. In order to purify the platinochlorid obtained as on page 279 , it is repeatedly recrystallized from a very small quantity of lukewarm water. This base has not been isolated in sufficient quantity to permit of a complete determination of its composition. It is probably a diamin, containing four or five carbon atoms, and hence is nearly related to some of the diamins already described. 
The platinochlorid, on analysis, gave: $\mathrm{Pt}=38.74, \mathrm{C}=10.83$, $\mathrm{H}=3.23$. It crystallizes in small needles, and is extremely soluble in water.

The hydrochlorid crystallizes with extreme difficulty, even on standing for some time in a desiccator. On exposure to the air it rapidly deliquesces.

Mydalein has an entirely specific action. Small quantities injected into guinea-pigs or rabbits produce, after a short time, a moistening of the under lip, and an abundant flow of secretion from the nose and eyes. The pupils dilate gradually to maximum and become reactionless; the ear-vessels become strongly injected, and the body temperature rises $1^{\circ}$ to $2^{\circ}$. The hairs bristle, and the animal occasionally shudders. Gradually the salivation ceases, the respiration and heart-action, which were at first hastened, now decrease, the temperature falls, the ears become pale, and the animal finally recovers. During the action of the poison the animal shows a tendency to sleep, and the peristaltic action of the intestines is heightened. Larger doses (0.050 gram) induce an exceedingly violent action which invariably results in the death of the animal. On post-mortem the heart is found to be stopped in diastole, and the intestines and bladder are contracted; otherwise nothing abnormal is observed.

A Toxic Base.-From human livers and spleens which were decomposing for two weeks in thorough contact with air there was isolated, besides cadaverin and putrescin, a small quantity of poisonous base (Brieger, II., 29, 48). The mercuric chlorid precipitate was decomposed, and the hydrochlorids were precipitated by gold chlorid (to remove cadaverin, which is soluble), and the aurochlorid was then changed into platinum salt, whereby the insoluble putrescin platinochlorid was removed. In the mother-liquor from the putrescin salt an easily soluble platinum compound was detected and found to contain 41.30 per cent. $\mathrm{Pt}$. It crystallized in fine needles. The hydrochlorid formed small, readily deliquescent needles, and did not produce a precipitate in alcoholic platinum chlorid. Injected into guinea-pigs and rabbits it induced an exalted peristalic action of the intestines, which lasted several days and produced in the animals, on account of the continuous evacuations, a condition of great weakness. No disturbance in the functions of the other organs was observed.

A Base was isolated from decomposing haddock which were exposed for five days during summer in an open iron vessel. Brieger (I., 42) found in the aqueous mercuric chlorid precipitate (see page 135) a base the hydrochlorid of which crystallized in well formed, small needles. The platinochlorid likewise crystallized in beautiful needles and gave, on analysis, 36.03 per cent. of $\mathrm{Pt} ; 7.81$ per cent. of $\mathrm{N}$. 
A substance of muscarin-like action was obtained by Brieger (I., 59) from putrefying gelatin, ten days at $35^{\circ}$, though in insufficient quantity to permit a determination of its character. The residue containing this substance gave, on distillation with alkali, only ammonia.

A Base was obtained by Bocklisch (III., 52, 53) from herring which had undergone putrefaction for twelve days. It was found in the distillate, together with trimethylamin and dimethylamin, obtained by distilling the mercuric chlorid filtrate, after the removal of the mercury, with sodium hydrate. The platinochlorid was easily soluble, and crystallized in large thin plates. On analysis it gave: $\mathrm{Pt}=28.57, \mathrm{C}=22.34, \mathrm{H}=4.66$. The hydrochlorid was easily soluble in water and in absolute alcohol, and besides with platinum gave only with phosphomolybdic acid a yellow precipitate which was soluble in excess and with ammonia developed an immediate blue color. It immediately reduced a mixture of ferric chlorid and potassium ferricyanid with formation of Berlin blue; and similarly threw down metallic gold from solutions of gold chlorid.

From poisonous mussel, Brieger (III., 79) obtained an aurochlorid of a base crystallizing in needles. The quantity isolated was insufficient for analysis. It is interesting because of its property of inducing salivation, a symptom which was observed by Schmidtmann and by Crumpe in some cases of mussel poisoning.

A Base was obtained by Guareschi and Mosso (Journ. für praktische Chem., 28, 508) from fresh beef, in the alkaline ether extract obtained by Dragendorff's method. It formed a yellowish alkaline fluid, of unpleasant odor, and after a time gave a deposit of microscopic crystals. The hydrochlorid gave the following reactions: Gold chlorid, yellow crystalline precipitate; platinum chlorid, precipitate; potassium iodid and iodin in hydriodic acid, kermes-red precipitate; phosphotungstic acid, nothing; phosphomolybdic acid, an abundant yellow precipitate; tannic acid, heavy, grayish precipitate, same with Mayer's reagent; picric acid, yellow precipitate; Marmés reagent, precipitate soluble in excess ; potassium bichromate, nothing; potassium permanganate and sulphuric acid, violet color ; potassium ferricyanid and ferric chlorid, Prussian-blue precipitate.

By giving a precipitate with tannin, and not with phosphotungstic acid, it resembles neurin.

Ch. Gram has studied the decomposition of yeast under the influence of an infusion of hay. The yeast was allowed to putrefy for fourteen days, and was then treated with zinc sulphate. The latter was precipitated by barium hydrate, and the filtrate after the removal of the barium by sulphuric acid was evaporated to dryness and extracted with absolute alcohol. The alcoholic solution was evaporated, 
and the residue again extracted with alcohol. The extraction residue was taken up with water, and again subjected to the above treatment with zinc sulphate, barium hydrate, etc.

The filtrate was poisonous and produced, in frogs, paralysis and stoppage of the heart in diastole. Addition of platinum chlorid and alcohol precipitated two bases. One of these, although possessing a curara-like action, did not affect the heart. When its solution was heated for twenty-four hours on a water-bath it caused general paralysis and stoppage of the heart. The platinochlorid contained 38.05 per cent. of platinum.

The other base also possessed a slight curara-like action and its platinochlorid gave, on analysis, 40.92 and 39.4 per cent. of platinum.

Brieger found a basic substance in small quantities in cultures of the staphylococcus pyogenes aureus on bouillon and beef-broth (II., 74). The hydrochlorid formed groups of colorless non-deliquescent needles. With platinum chlorid it yielded a double salt, crystallizing in needles, and containing 32.93 per cent. of $\mathrm{Pt}$. For its reactions, see Table $I$.

From aqueous as well as alcoholic extracts of cultures of staphylococcus aureus, Leber (1888) isolated a crystalline substance which he named phlogosin. The composition of this substance is not known. It does not seem to contain nitrogen, and inasmuch as it blackens silver it probably contains sulphur. It crystallizes in fine needles which are soluble in ether and in alcohol; difficultly soluble in water. It sublimes in needles. Alkalis precipitate it as amorphous yellow floccules which are soluble in acid and then can be recrystallized. With potassium ferricyanid and ferric chlorid it yields a blue color, and with potassio-mercuric, cadmic, and bismuth iodids precipitates which are soluble in excess. No precipitate is produced by gold or platinum chlorid, phosphotungstic, or molybdic, tannic, or picric acids.

A small quantity applied to the conjunctiva produces intense inflammation, suppuration, and necrosis. Introduced into the anterior chamber it induces intense suppuration and keratitis. The substance is entirely distinct from the base obtained by Brieger and described above.

A Base-boiling-point about $284^{\circ}$ - was obtained by Brieger (II., 61) from human livers and spleens which were putrefying for two or three weeks. It occurs in the mercuric chlorid filtrate, as described under saprin, page 279 , together with some mydalein, trimethylamin, and a hydrocarbon. The filtrate, after the mercury is removed by hydrogen sulphid, is evaporated to dryness, and finally the last traces of water are removed in a vacuum. The residue is then treated with absolute alcohol, and from this alcoholic solution the mydalein is 
precipitated by the addition of alcoholic mercuric chlorid. The trimethylamin is separated by distillation of the alkaline filtrate, previously deprived of its mercury by hydrogen sulphid; while the mother-liquor yields an oily mixture of hydrocarbons and bases. The latter were separated by fractional distillation, whereby only one of the bases was obtained in sufficient quantity for study. It boiled at about $284^{\circ}$, and gave with hydrochloric acid, on evaporation, a salt crystallizing in beautiful, long needles which were very easily soluble in perfectly absolute alcohol. With gold chlorid and picric acid it gave only oily products; with ferric chlorid and potassium ferricyauid, an intense blue; with platinum chlorid, an extremely easily soluble double salt which appeared under the microscope in the form of very fine needles; while from alcohol-ether the double salt slowly separated in thin plates which contained 30.36 per cent. of platinum. The free base showed a slight fluorescence. It is not poisonous and, according to Brieger, is probably a pyridin derivative.

Other non-poisonous bases were present in very small quantity in the mother-liquor described above, after the separation of the oily mixture.

Peptotoxin.-By this name Brieger (I., 14-19) designated a poisonous base which he found in some peptons, and hence in the digestion of fibrin; in putrefying albuminous substances, such as fibrin, casein, brain, liver, and muscles. It is a well known fact that animal tissues, in the early stages of putrefaction, possess strong toxic properties, even before the decomposition has advanced far enough to effect a marked splitting up of the proteid and carbohydrate molecules. Brieger and others have tried to seek an explanation of this toxicity by connecting it with an early peptonization of the proteids brought about by the action of enzymes which are distributed throughout the tissues, and which begin their activity immediately after death. This poison has not been definitely isolated, but its general properties and action have been studied by Brieger, who prepared it by digesting fibrin for twenty-four hours with gastric juice at the temperature of the blood. The perfectly fresh pepton thus obtained was evaporated to a syrupy residue, and this was then extracted with boiling alcohol. The residue left on evaporation of the alcoholic solution was digested for some time with amyl alcohol which on subsequent evaporation gave amorphous brownish masses. This extract was then purified by neutral lead acetate. The filtrate, after the removal of the lead by hydrogen sulphid, was repeatedly extracted with ether, then evaporated to dryness and extracted, as before, with amyl alcohol. This final extract was evaporated to drive off the alcohol, taken up with water and filtered. The colorless aqueous solution thus obtained contains the poisonous substance 


\section{CeI}

holin $\mathrm{C}$

$\mathrm{P}_{6} \mathrm{H}_{14} \mathrm{~N}$

ite pres

insolub

iter, on

ing hec

crystal

slumine

cipite

lite cur

cipit:

Nop

rty-wh

culent

luble in

ed amo

precipi

ellowis

alline $\mathrm{p}$

tat

hite crs

luble in

ellow 1

ate, soli

bot w:

No. I

hite g1

cryst.

tate, ap:

slow
rown g?

precip

precip

fine ne 
Table I-Tabular Yiew of the Reactions of Certain Ptomaíns,

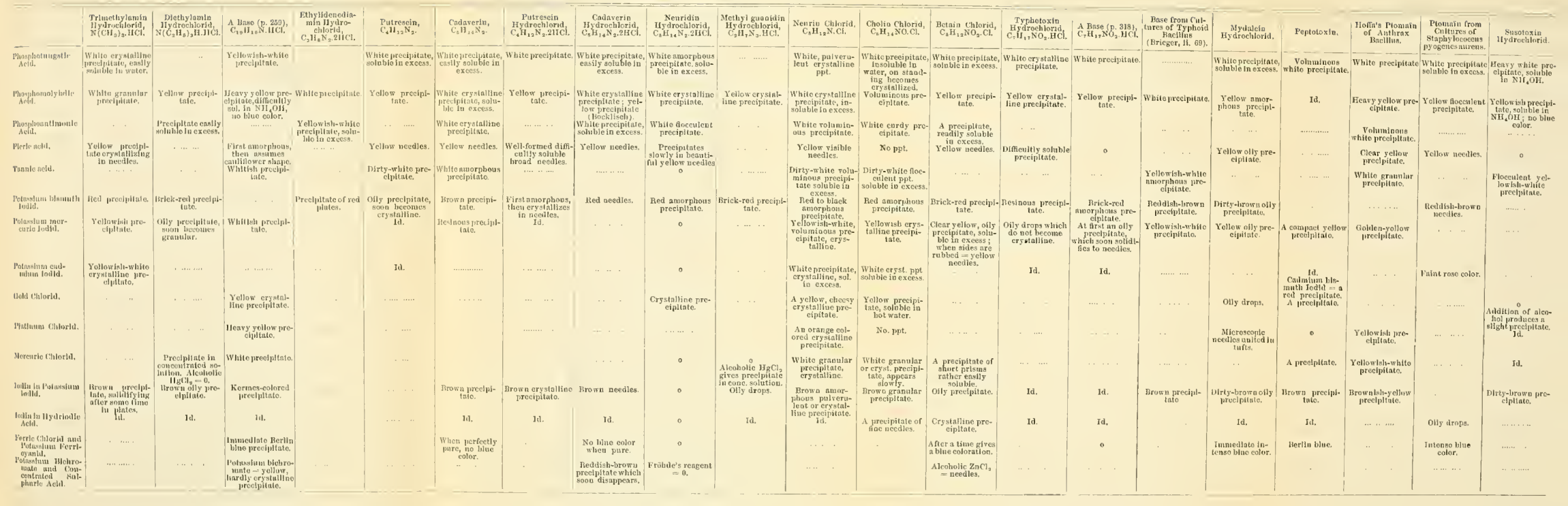


which, however, can only with extreme difficulty be brought to crystallization in vacuo.

Salkowski in eight digestion experiments with fresh fibrin obtained a poisonous extract in but one case. On the other hand, putrid fibrin or prolonged digestion, both implying bacterial activity, yield a poisonous product. Peptic digestion of serum-albumin, eggalbumin, and meat likewise gave negative results. In view of these facts as well as its presence in putrefying proteids, Salkowski concludes that a peptotoxin (in the sense of Brieger) does not exist. That poisonous products develop in meat and in proteids on putrefaction is well established. In 1891 Brieger ascribed a proteid nature to peptotoxin, excluding it from the bases. Stadthagen examined normal urine for peptotoxin, but failed to find it.

From animals with extensive burns Kijanitzin has isolated, by means of Brieger's method for peptotoxin, from the urine, blood, and especially the organs, a substance resembling peptotoxin in chemical and physiological behavior. Atropin antagonizes its action. The clinical symptoms in cases of extensive burns are closely allied to those observed in animals when a part or whole of the body is varnished. Rabbits die when $\frac{1}{8}-\frac{1}{6}$ of the body-surface is varnished. Death in these cases has been explained by excessive loss of heat or by lack of excretion of waste products of the skin. Kijanitzin holds that the varnishing alters the chemical products of cells of the skin so that poisons are formed and carried thronghout the body as in skin-burns.

Peptotoxin, when in its purest condition, as shown by its failure to give the biuret test, possesses a neutral reaction. Its behavior to Millon's reagent is quite characteristic ; it gives a white precipitate which on boiling becomes intensely red. Because of this reaction, Brieger is inclined to regard this substance as a hydroxyl or an amido derivative of benzol. The ptomaïn can be extracted from acid as well as alkaline solution by amyl alcohol-more difficultly in the cold than on heating. It is absolutely insoluble in ether, benzol, and chloroform; very soluble in water. It is not destroyed by boiling, by passing hydrogen sulphid, or by strong alkalis ; but is destroyed, however, when the putrefaction lasts longer than eight days. For its behavior to reagents, see Table I.

Various observers have shown that pepton possesses a toxic action, and some have been led to regard this toxicity as not due to the pepton itself, but rather to the presence of this or some other ptomaïn. At least Brieger found one specimen of dry Witte's pepton to be perfectly harmless ; whereas, the fresh pepton formed by fibrin digestion possessed strong toxic powers. Moreover, this non-poisonous pepton when exposed to the action of gastric juice was found to yield the poisonous substance. It is indeed probable that peptotoxin is not a distinct compound but rather a mixture as in the case 
of the toxalbumins. The poisonous nature of proteids and the physiological action of this base will be described later.

Pyocyanin, $\mathrm{C}_{14} \mathrm{H}_{14} \mathrm{~N}_{2} \mathrm{O}$, is the coloring matter of blue pus, and is produced by the action of the bacillus pyocyaneus. It was isolated by Ledderhose (1887) and is said to be an anthracene derivative. On contact with the air it is oxidized to pyoxanthose, a yellow substance. According to Kunz, it contains nitrogen and sulphur. The picrate is of a dark reddish-brown color; the platinum salt is black, and sometimes is obtained as glittering fine golden needles.

Table of Ptomaïns.

\begin{tabular}{|c|c|c|c|}
\hline Formula. & Name. & Discoverer. & $\begin{array}{l}\text { Physiological } \\
\text { Action. }\end{array}$ \\
\hline $\begin{array}{l}\mathrm{C} \mathrm{H}_{5} \mathrm{~N} \\
\mathrm{C}_{2} \mathrm{H}_{7} \mathrm{~N} \\
\mathrm{C}_{3} \mathrm{H}_{9} \mathrm{~N} \\
\mathrm{C}_{2} \mathrm{H}_{5} \mathrm{~N} \\
\mathrm{C}_{2} \mathrm{H}_{7} \mathrm{~N} \\
\mathrm{C}_{4} \mathrm{H}_{11} \mathrm{~N} \\
\mathrm{C}_{6} \mathrm{H}_{15} \mathrm{~N} \\
\mathrm{C}_{3} \mathrm{H}_{9} \mathrm{~N} \\
\mathrm{C}_{4} \mathrm{H}_{11} \mathrm{~N} \\
\mathrm{C}_{5} \mathrm{H}_{11}^{\mathrm{N}}(?) \\
\mathrm{C}_{5} \mathrm{H}_{13} \mathrm{~N} \\
\mathrm{C}_{6} \mathrm{H}_{15} \mathrm{~N} \\
\mathrm{C}_{7} \mathrm{H}_{11} \mathrm{~N} \\
\mathrm{C}_{8} \mathrm{H}_{11} \mathrm{~N} \\
\mathrm{C}_{8} \mathrm{H}_{11} \mathrm{~N} \\
\mathrm{C}_{8} \mathrm{H}_{13} \mathrm{~N} \\
\mathrm{C}_{9} \mathrm{H}_{13} \mathrm{~N} \\
\mathrm{C}_{10} \mathrm{H}_{15} \mathrm{~N} \\
\mathrm{C}_{10} \mathrm{H}_{15} \mathrm{~N} \\
\mathrm{C}_{32} \mathrm{H}_{31} \mathrm{~N} \\
\mathrm{C}_{2} \mathrm{H}_{8} \mathrm{~N}_{2} \\
\mathrm{C}_{3} \mathrm{H}_{6} \mathrm{~N}_{2} \\
\mathrm{C}_{3} \mathrm{H}_{8} \mathrm{~N}_{2} \\
\mathrm{C}_{4} \mathrm{H}_{12} \mathrm{~N}_{2} \\
\mathrm{C}_{3} \mathrm{H}_{12} \mathrm{~N}_{2} \\
\mathrm{C}_{6} \mathrm{H}_{11} \mathrm{~N}_{2} \\
\mathrm{C}_{6} \mathrm{H}_{14} \mathrm{~N}_{2} \\
\mathrm{C}_{6} \mathrm{H}_{16} \mathrm{~N}_{2} \\
\mathrm{C}_{7} \mathrm{H}_{10} \mathrm{~N}_{2} \\
\mathrm{C}_{10} \mathrm{H}_{26} \mathrm{~N}_{2}(?) \\
\mathrm{C}_{2} \mathrm{H}_{7} \mathrm{~N}_{3} \\
\mathrm{C}_{19} \mathrm{H}_{27} \mathrm{~N}_{3} \\
\mathrm{C}_{13} \mathrm{H}_{20} \mathrm{~N}_{4} \\
\mathrm{C}_{13} \mathrm{H}_{38} \mathrm{~N}_{4} \\
\mathrm{C}_{25} \mathrm{H}_{32} \mathrm{~N}_{4} \\
\mathrm{C}_{6} \mathrm{H}_{13} \mathrm{~N} \mathrm{O} \\
\mathrm{C}_{8} \mathrm{H}_{11} \mathrm{~N} \mathrm{O} \\
\mathrm{C}_{5} \mathrm{H}_{11} \mathrm{~N} \mathrm{O}_{2} \\
\mathrm{C}_{5} \mathrm{H}_{15} \mathrm{~N} \mathrm{O}_{2} \\
\mathrm{C}_{6} \mathrm{H}_{13} \mathrm{~N} \mathrm{O}_{3}\end{array}$ & $\begin{array}{l}\text { Methylamin. } \\
\text { Dimethylamin. } \\
\text { Trimethylamin. } \\
\text { Spermin (?). } \\
\text { Ethylamin. } \\
\text { Diethylamin. } \\
\text { Triethylamin. } \\
\text { Propylamin. } \\
\text { Butylamin. } \\
\text { Tetanotoxin. } \\
\text { Amylamin. } \\
\text { Hexylamin. } \\
\text { Di-hydrolutidin. } \\
\text { Collidin (?). } \\
\text { Pyridin base (?). } \\
\text { Hydrocollidin (?). } \\
\text { Parvolin (?). } \\
\text { Unnamed. } \\
\text { Pyridin base (?). } \\
\text { Unnamed. } \\
\text { Ethylidenediamin (?). } \\
\text { Anthracin. } \\
\text { Trimethylenediamin (?). } \\
\text { Putrescin. } \\
\text { Cadaverin. } \\
\text { Neuridin. } \\
\text { Saprin. } \\
\text { Hexamethylenediamin. } \\
\text { Unnamed. } \\
\text { Susotoxin. } \\
\text { Methyl guanidin. } \\
\text { Morrhuin. } \\
\text { Unnamed. } \\
\text { “ } \\
\text { Asellin. } \\
\text { Neurin. } \\
\text { Mydin. } \\
\text { d-amido-valerianic acid. } \\
\text { Cholin. } \\
\text { Mydatoxin. }\end{array}$ & 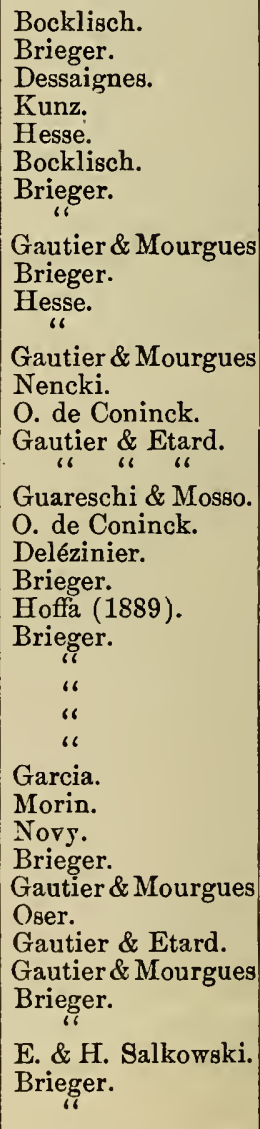 & 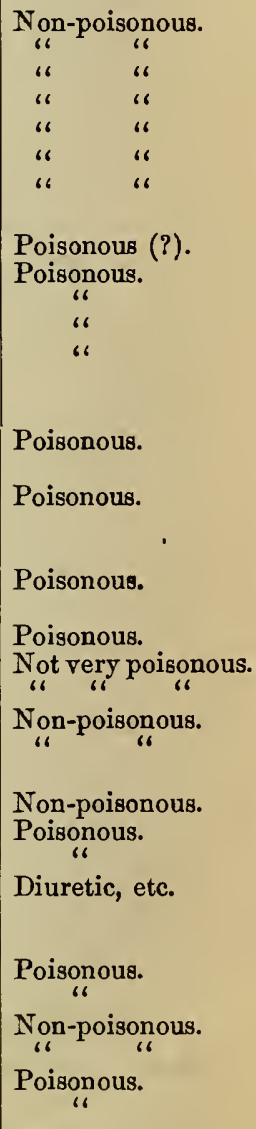 \\
\hline
\end{tabular}

${ }^{1}$ Only those bases are here denoted as poisonous which possess a decided toxicity. 


\section{MINATIONS.}

ä BNer's Inaugural Dissertation.

\begin{tabular}{|c|c|c|c|c|}
\hline \multicolumn{3}{|c|}{ Brouardel and Boutmy. } & \multirow{2}{*}{$\begin{array}{c}\text { Baumert } \\
\text { (Liebermann). }\end{array}$} & \multirow{2}{*}{ Otto. } \\
\hline $\begin{array}{l}\text { Substdaver. } \\
\text { auby hydro- } \\
\text { lic acid. }\end{array}$ & $\begin{array}{c}\text { Cadaver. } \\
\text { In water } 18 \text { months. }\end{array}$ & $\begin{array}{l}\text { Parts of } \\
\text { putrefying goose } \\
\text { and cadaver. }\end{array}$ & & \\
\hline $\begin{array}{l}\text { Solveut......... } \\
\text { Residue........ }\end{array}$ & Alkaline. & $\begin{array}{l}\text { Alkaline, volatile } \\
\text { liquid ; odor that } \\
\text { of urine of mice. }\end{array}$ & $\begin{array}{l}\text { Ether (acid). } \\
\text { Yellow, amorphous; } \\
\text { taste sharp, bitter. }\end{array}$ & $\begin{array}{l}\text { Petroleum-ether } \\
\text { (alkaline). } \\
\text { Bright yellow oil. }\end{array}$ \\
\hline $\begin{array}{l}\text { Tannic. } \\
\text { Gold Ch. }\end{array}$ & ....................... & $\begin{array}{l}\text { White precipitate. } \\
\text { Violet precipitate. }\end{array}$ & $\begin{array}{l}\text { Same as colchicin; } \\
\text { soluble in alcohol. } \\
\text { Same as colchicin. }\end{array}$ & A precipitate. \\
\hline Platinu & $\ldots \ldots \ldots$ & ............ & A precipitate, & Id. \\
\hline $\begin{array}{l}\text { Iodiu initate. } \\
\text { sium } \\
\text { Frölide' }\end{array}$ & (n).... & $\begin{array}{l}\text { Kermes-brown } \\
\text { precipitate. } \\
\text { Orange-red. }\end{array}$ & Same as colchicin. & (n)..... \\
\hline $\begin{array}{l}\text { Phosplic....... } \\
\text { Acid. }\end{array}$ & $\ldots \ldots . . .$. & (............. & $\begin{array}{l}\text { Same as colchicin, } \\
\text { blue with } \mathrm{NH}_{4} \mathrm{OH} \text {. }\end{array}$ & $\ldots \ldots \ldots \ldots$ \\
\hline $\begin{array}{l}\text { Sulphurne cold, } \\
\text { ish-violet. }\end{array}$ & $\begin{array}{l}\text { Colorless; on } \\
\text { warming, violet. }\end{array}$ & . $\quad$............. & Yellow color. & (.......... \\
\hline $\begin{array}{l}\text { Sulphur } \\
\text { aud P } \\
\text { Bichrı }\end{array}$ & ............ & $\begin{array}{l}\text { No odor of butyric } \\
\text { acid. }\end{array}$ & ............ & ........... \\
\hline Nitric $/$ & .......... & …........ & $\begin{array}{l}\text { Dark-yellow color, } \\
\text { concentrated } \mathrm{HNO}_{3} \\
\text { = carmine red, } \\
\text { which with water } \\
\text { gives yellow. }\end{array}$ & ........... \\
\hline $\begin{array}{l}\text { IIydroc: } \\
\text { Acid. } \\
\text { Mercuri }\end{array}$ & $\begin{array}{l}\text { Cherry-red on } \\
\text { heating. }\end{array}$ & $\begin{array}{c}\text { Colorless. } \\
\\
\ldots\end{array}$ & . & A precipitate. \\
\hline $\begin{array}{l}\text { Potassit: } \\
\text { C'hlor: } \\
\text { Chlorin }\end{array}$ & $\begin{array}{c}\text { Abundant } \\
\text { precipitate. }\end{array}$ & ............ & $\begin{array}{c}\text { Same as colchicin. } \\
\text { A precipitate, }\end{array}$ & \\
\hline Picric A o & $\begin{array}{c}\text { Potassium ferri- } \\
\text { cyanid is reduced, } \\
\mathrm{H}_{2} \mathrm{SO}_{4}+\mathrm{BaH}_{2} \mathrm{O}_{2}= \\
\text { brick red; on } \\
\text { warming, violet. }\end{array}$ & .......... & $\begin{array}{c}\text { Zeisel's reaction }=0, \\
\text { colchicin, green. } \\
\text { Ferric salts=blue. } \\
\text { Irillon's reagent } \\
\text { showed presence of } \\
\text { pepton. }\end{array}$ & ............ \\
\hline $\begin{array}{l}\text { Physiol, produces } \\
\text { Action of heart, } \\
\text { is, death. }\end{array}$ & Non-poisonous. & Nor-poisonous. & Non-poisonous. & $\begin{array}{l}\text { Intensely } \\
\text { poisonous. }\end{array}$ \\
\hline
\end{tabular}


Table II.-Ptomains in Toxicological Examinations.

Note.-The Greater Part of this Table has been Taken Direct from Gräbner's Inaugural Dissertation.

\begin{tabular}{|c|c|c|c|c|c|c|c|c|c|c|c|}
\hline \multirow[b]{2}{*}{$\begin{array}{l}\text { Substauce ex- } \\
\text { atnined. }\end{array}$} & \multirow{2}{*}{$\begin{array}{l}\text { Rärsch } \\
\text { and Fasshender. } \\
\begin{array}{l}\text { Iiver of decompos- } \\
\text { ing eadaver and of } \\
\text { fresh liver of ox. }\end{array}\end{array}$} & \multirow{2}{*}{ 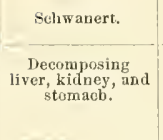 } & \multirow{2}{*}{$\begin{array}{c}\text { Liebermasn. } \\
\begin{array}{c}\text { Putrefying } \\
\text { stomaah and } \\
\text { contents. }\end{array}\end{array}$} & \multirow{2}{*}{ 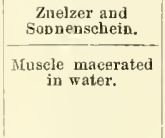 } & \multirow{2}{*}{ 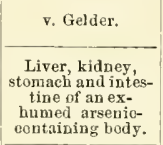 } & \multicolumn{4}{|c|}{ Brouardel and Boutmy. } & \multirow{2}{*}{$\begin{array}{c}\begin{array}{c}\text { Baumert } \\
\text { (Liebermann). }\end{array} \\
\end{array}$} & \multirow{2}{*}{$\begin{array}{c}\text { Otto. } \\
\end{array}$} \\
\hline & & & & & & $\begin{array}{c}\text { Cadaver. } \\
\text { Death by asphy xia. }\end{array}$ & $\begin{array}{l}\text { Cadaver. } \\
\text { Death by hydro- } \\
\text { cy anic acid. }\end{array}$ & $\begin{array}{l}\text { Cadaver. } \\
\text { in water } 18 \text { months. }\end{array}$ & $\begin{array}{l}\text { Parts of } \\
\text { putrefying goose } \\
\text { and cadiver. }\end{array}$ & & \\
\hline $\begin{array}{l}\text { Solvent. } \\
\text { Residue. }\end{array}$ & $\begin{array}{l}\text { Ether (acid and } \\
\text { alkaline). } \\
\text { Amoruhous, not } \\
\text { bitter. }\end{array}$ & $\begin{array}{l}\text { Ether (alkalue). } \\
\text { Liquid, volatile; } \\
\text { rejulsive taste. }\end{array}$ & $\begin{array}{l}\text { Ether (acid and } \\
\text { alkaline). } \\
\text { Resinons, brown- } \\
\text { ish, soluble in } \\
\text { water; acid taste. }\end{array}$ & $\begin{array}{c}\text { Ether (alkaline). } \\
\text { Greasy brownish } \\
\text { mass, with erystals. }\end{array}$ & Brown extract. & …... & $\ldots+\ldots$ & Alkaliue. & $\begin{array}{l}\text { Alkaliot, volatile } \\
\text { liquid; odor that } \\
\text { of urine of mice. }\end{array}$ & $\begin{array}{c}\text { Ether (acid). } \\
\text { Yellow, amorphous; } \\
\text { taste sharp, bittex. }\end{array}$ & $\begin{array}{l}\text { Petroloum-ether } \\
\text { (alkaline). } \\
\text { Bright yellow oil. }\end{array}$ \\
\hline $\begin{array}{l}\text { Tannic Acid. } \\
\text { foold fhlorid. }\end{array}$ & White precipitate. & $\begin{array}{c}\text { Graduai } \\
\text { cloudiness, } \\
\text { Bluish-yellow }\end{array}$ & White precipitate. & $\begin{array}{l}\text { White precipitate. } \\
\text { Yellow crystalline }\end{array}$ & $\begin{array}{l}\text { White preoipitate. } \\
\text { Brown precipitate. }\end{array}$ & $\begin{array}{l}\text { White pre } \\
\text { Yellow pre }\end{array}$ & recipitate. & ......... & $\begin{array}{l}\text { White precipitate. } \\
\text { Violet precipitute. }\end{array}$ & $\begin{array}{l}\text { Same as coleldicin; } \\
\text { soluble iu nleobolool. } \\
\text { Same as colchioin. }\end{array}$ & A precipitate. \\
\hline Plativum Chlorid. & $\begin{array}{l}\text { Yellowisi precipi- } \\
\text { tate. }\end{array}$ & $\begin{array}{l}\text { Dirty yellow six- } \\
\text { sided stars. }\end{array}$ & $\ldots \ldots \ldots . .$. & $\begin{array}{l}\text { precpiphere } \\
\text { Brownish-yeliow } \\
\text { precipitate. }\end{array}$ & Id. & & & $\ldots \ldots \ldots$ & $\ldots \ldots \ldots .$. & $\begin{array}{l}\text { A precipitate, } \\
\text { colohien }-0 .\end{array}$ & It. \\
\hline $\begin{array}{l}\text { Iodhn in Potas- } \\
\text { siumm Jodid. } \\
\text { lirülude's Reagent. }\end{array}$ & $\begin{array}{l}\text { Yellowish-brown } \\
\text { precipitate. } \\
\text { ….... }\end{array}$ & $\begin{array}{l}\text { Clear brown pre- } \\
\text { cipitate. } \\
\text { spendid bue, } \\
\text { later green. }\end{array}$ & $\begin{array}{l}\text { Yellow to dark } \\
\text { hrown. }\end{array}$ & $\begin{array}{l}\text { Kermes-brown } \\
\text { precipitate. } \\
\text {......... }\end{array}$ & Id. & Kermes-brown & itat & & $\begin{array}{l}\text { Kermes-brown } \\
\text { precipitite. } \\
\text { Orauge-red. }\end{array}$ & Same as colehiein. & ...... \\
\hline $\begin{array}{l}\text { Ploosplomolybdie } \\
\text { Aeld. } \\
\text { Sulphuric Aeid. }\end{array}$ & $\begin{array}{l}\text { Yellow precipitate, } \\
\text { on warming green; } \\
\text { with N11,011, blue. }\end{array}$ & $\begin{array}{c}\text { Yellow procipitate; } \\
\text { with N N11, } \\
\text { blue. } \\
\text { Dirty brownisb- }\end{array}$ & $\begin{array}{l}\text { Yellow preeipitate. } \\
\text { Colorlesy, the }\end{array}$ & $\begin{array}{c}\text { Heavy flocoulent } \\
\text { precipitate. }\end{array}$ & $\begin{array}{c}\text { Yellow preeiyitate. } \\
\text { On warming }\end{array}$ & $\begin{array}{l}\text { White precipitate. } \\
\text { On warming, violet. }\end{array}$ & In the cold, & ……... & $\ldots+\ldots \ldots$. & $\begin{array}{c}\text { Same as colohicin, } \\
\text { blue with } \mathrm{NH}_{4} \mathrm{Orr} \\
\text { Yellow color. }\end{array}$ & ......... \\
\hline $\begin{array}{l}\text { Sulphuric Acic } \\
\text { auld Potissinum } \\
\text { Bhehromate. }\end{array}$ & ... & $\begin{array}{l}\text { yellow, unclianged } \\
\text { on warming. } \\
\text { Reddish-brown, } \\
\text { then grass-green. }\end{array}$ & $\begin{array}{l}\text { slight reddish-violet } \\
\text { color. } \\
\text { …. }\end{array}$ & $\ldots \ldots \ldots . .$. & becomes yellow. & Intense & $\begin{array}{l}\text { browuish-violet. } \\
\text { green. }\end{array}$ & warming, violet. & $\begin{array}{l}\text { No odor of butyric } \\
\text { neid. }\end{array}$ & ............ & ........... \\
\hline Nitrie Seid. & .. & Yellow. & $\begin{array}{l}\text { Yellow spots on } \\
\text { evaporation. }\end{array}$ & ......... & ld. & Golden-y & -yellow. & & Colorloge & $\begin{array}{l}\text { Dark-yellow oolor, } \\
\text { eoncentrated 11NO } \\
\text { =curminie red, } \\
\text { which with water } \\
\text { gives yollow. }\end{array}$ & A necinita to \\
\hline $\begin{array}{l}\text { Hydrocthloric } \\
\text { Acid. } \\
\text { Mereurle thitorut. }\end{array}$ & .. & $\begin{array}{c}\text { Deliquesceut } \\
\text { white needles, } \\
\text { White crystalline }\end{array}$ & White cloudiuess. & Curily white pre- & White precipitate. & $\cdots$ & . & $\begin{array}{l}\text { Cherry-red on } \\
\text { lifating. }\end{array}$ & Colorless. & & A prooipitate. \\
\hline $\begin{array}{l}\text { Polassium moreuric } \\
\text { (chlisritl. } \\
\text { Chlorin wuter. }\end{array}$ & & $\begin{array}{l}\text { precipitate. } \\
\text { Dirty white pre- } \\
\text { cipitate. }\end{array}$ & Heavy white pre- & $\begin{array}{r}\text { oipitate. } \\
\ldots \ldots \ldots . . . \\
\ldots \ldots \ldots . . .\end{array}$ & $\begin{array}{l}\text { Id. } \\
\text { Id. }\end{array}$ & Yellow pre & ecipitate. & $\begin{array}{c}\text { Abundant } \\
\text { precipitate. } \\
\text {.......... }\end{array}$ & ….. & $\begin{array}{c}\text { Same as eolchioin. } \\
\text { A preoipitate, }\end{array}$ & $\ldots \ldots \ldots$ \\
\hline Picric $A$ cid. & & = & & $\ldots \ldots$. & ..... & \begin{tabular}{|} 
Abundant yellow \\
precipitate. Iodio \\
aoid is redueed. \\
Silver nitrate, white \\
precipitate with \\
reduction of \\
silver. Ferric \\
chlorid
\end{tabular} & 0 & $\begin{array}{c}\text { Potassium ferri- } \\
\text { oyanid is reduced, } \\
\mathrm{II}_{2} \mathrm{SO}_{4}+\mathrm{BuH}_{4} \mathrm{O}_{2} \\
\text { brick red ou } \\
\text { warming, violet. }\end{array}$ & & $\begin{array}{c}\text { colchicin }=0 . \\
\text { Zeisel's reaction=0, } \\
\text { colechiein, green, } \\
\text { Forrio salts =blue. } \\
\text { Millon's reagent } \\
\text { showed presenee of } \\
\text { popton. }\end{array}$ & ..... \\
\hline $\begin{array}{l}\text { Physlological } \\
\text { Aotion. }\end{array}$ & & & $\begin{array}{l}\text { When fed } \\
\text { to pigeons no } \\
\text { etject. }\end{array}$ & $\begin{array}{l}\text { Causes mydriasis } \\
\text { and increase in the } \\
\text { rate of heart-bent. }\end{array}$ & & & $\begin{array}{l}\text { In frogs, produces } \\
\text { slowing, of heart, } \\
\text { paralysis, death. }\end{array}$ & Nod-poisonous. & $\mathrm{N}_{0 \mathrm{H}-\mathrm{p} o \mathrm{is} \text { sornous. }}$ & Nou-poisonous, & $\begin{array}{l}\text { Jutensely } \\
\text { poisouous. }\end{array}$ \\
\hline
\end{tabular}




\section{Table III.—Reactions of Selmi's Ptomaïns.}

\begin{tabular}{|c|c|c|c|c|}
\hline Solvent. & $\begin{array}{c}\text { Ether } \\
\text { (acid). } \\
\text { } \iota .\end{array}$ & $\begin{array}{c}\underset{\text { Ether }}{\text { (alkaline) }} \\
b .\end{array}$ & $\begin{array}{c}\text { Chloroform } \\
\text { (alkaliue). } \\
c .\end{array}$ & $\begin{array}{l}\text { Amyl dlcohol } \\
\text { (alkaline). } \\
d .\end{array}$ \\
\hline Tannic Acid. & Precipitate. & & & \\
\hline Iodin in $\mathrm{Hydriodic} \mathrm{Acld.}$ & & $\begin{array}{l}\text { Precipitates in } \\
\text { two crystalline } \\
\text { forms. }\end{array}$ & $\begin{array}{l}\text { Crystalline } \\
\text { precipitate. }\end{array}$ & $\begin{array}{l}\text { Reddish-brown } \\
\text { precipitate with } \\
\text { deliquescing } \\
\text { crystals. }\end{array}$ \\
\hline Gold Chlorid. & Id. & A precipitate. & ....... & $\begin{array}{l}\text { Yellowish pre- } \\
\text { cipitate. }\end{array}$ \\
\hline Mercuric Chlorid. & Id. & $\begin{array}{l}\text { White } \\
\text { precipitate. }\end{array}$ & $\cdots \cdots$ & $\begin{array}{l}\text { Whitisb pre- } \\
\text { cipitate. }\end{array}$ \\
\hline Phosphomolybdic Acid. & (....... & $\begin{array}{l}\text { Violet or dark } \\
\text { blue. }\end{array}$ & ...... & n..... \\
\hline $\begin{array}{l}\text { Concentrated Sulphuric } \\
\text { Acid (warmed). }\end{array}$ & Violet red. & $\begin{array}{l}\text { Violet or yel- } \\
\text { lowish-brown. }\end{array}$ & $\begin{array}{l}\text { Reddisb } \\
\text { coloration. }\end{array}$ & $\cdots \cdots$ \\
\hline $\begin{array}{l}\text { Sulphuric Acid and Potas- } \\
\text { sium Dichromate. }\end{array}$ & ....... & $\begin{array}{l}\text { Gradually } \\
\text { passes into } \\
\text { green. }\end{array}$ & (0) & ....... \\
\hline $\begin{array}{l}\text { Iodic Acid. } \\
\text { Iodic Acid + Sulphuric }\end{array}$ & $\begin{array}{l}\text { Peductiou. } \\
\text { Rose- }\end{array}$ & Reduction. & Reduction. & Reduction. \\
\hline Acid + Solla. & colored salt. & & .......... & 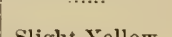 \\
\hline Nitric Acid. & $\begin{array}{l}\text { Yellow (on } \\
\text { warming }\end{array}$ & .. & $\cdots \cdots$ & Slight Yellow. \\
\hline $\begin{array}{l}\text { Hydrochloric Acid + Sul- } \\
\text { phuric Acid. }\end{array}$ & $\begin{array}{l}\text { stronger). } \\
\text { Slight } \\
\text { violet. }\end{array}$ & $\begin{array}{l}\text { Violet color } \\
\text { with odor }\end{array}$ & $\ldots$ & ...... \\
\hline Sulphnric Acid + Bromin. & ...... & $\begin{array}{l}\text { of hawthorn. } \\
\text { Red } \\
\text { (permanent). }\end{array}$ & $\begin{array}{l}\text { As with } \\
\text { sulphuric } \\
\text { acid. }\end{array}$ & - \\
\hline Frōhde's Reagent. & .. & $\begin{array}{l}\text { Yellowish- } \\
\text { brown to violet } \\
\text { or yellow. }\end{array}$ & Fied. & (........ \\
\hline Ferric Chlorid. & ...... & Cloudiness. & $\cdots \cdot$ & $\begin{array}{l}\text { Rose-colored } \\
\text { precipitate. }\end{array}$ \\
\hline Hydrochloric Acid. & .... & $\begin{array}{l}\text { Slight violet, } \\
\text { more distinct } \\
\text { on standing. }\end{array}$ & & -.... \\
\hline Phosphoric Acid (diluted). & ....... & $\begin{array}{l}\text { On warming, } \\
\text { violet color. }\end{array}$ & -. & $\cdots \cdots$ \\
\hline Sulphuric Acid (diluted). & $\begin{array}{l}\text { Violet pass- } \\
\text { ing into yel- } \\
\text { low; odor of } \\
\text { hawthorn. }\end{array}$ & $\begin{array}{l}\text { Violet, if } \\
\text { stirred becomes } \\
\text { black. }\end{array}$ & & $\cdots$ \\
\hline $\begin{array}{l}\text { Potassium Platinic Cyanid. } \\
\text { Potassium Argentic } \\
\text { Cyanid. } \\
\text { Platinum Cblorid. } \\
\text { Potassium Dichromate. }\end{array}$ & 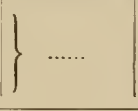 & $\left\{\begin{array}{l}\text { Occasionally } \\
\text { precipitates. }\end{array}\right.$ & \} & $\ldots \ldots$ \\
\hline
\end{tabular}



Table of Ptomä̈s.-Continued.

\begin{tabular}{|c|c|c|c|}
\hline Formula. & Name. & Discoverer. & $\begin{array}{l}\text { Physiological } \\
\text { Action. }\end{array}$ \\
\hline 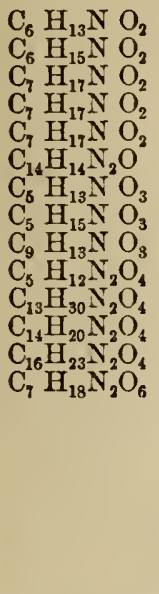 & $\begin{array}{l}\text { Unnamed. } \\
\text { Mytilotoxin. } \\
\text { Gadinin. } \\
\text { Typhotoxin. } \\
\text { Unnamed. } \\
\text { Pyocyanin. } \\
\text { Betain. } \\
\text { Muscarin. } \\
\text { Morrhuic acid. } \\
\text { Unnamed. } \\
\text { Tetanin. } \\
\text { Unnamed. } \\
\quad \text { " } \\
\quad \text { " } \\
\text { Tyrotoxicon. } \\
\text { Mydalein. } \\
\text { Spasmotoxin. } \\
\text { A diamin (?). } \\
\text { Peptotoxin. } \\
\text { Phlogosin. }\end{array}$ & $\begin{array}{l}\text { Brieger, } 1888 . \\
\text { Brieger. } \\
\text { "، } \\
\text { "، } \\
\text { Ledderhose. } \\
\text { Brieger. } \\
\text { " } \\
\text { Gautier \& Mourgues } \\
\text { Pouchet. } \\
\text { Brieger. } \\
\text { Guareschi. } \\
\text { Lepierre. } \\
\text { Pouchet. } \\
\text { " } \\
\text { Vaughan. } \\
\text { Brieger. } \\
\text { " } \\
\text { " } \\
\text { Leber. }\end{array}$ & $\begin{array}{l}\text { Non-poisonous. } \\
\text { Poisonous. } \\
\text { " } \\
\text { " } \\
\text { Non-poisonous. } \\
\text { Poisonous. } \\
\text { Poisonous. } \\
\text { " } \\
\text { Poisonous. } \\
" \\
" \\
" \\
" \\
" \\
\text { Inflammatory. }\end{array}$ \\
\hline
\end{tabular}

1 Only those bases are here denoted as poisonous which possess a decided toxicity. For Griffiths' bases, see page 261 . 


\section{CHAP'TER XV.}

\section{CHEMISTRY OF THE LEUCOMAÏNS.}

UNDER this head are classed those basic substances which exist preformed in the proteids or other constituents of the body or are produced as products of tissue metabolism. The proteid molecule is usually the parent of the ptomaïns and also of the leucomains. The distinction between these two groups is therefore necessarily imperfect and unsatisfactory. In the one case bacteria are at work and split up the proteid, giving rise to ptomaïns. The latter are essentially cleavage products and the differences which exist amoug these bases are to be sought largely in the peculiar composition of the proteid acted upon and perhaps to less extent in the individual germ which is the active agent of destruction. The fact that few, if any, of the ptomaïns can be shown to be synthetic products indicates that the bacteria or their enzymes act largely as hydrolytic agents.

Obviously, the living animal cell, through its enzyme, may produce the same or similar basic products which, however, being non-bacterial in origin are designated as leucomaïns. Furthermore, these basic substances may be liberated by the direct action of hydrolytic agents other than enzymes. Thus, to illustrate, the nuclein molecule when acted upon by bacteria yields the xanthin bases. The latter are formed in the living animal and plant in tissue metabolism and also result by the action of dilute mineral acids. The hexon bases, while they have not been as yet found to be bacterial products, will undoubtedly be shown to be such, for the reason that they are formed by the enzymes of yeast, of germinating plants, of pancreas and of the liver, and because they are easily split off from proteids by the action of acids. Again lecithin, the parent substance of cholin, is widely distributed in plants and animals. It yields cholin when acted upon by bacteria or by acids, or when it undergoes metabolism in the living plant or animal. The same is true for the well known ptomaïns, putrescin and cadaverin, which according to Lawrow are formed, undoubtedly from lysin and arginin, in the sterile autodigestion of pigs' stomachs.

The first attempt at the systematic study and generalization of these basic substances was made by Gautier, who applied to them the name leucomaïns, a term derived from the Greek, $\lambda \varepsilon^{\prime} \gamma \omega \mu \alpha$, signifying white of eggs. Under this name he included all those basic substances which are formed in animal tissues during life, in contradistinction to the ptomaïns or basic products of putrefaction. Many 
of these bases exist preformed in plants or can be readily prepared from them.

Thus vegetable tissues are known to contain not only what are ordinarily designated as ptomaïns, such as cholin, but also leucomains, of the hexon and purin group. Indeed, in this latter group must be placed, on account of their relation to xanthin, those well defined alkaloidal bases, caffein, theophyllin and theobromin. Not only are the representatives of these two divisions of basic substances common to both kingdoms, but their parent bodies, lecithin, nuclein, and proteids occur in both, and hence give rise to the same bases on decomposition.

Gautier believed that the leucomains are being formed continuously and incessantly in the animal tissues side by side with the formation of urea and carbonic acid, and at the expense of the nitrogenous elements. It is quite probable, as Kossel has pointed out, that some of these products are in themselves antecedents of endproducts of metabolism. This is unquestionably true of the amido group, which exists in the adenin and guanin molecules, and through vital or putrefactive processes is split off, giving rise to ammonia which in turn serves to form urea. More than that, the researches of the past few years have shown that the purin and hexon bases, while primary cleavage bodies, are largely broken down into more simple metabolic products. Thus, the uric acid which is formed in certain organs in the body of mammals is chiefly, if not wholly, derived by the oxidation of the purin bases or of their antecedent. The kidneys, muscles and even the liver itself may destroy uric acid, and for that reason the amount of uric acid excreted does not indicate the amount that is formed (Wiener). The cleavage of uric acid into allantoin, oxalic acid and urea is well known, and Minkowski has recently shown that allantoin is also formed after the administration of purin bases, as well as of thymus glands.

Bouchard endeavored to explain the presence of leucomains in the urine, by supposing that they were originally formed in the intestinal tract, from which they were absorbed into the system, to be subsequently eliminated by the kidneys. This view has also been brought forward by Schär (1886) who held that these bases may be formed by putrefactive changes in the intestinal tract, in which case they are absorbed into the circulatory system, whence they may be partly eliminated by the kidneys or may be partly deposited in the tissues themselves.

The views of Bouchard and Schär have, to a certain extent, been confirmed by the investigations of Udránszky and Baumann, who showed that the well known ptomaïns cadaverin and putrescin occur in the urine in cystinuria, and are formed by putrefactive changes induced in the intestinal tract, probably by specific microörganisms. Under this same head fall the observations of Wolkow and Bau- 
mann, that alkapton is produced from tyrosin by similar changes in the intestines. The production of intestinal products, their absorption and excretion by the kidneys, is likewise seen in such well known compounds as phenol, indol, skatol, etc. The origin of the true leucomaïns cannot, however, be accounted for in this manner, for they are indissolubly connected with the metabolism of the cell itself, and are, therefore, formed in the tissues and organs proper. As such they are endogenous whereas the intestinal products are chiefly the result of bacterial action and are therefore of exogenous origin.

The leucomains have been credited by many as playing the chiet rôle in auto-intoxications. It should be noted, however, that most of the basic bodies which have been studied up to the present are far from being poisonous. The antecedents of these bases, however, such as the nucleinic acids and more especially the histons and protamins exert a pronounced toxic action and hence may play a most important part in auto-intoxications. Of course, it must be admitted that there may be generated within the body toxic leucomaïns which, as yet unknown, normally undergo prompt destruction. The fact that amino derivatives of purin and pyrimidin are highly toxic is deserving of attention.

The known sources of leucomaïns are the nucleins of the nuclei and the proteids of the protoplasm of the cells. The former gives rise to the purin and pyrimidin bases whereas the latter generate the hexon bases. In addition to these there is a fairly large number of substances, as yet but poorly studied, which remain to be accounted for. Cholin and allied bodies may be looked upon as leucomains derived from lecithin. They have been described in the preceding chapter.

The leucomaïns may be divided into the following groups: (1) the purin or uric acid group ; (2) the pyrimidin group ; (3) the hexon bases; (4) the creatinin group.

The first of these divisions contains a number of well known bases which are derivatives of purin and for that reason are closely allied to uric acid.

PURIN,
ADENIN,
HYPOXANTHIN,
GUANIN,
METHYL GUANIN,
XANTHIN,
(URIC ACID,
1-METHYL XANTHIN,
3-METHYL XANTHIN,
7-METHYL XANTHIN,
1-7 DI-METHYL XANTHIN,
1-3 DI-METHYL XANTHIN,
3-7 DI-METHYL XANTHIN,
1-3-7 TRI-METHYL XANTHIN,
CARNIN,
CARNOSIN,
CYTOSIN,

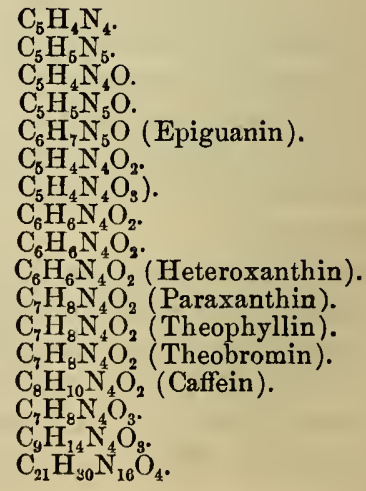


The pyrimidin group may be considered as antecedents or perhaps more correctly as cleavage products of the purin bodies. It is represented by the following:

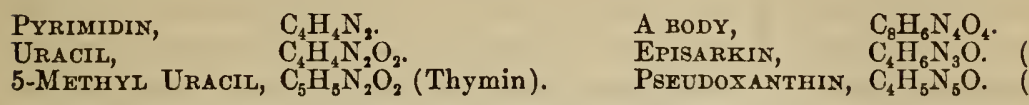

The hexon bases proper are only three in number. To these may be appended a lower homologue, two amines and a related acid which may be looked upon as cleavage products of the hexons. The third group then will include :
ARginin, $\mathrm{C}_{6} \mathrm{H}_{14} \mathrm{~N}_{4} \mathrm{O}_{2}$
Histidin, $\mathrm{C}_{6} \mathrm{H}_{9} \mathrm{~N}_{3} \mathrm{O}_{2}$
Lysin, $\mathrm{C}_{6} \mathrm{H}_{14} \mathrm{~N}_{2} \mathrm{O}_{2}$
ORNITHIN, $\mathrm{C}_{5} \mathrm{H}_{12} \mathrm{~N}_{2} \mathrm{O}_{2}$.
$\begin{array}{ll}\text { Pyrrolidin Carbonic AcId, } & \mathrm{C}_{5} \mathrm{H}_{2} \mathrm{NO}_{2} . \\ \text { Gerontin, } & \mathrm{C}_{5} \mathrm{H}_{24} \mathrm{~N}_{2} \text {. } \\ \text { SPERMin, } & \mathrm{C}_{2} \mathrm{H}_{5} \mathrm{~N} \text { (?) }\end{array}$
METHYL QUINOLIN,
$\mathrm{C}_{2} \mathrm{H}_{5} \mathrm{~N}$ (?)

The members of the fourth group have all been discovered by Gautier and by him are regarded as allied to creatin and creatinin. These two substances, especially the latter, have been hitherto regarded as strongly basic in character, but Salkowski has shown that creatinin, when perfectly pure, possesses little or no alkaline reaction, and, moreover, does not combine with acids. In structure and properties it approximates the pyrimidin bodies. The bases in this group are :

\begin{tabular}{|c|c|}
\hline $\begin{array}{l}\text { (CREATININ, } \\
\text { /CREATIN, } \\
\text { CRUSO-CREATININ, } \\
\text { XANTHO-CREATININ, } \\
\text { AMPHI-CREATIN, } \\
\text { BASE, } \\
\text { BASE,; }\end{array}$ & $\begin{array}{l}\left.\mathrm{C}_{4} \mathrm{H}_{7} \mathrm{~N}_{3} \mathrm{O}\right) \\
\left.\mathrm{C}_{4} \mathrm{H}_{9} \mathrm{~N}_{3} \mathrm{O}_{2}\right) \\
\mathrm{C}_{5} \mathrm{H}_{8} \mathrm{~N}_{4} \mathrm{O} \\
\mathrm{C}_{5} \mathrm{H}_{10} \mathrm{~N}_{4} \mathrm{O} \\
\mathrm{C}_{9} \mathrm{H}_{19} \mathrm{~N}_{7} \mathrm{O}_{4} \\
\mathrm{C}_{11} \mathrm{H}_{24} \mathrm{~N}_{10} \mathrm{O}_{5} \\
\mathrm{C}_{12} \mathrm{H}_{25} \mathrm{~N}_{11} \mathrm{O}_{5}\end{array}$ \\
\hline
\end{tabular}

Besides these two general classes of leucomains, there may be made a fifth class of undetermined leucomains, embracing those bases which have been observed, but studied more or less incompletely, in the various physiological secretions of the body.

\section{PURIN BASES.}

The substances included under this head were formerly spoken of as xanthin bases because of their relation to xanthin, the earliest known member of the group. Subsequently, the term nuclein bases was employed to designate the original four xanthin bases, inasmuch as they are derived from the nucleins. The latter on decomposition with acids yields one or more of these basic products. Kossel and Krüger, in 1894, suggested a new term based upon the fact that the xanthin bases like uric acid contain alloxan and urea groups. They designated therefore these as alloxuric bases. On the other hand the expression "alloxuric bodies" was made to include uric acid as well as the xanthin bases. 
The studies of Fischer in 1884 on uric acid led him to consider this substance as a derivative of a hypothetical body, $\mathrm{C}_{5} \mathrm{H}_{4} \mathrm{~N}_{4}$, to which he gave the name purin. The remarkable researches of this investigator during the past few years have shown that the xanthin bases are actually related to uric acid and that they also are derivatives of purin. This parent substance of uric acid and of the xanthin bases was not prepared until 1898, although as stated, its existence was pointed out fifteen years before. The term purin bodies therefore includes purin and all of its derivatives. The purin bodies formed in the animal organism and appearing in the urine are uric acid and the several xanthin bases. The expression purin bases implies the same as the older terms xanthin, nuclein or alloxuric bases.

Before considering the individual purin bases it is desirable to point out the main facts bearing upon the constitution or structure of the members of this group. A thorough understanding of the structural relationship of these several bodies is necessary in order to appreciate the rôle which they play in tissue metabolism. The waste product uric acid differs from xanthin in that it contains one atom of oxygen more than does the latter. Xanthin in turn differs in the same way from hypoxanthin. For some time the belief has prevailed that the nuclei of cells contain the physiologically important xanthin bases. These bases do not exist free but rather in combination with proteid substances forming the so-called nucleinic acids, or the more complex nucleins. When the nucleins undergo metabolism the purin bases are set free, suffer more or less oxidation and leave the body partly as uric acid and partly as cleavage products such as the pyrimidin bodies, creatinin, allantoin, urea, oxalic acid, etc. These successive changes may be rendered apparent by comparing the formulæ of these bodies-

$\begin{array}{ll}\text { Purin, } & \mathrm{C}_{5} \mathrm{H}_{4} \mathrm{~N}_{4} . \\ \text { Hypoxanthin, } & \mathrm{C}_{5} \mathrm{H}_{4} \mathrm{~N}_{4} \mathrm{O} . \\ \text { Xanthin, } & \mathrm{C}_{5} \mathrm{H}_{4} \mathrm{~N}_{4} \mathrm{O}_{2} . \\ \text { Uric ACID, } & \mathrm{C}_{5} \mathrm{H}_{4} \mathrm{~N}_{4} \mathrm{O}_{8} .\end{array}$

Although these four substances form a homologous group it does not follow that uric acid can be readily prepared by oxidation from either of the preceding or that vice versa it can be reduced to these bases. The statement made by Strecker that hypoxanthin by the action of fuming nitric acid yields a nitro-product, which on reduction gives xanthin, was taken to indicate that hypoxanthin possessed a constitution similar to that of xanthin. Kossel, however, showed in 1882 that this conversion of hypoxanthin into xanthin did not take place, and in this he was confirmed by Fischer in 1884. In the same way the observation of Rheineck, reported by Strecker, that uric acid is converted by sodium amalgam into xanthin, and this still further reduced to hypoxanthin, has been shown to be incorrect 
by Fischer, who failed to change xanthin into uric acid or uric acid into xanthin. While therefore uric acid, xanthin, and hypoxanthin contain three, two, and one atoms of oxygen, respectively, and are structurally closely allied, nevertheless thus far it has not been possible to convert directly any one of those compounds into the others.

Only very recently Sundwik has attempted the reduction of uric acid by boiling it with sodium hydrate and chloroform. By this procedure he believes that he has prepared xanthin and bypoxanthin, but a confirmation of these results is necessary.

By electrolytic reduction of uric acid Tafel ${ }^{1}$ obtained a substance which he has designated as puron, $\mathrm{C}_{5} \mathrm{H}_{8} \mathrm{~N}_{4} \mathrm{O}_{2}$. This body is neither acid nor basic in character and is therefore a fully saturated compound. It readily changes into an unsaturated isomer-iso-puron. A tetrahydrouric acid, $\mathrm{C}_{5} \mathrm{H}_{8} \mathrm{~N}_{4} \mathrm{O}_{3}$, was also obtained which with baryta gave $\alpha-\beta$ diamino-propionic acid, $\mathrm{C}_{3} \mathrm{H}_{8} \mathrm{~N}_{2} \mathrm{O}_{2}$; and with nitrous acid a base $\mathrm{C}_{4} \mathrm{H}_{7} \mathrm{~N}_{3} \mathrm{O}_{2}$.

The classical investigations of Fischer, however, have shown that indirectly uric acid may be changed to xanthin or to hypoxanthin and these bases in turn by a similar indirect process can be converted into uric acid. Thus, on treating 1-3 di-methyl uric acid with phosphorus pentachlorid Fischer obtained chlor-theophyllin, which on reduction with hydriodic acid gave theophyllin or di-methyl xanthin. By introducing a methyl group into the latter caffein or trimethyl xanthin results. In a similar way caffein may be prepared from 1-3-7 tri-methyl uric acid. Inasmuch as the di- and tri-methyl uric acids can be prepared artificially it follows that the complete synthesis of theophyllin and of caffein was effected by this transformation.

Subsequently (1897) Fischer converted potassium urate by means of phosphorus oxychlorid into an oxy-di-chlor purin and this into tri-chlor purin. The latter by a series of transformations which it is unnecessary to detail was changed into xanthin, hypoxanthin, adenin, guanin, heteroxanthin and paraxanthin. With the exception of methyl xanthin all of the known purin bases found in the urine have been therefore not only made from uric acid but their complete synthesis has also been effected.

The starting point in the consideration of the structure of the purin bodies is uric acid, which is not only the earliest known, but also the most easily obtainable and best studied member of this group. The structure of uric acid as given by Medicus in 1875 was verified and fully established by the studies of Fischer in 1884 . On the other hand, these early investigations of Fischer on the xanthin bases led him to ascribe to these somewhat different formulæ than did Medicus. When, however, Fischer in 1895 succeeded in converting di-methyl uric acid into chlor-theophyllin it was evident

${ }^{1}$ Berichte, 34, 258, 1181 (1901). 
that the xanthin bases possessed essentially the same structure as uric acid. This fact was rendered still more evident when hydroxy-caffein not only was recognized to be a tri-methyl uric acid, but on treatment with methyl iodid was shown to form the tetra-methyl uric acid. Fischer therefore abandoned the formulæ of caffein and xanthin which he proposed in 1883 and accepted those which Medicus had previously suggested.

The formula which Medicus suggested and which shows best the various decompositions of uric acid is that given below (1). The tautomeric form (2) has a great deal in its favor, but is not used. Similar tautomeric formulæ can be deduced for purin, trichlor purin, adenin and other bases.

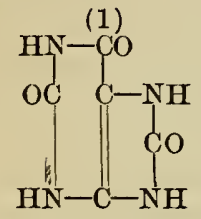

URIC ACID.

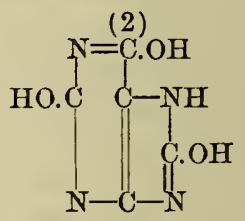

The group $\mathrm{C}_{5} \mathrm{~N}_{4}$ in the above formulæ is designated as the purin nucleus and from it purin itself, $\mathrm{C}_{5} \mathrm{H}_{4} \mathrm{~N}_{4}$, is easily derived. The nomenclature of purin bodies is based upon the order of substitution in the purin nucleus, $\mathrm{C}_{5} \mathrm{~N}_{4}$. Nine positions are possible as shown in the second formula below :
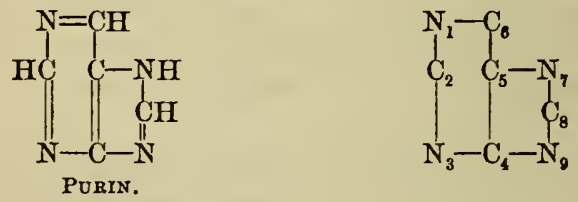

Uric acid becomes 2-6-8 tri-oxypurin. By the introduction of a single methyl group into uric acid a methyl uric acid results. On examining the formula of uric acid it will be seen that theoretically four methyl uric acids are possible. Strange to say there are present six known mono-methyl uric acids; in other words there are two more than are theoretically possible.
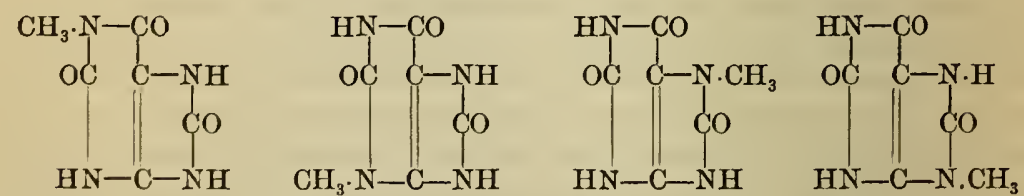

1-Methyl Uric Acid. 3-Methyl Uric Acid. 7-Methyl URIC ACID. 9-Methyl URic Acid.

By substituting two methyl groups into uric acid a di-methyl uric acid results. Six di-methyl uric acids are possible and all are known, of the tri-methyl uric acids four are possible and are like- 
wise all known. One tetra-methyl uric acid is possible and is known but in addition an isomer exists in methoxy-caffein.

Xanthin. - The successful synthesis of xanthin by Fischer (1897) has shown that this interesting base is 2-6-di-oxypurin and as such possesses the formula originally deduced by Medicus. A consideration of the purin formula will show that three di-oxypurins are possible. Xanthin therefore has two isomers, 6-8 and 2-8 dioxypurins, the latter of which is as yet unknown although methyl derivatives have been prepared.<smiles>O=C1NC2NC=NC(=O)C2N1</smiles>

Xanthin.<smiles></smiles>

GUANIN.

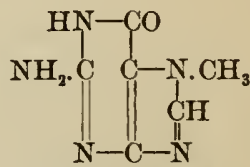

EPIGUANIN OR 7-METHYL GUANIN.

The methyl derivatives of xanthin are of great interest inasmuch as they make up the greater part of the natural purin bases. By replacing a hydrogen atom in either one of the three imido groups 1,3 , or 7 , in xanthin by a methyl group a mono-methyl xanthin results. All three of the possible mono-methyl xanthins are known. The 1-methyl xanthin was discovered in urine by Krüger and Salomon in 1897, but as yet it has not been synthesized. Heteroxanthin, which is 7-methyl xanthin (Krüger and Salomon 1895), was likewise isolated from urine by Salomon in 1884. The 3-methyl xanthin has been isolated from the urine after administration of large doses of caffein.<smiles>Cn1c(=O)[nH]c2ncnc(N)c21</smiles>

1-Methy XaNthin.<smiles>CNC(=O)C1NC(=O)NCN1C</smiles>

2-METHYL XANTHIN.

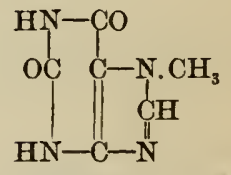

HETEROXANTHIN OR 7-METHYL XANTHIN.

Only three di-methyl xanthins are possible and these have been known to exist in nature for many years. They are theobromin, the active principle of theobroma cacao; theophyllin, which exists in tea, and paraxanthin which occurs in the urine. The relationship which exists between these three isomers is readily seen from the formulæ,<smiles></smiles>

ThEOPHYLLIN OR 1-8 DI-METHYL XANTHIN.

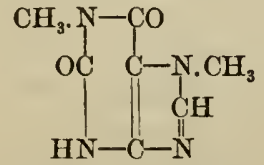

PARAXANTHIN OR Di-METHYL XaNTHIN.<smiles></smiles>

THEOBROMIN OB 
Only one tri-methyl xanthin is possible and that derivative is the active principle of tea and coffee-caffein.

If the tautomeric formula of xanthin, with the imido group transferred from position 7 to 9 , is considered it will be seen that additional derivatives of xanthin are possible, such as a 9 -methyl xanthin, 1-9 and 3-9 di-methyl xanthins and a 1.3.9-tri-methyl xanthin. The latter is an isomer of caffein but unlike the latter possesses but little action upon the muscle (Schmiedeberg, Berichte, 34, 2556).

In 1881 Fischer showed that caffein possessed the formula given below. In the following year he succeeded in changing xanthin into theobromin which in turn can be easily methylated to caffein. In 1897 Fischer successfully synthesized all of the natural xanthins except 1-methyl xanthin. The origin of the di-methyl and monomethyl xanthins was not understood until but recently. Unlike xanthin, these derivatives when found in the urine cannot be traced to tissue metabolism, but on the contrary they result by the splitting off of one or more methyl groups from the caffein or theobromin which is ingested. Thus, when very large doses of caffein are given to a dog all three methyl groups are attacked at once. The one in position 7 is the least firm and hence disappears quite readily. Heteroxanthin or 7-methyl xanthin is therefore present in minimal amounts and for the same reason theophyllin or 1-3 di-methyl xanthin is found in larger amounts than paraxanthin or theobromin. It is of singular interest to note that caffein in the body yields not only paraxanthin but also theophyllin and theobromin which have been known heretofore to exist only in the vegetable kingdom. The purin bases which result from the cleavage of caffein in the body vary according to the animal used. Thus, while feeding caffein to a dog yields chiefly theophyllin (1-3) and 3-methyl xanthin, in a rabbit it yields chiefly paraxanthin (1-7), 1-methyl xanthin and heteroxanthin (7).

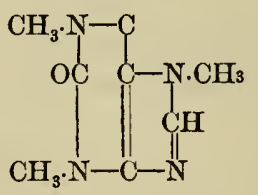

CATFETN.

Guanin.-The fact that guanin is readily changed into xanthin shows that the two bases are closely related. Indeed, the relation is the same as that between adenin and hypoxanthin. By decomposition with concentrated hydrochloric acid Wulff obtained the same products as are given by xanthin. On oxidation, however, with chlorin neither Strecker nor Fischer were able to obtain alloxan and urea, but instead obtained parabanic acid, guanidin, and carbonic acid. A guanidin residue is contained in the molecule in place of the urea residue in xanthin. A striking difference between 
xanthin and guanin is seen in the difficulty of preparing alkyl derivatives of guanin, whereas with xanthin the result is easily obtained. Fischer and Reese failed in preparing alkyl derivatives, and Wulff succeeded only with ethyl guanin. Recently, however, Fischer lias succeeded in effecting the synthesis of two methyl derivatives and of guanin itself. The structural formulæ, thus deduced, for guanin and epiguanin are given on p. 339. It will be seen that guanin is 2 -amino-6-oxypurin, while epiguanin is 7 -methyl guanin.

Two of the five possible isomers of guanin have been prepared synthetically by Fischer. One of these, 6-amino-2-oxypurin, very closely resembles guanin, so much so that it can be easily mistaken for this base.

Hypoxanthin.-This is 6-oxypurin and inasmuch as it can be readily prepared from adenin by the action of nitrous acid, it follows that the latter is 6-aminopurin. Of the four possible formulæ for adenin Fischer selects the one given below. Two isomers of adenin are possible.

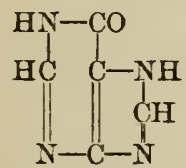

MrPOXANTHIN.

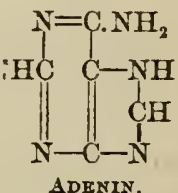

ADENIN.

It should be remembered that there are isomers of both of these bases and that tautomeric forms may exist as in the case of uric acid.

The relation of adenin and hypoxanthin to uric acid and xanthin was clearly shown by Krüger in 1891 and 1893, although the structure of these bases was not fixed by Fischer until 1897. Thus, the bromin derivatives of adenin and hypoxanthin correspond to those of xanthin, guanin and caffein, and on oxidation with chlorin yield alloxan and urea. The bromiu derivatives of hypoxanthin, adenin, xanthin, guanin, and caffein result by the substitution of bromin in the $\mathrm{CH}$ group of xanthin, $i$. e., in position 8 . The analogy to the other members of the uric acid group is seen from the following equations:

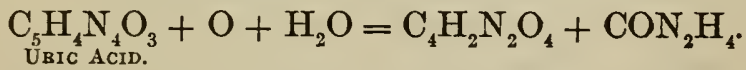

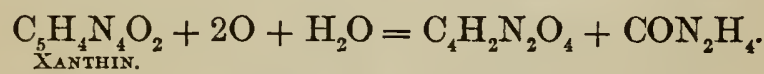

$$
\begin{aligned}
& \mathrm{C}_{5} \mathrm{H}_{4} \mathrm{~N}_{4} \mathrm{O}+3 \mathrm{O}+\mathrm{H}_{2} \mathrm{O}=\mathrm{C}_{4} \mathrm{H}_{2} \mathrm{~N}_{2} \mathrm{O}_{4}+\mathrm{CON}_{2} \mathrm{H}_{4} \text {. } \\
& \underset{\text { GUANIN. }}{\mathrm{C}_{5} \mathrm{H}_{5} \mathrm{~N}_{5} \mathrm{O}}+3 \mathrm{O}+\mathrm{H}_{2} \mathrm{O}=\underset{\text { PABABANIC ACID. }}{\mathrm{C}_{3} \mathrm{H}_{2} \mathrm{~N}_{2} \mathrm{O}_{3}}+\underset{\text { GUANIDIN. }}{\mathrm{CH}} \mathrm{NH}_{2} \mathrm{H}_{4}+\mathrm{CO}_{2} \text {. } \\
& \underset{\text { ADENIN. }}{\mathrm{C}_{5} \mathrm{H}_{5} \mathrm{~N}}+3 \mathrm{O}+2 \mathrm{H}_{2} \mathrm{O}=\mathrm{C}_{4} \mathrm{H}_{2} \mathrm{~N}_{2} \mathrm{O}_{4}+\mathrm{CON}_{2} \mathrm{H}_{4}+\mathrm{NH}_{3} \text {. (?) }
\end{aligned}
$$


The equations for adenin and hypoxanthin are not fully established, but the bromin compounds are decomposed into alloxan. In addition to this urea and oxalic acid are formed, both of which may be derived from alloxan by decomposition, though some of the urea may be independent of the breaking down of the alloxan group.

Again, adenin and hypoxanthin on decomposition with concentrated hydrochloric acid yield the same products (glycocoll, ammonia, formic and carbonic acids) as xanthin, guanin, etc. This decomposition is best seen from the following equations :

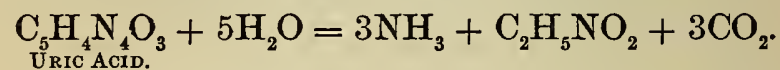

$$
\begin{aligned}
& \mathrm{C}_{5} \mathrm{H}_{4} \mathrm{~N}_{4} \mathrm{O}_{2}+6 \mathrm{H}_{2} \mathrm{O}=3 \mathrm{NH}_{3}+\mathrm{C}_{2} \mathrm{H}_{6} \mathrm{NO}_{2}+2 \mathrm{CO}_{2}+\mathrm{CH}_{2} \mathrm{O}_{2} \text {. } \\
& \text { - } \mathrm{C}_{5} \mathrm{H}_{5} \mathrm{~N}_{5} \mathrm{O}+7 \mathrm{H}_{2} \mathrm{O}=4 \mathrm{NH}_{3}+\mathrm{C}_{2} \mathrm{H}_{5} \mathrm{NO}_{2}+2 \mathrm{CO}_{2}+\mathrm{CH}_{2} \mathrm{O}_{2} \text {. } \\
& \underset{\text { HYPOX }}{\mathrm{C}_{5} \mathrm{H}_{4} \mathrm{~N}_{4} \mathrm{O}}+7 \mathrm{H}_{2} \mathrm{O}=3 \mathrm{NH}_{3}+\mathrm{C}_{2} \mathrm{H}_{5} \mathrm{~N}_{2} \mathrm{O}+\mathrm{CO}_{2}+2 \mathrm{CH}_{2} \mathrm{O}_{8} \text {. }
\end{aligned}
$$

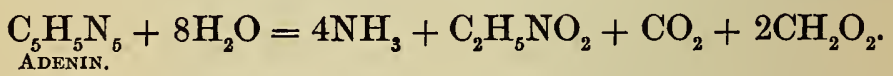

Uric acid has no CH group, hence does not yield formic acid, whereas xanthin and guanin have each one $\mathrm{CH}$ group and yield one molecule of formic acid. Hypoxanthin and adenin therefore have two $\mathrm{CH}$ groups.

Furthermore, uric acid contains three $\mathrm{CO}$ groups and yields three $\mathrm{CO}_{2}$ molecules, whereas xanthin and guanin have two $\mathrm{CO}$ groups and yield two $\mathrm{CO}_{2}$ molecules. Hence hypoxanthin has but one $\mathrm{CO}$ group, since it yields but one $\mathrm{CO}_{2}$ molecule. The corresponding carbon atom in adenin is likewise split off as carbonic acid.

The changes which the purin bodies undergo in the animal economy have been the subject of numerous investigations. As is well known, in birds the greater part of the waste nitrogen passes out in the form of uric acid. This large amount of uric acid is derived from two sources: (1) in small part from the nuclein metabolism of the tissue ; (2) chiefly by synthetic transformation of simple nitrogenous substances. Thus, after the administration of urea or of ammonium salts of organic acids birds eliminate uric acid. This synthesis, according to Minkowski, takes place within the liver, since after the removal of that organ the change does not take place. Instead the administered nitrogen is excreted as ammonium salts while the amount of uric acid falls to a minimum corresponding to that formed by actual nuclein metabolism.

In mammals on the other hand the purin bodies carry off only a very small part of the waste nitrogen. The chief if not the sole source of the uric acid is tissue metabolism, since the recent studies 
of Minkowski (1898) render it very doubtful that a uric acid synthesis occurs in mammals in a manner similar to that in birds. In dogs the administration of urea, ammonium lactate and allantoin is not followed by excretion of uric acid. Nevertheless it is obvious that purin compounds are synthesized in the body out of the food supplied. The growing cell can undoubtedly build up the purin molecule out of simple material and is in no wise restricted to the preformed nucleins or purins in the food. The amount thus synthesized in mammals may be considered as not in excess of that necessary for the purpose of the cells and hence the synthetic elimination of uric acid is not recognized. In birds on the other hand it is conceivable that the pronounced synthetic power of the liver is directed not at a mere elimination of uric acid but rather at the formation of antecedents (purins?) which are utilizable by the cells and stored up as nuclein. The supply of such antecedents being in great excess of the actual needs of the economy leads to their oxidation and as a result we have increased uric acid elimination which from this standpoint would correspond to that of urea. The greater part of the latter is believed to result from the breaking down of the circulating proteids when these are in excess of the needs of the tissues.

When nucleinic acid is fed to mammals the amount of uric acid eliminated is increased. The same is true as Taylor and others have shown after a diet of sweet-breads which are rich in nucleins. When, however, the actual amount of uric acid eliminated is compared with the amount of nuclein present it is evident that by far the greater part of the purin bodies is broken up and disappears. Two explanations are offered on this point. In the first place it is possible that the larger part of the purin bodies in nuclein is oxidized direct into urea and only a small part goes to form uric acid. Or, the second possibility is that the greater part is oxidized into uric acid; this in turn is largely broken down into urea, and only a small portion escaping this cleavage appears in the urine.

It is well known that uric acid when fed to mammals is broken down and excreted as urea, allantoin (Salkowski), oxalic acid, glycocoll and other products. If the amount of uric acid fed is excessive allantoin can be detected in the urine, otherwise the cleavage is carried farther, as is seen in the fact that no allantoin can then be isolated. The studies of Wiener (1899) have shown that certain organs in the body when crushed may destroy while others apparently make uric acid. Moreover the action of a given organ was found to vary with the species of the animal. Thus, the liver, spleen and thymus of beef were found to make uric acid whereas the liver of $\mathrm{dog}$ and hog actually destroyed this substance. Again, the kidney and muscles of the beef and horse were shown likewise to destroy uric acid whereas the kidney of a dog did not. In these experiments the antecedent of uric acid is undoubtedly nuclein since hypoxanthin 
by the action of the crushed liver is changed to uric acid. In beefliver, however, Wiener obtained an alcohol-soluble antecedent which was not a purin. It is very doubtful as to whether uric acid can thus be synthesized from urea, glycocoll and similar compounds. One fact is evident and that is that uric acid and hence other purin bodies are broken down in the body and that this change takes place especially in certain organs.

The amount of uric acid actually carried to the kidneys to be eliminated $(a)$ therefore will be the algebraic sum of that formed in nucleiu metabolism $(x)$ (tissue and food), that formed by synthesis $(y)$, and that destroyed in the body (z). Thus,

$$
x+y-z=a .
$$

The actual anount of uric acid eliminated, however, may be considerably less than that represented by $a$ since the kidney may be incapable of effecting the complete excretion. In which case deposits of uric acid will occur in the joints, as in rheumatism; and in the kidneys as in adenin poisoning and in the kidney infarcts of the new-born (Spiegelberg).

The purin bases may be looked upon as undergoing changes similar to those of uric acid. Hypoxanthin apparently is the only member of the group which when fed to birds, dogs or man (Minkowski) is eliminated as uric acid or as its cleavage product allantoin. Moreover the crushed beef liver is capable of effecting this conversion into uric acid (Wiener). Adenin on the other hand in dogs does not give rise to uric acid or to allantoin. A small amount appears in the urine unchanged (Kossel, Minkowski) the remainder being undoubtedly broken up into simple products. It is significant that the thymus gland which is rich in an adenin-producing nuclein should increase the amount of uric acid and even give rise to allantoin, whereas adenin itself is without this effect. It may be that the adenin while still in a nascent condition, as it were, is more readily oxidized than when definitely formed. At all events its antecedent behaves quite differently.

According to Krüger and Schmid ${ }^{1}$ the purin bases when administered to man are largely eliminated as uric acid. Thus, 62.3 per cent. of the hypoxanthin given was excreted as uric acid and only 0.15-0.25 per cent. as purin bases. Adenin gave a similar result, 40.7-41.2 per cent. appearing as uric acid and 2.6-3.7 per cent. being unchanged. With xanthin 10 per cent. formed uric acid and 1 per cent. was eliminated unchanged. Guanin perhaps also yields uric acid but this is not definitely settled.

Nothing definite can be said regarding the fate of xanthin and guanin in the animal body. It has been shown in pancreatic and 
yeast auto-digestions that hypoxanthin and xanthin are readily destroyed by the action of the enzyme whereas adenin and guanin resist. The xanthin derivatives however have been studied carefully and the results are extremely interesting. Thus, the caffein and theobromin of the food are progressively broken up into simpler xanthin bodies and a large part of the xanthin molecule is completely destroyed. The cleavage of caffein will be described in detail later but in this connection it is desirable to point out that most of the purin bases found in the urine owe their origin not to direct tissue metabolism but rather to the cleavage of caffein and similar bases introduced with the food. Inasmuch as the increase of purin bases is not proportional to the amount of caffein ingested it is evident that considerable of the latter is destroyed. Moreover it may be considered as an established fact that caffein does not increase the amount of uric acid although the use of coffee according to Taylor is followed by a marked increase in the elimination of this product.

The pharmacological action of the purin derivatives has been recently (1901) investigated by Schmiedeberg. Owing to the extremely slight solubility of some of these bases it follows that their effects are but slight. In general the well known effects of caffein are reproduced, although obviously differences exist. The action of caffein is directed upon the central nervous system, the muscles and the kidneys. The effects on the former are seen in the increased reflex irritability which, as in the case of strychnin, may lead to complete tetanus and even paralysis. The muscles contract more easily and more effectively and with large doses they become permanently contracted, passing into a condition of coagulation like that caused by heat and cold (see Cushny's Pharmacology). The action on the kidney is seen in the marked diuresis.

Purin exerts a slower coagulation effect upon muscles and causes increased reflex irritability. The resemblance to caffein is even more marked with methyl purin.

Hypoxanthin was found to have no action upon muscle, but the effect upon the nervous system was seen in the increased irritability and tetanus. The di-methyl hypoxanthin had a slight action upon the muscles and also tetanized. On the other hand 8-oxypurin, the isomer of hypoxanthin, did coagulate muscle, but showed no tetanic action, whereas its di-methyl derivative showed both effects and was about as active as theobromin.

Xanthin agrees in its action fully with the above 8-oxypurin; that is it causes muscular rigor and general paralysis, but no increased irritability. The alkyl derivatives of xanthin cause greater rigor of the muscles and otherwise act like caffein and theobromin. Of the di-methyl xanthins theobromin showed less action on muscles than theophylliu, while paraxanthin was the most active. 
The diuretic action of the purin bodies apparently parallels the effect upon muscles. Thus, paraxanthin is more diuretic than theophyllin while theobromin is less effective than the latter. The increased reflex irritability, as with ammonia compounds, is due to the nitrogen groups, whereas the action upon muscle is peculiar to the purin molecule.

Adenin, unlike the other purin bases, has been shown by Minkowski to be a violent poison. The profound effects induced, which will be described later, are apparently due to the presence of the amino group. Steudel has also observed a marked toxicity following the introduction of the amino group into pyrimidin.

\section{The Purin Bases.}

Purin, $\mathrm{C}_{5} \mathrm{H}_{4} \mathrm{~N}_{4}$, was successfully prepared by Fischer ${ }^{1}$ in 1898 . It is of interest because it is the prototype of the group to which it lends the name. Although as yet it has not been met with in the body or among the cleavage products of proteids, it is not unlikely but that improved methods will reveal the presence of purin and its methyl derivatives. A brief description of its properties which clearly place it in the same series with hypoxanthin, xanthin and uric acid, will therefore be appropriate.

Purin forms colorless microscopic needles often united in spherical aggregates. The melting point is $211-212^{\circ}$. It is very easily soluble in cold water and the solutions do not react toward litmus or curcuma. It is also very easily soluble in warm alcohol, from which it slowly crystallizes in small densely felted needles. It is more difficultly soluble in acetic ether and in aceton, and especially so in ether or in chloroform. It forms salts with acids as well as with bases.

The nitrate, $\mathrm{C}_{5} \mathrm{H}_{4} \mathrm{~N}_{4} \cdot \mathrm{HNO}_{3}$, is very easily soluble in hot water; rather difficultly so in hot alcohol. It crystallizes in roundish aggregates. On warming with dilute nitric acid it becomes yellow. It melts at $205^{\circ}$ with decomposition. The chlorid, iodid and sulphate are extremely soluble in water.

The picrate is difficultly soluble, requiring about twenty parts of boiling water. It forms yellow glistening needles which melt at $208^{\circ}$.

The platinochlorid forms, upon the addition of platinum chlorid to fairly concentrated solutions of the chlorid, as fine yellow needles which readily dissolve on heating. Gold chlorid gives an oily or resinous precipitate, which in time forms a solid yellow, granular mass which is also readily soluble on heating.

The sodium salt is very soluble in water, difficultly so in concentrated sodium hydrate from which it crystallizes in bundles of fine needles. The potassium and barium salts are likewise very soluble.

${ }^{1}$ Berichte, 31, 2550; 32, 493. 
With ammoniacal silver solution it gives at once a colorless amorphous precipitate, which is not blackened by boiling or by light. Nentral silver nitrate produces a white precipitate which is soluble in hot dilute nitric acid and separates out on cooling as a white granular or crystalline powder. Ammoniacal zinc solutions precipitate purin, especially on heating, in a fine pulverulent form. Mercuric chlorid gives an amorphous precipitate which on boiling becomes crystalline. Phosphotungstic acid produces an extremely fine precipitate, imparting a milky appearance to the fluid. Tannin produces a colorless flocculent deposit, while bismuth iodid in acid solution yields a red granular precipitate. Potassium iodid, ferrocyanid and Nessler's solution produce no precipitate.

With bromin it yields a beautiful yellowish-red, crystalline mass, which dissolves on heating and recrystallizes on cooling. It is very resistant to oxidation and with nitric acid it does not give the murexid reaction.

The 7-methyl and 9-methyl purins have also been prepared. Of especial interest is the fact that starting out with methyl uracil, a pyrimidin derivative, Gabriel and Colman ${ }^{1}$ (1901), succeeded in converting it into purin bodies-6-methyl purin and 6-2-methylamido purin.

The pharmacological action of purin is referred to on p. 345 .

Adenin, $\mathrm{C}_{5} \mathrm{H}_{5} \mathrm{~N}_{5}$, was discovered by Kossel in 1885 . It was prepared synthetically by Fischer ${ }^{2}(1)$ by treating tri-chlor-purin with ammonia and subsequent reduction of the dichlor-adeniu ; (2) by similar treatment of the methyl derivatives of oxy-di-chlor-purin. The 7-and 9-methyl adenins were incidentally prepared.

This base was first prepared from pancreatic glands-hence the term adenin, which is derived from the Greek word aory, meaning a gland. It has since been shown to occur together with guanin, hypoxanthin, etc., as a decomposition product of nuclein, and, therefore, it may be obtained from all tissues and organs, animal or vegetable, rich in nucleated cells. Accordingly it has been found in the kidneys, spleen, paucreatic, thymus, and lymphatic glands, in beeryeast, in spermatic fluids, but not in testicles of the steer ; occurs also in tea-leaves. In the latter adenin appears to exist in a preformed condition, since it can be extracted without the use of acid reagents (bypoxanthin absent, Krüger, 1896). Tea-extract yields about 6 grams of adeuin per liter (Krüger).

The thymus gland, as a prototype of embryonic, highly cellular tissue, yields a considerable amount of adenin, but no xanthin (Inoko); that from a calf, for instance, was found by Schindler to contain 0.18 per cent. The thymus nucleinic acid (adenylic acid)

${ }^{1}$ Berichte, 34, 1246, 1256.

2 Berichte, 30, 2238. 
was at first believed to yield only adenin but eventually was found to give also guanin besides cytosin and thymin (Kossel and Neumaun $\left.{ }^{1}\right)$. Although the pancreas was the organ from which originally adenin was isolated, yet according to Bang ${ }^{2}$ it yields a nucleinic acid (guanylic) which on decomposition gives rise to only one nuclein base-guanin, besides glycerin, phosphoric acid and pentose. On the other hand Levene ${ }^{3}$ obtained from a pancreas nucleinic acid, adenin, guanin and traces of xanthin and hypoxanthin. He also found that during auto-digestion of the pancreas the hexon bases and uracil and possibly thymin formed. According to Kutscher ${ }^{4}$ in pancreatic and yeast auto-digestion the purin bases are set free but the xanthin fraction is soon destroyed, leaving only adenin and guanin.

From tubercle bacilli Ruppel isolated a protamin, "tuberculosamin" and a nucleinic acid, tuberculinic acid. According to Levene the nucleinic acid from this germ is less stable than that from other sources. It contains iron and on decomposition yields guanin and adenin.

The auto-digestion of yeast is essentially the same as that of the pancreas and like the latter is due to a tryptic ferment. The products are leucin, tyrosin, asparaginic acid, ammonia, adenin, guanin, at times hypoxanthin and xanthin, also the three hexon bases. Carnin and butalanin have also been isolated. Kutscher has also isolated a new body, $\mathrm{C}_{8} \mathrm{H}_{6} \mathrm{~N}_{4} \mathrm{O}_{4}$, which probably corresponds to the uracil that Levene obtained from auto-digested pancreas. Geret and $\mathrm{Hahn}^{5}$ had previously shown that the yeast plasma contained an energetic proteolytic enzyme which digested fibrin, egg albumin and pepton. Leucin, tyrosin and hypoxanthin were formed. Similar enzymes were observed in tubercle and typhoid bacilli, in sarcine, and in lupine sprouts. In tryptic digestion of nuclein containing proteid it is evident that adenin and other xanthin bases may be set free the same as if the cleavage had been brought about by an acid. The bases then would appear in the "antipepton" (p. 424). In autodigested adrenals adenin is probably present (p. 386).

Adenin, guanin and xanthin together with cholin, diamins (?), uric acid and urea have been obtained from brains (Gulewitsch). ${ }^{6}$

From 10,000 liters of urine Krüger and Salomon obtained $3.54 \mathrm{~g}$. of adenin besides large amounts of xanthin and its derivatives.

It has also been observed in the liver and urine of leucocythæmic patients (Stadthagen); its occurrence in this disease will be readily understood when it is remembered that leukæmia is characterized by the presence in the blood of an unusual proportion of the nucleated

${ }^{1}$ Zeits. physiol. Chem., 22, 74.

2 Zeits. physiol. Chem., 31, 411.

${ }^{3}$ Zeits. physiol. Chem., 32, 541.

4 Zeits. physiol. Chem., 32, 66.

- Berichte, 31, 2336 (1898).

${ }^{6}$ Zeits. physiol. Chem., 27, 50. 
white blood corpuscles, which owing to various unfavorable conditions, become destroyed in time, and the contained nuclein, as a result, splits up into adenin and guanin. These two bases may, therefore, be expected in all pathological conditions where there is an abnormal accumulation of pus. Indeed, as early as 1865, Naunyn extracted from pus, obtained from the pleural cavity, a considerable quantity of a substance which was probably either adenin or guanin, or both. Neither uric acid nor xanthin bases in recognizable amounts are present in fresh human blood (100-300 c.c.); both are present in exudates and transudates (Jaksch). In the blood of leukæmics and in blood after a diet of thymus glands uric acid is present in increased quantity (Petren).

Adenin does not occur, or only in minute traces, in meat extract; and in this it resembles guanin, which is present only in traces. This may be due to the fact that adenin and guanin are readily converted into hypoxanthin and xanthin respectively, as bas been shown in the putrefaction experiments of Schindler. This conversion of adenin and guanin into hypoxanthin and xanthin takes place in the pancreas immediately after death, so that the amount of adenin found may be quite small. They may be considered as transitional products of cell metabolism, the amido group contained in each readily being replaced by oxygen, and giving rise to ammonia, and this in turn to urea. Kossel, however, explained this fact on the ground that the muscle tissue is very poor in nucleated cells, $i$. e., in nuclein. It would seem that the muscle cell in losing the morphological character of a cell has also suffered a corresponding loss in its chemical properties. For while the decomposition products of nuclein-hypoxanthin, xanthin, phosphoric acid, etc.-are found in the muscle tissue, they do not exist in combination as they do in the nuclein molecules. This is seen in the fact that the bases exist in the free condition, since they can be extracted by water; and again, the phosphoric acid is present in the muscle tissue, not in organic combination, but as a salt. In the nucleated cell, adenin, guanin, etc., do not exist in a free condition, but form, in part at least, with albumin and phosphoric acid, a loose combination which is readily decomposed by the action of acids at the boiling temperature. This same change takes place spontaneously after death. It is quite possible that the existence of these bases in muscles, in the free condition, is due to the action of enzymes.

There can be no doubt that adenin and guanin play an important part in the physiological function of the cell nucleus, which, from recent abservations, appears to be necessary to the formation and building up of organic matter. It is now known that non-nucleated cells, though capable of living, are not capable of reproduction. We must look, therefore, to the nucleus as the seat of the functional activity of the cell-indeed, of the entire organism. Nuclein, the 
parent-substance of adenin and guanin, is the best known and probably most important constituent of the nucleus, and as such it has been already credited with a direct relation to the reproductive powers of the cell. This chemical view has already been confirmed by Zacharias, who showed that chromatin of histologists is identical with nuclein. More recently Mathews ${ }^{1}$ has shown that the chromatin in herring spermatozoa is a salt-like combination of protamin and nucleinic acid. Liebermann has questioned nuclein as being the source of xanthin compounds, but in this he is not supported by the mass of evidence. In the ease of birds, however, it must be conceded that uric acid results by the oxidation of the purin bases and above all by synthesis from urea, amido acids, etc. In mammals uric acid is probably wholly derived by oxidation of the purin bases originating from nuclein. Wiener, ${ }^{2}$ however, has rendered it probable that the synthetic function is also present though to less degree (p. 343). Whether purin bases as well as uric acid may be formed synthetically in birds remains to be demonstrated.

According to Minkowski (1898) adenin is a powerful poison. When injected subcutaneously it induces increased heart action and rapid death. On feeding it affects the digestive tract and the kidneys. The constant vomiting induced is probably due to the intense inflammation of the duodenal mucous membrane which may lead to actual destruction of tissue. The effect upon the kidney is seen in the presence in the urine of casts, albumin and of minute sphæroliths of uric acid. On section the kidney shows changes similar to those observed after injection of urates. The cortex especially reveals the presence of numerous grayish white points which under the microscope are found to consist of bundles of crystals or burs or more often of balls which show a concentric or radial marking. These deposits are undoubtedly uric acid but their origin is unknown. The excessive uric acid may be ascribed to the oxidation of the adenin but this is improbable. It is more likely that adenin favors the accumulation of uric acid by interfering with the destruction of this product in the liver. The studies of Wiener have shown that the crushed liver of the dog (p. 343) does destroy uric acid. Adenin is not as poisonous to rabbits and man as to dogs (Krüger and Schmid, 1902).

A continued diet of sweet-breads which as is known are rich in antecedents of adenin produced symptoms of intoxications such as diarrhœa, anorexia, nausea, beadache, malaise and abdominal pains (Taylor). The observation of Kossel that adenin is in part excreted unchanged has been confirmed by Minkowski and others.

Lilienfeld, in his study of the chemistry of the lencocytes, showed that the nuclei of these cells contain a complex body, nucleohiston,

1 Zeits. physiol. Chem., 23, 399.

${ }^{2}$ Archives f. exp. Path. u. Pharm., 42, 898 (1899). 
which is decomposed by acids into histon and leuconuclein. The latter in turn can be decomposed into albumin and nucleinic acid, which on heating with mineral acids yields phosphoric acid, and the nuclein bases (adenin, hypoxanthin) and unknown products. As Kossel has pointed out, it is probable that ordinary nucleinic acid is a mixture of several, since two or more nuclein bases form on decomposition. The existence, however, of nucleohiston, has been questioned by Bang and others.

Adenin, when crystallized from warm or impure solutions, is obtained either as an amorphous substance, pearly plates, or in the form of very small microscopic needles ; from dilute cold solutions it separates in long, needle-shaped crystals containing three molecules of water. This water of crystallization is lost on exposure to the air or on heating to $53^{\circ}$, and the crystals become opaque. By precipitating a concentrated solution of the hydrochlorid with ammonia adenin may be obtained as anhydrous, small whetstone-shaped crystals, which, recrystallized from hot water, form large, regular, four-sided pyramids, single or bur-shaped. It is soluble in about 1086 parts of water at the ordinary temperature; more easily in hot water, from which on cooling it recrystallizes. The aqueous solution possesses a neutral reaction. The free base is insoluble in ether, chloroform, and alcohol; soluble in glacial acetic acid, and somewhat in hot alcohol. It dissolves readily in mineral acids, yielding well crystallizable salts. The fixed alkalis dissolve it with ease, but on neutralization of the solution it is reprecipitated ; from such solutions in alkalis anhydrous large crystals are thrown down by acetic or carbonic acid (Krüger). In aqueous ammonium hydrate it is more readily soluble than guanin (which is insoluble, Schindler; somewhat soluble, Wulff), and more difficultly soluble than hypoxanthin-a fact which is made use of to effect a separation from those bases. It is but slightly soluble in sodium carbonate.

Adenin can be heated to $278^{\circ}$ without melting; at this temperature it becomes slightly yellow, and yields a white sublimate. It can be completely volatilized without decomposition, by heating on an oil-bath at $220^{\circ}$; the sublimate consists of pure, white, plumose needles of adenin, but at $250^{\circ}$ partial decomposition occurs, and some hydrocyanic acid forms. According to Fischer, when heated rapidly, in a capillary in a paraffin-bath, to $360^{\circ}-365^{\circ}$ adenin suddenly melts and evolution of gas takes place. When heated with potassium hydrate to $200^{\circ}$ on an oil-bath, it yields a considerable quantity of potassium cyanid. Adenin is quite indifferent to the action of acids, alkalis, and even oxidizing agents. Thus, it may be boiled for hours with baryta, potash, or hydrochloric acid, without suffering decomposition. But when heated with dilute hydrochloric acid at $135^{\circ}$ for several days, or with concentrated hydrochloric acid, in a sealed tube at a temperature exceeding $100^{\circ}$, adenin is 
completely decomposed, with formation of carbonic acid and ammonia :

$$
\mathrm{C}_{5} \mathrm{H}_{5} \mathrm{~N}_{5}+5 \mathrm{H}_{2} \mathrm{O}+5 \mathrm{O}=5 \mathrm{CO}_{2}+5 \mathrm{NH}_{3} \text {. }
$$

When heated with 20 per cent. sulphuric acid in an autoclave at $150^{\circ}$ for two hours adenin is decomposed (Jones).

On heating adenin with concentrated hydrochloric acid to $180^{\circ}-$ $200^{\circ}$ for $12-14$ hours, Krüger obtained ammonia, carbon dioxid, carbon monoxid, and glycocoll. The carbon monoxid results from the splitting up of formic acid. This decomposition is strictly analogous to that of hypoxanthin, xanthin, etc.

$$
\mathrm{C}_{5} \mathrm{H}_{5} \mathrm{~N}_{5}+8 \mathrm{H}_{2} \mathrm{O}=4 \mathrm{NH}_{3}+\mathrm{CO}_{2}+2 \mathrm{CH}_{2} \mathrm{O}_{2}+\mathrm{C}_{2} \mathrm{H}_{8} \mathrm{NO}_{2} \text {. }
$$

The free base, as well as benzoyl adenin, is unaffected by the weak oxidizing action of potassium permanganate, but on stronger oxidation it is wholly destroyed. Bromin water produces in aqueous solutions of adenin an oily precipitate which, on contact with potassium hydrate or ammonia, gives a beautiful red or violet color. Sodium amalgam and zine chlorid appear to have no action; but on boiling with zinc and hydrochloric acid it yields a very unstable reduction product which, in the presence of oxygen, in alkaline solution, first assumes a red color and finally throws down a reddishbrown precipitate. This brown substance appears to be identical with azulmic acid, which has been known for a long time as a product of the polymerization of hydrocyanic acid.

Adenin and hypoxanthin do not give the xanthin reaction; that is to say, when adenin is evaporated on a water-bath with dilute or fuming nitric acid it gives a white residue which fails to give any coloration with sodium, ammonium, or barium hydrate (xanthin reaction). Similarly, it does not give the so-called Weidel's reaction on heating with fresh chlorin water and a trace of nitric acid as long as gas is given off, then evaporating to dryness on a water-bath and exposure of the residue to an ammoniacal atmosphere. In this respect it resembles hypoxanthin which, when pure, does not answer to either of these tests. When either of these bases, however, is evaporated on a water-bath with bromin water and nitric acid a residue is obtained which with alkalis is colored red (Kossel). Another test for adenin, which is given also by hypoxanthin, but not by guanin, caffein, and episarkin, is as follows: The substance to be tested is digested for half an hour with zinc and hydrochloric acid in a test-tube on a water-bath. If adenin is present, the solution will assume on standing, more rapidly on shaking, a ruby-red coloration which in time disappears. The colorless liquid on dilution and addition of sodium hydrate becomes again red, which later on turns into a brownish-red (Kossel). This reaction depends upon the formation of a reduction product which, owing to its unstable nature, 
is soon oxidized by the oxygen of the atmosphere into a brownish, amorphous substance, apparently identical with azulmic acid.

Ferric chlorid imparts to an aqueous solution of adenin an intense red color which is not affected by heating. Copper sulphate produces an amorphous grayish-blue precipitate, which is easily soluble in dilute acids and ammonia. The light-blue solution in fixed alkalis on warming gives a precipitate of copper oxid.

Drechsel's reaction. In 1892 Drechsel showed that certain xanthin bases are precipitated by an ammoniacal solution of cuprous chlorid; or from fixed alkaline solution by Fehling's solution in the presence of a reducing substance. In addition to uric acid, which has been known to give this reaction, xanthin, guanin, hypoxanthin, creatin, and creatinin, the latter on boiling, reacted. Balke applied the test to fixed alkaline solutions, using Fehling's solution, and as reducing substances hydroxylamin hydrochlorid, or dextrose. $\mathrm{He}$ found that adenin, hypoxanthin, xanthin, heteroxanthin, paraxanthin, carnin, protamin, and uric acid gave precipitates, whereas theobromin and caffein did not. Krüger employed copper sulphate and sodium bisulphite, the advantage being that the precipitation can take place in neutral, acid, or alkaline solutions. The results differ somewhat with the kind of reducing agent employed. Thus copper sulphate and sodium bisulphite precipitate uric acid, adenin, methyl adenin, hypoxanthin, guanin, also dimethyl hypoxanthin from cold concentrated solution; theobromin, caffein, creatin, creatinin are not precipitated. With copper sulphate and sodium hyposulphite, adenin, methyl adenin, and guanin are readily precipitated; hypoxanthin only on heating (separation from adenin), whereas the other six compounds are not precipitated. The precipitates are soluble in excess of sodium hyposulphite.

Adenin and hypoxanthin can, therefore, be completely precipitated, especially by the aid of heat, from their solution by copper sulphate and sodium bisulphite. Hence this reagent could be used as a substitute for ammoniacal silver solution in the method of separation and even of estimation by determining either the amount of copper, or of nitrogen by Kjeldahl's method. The adenin precipitate is colorless and gelatinous; changes on exposure to a light or brownish green, and on drying it becomes dark green. It is easily soluble in mineral acids, especially nitric; slowly soluble in hot acetic acid. It is not decomposed with sodium hydrate; readily decomposed with alkali sulphids, and is readily soluble in ammonia. It is soluble in about 200,000 parts of hot water.

On treatment with nitrous acid it is converted into hypoxanthin according to the equation :

$$
\mathrm{C}_{5} \mathrm{H}_{5} \mathrm{~N}_{5}+\mathrm{HNO}_{2}=\mathrm{C}_{5} \mathrm{H}_{4} \mathrm{~N}_{4} \mathrm{O}+\mathrm{N}_{2}+\mathrm{H}_{2} \mathrm{O} \text {. }
$$

Kossel obtained 72 per cent. of the theoretical yield. Since then 23 
Krüger, by modifying the experimental conditions, adding sodium nitrite in small portions to a solution of adenin in dilute sulphuric acid at $70^{\circ}$, obtained an almost quantitative conversion.

This formation of hypoxanthin from adenin is analogous to Strecker's transformation of guanin into xanthin by a similar action of nitrous acid (see guanin). In both cases the change of a highly nitrogenized into a less nitrogenized body is accomplished by replacing an $\mathrm{NH}_{2}$ group by $\mathrm{O}$. The change is somewhat analogous to that seen in the conversion of primary amins into primary alcohols. Thus,

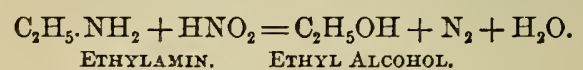

In the extraction of adenin from the mother liquor of tea leaves after removal of caffein, if urea is not added to the nitric acid, nearly one-half of the adenin may be converted into hypoxanthin. By processes of putrefaction adenin is converted into hypoxanthin and guanin into xanthin (Schindler). A similar conversion of adenin and guanin takes place rapidly in the pancreas after death and it is not unlikely that this change is due to the action of nitrous acid produced by bacteria. Adenin undergoes this decomposition more rapidly than do the other purin bases. In view of the ease with which this conversion of adenin and guanin takes place it is quite probable that similar changes may take place within the cell nucleus proper and as a result hypoxanthin and xanthin form in greater or less amount. The formation of xanthin from guanin represents the conversion of a guanidin residue into a urea group. The amido group in all probability is split off as ammonia and goes to make urea.

Adenin unites with bases, acids, and salts. The salts of adenin with mineral acids can be recrystallized, thus differing from the corresponding salts of guanin and hypoxanthin, which are dissociated by the action of water. The solutions of the salts, however, show an acid reaction to litmus, but not to methyl orange. The addition of ammonia to concentrated aqueous solutions of its salts yields crystalline adenin.

The hydrochlorid, $\mathrm{C}_{5} \mathrm{H}_{5} \mathrm{~N}_{5} \cdot \mathrm{HCl}+\frac{1}{2} \mathrm{H}_{2} \mathrm{O}$, forms colorless, transparent, strongly refracting crystals. One part of the anhydrous salt is soluble in 41.9 parts of cold water. Microscopically it is distinct from that of hypoxanthin and adenin-hypoxanthin. From the composition of the gold salt it is highly probable that a hydrochlorid, $\mathrm{C}_{5} \mathrm{H}_{5} \mathrm{~N}_{5} .2 \mathrm{HCl}$, exists analogous to that of guanin. In the course of the synthesis of adenin Fischer prepared a di-iodid.

The nitrate, $\mathrm{C}_{5} \mathrm{H}_{5} \mathrm{~N}_{5} . \mathrm{HNO}_{3}+\frac{1}{2} \mathrm{H}_{2} \mathrm{O}$, crystallizes from the aqueous solution in fine, stellate needles. One part of the dry salt dissolves in 110.6 parts of water.

The sulphate, $\left(\mathrm{C}_{5} \mathrm{H}_{5} \mathrm{~N}_{5}\right)_{2} \cdot \mathrm{H}_{2} \mathrm{SO}_{4}+2 \mathrm{H}_{2} \mathrm{O}$, can be obtained from the 
aqueous solution in two different crystalline forms. This may possibly be due to the presence of the adenin-hypoxanthin compound (Bruhns). It is easily soluble in hot water, and at the ordinary temperature it is soluble in 153 parts of water (156, Fischer).

The oxalate, $\mathrm{C}_{5} \mathrm{H}_{5} \mathrm{~N}_{5} \cdot \mathrm{C}_{2} \mathrm{H}_{2} \mathrm{O}_{4}+\mathrm{H}_{2} \mathrm{O}$, is obtained by dissolving adenin in hot, dilute, aqueous oxalic acid, from which solution, on cooling, it separates as a voluminous difficultly soluble precipitate of roundish masses which are composed of long, delicate needles. The oxalates of guanin, hypoxanthin, and xanthin are more easily soluble than that of adenin, and exhibit, moreover, a different appearance. Adenin bichromate, $\left(\mathrm{C}_{5} \mathrm{H}_{5} \mathrm{~N}_{5}\right)_{2} \cdot \mathrm{H}_{2} \mathrm{C}_{2} \mathrm{O}_{7}$. This compound separates in a few hours from a mixture of adenin and chromic acid solutions in well formed yellowish-red crystals (Bruhns). According to Krüger, it forms six-sided plates, is easily soluble in hot water, difficultly in cold, and is unchanged by heating to $150^{\circ}$. The corresponding salt of guanin readily dissociates.

Adenin metaphosphate, $\mathrm{C}_{5} \mathrm{H}_{5} \mathrm{~N}_{5} \cdot \mathrm{HPO}_{3}$. According to Kossel, adenin is not precipitated with metaphosphoric acid, but this is not strictly true. Aqueous, or even cold saturated solutions of adenin give on the addition of a few drops of metaphosphoric acid an amorphous precipitate, appearing under the microscope as fine round granules or extremely thin membranous masses. It has not been obtained in a crystalline condition. Like the corresponding guanin compound, it is difficultly soluble in cold water. It is easily soluble in alkalis and in ammonia ; is more or less soluble in dilute acids according to the concentration, and is soluble in excess of metaphosphoric acid. Hence a strongly acid, not too concentrated, solution of adenin is not precipitated (Wulff). Adenin is precipitated less completely than guanin, whereas hypoxanthin does not give a difficultly soluble metaphosphate.

The chloracetate, $\mathrm{C}_{5} \mathrm{H}_{5} \mathrm{~N}_{5} \cdot \mathrm{ClCH}_{2} \cdot \mathrm{CO}_{2} \mathrm{H}$, was prepared by Krüger by adding an excess of chloracetic acid to a hot aqueous solution of adenin. On cooling it crystallizes in right-angled plates and in stellate four-sided prisms. It is easily soluble in water and in hot aqueous alcohol; difficultly in cold alcohol. At $162^{\circ}-163^{\circ}$ it melts, giving off hydrochloric acid and forming a yellowish-red fluid which gradually becomes intensely red.

Potassium ferro- and ferricyanid produce no precipitate in a solution of adenin, but if acetic acid is then added the former gives rise to a precipitate of thin plates; the latter, to a precipitate of light-brown crystals grouped in bunches (Krüger). According to Bruhns, adenin gives with potassium ferricyanid brownish-green needles.

The picrate, $\mathrm{C}_{5} \mathrm{H}_{5} \mathrm{~N}_{5} \cdot \mathrm{C}_{6} \mathrm{H}_{2}\left(\mathrm{NO}_{2}\right)_{3} \mathrm{OH}+\mathrm{H}_{2} \mathrm{O}$, is thrown down as a bright-yellow flocculent precipitate when aqueous solutions of adenin salts are treated with sodium picrate. Recrystallized from hot water 
it forms bright-yellow, very voluminous bunches of long, fine needles which, on drying, acquire a silky luster and form a felted mass. It is difficultly soluble in cold water $(1: 3500)$; more readily in hot water and in alcohol (96 per cent.); is insoluble in dilute acids. It dissolves readily in a solution of sodium phosphate, from which solution it is precipitated by hydrochloric acid. Other salts of adenin, as the metaphosphate, behave in the same way. Uric acid is also dissolved by sodium phosphate (Wulff). The water of crystallization is not lost on exposure to air, but is driven off at $100^{\circ}$; the salt then remains unchanged even at $220^{\circ}$. A cold concentrated aqueous solution of the salt treated with one-tenth its volume of cold concentrated solution of sodium picrate produces a precipitate of short, fine needles, consisting of most of the adenin picrate (five-sevenths). The solubility of the picrate can thus be reduced to as low as $1: 13750$, and on this fact is based the quantitative method of Bruhns. The salt can also be obtained in its characteristic groups by combining cold saturated aqueous adenin solution $(1: 1086)$ with picric acid; with sodium picrate, however, adenin gives no precipitate, since the picrate is soluble in an equivalent quantity of sodium hydrate. Thus is explained Kossel's statement that adenin forms an easily soluble compound with picric acid. Heated on platinum foil it burns slowly and leaves considerable carbon residue. The very bright yellow color of the salt serves to distinguish it from most of the other picrates, especially guanin picrate. Adenin may be isolated from its picrate by extraction of the hydrochloric acid solution with ether, by precipitation of the ammoniacal solution with silver nitrate, and best, according to Krüger, by dissolving the picrate in hot dilute ammonia, and when cold precipitating most of the picric acid with ammoniacal copper sulphate solution. The filtrate can then be evaporated, dissolved in dilute $\mathrm{H}_{2} \mathrm{SO}_{4}$, and the last traces of picric acid removed with ether.

It may be noted that adenin and guanin form difficultly soluble picrates, whereas xanthin and hypoxanthin form relatively easily soluble compounds.

The platinochlorid, $\left(\mathrm{C}_{5} \mathrm{H}_{5} \mathrm{~N}_{5} \cdot \mathrm{HCl}\right)_{2} \mathrm{PtCl}_{4}$, crystallizes from dilute aqueous solution in small yellow needles. The concentrated aqueous solution of this salt, when boiled for some time, decomposes, with the separation of a clear, yellow powder, which is but slightly soluble in cold water, and has the composition $\mathrm{C}_{5} \mathrm{H}_{5} \mathrm{~N}_{5} \cdot \mathrm{HCl}_{2} \mathrm{PtCl}_{4}$.

The aurochlorid, on evaporation, yields very characteristic forms. It has been more recently studied by Wulff, and found to possess the formula $\mathrm{C}_{5} \mathrm{H}_{5} \mathrm{~N}_{5} \cdot(\mathrm{HCl})_{2} \cdot \mathrm{AuCl}_{3}+\mathrm{H}_{2} \mathrm{O}$. From the hydrochloric acid solution of adenin and gold chlorid, on sufficient concentration, or from dilute solutions by gradual evaporation, it separates in l)right, well formed orange-colored crystals, which may attain a length of $1.2 \mathrm{~cm}$. As pointed out by Kossel, this salt is well 
adapted for the qualitative recognition of adenin, especially in the presence of guanin, which gives no such compound.

Adenin lead was prepared by Krüger by adding a solution of adenin and sodium hydrate to an aqueous solution of lead acetate. It forms lusterless needle-shaped crystals. The composition appears to be $\mathrm{C}_{5} \mathrm{H}_{3} \mathrm{PbN}_{5}$. On friction it becomes strongly electric. Heated with methyl iodid it gives rise to addition products (see page 363 ).

The silver salt of adenin, $\mathrm{C}_{5} \mathrm{H}_{4} \mathrm{AgN}_{5}$, is formed when silver nitrate is added in molecular proportion to a boiling ammoniacal solution of adenin. On heating this compound for thirteen hours at $130^{\circ}$ with methyl iodid no appreciable change results (Krüger), although Thoiss obtained a compound, presumably a methyl addition product. An excess of silver nitrate produces, in the cold, the compound $\mathrm{C}_{3} \mathrm{H}_{3} \mathrm{Ag}_{2} \mathrm{~N}_{5}+\mathrm{H}_{2} \mathrm{O}$, which is converted slowly in the cold, immediately on warming, into the other salt, according to the equation :

$$
2\left(\mathrm{C}_{5} \mathrm{H}_{3} \mathrm{Ag}_{2} \mathrm{~N}_{5}+\mathrm{H}_{2} \mathrm{O}\right)=2 \mathrm{C}_{5} \mathrm{H}_{2} \mathrm{AgN}_{5}+\mathrm{Ag}_{4} \mathrm{O}+\mathrm{H}_{2} \mathrm{O} \text {. }
$$

Owing to this instability the two compounds are always found together in varying proportion. Both are difficultly soluble in water, and in ammonia even at the boiling-point. The precipitation of adenin by an ammoniacal silver solution is complete, and is therefore available for quantitative estimation. The precipitate of adenin, as well as of other xanthin bases, is soluble in excess of sodium hyposulphite (Kossel).

Adenin silver nitrate, $\mathrm{C}_{5} \mathrm{H}_{5} \mathrm{~N}_{5} \cdot \mathrm{AgNO}_{3}(\mathrm{Ag}=35.4$ per cent.), corresponds to the similar hypoxanthin and guanin salts. It is obtained by dissolving the above silver compound in hot nitric acid; and from this solution, on cooling, it separates in needle-shaped crystals, which are not permanent. This lack of stability, as compared with the permanent hypoxanthin silver nitrate, was first pointed out by Kossel and was thought to be due to the loss of nitric acid in washing, and also by heating at $100^{\circ}$. Bruhns, however, has shown that the acidity of the wash-water is indicated by litmus, but not by methyl orauge, which is not colored red by silver nitrate. It would seem that adenin, as well as hypoxanthin, and possibly xanthin, form silver compounds containing one and two molecules of silver nitrate; the greater the quantity of silver nitrate used the higher is the per cent. of silver, $i$. e., the more of the latter compound is formed. These are very unstable, and are decomposed by dilute nitric acid, more so by water, into silver nitrate, and the compound containing one molecule of silver nitrate. We have in this behavior an interesting case of mass action and chemical equilibrium between adenin, silver nitrate, nitric acid and water. Ammonium hydrate removes the nitric acid from this as easily as from the hypoxanthin compound, and there is formed, according to the com- 
position of the original salt, a varying mixture of $\mathrm{C}_{5} \mathrm{H}_{4} \mathrm{AgN}_{5}$ and $\mathrm{C}_{5} \mathrm{H}_{3} \mathrm{Ag}_{2} \mathrm{~N}_{5}+\mathrm{H}_{2} \mathrm{O}$. The solubility in nitric acid is about the same as that of hypoxanthin silver nitrate.

Adenin silver picrate, $\mathrm{C}_{5} \mathrm{H}_{4} \mathrm{AgN}_{5} \cdot \mathrm{C}_{6} \mathrm{H}_{2}\left(\mathrm{NO}_{2}\right)_{3} \mathrm{OH}+\mathrm{H}_{2} \mathrm{O}$, is obtained as an amorphous voluminous yellow precipitate when silver nitrate is added to a cold aqueous solution of adenin picrate. If the latter solution is previously raised to the boiling-point, the precipitate soon becomes crystalline and rapidly subsides. The adenin can thus be almost wholly removed from solution. The crystalline form loses its water of crystallization at $120^{\circ}$, while the amorphous form does not appreciably decrease in weight, and its composition does not appear to be so constant as that of the corresponding hypoxanthin compound. On treatment with ammonium hydrate the picric acid is removed, and adenin silver, $\mathrm{C}_{5} \mathrm{H}_{4} \mathrm{AgN}_{5}$, is left, stained yellow by picric acid.

Adenin mercury picrate, $\left(\mathrm{C}_{5} \mathrm{H}_{4} \mathrm{~N}_{5}\right)_{2} \mathrm{Hg} \cdot 2 \mathrm{C}_{6} \mathrm{H}_{2}\left(\mathrm{NO}_{2} \mathrm{O}\right)_{3} \mathrm{OH}$, can be prepared by treating a hot concentrated aqueous solution of adenin picrate with an excess of sodium picrate, and then with mercuric chlorid. It forms a yellow granular, crystalline precipitate (nicroscopic needles) which rapidly subsides and increases in quantity as the solution cools. Its composition apparently varies, containing one to two molecules of water, according to the temperature of the solution. One molecule is given off at $100^{\circ}$ and the second at $105^{\circ}-120^{\circ}$. The latter preparation, then, on exposure to the air, rapidly absorbs one molecule of water. The object of the sodium picrate in the precipitation is to combine with the hydrochloric acid, which is set free. The precipitate produced by mercuric chlorid in cold adenin picrate solution shows yellow and white granules, and is not homogeneous. Bruhns considers it to be a mixture of the adenin mercury picrate and the compound $\mathrm{C}_{5} \mathrm{H}_{4} \mathrm{~N}_{5} \mathrm{Hg}_{2} \mathrm{Cl}_{3}$; if sodium picrate is added, however, the pure adenin mercury picrate forms, since no hydrochloric acid is set free.

Adenin mercuric chlorid, $\mathrm{C}_{5} \mathrm{H}_{4} \mathrm{~N}_{5} \mathrm{HgCl}$, is thrown down as a white, finely granular precipitate when a boiling aqueous adenin solution is treated gradually with concentrated mercuric chlorid solution (Bruhns). On neutralizing the filtrate from this precipitate a second deposit forms. According to Krüger, the reaction that takes place is as follows:

$$
\begin{gathered}
2 \mathrm{C}_{5} \mathrm{H}_{5} \mathrm{~N}_{5}+2 \mathrm{HgCl}_{2}=\mathrm{C}_{5} \mathrm{H}_{4} \mathrm{~N}_{5} \cdot \mathrm{HgCl}+\mathrm{C}_{5} \mathrm{H}_{5} \mathrm{~N}_{5} \cdot \mathrm{HCl} \cdot \mathrm{HgCl}_{2} \cdot \\
\mathrm{C}_{5} \mathrm{H}_{5} \mathrm{~N}_{5} \cdot \mathrm{HCl} \cdot \mathrm{HgCl}_{2}+\mathrm{Na}_{2} \mathrm{CO}_{3}=\mathrm{C}_{5} \mathrm{H}_{4} \mathrm{~N}_{5} \cdot \mathrm{HgCl}+2 \mathrm{NaCl}+\mathrm{CO}_{2}+\mathrm{H}_{2} \mathrm{O} .
\end{gathered}
$$

Heated with alkyl iodids it does not give rise to substitution compounds. Free hydrochloric acid is indicated by the reaction with methyl orange. Treated with ammonium hydrate the chlorin is removed, and there is formed apparently the compound $\mathrm{C}_{5} \mathrm{H}_{4} \mathrm{~N}_{5} \mathrm{HgOH}$. If dissolved in warm dilute hydrochloric acid and allowed to crystal- 
lize, the double salt $\mathrm{C}_{5} \mathrm{H}_{5} \mathrm{~N}_{5} \cdot \mathrm{HCl} \cdot \mathrm{HgCl}_{2}+2 \mathrm{H}_{2} \mathrm{O}$ separates in long, stellate, silky needles.

Another mercury compound, $\mathrm{C}_{5} \mathrm{H}_{4} \mathrm{~N}_{5} \mathrm{Hg}_{2} \mathrm{Cl}_{3}$, is obtained when the precipitation takes place in the cold. The precipitate is white, flocculent, and anhydrous. In this reaction, as above, for each adenin molecule an equivalent of hydrochloric acid is set free. This same body is also produced when an adenin solution is boiled with a large excess of mercuric chlorid and as little hydrochloric acid as possible to effect solution. On cooling small stellate needles separate out, which do not lose their weight at $110^{\circ}$. It can also be obtained by boiling the following compounds with water.

When adenin is boiled with a large excess of mercuric chlorid and much hydrochloric acid to dissolve completely the precipitate that first forms, there is deposited on cooling a crystalline product, which is variable in its composition, and apparently consists of double salts of adenin and mercuric chlorid, such as $\mathrm{C}_{5} \mathrm{H}_{5} \mathrm{~N}_{5} \cdot \mathrm{HCl} .5 \mathrm{HgCl}_{2}$ and $\mathrm{C}_{5} \mathrm{H}_{5} \mathrm{~N}_{5} \cdot \mathrm{HCl} .6 \mathrm{HgCl}_{2}$. On boiling with water these rapidly decompose, forming the compound $\mathrm{C}_{5} \mathrm{H}_{4} \mathrm{~N}_{5} \cdot \mathrm{Hg}_{2} \mathrm{Cl}_{3}$. The formation of a double salt, $\mathrm{C}_{5} \mathrm{H}_{5} \mathrm{~N}_{5} . \mathrm{HCl} . \mathrm{Hg}_{2} \mathrm{Cl}_{2}+2 \mathrm{H}_{2} \mathrm{O}$, is described above.

Adenin mercury cyanid, $\left(\mathrm{C}_{5} \mathrm{H}_{5} \mathrm{~N}_{5}\right)_{2} \cdot \mathrm{Hg}(\mathrm{CN})_{2}$, separates as stellate needles and plates when a mixture of hot solutions of adenin and mercuric cyanid are allowed to cool.

An adenin bismuth iodid, $\mathrm{C}_{5} \mathrm{H}_{5} \mathrm{~N}_{5} \cdot \mathrm{HI} .2 \mathrm{BiI}_{3}+2 \mathrm{H}_{2} \mathrm{O}$, is obtained when an aqueous adenin solution is treated with potassium bismuth iodid containing free hydriodic acid. The heavy precipitate, which in color resembles carbon monoxid hæmoglobin, consists of microscopic glittering red needles. On contact with much water it partly decomposes, forming light reddish-yellow amorphous floccules, which become darkish brown at $100^{\circ}$.

Chlor-adenin has not been obtained, since chlorin passed over dry adenin in the cold, or at $100^{\circ}$, or into a boiling chloroformic suspension of adenin is without effect. Phosphorus pentachlorid heated with adenin at $160^{\circ}-170^{\circ}$ for some hours gave a light-brown body of uncertain composition.

The synthetic di-chlor adenin is derived from tri-chlor purin.

Brom-adenin. By treating well dried adenin with excess of dried bromin a dark-red body is obtained which appears to contain six atoms of bromin, $\mathrm{C}_{5} \mathrm{H}_{5} \mathrm{~N}_{5} \cdot \mathrm{Br}_{6}$ (Bruhns). On mere exposure to the air, more rapidly on heating at $100^{\circ}-120^{\circ}$, it becomes light-yellow and decomposes, yielding bromin, brom-adenin, $\mathrm{C}_{5} \mathrm{H}_{4} \mathrm{BrN}_{5}$, and its hydrobromid, $\mathrm{C}_{5} \mathrm{H}_{4} \mathrm{BrN}_{5}$. $\mathrm{HBr}$. Brom-adenin is white, difficultly soluble in cold water $(1: 10,000)$, more readily in hot water, very easily in ammonia and in fixed alkalis. It crystallizes from water or dilute ammonia in stellate needles or very thin plates which, when dried in air, often assume a silky luster. The crystals contain a variable amount of water depending on the temperature at 
which the crystallization takes place. Thus, the crystals may contain almost two molecules of water, whereas when crystallization occurs at above $60^{\circ}$ the crystals are auhydrous. It is a rather strong base and forms well characterized salts which are difficultly soluble in cold water, more easily in the presence of an excess of acid, from which it is thrown down as a white micro-crystalline precipitate by addition of ammonia. It is also formed from the original dark-red body by treatment with sodium bisulphite, or better by dissolving the body in ammonium hydrate, or, according to Krüger, by heating to $130^{\circ}$, then dissolving in sodium hydrate and precipitating with carbonic or acetic acid. It is only difficultly attacked by boiling alcohol or aqueous potash or alcoholic ammonia. The atom of bromin cannot therefore be replaced by an amido or by a hydroxyl group. Sodium alcoholate heated with brom-adenin at $145^{\circ}$ for hours has no effect.

Brom-adenin is very easily and completely chauged to adenin by the action of sodium amalgam in the cold, or by boiling for several hours with zinc dust. No azulmic acid is formed. It is not affected by iron dust (Bruhns). A ccording to Krüger, it is affected by heating with concentrated potash at $180^{\circ}-190^{\circ}$, and the bromin is not replaceable by radicals as phenol.

The study by Bruhns of the decomposition of the dark-red body, mentioned above, has shown that it is very probably a hydrobromid of brom-adeuin, tetra-bromid, $\mathrm{C}_{5} \mathrm{H}_{4} \mathrm{BrN}_{5} \cdot \mathrm{Br}_{4} \cdot \mathrm{HBr}$. According to Krüger, this compound does not always form by the addition of bromin to adenin. Ordinarily the hydrobromid of brom-adenin forms, unless a very large excess of bromin is used. Compounds similar to brom-adenin are formed by hypoxanthin, guanin, xanthin, and caffein. Azulmic acid reacts with bromin in much the same way as adenin.

The hydrochlorid, sulphate, and nitrate of brom-adenin have been prepared and analyzed by Bruhns.

Brom-adenin picrate, $\mathrm{C}_{5} \mathrm{H}_{4} \mathrm{BrN}_{5} \cdot \mathrm{C}_{6} \mathrm{H}_{2}\left(\mathrm{NO}_{2}\right)_{3} \mathrm{OH}+\mathrm{H}_{2} \mathrm{O}$, resembles that of adenin, but is more voluminous. It is precipitated under the same conditions as adenin. The solubility in cold water is about the same $(1: 3220)$. It is likewise almost completely thrown out of solution by sodium picrate. Under the microscope, however, it can be readily distinguished from adenin picrate, since it does not form distinct crystals, but rather bundles of thin thread-like needles.

The metal derivatives of brom-adenin are analogous to those of adenin. Thus, ammoniacal silver solution gives rise to a mixture of $\mathrm{C}_{5} \mathrm{H}_{3} \mathrm{AgBrN}$ and $\mathrm{C}_{5} \mathrm{H}_{2} \mathrm{Ag}_{2} \mathrm{BrN}_{5} . \mathrm{H}_{2} \mathrm{O}$. Silver nitrate produces a gelatinous precipitate which, like the adenin silver nitrate, has an inconstant composition; on careful heating with nitric acid (1.1 sp. g.) it can be obtained in needles which resemble exactly those of the 
adenin compound. Prolonged boiling with nitric acid results in the separation of silver bromid. Mercuric chlorid, cadminm chlorid, potassium bismuth iodid, etc., give precipitates with brom-adenin the same as with adenin.

Brom-adenin gives the xanthin reaction, whereas adenin itself does not. Thus, if evaporated with strong nitric acid on the waterbath to dryness, and the cold yellowish or reddish residue is touched with sodium hydrate, a bluish-violet color forms. With ammonia it is a purple-red; with baryta water a pure violet.

Dry chlorin gas passed over warm dry brom-adenin has no effect. If, however, the brom-adenin is moist, decomposition and solution result. On evaporation of the solution the residue gives with potassium hydrate an intense violet-red color; baryta produces a bluishgreen precipitate.

It is therefore evident from the above reactions with nitric acid and chlorin that brom-adenin is more readily destroyed or oxidized than adenin. Inasmuch as all attempts at obtaining oxidation products of adenin which would shed light on its constitution failed, the study of the oxidation products of brom-adenin therefore possessed special interest. Krüger succeeded in oxidizing brom-adenin with hydrochloric acid and potassium chlorate in warm solution, into alloxan, urea, and oxalic acid. A reddish substance which dissolved in alkalis with a purple-red color was also produced in small amounts. Its alkaline solution gave a dirty-blue precipitate with baryta. The amount of alloxan found was very small ; indeed, in one experiment it was entirely absent. Nevertheless, it was sufficient to prove that in adenin, and hence in hypoxanthin, an alloxan group and probably an urea residue were present as in uric acid and xanthin.

When adenin is treated with zinc and hydrochloric acid in the cold it forms a difficultly soluble crystalline double salt which has not been obtained in the pure state. This double salt is not obtained by direct treatment of adenin hydrochlorid with zine chlorid.

One of the hydrogen atoms of adenin is capable of replacement by organic radicals, as seen from the following compounds:

Acetyl adenin, $\mathrm{C}_{5} \mathrm{H}_{4} \mathrm{~N}_{5}$. $\mathrm{CO} \cdot \mathrm{CH}_{3}$, can be obtained by heating the anhydrous base with an excess of acetic anhydrid for some time, in an oil-bath, at $130^{\circ}$. It crystallizes in small white scales which dissolve but slightly in cold water and in alcohol; more readily in hot water, in dilute acids and alkalis. Heated to $260^{\circ}$ it becomes yellow, but does not melt.

Benzoyl adenin, $\mathrm{C}_{5} \mathrm{H}_{4} \mathrm{~N}_{5} . \mathrm{CO} \cdot \mathrm{C}_{6} \mathrm{H}_{5}$, is obtained by the action of benzoic anhydrid, but not of benzoyl chlorid or adenin. It crystallizes from water in long, lustrous, thin needles which sometimes are grouped in bundles and melt at $234^{\circ}-235^{\circ}$. It is easily soluble in hot alcohol, from which it recrystallizes on cooling; also in dilute 
acids and in ammonia. With ammoniacal silver nitrate it gives a precipitate resembling that of adenin, but is more readily soluble in ammonia. This compound is quite stable, since it decomposes very slowly on boiling with hydrochloric acid; on protracted boiling with water it is changed into adenin and benzoic acid.

Mono-benzyl adenin, $\mathrm{C}_{5} \mathrm{H}_{4} \mathrm{~N}_{5} \cdot \mathrm{CH}_{2} \cdot \mathrm{C}_{6} \mathrm{H}_{5}$, was obtained by Thoiss by heating well dried adenin with benzyl chlorid to boiling $\left(178^{\circ}\right)$ on an oil-bath. It can also be obtained, according to Krüger, by heating adenin in a flask with benzyl chlorid in a sulphuric acid bath; also by heating adenin with alcohol, potassium hydrate, and benzyl chlorid under an inverted condenser. It crystallizes from alcohol in short, glistening prisms, frequently in small pointed crystals grouped in plate-like aggregations. A ten per cent. alcoholic solution gives reactions with silver nitrate, ammoniacal silver solution, mercuric chlorid, picric acid and platinum chlorid. Gold chlorid gives no precipitate. The compound forms pure white microscopic crystals and melts at $259^{\circ}$. It is easily soluble in hot water and in hot alcohol; but little in ether. Its solubility in water at $15^{\circ}$ is $1: 2250$; in water at $100^{\circ}$ is $1: 320$. With acids it forms salts from which alkalis throw down the base. The hydrochlorid $\mathrm{C}_{5} \mathrm{H}_{4}\left(\mathrm{C}_{7} \mathrm{H}_{7}\right) \mathrm{N}_{5} \cdot \mathrm{HCl}$, forms fine glassy needles or four-sided glassy prisms with inclined end-surfaces, which are readily soluble in alcohol and in water, but not in ether. The sulphate and nitrate possess similar properties. The sulphate, $\left(\mathrm{C}_{5} \mathrm{H}_{4}\left(\mathrm{C}_{7} \mathrm{H}_{7}\right) \mathrm{N}_{5}\right)_{2} \cdot \mathrm{H}_{2} \mathrm{SO}_{4}$, forms glassy long prisms containing five molecules of water, four of which easily pass off at $100^{\circ}$, and the fifth at $110^{\circ}$. Like adenin, it yields a silver compound which is insoluble in ammonia. On reduction with zinc and hydrochloric acid it forms an amorphous red unstable compound. Treated with nitrous acid, benzyl adenin is reduced to benzyl hypoxanthin, thus showing that the benzyl group replaces a hydrogen atom in the group $\mathrm{C}_{5} \mathrm{H}_{4} \mathrm{~N}_{4}$, which Kossel has called adenyl (see page 336 ).

Benzyl adenin picrate, $\mathrm{C}_{12} \mathrm{H}_{11} \mathrm{~N}_{5} \cdot \mathrm{C}_{6} \mathrm{H}_{2}\left(\mathrm{NO}_{2}\right)_{3} \mathrm{OH}$, is obtained as fine felted yellow needles, which are fairly soluble in water and in alcohol ; insoluble in ether.

Like adenin, the benzyl compound is very resistant to oxidation with potassium permanganate. On treatment with sulphuric acid and chromic acid a part is completely oxidized and the remainder is unchanged. Bromin acts energetically, forming a dark-red sticky mass, which at $120^{\circ}$ only gradually gives off a part of the bromin and becomes dark-yellow in color and firmer in consistency. Apparently four atoms of bromin unite with one molecule.

On decomposition with concentrated hydrochloric acid at $180^{\circ}-$ $200^{\circ}$ it yields glycocoll, volatile bases, and a resinous body, $\mathrm{C}_{14} \mathrm{H}_{12}$, identical with that obtained by Cannizzaro by the action of dehydrating agents on benzyl alcohol (Krüger). 
Dibenzyl adenin, $\mathrm{C}_{5} \mathrm{H}_{3}\left(\mathrm{C}_{7} \mathrm{H}_{7}\right)_{2} \mathrm{~N}_{5}$, is produced, according to Krüger, in small amount in the preparation of the mono-benzyl compound. It is best obtained by treating mono-benzyl adenin with benzyl chlorid; or by the action of benzyl chlorid on an alcoholic solution of adenin and potassium hydrate. The free base is obtained by precipitating a solution of the hydrochlorid with ammonium hydrate. It forms fine silky needles which melt at $171^{\circ}$ to a yellow fluid. It is easily soluble in ether, very easily in alcohol. In cold water at $13.5^{\circ}$ the solubility is $1: 13,300$; in water at $100^{\circ}$ it is $1: 1300$. A 1 per cent. alcoholic solution gives reactions with silver nitrate, ammoniacal silver solution, mercuric chlorid, copper sulphate, platinum chlorid, and picric acid. Gold chlorid, lead acetate, basic lead acetate, give no precipitate.

The hydrochlorid, $\mathrm{C}_{5} \mathrm{H}_{3}\left(\mathrm{C}_{7} \mathrm{H}_{7}\right)_{2} \mathrm{~N}_{5} \cdot \mathrm{HCl}$, crystallizes in fine silky needles, frequently in long prisms with a silky luster resembling that of caffein. It is easily soluble in water and in alcohol; insoluble in ether. It is thrown out of water solution, in part, by the addition of hydrochloric acid-a reaction not given by the mono-benzyl compound. The melting-point is at $219^{\circ}-220^{\circ}$.

The nitrate, $\mathrm{C}_{5} \mathrm{H}_{3}\left(\mathrm{C}_{7} \mathrm{H}_{7}\right)_{2} \mathrm{~N}_{5}$. $\mathrm{HNO}_{3}$, crystallizes in fine, long glistening needles, which are difficultly soluble in cold dilute nitric acid. It melts at $167^{\circ}$ with evolution of gas. On decomposition with concentrated hydrochloric acid at $180^{\circ}-200^{\circ}$ it yields the same products as mono-benzyl adenin (Krüger).

9 -methyl adenin, $\mathrm{C}_{5} \mathrm{H}_{4}\left(\mathrm{CH}_{3}\right) \mathrm{N}_{5}$, was first prepared in a condition of purity by Krüger. In 1897, Fischer ${ }^{1}$ prepared the 7- and 9methyl adenins synthetically. The methyl adenin described by Thoiss and prepared by the action of methyl iodid on adenin-silver at $100^{\circ}$ is probably an addition product, not methyl adenin. The introduction of the methyl group in this way does not take place readily, according to Krüger, owing to the formation of addition products. This addition of methyl iodid can be overcome readily by the presence of sodium alcoholate. This can be done by dissolving adenin in alcoholic sodium hydrate and then adding methyl iodid and allowing to stand for some months, or better, by warming under an inverted condenser. It crystallizes from water in anhydrous, long, silky needles or glassy prisms resembling caffein. On drying in the air the silky luster disappears. It may crystallize with one and a half molecules of water and does not melt at or below $270^{\circ}$. According to Fischer, it melts at $308^{\circ}-310^{\circ}$ (corr.) without decomposition. It is rather difficultly soluble in water, equally so in alkalis; and is soluble in 14 parts of hot water. The gold salt of methyl adenin crystallizes in fine yellow lusterless needles. The platinochlorid is rather difficultly soluble in cold water and crystallizes in bright four- or six-sided rhombic plates. A 1 per cent.

${ }^{1}$ Berichte, 30, 2250 ; 31, 104, 112. 
aqueous solution of methyl adenin gives reactions with picric acid, silver nitrate, ammoniacal silver solution, mercuric chlorid, copper sulphate, and sodium bisulphite. The precipitate by the latter reagent is more soluble than that of adenin. Lead acetate and basic lead acetate give no precipitate. Like adenin, it forms a mono-brom derivative (Krüger). The methyl compound of Thoiss gave reactions with baryta water, alcoholic zinc chlorid, mercuric nitrate, and cadmium chlorid, while basic lead acetate was without effect.

On decomposition with concentrated hydrochloric acid at $180^{\circ}-$ $200^{\circ}$ it yields ammonia, methylamin (distinction from adenin), carbonic acid, formic acid, and glycocoll, according to the equation :

$$
\begin{gathered}
\mathrm{C}_{5} \mathrm{H}_{5}\left(\mathrm{CH}_{3}\right) \mathrm{N}_{4}+8 \mathrm{H}_{2} \mathrm{O}=3 \mathrm{NH}_{3}+\mathrm{CH}_{3} \mathrm{NH}_{2}+\mathrm{CO}_{2}+ \\
2 \mathrm{H} . \mathrm{CO} . \mathrm{OH}+\mathrm{NH}_{2} \cdot \mathrm{CH}_{2} \cdot \mathrm{COOH} .
\end{gathered}
$$

Mono-methyl adenin methyl iodid, $\mathrm{C}_{5} \mathrm{H}_{4}\left(\mathrm{CH}_{3}\right) \mathrm{N}_{5} \cdot \mathrm{CH}_{3} \mathrm{I}$, is obtained by the action of methyl iodid on adenin-lead. It crystallizes from alcohol in coarse, glassy small crystals which are easily soluble in alcohol and in water; insoluble in ether. Simple substitution products do not form readily, but rather addition products.

The 7-methyl adenin was prepared synthetically by Fischer. It melts at $351^{\circ}$ (corr.) and sublimes at a higher temperature. It is soluble in 29 parts of boiling water and separates out on cooling as a granular powder, only occasionally bent needles being present. It is difficultly soluble in alcohol and forms salts with acids and metals like its isomer.

Both methyl adenins can be readily changed into the corresponding methyl hypoxanthins.

Ethyl adenin, $\mathrm{C}_{5} \mathrm{H}_{4}\left(\mathrm{C}_{2} \mathrm{H}_{5}\right) \mathrm{N}_{5}$, was prepared by Krüger according to the principle employed for the preparation of the methyl derivative. It is easily soluble in water and alcohol, and its aqueous solution gives reactions with silver nitrate, ammoniacal silver nitrate, mercuric chlorid, copper sulphate, and sodium bisulphite, picric acid, platinum chlorid, gold chlorid. The sulphate crystallizes from concentrated solutions in roundish masses of crystals.

Iso-amyl adenin, $\mathrm{C}_{5} \mathrm{H}_{4}\left(\mathrm{C}_{5} \mathrm{H}_{11}\right) \mathrm{N}_{5}$, was also obtained by Krüger by heating adenin with alcohol, sodium hydrate, and iso-amyl iodid. It is easily soluble in alcohol, acetone, chloroform, hot benzol ; difficultly soluble in ether and carbon disulphid. The solubility in water at ordinary temperature is $1: 1430$, and is not increased by sodium or ammonium hydrate. It is easily soluble in acids. It forms large bright irregular plates which melt at $148^{\circ}-150^{\circ}$. A 0.7 per cent. aqueous solution reacts with silver nitrate, ammoniacal silver solution, mercuric chlorid, picric acid, and gold chlorid. Platinum chlorid does not give a precipitate.

The 9-phenyl adenin has been synthesized by Fourneau (1901) from the corresponding uric acid. 
Adenin-hypoxanthin, $\mathrm{C}_{5} \mathrm{H}_{5} \mathrm{~N}_{5}+\mathrm{C}_{5} \mathrm{H}_{4} \mathrm{~N}_{4} \mathrm{O}$. - The occurrence of this compound was observed by Kossel, but it was isolated and studied for the first time by Bruhns. It can be prepared by cooling a hot aqueous solution of equal parts of the two bases. At first it is obtained as thick, starch-like, semi-transparent masses, which later in part become white and chalky. By spontaneous evaporation of its solution in very dilute ammonia it forms pearly aggregates of very small radially arranged needles, which contain water of crystallization. These effloresce somewhat and lose the water at $100^{\circ}$. The compound is more readily soluble in water than its components, but an exact determination of its solubility is impossible, inasmuch as the separation from hot solutions is not completed for some weeks. Any adenin present can be separated by recrystallization. It forms a distinct erystalline hydrochlorid, which should be borne in mind when examining microscopically for the two bases; but the combination is loose, since addition of gold chlorid brings down the characteristic gold salt of adenin. Ordinarily it does not form salts with sulphuric or nitric acid, but more often is decomposed by these, so that the difficultly soluble adenin crystallizes out. Once, however, Brubns obtained a sulphate which differed from the pure adenin and hypoxanthin sulphates. This perhaps explains the observation of Kossel that adenin sulphate forms crystals belonging to two systems. The compound can be decomposed into its constituents by fractional crystallization of the sulphate or nitrate; but better by forming the picrates, which are very unequally soluble in water. The existence of this compound undoubtedly explains many of the mistakes and discrepancies concerning the properties of lyppoxanthin, which it resembles more than adenin, and for the same reason, perhaps, adenin was so often overlooked.

Adenin-theobromin, $\mathrm{C}_{5} \mathrm{H}_{5} \mathrm{~N}_{5} \cdot \mathrm{C}_{7} \mathrm{H}_{8} \mathrm{~N}_{4} \mathrm{O}_{2}$. - This compound resembles the preceding, and was prepared by Krüger (1896) from tea-extract. It is easily soluble in hot, more difficultly in cold water. On recrystallization from water partial decomposition takes place. From aqueous solution picric acid throws down adenin, while theobromin remains in solution. It can be obtained, by crystallizing an aqueous solution of equal molecules of the two bases, as fixe, long prisms of marked silky appearance.

Hypoxanthin, $\mathrm{C}_{5} \mathrm{H}_{4} \mathrm{~N}_{4} \mathrm{O}$, sometimes also known as sarcin or sarkin, was discovered by Scherer (1850) in splenic pulp and in the muscles of the heart, and was named thus because it contains one atom of oxygen less than xanthin.

The base has been prepared synthetically by Fischer. ${ }^{1}$ Thus, trichlor purin with alkali yields di-chlor hypoxanthin which on reduc-

${ }^{1}$ Berichte, 30, 2226. 
tion yields hypoxanthin. It, as well as its methyl derivatives, may be also prepared from the synthetic adenin and its methyl derivatives by treatment with nitrous acid.

It has been obtained, usually accompanying adenin and guanin, from nearly all of the animal tissues and organs rich in nucleated cells, $i$. e., in nuclein. It has been found in blood after death, but not in blood when flowing through the blood vessels. Salomon has recently shown it to be a normal constituent of urine, present, however, in an exceedingly minute quantity.

From 10,000 liters of urine Krüger and Salomon obtained $8.5 \mathrm{~g}$. of hypoxanthin. For the other bases present, see xanthin, p. 389.

In the blood and urine of leucocythæmic patients it occurs in increased quantity owing to the abnormally large number of nucleated white blood corpuscles in circulation (p. 348). Bence Jones observed in the urine of a boy, who about three years before showed the symptoms of renal colic, a deposit of characteristic whetstonelike crystals, resembling uric acid, but differing from the latter by dissolving readily on the application of heat, while from hydrochloric acid it crystallized in elongated six-sided plates. These crystals be believed to be those of xanthin, but Scherer and others considered them to be hypoxanthin. It is therefore quite possible, though very rare, for this base to form a deposit in the urine and to be confounded in shape with uric acid. Thudichum obtained it from the urine of persons sick with liver or kidney diseases. According to Jaksch, it is present in exudates and transudates with uric acid.

Among other places it has been found in the brain, muscle, serum, marrow of bones, kidney, heart, spleen, liver, peripheral muscles (sarkin of Strecker), in the adrenals (Holm, Okerblom); in the spawn of salmon (Piccard), in the testicles of the bull (Salomon), in the nuclein of pus and red corpuscles (Kossel), in developing eggs, and in putrefaction of albumin (Salomon). It has also been found in the spores of lycopodium, and in the pollen of various plants, in seed of black pepper, in grass, clover, oats, bran of wheat, larvæ of ants; in the juice of potato (Schulze); in certain wines (Kayser); in the aqueous decoction of beer yeast (Schützenberger); and also in the liquid in which yeast is grown (Bechamp). In the autodigestion of yeast hypoxanthin and xanthin disappear and only adenin and guanin remain (Kutscher) (see p. 348).

Demant has shown it to be relatively abundant in the muscles of pigeons in a state of inanition, while in muscles of well fed pigeons it is said to be entirely absent. Salomon found hypoxanthin and xanthin in the cotyledons of lupine, as well as in the sprouts of malt, while Reinke and Rodewald observed these two bases together with guanin in Ethalium septicum. With adenin, xanthin, and theophyllin, it occurs in tea-leaves (Kossel); but Krüger (1896) showed 
that it is present in traces, if at all, when the copper method is used. In other words, by the action of nitrous acid, even in presence of urea, adenin is partially changed to hypoxanthin when the silver salts obtained by the old method are treated with nitric acid. Balke has found it in malt-sprouts by the copper method. In the pollen of the fir (Pinus sylvestris) Kresling found hypoxanthin, xanthin, and guanin, but not adenin. In the seeds of Randia dumetorum Vogtherr found hypoxanthin and guanin, but no xanthin.

Hypoxanthin has beeu extracted from the pancreas. Adenin and guanin in the pancreas readily change after death into hypoxanthin and xanthin (Inoko). Inasmuch as in the sterile auto-digestion of yeast, as pointed out above, only adenin and guanin remain it would seem as if trypsin readily destroyed hypoxanthin and xanthin. On the other hand, in the presence of bacteria, possibly owing to the formation of nitrous acid, adenin and guanin are converted, as pointed out by Inoko, into hypoxanthin and xanthin. The otherwise discrepant results are thus readily explained.

For the accidental formation of hypoxanthin from adenin by nitrous acid in the silver method, see page 409 .

When a mixture of guanin, xanthin, and hypoxanthin is allowed to putrefy, the bases decompose and disappear in the order named. Hypoxanthin resists bacterial action the longest, and this corresponds with its behavior to reagents (Baginsky). Adenin during putrefaction, in the absence of air, is converted into hypoxanthin and guanin is correspondingly changed into xanthin (Schindler). An amido group is, therefore, replaced by oxygen, and probably goes to form urea. This conversion is a very important fact, since the process of putrefaction, as Hoppe-Seyler has repeatedly pointed out, is analogous to the vital process, and the same chemical change may take place in the animal organs. The same change very probably takes place in the auto-digestion of yeast provided living yeast, and especially bacteria, are present. In sterile auto-digestions, as carried on in the presence of an antiseptic, only adenin and guanin are present in appreciable amount. Its formation from adenin can be represented thus:

$$
\mathrm{C}_{5} \mathrm{H}_{5} \mathrm{~N}_{5}+\mathrm{H}_{2} \mathrm{O}=\mathrm{C}_{5} \mathrm{H}_{4} \mathrm{~N}_{4} \mathrm{O}+\mathrm{NH}_{3} \text {. }
$$

Hypoxanthin occurs frequently in plants together with the other members of this group, namely, adenin, guanin, xanthin and its methyl derivatives. The widely distributed character of these bases is due to the presence of a parent substance, viz., nuclein, the necessary constituent of all cells capable of development, which under the influence of acids, and probably likewise of cellular enzymes, decomposes into the above mentioned bases. They may, therefore, be considered as the first steps in the retrograde metamorphosis of nearly all of the tissues of animals and plants. 
Recent advances in biological chemistry have shown that the undeveloped eggs of various insects and birds yield much less xanthin bodies (hypoxanthin, xanthin, etc.) on treatment with dilute acid than do the partially developed eggs (Tichomiroff, Kossel). This is dependent upon the remarkable fact observed by Kossel that the nuclein of undeveloped chicken eggs differs from the nuclein of cell nuclei and resembles that obtained from milk. For, while the nuclein from the cell nuclei decomposes into adenin, guanin, hypoxanthin, etc., that from undeveloped eggs and from milk yields no nitrogenous bases on treatment with acids. But as the egg develops, $i$. e., the nucleated cells increase in number, this latter nuclein is gradually converted or gives way to the ordinary cell nuclein, and hence it is that the chick embryo yields guanin, hypoxanthin, and possibly adenin.

Unquestionably, the presence of hypoxanthin, etc., in developing cells is due to the presence of the nuclein molecule from which it is readily split off. In muscle, however, hypoxanthin and xanthin appear to exist preformed, and bear no relation to nuclein, since they are in the free condition, and can be extracted from the tissue by water. This condition is analogous to that observed in plants, such as tea, and in sprouts and is undoubtedly due to an enzyme action on nuclein (p. 348).

According to the observations of Salomon and Chittenden, hypozanthin is formed by the digestion of blood fibrin with gastric juice, pancreatic juice, or on heating with water or dilute acids. Egg albumin under the same conditions does not yield any hypoxanthin, except when treated with pancreatic juice. These observations require repetition, inasmuch as the fibrin used undoubtedly contained nuclein, which, as we now know, readily decomposes under those conditions into its characteristic nitrogenous bases.

Hypoxanthin can be readily obtained from a number of closely related substances. Thus, carnin, by the action of oxidizing agents, is converted into hypoxanthin. For this reason Weidel and Schützenberger regarded hypoxanthin as derived from carnin and this view may be in part correct for it is not improbable but that carnin which is a purin base exists at times in the nuclein molecule or is formed during the metabolism of the purin radical.

Again, it can be obtained from adenin (p. 353) by the action of nitrous acid. The relation that hypoxanthin bears to uric acid had not been definitely established until Krüger showed that the constitution of hypoxanthin was closely connected with that of uric acid and the xanthin compounds. Streckler's belief that hypoxanthin by oxidation yields xanthin, and that uric acid by reduction with sodium amalgam yields first xanthin and then hypoxanthin, was not confirmed by Kossel or by Fischer. The reported change of uric acid into xanthin and hypoxanthin by 
Sundwik (p. 337) will require confirmation. Nevertheless, the researches of Fischer have shown that at least indirectly the purin bases may be converted into uric acid and vice versa (p. 337).

Hypoxanthin was once regarded as a step lower than guanin in the series of nitrogenous products of regressive metamorphosis, and consequently was considered as derived from guanin. The investigations of Kossel, however, show that it arises not from guanin but from adenin. On the other hand, guanin is to be looked upon as the source of xanthin. It is probable that in the organism it is oxidized as soon as it is set free from the nuclein, forming successively xanthin, uric acid, urea, etc., and the small quantity present in the urine is all that has escaped oxidation. 'The fact that hypoxanthin is so widely distributed in the organism, and in much larger quantities than was formerly supposed, shows that it may constitute, together with the closely related bodies creatin, xanthin, guanin, etc., a part of the antecedents of urea and of uric acid. This view is furthermore strengthened since hypoxanthin is especially abundant in those organs which are most active in producing metabolic changes in the body, viz., the liver and spleen. The fact that tryptic ferments apparently decompose hypoxanthin and xanthin has been already pointed out (p. 348). The conversion of hypoxanthin into uric acid by an emulsion of liver cells has been shown by Wiener (p. 343).

When fed to dogs, it was observed that the amount of hypoxanthin present in the urine decreased, and even became less in amount than before the experiment; but, on the other hand, the amount of xanthin and especially of uric acid in the urine was found to have increased above the normal. This shows that hypoxanthin in the body is oxidized probably first to xanthin, then into uric acid. According to Robert, hypoxanthin is a true muscle stimulant (see p. 345).

Hypoxanthin is a white, colorless, crystalline powder, sometimes in part amorphous; according to Bruhns, pure hypoxanthin does not form floccules and bunches of microscopic needles, but usually coherent crusts, which consist of roundish, sharp-cornered granules; some resemble octahedra. The synthetic hypoxanthin separates from hot aqueous solution as a colorless crystalline powder (Fischer). It is soluble in about 300 parts of cold water (Strecker), but according to Fischer the synthetic base is soluble at $19^{\circ}$ in 1415 and at $23^{\circ}$ in 1370 parts of water. This agrees fairly with Scherer's result $1: 1090$. The base separates slowly from aqueous solutions, and when pure the solubility, even in the beginning, is less than $1: 300$. At the end of four days Bruhns found it to be $1: 1880$. It is more easily soluble in boiling water (78 parts Strecker, 69.5 parts Fischer), and, on cooling, separates in the form of white, crystalline floccules, thus differing from xanthin which is amorphous. According to Scherer the solubility in warm water is 1 to 180 . The solubility in 
cold alcohol is very slight, about $1: 1000$. It dissolves in acids and alkalis without decomposition, and from solutions in the latter it can be precipitated by passing carbonic acid, or by the addition of acetic acid. The aqueous solution possesses a neutral reaction.

The free base can be heated up to $150^{\circ}$ without suffering decomposition, but above this temperature it sublimes, and partially decomposes, with evolution of hydrocyanic acid. When heated with potassium hydrate to $200^{\circ}$ it yields ammonia and potassium cyanid. Heated with water to $200^{\circ}$ it decomposes into carbonic acid, formic acid, and ammonia, and in this respect it agrees with adenin (page 342). The properties of Strecker's sarkin agree closely with those of adenin-hypoxanthin; and, inasmuch as the latter has been often described as hypoxanthin, it is very desirable that the properties of hypoxanthin be redetermined.

When evaporated with an oxidizing agent, chlorin water and nitric acid, the residue is said to give on contact with ammonia vapors a rose-red color (Weidel test). Kossel, however, has shown that this is due to the presence of xanthin, and that pure hypoxanthin does not give either the murexid test or the xanthin reaction. According to Strecker, concentrated nitric acid converts hypoxanthin into a nitro compound, which in turn, by the action of a reducing agent, is changed into xanthin. This statement has not been confirmed either by Fischer or by Kossel. It does not give a green color with sodium hydrate and chlorid of lime-distinction from xanthin (page 392). Potassium permanganate when added to an acid solution of the base is very slowly decolored and hence can be used for its purification (Kossel).

Like adenin (page 352), when evaporated with bromin water and nitric acid on a water-bath it gives a residue which with alkalis turns red, whilst nitric acid alone, as given above, has no effect (Kossel). An additional similarity to adenin is seen in its behavior to zinc and hydrochloric acid. The addition of sodium hydrate after reduction produces a red color (page 352) which is not as pronounced as in the case of adenin (Fischer). ${ }^{1}$

For the behavior of hypoxanthin and other bases to Drechsel's reaction, see p. 353 . With copper sulphate and sodium bisulphite it forms a whiter, more flocculent precipitate than adenin, soluble in 250,000 parts of hot water (Krüger). Its solubility and properties are about the same as those of the adenin compound. 0.5 per cent., and even stronger solutions, are not precipitated in the cold by copper sulphate and sodium hyposulphite. It is, however, precipitated on heating, whereas uric acid is not. It is, therefore, possible to separate uric acid from adenin and hypoxanthin by precipitating the latter two bases in hot solution with copper sulphate and sodium hyposulphite. The method, however, is of little practical value, 'Berichte, 30, 2230, 2241. 
since uric acid can be readily separated from these two bases with dilute acids. The separation may be useful for guanin and xanthin, which are less soluble in dilute acids, and hence difficult to separate from uric acid. By effecting the precipitation in cold solution of the two bases, adenin can be separated from hypoxanthin.

With acids it yields crystallizable compounds, and, like the amido acids, it forms compounds with bases and also with metallic salts, such as silver nitrate and copper acetate.

The hydrochlorid, $\mathrm{C}_{5} \mathrm{H}_{4} \mathrm{~N}_{4} \mathrm{O} . \mathrm{HCl}+\mathrm{H}_{2} \mathrm{O}$, crystallizes in needles, and, like the nitrate and sulphate, it is dissociated on contact with water. The crystalline form is characteristic and distinct from that of adenin, as well as adenin-hypoxanthin. The nitrate forms thick prisms or roundish masses readily soluble in water and ammonia. Platinum chlorid forms a yellow crystalline double salt, having the composition $\mathrm{C}_{5} \mathrm{H}_{4} \mathrm{~N}_{4} \mathrm{O} . \mathrm{HCl}$. $\mathrm{PtCl}_{4}$.

It does not form a difficultly soluble metaphosphate as adenin or guanin (see p. 355).

The picrate forms bright yellow prisms easily soluble in hot water, which solution is not affected as that of adenin by sodium picrate. According to Wulff, it possesses the formula $\mathrm{C}_{5} \mathrm{H}_{4} \mathrm{~N}_{4} \mathrm{O}_{2} \mathrm{C}_{6} \mathrm{H}_{2}\left(\mathrm{NO}_{2}\right)_{3} \mathrm{OH}$ $+\mathrm{H}_{2} \mathrm{O}$. It is obtained by addition of picric acid to a solution of adenin, or of sodium picrate to an acid solution of adenin. Depending on the concentration, it precipitates in greater or less length of time. It is difficultly soluble in cold water; easily in alkaline, also in ammonia. The estimation of adenin or guanin by picric acid in the presence of hypoxanthin is likely to give a high result.

Hypoxanthin-lead can be prepared, according to Krüger, by adding a solution of hypoxanthin in sodium hydrate to a solution of lead acetate. It is amorphous.

Hypoxanthin silver, $\mathrm{C}_{5} \mathrm{H}_{2} \mathrm{Ag}_{2} \mathrm{~N}_{4} \mathrm{O} . \mathrm{H}_{2} \mathrm{O}$, forms an amorphous colorless precipitate when silver nitrate is added to an ammoniacal solution of the base. All attempts to obtain a compound containing but one atom of silver in the molecule, corresponding to the adenin compound $\mathrm{C}_{5} \mathrm{H}_{4} \mathrm{AgN}_{5}$, have failed. The above compound was first prepared by Strecker, and given the formula $\mathrm{C}_{5} \mathrm{H}_{4} \mathrm{~N}_{4} \mathrm{O} \cdot \mathrm{Ag}_{2} \mathrm{O}$; but the former is preferable, since on heating at $120^{\circ}$ two and a half molecules of water are lost and $2 \mathrm{C}_{5} \mathrm{H}_{2} \mathrm{Ag}_{2} \mathrm{~N}_{4} \mathrm{O}+\mathrm{H}_{2} \mathrm{O}(\mathrm{Ag}=60.2$ per cent.) results. At $140^{\circ}-150^{\circ}$ it loses again in weight and becomes gradually gray; on exposure to air it absorbs moisture. In this form hypoxanthin can be estimated quantitatively; the presence of sodium picrate does not interfere, but chlorids, etc., do. It is insoluble in hot water. The compound $\mathrm{C}_{5} \mathrm{H}_{2} \mathrm{Ag}_{2} \mathrm{~N}_{4} \mathrm{O} .3 \mathrm{H}_{2} \mathrm{O}$ is obtained in the form of microscopic needles, by treating pure hypoxanthin silver nitrate with excess of aqueous ammonia. On boiling with ammonia water it is but slightly dissolved, and appears to lose slowly a part of its water of crystallization. As a result of the 
decomposition one half of the hypoxanthin passes into solution, and can be recovered on boiling with addition of silver nitrate in the crystalline form ; or in the cold, as the usual amorphous precipitate, $\mathrm{C}_{5} \mathrm{H}_{2} \mathrm{Ag}_{2} \mathrm{~N}_{4} \mathrm{O} \cdot \mathrm{H}_{2} \mathrm{O}$.

Hypoxanthin silver nitrate, $\mathrm{C}_{5} \mathrm{H}_{4} \mathrm{~N}_{4} \mathrm{O} . \mathrm{AgNO}_{3}(\mathrm{Ag}=35.20$ per cent.), is the best known compound; its formula was established by Strecker. It is obtained by dissolving the above precipitate, produced by addition of silver nitrate to an ammoniacal solution of the base, in hot nitric acid, specific gravity 1.1 ; on cooling the hypoxanthin silver nitrate crystallizes in the form of tufts of microscopic needles or plates. Heated at $100^{\circ}-120^{\circ}$ it remains constant in weight; the quantity of silver present, when determined, is always somewhat higher than the theoretical, especially if an excess of silver nitrate is employed in the precipitation. The explanation of this fact is probably that given under adenin, though presence of silver chlorid may partly be the cause. On treatment with ammonia it loses not only nitric acid, but also half of the hypoxanthin, and $\mathrm{C}_{5} \mathrm{H}_{2} \mathrm{Ag}_{2} \mathrm{~N}_{4} \mathrm{O} .3 \mathrm{H}_{2} \mathrm{O}$ forms. The change takes place readily even in the cold, and if during the digestion an excess of silver nitrate is added, the hypoxanthin set free is converted into this compound, which is wholly constant in composition compared with the hypoxanthin silver nitrate. The conversion is quantitative. Very dilute hydrochloric acid, as well as hydrogen sulphid, removes the silver from this compound.

Hypoxanthin silver picrate, $\mathrm{C}_{5} \mathrm{H}_{3} \mathrm{AgN} \mathrm{N}_{4} \mathrm{O}_{6} \mathrm{H}_{2}\left(\mathrm{NO}_{2}\right)_{3} \mathrm{OH} \quad(\mathrm{Ag}$ $=22.88$ per cent.), is gradually formed by adding silver nitrate to a boiling solution of hypoxanthin picrate. The precipitate is granular and of a lemon-yellow color, and consists of aggregations of fine, short needles. It is slightly soluble in hot, insoluble in cold water. It is, therefore, applicable for a quantitative determination of the base. Aqueous ammonia very readily and completely removes the picric acid from the compound, and the residue is hypoxanthin silver, which is slightly colored yellow by a trace of picric acid; half of the hypoxanthin passes into solution. Nitric acid with difficulty converts it into hypoxanthin silver nitrate.

Hypoxanthin mercuric chlorid, $\mathrm{C}_{5} \mathrm{H}_{3} \mathrm{~N}_{4} \mathrm{OHgCl}$, is obtained by adding an equivalent quantity of mercuric chlorid to a boiling solution of hypoxanthin. The precipitate, which increases on cooling, is crystalline.

A second compound, $\mathrm{C}_{5} \mathrm{H}_{3} \mathrm{~N}_{4} \mathrm{OHg}_{2} \mathrm{Cl}_{3}$, is produced by adding a strong excess of mercuric chlorid, in the cold, to an aqueous solution of hypoxanthin. It forms a heavy granular micro-crystalline precipitate, which contains some water of crystallization.

By boiling the preceding compound with just sufficient hydrochloric acid to effect complete solution, there is formed on standing a precipitate of white roundish aggregates of leafy or needle-shaped 
glittering crystals which have the composition $\mathrm{C}_{5} \mathrm{H}_{4} \mathrm{~N}_{4} \mathrm{O} \cdot \mathrm{HgCl}_{2}$ $+\mathrm{H}_{2} \mathrm{O}$.

The following table of Bruhns illustrates the analogy existing between the mercury compounds of adenin and hypoxanthin and similar derivatives of ammonium :

\begin{tabular}{|c|c|c|}
\hline 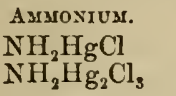 & $\begin{array}{l}\mathrm{C}_{5} \mathrm{H}_{4} \mathrm{~N} \\
\mathrm{C}_{5} \mathrm{H}_{4} \mathrm{~N}\end{array}$ & $\begin{array}{l}\mathrm{C}_{3} \mathrm{H}_{3} \\
\mathrm{C}_{5} \mathrm{H}_{3}\end{array}$ \\
\hline & $\mathrm{DH}$ & $\mathrm{C}_{5} \mathrm{H}_{4} \mathrm{~N}_{4} \mathrm{OHgC}$ \\
\hline
\end{tabular}

Hypoxanthin, as well as guanin and xanthin, forms readily soluble compounds with fixed alkalis. From these solutions the alkali compounds tend to crystallize on gradual evaporation, in rosettes or bundles of needles-difference from heteroxanthin and paraxanthin (Salomon). The alkali solutions of the xanthin bases are precipitated by carbonic acid and behave with acid salts, bicarbonates, and ammonium salts the same as the heteroxanthin-sodium compound.

According to Bruhns, hypoxanthin and uric acid are unaffected by the action of dry bromin, even at $100^{\circ}$, but Krüger has shown this statement to be incorrect. Bromin has no action on hypoxanthin at ordinary temperatures, but at $100^{\circ}$ and as high as $150^{\circ}$ the latter is changed quantitatively into brom-hypoxanthiu. A dark-red crystalline mass is obtained which contains six atoms of bromin to one molecule of hypoxanthin. It is a tetra-bromid of brom-hypoxanthin hydrobromid, $\mathrm{C}_{5} \mathrm{H}_{3} \mathrm{BrN}_{4} \mathrm{O} . \mathrm{HBr} . \mathrm{Br}_{4}$, analogous to the similar compound of adenin. It loses bromin slowly in the cold, rapidly at $120^{\circ}$, and brom-hypoxanthin hydrobromid remains, $\mathrm{C}_{5} \mathrm{H}_{3} \mathrm{BrN}_{4} \mathrm{O} . \mathrm{HBr}$. From this salt the free base can be obtained after conversion into the sodium compound. For this purpose the solution of the salt is treated with sodium hydrate or carbonic acid, or saturated direct with sodium carbonate, then concentrated to crystallization.

Brom-hypoxanthin, $\mathrm{C}_{3} \mathrm{H}_{3} \mathrm{BrN}_{4} \mathrm{O}+2 \mathrm{H}_{2} \mathrm{O}$. This can be prepared as just lescribed, or by the action of nitrous acid on brom-adenin at $70^{\circ}$. It forms a heavy powder of small, coarse crystals ; may form spherical groups of long, hair-like needles containing $1 \frac{1}{2}$ molecules of water. It is difficultly soluble in water; easily soluble in acids and alkalis. The aqueous solution reacts strongly acid. It has the properties of a base and an acid, behaving with alkalis and alkali carbonates the same as uric acid, setting free carbonic acid from the latter.

An aqueous solution of brom-hypoxanthin reacts with silver nitrate, ammoniacal silver solution, mercuric chlorid, copper sulphate, and sodium bisulphite. In acid solution it is precipitated by tannic acid, phosphotungstic acid, phosphomolybdic acid, by basic lead acetate; and is not precipitated by lead acetate or by baryta water. 
On heating brom-hypoxanthin with sodium carbonate, or by passing carbonic acid into a solution of the base in sodium hydrate, the sodium compound of brom-hypoxanthin forms, $\mathrm{C}_{5} \mathrm{H}_{2} \mathrm{NaBrN}_{4} \mathrm{O}+$ $2 \mathrm{H}_{2} \mathrm{O}$. It is easily soluble in hot water; rather difficultly in cold water. The solution has an alkaline reaction. The free base can be obtained by adding the calculated quantity of acid. The corresponding barium compound is obtained by passing carbonic acid into a solution of brom-hypoxanthin in barium bydrate. On concentration it crystallizes in fine white needles, and has the composition $\left(\mathrm{C}_{5} \mathrm{H}_{2} \mathrm{BrN}_{4} \mathrm{O}\right)_{2} \mathrm{Ba}$. The lead compound forms, on the addition of lead acetate to a solution of the base in sodium hydrate, as an amorphous precipitate.

The bromin in brom-hypoxanthin is held as firmly as that in bromadenin. Thus it is not affected by heating with alcoholic potash for three hours.

On decomposition of brom-hypoxanthin with hydrochloric acid and potassium chlorate Krüger obtained alloxan and urea. The yield of alloxan is not greater than that from adenin, and much less than that from xanthin, due undoubtedly to the alloxan nucleus splitting up into simpler bodies because of a different arrangement of bonds.

The synthetic dichlorhypoxanthin was prepared by Fischer from trichlorpurin (p. 337).

Benzyl hypoxanthin, $\mathrm{C}_{5} \mathrm{H}_{3} \mathrm{~N}_{4} \mathrm{O} \cdot \mathrm{CH}_{2} \cdot \mathrm{C}_{6} \mathrm{H}_{5}$, was obtained by Thoiss by the action of nitrous acid on benzyl adenin. It forms a white crystalline mass which under the microscope consists of thin plates. It is easily soluble in hot water, dilute alcohol, and in acetic ether; insoluble in ether and chloroform. It melts at $280^{\circ}$. It appears, as Kossel pointed out, that adenin and hypoxanthin contain a group, $\mathrm{C}_{5} \mathrm{H}_{4} \mathrm{~N}_{4}$, which he named adenyl. This, it will be seen, corresponds to Fischer's purin.

Two of the possible 3-methyl hypoxanthins are known. The 7and 9-methyl derivatives can be prepared from the corresponding compounds of adenin by the action of nitrous acid (Fischer). ${ }^{1}$

1-7-dimethyl hypoxanthin, $\mathrm{C}_{5} \mathrm{H}_{2}\left(\mathrm{CH}_{3}\right)_{2} \mathrm{~N}_{4} \mathrm{O}$. This compound was prepared by Bruhns, in the same way as methyl adenin, by heating hypoxanthin with alcohol, sodium alcoholate, and methyl iodid. Fischer ${ }^{2}$ prepared the same derivative from dichlorhypoxanthin and from 7-methyl hypoxanthin. A compound of dimethyl hypoxanthin with sodium iodid, $\mathrm{C}_{5} \mathrm{H}_{2}\left(\mathrm{CH}_{3}\right)_{2} \mathrm{~N}_{4} \mathrm{O} \cdot \mathrm{NaI}+3 \mathrm{H}_{2} \mathrm{O}$, formed first which, crystallized from alcohol, gave prismatic crystals, easily soluble in water and in hot alcohol, insoluble in ether. The synthetic hypoxanthin forms this same characteristic double salt. Other bases of the uric acid group are not known to form similar compounds.

1 Berichte, 30, $2409 ; 31,113$.

${ }^{2}$ Berichte, 30, 2230, 2411. 
Even caffein, which resembles dimethyl hypoxanthin, does not enter into such a combination.

The free base is obtained by treatment of this compound with freshly precipitated silver oxid. It melts at $244^{\circ}-246^{\circ}$ (Fischer). From chloroform it crystallizes in fine silky needles, containing three molecules of water of crystallization. From alcohol it crystallizes in groups of small pointed anhydrous crystals. It is easily soluble in water and chloroform; less so in alcohol. A strong solution of the base reacts with silver nitrate, with nitric acid and silver nitrate, with copper sulphate and sodium bisulphite (in the cold, not on warming). Copper sulphate and sodium thiosulphate do not give a precipitate. A one per cent. solution of the base gives precipitates with mercuric chlorid, platinum chlorid, and gold chlorid, but not with lead acetate, basic lead acetate, picric acid, and ammoniacal silver solution.

On account of the solubility of this base in water and chloroform ; the fact that it is not precipitated by ammoniacal silver solution, which precipitates all bases of the uric acid group containing a replaceable imido group; also on account of its behavior with copper sulphate and mercuric chlorid, Krüger concluded that hypoxanthin contained only two imiado groups capable of substitution. In addition to these adenin contains a third amido group, which is not replaceable, and which with nitrous acid is replaced by oxygen. The nitrogen in position 9 , however, is capable of forming methyl compounds.

On decomposition with concentrated hydrochloric acid at $180^{\circ}-$ $200^{\circ}$ it yields one molecule of methylamin and two of ammonia and one of sarkosin, according to the equation :

$$
\begin{gathered}
\mathrm{C}_{5} \mathrm{H}_{2}\left(\mathrm{CH}_{3}\right)_{2} \mathrm{~N}_{4} \mathrm{O}+7 \mathrm{H}_{2} \mathrm{O}=2 \mathrm{NH}_{3}+\mathrm{CH}_{3} \mathrm{NH}_{2}+\mathrm{CO}_{2}+ \\
2 \mathrm{HCOOH}+\mathrm{CH}_{3} \cdot \mathrm{NH} \cdot \mathrm{CH}_{2} \cdot \mathrm{COOH} .
\end{gathered}
$$

The isomer 1-9-di-methyl hypoxanthin is probably formed from 9-methyl hypoxanthin (Fischer).

Diethyl hypoxanthin ethyl iodid, $\mathrm{C}_{5} \mathrm{H}_{2}\left(\mathrm{C}_{2} \mathrm{H}_{5}\right)_{2} \mathrm{~N}_{4} \mathrm{O} \cdot \mathrm{C}_{2} \mathrm{H}_{5} \mathrm{I}$. This compound was also prepared by Krüger, by heating hypoxanthinlead with ethyl iodid. It crystallizes from alcohol in beautiful foursided glistening prisms, which are easily soluble in water and in hot alcohol ; insoluble in ether.

Iso-amyl hypoxanthin, $\mathrm{C}_{5} \mathrm{H}_{3}\left(\mathrm{C}_{5} \mathrm{H}_{11}\right) \mathrm{N}_{4} \mathrm{O}$, was likewise prepared by Krüger by heating hypoxanthin in the presence of sodium hydrate and alcohol with amyl iodid. It forms six-sided rhombic plates and is easily soluble in chloroform, difficultly in cold water, insoluble in ether.

Di-iso-amyl hypoxanthin, $\mathrm{C}_{5} \mathrm{H}_{2}\left(\mathrm{C}_{5} \mathrm{H}_{11}\right)_{2} \mathrm{~N}_{4} \mathrm{O}$, is formed in small amount at the same time as the preceding. It yields a hydrochlorid crystallizing in small needles. The base is set free from solu- 
tions of the salt as an oil which on cooling becomes crystalline. The formation of these two compounds is analogous to the benzyl substitutions in adenin.

Ethyl chloro-carbonate, acting on hypoxanthin in the presence of sodium hydrate, produces a precipitate which, recrystallized from hot water, forms elongated sharp-angled plates which melt at $185^{\circ}-$ $190^{\circ}$. It is insoluble or difficultly soluble in cold or hot alcohol, in ether, and in cold water; easily soluble in hot water, in sodium hydrate, and in hydrochloric acid. Its formula corresponds to $\mathrm{C}_{5} \mathrm{H}_{3} \mathrm{~N}_{4} \mathrm{O}$.CO. $\mathrm{C}_{2} \mathrm{H}_{5}$. It is, therefore, considered by Kossel to be a urethan of hypoxanthin.

Phosphomolybdic acid precipitates hypoxanthin from acid solution, and in general the base gives the ordinary alkaloidal reactions.

It is not precipitated by ammoniacal basic lead acetate. Copper acetate does not precipitate it in the cold, but does on boiling. This fact has been made use of in the isolation of hypoxanthin. Mercuric chlorid, as well as mercuric nitrate, produces a flocculent precipitate.

Altogether, in its behavior to reagents it resembles xanthin to a very considerable degree. The two can be separated, however, by the different solubilities of the hydrochlorids in water, and more especially by that of the silver salts in nitric acid.

Physiological Action.-25-100 mg. begin to act on frogs in from six to twenty-four hours, and produce increased reflex excitability and convulsive attacks; 5-100 mg. are fatal (Filehne). (See also page 345.) When injected subcutaneously into hepatotomized geese or chickens, or when fed to chickens, a corresponding increase in uric acid secretion is observed (v. Mach). A similar conversion of hypoxanthin into uric acid is effected by emulsions of the liver of certain mammals (Wiener, page 343). This conversion is analogous to that observed by Stadthagen in the case of guanin (pages 344,379 ), and shows that in the xanthin bodies we may have antecedents of uric acid apart from the synthesis of the latter from ammonia in the liver, or from the direct decomposition of nucleins. The process by which this change is effected is undoubtedly one of oxidation.

Guanin, $\mathrm{C}_{5} \mathrm{H}_{5} \mathrm{~N}_{5} \mathrm{O}$, was discovered, in 1844 by Unger, as a constituent of guano, in which it is present in varying quantities according to the region from which the guano comes. Thus the Peruvian guano is reported as containing the largest proportion of this base, and on that account this variety is employed when it is desired to prepare guanin.

Fischer accomplished the synthesis of guanin by changing di-chlor hypoxanthin with ammonia into an amino-oxychlor purin which on reduction with hydriodic acid gave guanin. Its isomer $^{1}$ (p. 341),

${ }^{1}$ Berichte, 30, 2247. 
prepared from di-chlor adenin, resembles guanin very much and inasmuch as it may be looked upon as an oxidation product of adenin Fischer is inclined to believe that it may be found in the animal body. From guanin it is distinguished by the fact that the sulphate contains one molecule of water which is not driven off at $120^{\circ}$, and also by not yielding guanidin on cleavage.

More recently Traube ${ }^{1}$ has effected the synthesis of guanin by starting out with guanidin and cyanacetic acid ethyl ester. A pyrimidin body was obtained which with formic acid gave the purin base guanin.

Guanin has been met with in a very large number of tissues, both animal and vegetable; in the liver, pancreas, lungs, retina, in the thymus gland of the calf, and in the testicle substance of the bull; in the scales of the bleak, and in the swimming bladder of fish, as well as in the excrements of birds, of insects, as the garden spider, in which it occurs with a small quantity of uric acid (Weinmanu), and is to be regarded as a decomposition product of proteids in the tissues of the spider. It is also found in the spawn and testicle of salmon, and Schulze and others have shown it to be present in the young leaves of the plane-tree, of vine, etc., also in grass, clover, oats, as well as in the pollen of various plants. Kresling found it in the pollen of the fir with hypoxanthin and xanthin. Guanin and hypoxanthin, but no xanthin, are present in the seeds of Randia dumetorum (Vogtherr). Schützenberger isolated it, together with hypoxanthin, xanthin, and carnin, from yeast which had been allowed to stand in contact with water at or near the body temperature. On the other hand in sterile auto-digestion of yeast and of pancreas Kutscher found only adenin and guanin; in the former also the hexon bases, ammonia, leucin, tyrosin, asparaginic acid and the base $\mathrm{C}_{8} \mathrm{H}_{6} \mathrm{~N}_{4} \mathrm{O}_{4}$. As pointed out on p. 367 in the presence of bacteria, perhaps owing to the formation of nitrous acid, the guanin is changed to xanthin. Levene obtained guanin and adenin from the nucleinic acids prepared from pancreas and from tubercle bacilli.

Pathologically, it occurs in the muscles, ligaments and joints of swine suffering from the disease known as guanin gout. Normally, guanin, like adenin, is present in muscle tissue only in traces. It has never been found in the urine, though xanthin has been mistaken for guanin by some. In their extensive studies upon the alloxuric bases Krüger and Salomon failed to obtain guanin from 10,000 liters of urine. Possibly the base was changed to xanthin by the nitrous acid formed in the process, although this is not the case with adenin which was fonnd in the urine. Altogether the presence of guanin in urine remains yet an open question. In some cases of exudates and trausudates guanin is present in considerable amounts (Jaksch). 
The pancreas according to Hammarsten yields a nucleo-proteid which on decomposition gives chiefly guanin. Bang has prepared a nucleinic acid, presumably from this proteid, which on hydrolysis yields guanin ( 34.35 per cent.) and no other base. Glycerin, phosphoric acid and pentose are the other products. Should Bang's work be confirmed it will go to show that there are several nucleinic acids in the pancreas, inasmuch as adenin and traces of xanthin and hypoxanthin have been obtained from this organ (Kossel, Levene).

As to the origin of this substance in the organism very little has been known up to within a few years, except so far as it has been shown to be, together with other members of this group, a transitory product in the metabolism of nitrogenous foods and tissues. In the case of the lower animals it is evidently the end-product of all change, inasmuch as it is excreted as such. Our knowledge as to the immediate origin of this and the other allied bases has been extended by the brilliant researches of Kossel and his pupils on the decomposition products of nuclein, in which he has shown that this essential constituent of all nucleated cells, whether animal or vegetable, decomposes under the action of water, dilute acids or enzymes into adenin, guanin, hypoxanthin, and xanthin. We know that the first two bases are readily converted by the action of nitrous acid into the other two; that is to say, a $\mathrm{NH}_{2}$ group in these bases is replaced by an atom of $\mathrm{O}$-a change which it is not at all unlikely takes place in the tissues, perhaps in every cell nucleus. That such a change is quite probable is shown by the putrefaction experiments of Schindler, whereby adenin and guanin were converted respectively into hypoxanthin and xanthin. If this explanation is correct, then adenin and guanin are primary transitional products between the complex nucleo-proteid on the one hand, and hypoxanthin and xanthin on the other. These two, in turn, may form the connecting link between the former and the final waste products uric acid and urea.

Schulze and Bosshard (1886) found in young vetch, clover, ergot, etc., a new base, to which they have given the name vernin. It has the formula $\mathrm{C}_{16} \mathrm{H}_{20} \mathrm{~N}_{8} \mathrm{O}_{8}$, and is of especial interest at this point, since on heating with hydrochloric acid it apparently yields guanin. We have, therefore, at least two well defined sources of guanin, the nucleins and vernin.

Neither adenin nor guanin occurs in normal muscle further than in mere traces, a fact which can only be explained on the ground that the muscle tissue is poor in nucleated cells, and hence in nuclein. Just as the muscle cell has become morphologically differentiated from the typical cell, it may be looked upon also as having undergone a concomitant chemical differentiation (page 349).

Guanin and creatin apparently mutually replace one another. Thus, in the muscle, as just stated, guanin occurs only in traces whereas creatin is especially abundant. This may find its explana- 
tion in the fact that both are substituted guanidins. Creatin is regarded by Hoppe-Seyler as an intermediate product in the formation of urea, and a similar róle probably belongs to guanin. It has already been pointed out (p. 336) that the purin bases may break up in part into pyrimidin bodies and these in turn may yield simpler products. It is therefore possible for guanin to thus yield creatinin which on hydration would give creatin. The formation of the latter from hexon bases such as arginin is a like possibility. The observation of Wiener that uric acid is destroyed by the muscle and kidney of herbivora (beef, horse) and by the liver of carnivora (dog, hog) emphasizes a like action of the muscles with respect to the purin bases. From Stadthagen's experiments on dogs we know that guanin ingested produces an increase in the amount of uric acid and urea excreted, and the same is also true of the nuclein derived from yeast. These results have led him to the conclusion that in mammals uric acid is a direct, or more or less altered cleavage product of proteids, notwithstanding the fact that in birds it is the result of synthesis in the liver (page 344).

Guanin as ordinarily obtained, when ammonia is added to a solution of a salt, forms a white amorphous powder. According to Horbaczewski ${ }^{1}$ it can be obtained in a crystalline form by dissolving the powder in a warm dilute alkali (1-2000), adding about one third volume of alcohol and then acidulating with acetic acid. Any cloudiness that forms is removed by filtration, after which the clear filtrate is allowed to stand. In time the guanin crystallizes in rather large roundish or irregular aggregates which resemble those of creatinin zinc chlorid and consist of long prisms or pyramids microscopically quite unlike those of xanthin. The crystals contain no water of crystallization.

The free base is insoluble in water, alcohol, ether, and ammonium hydrate ; easily soluble in mineral acids, fixed alkalis, and in excess of concentrated ammonium hydrate. It can be heated to above $200^{\circ}$ without undergoing decomposition. When evaporated with strong nitric acid it gives a yellow residue, and this on the addition of sodium hydrate assumes a red color, which on heating becomes purple, then indigo-blue; on cooling it returns to a yellow, passing through purple and reddish-yellow shades, due, according to v. Brücke, to absorption of water. This, the so-called xanthin reaction, was supposed to be due to the formation of xanthin and a nitroproduct. It is given best by guanin, then by xanthin, and is not given by either hypoxanthin or adenin.

Nitrous acid converts it directly into xanthin, thus :

$$
\mathrm{C}_{5} \mathrm{H}_{5} \mathrm{~N}_{5} \mathrm{O}+\mathrm{HNO}_{2}=\mathrm{C}_{5} \mathrm{H}_{4} \mathrm{~N}_{4} \mathrm{O}_{2}+\mathrm{N}_{2}+\mathrm{H}_{2} \mathrm{O} \text {. }
$$

This reaction is identical with that of adenin, whereby hypo${ }^{1}$ Zeits. physiol. Chem., 23, 226, 1897. 
xanthin is formed (see p. 353). By putrefaction in the absence of air it forms xanthin (Schindler). The change can be represented by the equation :

$$
\mathrm{C}_{5} \mathrm{H}_{5} \mathrm{~N}_{5} \mathrm{O}+\mathrm{H}_{2} \mathrm{O}=\mathrm{C}_{5} \mathrm{H}_{4} \mathrm{~N}_{4} \mathrm{O}_{2}+\mathrm{NH}_{3} \text {. }
$$

On oxidation with potassium permanganate it yields urea, oxalic acid, and oxy-guanin. On heating with hydrochloric acid and potassium chlorate it is oxidized to carbonic acid, guanidin, and parabanic acid, according to the equation :

$$
\mathrm{C}_{5} \mathrm{H}_{5} \mathrm{~N}_{5} \mathrm{O}+\mathrm{H}_{2} \mathrm{O}+\underset{\text { PARABANIC ACID. }}{\mathrm{CO}}=\underset{\text { GUANIDIN. }}{\mathrm{CO}-\mathrm{NH}}>\mathrm{NH}+\underset{\mathrm{H}_{2} \mathrm{~N}}{\mathrm{H}}>\mathrm{C}=\mathrm{NH}+\mathrm{CO}_{2} .
$$

This decomposition, noted by Strecker, was repeated and confirmed by Fischer who was unable to obtain alloxan and urea, as in the case of xanthin, but instead isolated parabanic acid and guanidin. The latter may be identified as a picrate (Emich).

According to Strecker, a small amount of xanthin is formed in this reaction, and it is quite possible that this base is also formed on oxidation with nitric acid, especially if it contains nitrous acid.

On decomposition with concentrated hydrochloric acid at $180^{\circ}-$ $200^{\circ} \mathrm{Wulff}$ obtained cleavage products similar to those of xanthin, namely, ammonia, glycocoll, carbonic acid, and formic acid. The reaction is as follows:

$$
\mathrm{C}_{5} \mathrm{H}_{5} \mathrm{~N}_{5} \mathrm{O}+7 \mathrm{H}_{2} \mathrm{O}=4 \mathrm{NH}_{3}+\mathrm{C}_{2} \mathrm{H}_{5} \mathrm{NO}_{2}+2 \mathrm{CO}_{2}+\mathrm{CH}_{2} \mathrm{O}_{2} \text {. }
$$

Guanin combines with acids, bases and salts. It is a very feeble base as seen from the fact that some of its salts, as the hydrochlorid, bichromate, etc., readily dissociate on contact with water, especially at higher temperatures. On the other hand, adenin is a much stronger base. It unites with bases to form crystalline compounds; and with one or two equivalents of acids to form crystallizable salts. Thus, with hydrochloric acid it forms the two salts, $\mathrm{C}_{5} \mathrm{H}_{5} \mathrm{~N}_{5} \mathrm{O} .(\mathrm{HCl})_{2}$ and $\mathrm{C}_{5} \mathrm{H}_{5} \mathrm{~N}_{5} \mathrm{O} . \mathrm{HCl}+\mathrm{H}_{2} \mathrm{O}$. Similar combinations can be obtained with nitric acid. The sulphate, $\left(\mathrm{C}_{5} \mathrm{H}_{5} \mathrm{~N}_{5} \mathrm{O}\right)_{2} \cdot \mathrm{H}_{2} \mathrm{SO}_{4}$, crystallizes in long needles, and, like the other salts, is decomposable by water. It contains two molecules of water, a fact which distinguishes guanin from its isomers. The iodid, nitrate, oxalate, and tartrate are also known.

The platinochlorid, $\left(\mathrm{C}_{5} \mathrm{H}_{5} \mathrm{~N}_{5} \mathrm{O} \cdot \mathrm{HCl}\right)_{2} \mathrm{PtCl}_{4}+2 \mathrm{H}_{2} \mathrm{O}$, is readily obtained in a crystalline condition. The silver compound is soluble in hot nitric acid, and on cooling separates out in fine, needle-shaped crystals, having the composition $\mathrm{C}_{5} \mathrm{H}_{5} \mathrm{~N}_{5} \mathrm{O} \cdot \mathrm{AgNO}_{3}$. For its behavior to Dreclsel's reaction, precipitation with copper solution in the presence of reducing substances, see page 353 . The compound forms as a white flocculent precipitate, which soon turns greenish and tends to disso- 
ciate on contact with water (Balke). It probably has the formula $\mathrm{C}_{5} \mathrm{H}_{5} \mathrm{~N}_{5} \mathrm{O} \cdot \mathrm{Cu}_{2} \mathrm{O}$. As it has more imido groups than adenin and hypoxanthin, it is probable that its solubility is less than that of the copper compound of these bases.

The solutions of the hydrochlorid are precipitated by mercuric chlorid and nitrate, potassium chromate, potassium ferricyanid, and by picric acid. Basic lead acetate gives a precipitate only on addition of ammonium hydrate.

Guanin bichromate, $\left(\mathrm{C}_{5} \mathrm{H}_{5} \mathrm{~N}_{5} \mathrm{O}\right)_{2} \cdot \mathrm{H}_{2} \mathrm{Cr}_{2} \mathrm{O}_{7}$, is in composition analogous to that of adenin, and was obtained by Wulff by adding potassium bichromate to a hydrochloric acid solution of guanin. It appears as well formed, bright, orange-colored, elongated, four-sided prisms, with truncated ends. On contact with water it readily dissociates, especially at $100^{\circ}$. When heated above $100^{\circ}$ it gives off water whereas adenin, which is a stronger base, does not dissociate on contact with water, and is permanent at above $100^{\circ}$.

Guanin metaphosphate, $\mathrm{C}_{5} \mathrm{H}_{5} \mathrm{~N}_{5} \mathrm{O} \cdot \mathrm{HPO}_{3}+\mathrm{H}_{2} \mathrm{O}$, has also been studied by Wulff. It is characterized by extremely slight solubility in water and in dilute acids. Pohl first observed that sodium metaphosphate gave with guanin hydrochlorid a precipitate insoluble in excess of acids, but soluble easily in alkalis. Liebermann obtained a precipitate by adding metaphosphoric acid to a solntion of guanin in sodium hydrate. The same precipitate, according to Wulff, forms when an acid solution of guanin is treated with metaphosphoric acid. Contrary to Liebermann, the salt has a definite composition, and is not prone to dissociation as is the case with other compounds of guanin. The precipitation of guanin is so complete that in the filtrate picric acid gives no reaction, and silver nitrate produces only a slight flocculent precipitate. Inorganic salts, as magnesium sulphate, may prevent or retard the precipitation. It forms an amorphous, porcelain-like mass which is ignited with difficulty. Dried at $120^{\circ}$ it still retains one-half molecule of water. In the presence of water at high temperature, as in drying, a part of the compound is converted apparently into the orthophosphate, so that very accurate quantitative results are not possible.

Liebermann's view that nuclein is a metaphosphate of albumin containing mechanical admixtures of metaphosphates of xanthin bases has been shown to be wrong. As additional evidence is the fact that guanin metaphosphate is very difficultly soluble in ammonium hydrate, while nuclein is extremely soluble in dilute ammonia.

Guanin ferricyanid, $\left(\mathrm{C}_{5} \mathrm{H}_{5} \mathrm{~N}_{5} \mathrm{O}\right)_{4} \cdot \mathrm{H}_{3} \mathrm{Fe}(\mathrm{CN})_{6}+8 \mathrm{H}_{2} \mathrm{O}$. This compound separates slowly, when potassium ferricyanid is added to a solution of the hydrochlorid, as small, bright, brownish-yellow four-or sixsided prisms. At $100^{\circ}$ it slowly loses weight, and heating for several hours at $220^{\circ}-130^{\circ}$ is necessary to expel all the water. The composition of this salt is noteworthy, for one molecule of acid com- 
bines with four of guanin. The trivalent ferricyanic acid does not always exist as such in combination, as in the case of the salt of guanin. On the other hand, as pointed out by Wulff, guanin, though usually a monacid base, may unite with different proportions of acid. Thus four nitrates are known, while in the oxalate and tartrate three molecules of guanin unite with two molecules of acid.

Guanin also forms a compound with ferrocyanic acid, which appears as almost colorless needles.

Guanin nitroferricyanid, $\left(\mathrm{C}_{5} \mathrm{H}_{5} \mathrm{~N}_{5} \mathrm{O}\right)_{2} \cdot \mathrm{H}_{2} \mathrm{NOFe}(\mathrm{CN})_{5}+1 \frac{1}{2} \mathrm{H}_{2} \mathrm{O}$. This is likewise obtained by adding sodium nitroprussid to a solution of guanin hydrochlorid. It forms large, glistening, brick-red, four-sided prisms with pointed ends.

Potassium bismuth iodid produces in even very dilute solution of the salts of guanin a precipitate which consists of fine, rather long red needles. When dry it forms a loose, deep-red mass. On heating even below $100^{\circ}$ water is given off and the color changes to a dark violet. It dissociates readily on contact with water. The formula as determined by Wulff is $\mathrm{C}_{5} \mathrm{H}_{5} \mathrm{~N}_{5} \mathrm{O} . \mathrm{HI} \cdot 2 \mathrm{BiI}_{3}+2 \mathrm{H}_{2} \mathrm{O}$.

Guanin picrate, $\mathrm{C}_{5} \mathrm{H}_{5} \mathrm{~N}_{5} \mathrm{O} \cdot \mathrm{C}_{6} \mathrm{H}_{2}\left(\mathrm{NO}_{2}\right)_{3} \mathrm{OH}+\mathrm{H}_{2} \mathrm{O}$. The reaction with picric acid (Capranica) is said to be very characteristic, and a means of distinguishing this base from xanthin and hypoxanthin. It is best obtained by adding a cold, saturated solution of picric acid or of sodium picrate to the warm acidulated solution of guanin when a light, crystalline precipitate forms. Under the microscope it appears in pencil-shaped, fern-like tufts of fine, orange-yellow needles, rarely in bunches of large needles. Adenin picrate has a lighter color. This compound is characterized by its crystalline form and its extreme insolubility in cold water and in dilute acids. Guanin solutions, 1:30,000, are still precipitated by picric acid, though after some time. When dry it has a golden-yellow color, felt-like consistency, and silky luster. On heating it becomes almost orange-red, and on cooling the original color returns. At $110^{\circ}$ it loses water of crystallization, the silky luster, and becomes light-yellow; at $190^{\circ}$ it begins to decompose. It dissolves easily on warming in fixed alkalis and carbonates. A solution of guanin-sodium is therefore precipitated only by excess of picric acid. It is rather easily soluble in warm dilute acids; difficultly in cold acids. It is dissociated by water, alcohol, and ammonia, especially when warmed (Wulff).

Guanin silver picrate, $\mathrm{C}_{5} \mathrm{H}_{4} \mathrm{AgN} \mathrm{N}_{5} \mathrm{O} . \mathrm{C}_{6} \mathrm{H}_{2}\left(\mathrm{NO}_{2}\right)_{3} \mathrm{OH}+1 \frac{1}{2} \mathrm{H}_{2} \mathrm{O}$, is thrown down from a boiling solution of a guanin salt, treated with excess of picric acid, by silver nitrate as a voluminous, lemon yellow amorphous precipitate. It is very difficultly soluble in hot water, almost insoluble in cold water. It tends to become dissociated on contact with water-picric acid being removed. Ammonia removes the picric acid easily and completely, leaving guanin-silver (Wulff).

Guanin sodium dissociates on contact with water. 
Brom-guanin, $\mathrm{C}_{5} \mathrm{H}_{4} \mathrm{BrN}_{5} \mathrm{O}$, was prepared by Fischer and Reese in 1883 , and is analogous to brom-caffein, adenin, and hypoxanthin. The hydrochlorid dissociates at ordinary temperature. By the action of nitrous acid it is changed into brom-xanthin just as brom-adenin is converted into brom-hypoxanthin. On prolonged heating with strong hydrochloric acid it yields 2-amino 6.8 di-oxy purin (Fischer).

Acetyl guanin, $\mathrm{C}_{5} \mathrm{H}_{4} \mathrm{~N}_{5} \mathrm{O} \cdot \mathrm{CO} \cdot \mathrm{CH}_{3}$, was prepared by Wulff by heating dry powdered guanin with acetic anhydrid. It forms small, colorless, silky needles which are very difficultly soluble in cold water-in about 4,000 parts; more difficultly in cold alcohol and almost insoluble in ether. It is soluble in about 150 parts of boiling water; less easily in hot alcohol. It is easily soluble in dilute acids, alkalis, and ammonia, especially on warming. On heating with acids and alkalis it is completely saponified. From a solution in cold dilute sodium hydrate it is precipitated unchanged by carbonic acid. It is likewise unaffected by boiling water. At $260^{\circ}$ it is apparently unchanged.

Propionyl guanin, $\mathrm{C}_{5} \mathrm{H}_{4} \mathrm{~N}_{5} \mathrm{O} \cdot \mathrm{CO} \cdot \mathrm{CH}_{2} \cdot \mathrm{CH}_{3}$, was also prepared by Wulff by heating dried guanin with propionic anhydrid. Only a small part of the guanin enters into combination. It forms peculiar crystals, which under the microscope appear as rather long plates or scales, frequently with notched edges. The precipitate is very voluminous and when dried forms a light, felt-like, white mass having a mother of pearl luster. Its properties correspond to the acetyl compound. It is likewise apparently unaffected at $260^{\circ}$.

Benzoyl guanin, $\mathrm{C}_{5} \mathrm{H}_{4} \mathrm{~N}_{5} \mathrm{O}$.CO. $\mathrm{C}_{6} \mathrm{H}_{5}$. Benzoic anhydrid reacts even less energetically than propionic anhydrid on guanin, and only a very small quantity of the ester forms. It appears as small round masses, which consist of fine stellate or bunched needles. It is difficultly soluble in hot water and alcohol, insoluble in ether. At very high temperature it decomposes with brown coloration. It is rather resistant to the action of boiling water, but is readily saponified by hot dilute acids. Attempts to obtain this compound by the action of benzoyl chlorid on guanin or on guanin-silver failed.

Alkyl derivatives of guanin. Fischer and Reese attempted unsuccessfully to prepare alkyl derivatives of guanin by the action of methyl iodid on the lead and silver compounds of guanin. Wulff endeavored to prepare benzyl guanin in the same way that Thoiss made benzyl adenin, but failed. Even heating benzyl chlorid with guanin in the presence of sodium hydrate failed. Attempts to prepare a dimethyl derivative were likewise resultless. Recently, however, Fischer has been able to prepare synthetically the 7-methyl and the 1-7-dimethyl guanins. The former is identical with Krüger and Salomon's epiguanin which will be next described. The latter on oxidation yields methyl guanidin, and corresponds to heteroxanthin. 
Ethyl guanin, $\mathrm{C}_{5} \mathrm{H}_{4} \mathrm{~N}_{5} \mathrm{O} \cdot \mathrm{C}_{2} \mathrm{H}_{5}$, was obtained by Wulff by heating guanin with ethyl iodid in the presence of sodium hydrate and alcohol. It forms small needle-shaped crystals. When dry it yields a light, dry mass. It is difficultly soluble in water, very difficultly in alcohol, easily in mineral acids. In general it gives the same reactions as guanin. Thus, it yields a silver compound difficultly soluble in ammonium hydrate; a finely crystalline picrate, etc. The boiling-hot aqueous solution is precipitated by gold chlorid. Apparently it is not altered by heating at $280^{\circ}$.

Physiologically guanin, like uric acid, is inert (Filehne). This may be due to the extreme insolubility of the base (p. 345).

Guanin may be readily prepared from Peruvian guano by boiling it repeatedly with milk of lime until the liquid becomes colorless. The residue, consisting largely of uric acid and guanin, is boiled with a solution of sodium carbonate, filtered, and the filtrate, after the addition of sodium acetate, is strongly acidulated with hydrochloric acid. This precipitates the guanin, together with some uric acid. The precipitate is dissolved in boiling hydrochloric acid, and the guanin then thrown out of solution by the addition of ammonium hydrate.

A more convenient method of isolation of guanin from Peruvian guano is that of Wulff. The guano is boiled with about 5 per cent. sulphuric acid for 4-6 hours, then cooled, and at once filtered. The filtrate is rendered alkaline with sodium hydrate and again filtered. This filtrate, containing guanin and a little uric acid, is now precipitated with ammoniacal silver solution. The voluminous precipitate, after standing twelve hours, is transferred to a thick plaited filter, and washed first with cold then with hot water. While still moist the precipitate is removed from the filter and introduced gradually into hot, dilute hydrochloric acid. The silver chlorid is filtered off, and the filtrate digested on a water-bath with animal charcoal. The clear solution is then saturated with ammonium hydrate to precipitate the guanin. In order to destroy the traces of uric acid which accompany the guanin the precipitate is dissolved together with a small amount of urea, in boiling 20 per cent. nitric acid, then set aside to crystallize. The nitrate of guanin is now dissolved in dilute sodium hydrate, and reprecipitated by addition of ammonium chlorid, thus removing any traces of xanthin that may be present.

Inasmuch as the guanin is present in guano in combination partly with calcium, partly as a nuclein-like body, it is not all set free by a single boiling with dilute acid. The extraction should, therefore, be repeated until it ceases to be given off.

Guanin is also obtained in the decomposition of nuclein with dilute acids, and can, therefore, be prepared from such cellular organs as the spleen, pancreas, etc. It can also be prepared from auto-digested pancreas or yeast (Kutscher). 
It should be noted here that in the decomposition of the mixed silver compounds with hydrogen sulphid or ammonium sulphid (Schindler) the guanin often only in part passes into solution with adenin and hypoxanthin, and the remainder is held back in the silver sulphid precipitate. The latter should, therefore, be boiled with dilute hydrochloric acid, and on saturating the filtrate with ammonia the guanin after a while separates out. That portion of the guanin which does pass into solution with the other two bases is separated from them by digestion with ammonia on a water-bath. The two portions are then combined, tranferred to a filter, previously dried at $110^{\circ}$, and weighed, washed well with ammonia, then dried and weighed.

Owing to the slight solubility of guanin picrate it has been proposed by Wulff as a means for the estimation of guanin. For this purpose the neutral or acid guanin solution is precipitated while warm, with a sufficient amount of cold saturated picric acid solution. After standing twenty-four hours the solution is filtered through a hardened filter, and the precipitate well drained. It is then washed with one per cent. picric acid and allowed to drain, after which it is placed between two watch-glasses, and dried by slowly raising the temperature, finally for one and a half hours at $110^{\circ}$. A deduction is made for the free picric acid by determining the amount of water in the precipitate from the difference in the weights before and after drying. Allowance should be made for one molecule of water of crystallization that is driven off. A further correction for the solubility of the guanin salt should be made by adding 0.0035 for each 100 c.c. of the combined filtrate and wash-water. The results thus obtained are quite satisfactory. Bruhns has employed the picrate of adenin in the estimation of adenin. Xanthin and hypoxanthin were supposed to yield soluble compounds with picric acid, so that either adenin or guanin, or both, could be separated from these bases in this way. Wulff, however, has shown that when guanin is precipitated by picric acid in the presence of hypoxanthin some of the latter is also precipitated, so that it is not possible to separate the two bases in this way. The same is true of adenin and hypoxanthin if the picrate is not filtered off until after some hours.

In the separation of adenin and hypoxanthin from guanin by heating with ammonium hydrate, some guanin is dissolved, so that the filtrate cannot be used with accuracy for the separation of adenin from hypoxanthin by Bruhns' method. Wulff has endeavored to replace the ammonia with metaphosphoric acid. Guanin is precipitated from feebly acid solutions by metaphosphoric acid almost completely. The precipitate filtered off, washed with cold water, dried at $110^{\circ}$, and weighed as $\mathrm{C}_{5} \mathrm{H}_{5} \mathrm{~N}_{5} \mathrm{O} \cdot \mathrm{HPO}_{3}+\frac{1}{2} \mathrm{H}_{2} \mathrm{O}$, gives usually slightly low results, owing to the difficulty of washing the precipitate, and the fact that the amount of water retained in drying varies 
through the partial conversion into orthophosphate. Where more accurate results are desired the precipitate can be transferred to a Kjeldahl flask, and the nitrogen determined. The guanin can then be calculated from the amount of nitrogen found.

Although adenin is precipitated slowly by metaphosphoric aoid, it does not interfere with the separation of guanin, since it is soluble in large excess of reagent. Hypoxanthin does not give rise to a difficultly soluble metaphosphate, hence does not interfere with the precipitation of guanin. In the filtrate from guanin metaphosphate the hypoxanthin can be determined directly by Bruhns' hypoxanthin silver picrate method, though on account of the excess of metaphosphoric acid in the filtrate it is not to be recommended. It would be better to precipitate the filtrate with ammoniacal silver solution, to decompose the silver salts with dilute hydrochloric acid, and then in the filtrate to separate, according to Bruhns, the adenin from the hypoxanthin. The precipitation of guanin in the presence of adenin should be carried out in very dilute solutions, and, as stated, an excess of reagents should be employed. The method possesses decided advantages over the ammonia method of separation, owing to the solubility of guanin in ammonia, especially when hot. According to Wulff, 100 c.c. of a five per cent. solution of ammonia dissolves in the cold $0.01 \mathrm{~g}$. of guanin. A volumetric method for the estimation of guanin by means of copper was suggested by Balke.

Epiguanin, $\mathrm{C}_{6} \mathrm{H}_{7} \mathrm{~N}_{5} \mathrm{O}$, was isolated from urine by Krüger and Wolff in 1893, but it was not until five years later that its composition and structure was definitely determined by comparison with the synthetic 7 -methyl guanin ${ }^{1}$ (p. 341), with which it is identical. This same base was unquestionably isolated by Salomon ${ }^{2}$ as early as 1884 from hog's urine; later from ox urine and leukemic urine. The amount of epiguanin obtained by Krüger and Salomon ${ }^{3}$ from 10,000 liters of urine is about the same as that of adenin $(3.40 \mathrm{~g}$.$) and is$ considerably less than that of xanthin and its derivatives (p. 389).

It is important to note that this base is probably present with adenin and with xanthin, 1-methyl xanthin and hypoxanthin in the adrenals $\left(\right.$ Okerblom $\left.^{4}\right)$. Krüger and Salomon incline to the belief that epiguanin, like the methylated xanthins, results by the cleavage of higher methylated homologues of guanin which are probably present in the food or else it is a constituent of the food and as such passes through the body unchanged. Some epiguanin may unquestionably be derived directly or indirectly from the food, but the probable isolation of epiguanin and the separation of 1-methyl xanthin from the ad-

${ }^{1}$ Berichte, 30, 2411 ; 31, 544.

2 Zeits. physiol. Chem., 18, 207 (1893); 24,390.

'Zeits. physiol. Chem., 24, 387; 26, 367, 389.

- Zeits. physiol. Chem., 28, 60, 1899. 
renals would seem to indicate the existence of di- or trimethyl derivatives of guanin in the body as products of nuclein metabolism. A 1-7 dimethyl guanin for example might give rise to epiguanin, which in turn would yield heteroxanthin (7-methyl xanthin). Similarly a 1-methyl guanin is possible, which apparently is the source of 1-methyl xanthin. Finally paraxanthin (1-7-dimethyl xanthin) may in part be directly derived from the corresponding guanin derivative. Additional light upon the origin of epiguanin has been gained by the observation of Fischer (1898), that it arises from an adenin derivative apparently by intramolecular migration.

The natural as well as the synthetic base is precipitated from concentrated solution of its salts by addition of ammonia and immediate cooling as whetstone-shaped crystals. Heated rapidly in a capillary it begins to color at about $390^{\circ}$ and later chars without melting. The 1-7-dimethyl guanin melts without decomposition at $338^{\circ}-340^{\circ}$ (Fischer). Similar crystals are given by Balke's episarkin, which in many other respects resembles epiguanin. Episarkin, however, is not precipitated by picric acid, but is thrown down by mercuric chlorid and by ammoniacal lead acetate; nor does it give the xanthin reaction. From solutions in sodium hydrate it is precipitated by acids in the form of splendid, matted silky prisms which if agitated in water give a peculiar asbestos-like appearance. The whetstone-like forms on recrystallization from water yield the prismatic type.

It is difficultly soluble in hot water and in ammonia; almost insoluble in alcohol and in cold water, a fact which is of value in the isolation of the base. The solubility in boiling water is 1 to 900 (Fischer). It is easily soluble in dilute alkali and in hydrochloric and sulphuric acids, difficultly in dilute nitric acid from which the nitrate separates on cooling in polyhedric crystals. The sulphate forms fine bent needles, which quickly change to elongated often six-sided plates. From 33 per cent. sodium hydrate solution on cooling it crystallizes, probably as a sodium compound, in broad, glistening pointed needles. Carbonic acid precipitates the free base from its solution in dilute alkali (Fischer).

The picrate, $\mathrm{C}_{6} \mathrm{H}_{7} \mathrm{~N}_{5} \mathrm{O} \cdot \mathrm{C}_{6} \mathrm{H}_{2}\left(\mathrm{NO}_{2}\right)_{3} \mathrm{OH}$, is difficultly soluble in water and is useful for the identification of epiguanin. It forms a voluminous precipitate which consists of anhydrous, peculiarly bent prisms arranged in fan-like bundles, or of rhombic or six-sided plates. At $18^{\circ}$ it is soluble in 2708-2732 parts of water (Krüger and Salomon); at $16^{\circ}$ it is 1 to 3049 . It begins to sinter at about $253^{\circ}$ and decomposes suddenly with evolution of gas at $257^{\circ}$. From a slightly alkaline, warmed solution of the picrate the free base is precipitated at once upon the addition of ammonium chlorid.

The platinochlorid crystallizes from concentrated solution in long glistening, six-sided orange-colored prisms which on warming with 
the mother-liquor change, like the sulphate, to a different form (Fischer). Gold chlorid yields a precipitate of fine yellow needles which, if dissolved in warm water and the solution allowed to evaporate slowly in the cold, form macroscopic, glistening, polyhedric crystals.

Potassium bichromate when added to a solution of the chlorid yields in a short time fine, glistening, four-sided yellow prisms.

With silver nitrate and ammonia the aqueous solution of the base gives a gelatinous precipitate. Silver nitrate added to the nitric acid solution of the base gives a colorless amorphous precipitate which when recrystallized from hot dilute acid forms fine needles (Fischer). Copper sulphate and sodium bisulphite in the cold likewise produce a gelatinous precipitate, which in warm solutions however is flocculent. Copper sulphate and sodium thiosulphate produce in warm solutions a flocculent white precipitate which later turns to brown.

While mercuric chlorid produces in solutions of adenin and of hypoxanthin (0.1 to 500$)$ an immediate flocculent precipitate, with epiguanin a considerable excess of the reagent is necessary and then only a cloudiness results.

An aqueous solution of the base is not thrown down by lead acetate, neutral or basic; nor by lead acetate and ammonia in which respect it behaves like adenin whereas hypoxanthin is completely precipitated by this reagent as a heavy, flocculent or gelatinous precipitate. As Krüger and Salomon point out an ammoniacal solution of lead acetate may by itself on standing give a deposit which should not be mistaken for a precipitate of the base.

On evaporation with concentrated nitric acid it leaves a yellow residue which with sodium hydrate becomes orange-red; on heating this becomes darker and even slightly violet (xanthin reaction). Evaporated with hydrochloric acid and a chlorate it gives a white residue which with ammonia vapors become violet red (murexid test).

With nitrous acid it readily yields heteroxanthin (7-methyl xanthin), and on cleavage with chlorin it gives guanidin (Fischer ${ }^{1}$ ).

Xanthin, $\mathrm{C}_{5} \mathrm{H}_{4} \mathrm{~N}_{4} \mathrm{O}_{2}$, was discovered by Marcet in 1817 in a urinary calculus and since then it has been frequently found as the only or chief constituent of many calculi. It was not synthesized until 1897 when Fischer ${ }^{2}$ prepared it in two ways from tri-chlorpurin. In 1900 Trambe $^{3}$ prepared it, as well as guanin, uric acid and the methyl xanthins, from urea and cyanacetic acid.

Although obviously present in the urine it was not isolated therefrom until very recently. It is a normal constituent but is present

${ }_{1}^{1}$ Berichte, 30, 2413.

${ }^{2}$ Berichte, 30, 2232 ; 31, 2562.

${ }^{3}$ Berichte, 33, 1371, 3035 . 
only in extremely minute quantities. Thus, from 10,000 liters of urine, Krüger and Salomon ${ }^{1}$ obtained $10.11 \mathrm{~g}$. of xanthin, $22.35 \mathrm{~g}$. of heteroxanthin, $31.29 \mathrm{~g}$. of 1-methyl xanthin, $15.31 \mathrm{~g}$. of paraxanthin, $8.50 \mathrm{~g}$. of hypoxanthin, $3.54 \mathrm{~g}$. of adenin and $3.40 \mathrm{~g}$. of epiguanin. It is evident from this that xanthin and its methyl derivatives make up the greater part of the purin bases found in the urine. Hitherto these urinary bases have been considered as metabolic products of the nuclein of the tissues but recent investigations have demonstrated that there is another and indeed principal source for xanthin and its derivatives. It is now known that caffein and theobromin undergo cleavage in the body and yield the less methylated xanthins. With the fact in mind that a large part of the purin bases in the urine are not derived from the nuclein of the tissues but owe their origin to more complex bases in the food it is evident that the determination of the ratio of the nitrogen of uric acid to that of the purin bases is no criterion of cellular metabolism unless the presence of these bases in the food is first eliminated.

During the use of sulphur baths, or after the thorough application of sulphur salves, the quantity of xanthin in the urine is said to be considerably increased. It is likewise more abundant in the urine of leukemic patients, for the reasons already given on p. 348 . In small amounts of lenkemic urine (two cases) Salomon, however, was not able to detect it by means of the sodium reaction. It was found in one out of four pneumonic urines; and in two out of ten normal urines from which at times it may be wholly absent. Baginsky holds that the amount of xanthin normally present in the urine may be increased tenfold in the case of acute nephritis. Bence Jones observed in the urine of a child sick with renal colic a deposit of crystals which he considered to be xanthin, but other observers are inclined to regard the crystals as those of hypoxanthin. Vaughan has reported the presence of xanthin in deposits from the urine of patients with enlarged spleen.

Xanthin, like the preceding three bases, is widely distributed in animal tissues and in plants. In muscle and in the pancreas it was first detected by Scherer (1859) and since then it has been obtained, though often in mere traces, from all nucleated tissues. Thus, according to Schindler, in the thymus gland and in the spermatozoa of the carp there are but very small amounts, if any, of xanthin, whereas the sarkin bases, especially adenin, are present in abundance. This observation has been confirmed by Inoko. The xanthin bases (xanthin and guanin) are obtained in variable but greater amount than the sarkin bases (adenin and hypoxanthin) from the pancreas and from spermatozoa of the bull, boar and salmon. The bases rich in oxygen, as hypoxanthin and xanthin, are more abundant (2 to 1) than those rich in nitrogen (adenin and guanin, Inoko). Inoko

${ }^{1}$ Zeits. f. physiol. Chem., 26, 367, 1898. 
found in the semen of bull the four bases, adenin, guanin, xanthin, and hypoxanthin. In the nucleinic acid from bull's testicles 6.039 per cent. of xanthin was found; guanin was absent. The amount of hypoxanthin was also large, 1.96 per cent. against 0.736 per cent. of adenin. Drechsel isolated xanthin and cystin from the liver of the horse. Unger and Phipson have extracted it from guano.

From fresh adrenals Okerblom obtained less xanthin than when these were digested for two days at $37^{\circ}$ in the presence of chloroform. In the latter case chiefly xanthin, some 1-methyl xanthin and hypoxanthin and traces of epiguanin and adenin were found. On the other hand in auto-digested pancreas and yeast Kutscher found only traces of xanthin and hypoxanthin (p. 348) although Schützenberger found both of these bases and also carnin. Salomon found it among the products of the pancreatic digestion of fibrin but it is clear that the latter must have contained an admixture of nuclein (p. 368).

Together with hypoxanthin, guanin, and possibly adenin, it occurs in many plants, among which may be mentioned lupine, æthalium, sprouts of malt (Salomon, Balke), tea-leaves (Baginsky), gourd seeds, soja beans, etc., in sprouts of Cicer arietinum (Belsung). Xanthin bases are found in sprouts of lupine and of the gourd; with hypoxanthin and guanin it occurs in the fir, Pinus sylvestris. Its presence in auto-digested yeast has already been referred to. As pointed out above the presence of xanthin and its derivatives in food, especially in coffee, tea, cocoa, constitutes the chief source of xanthin and of the allied bases in the urine.

Xanthin on electrolytic reduction yields desoxyxanthin, $\mathrm{C}_{5} \mathrm{H}_{6}$ $\mathrm{N}_{4} \mathrm{O}$ (Tafel and Ach, 1901).

It was held by Strecker that xanthin may be obtained by reduction of uric acid with sodium amalgam according to the equation:

$$
\underset{\text { URIC Acid. }}{\mathrm{C}_{5} \mathrm{H}_{4} \mathrm{~N}_{4} \mathrm{O}_{3}}+\mathrm{H}_{2}=\underset{\text { XaNTHin. }}{\mathrm{C}_{5} \mathrm{H}_{4} \mathrm{~N}_{4} \mathrm{O}_{2}}+\mathrm{H}_{2} \mathrm{O} \text {. }
$$

This view, however, was not confirmed by Fischer (p. 336). More recently Sundwik claims to have effected the reduction of uric acid to xanthin and to hypoxanthin (p. 337). The reverse operation, the conversion of hypoxanthin into xanthin, likewise reported by Strecker, has not been confirmed by Fischer or by Kossel. It is, therefore, evident that while these bodies apparently form a continuous oxidation series with uric acid as the final product, and although this change undoubtedly goes on in the animal economy, yet all attempts to reproduce it in the laboratory by oxidation with potassium permanganate or with nitric acid have yielded only negative results. Nevertheless, in a more indirect way it is possible to convert uric acid into the purin bases and vice versa. This was 
demonstrated in 1895 when Fischer effected the synthesis of caffein (trimethyl xanthin) from dimethyl uric acid; also, the conversion of theobromin into the same uric acid derivative.

Xanthin can be obtained most conveniently by the action of nitrous acid on guanin. The change may be represented by this equation :

$$
\mathrm{C}_{6} \mathrm{H}_{5} \mathrm{~N}_{5} \mathrm{O}+\mathrm{HNO}_{2}=\underset{5}{\mathrm{C}_{5} \mathrm{H}_{4} \mathrm{~N}_{4} \mathrm{O}_{2}}+\mathrm{N}_{2}+\mathrm{H}_{2} \mathrm{O} \text {. }
$$

This reaction, first described by Strecker (1858), and later by Fischer,' corresponds exactly to the one by which Kossel transformed adenin into hypoxanthin (see page 353 ).

By putrefaction guanin is also changed into xanthin, probably because of the action of the nitrous acid elaborated by bacteria (p. 367).

Gautier, starting out on the hypothesis that xanthin is a polymerization product of hydrocyanic acid, endeavored to prepare it directly from this compound. Indeed, he claimed to have succeeded in effecting the synthesis of not only xanthin, but also of its homologue, by simply heating hydrocyanic acid in a sealed tube with water and a little acetic acid, the latter being added to neutralize any ammonia that might form. He expressed the reaction as follows :

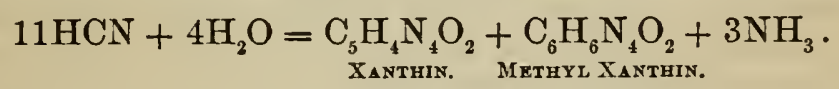

Fischer ${ }^{2}$ on repeating this experiment failed to obtain xanthin and since then Gautier has not demonstrated the correctness of his former view.

Xanthin is a white, granular, amorphous body, and is deposited from hot aqueous solution on cooling in colorless floccules, or as a fine powder, which under the microscope is seen to consist of rounded granules. According to Horbaczewski ${ }^{3}$ it can be easily obtained in the crystalline form by adding acetic acid to an alkaline solution of the base after previously diluting with hot water till the solution is about 1 to 2000 . The clear liquid, filtered if necessary, yields in a few days a deposit upon the sides and bottom of the dish of groups of crystals. These are thin, large, glistening, rhombic plates. If the material is impure lencin-like balls or whetstone-shaped crystals may form. The crystals contain one molecule of water, which is not lost at $110^{\circ}$, but is given off at $125^{\circ}-130^{\circ}$. The synthetic xanthin forms a colorless crystalline powder (Fischer).

When occurring in calculi, it forms compact, moderately hard yellow, or brown fragments which on being rubbed with the fingernail, assume a wax-like appearance; when isolated from the urine

inn. d. Chem., 215, 309.

Berichte, 30, 3131; 31, 449.

3 Zeit. physiol. Chem., 23, 226 (1897). 
it is yellowish, and even when prepared from guanin it is still colored. The decoloration can be effected, according to Balke, by converting the xanthin into the mono-sodium compound which can be recrystallized and then decomposed with acetic acid. The base is thus obtained in snow-white floccules.

It is difficultly soluble in cold water (about 14,000 parts), alcohol, and ether ; somewhat more soluble in boiling water (about 1,200 parts). It is soluble in alkalis and alkali carbonates, not bicarbonates, and from these solutions it is precipitated on neutralization with acids, or by passing carbonic acid. In warm ammonia it dissolves more readily than does uric acid or guanin, and on cooling the ammonium compound recrystallizes. The solubility in cold, even strongly acid solutions, is very slight (Wulff). It acts as a weak base and as a weak acid; with salts of the heavy metals it forms difficultly soluble or insoluble compounds. Its basic properties, however, are weaker than those of hypoxanthin or guanin.

When xanthin is evaporated with nitric acid it leaves a lemonyellow residue (hence its name), which is not changed by ammonium hydrate-distinction from uric acid-but with potassium hydrate becomes yellowish-red, on heating purple-red. This, the so-called xanthin reaction, is not given by hypoxanthin or by adenin. On the other hand, when evaporated with chlorin water, or with hydrochloric acid and a chlorate, it yields a residue which with ammonia gives the murexid test (Fischer, ${ }^{1}$ Kossel).

When added to a mixture of bleaching powder and sodium hydrate in a watch-glass the solution becomes covered by a dark-green scum, which changes to a brown, and soon disappears-distinction from hypoxanthin.

On heating xanthin a small portion volatilizes; the greater part decomposes into ammonium carbonate, cyanogen, and hydrocyanic acid. Heated at $200^{\circ}$ with hydrochloric acid, it decomposes with the formation of ammonia, carbonic acid, formic acid, and glycocoll (E. Schmidt, see p. 342). When bromin is allowed to act on xanthin, there is formed a substitution compound, having the formula $\mathrm{C}_{5} \mathrm{H}_{3} \mathrm{BrN}_{4} \mathrm{O}_{2}$. It may also be obtained by the action of nitrous acid on bromguanin (p. 383). Brom-xanthin is easily prepared and can be readily changed to brom-caffein which in turn can be converted into ethoxy- and hydroxy-caffein or into caffein itself. Obviously this is a crucial test for the recognition of xanthin (Fischer). ${ }^{2}$ The synthetic chlor-xanthin is easily converted in like manner. With potassium chlorate and hydrochloric acid it yields alloxan and urea (p. 341).

Xanthin is a weak base, which dissolves in acids with the formation of salts.

The hydrochlorid, $\mathrm{C}_{5} \mathrm{H}_{4} \mathrm{~N}_{4} \mathrm{O}_{2} \cdot \mathrm{HCl}$, is difficultly soluble in water,

Berichte, 30, 2236.

${ }^{2}$ Berichte, 31, 2563. 
more so than the corresponding salt of hypoxanthin, and is deposited in glistening six-sided plates, often forming aggregations. Its solution does not precipitate platinum chlorid. The nitrate forms fine yellow crystals which when pure are colorless.

The sulphate, $\mathrm{C}_{5} \mathrm{H}_{4} \mathrm{~N}_{4} \mathrm{O}_{2} \cdot \mathrm{H}_{2} \mathrm{SO}_{4}+\mathrm{H}_{2} \mathrm{O}$, crystallizes in microscopic, glistening, rhombic plates, decomposable by water.

With baryta water xanthin forms the difficultly soluble compound $\mathrm{C}_{5} \mathrm{H}_{4} \mathrm{~N}_{4} \mathrm{O}_{2} \cdot \mathrm{Ba}(\mathrm{OH})_{2}$, which corresponds to the hypoxanthin salt $\mathrm{C}_{5} \mathrm{H}_{4} \mathrm{~N}_{4} \mathrm{O} \cdot \mathrm{Ba}(\mathrm{OH})_{2}$, and to that of guanin.

On the addition of a very small amount of sodium hydrate to xanthin it dissolves, and very soon small white needles separate. The crystals dissolve in excess of alkali. This xanthin-sodium compound, $\mathrm{C}_{5} \mathrm{H}_{3} \mathrm{NaN}_{4} \mathrm{O}_{2}+\mathrm{H}_{2} \mathrm{O}$, is also obtained by passing carbonic acid into an alkaline solution of xanthin. It forms small bunched needles, which are rather easily soluble in water, imparting an alkaline reaction. On the addition of acetic acid the pure white base is thrown down. The compound is partly dissociated by hot water, and resembles the corresponding primary uric acid salts. It is probable that xanthin can form, like uric acid, a soluble secondary salt, since with excess of sodium hydrate it forms a readily soluble compound which probably contains two atoms of sodium. For the reactions of this compound, see page 373. (See heteroxanthin and paraxanthin.) It does not give a mono-methyl xanthin by heating with methyl iodid (Balke). The water of crystallization is expelled only at $190^{\circ}-200^{\circ}$.

From ammoniacal solution silver nitrate precipitates the colorless compound $\mathrm{C}_{5} \mathrm{H}_{4} \mathrm{~N}_{4} \mathrm{O}_{2} \cdot \mathrm{Ag}_{2} \mathrm{O}$, which is unaltered by short boiling and is insoluble in ammonia, but soluble in hot nitric acid. From the nitric acid solution, on long standing, there separates the compound $\mathrm{C}_{5} \mathrm{H}_{4} \mathrm{~N}_{4} \mathrm{O}_{2} \cdot \mathrm{AgNO}_{3}$, which, on contact with water, decomposes, giving off nitric acid. The ammoniacal solution is also precipitated by lead acetate-separation from hypoxanthin-also by calcium and zinc chlorids. Cupric acetate gives a precipitate only on boiling. The aqueous solution is not precipitated by lead acetate, but is by phosphomolybdic and phosphotungstic acids, by mercurous and mercuric salts. Picric acid gives an easily soluble compound, which resembles that of hypoxanthin, but differs from that of guanin. Xanthin gives with a copper solution and a reducing substance (Drechsel's reaction, see page 353 ) a milk-white precipitate which eventually becomes bluish-green (Balke). Since xanthin has more imido groups than adenin or hypoxanthin and less than uric acid, it is probable that the solubility of the copper compound will be between the solubilities of the corresponding compounds of hypoxanthin and uric acid ; that is, between $1: 250,000$ and $1: 360,000$, the solubilities respectively in hot water. The copper compound of uric acid is soluble in 560,000 parts of cold water (Krüger). 
As to the physiological relation of xanthin very little need be said (page 369). It bears the same relation to guanin that hypoxanthin does to adenin, and, like the latter, is to be looked upon as an intermediate compound, a step lower than guanin, and nearer the limit of oxidation-uric acid. It is quite probable that in the body it is oxidized about as rapidly as it is formed. Like hypoxanthin, it is to be regarded as a true muscle stimulant, especially of the heart (Baginsky). According to Filehne, it produces in frogs a decided muscular rigor and paralysis of the spinal cord. The heart muscle is also affected, which is not the case with caffein or theobromin. The fatal dose is less than one-half pro mille. In its action it is stronger than theobromin, while caffein is weaker than either of the two. Paschkis and Pal hold that the reverse is true.

As pointed out on p. 339 xanthin may form three mono-methyl derivatives - 1-, 3-, and 7-methyl xanthins ; likewise three di-methyl compounds, namely, theobromin, theophyllin and paraxanthin ; and one tri-methyl derivative, which is caffein. With the probable exception of caffein, all of these methyl xanthin derivatives appear in the urine, where they are to be looked upon not as cleavage products of tissue nuclein, but rather as hydrolytic bodies derived from the caffein and other higher methyl derivatives present in the food. For the existence of other methyl derivatives see page 340 .

1-Methyl xanthin, $\mathrm{C}_{6} \mathrm{H}_{6} \mathrm{~N}_{4} \mathrm{O}_{2}$, were isolated from urine in 1897 by Krüger and Salomon. ${ }^{1}$ As shown on page 389 , it is by far the most abundant of the purin bases in urine. Okerblom has also obtained this base from adrenals (p. 390), which fact demonstrates that 1-methyl xanthin may be derived from higher homologues of xanthin contained within the tissue nuclein, as well as from those contained in the food. As shown below it appears in rabbits' urine after feeding with paraxanthin. The constitution of this base cannot be said to be definitely established. The conclusion as to its structure was reached by comparison with the 3 -and 7 -methyl xanthins, with neither of which it was identical. Hence it was given the structure assigned. The base has not been synthesized.

On treatment with methyl iodid it is easily changed into caffein and into theophyllin (1-3-di-methyl xanthin) which can be identified by the sodium salt which is rather difficultly soluble in ten per cent. sodium hydrate (Krüger ${ }^{2}$ ).

The free base crystallizes best from acetic acid forming very thin, superposed, six-sided, rarely four-sided rhombic plates. It separates from water as dull, colorless crystalline powder which shows rosettelike groups.

It is difficultly soluble in cold water, but more easily than xanthin.

'Zeits. physiol. Chem., 24, 380 ; 26, 367.

${ }^{2}$ Berichte, 33, 3665 (1900). 
It is easily soluble in anmonia and in sodium hydrate, and unlike heteroxanthin it forms an easily soluble sodium compound. The barium salt unlike that of 3-methyl xanthin is very soluble. Unlike 3 -methyl xanthin it is easily soluble in dilute mineral acids and from these solutions on slow evaporation in the cold the corresponding salts are obtained.

The chlorid forms beautiful glassy rhombic plates or prisms. The nitrate forms long four-sided prisms at times shortened to resemble six-sided plates. These salts like those of xanthin and heteroxanthin, are easily dissociated by water.

The aurochlorid forms glistening rhombic prisms. The platinum double salt crystallizes from concentrated solutions in stellate needles or in prisms.

With ammonia and silver nitrate it gives a gelatinous precipitate. The silver nitrate compound crystallized from dilute nitric acid (1.1 sp. g.) forms, like xanthin, fine needles grouped in rosettes. The solubility and crystalline form is such that the two bodies cannot be distinguished and as pointed out by Krüger and Salomon it is probable that the xanthin as heretofore obtained may contain an admixture of the methyl derivative. Copper sulphate and sodium bisulphite produce in the cold a voluminous precipitate, and in warm solutions a white flocculent deposit. Copper sulphate and sodium thiosulphate precipitate only on heating. Mercuric chlorid produces a cloudiness which disappears on heating ; but on the addition of soda a white flocculent precipitate results.

On evaporation with concentrated nitric acid it gives the xanthin reaction. With concentrated hydrochloric acid and a chlorate it gives an intense murexid test. In the latter test the addition of sodium hydrate also gives a purple red color which, when the dish is cool, on the addition of a drop or two of water turns to a splendid bluish violet. The latter disappears on heating. The base yields a bromin derivative which is no longer basic and is difficultly soluble in water and in dilute acids and is not altered at $295^{\circ}$. It dissolves easily in alkalis and in ammonia. The sodium salt crystallizes in six-sided plates.

When paraxanthin or 1-7-di-methyl xanthin is fed to rabbits a part appears in the urine unchanged and in addition 1-methyl xanthin is present (Krüger and Schmidt ${ }^{1}$ ). The 7 -methyl group therefore is split off in the rabbit and this fact supports the view of Krüger and Salomon that this base in man is derived from paraxanthin.

3-methyl xanthin, $\mathrm{C}_{6} \mathrm{H}_{6} \mathrm{~N}_{4} \mathrm{O}_{2}$, has not been obtained from the urine of man; possibly because the methyl group in position 3 is easily split off in the body. However, the line of cleavage depends largely upon the species of animal. It was first found in the urine

${ }^{1}$ Berichte, 32, 2680 (1899). 
of dogs after feeding with caffein (Albanesi, ${ }^{1} \mathrm{Krüger}^{2}$ ). Krüger and Schmidt ${ }^{3}$ found it in the dog and rabbit after feeding with theobromin (see p. 406, caffein).

Before its discovery in urine this base had been prepared synthetically by Fischer and $\mathrm{Ach}^{4}$ out of 3-methyl uric acid in the same way that theophyllin was first prepared from 1-3 di-methyl uric acid.

The free base is readily obtained from its salts which are dissociated on the addition of water or by addition of ammonia and evaporation to dryness. It forms a crystalline powder. From boiling water it crystallizes in fine glistening needles, or in small obliquely truncated prisms. From solution in alkali it is precipitated by addition of acetic acid.

When heated it turns yellow at about $360^{\circ}$ and at higher temperature it gradually decomposes without melting. It is soluble in 350 parts of boiling water (1-200, Albanesi), at $18^{\circ}$ it is soluble in 1,110 parts of water (Albanesi). It is more difficultly soluble in absolute alcohol, and still more so in chloroform and in acetic ether. It is very easily soluble in dilute alkalis and in ammonia. Concentrated alkali precipitates on cooling the sodium salt in the form of very fine bent needles. On boiling with baryta it forms a very difficultly soluble compound $(1: 8,924)$ which crystallizes in very thin, glistening, sixsided plates. This fact distinguishes 3 -methyl xanthin from 1-methyl xanthin just as the solubility of the sodium salt distinguishes it from 7-methyl xanthin.

The base forms with mineral acids crystallizable but unstable salts. The chlorid crystallizes from warm acid solution in fine needles. The iodid forms rather coarse prisms. The nitrate separates from warm acid solution (1.16 sp. g.) as coarse irregular crystals, and because of its difficult solubility it can be used for the purification of the base (distinction from 1-methyl xanthin).

On the addition of barium chlorid to an ammoniacal solution of the base a precipitate forms which when dry dissolves in 480 (516) parts of boiling water ; in $750(862)$ parts of water at $18^{\circ}$.

Silver nitrate added to a nitric acid solution of the base produces a crystalline precipitate, largely consisting of needles which redissolve on heating and on subsequent slow cooling long, thin prisms form. When silver nitrate is added to an ammoniacal solution of the base it yields a white amorphous precipitate which is not altered by heat. The base is thrown down by copper sulphate and sodium bisulphite.

On oxidation with chlorin it yields, like all xanthins, the murexid test.

1 Arch. f. exp. Path. u. Pharm., 35, 449 ; Berichte, 32, 2280.

${ }^{2}$ Berichte, 32, 2818 (1899).

${ }^{3}$ Berichte, 32, 2677.

- Berichte, 31, 1980 (1898). 
On treatment with methyl iodid it can be readily converted into theobromin and into caffein. Like heteroxanthin it can be subjected to electrolytic reduction.

According to Albanesi it is more diuretic than 7-methyl xanthin (heteroxanthin). When fed to rabbits it is in part excreted as such and is unaccompanied by xanthin (Krüger and Schmidt). The greater portion is probably broken up into products simpler than the purins. (See also p. 407.)

Heteroxanthin or 7-methyl xanthin, $\mathrm{C}_{6} \mathrm{H}_{6} \mathrm{~N}_{4} \mathrm{O}_{2}$, was isolated from urine in 1884 by Salomon, and again in 1893 by Balke. The amount present constitutes about two-thirds of that of 1-methyl xanthin (p. 389). It is a remarkable fact that this base occurs in dog's urine unaccompanied by paraxanthin, and the same seems to hold true for the urine of leucocythæmic persons. Salomon examined the liver, muscles, and kidneys of a dog, but was unable to obtain any heteroxanthin or paraxanthin, and the total amount of xanthin bodies present was about normal. For that reason he inclined to the belief that these two bases may possibly have their origin in the kidney. A certain amount of heteroxanthin may unquestionably be derived from the corresponding guanin derivative-epiguanin (p. 386); and its presence in normal dog's urine would indicate that it is in part a product of the metabolism of nuclein. The greater part of the heteroxanthin is known, however, to result from the cleavage of higher homologues present in the food such as caffein and theobromin (p. 406).

Paraxanthin and heteroxanthin are not present in the urine or kidneys of the cow. Salomon found this base in the urine of one out of two leukæmic patients; in one with splenic tumor, and three times out of ten normal urines. The amount of xanthin bodies present in the urine is unaffected by phosphorus poisoning. Unlike the other xanthin bodies, heteroxanthin has not as yet been isolated from plants, meat extract, or guano. Neither it or paraxanthin have been found in bull's testicles (Inoko).

Heteroxanthin has been prepared synthetically by Fischer ${ }^{1}$ out of theobromin. With phosphorus oxychlorid this yields a 7-methyl 2-6-dichlorpurin which on heating with concentrated hydrochloric acid gives heteroxanthin. With slight modification the same method yields paraxanthin, 7-methyl hypoxanthin, 7-methyl guanin, and 1-7-dimethyl guanin. A similar transformation of theobromin into heteroxanthin occurs in the animal body, the methyl group in position three being usually least firmly held in place. Heteroxanthin can be readily methylated to theobromin and to caffein.

Heteroxanthin forms a white amorphous powder, which sometimes on prolonged contact with water forms microscopic crystalline

${ }^{1}$ Berichte, 30, 2400. 
tufts. Balke also described a crystalline modification. The synthetic base forms a colorless indistinctly crystalline powder.

It is very difficultly soluble in cold water; much more easily in hot water, and the solution thus obtained is neutral in reaction. According to Fischer the solubility in boiling water is 1:142. Bondzynski and Gottlieb tested a heteroxanthin which undoubtedly contained 3-methyl xanthin (p. 406) and found it to be more soluble in boiling water (1-109); in water at $18^{\circ}$ the solubility was 1 to 1,592. It was more difficultly soluble in absolute alcohol and was insoluble in chloroform and in ether. It is easily soluble in ammonium hydrate and like uric acid dissolves in piperazin. In 3.3 per cent. sodium hydrate the solubility is about 1 to 2,100 (Krüger and Salomon).

When slowly heated it partially melts and decomposes at $341^{\circ}-$ $342^{\circ}$ but on rapid heating it begins to sinter at about $360^{\circ}$, then colors and finally melts at $380^{\circ}$ with evolution of gas (Fischer).

The hydrochlorid is characterized by its rather difficult solubility and ready crystallization (a distinction from the paraxanthin salt). The salt forms large colorless tufts of crystals, which on contact with water soon lose their transparency and become opaque; gradually their crystalline form disappears, till finally they completely decompose with the formation of heteroxanthin. This decomposition is hastened by warming, either with or without addition of ammonia. The nitrate is more difficultly soluble and crystallizes from ten per cent. acid in bent rhombic plates. Platinum chlorid produces in the hydrochloric acid solution a precipitate of crystalline double salt.

On evaporation with nitric acid on the water-bath (xanthin reaction) it remains as a pure white residue, which on contact with sodium hydrate develops only a trace of reddish coloration or none at all. Weidel's test, with concentrated hydrochloric acid and a chlorate (page 392) produces a splendid red color, which becomes blue on the addition of sodium hydrate. Simple evaporation with chlorin water gives a similar though not so strong a color reaction. By electric reduction it yields desoxyheteroxanthin, $\mathrm{C}_{6} \mathrm{H}_{8} \mathrm{~N}_{4} \mathrm{O}$ (Tafel and Weinschenk ${ }^{1}$ ).

On heating with concentrated hydrochloric acid, or with dilute sulphuric acid, at $180^{\circ}-200^{\circ}$ it decomposes and yields sarkosin and other products, according to the equation (Krüger and Salomon) :

$$
\begin{gathered}
\mathrm{C}_{6} \mathrm{H}_{6} \mathrm{~N}_{4} \mathrm{O}_{2}+5 \mathrm{H}_{2} \mathrm{O}=2 \mathrm{CO}_{2}+\mathrm{CO}+3 \mathrm{NH}_{3}+ \\
\mathrm{CH}_{3} \cdot \mathrm{NH} \cdot \mathrm{CH}_{2} \cdot \mathrm{COOH} .
\end{gathered}
$$

On treatment with methyl iodid it yields caffein.

Silver nitrate produces in ammoniacal as well as in nitric acid

'Berichte, 33, 3369. 
solutions a precipitate which readily dissolves on warming in even very dilute nitric acid; from this solution, if not too concentrated, the heteroxanthin silver nitrate compound crystallizes in characteristic well formed plate-like prismatic crystals which are often crossed. They are soluble in about 2,800 parts of nitric acid of 1.1 specific gravity (Krüger and Salomon). Copper acetate produces in the cold, in solutions of heteroxanthin, a clear green precipitate. The base is also precipitated by phosphotungstic acid, and by ammoniacal basic lead acetate. Picric acid does not give a yellow-colored precipitate in solutions of the hydrochlorid.

Mercuric chlorid readily precipitates heteroxanthin in the form of a grayish-yellow compound, which on standing twelve or twenty-four hours becomes converted into pure white crystalline aggregations. This mercuric compound can be converted directly into the corresponding silver compound by the addition of silver nitrate and ammonia, as described under paraxanthin. For Drechsel's reaction with copper, see page 353 . The precipitate that forms is gelatinous, milk-white, but soon turns green, as in the case of guanin and xanthin. The solubility of the copper compound would be about the same as adenin and hypoxanthin, since it has two imido groups. The addition of barium chlorid to an ammoniacal solution of the base gives a gelatinous precipitate which is rather soluble in hot water and recrystallizes on cooling in rosettes or balls, $\left(\mathrm{C}_{6} \mathrm{H}_{5} \mathrm{~N}_{4} \mathrm{O}_{2}\right)_{2} \mathrm{Ba}$.

This base resembles paraxanthin in its property of yielding a difficultly soluble precipitate with fixed alkali. This reaction is best brought about by dissolving the heteroxanthin hydrochlorid in warm dilute sodium hydrate, when, on cooling, the corresponding sodium salt will crystallize out in oblique-angled plates; or clear long prisms, $\left(\mathrm{C}_{6} \mathrm{H}_{5} \mathrm{~N}_{4} \mathrm{O}_{2} \mathrm{Na}+5 \mathrm{H}_{2} \mathrm{O}\right)$, Krüger and Salomon. ${ }^{1}$ Long-pointed twin crystals are the most characteristic. The crystals show greater variations in form than those of paraxanthin. The behavior of the twin crystals with polarized light is of service in identification. These crystals dissolve easily in water, and on neutralization of the solution with an acid a dense pulverulent precipitate of heteroxanthin forms. This sodium compound, as well as that of paraxanthin, is permanent, non-deliquescent; on moderate heating becomes cloudy, and melts above $300^{\circ}$. It is more difficultly soluble in water than the paraxanthin compound; the solution has an alkaline reaction. It is soluble in mineral acids and ammonia; the latter redeposits it unchanged on evaporation. On neutralization of its solution with mineral acids or with lactic, acetic or carbonic acids, the pure base separates out as amorphous or crystalloidal roundish or rosette-like masses, while paraxanthin under the same conditions forms its characteristic crystals. The sodium is also removed from both compounds by borax, potassium bisulphate, biphosphate of sodium, bisulphite of

${ }^{1}$ Zeits. physiol. Chem., 24, 370. 
sodium and potassium bicarbonate, ammonium bitartrate. Ammonium chlorid, nitrate, sulphate, carbonate, oxalate, tartrate, are transposed to form sodium salts, and the free bases are thrown down. Similar decomposition of the sodium salts of the two bases occurs when placed in urine or in meat extract.

For illustrations of the sodium compounds of the two bases see Virchow's Archives, 125, 556.

The potassium compounds of heteroxanthin and paraxanthin are well crystallized bodies, of high melting-point, and are more soluble than the sodium compound. Their decompositions are the same as those of the sodium salt.

The reaction with sodium is the basis for Salomon's method of recognition of these bases in small quantities of urine. The other two mono-methyl derivatives form soluble sodium compounds.

It can thus be distinguished from paraxanthin, the sodium compound of which, on similar treatment, yields the characteristic crystalline form of the free base. This sodium reaction, therefore, distinguishes it at once from xanthin, hypoxanthin, guanin, and paraxanthin. It differs from the latter, as has already been indicated, in the solubility and amorphous character of the free base; in the behavior of the hydrochlorid and the sodium compound, and in not giving a precipitate with picric acid, nor the characteristic odor given by paraxanthin on heating.

The physiological action of heteroxanthin has been studied by Krüger and Salomon (1895). Its action is almost the same as that of paraxanthin (page 403), indicating a close chemical relation. Its action, however, is much less intense. A dose two or three times greater than that of paraxanthin must be injected into frogs to produce the same symptoms. It has a local action producing early contraction and rigor of the muscles. Its general action is seen in the gradual or rapid paralysis of respiration, according to the dose; in the loss of motion in the extremities, and in the decrease of reflexes. It is not as marked a diuretic as 3-methyl xanthin (pages $345,397)$.

Paraxanthin or 1-7-dimethyl xanthin (p. 339), $\mathrm{C}_{7} \mathrm{H}_{8} \mathrm{~N}_{4} \mathrm{O}_{2}$, was first isolated from urine by Thudichum (1879), who named it urotheobromin. It was again isolated in 1883 by Salomon, who has since shown it to be a constituent of normal urine, although present in exceedingly minute quantity. Thus from 1,200 liters of urine only 1.2 grams ( 0.0001 per cent.) of this substance were obtained. From 10,000 liters of urine the yield was only 15.31 g., or less than the amount of heteroxanthin (p. 389). It was also isolated in 1893 by Balke.

The first synthesis of paraxanthin was effected by Fischer, ${ }^{1}$ who ${ }^{1}$ Berichte, 30, 2408; 31, 2622. 
succeeded in transforming theobromin into this base. Later with Clemm he synthesized it direct from 1.7-dimethyl uric acid, which with phosphorus oxychlorid yields chlorparaxanthin.

The supposition of Fischer that paraxanthin in the urine was derived from caffein in the same way that heteroxanthin results from theobromin, $i$. e., by the splitting off of the methyl group in position 3 has been recently confirmed. When caffein is fed to a dog in small doses it is excreted as 3-methyl xanthin. If, however, large doses of caffein are administered some of the base passes through unchanged. In addition the urine contains all three dimethyl xanthins (paraxanthin, theophyllin and theobromin) and also 3-methyl xanthin (Krüger ${ }^{1}$ ). In view of this fact and inasmuch as paraxanthin has not been met with as a direct cleavage product of nuclein nor found in the urine of dogs or of leukemic patients it is evident that this base when present in the urine owes its origin to the caffein contained in coffee or tea.

Paraxanthin is obtained in colorless, glassy, generally six-sided plates which are arranged in tufts or rosettes. From very concentrated aqueous solutions it crystallizes in long, colorless, interwoven needles which on drying exhibit the silky luster of tyrosin. The crystals belong to the monoclinic system, and may crystallize with, as well as without, water (Salomon ${ }^{2}$ ).

If water is present, on careful heating $\left(110^{\circ}\right)$ the crystals lose their brilliancy and become whitish and opaque, and at $120^{\circ}-130^{\circ}$ the water is completely driven off. The conditions under which crystals containing water are formed are not known, probably by slow crystallization, whereas rapid crystallization from hot concentrated solution yields the anhydrous needles. At about $170^{\circ}-180^{\circ}$ sublimation takes place. The melting-point is at about $284^{\circ}$ (Kossel), $295^{\circ}-296^{\circ}$ (Fischer). Theophyllin melts at $264^{\circ}$, while theobromin sublimes without melting, and caffein melts at $232^{\circ}-233^{\circ}$. It can be heated to $250^{\circ}$ without melting or suffering any decomposition, but when heated more strongly it gives off white vapors which possess a distinct iso-nitril odor; at the same time it carbonizes and takes fire. When evaporated with concentrated nitric acid, as in the ordinary xanthin test, it gives only a slight yellow residue. On the other hand when evaporated with chlorin water or with an acid and a chlorate the dry residue on exposure to an ammoniacal atmosphere under a bell-jar, gives a beautiful rose-red color (so-called Weidel's test).

It is difficultly soluble in cold water (though more easily than xanthin); somewhat more readily soluble in hot water (24 parts, Fischer); insoluble in ether and alcohol. It is soluble in ammonium

${ }^{1}$ Berichte, 32, 2818.

${ }^{2}$ Virchow's Archives, 125, 554; Berichte, 16, 195 ; 18, 3406 ; Zeits. physiol. Chem., 11,$415 ; 13,187$. 
hydrate, hydrochloric acid, and nitric acid. Its solutions are neutral in reaction.

Silver nitrate produces in nitric acid, as well as in ammoniacal solutions, a flocculent or gelatinous precipitate, which in concentrated solutions forms an almost perfect jelly-like mass. This silver precipitate is soluble in warm nitric acid, from which on cooling it separates in white crystalline tufts possessing a silky luster. On decomposition with hydrogen sulphid the silver salt yields pure paraxanthin. Picric acid produces in the hydrochloric acid solution a precipitate consisting of densely felted yellow crystalline spangles. It is also precipitated by phosphotungstic acid and copper acetate; mercuric chlorid when added in excess gives after some time a precipitate composed of a mass of colorless prisms, which are rather difficultly soluble in water; easily in hot water. The crystals of paraxanthin mercuric chlorid when moderately heated become opaque from loss of water of crystallization; at a higher temperature they melt, undergoing at the same time partial decomposition, and on strong heating they evolve disagreeable nauseating vapors. The aqueous solution of this mercuric double salt gives with silver nitrate an abundant precipitate of silver chlorid, which disappears on the addition of ammonium hydrate, and is replaced by the flocculent gelatinous precipitate of silver paraxanthin. The hydrochloric acid solution of paraxanthin crystallizes with difficulty even when strongly concentrated, and on the addition of platinum chlorid it yields a well crystallizable orange-colored paraxanthin platinochlorid. It is not precipitated by basic lead acetate nor by mercuric nitrate. Copper solutions, in the presence of reducing substances, give a flocculent milk-white precipitate, which on washing turns greenish by oxidation (Balke, p. 353). Picric acid gives a yellow precipitate in a hydrochloric acid solution of the base.

Like heteroxanthin it yields a characteristic difficultly soluble sodium salt which can be made use of for purpose of identification and separation. The sodium salt has the formula $\mathrm{C}_{7} \mathrm{H}_{7} \mathrm{~N}_{4} \mathrm{O}_{2} \mathrm{Na}$ $+4 \mathrm{H}_{2} \mathrm{O}$. It forms, upon the addition of sodium hydrate to a concentrated solution of the base, a precipitate of long, glittering, crystalline spangles which under the microscope are seen to consist of delicate rectangular, often longitudinally striated plates which are isolated or united in tufts. The plates show double refraction. Besides these crystals there are also present hexagonal plates resembling cystin. The crystals are soluble in a little water, or on warming, but precipitate again on cooling. Paraxanthin, although it shares with heteroxanthin the property of forming a difficultly soluble compound with fixed alkalis, however can be distinguished from the latter by neutralizing with an acid the solution of the sodium or potassium compound, when, in the case of paraxanthin, there will be obtained a precipitate of the characteristic crystals of that base; 
whereas heteroxanthin on similar treatment gives a dense pulverulent precipitate. This reaction is not given by theophyllin.

Like the other xanthin derivatives on treatment with methyl iodid it yields caffein.

The physiological action of paraxanthin has been studied by Salomon and by Schmiedeberg. Injections into the muscles of 1-2 mg. produced almost at once a rigor mortis-like condition of the muscles affected, with diminished reflex excitability witlout previous increase; 6-8 mg. iutroduced into the lymph sac bring on a gradual loss of voluntary motion as well as of reflex excitability; the rigor is more marked in the anterior extremities, which have a wooden or waxy consistency. Dyspnœa is likewise an early symptom, but as soon as rigor sets in the respirations drop far below the normal and may be absent for several minutes. At times the lungs are enormously dilated, same as with theobromin. The heart's action is intact till the very last. In mice the reflexes are increased almost to a tetanus. An injection of $0.2 \mathrm{~g}$. in a $500 \mathrm{~g}$. guinea-pig produced convulsions and death in half an hour. The same dose introduced into the vein of a rabbit had no effect. The lethal dose for frogs, subcutaneously, was found to be $0.15-0.2$ per cent. of the body-weight-somewhat lower than that of theobromin and xanthin. The action of these three bases is very similar. They produce in common the slow creeping movements, followed by cessation of spontaneous muscle action, complete loss of reflex excitability without a previous rise. The heart's action is not affected till in the latest stages.

Krüger and Schmidt ${ }^{1}$ found that paraxanthin when fed to rabbits was excreted in part as such and in part as 1-methyl xanthin. From this and similar experiments with theobromin and caffein it would seem that at least in the bodies of rabbits the methyl group in position 3 is most easily split off, the 7 -methyl group next and lastly that in position 1. When, however, 3-methyl xanthin is fed to rabbits it is in part excreted as such but this may be due to the rather large doses administered. For the studies of Schmiedeberg see page 345 .

According to Rachford ${ }^{2}$ the xanthin bodies are important factors in the causation of migraine. From the urine passed immediately after an attack of this disease he obtained an extract which he believed to contain relatively large quantities of paraxanthin, and he ascribed the marked toxicity of such extracts to this base. Pfaff, however, failed to find any increase of paraxanthin and interpreted the toxicity of the extracts as due to the presence of ammonium salts resulting from the method employed. Our present knowledge regarding the origin of this base may be considered as definitely elimiting paraxanthin as a possible etiological factor.

${ }^{1}$ Berichte, 32, 2677.

2 Trans. Assoc. Amer. Physicians, 10, 11, 14, 224 (1899). 
Theophyllin or 1-3-di-methyl xanthin (p. 339), $\mathrm{C}_{7} \mathrm{H}_{8} \mathrm{~N}_{4} \mathrm{O}_{2}$, was isolated in 1888 by Kossel ${ }^{1}$ from the alcoholic extract of tea leaves. It has not been met with as a product of nuclein metabolism in the body nor even as a cleavage product. According to Albanesi a dimethyl xanthin, presumably theophyllin, was present in his urine after taking caffein. This, however, has been doubted by Krüger. ${ }^{2}$ Nevertheless, that theophyllin may be present in the urine is shown from Krüger's own experiments with caffein on dogs (p. 406). When large doses were administered all three di-methyl xanthins were present in the urine and of these theophyllin was the most abundant. It would seem therefore that in the dog, at least, caffein undergoes cleavage, yielding as the chief di-methyl xanthin, theophyllin, and on further cleavage this yields 3-methyl xanthin. In other words the methyl group in position 7 is the first to be split off and that in position 1 follows next. The large amounts of 1 - and 7-methyl xanthins in the urine of man would indicate that in the human body the cleavage is different in kind from that in the dog and is more nearly like that in rabbits.

Theophyllin was the first xanthin derivative synthesized by Fischer and Ach. ${ }^{3}$ 1.3-di-methyl uric acid with phosphorus oxychlorid yields chlor-theophyllin which on reduction yields the base (p. 337). Traube ${ }^{4}$ prepared theophyllin as well as the above uric acid out of di-methyl urea and cyanacetic acid. With methyl iodid it yields caffein.

In tea extract the base is associated with caffein, xanthin, adenin and hypoxanthin. Further investigations are necessary to determine whether the other di-methyl xanthins and the mono-methyl xanthins are present in the extract. The origin of these xanthin derivatives in the tea-plant is likewise involved in obscurity. They may be regarded as direct synthetic products or as cleavage forms from nuclein.

Inasmuch as theophyllin cannot be regarded as a product of tissue metabolism a detailed description of its properties in this connection is unnecessary. The reader is therefore referred to Kossel's original paper.

Theobromin or 3-7-di-methyl xanthin (p. 336), $\mathrm{C}_{7} \mathrm{H}_{8} \mathrm{~N}_{4} \mathrm{O}_{2}$, was isolated from cocoa in 1842 by Woskresensky, but its structure was not determined until 1883. It has been found only once in urine, and then in the case of a dog after administration of caffein in large doses (p. 406). The amount of theobromin was about the same as that of paraxanthin and was much less than that of theophyllin.

1 Zeits. physiol. Chem., 13, 298.

${ }^{2}$ Berichte, 32, 2819, 2280.

${ }^{3}$ Berichte, 28, 3135.

- Berichte, 33, 3052. 
Obviously theobromin may be expected under like conditions in human urine.

The base has been synthesized by Fischer ${ }^{1}$ out of 1-3-di-methyl uric acid; also from uric acid itself by converting this into 3-methyl uric acid. Traube ${ }^{2}$ likewise prepared theobromin by treating 3methyl xanthin with methyl iodid. Theobromin can be readily methylated to caffein.

The changes which theobromin undergoes in the body have been the subject of a number of very interesting investigations. Bondzynski and Gottlieb ${ }^{3}$ in 1895 showed that it underwent cleavage when fed to rabbit and to dogs. In rabbits only 19 per cent. of the theobromin was recovered unchanged, and 24.6 per cent. was obtained as a methyl xanthin which these workers identified with beteroxanthin, although as pointed out on p. 398 this substance must have been mixed with some 3 -methyl xanthin. Xanthin itself was not obtained. Similar though incomplete experiments with caffein seemed to give the same methyl xanthin. They also found that when theobromin was administered to a leukemic that it was excreted as such and as methyl xanthin in about the same amounts as in a healthy person. According to Rost's ${ }^{4}$ experiments the dog will excrete about 31.8 per cent. of the administered theobromin; the rabbit 28 per cent. and man 20 per cent. Unlike caffein, the base produces diuresis in the dog and hence the much greater elimination of theobromin (page 345).

About the same time Albanesi experimented with caffein and likewise obtained a methyl xanthin which was assumed to be heteroxanthin, but was eventually shown to be 3-methyl xanthin (p. 395). Krüger and Schmidt fed theobromin to dogs and found that they eliminated some unchanged base, chiefly 3 -methyl xanthin and some 7-methyl xanthin or heteroxanthin. In rabbits the results were qualitatively alike, but differed quantitatively. In addition to theobromin their urine contained chiefly 7 -methyl xanthin and but little 3-methyl xanthin. Later Krüger showed that caffein splits up in the dog into theobromin and other cleavage products (p. 406).)

Caffein or 1.3.7-tri-methyl xanthin (p. 340), $\mathrm{C}_{8} \mathrm{H}_{10} \mathrm{~N}_{4} \mathrm{O}_{2}$, is the active principle of tea and coffee and while it is not a product of tissue metabolism in the animal body it is of interest because of its relation to xanthin and its derivatives as well as to uric acid. Of the ordinary purin bases found in human urine only adenin, hypoxanthin and xanthin are cleavage products of the nucleins of the tissues. 1- and 7-methyl xanthins (heteroxanthin) and 1-7-di-methyl xanthin (paraxanthin) are undeniably derived from the caffein taken

${ }^{3}$ Berichte, 30, 1839 ; 31, 1980 ; 32, 470.

2 Berichte, 33, 3050.

${ }^{3}$ Archiv. Palh. u. Pharm., 36, 45, 133 ; 37, 385 ; Berichte, 28, 1113.

'Archiv. Path. u. Pharm., 36, 56. 
into the body. Furthermore 3-methyl xanthin and the other two di-methyl xanthins, theophyllin and theobromin, appear in the urine after caffein administration and are therefore likewise cleavage products of this base.

To Bondzynski and Gottlieb and to Albanesi is due the credit of having demonstrated that caffein and theobromin were demethylated within the animal body. The former worked chiefly with theobromin and showed that this base was changed in dogs, rabbits and in man to heteroxanthin. Krüger and Schmidt eventually showed that in addition to heteroxanthin also 3-methyl xanthin was formed.

Albanesi on feeding caffein to dogs obtained what he first believed to be heteroxanthin but which on further study by himself and by Krüger was shown to be 3-methyl xanthin. The investigations of the latter are the most exhaustive. They show that when caffein is given to the $d o g$ in small quantities it is excreted as 3-methyl xanthin. In large doses, however, the demethylation is not so complete and hence the several intermediate products appear. Some caffein passes through unchanged. Each of the three methyl groups are attacked in the organism and as a result the three dimethyl xanthins, theophyllin, theobromin and paraxanthin, appear in the urine. The first mentioned predominates and the other two are present in about equal amounts. 3-methyl xanthin apparently is the only mono-methyl derivative present. The demethylation is unquestionably different in different animals as can be seen from experiments with theobromin (p. 405), and with paraxanthin (p. 403). Albanesi's statement that caffein in rabbits was changed to xanthin, and in man to di-methyl xanthin (theophyllin ?) has been questioned by Krüger. While in dogs the cleavage of caffein yields chiefly theophyllin and 3-methyl xanthin, it would seem that in man paraxanthin and 1- and 7-methyl xanthins are the more resistant.

It seems to be well established that caffein does not increase the amount of uric acid in the urine. As shown above, however, it does materially increase the amount of the purin bases. Krïger and Schmid have shown that the increase of purin bases is not proportional to the amount of caffein administered. Thus, when only $0.05 \mathrm{~g}$. of caffein was given 33 per ceut. of its nitrogen appeared as purin bases. With $0.2 \mathrm{~g}$. of caffein only 19 per cent. of the nitrogen was found in this condition. The ratio of the nitrogen of uric acid to that of the purin bases is $13.5: 1$ or $12.7: 1$. After caffein administration it falls to $9.9: 1$ or $8.35: 1$.

The results with theobromin were in some respects very different. Like caffein it does not increase the amount of uric acid but it has a greater influence upon the purin bases-the ratio being 2.6:1. In other words 47 per cent. of the theobromin nitrogen appeared in the form of purin bases.

As yet there is no direct evidence going to show that xanthin is 
formed during the demethylation of caffein and of the other higher homologues. The possibility of this taking place must be conceded. On the other hand it is evident that the purin group undergoes to a large extent complete cleavage. These simpler products are as yet unknown but it is easy to see that pyrimidin derivatives and even creatinin may originate during the cleavage of purin bodies. Wiener's observations on uric acid show that this purin body is not only a nuclein cleavage product but that it may also be a synthetic one. Furthermore he has shown that uric acid is not only being made but is also being constantly destroyed within the body. This process of formation and of decomposition results in the normal minimum excretion of uric acid (see page 344). If, however, the latter change is diminished or inhibited the amount of uric acid becomes apparently increased and may lead to disease conditions such as rheumatism. The same view may be extended to the purin bases and the relatively large elimination of these bodies in leukemia may be due not only to increased formation but also to decreased destruction.

For the pharmacological action of caffein and other purins see page 345 .

Caffein was discovered in 1820 by Runge. It can be readily prepared from xanthin or from either of the mono-methyl or dimethyl derivatives. It has been synthesized from the mono-, di-, tri-, and tetra-methyl uric acids. In other words it can be prepared from uric acid direct (Fischer). It has also been synthesized from di-methyl urea and cyanacetic acid (Traube).

\section{Separation of the Purin Bases.}

The detection and separation of the purin bases in urine offers considerable difficulty which will be readily understood when it is remembered that as many as fourteen of these bases have been found in that secretion. Most of these, as indicated elsewhere, do not represent tissue metabolism proper but rather cleavage products of the purins contained in the food and drink.

Neubauer's method, which has been employed in some modification or other for the isolation of purin bases, depends upon the fact that they are precipitated from an ammoniacal solution by an ammoniacal silver solution. This reaction, as Krïger has pointed out, is given by all xanthin bases which contain an imido group capable of substitution. It is not given by caffein, the fully methylated xanthin, or by dimethyl hypoxanthin. The further separation of the bases is accomplished by dissolving the silver precipitate, together with a little urea, to destroy any nitrous acid, in boiling nitric acid of 1.1 specific gravity. The hot solution is filtered, and from the filtrate, on cooling, the "hypoxanthin fraction" of Salomon -hypoxanthin, guanin, carnin, adenin, and episarkin, crystallize as the corresponding silver nitrate compounds. That portion of 
silver salts remaining in solution in the nitric acid, the "xanthin fraction," consists of xanthin, paraxanthin, and heteroxanthin.

The urine, acidulated with hydrochloric acid, is precipitated with phosphotungstic acid; the precipitate is decomposed by warming with baryta, filtered, and the filtrate is freed from barium by the cautious addition of sulphuric acid. The solution is then made alkaline with ammonium hydrate, any traces of phosphates that appear are filtered off. Or, the urine may be rendered directly alkaline with ammonium hydrate and after standing 24 hours the precipitated phosphates removed by filtration. The filtrate finally is precipitated by the addition of ammoniacal silver nitrate $(0.5 \mathrm{~g}$. per liter). The precipitate which forms consists of the silver compounds of the xanthin bodies, and is purified by dissolving in boiling nitric acid of $1.1 \mathrm{sp} . \mathrm{g}$. The filtrate, on cooling, yields a precipitate of the silver nitrate compounds of adenin, hypoxanthin, and guanin. The xanthin compound remains in solution. The silver salts of the three bases mentioned are decomposed with dilute hydrochloric, or with hydrogen or sodium or ammonium sulphid, and the acid solution of the three bases is then heated on the water-bath with excess of ammonia. Guanin is thus thrown out of solution. In the ammoniacal filtrate the adenin is separated from the hypoxanthin and estimated by picric acid (see p. 411). The hypoxanthin is estimated as hypoxanthin silver picrate. Instead of the separation of guanin from the other bases by ammonia Wulff's metaphosphate method may be employed (p. 385). The adenin and hypoxanthin are then precipitated from the filtrate by ammoniacal silver solution. The silver salts decomposed with hydrochloric acid, and in the filtrate the adenin can be estimated as the picrate; the hypoxanthin as the hypoxanthin silver picrate.

A second method for the extraction of the xanthin bases is based upon Drechsel's reaction, namely, that they are precipitated by a copper solution in the presence of a reducing agent (see page 353). The precipitation is so complete that the filtrate does not react with ammoniacal silver nitrate. The reliability of the process has been quantitatively tested by Balke, who found the yield of xanthin bases from meat extract to be slightly greater by the copper method over Neubauer's silver nitrate process. In addition to cheapness the method possesses an advantage in the less bulky precipitates.

As reagents Balke employed Fehling's solution and hydroxylamin hydrochlorid, whereas Krüger made use of copper sulphate (13 per cent.) and sodium bisulphite (1-2). The latter solutions are obviously preferable.

The urine or other liquid to be examined is first freed from any albumin that may be present. It is then boiled and the copper and bisulphite solutions are added. Barium chlorid may be added to facilitate the settling of the precipitate, which after standing several 
hours is filtered off and repeatedly washed with warm water $\left(60^{\circ}\right)$. The precipitate is then suspended in acidulated water and decomposed with hydrogen sulphid. To destroy the uric acid which may be present the solution is acidulated with acetic acid and boiled with manganese dioxid, after which the dissolved manganese is precipitated by boiling with ammonium carbonate and hydrate. The purin bases may now be reprecipitated with copper sulphate and bisulphite solutions.

All the xanthin compounds containing an imido group capable of substitution, with the exception of theobromin, are precipitated. Caffein and dimethyl hypoxanthin contain no imido group, and are therefore not precipitated. Neither are urea, allantoin, amido acids, pepton, albumose, creatin and creatinin thrown down. The more imido groups present in a compound the less soluble is the precipitate.

Krïger and Wulff in 1894 applied the copper method for the determination of the relative amounts of purin bases and uric acid in urines (see Novy's Physiological Chemistry). The ratio of the nitrogen of the former to that of the latter is of little value when it is remembered that the greater part of the purin bases in the urine are derived from the caffein and similar bodies in the food and have nothing to do with cell metabolism.

The Neubauer method as ordinarily employed does not yield as satisfactory results as may be expected. As pointed out by Krüger and Salomon the use of nitric acid would cause an oxidation of carnin to hypoxanthin. Moreover, any nitrous acid present would convert more or less completely the guanin into xanthin and the adenin into hypoxanthin. This change as Krïger has shown is not prevented by the addition of even a large excess of urea. They thus explain their non-detection of carnin and guanin in the urine. A more serious objection was pointed out by them in connection with the separation of the purin bases into the two fractions by means of boiling nitric acid. The supposition that the silver salts of the xanthin fraction are easily soluble in the nitric acid is not correct. Thus, the silver salt of heteroxanthin requires 2,820 parts of nitric acid $(1.1 \mathrm{sp} . \mathrm{gr}$.) for solution. It follows therefore that the hypoxanthin fraction as ordinarily obtained always contains considerable amounts of the xanthin fraction.

In the course of their exhaustive investigation upon the urinary bases Krüger and Salomon ${ }^{1}$ recognized these defects and devised the following method of separation:

The purin bodies may be precipitated either by means of ammoniacal silver solution or by means of copper sulphate and sodium bisulphite as already described. In the former case the washed precipitate is transferred to a round bottom flask and decomposed on a

${ }^{1}$ Zeits. physiol. Chem., 26, 373 (1898). 
water-bath by cautious addition of dilute hydrochloric acid till the original voluminous precipitate is replaced by that of silver chlorid. The liquid is then heated over a flame and hydrochloric acid, equal to that already used, is added. The solution is filtered while hot and the precipitate, which contains most of the uric acid, is thoroughly washed with very dilute hydrochloric acid.

When the copper method is used the precipitate, after thorough washing with boiling water, is transferred to a flask, then warmed and rendered alkaline with ammonium hydrate. The liquid is then strongly acidulated with hydrochloric acid and the copper is precipitated by means of hydrogen sulphid. The precipitate is filtered while hot and freely washed. It contains the greater part of the uric acid.

The acid filtrate obtained by either method is concentrated on the water-bath, preferably at a low temperature and with constant stirring. To remove the acid from the syrupy residue this is twice taken up with water and reëvaporated. This operation is repeated with 96 per cent. alcohol till the acid is expelled and the residue becomes granular. This is then digested for several hours at $40^{\circ}$ with distilled water; the insoluble part is filtered off and washed with water, then with alcohol and with ether. The aqueous filtrate, evaporated and the residue treated as before, may yield a slight insoluble portion which is to be combined with the main quantity.

The insoluble residue contains xanthin, heteroxanthin, and 1-methyl xanthin. The aqueous filtrate contains adenin, hypoxanthin, paraxanthin and epiguanin with traces of heteroxanthin and 1-methyl xanthin. In other words xanthin and hypoxanthin fractions result the same as in Neubauer's method with the difference that paraxanthin appears in the latter.

1. In order to separate the three bases present in the xanthin fraction the insoluble residue is dissolved in fifteen parts of hot 3.3 per cent. sodium hydrate ( $\mathrm{HCl}$ free). Within 24 hours the sodium salt of heteroxanthin separates out pure and almost quantitatively.

This is filtered off and the filtrate is divided into portions of 60 c.c. and warmed to $60^{\circ}$. Each portion is then poured slowly and with constant stirring into a cold mixture of 20 c.c. of concentrated nitric acid and 20 c.c. of water. This dilute acid should be boiled before being used. The uric acid remaining in solution is thus destroyed and the solution on standing a few hours in the cold yields a precipitate of xanthin nitrate. This, if pure, is a heavy crystalline powder consisting of masses of plates. If impure, the airdried precipitate should be divided into portions of $3 \mathrm{~g}$., each of which is then dissolved in warm sodium hydrate and the solution diluted to 60 c.c. is treated as before. To obtain free xanthin the nitrate is dissolved in ammonia and from the solution when evaporated xanthin separates out in amorphous crusts. 
The filtrate from the xanthin nitrate saturated with ammonia and concentrated yields a satin-like mass consisting of microscopic plates of 1-methyl xanthin. The remainder in solution can be precipitated with ammoniacal silver solution or with copper sulphate.

2. The filtrate from the xanthin fraction on addition of a slight excess of ammonia yields at once a precipitate of glistening prisms - epiguanin. This is filtered off and the ammonia is expelled from the filtrate by heating. The solution which should not be too concentrated is then treated in the cold with a 1.1 per cent. solution of picric acid to a slight excess. The adenin picrate is at once removed by means of a suction pump.

The filtrate from the adenin is acidulated with sulphuric acid and extracted with benzol or toluol to remove picric acid. After which the remaining bases are precipitated with ammoniacal silver solution or with copper sulphate and bisulphite. The precipitate is decomposed with hydrogen sulphid after which the aqueous filtrate is evaporated to dryness. Portions of $3 \mathrm{~g}$. each, of the dry residue, are dissolved in 100 c.c. of hot dilute nitric acid $\left(90\right.$ c.c. $\mathrm{H}_{2} \mathrm{O}$ +10 c.c. conc. $\mathrm{HNO}_{3}$ ). On cooling pure hypoxanthin nitrate separates out.

The filtrate from this deposit contains small amounts of hypoxanthin, heteroxanthin, and 1-methyl xanthin besides paraxanthin. To separate these bases the entire method is repeated from the beginning. The above filtrate is precipitated with ammoniacal silver solution or with copper sulphate and sodium bisulphite. The hydrochloric acid filtrate, obtained by decomposing the precipitate in the manner previously described, is evaporated and the residue is extracted as before with as little cold water as possible. The insoluble portion contains heteroxanthin and 1-methyl xanthin, and these are separated by means of a 3.3 per cent. solution of sodium hydrate. The filtrate contains hypoxanthin and paraxanthin. In order to remove the hydrochloric acid these bases are again precipitated as silver or copper compounds. These are decomposed and the dry residue dissolved in a little hot dilute nitric acid (as above) yields on cooling hypoxanthin nitrate. The filtrate contains paraxanthin which can be obtained now as the sodium salt or as the free base.

Guanin, if present, is to be expected in both fractions. The xanthin fraction, treated with ammonia, would yield the insoluble guanin. From the hypoxanthin fraction guanin and epiguanin would be precipitated together by ammonia. It can be separated from the latter by treatment with hot water or hot dilute ammonia.

Inasmuch as caffein and theobromin are not precipitated by ammoniacal silver solution or by the copper sulphate method it is necessary in order to detect these bases to resort to precipitation with phosphotungstic acid. For this purpose the urine, acidulated with sulphuric acid, is treated with this reagent. The precipitate is de- 
composed in the cold with baryta water, treated with carbonic acid and finally the liquid is warmed on the water-bath and filtered hot. The filtrate is then precipitated with copper sulphate and sodium bisulphite to remove the ordinary purin bases. The filtrate from the copper precipitate is treated with hydrogen sulphid, the resulting clear solution is evaporated and the residue extracted with chloroform. This takes up the caffein and theobromin. To separate these the chloroform solution is evaporated and the residue is taken up with water, and the solution treated with silver nitrate and a slight excess of ammonia. The ammonia is then expelled by boiling and the solution on cooling yields a deposit of the theobromin silver compound. The filtrate from this deposit is acidulated with hydrochloric acid and extracted with chloroform, which on evaporation yields caffein.

The 3-methyl xanthin is separated from the purin bases as the insoluble barium salt. Theophyllin is separated from paraxanthin by recrystallizing the silver nitrate compounds from nitric acid (Krüger). ${ }^{1}$

Separation of uric acid from xanthin bases.

In the precipitation of uric acid by the silver method the xanthin bases if present are likewise thrown down. However, as adenin and hypoxanthin are readily soluble in acids and alkalis, they are easily separated after decomposition of the silver precipitate. Xanthin and guanin are less soluble, and hence may render the uric acid impure especially when this is isolated from animal organs. Wulff detected the presence of xanthin in a uric acid precipitate by destroying the uric acid with hot dilute nitric acid-the xanthin remaining unchanged. The substance is heated on the water-bath with dilute nitric acid (100 parts water and 5 parts nitric acid, $1.14 \mathrm{sp}$. g.), and after gas ceases to be given off it is boiled for a short time, rendered ammoniacal, and if xanthin is present it gives a precipitate with silver nitrate. The precipitate can be collected on a weighed filter, dried at $120^{\circ}$, and weighed as $\mathrm{C}_{5} \mathrm{H}_{4} \mathrm{~N}_{4} \mathrm{O}_{2} \cdot \mathrm{Ag}_{2} \mathrm{O}$; or from the weight of silver after ignition the amount of xanthin may be calculated. If the amount of xanthin is large, it can be weighed directly. For this purpose the solution is rendered slightly ammoniacal, after the oxidation with nitric acid, and then is treated with acetic acid and an equal volume of alcohol. After twelve hours filter, wash with dilute alcohol, dry at $110^{\circ}$, and weigh.

The destruction of uric acid by nitric acid and by manganese dioxid has been already referred to. Horbaczewski's method for the separation of uric acid from xanthin is as follows: The mixture of the two substances is dissolved with aid of gentle heat in a platinum dish in concentrated sulphuric acid (2 c.c. for 0.1 g. substance).

'Berichte, 32, 2681, 2823, 3336. 
The solution is diluted with four parts of water, stirred thoroughly, and set aside for 3-6 hours. The uric acid is thus precipitated, and is then collected on a small filter, washed with water, acidulated with sulphuric acid, then with pure water. Then it is transferred to the dish in which it was precipitated, dissolved in a little pure sodium hydrate (e natrio), strongly acidulated with hydrochloric acid, and evaporated to a fow c.c. After standing one hour it is filtered through Ludwig's glass-wool filter, washed with HCl-water, then with water, finally with alcohol and ether, dried at $110^{\circ}$, and weighed. The filtrate and wash-water are combined, and a correction is made for the solubility $(1: 16,000)$ of uric acid. This correction is added to the weighed amount. The results obtained by this method are excellent. The separation from guanin and xanthin is complete.

Carnin, $\mathrm{C}_{7} \mathrm{H}_{8} \mathrm{~N}_{4} \mathrm{O}_{3}$, was isolated in 1871 from American meat extract by Weidel, but it has not been obtained from muscle tissue itself. Balke, however, has isolated it from fresh horse-meat extract. It has also been obtained from yeast liquors by Schützenberger, and Pouchet claims to have isolated it from urine, but has not offered sufficient evidence on this point. Salomon (1893) obtained a body resembling carnin from leukæmic urine.

It can be separated from the meat extract by the following method originally employed by Weidel: The extract is dissolved in six or seven parts of warm water, then concentrated baryta water is added, avoiding, however, an excess. The filtrate is precipitated by basic lead acetate. The precipitate is collected, thoroughly washed and pressed, and finally it is repeatedly extracted with a large quantity of boiling water. The carnin lead salt is thus dissolved out; the filtrate, after removal of the lead by hydrogen sulphid, is evaporated to a small volume. The concentrated solution thus obtained is treated with silver nitrate, which gives a precipitate of silver chlorid and of the silver salt of carnin. By treatment with ammonium hydrate the silver chlorid can be completely removed from the precipitate, whereas the silver compound of carnin is insoluble in that reagent. To obtain pure carnin the silver salt is decomposed with hydrogen sulphid, and the filtrate, after purification by bone-black, is evaporated to crystallization. According to Weidel, carnin forms about 1 per cent. of the meat extract. Kemmerich (1893) has found only one-quarter of 1 per cent., or even less. The amount, therefore, varies considerably, and may be very small in the fresh extract.

Carnin forms white crystalline masses which on drying become loose and chalk-like. It is very difficultly soluble in cold water, easily and completely in boiling water, and recrystallizes on cooling. It is insoluble in alcohol and ether. The taste is decidedly bitter, and the reaction is neutral. The base is not precipitated by neutral 
lead acetate, but is precipitated by the basic salt as a flocculent white precipitate soluble in boiling water. On heating carnin decomposes and takes fire, and at the same time gives off a peculiar odor. It crystallizes with one molecule of water, which it loses at $100^{\circ}-110^{\circ}$.

The hydrochlorid, $\mathrm{C}_{7} \mathrm{H}_{8} \mathrm{~N}_{4} \mathrm{O}_{3}$. $\mathrm{HCl}$, forms pretty prisms (Kemmerich), and decomposes on heating with concentrated hydrochloric acid.

The platinochlorid, $\mathrm{C}_{7} \mathrm{H}_{8} \mathrm{~N}_{4} \mathrm{O}_{3} \cdot \mathrm{HCl} \cdot \mathrm{PtCl}_{4}$, forms a fine, sandy, golden-yellow powder.

With silver nitrate carnin unites to form a white flocculent precipitate, insoluble in nitric acid or in ammonia hydrate. Its formula corresponds to $2\left(\mathrm{C}_{7} \mathrm{H}_{7} \mathrm{AgN}_{4} \mathrm{O}_{3}\right)+\mathrm{AgNO}_{3}$.

With copper solution and reducing agent it gives a yellow precipitate which on washing becomes grass-green (Balke).

Carnin is not affected by prolonged boiling with concentrated barium hydrate. Bromin water decomposes it with the evolution of gas and the formation of hypoxanthin. This change takes place according to the following equation :

$$
\mathrm{C}_{7} \mathrm{H}_{8} \mathrm{~N}_{4} \mathrm{O}_{3}+2 \mathrm{Br}=\mathrm{C}_{5} \mathrm{H}_{4} \mathrm{~N}_{4} \mathrm{O} \cdot \mathrm{HBr}+\mathrm{CH}_{3} \mathrm{Br}+\mathrm{CO}_{2} \text {. }
$$

A similar decomposition into hypoxanthin is brought about by the action of nitric acid, though in this case oxalic acid and a yellow body are formed. Because of this decomposition carnin cannot be expected when Neubauer's method is used. When carnin is evaporated with chlorin water containing a little nitric acid, the residue, on contact with ammonia, gives a rose-red color (murexid test). This is due, according to Weidel, to the formation of hypoxanthin, but it has since been shown that the latter base does not give this reaction which, however, is given by xanthin and its derivatives.

The constitution of carnin is not known. It is undoubtedly an ester of hypoxanthin. The above decompositions may be taken to indicate the presence of an acetic group in position 7 ; otherwise it must be looked upon as a methyl carboxyl derivative of hypoxanthin.

The physiological action of carnin has been examined somewhat by Brücke, and according to him the base is not very poisonous. The only effect observed, when taken internally, was a fluctuation in the rate of the heart-beat, though even this was by no means definite in its nature.

Carnosin, $\mathrm{C}_{9} \mathrm{H}_{14} \mathrm{~N}_{4} \mathrm{O}_{3}$, is a new base isolated in 1900 by Gulewitsch and Amiradzibi ${ }^{1}$ from Liebig's meat extract. It has not as yet been studied very thoroughly and nothing definite can be said as to its structure. The silver compounds show, however, a striking resemblance to arginin. It may be looked upon as a di-methyl 
carnin in which case it would contain less hydrogen than given above.

The free base is very easily soluble in water and has a strong alkaline reaction. It forms minute needle-shaped crystals and melts with decomposition at $239^{\circ}$.

The nitrate on evaporation yields radiating crystals and is also precipitated in stellate masses of needles by the addition of alcohol to a hot aqueous solution of the salt. It is dextro-rotatory and melts with decomposition at $211^{\circ}$.

On boiling with copper carbonate it yields a copper compound $\left(\mathrm{C}_{9} \mathrm{H}_{14} \mathrm{~N}_{4} \mathrm{O}_{3} . \mathrm{CuO}\right)$ which is difficultly soluble in cold, rather easily soluble in hot water from which it crystallizes in characteristic sixsided plates. It decomposes without melting at $220^{\circ}$.

Cytosin, $\mathrm{C}_{21} \mathrm{H}_{30} \mathrm{~N}_{16} \mathrm{O}_{4}+5 \mathrm{H}_{2} \mathrm{O}$, was obtained by Kossel and Neumann by the decomposition of adenylic acid (from thymus glands) by heating with 20 per cent. sulphuric acid in a sealed tube at $150^{\circ}$; also by the action of water at $170^{\circ}$. The yield is about 2 per cent. It forms rectangular plates, often with blunted corners. On slow separation the crystals may attain a length of a centimeter. The water of crystallization is expelled at $100^{\circ}$. It is easily soluble in hot water, from which it separates on cooling; difficultly soluble in alcohol ; insoluble in ether.

It forms well crystallized salts. Thus, the sulphate forms needles ; the chlorid is easily soluble and appears in prisms. The nitrate, platinochlorid, and aurochlorid likewise crystallize easily. A brickred precipitate forms in even very dilute acidulated solution by the addition of potassium bismuth iodid. Silver nitrate produces a precipitate which is increased by addition of a little ammonia, but is dissolved gradually by an excess, especially on warming, but it reappears in crystalline form on cooling.

The picrate, $\mathrm{C}_{21} \mathrm{H}_{30} \mathrm{~N}_{16} \mathrm{O}_{4} \cdot 2 \mathrm{C}_{6} \mathrm{H}_{3} \mathrm{~N}_{3} \mathrm{O}_{7}$, is difficultly soluble and crystallizes in yellow needles.

It is evident that cytosin is still subject to investigation. In some respects it suggests a relationship to the pyrimidin bodies. At all events the formula as given is more complex than might be expected by comparison with the other well studied products of nucleins.

\section{PYRIMIDIN GROUP.}

Two substances belonging to this group have been isolated from nucleinic acids by hydrolysis. Although they are neither basic nor acid in character, yet their relation to the purin bodies and hence to nuclein is such as to render their consideration necessary. They have been regarded as antecedents of the purin bodies, rather than as decomposition products of these substances. Nevertheless, it 
would seem as if the latter view was the correct one. The reason for this belief is twofold. In the first place the hydrolytic process is so energetic that adenin and presumably the other purin bases as well are completely broken up. Again, if the pyrimidin bodies are antecedents, it might be expected that on feeding to animals purin bodies would be eliminated in the urine. Steudel (1901), however, has shown that a number of synthetic pyrimidin bodies can be given to the dog with the result that some of these are destroyed while others are eliminated as such, and at no time are purin bodies formed. Thymin, a natural pyrimidin body, is split up and yields urea, whereas its isomer the synthetic methyl uracil is excreted unchanged.

Steudel's investigation further shows that the di-and tri-amino derivatives of pyrimidin are highly toxic, while the others are not. These poisonous substances yield insoluble compounds which are deposited in the tubules of the kidney. It is noteworthy that the really only poisonous natural purin body is adenin which, as already shown, is 6-amino purin (see pages 341,350 ). It would seem as if the introduction of the amino group into the purin or pyrimidin nucleus rendered such bodies poisonous.

Thymin.-In 1893, Kossel and Neumann showed that the nucleinic acid from the thymus gland of the calf on boiling with dilute sulphuric acid yields a crystalline body ( 8 per cent.) which they designated as thymin. At first they ascribed it the formula $\mathrm{C}_{23} \mathrm{H}_{26} \mathrm{~N}_{8} \mathrm{O}_{6}$ but subsequent investigations showed that the original preparations were not pure and that thymin was $\mathrm{C}_{5} \mathrm{H}_{6} \mathrm{~N}_{2} \mathrm{O}_{2}$.

Thymin was also obtained by Kossel and Neumann by similar hydrolysis of the nucleinic acid of ox spleen. The "nucleosin" which Miescher isolated from a nucleinic acid derived from the spermatozoa of the salmon is according to Kossel identical with thymin.

In 1896 Kossel isolated it from the spermatozoa of the sturgeon and three years later Gulewitsch obtained it from the testicles of the herring. Its possible presence in the auto-digestion of the pancreas has been indicated by Levene. Kutscher obtained thymin and possibly uracil in the auto-digestion of thymus glands. Fischer and Roeder ${ }^{1}$ in 1901 prepared thymin and uracil synthetically.

Thymin forms four or six-sided colorless plates resembling those of cholesterin and is doubly refractive. Illustrations of these are given by Gulewitsch (Zeit. physiol. Chem., 27, 295). On careful heating it can be sublimed and, as Kossel has shown, its presence in nucleinic acid can thus be directly demonstrated by heating some of the acid between two watch glasses. The melting point is above $290^{\circ}$. When heated rapidly it sinters at $318^{\circ}$ and melts at about $321^{\circ}$ with evolution of gas becoming yellowish (Fischer and ${ }^{1}$ Berichte, 34, 3751, 4129. 
Roeder). It is difficultly soluble in cold, easily so in boiling water, less easily in alcohol than in ether. Although it is neither acid nor basic in character a potassium thymin compound can be obtained (Steudel), with mercuric nitrate it yields a voluminous precipitate. Mercuric chlorid gives no precipitate but does form one if the liquid containing the mercury salt is rendered slightly alkaline with sodium hydrate. In like manner silver nitrate does not yield a precipitate but the subsequent addition ammonium or barium hydrate produces a gelatinous precipitate which redissolves in excess of the alkali.

Thymin decolors bromin water and eventually forms a bromin derivative. According to Jones (1900) this change takes place according to the equation :

$$
\mathrm{C}_{5} \mathrm{H}_{6} \mathrm{~N}_{2} \mathrm{O}_{2}+2 \mathrm{Br}+\mathrm{H}_{2} \mathrm{O}=\mathrm{C}_{5} \mathrm{H}_{7} \mathrm{~N}_{2} \mathrm{O}_{3} \mathrm{Br}+\mathrm{HBr} \text {. }
$$

On heating with phosphorus oxychlorid Steudel and Kossel (1900), obtained a dichlorthymin which was different from that obtained from the isomer methyl uracil. By heating potassium thymin with methyl iodid Steudel obtained a dimethyl thymin which also differed from the isomeric trimethyl uracil of Behrend.

Although at the time of the discovery of thymin Kossel suggested that it possibly might be a pyrimidin body, yet positive proof of this and the demonstration of its constitution was not supplied until recently. The pyrimidin character was demonstrated by Steudel (1901), who succeeded in obtaining a nitro-thymin and this on reduction with tin and hydrochloric acid gave a body which with chlorin water and ammonia yielded an intense murexid reaction, thus indicating the presence of the alloxan group. This was further corroborated by the oxidation of thymin with barium permanganate, urea being obtained. In view of these reactions thymin is a pyrimidin body isomeric with methyl uracil. The di-substitution products indicate that thymin possesses the following structural formula:
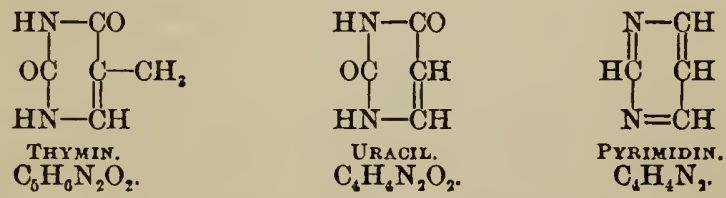

The position of the carbon and nitrogen is indicated by numbers in the same way as in the case of purin (p. 338). Thymin is therefore 5-methyl 2-6-dioxypyrimidin. Methyl uracil, the isomer synthesized by Behrend and Roosen, has the methyl group in position $4(=6)$. It is prepared by the action of urea on acetacetic ether. Gabriel and Colman ${ }^{1}$ changed methyl uracil into $4(=6)$ methyl pyrimidin and eventually into pyrimidin. From the 4.5.6-methyl 
diamido pyrimidin by the action of formic acid they prepared 6methyl purin, ${ }^{1}$ thus effecting the conversion of a pyrimidin into a purin body. Obviously the reverse change is equally possible, in which case the origin of the pyrimidin bodies is easily accounted for. Pyrimidin has also been synthesized by Emery. ${ }^{2}$ Several amido and methyl derivatives have been recently described. ${ }^{3}$

From the above constitution of pyrimidin it will be seen that it may be looked upon as benzol, in which the nitrogen occupies the meta position. It therefore has two isomers. Thus, when the nitrogen is in the ortho position pyridazin results; when in the para position pyrazin.

Uracil.-From yeast nucleinic acid Kossel and Neumann in 1893 isolated a thymin-like substance but the amount obtained was insufficient for identification. Ascoli, in 1901, demonstrated that this substance was not thymin but the next lower homologue, $\mathrm{C}_{4} \mathrm{H}_{4} \mathrm{~N}_{2} \mathrm{O}_{2}$, which corresponds to the hitherto unknown uracil. A similar, if not identical body, with possibly thymin, is formed in the autodigestion of the pancreas (Levene, 1901) and it is probable that Kutscher met with this body although he gave it the formula $\mathrm{C}_{8} \mathrm{H}_{6} \mathrm{~N}_{4} \mathrm{O}_{4}$ in the auto-digestion of yeast. Uracil has been synthesized by Fischer and Roeder. ${ }^{4}$

Uracil crystallizes in needles, arranged in rosettes. It does not sublime as easily as does thymin. The synthetic product on rapid heating becomes brown at $280^{\circ}$ and melts at about $335^{\circ}$ with evolution of gas. It is easily soluble in hot, rather difficultly in cold water and is almost insoluble in alcohol and in ether. It is easily soluble in ammonia but it does not unite with hydrochloric or with nitric acids. When treated in the same way as thymin it yields Weidel's reaction. It therefore in all probability possesses the structure, as given above, in which case it is 2-6 dioxypyrimidin.

A Body, $\mathrm{C}_{8} \mathrm{H}_{6} \mathrm{~N}_{4} \mathrm{O}_{4}$, was isolated by Kutscher ${ }^{5}$ from yeast undergoing sterile auto-digestion. It was precipitated from the ammoniacal silver filtrate, after removal of puriu bases, on neutralization with nitric acid. When purified, however, the substance is not precipitable by silver nitrate in acid solution and is thrown down by an ammoniacal silver solution but the precipitate is soluble in excess of ammonia and in nitric acid. After treatment with boiling, dilute nitric acid it is precipitated by silver nitrate. The amorphous precipitate thus obtained is soluble in boiling nitric acid and separates out on cooling as fine needles.

The substance is neutral in reaction and is precipitated by phos-

${ }^{1}$ Berichte, 34, 1246.

${ }^{2}$ Berichte, 34, 4178.

${ }^{3}$ Berichte, 34, 2812, 3362, 3956.

4 Berichte, 34, 3751, 4129.

${ }_{5}$ Zeits. physiol. Chem., 32, 67. 
photungstic acid. Inasmuch as Levene obtained uracil, $\mathrm{C}_{4} \mathrm{H}_{4} \mathrm{~N}_{2} \mathrm{O}_{2}$, in a similar auto-digestion of pancreas it may be that Kutscher's body has a simpler formula than that given above and moreover it may be related to, if not identical with, uracil. Its behavior to silver is very much like that of thymin.

The pyrimidin bodies may be obtained by the hydrolysis of pure nucleinic acid but the better procedure is to separate them from the hydrolytic products of the original tissue or material. The method perfected by Jones is especially useful for this purpose. He employed the testicles of the herring from which the protamin had been previously removed with an acid. This material was mixed with 20 per cent. sulphuric acid and the mixture was autoclaved for 2 hours at $150^{\circ}$. This heating completely destroys the adenin but is without effect on the thymin. After removal of the humus substances by filtration, powdered barium hydrate is added till a slight but distinct alkaline reaction is obtained. The precipitate of barium sulphate is filtered off and thoroughly washed with boiling water. The combined filtrates are then concentrated so that for each $100 \mathrm{~g}$. of dry testicles there is 500 c.c. of liquid.

A preliminary test is now made to ascertain the exact amount of silver nitrate necessary. For this purpose, a small but known amount of the liquid is taken and after slightly acidulating a 2 per cent. solution of silver nitrate is added from a burette till a drop of the liquid added to an excess of baryta water yields a yellow and not a white precipitate. The amount of silver solution necessary for portions of 500 c.c. of the liquid can then be calculated.

The original liquid is then slightly acidulated with nitric acid and divided into portions of 500 c.c. each. To each of these the requisite amount of silver nitrate solution is then added. After removal of the precipitate, the filtrate is rendered slightly alkaline with baryta. This precipitate is washed by decantation and finally drained over a pump. It is then decomposed with hydrogen sulphid, under pressure. The united filtrates on evaporation yield crystals of thymin. These are decolored with charcoal and recrystallized from hot water. In the case of herring testicles the yield of thymin is about 2 per cent.

It has already been pointed out that the pyrimidin bodies may be considered as antecedents of the purin bodies formed in the animal organism or more correctly as cleavage products of the latter. The relation of the pyrimidin and purin bodies can best be shown by equations representing the synthesis of uric acid. Behrend and Roosen prepared uric acid by combining urea with a pyrimidin body - iso-dialuric acid. 


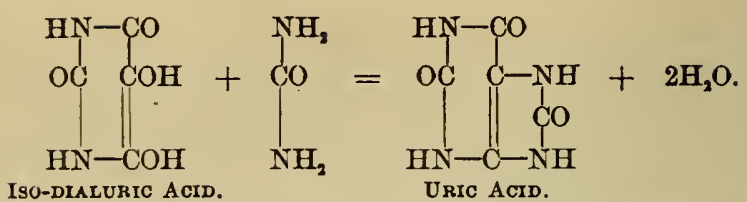

Fischer and Ach prepared uric acid from the pyrimidin body pseudo-uric acid. This on fusion with oxalic acid or better on boiling with 20 per cent. hydrochloric acid yields uric acid.

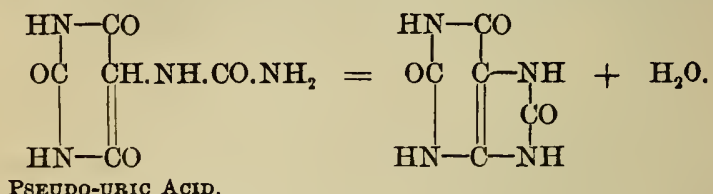

The interesting syntheses of uric acid and of the purin bases by Traube (1900) are also affected through the intermediate formation of pyrimidin bodies.

Episarkin, $\mathrm{C}_{4} \mathrm{H}_{6} \mathrm{~N}_{3} \mathrm{O}$.-This base was isolated by Balke in 1893 from urine. From about 1,600 liters of urine only about 0.4 g. was obtained. It accompanied the hypoxanthin silver nitrate. The mixture on digestion with dilute ammonia on a water-bath gave hypoxanthin-silver, whereas episarkin, in part, passed into solution. The solution was filtered, and the slightly ammoniacal filtrate on standing for twelve hours gave a crop of small needles of episarkin. The hypoxanthin-silver still contained an admixture of the new base, as was found by decomposition with hydrogen sulphid and concentration.

The crystals are more difficultly soluble in water and their crystalline form is distinctly different from that of bypoxanthin. A separation can best be accomplished by dissolving the mixture of the two bases in as little dilute ammonia as possible, then on saturating this solution with carbonic acid small whetstone-shaped needles separate. In this respect it resembles epiguanin. When recrystallized from hot water it yields small prisms or needles which may attain a length of $1 \mathrm{~cm}$. and may be grouped in bunches. They are white and glassy, and when dry form a felt-like mass. The crystals are permanent and do not effloresce.

On evaporation with concentrated nitric acid, it gives a yellow residue which with sodium hydrate becomes paler. On evaporation with nitric acid and chlorin water (Weidel's reaction), it gives a white residue unaltered by ammonia. When evaporated on a waterbath with hydrochloric acid and potassium chlorate it leaves a white residue, which becomes colored an intense violet by ammonia. This reaction certainly shows that this base is related either to the purin or to the pyrimidin bodies. 
It is difficultly soluble in hot water; almost wholly insoluble in cold water $(1: 13,000)$. It is readily soluble in dilute hydrochloric acid, from which, on evaporation, the hydrochlorid crystallizes in easily soluble, pretty needles. The base does not yield an insoluble compound with sodium hydrate, though it is possible that the whetstone-shaped crystals mentioned above represent an ammonium salt. With silver nitrate it gives a precipitate which is insoluble in nitric acid, easily soluble in ammonia. In other respects the salt resembles that of hypoxanthin. Thus, by boiling with nitric acid (1.1 sp. g.) a crystalline silver nitrate compound forms which probably contains one atom of silver, and on digestion with dilute ammonia yields a silver compound with two atoms of silver-since a part of the episarkin passes into the filtrate and crystallizes out on cooling. The base gives white precipitates with phosphotungstic acid, mercuric chlorid, and ammoniacal lead acetate.

It is distinguished from adenin and hypoxanthin by its almost complete insolubility in cold water; from the latter by not being precipitated by picric acid, and by not giving the characteristic rubyred coloration in the alkalinized filtrate, after reduction with zinc and hydrochloric acid (page 370). From ranthin it is distinguished by the absence of the xanthin reaction; from hetero- and paraxanthin by the absence of the insoluble sodium compound from guanin and adenin by not clouding at $53^{\circ}$, and by the picric acid reaction. The fact that it is not precipitated by picric acid and is precipitated by ammoniacal lead acetate distinguishes it from epiguanin.

Krüger, in 1895, from tea extract isolated a base resembling somewhat episarkin. It differed, however, from the latter in giving with picric acid a very fine crystalline compound. It was more soluble in water, and gave different color reactions.

Nothing definite can be stated regarding the constitution of episarkin. The formula as given by Balke is open to the objection that the sum of the hydrogen and nitrogen atoms is an odd number. Changing for this reason the number of hydrogen atoms to five, we would then have a compound, $\mathrm{C}_{4} \mathrm{H}_{7} \mathrm{~N}_{3} \mathrm{O}$. Such a body may be looked upon as an amino derivative of uracil to which it would bear the same relation that guanin bears to xanthin. This relationship to the pyrimidin group would be expressed by the structural formula :<smiles>Nc1ncccc1NC(=O)O</smiles>

According to this structure episarkin with nitrous acid should yield uracil and on cleavage should give guanidin. Furthermore, it would appear to be derived from guanin. Thymin itself may be looked upon as a cleavage and oxidation product of epiguanin. 
A Base, $\mathrm{C}_{4} \mathrm{H}_{5} \mathrm{~N}_{5} \mathrm{O}$, was obtained by Gautier from fresh muscle tissue of beef, according to the method given on page 455 , and on account of a resemblance in some of its properties with xanthin he named it pseudoxanthin. This name is very inappropriate, not only because it differs so much in its empirical formula from that of xanthin, $\mathrm{C}_{5} \mathrm{H}_{4} \mathrm{~N}_{4} \mathrm{O}_{2}$, but also because the term pseudoxanthin has already been applied by Schultzen and Filehne to a body isomeric with xanthin, which was obtained by the action of sulphuric acid on uric acid.

The free base forms a light yellow powder, slightly soluble in cold water, soluble in weak alkali and in hydrochloric acid. The hydrochlorid is very soluble, and it forms stellate prisms with curved faces, which resemble the corresponding salt of hypoxanthin, and to some extent, also, the whetstone-shaped crystals of uric acid.

Like xanthin, its aqueous solution is precipitated in the cold by mercuric chlorid, silver nitrate, and by ammoniacal lead acetate, but not by normal lead acetate. On evaporation with nitric acid, the residue gives, on contact with potassium hydrate, as in the case of xanthin, a beantiful orange-red coloration (xanthin reaction). It differs from xanthin, not only in its empirical composition, but also in its greater solubility and in its crystalline form. It is possible that this base, on account of its great resemblance to xanthin, may have been mistaken, at different times, for that compound. It is to be noted, however, that this base has not been met since Gautier's work. If the formula given is correct, the base may be considered as a pyrimidin body, such as a di-imido derivative of the preceding.

\section{HEXON BASES.}

The purin bases through the studies of Kossel have been shown to be derivatives of the nuclein which exist within the nuclei of cells. They are therefore to be looked upon as essentially products of nuclear metabolism and more especially of the nucleinic acids. If the results obtained by Bang with guanylic acid, a nucleinic acid from the pancreas, be correct and hold true for the other nucleinic acids then all the nitrogen contained in these acids is in the purin form and hence would leave the body to some extent as purin bases, but chiefly in the oxidized form as uric acid. A portion of the purin bodies, however, may undergo hydrolytic change and appear in the urine as pyrimidin derivatives or even in the more simple form of allantoin, creatinin and urea. The presence of allantoin and oxalic acid in urine and even in sprouts is thus accounted for.

On the other hand extensive investigations carried on during the past ten years have shown the existence of a group of basic substances characteristic of proteid bodies and in no wise related to the nucleinic acids and their products. These basic substances have 
been designated by Kossel as the hexon bases inasmuch as they contain six carbon atoms.

Arginin the first known, and perhaps the most important member of this group, was discovered by Schulze and Steiger ${ }^{1}$ in 1886 in lupine sprouts wherein it is formed as a result of the energetic proteid disintegration which takes place in the developing plant. The base exists preformed in the cotyledons inasmuch as it can be extracted from these by water alone without the aid of hydrolytic agents. In this way the dry sprouts yielded as much as 3.5 per cent. of arginin. Nine years later Hedin ${ }^{2}$ demonstrated that arginin could be obtained by the hydrolytic cleavage of a number of proteid substances. On decomposing these by boiling with hydrochloric acid and tin, he obtained variable amounts of the base as shown below and at no time was the quantity as large as that obtained by Schulze from sprouts.

The yield was as follows:

$\begin{array}{lllll}\text { Conglutin, } & 2.75 & \text { per cent. } & \text { Egg albumin, } & 0.8 \text { per cent. } \\ \text { Gelatin, } & 2.6 & \text { " } & \text { " } & \text { Blood serum, dried, } 0.7 \text { " } \\ \text { Horn, } & 2.5 & \text { " } & \text { " } & \text { Casein, } \\ \text { Yolk albumin, } 2.3 & \text { " } & \text { " } & & \end{array}$

As yet no proteid is known which does not yield arginin on hydrolysis. Elastin which was studied by Berg and by Hedin was supposed to be free from arginin but Kossel and Kutscher ${ }^{3}$ showed by means of improved methods, that it did contain about 0.3 per cent. of the base. Subsequently lysin was also shown to be present. Of the total nitrogen contained in the young sprouts of pine and fir 21 to 29 per cent., according to Schulze, ${ }^{4}$ is represented by arginin while the proteid from the seeds of the same plants on cleavage with hydrochloric acid and tin yield as much as 10 per cent. of the base. On a subsequent occasion a yield of only 6 per cent. was obtained from fir seeds. The proteids contained in the seeds of plants are apparently extremely rich in arginin which is set free during the active proteid destruction which ensues during germination. This becomes readily comprehensible when it is remembered that tryptic ferments are brought into being in the process of germination (Butkewitch) and that the hydrolytic cleavage which these ferments induce is analogous to that produced by acids, and corresponds to the pancreatic digestion of proteids. This fact will be rendered the more apparent on comparison of the digestion products formed by trypsin and the following bodies which Schulze ${ }^{5}$ has isolated and

\footnotetext{
${ }^{1}$ Zeits. physiol. Chem., 11, 44 (1886).

${ }^{2}$ Zeits. physiol. Chem., 21, 155 (1895); 20, 186 (1894).

${ }^{3}$ Zeits. physiol. Chem., 25, 551 (1898); 31, 205.

- Zeits. physiol. Chem., 22, 435 ; 25, 360.

${ }^{5}$ Zeits. physiol. Chem., 28, 470.
} 
shown to exist preformed in germinating plants: Leucin, tyrosin, phenyl alanin, amido valerianic acid, asparagin, glutamin, arginin, histidin and lysin.

The three last mentioned substances, the hexon bases, have been only recently shown to be products of tryptic digestion. Indeed, the studies of Kutscher ${ }^{1}$ bave shown that the so-called antipepton is not a chemical substance possessing a definite formula, $\mathrm{C}_{10} \mathrm{H}_{15} \mathrm{~N}_{3} \mathrm{O}_{5}$, as Siegfried believes, but is rather a heterogeneous mixture from which he was able to isolate leucin, tyrosin, aspartic and glutamic acids, arginin, histidin and lysin. In other words trypsin, like sulphuric acid, if given sufficient time will completely split up the proteid molecule and will not stop short, as has been heretofore held with a resistant antipepton. An exactly similar condition is met with in the auto-digestion of yeast where not only xanthin bases and amido acids, but also hexon bases form $\left(\mathrm{Kutscher}^{2}\right)$. It is of interest to note that according to Levene ${ }^{3}$ arginin, histidin and uracil, and possibly thymin, are formed during the auto-digestion of the pancreas. From the liver of a case of acute yellow atrophy Taylor obtained leucin and asparaginic acid but no hexon or purin bases. Hedin ${ }^{4}$ was the first to point out the presence of lysin in tryptic digestion of fibrin and Kossel and Mathews found the three hexon bases in the similar digestion of sturin.

The investigations of Lawrow ${ }^{5}$ show that the proteid molecule is also destroyed in peptic digestion. Thus in the sterile auto-digestion of pigs' stomachs he obtained leucin, asparaginic and amido valerianic acids but no tyrosin. Hexon bases were not present but instead large amounts of cadaverin and putrescin. The latter two bases undoubtedly resulting by the pepsin hydrolysis of the proteid. A somewhat similar result was obtained by Kutscher ${ }^{6}$ in the auto-digestion of the thymus gland. Of still greater significance is the observation of Kutscher and Seemann ${ }^{7}$ that in the small intestines albumoses and peptons are nearly absent and that leucin, tyrosin, lysin and arginin are present as a result of energetic tryptic action.

Haslam ${ }^{8}$ has shown that the three bases exist in different amounts in deutero- and hetero-albumose. Hart $^{9}$ found that the yield of hexon bases was about the same in syntonin and protalbumose whereas in heteroalbumose the relative amounts were different and to a certain extent depended upon the quantity of salt present.

According to Emmerling ${ }^{10}$ the enzyme papayotin acting on blood

${ }^{1}$ Zeits. physiol. Chem., 25, 195; 26, 110 ; 28, 88 (1899).

2 Zeits. physiol. Chem., 32, 59.

3 Zeits. physiol. Chem., 32, 540 (1901).

- Zeits. physiol. Chem., 21, 298.

5 Zeits. physiol. Chem., 26, $512 ; 33,312$.

6 Zeits. physiol. Chem., 34, 117.

TZeits. pliysiol. Chem., 34, 543.

${ }^{8}$ Zeits. physiol. Chem., 32, 54.

${ }^{9}$ Zeits. physiol. Chem., 33, 347.

${ }^{10}$ Berichtc, 35, 695, 700 (1902). 
fibrin in the presence of toluol yields not only albumose and pepton but also simpler cleavage products such as arginin, leucin, tyrosin, glycocoll, alanin, phenyl alanin, asparaginic and glutamic acids. The enzyme of B. fluorescens liquefaciens under like conditions gave arginin, lencin and asparaginic acids.

It is evident from what has been said that arginin and the other hexon bases exist preformed in the proteid molecule and that they are liberated or split off by the action of acids and of tryptic enzymes and by oxidation with potassium permanganate (Jolles). A most interesting question arises as to whether these hexon bases are also liberated in the tissue metabolism of the body. Already Gulewitsch $^{1}$ has shown that the spleen, on standing with thymol for eighteen hours, contains arginin. This may be due to initial hydrolysis, especially since it is known that the spleen and other organs of the body contains an active proteolytic enzyme (Hedin and Rowland). ${ }^{2}$ As yet the hexon bases have not been detected in the urine, but it is not unreasonable to suppose, inasmuch as they are unquestionably antecedents of urea, that they will appear in the urine when normal urea formation is interfered with. The marked resemblance which arginin bears to creatinin and to the leucomaïns of that group was pointed out from the first by Schulze.

The second member of the hexon group, lysin, we owe to the brilliant investigation of Drechsel $^{3}(1890)$ on the cleavage products of casein. On boiling casein with hydrochloric acid and tin he obtained ammonia, amido acids, and two bases lysin and lysatinin. Fischer, a pupil of Drechsel, obtained the same products by hydrolyzing gelatin in like manner (Inaug. Diss. 1890). Subsequently, Siegfried ${ }^{4}$ showed that a number of proteids, conglutin, glutenfibrin, hemiprotein, oxyprotosulfonic acid and egg albumin also yielded the two bases found by Drechsel. Hedin isolated lysin from horn, casein, conglutin, egg albumin, yolk albumin, and blood serum. By employing the same method Schwarz ${ }^{5}$ obtained from the elastin of the aorta some lysatinin. In 1895, however, Hedin ${ }^{6}$ showed that lysatinin was really a mixture of equal molecules of lysin and arginin. Since then lysin has been found associated with arginin and histidin in various proteids of animal origin with the exception of the several protamins indicated in the subjoined table. Its presence in "antipepton" and in tryptic digestion of fibrin and sturin has already been referred to. Schulze ${ }^{7}$ rounded out his studies on arginin in plants by showing that both lysin and histidin were also present in lupine sprouts and in the seeds of the fir. The

\footnotetext{
1 Zeits. physiol. Chem., 30, 533 (1900).

${ }^{2}$ Zeits. physiol. Chem., 32, 341, 531, 1901.

3 Berichte, 23, 3096.

- Berichte, 24, 418, 1891.

- Zeits. physiol. Chem., 18, 487.

6 Zeits. physzol. Chem., 21, 297, 1895 ; 20, 186.

7 Zeits. physiol. Chem., 28, 459, 465.
} 
three bases therefore result as a rule simultaneously in the hydrolytic cleavage of proteids irrespective as to whether this is induced by acids or by enzymes. From the table given below it will be seen that lysin is not only absent from several protamins but also from the alcohol soluble proteids of wheat and maize. In the latter case it is noteworthy that the absence of lysin is associated with an unusually large amount of ammonia. Levene and Mendel, ${ }^{1}$ it should be noted, obtained by hydrolysis of edestin, the crystalline proteid from hemp-seed, arginin, histidin and undoubtedly lysin. According to Schulze and Winterstein ${ }^{2}$ edestin and the proteid from seeds of conifers yields more arginin than do other vegetable proteids. Edestin gave 11 per cent. arginin ; 1.17 per cent. histidin and 1.3 per cent. lysin.

The splendid researches of $\mathrm{Kossel}^{3}$ on the protamins and their cleavage products led to the discovery of histidin, the third hexon base. Undoubtedly, histidin was obtained by Siegfried in his studies on the cleavage products of proteids (1891), but it remained for Hedin $^{4}$ to clearly demonstrate its presence among the hydrolytic products of casein, egg albumin, blood serum and horn. Since then it has been found as a constant cleavage product of the more complex animal and plant proteids.

Recently Kossel and Kutscher ${ }^{5}$ have carried out a most careful study upon the quantitative distribution of the hexon bases in the different proteids. Their results in per cent. are given in the following table:

\begin{tabular}{|c|c|c|c|c|c|c|c|c|c|c|c|c|}
\hline & 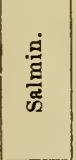 & 䒕 & 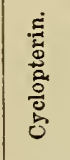 & 离 & 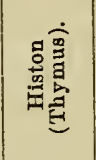 & 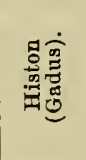 & 矛 & 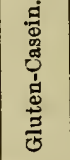 & 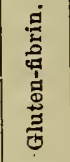 & 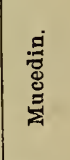 & 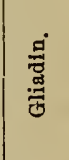 & 胥 \\
\hline $\begin{array}{l}\text { Arginin. } \\
\text { Lysin. } \\
\text { Histidin. } \\
\text { Ammonia. }\end{array}$ & \begin{tabular}{|c|}
84.3 \\
0 \\
0 \\
0 \\
\end{tabular} & $\begin{array}{c}82.2 \\
0 \\
0 \\
0\end{array}$ & $\begin{array}{c}62.5 \\
0 \\
0 \\
-\end{array}$ & $\begin{array}{c}58.2 \\
12.0 \\
12.9 \\
0\end{array}$ & \begin{tabular}{|c|}
14.36 \\
7.7 \\
1.21 \\
1.66 \\
\end{tabular} & $\begin{array}{r}15.52 \\
8.30 \\
2.34 \\
0.74\end{array}$ & $\begin{array}{c}9.3 \\
5-6 \\
\text { small } \\
0.3\end{array}$ & \begin{tabular}{|l|}
4.4 \\
2.15 \\
1.16 \\
2.45 \\
\end{tabular} & $\begin{array}{c}3.05 \\
0 \\
1.53 \\
3.89\end{array}$ & $\begin{array}{c}3.13 \\
0 \\
0.43 \\
4.23\end{array}$ & \begin{tabular}{|l}
2.75 \\
0 \\
1.20 \\
4.1
\end{tabular} & $\begin{array}{c}1.82 \\
0 \\
0.81 \\
2.56 \\
\end{array}$ \\
\hline
\end{tabular}

Arginin, it will be seen, is a constant constituent, and is by far the most abundant of the hexon bases. The amounts found in several instances are higher than those which Hedin obtained (p. 423). Hedin's results represent the minimum values obtained by a somewhat imperfect method. For the amounts of arginin in seeds, sprouts and in elastin see p. 423.

${ }^{1}$ Am. Journ. Physiol., 6, 48, 1901.

2 Zeits. physiol. Chem., 33, 557.

'Zeits. physiol. Chem., 22, 178, 1896.

4 Zeits. physiol. Chem., 22, 191.

${ }^{5}$ Zeits. physiol. Chem., 31, 207, 1900. 
The method which Kossel and Kutscher 1 have employed for the isolation and separation of the hexon bases in a very condensed form is as follows: For every gram of proteid 3 c.c. of concentrated $\mathrm{H}_{2} \mathrm{SO}_{4}$ and 6 c.c. of water are taken and the mixture is boiled for 14 hours under an inverted condenser. The liquid is then made up to a definite volume and the total nitrogen in a definite portion can be determined. Hot, saturated baryta is then added till only a slight acidity remains, after which the $\mathrm{BaSO}_{4}$ is drained and thoroughly washed. The filtrate and wash-water are combined and the total nitrogen in a portion, is again determined. The difference represents the nitrogen in the humin substances, which are dragged down with the $\mathrm{BaSO}_{4}$.

In another portion the ammonia is determined by distillation with magnesia. The ammonia is expelled from the remaining total thid by evaporation with magnesia. The combined ammonia-free fluids are then rendered strongly alkaline with baryta and the resultant precipitate is thoroughly washed. The filtrate and wash-water are combined and acidulated with $\mathrm{H}_{2} \mathrm{SO}_{4}$. The precipitate which forms is likewise thoroughly washed and the liquids are united, made up to a definite volume and the total nitrogen in a portion again determined $=$ Humin nitrogen $I I$.

To separate the bases present arginin and histidin are precipitated together, while the lysin remains in solution. For this purpose the acid liquid is diluted, heated on the water-bath and silver sulphate is added till a drop of the liquid added to an excess of baryta yield a yellow precipitate. When this point is reached the liquid is cooled to $40^{\circ}$ and saturated with baryta. The precipitate of arginin- and histidin-silver is removed at once and washed thoroughly with baryta.

To separate the arginin and histidin, the precipitate is suspended in dilute sulphuric acid and decomposed with hydrogen sulphid. The sulphid precipitate is removed and thoroughly washed. The combined filtrates are evaporated to expel the gas, then made up to a definite volume and the total nitrogen in a portion is determined. The solution is then neutralized with baryta, after which barium nitrate is added till all the sulphuric acid is precipitated. The precipitate is removed and well washed. The filtrates are united, concentrated to about $300 \mathrm{c.c}$., after which silver nitrate is added until a drop of the liquid when added to an excess of baryta gives a yellow precipitate. The solution is carefully neutralized with baryta, after which the reagent is added from a burette in small portions at a time till all the histidin has been precipitated. This point is reached when a portion of the liquid slowly added to weak ammoniacal silver nitrate yields no precipitate. If a precipitate forms and is soluble in excess of ammonia, histidin is present.

The histidin-silver precipitate is filtered off and washed well. The filtrates contain arginin. T'o separate the histidin the precipitate is suspended in water, acidulated with $\mathrm{H}_{2} \mathrm{SO}_{4}$, and decomposed with hydrogen sulphid. The combined filtrate and wash-water are then freed from sulphuric acid by means of baryta. The latter is removed and the final filtrate is evaporated to dryness. The residue is taken up with silver nitrate solution to which a drop of nitric acid was added and the solution is filtered. The filtrate on evaporation yields the crystalline histidin dichlorid, which is dried in a vacuum at $40^{\circ}$ and weighed.

The above arginin filtrate is saturated with powdered baryta and the resultant precipitate is well washed, after which it is suspended in acidulated water and decomposed with hydrogen sulphid. The combined filtrates are evaporated and then made up to a definite volume. The nitrogen in a portion is determined and the total amount of arginin is calcu-

${ }^{1}$ Zeits. physiol. Chem., 31, 166-178; 25, 178. 
lated. The liquid is then freed from acid by baryta and from the latter by carbonic acid, after which it is neutralized with nitric acid and evaporated. When dried in a vacuum it can be weighed as the neutral nitrate $\mathrm{C}_{6} \mathrm{H}_{14}$ $\mathrm{N}_{4} \mathrm{O}_{2} \cdot \mathrm{HNO}_{3}+\frac{1}{2} \mathrm{H}_{2} \mathrm{O}$. The arginin may also be estimated polarimetrically, or as the acid di-nitrate.

The lysin is contained in the original filtrate from the histidin and arginin silver precipitate. To isolate it, the solution is acidulated and the silver removed with hydrogen sulphid. The combined filtrate and washwater is concentrated to about 500 c.c., and after sulphuric acid is added to make a 5 per cent. solution it is precipitated with phosphotungstic acid. The precipitate is thoroughly washed. It contains most of the lysin and a small amount of mono-amido acids. The bulk of the latter is contained in the filtrate. The precipitate is decomposed with baryta and filtered. The combined filtrate after removal of excess of barium with carbonic acid is evaporated to dryness. The residue is taken up in water, filtered, and the filtrate is again evaporated to dryness. The residue is now taken up in alcohol and an alcoholic picric acid solution is added, carefully avoiding an excess. The precipitate is filtered off, washed with a little absolute alcohol and then is dissolved in boiling water. The solution after concentration and cooling yields needle-shaped crystals which are filtered off, washed and weighed as lysin picrate. Some lysin remains in the mother liquors.

Instead of decomposing the phosphotungstic acid precipitates with baryta Winterstein advises extraction with ether and hydrochloric acid $(Z, P$. C., 34, 154).

It should be noted in this connection that the amount of lysin and or ammonia in proteid cleavage depends upon the amount of salt present. When salt is present the nitrogenous humin substances are decreased and the yield of lysin and ammonia is increased (Hart, Z. P. C., 33, 347).

\section{PROTAMINS AND HISTONS.}

By far the most fruitful result of the study of the hexon bases is the light which has been shed upon the composition of the proteids. Moreover a better insight has been gained into the nitrogen metabolism of the body. The fact that arginin on hydration with baryta yields urea indicates the existence of a urea or rather guanidin group in that base and hence in every proteid. From the constitution of arginin and lysin (p. 437, 444) it will be seen that these represent respectively diamidovalerianic and diamidocaproic acids. It is by the cleavage of these acids that bacteria (and enzymes, Lawrow) yield the well known ptomains cadaverin and putrescin. The presence of these ptomaïns in cystinuria is now more readily understood. The monamido acids represented by leucin, tyrosin, asparaginic acid and phenyl alanin, make up a third type of nitrogen combination in the proteid molecule. The liberation of ammonia by hydrolytic change, as seen in the table on p. 426 , indicates the existence of a fourth as yet unknown nitrogen group. The bulk of the proteid nitrogen is therefore found in the four groups mentioned. The fact that ammonia and amido acids are changed in the body into urea, when applied to the proteid molecule as thus understood, readily explains the transformation of the greater part of the nitrogen of our food into the final waste product, urea. 
The simplest group of proteids are the protamins. We owe the discovery of these bodies as well as of nucleins to Miescher ${ }^{1}$ who in 1874 isolated the first known representative of this group. By means of dilute hydrochloric acid Miescher extracted from the spermatozoa of the salmon two new bodies protamin, a base, and nucleinic acid. Piccard ${ }^{2}$ in the same year extended somewhat the investigation of Miescher by ascribing a different formula and demonstrating the presence of xanthin bases in Miescher's preparations. During the ensuing twenty years protamin was apparently wholly forgotten and its nature remained therefore uncertain. Since 1894, however, we know through the labors of Kossel and his pupils that Miescher's substance is one of a number of closely allied bodies. For this group Kossel ${ }^{3}$ reserves the original term protamin and designates the individual compounds according to their source. Thus, Miescher's protamin since it is derived from the salmon is known as salmin; that from the sturgeon becomes sturin; while the herring yields clupein. To these may be added scombrin ${ }^{4}$ from the spermatozoa of the mackerel; cyclopterin ${ }^{5}$ from the Cyclopterus lumpus (sea-hare); accipenserin ${ }^{6}$ from Accipenser stellatus; and silurin from the shad (Silurus glanis). From tubercle bacilli Ruppel ${ }^{7}$ isolated a protamin to which he has given the name tuberculosamin. A similar body it may be stated has been obtained from the colon bacillus by Vaughan. The closely related histon bodies are considered on p. 434 .

From the evidence thus far gathered it is clear that the protamins are by no means widely distributed in nature. As a rule they are present in the mature spermatozoa of fish. The immature spermatozoa on the other hand are believed to contain chiefly histon. Apparently, protamins are not present in the spermatozoa of the higher animals. Miescher failed to find it in those of the steer and Math$\mathrm{ews}^{8}$ likewise failed to isolate it from the steer and boar.

The one property which is common to all proteids, so far as our knowledge at present extends, is the formation of hexon bases on cleavage. From the table on p. 426 it will be seen that arginin is present in remarkably large quantity in the several protamins. Moreover, these bodies yield the biuret reaction and are converted by trypsin into "protons," the analogues of pepton, and eventually into the hexon bases. It is because of these facts that the protamins are regarded as the simplest of proteids. In the spermatozoa protamin undoubtedly exists in a salt-like combination with nucleinic

1 Zeits. physiol. Chem., 22, 177.

${ }^{2}$ Berichte, 7, 1714.

${ }^{3}$ Zeits. physiol. Chem., 22, 180, 1896.

- Zeits. physiol. Chem., 26, 524, 1898.

${ }^{5}$ Zeits. physiol. Chem., 28, 313, 1899.

6 Zeits. physiol. Chem., 32, 200, 1901.

7 Zeits. physiol. Chem., 26, 231, 1898.

8 Zeits. physiol. Chem., 23, 399, 1897. 
acid. From the investigations of Mathews it would seem that the chromatin of these cells in the case of the herring is a combination of equal molecules of clupein, $\mathrm{C}_{30} \mathrm{H}_{57} \mathrm{~N}_{17} \mathrm{O}_{6}$, and nucleinic acid, $\mathrm{C}_{40} \mathrm{H}_{54} \mathrm{~N}_{4} \mathrm{P}_{4} \mathrm{O}_{27}$.

According to Kossel ${ }^{1}$ the group or combination which yields the hexon bases is characteristic of all proteids and is therefore to be considered as the nucleus of the proteid molecule. In the simplest proteids like salmin and clupein this nucleus yields but one base, arginin. In others, as shown in the table on p. 426, this nucleus is a trifle more complex since it yields two bases, arginin and histidin. And lastly, as it exists in the majority of proteids it yields all three hexon bases.

The simplest protamins are rendered a trifle more complex by the addition of monoamido acids to the nuclear group. Thus, clupein contains amido valerianic acid but no lysin. Again, cyclopterin unlike other protamins contains an aromatic group which is split off as tyrosin ( 8.3 per cent.). The complexity of the molecule may continue to grow by the addition of other monamido acids like leucin, glycocoll, tyrosin, asparginic and glutamic acid. Eventually, the molecule is increased by the introduction of groups containing other elements, such as sulphur, iron, and even iodin. The growth of more complex proteids is seen in the fact observed by Kossel ${ }^{2}$ that protamins precipitate albumin and albumoses and give rise to histonlike bodies. Kutscher has observed a similar behavior between albumoses and other proteids. Again, the relatively simple proteids unite with entirely different groups, such as carbohydrates yielding glycoproteids; or with nuclein yielding nucleoproteids; with hematin giving rise to hemoglobins.

The protamins are isolated according to the method of Kossel ${ }^{3}$ For this purpose the mature testicles are finely divided, shaken up with water and strained through gauze. The milky liquid is precipitated by careful addition of acetic acid. The precipitated spermatozoa are boiled with alcohol and finally extracted with ether. 500 c.c. of a one per cent. solution of sulphuric acid are added to each $100 \mathrm{~g}$. of the dried spermatic cells and the mixture thoroughly shaken for one quarter of an hour. The liquid is filtered off and the residue is extracted six times in the same way. The addition of salt favors the deposition of the precipitate. The combined aqueous extracts are precipitated by the addition of three volumes of alcohol. After decantation the precipitate of protamin sulphate is drained, then dissolved in hot water and re-precipitated with alcohol. To remove traces of nucleinic acid, the precipitate is dissolved in warm water and reprecipitated with sodium picrate. The deposit is

${ }^{1}$ Zeits. physiol. Chem., 25, 186, 1898.

${ }^{2}$ Zeits. vhysiol. Chem., 22, 178.

${ }^{3}$ Zeits. physiol. Chem., 22, 178 ; 25, 166 ; 32, 198. 
washed thoroughly, dissolved in sulphuric acid and from the solution the picric acid is removed with ether. The protamin sulphate is then thrown down with alcohol and the whole process is repeated if necessary till the substance forms a white flocculent precipitate.

The protamins are strong bases and the aqueous solution possesses a marked alkaline reaction. With acids they form well defined salts. The sulphates usually form a white powder which, in the case of sturin and accipenserin, is very soluble in water. The sulphate of clupein is soluble in warm water and separates out on cooling as oily globules. The solutions of protamins are levo-rotatory and give the biuret reaction in the cold. Cyclopterin is the only protamin which gives the Millon test thus indicating the presence of an aromatic group. From it Kossel and Kutscher obtained 8.3 per cent. of tyrosin. Clupein gave amido valerianic acid. In the presence of ammonia they precipitate pepton solutions. It is of interest to note that the sulphates are precipitated by saturated salt or ammonium sulphate solutions. The protamins are precipitated by the usual alkaloidal reagents even in neutral or slightly alkaline solutions. In this respect they differ from the peptons and albumoses which require an acid reaction. Sodium tungstate, phosphotungstate, chromate, ferrocyanid, picrate and solutions of iodin and bromin give precipitates. Benzoyl chlorid, silver nitrate and mercuric chlorid likewise precipitate the protamins. They are also thrown down as cuprous compounds by copper sulphate and sodium hyposulphite.

On boiling with dilute sulphuric acid for a short time the protamins hydrolyze partially, forming substances which may be compared to the peptons. To these primary cleavage products Kossel has applied the term proton. On prolonged boiling with acid these are decomposed, giving rise to the hexon bases. Kossel and Mathews have shown that while pepsin is without effect on the protamins, trypsin decomposes them, like an acid, yielding protons and eventually the hexon bases. In this case the biuret reaction disappears as in the auto-digestion of the pancreas. In this respect the digestion of protamins is similar to that of the more complex proteids (see page 424).

Salmin and clupein according to Kossel ${ }^{1}$ are identical and possess the formula $\mathrm{C}_{30} \mathrm{H}_{57} \mathrm{~N}_{17} \mathrm{O}_{6}$. The composition of sturin is represented by $\mathrm{C}_{36} \mathrm{H}_{69} \mathrm{~N}_{19} \mathrm{O}_{7}$ and that of accipenserin by $\mathrm{C}_{35} \mathrm{H}_{72} \mathrm{~N}_{18} \mathrm{O}_{9}$.

As to the inner structure of the protamin molecule very little that is definite can be said. The large amount of arginin present in these substances indicates that this base forms an important part of the molecule. The hexon bases contain each 6 carbon atoms and the comparison of these by Kossel to the hexoses of the carbohydrate group is certainly very ingenious. Usually on hydration the

1 Zeits. physiol. Chem., 25, 168, 173. 
carbohydrates yield the characteristic hexose unit. On similar treatment the protamins, and to less extent the more complex proteids, may be considered as yielding the equally characteristic hexon unit. In the case of sturin Kossel and Kutscher ${ }^{1}$ have rendered it probable that a molecule of this protamin breaks up into four molecules of arginin and one each of histidin and lysin.

In view of the marked germicidal properties possessed by nucleinic acid it is of great interest to note that the protamins are likewise destructive to bacteria. This fact has been shown by H. Kossel ${ }^{2}$ who tested the action of sturin and of its carbonate upon a number of germs. The most noticeable effect was obtained with the cholera vibrio. These, in water suspension, were killed within five minutes by sturin carbonate in dilutions as high as 1 to 10,000. Even a 1 to 50,000 solution exerted a marked action, though the time required was several hours. Typhoid bacilli and staphylococci were obviously not destroyed as readily, but a marked diminution was observed when the protamin was allowed to act for twenty-four hours. Spores of anthrax however were not affected. Solutions of protamin carbonate in ox-serum in a strength of 1 to 5,000 destroyed anthrax bacilli within four hours. On the other hand cholera and typhoid bacilli, though greatly diminished in numbers, were still present in viable condition at the end of that time.

It is evident from the above quoted experiments that the animal cell possesses germicidal substances other than nucleinic acid. It should be remembered, however, that the protamins are by no means widely distributed; on the contrary they appear to be restricted to the spermatozoa of certain animals.

Another fact brought out by $\mathrm{H}$. Kossel is the marked poisonous action of protamins and of histon. Injected subcutaneously the protamins produce in guinea-pigs severe local inflammation which brings on extensive exudate and finally pronounced necrosis. When injected intraperitoneally or intravenously both substances cause death in a few minutes. The fact that histon possessed toxic properties was first pointed out by Novy. ${ }^{3}$ When thymus histon was injected subcutaneously into guinea-pigs a slight temporary depression of temperature followed. This was followed in a few hours by an increase of 1 to $1.5^{\circ}$, which would persist for two or more days. Necrosis at the point of inoculation was frequently observed. In rabbits intravenous injections produced exceedingly rapid effects, death often resulting in a few minutes.

Since then the toxic action of histon has been recognized by Kossel and by Thompson. The fall in blood pressure, the change in respiratory movements, the retarding effect upon coagulation and the

${ }^{1}$ Zeits. physiol. Chem., 31, 185 ; 25, 184.

${ }^{2}$ Zeits. f. Hygiene, 27, 36, 1898.

${ }^{3}$ Journ. Exp. Med., 1, 709, 1896. 
agglutination of corpuscles together with the decrease in leucocytes is no wise different from that of the protamins. This interesting fact is readily explainable when it is remembered that the histons are in all probability compounds of protamins and proteids. According to Bang ${ }^{1}$ the nucleoproteid from the pancreas and its cleavage product guanylic acid possess a physiological action not unlike that of histon and protamin.

The physiological action of protamins has been the subject of a careful study by Thompson ${ }^{2}$ who showed that clupein and salmin, which are probably identical, and scombrin show no difference in their effects whereas the more complex sturin is somewhat less poisonous. A marked and relatively rapid fall in blood pressure was observed shortly after the injection of the protamins. In dogs death rapidly followed if the dose exceeded $0.15-0.18 \mathrm{~g}$. of clupein per 10 kilograms of body weight. The limit in the case of sturin was about $0.20-0.25 \mathrm{~g}$. With non-fatal doses the blood pressure returns to the normal in about half an hour and a subsequent injection of an otherwise fatal dose is tolerated. According to Thompson the protamins, like the albumoses, act directly upon the walls of the bloodvessels. The effect upon the respiration is extremely marked and when death occurs it is due to paralysis of the respiratory movements. The drop in the blood pressure is accompanied by an increase in the depth and frequency of both the abdominal and thoracic respiratory movements. During the period of minimum blood pressure the respiratory movements cease. With the rise in pressure the abdominal respiration is resumed but the thoracic movements are held in check as long as the poison continues to act. The effect upon the coagulation of blood was also shown to be marked. While a single injection shows but little effect, two or three injections delay the coagulation for many hours. A retardation was also observed when the blood was added to protamin in a test-tube. It is worthy of note that the blood became more transparent, and granular. The corpuscles thus agglutinated settle rapidly, leaving a clear supernatant plasma. In this respect the protamins behave like albumose, histon, venoms, ricin, guanylic acid and other agglutinants. The number of circulating leucocytes was appreciably decreased.

From the above it is evident that the protamins and histons are active poisons. The toxicity is connected with the protamin molecule as a whole inasmuch as it disappears completely on cleavage. Thus, the very earliest hydrolytic products, the proton, or pepton of the protamins, exerts but very little effect in $0.5 \mathrm{~g}$. doses, and the final products of cleavage, the hexon bases, according to Thomson show no action whatsoever upon the blood pressure or upon the respiration. Arginin and histidin, like antipepton, hasten the coag-

1 Zeits. physiol. Chem., 32, 201, 1901.

8 Zeits. physiol. Chem., 29, 1, 1900. 
ulation of blood. The inertness of arginin, it may be added, was first pointed out by Schmiedeberg ${ }^{1}$ in 1896 . Matthews has shown that arginin in large amount exerts a distinct retarding effect upon tryptic digestion.

It has already been pointed out that the protamins precipitate solutions of albumose or pepton and give rise to histon, or at all events to histon-like bodies. Inasmuch as the natural histons show many points of resemblance to the protamins it is quite probable that these substances represent the next higher group of proteids. A brief consideration of the histons in this connection is therefore by no means out of place.

The first representative of this group was discovered in 1884 by Kossel. ${ }^{2}$ Inasmuch as Miescher had previously shown that the spermatozoa of the salmon contained a salt-like combination of nuclein and protamin, the thought suggested itself that a similar compound of nuclein and a basic body might be present in the red corpuscles of the blood of geese. On extracting the well washed stroma, perfectly free from hemoglobin, by means of dilute hydrochloric acid he obtained a pepton-like body to which he gave the name histon. Ten years later Lilienfeld ${ }^{3}$ showed that the nucleohiston from the leucocytes of the thymus gland on treatment with dilute hydrochloric acid was split up into nuclein and histon. The latter, although coagulable by heat, he regarded as identical with the histon of Kossel. Very soon thereafter histon was reported to be present in febrile urine (Krehl and Matthes ${ }^{4}$ ) and in leukemic urine (Kolisch and Burian ${ }^{5}$ ).

An examination of the spermatozoa of the sea-urchin for protamin by Mathews showed that ${ }^{\circ}$ these cells contained a compound of nuclein and of a histon-like body to which he gave the name arbacin. This interesting observation is substantiated by that of Miescher, ${ }^{7}$ who first pointed out that the salmon spermatozoa if immature did not contain protamin, but instead a histon-like substance-albuminose. A similar condition was observed by Bang ${ }^{8}$ in connection with mackerel spermatozoa. These, when mature, as already pointed out, contain the protamin scombrin, but the immature spermatozoa do not contain the substance, but instead a histon, which Bang designates as scombron. Shortly thereafter Kossel and Kutscher ${ }^{9}$ showed that the same was true for the mature spermatozoa of the cod (Gadus morrhua). Ehrström ${ }^{10}$ arrived at a similar result with the

${ }_{1}$ Berichte, 29, 355.

2 Zeits. physiol. Chem., 8, 511.

${ }^{3}$ Zeits. physiol. Chem., 18, 482, 1894.

4 Archiv. exp. Path. u. Pharm., 36, 441, 1895.

5 Zeits. klin. Med., 29, 374, 1896.

'Zeits. physiol. Chem., 23, 399, 1897.

7 Archiv. exp. Path. u. Pharm., 37.

8 Zeits. physiol. Chem., 27, 466, 1899.

Y Zeits. physiol. Chem., 31, 191, 1900.

10 Zeits. physiol. Chem., 32, 350, 1901. 
mature spermatozoa of the Lota vulgaris, a fish closely related to the former.

It is evident from these investigations that while the mature spermatozoa of some fish contain a salt-like combination of nucleinic acid and a protamin, the same spermatozoa in the immature condition contain a similar componnd in which, however, the protamin is replaced by histon. Again, in some fish as the cod tribe protamin is absent even from the mature cells and its place is taken by histon. Our knowledge of the composition of the spermatozoa of higher animals is extremely deficient. According to Mathews the spermatic cells of the steer and boar contain neither histon nor protamin, while according to Bang the liver, kidney, pancreas and testes contain no histon. At least no such body could be extracted from these organs by dilute acid. On the other hand, the researches of Schulz ${ }^{1}$ show that the proteid constituent of hemoglobin, globin, is a histon. In this instance the histon occurs in combination with the acid hematin, while in all other instances it is combined with nucleinic acid. Thus far, then, histon bodies have been shown to be present in the stroma of red blood cells of geese, in hemoglobin, in the leucocytes of the thymus gland, usually in the immature and at times in mature spermatozoa of certain animals.

Obviously, as in the case of the protamins, we have to deal with a group of histons and not with a single individual. With this fact in mind it becomes easy to reconcile the divergent statements which have been made by different investigators regarding the chemical reactions of these substances. Like the protamins, the histons possess marked basic properties and on decomposition with dilute acid they yield the hexon bases. The amount of bases is by no means as large as that obtained from the protamins, but at the same time it is much greater than that from other proteids. Looked at from the standpoint of the amount of hexon bases contained in the molecule the protamins occupy the first, the histons the second and the gelatins the third place. The thymus histon, according to Kossel, yields about 40 per cent. of its nitrogen in the form of hexon bases, while according to Lawrow ${ }^{2}$ these bases make up about 25 per cent. of its weight. The hemoglobin of the horse, on cleavage with hydrochloric acid and tin, yields, according to the same observer, ${ }^{3}$ three hexon bases and the amount corresponds to about 20 per cent. of the globin present. The Lota histon gave Ehrström 12 per cent. arginin, 3.17 per cent. lysin, 2.85 per cent. histidin and 0.66 per cent. ammonia. Analyses of gadus and thymus histons are given in the table on p. 426.

The histons yield several characteristic reactions. Neutral solu-

${ }_{1}^{1}$ Zeits. physiol. Chem., 24, 449, 1898.

2 Zeits. physiol. Chem., 28, 390, 1899.

${ }^{3}$ Berichte, 34, 101, 1901. 
tions, free from salts of ammonia, are precipitated by ammonia, but the precipitate redissolves in excess of the reagent. The scombron precipitate however does not redissolve. In the presence of ammonia salts the precipitate induced by ammonia is permanent. As pointed out by Bang the acid albuminates and vitellin are likewise precipitated by ammonia. The fixed alkalis also precipitate histon. While pure solutions of histon are not coagulated on boiling this does occur in the presence of a small amount of salt. As Kossel first showed histon may be precipitated from acid solution by saturation with sodium chlorid or other salts. With nitric acid the histons give the albumose reaction; likewise the xanthoproteic reaction. The biuret test is given by all. With Millon's reagent the reaction is slight but distinct. Salts of mercury precipitate some histons, but not others. The most important reaction is based upon the fact that while ordinary proteids are precipitated by certain alkaloidal reagents in acid but not in neutral solution, the histons like protamins, because of their pronounced basic character, are precipitated by these reagents from neutral as well as acid solutions. Sodium ferrocyanid, picrate, phosphotungstate and phosphomolybdate can be thus used. Again, like the protamins, histon precipitates proteids, provided free alkali is not present in excess. This property is likewise seen in the marked agglutinating action which histon exerts on bacteria. On digestion with pepsin thymus histon is split up and a protamin-like body results (Bang).

The physiological action of histon is very similar to that of prota$\min ($ p. 432). The action of thymus histon on toxins and on bacteria with special reference to immunity has been studied by Novy. ${ }^{1}$

Histon may be prepared direct from the thymus gland. ${ }^{2}$ For this purpose the gland is finely divided and extracted with water. To the aqueous solution hydrochloric acid is added to 0.8 per cent. concentration. The resultant precipitate is removed by centrifugation and from the clear filtrate the histon is thrown down by addition of ammonia. The precipitate is washed with ammoniacal water, then with alcohol and finally with ether.

According to Fleroff ${ }^{3}$ the thymus gland on extraction with dilute sulphuric acid, as in the method for protamins, yields histon and para-histon. The latter is soluble in ammonia and water and is thus separated from histon. For the separation of histon from spermatozoa, see Kossel (Z.P. C., 31, 192); from hemoglobin, see Schulz (Z. P. C., 24, 456).

Arginin, $\mathrm{C}_{6} \mathrm{H}_{14} \mathrm{~N}_{4} \mathrm{O}_{2}$. - The study of the constitution of this base has revealed several important facts which enable us to explain satisfactorily a number of vital reactions. The observation of Drech-

1 Journ. Exp. Med., 1, 709, 1896.

Z Zeits. physiol. Chem., 31, 189; 28, 388.

${ }^{3}$ Zeits. physiol. Chem., 28, 307. 
sel in 1890 that lysatinin (p. 425) on boiling with baryta gave urea led Schulze and Likiernik to make a similar experiment with arginin. They also obtained urea. As already pointed out lysatinin was eventually shown to be a mixture of arginin and lysin. This was the first time that urea was successfully prepared by the mere hydration of a cleavage product of proteids. It indicated that arginin was without doubt a guanidin derivative.

Subsequently Schulze and Winterstein ${ }^{1}$ demonstrated that the cleavage of arginin by means of baryta or other alkali yielded urea and oruithin, this change being represented by the equation :

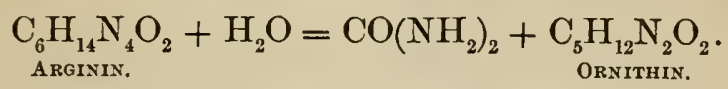

Ornithin had been demonstrated by Jaffé, twenty years before, to be an intermediate waste product in the metabolism in chicken. When benzoic acid is introduced into the body of mammals, as is well known, it unites with glococoll and is eliminated as hippuric acid. In birds it combines with ornithin and is excreted as ornithuric acid, $\mathrm{C}_{19} \mathrm{H}_{20} \mathrm{~N}_{2} \mathrm{O}_{4}$. The latter was shown by Jaffé to be a dibenzoyl ornithin and the base itself he regarded as a diamido valerianic acid although at the time no proof of this could be adduced. On treatment with nitrous acid Schulze and Winterstein demonstrated the presence of two amido groups in ornithin. This fact aud the formation of urea led them to ascribe to arginin the following structure which subsequent investigations proved to be correct.

$$
\begin{aligned}
& \mathrm{NH}_{2} \\
& \mathrm{NH} \cdot \mathrm{CH}_{2} \cdot \mathrm{CH}_{2} \cdot \mathrm{CH}_{2} \cdot \mathrm{CH}\left(\mathrm{NH}_{2}\right) \cdot \mathrm{COOH} .
\end{aligned}
$$

It is evident from this structure that arginin is closely related to creatin.

$$
\begin{aligned}
& \mathrm{NH}_{2} \\
& \mathrm{~N} \cdot \mathrm{NH} \\
& \mathrm{N}\left(\mathrm{CH}_{3}\right) \cdot \mathrm{CH}_{2} \cdot \mathrm{COOH} .
\end{aligned}
$$

Analogous to the synthesis of creatin from cyanamid and sarkosin, it is possible to effect the synthesis of arginin, as Schulze and Winterstein ${ }^{2}$ have done, by the action of cyanamid on ornithin. These investigators supplied additional evidence in favor of the correctness of the structure above given by converting ornithin by dry distillation and hence closing the chain, into a pyrrolidin-like body.

In 1889 Udránszky and Baumann ${ }^{3}$ pointed out the close relation

1 Zeits. physiol. Chem., 26, 1, 1898 ; 34, 144.

${ }^{2}$ Berichte, 32, 3191 (1899) ; Zeits. physiol. Chem., 34, 128.

${ }^{3}$ Zeits. physiol. Chem., 13, 590. 
between ornithin and the ptomain putrescin and the possibility of the latter being formed by splitting off carbonic acid. Although they had in mind experiments in this direction yet nothing was done until Ellinger ${ }^{1}$ effected the transformation. On decomposing ornithin by the aid of bacteria, especially in the absence of oxygen, he obtained putrescin and thus not only furnished a correct explanation of the origin of this ptomain but also confirmed the position of the amido groups in ornithin and hence in arginin. The cleavage of proteids by bacteria therefore can be traced through arginin and ornithin to putrescin. The structural relation of these bodies can be seen from the equation :

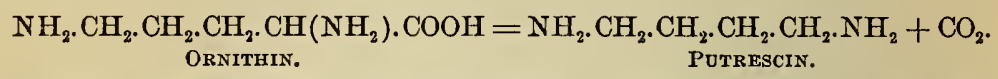

From this it is evident that ornithin is $\alpha-\delta$ diamido valerianic acid and is the next lower homologue of lysin (see p. 444). This diamido valerianic acid in the inactive or racemic form has been recently synthesized by Fischer. ${ }^{2}$ Like ornithin it has a strong alkaline reaction and gives a white precipitate with mercuric chlorid ; with phosphotungstic acid it yields a heavy crystalline precipitate which can be recrystallized from hot water in colorless needles. With benzoyl chlorid it forms monobenzoyl and di-benzoyl derivatives corresponding to those of ornithin. While Fischer found the natural ornithuric acid to be dextro-rotatory, the synthetic one was inactive, otherwise the two showed the greatest resemblance. The synthetic ornithuric acid melts at $184-185^{\circ}$ whereas the natural acid melts at $182^{\circ}$ (Jaffe) ; $184^{\circ}$ (Schulze and Winterstein). Both yield the characteristic calcium salt. The $d$-amido valerianic which Salkowski isolated from putrefying proteids in all likelihood is derived from the former.

On oxidation with potassium permanganate arginin is destroyed but by employing the barium salt Benech and Kutscher ${ }^{3}$ succeeded in oxidizing the base with the formation of guanidin, thus demonstrating positively the presence of this group in the arginin molecule. Just as creatin is a methyl guanidin acetic acid so arginin becomes guanidin- $\alpha$-amido valerianic acid. On further examination of the oxidation products Kutscher ${ }^{4}$ obtained $\gamma$-guanidin butyric acid and also succinic acid. The oxidation of arginin can be shown as follows:

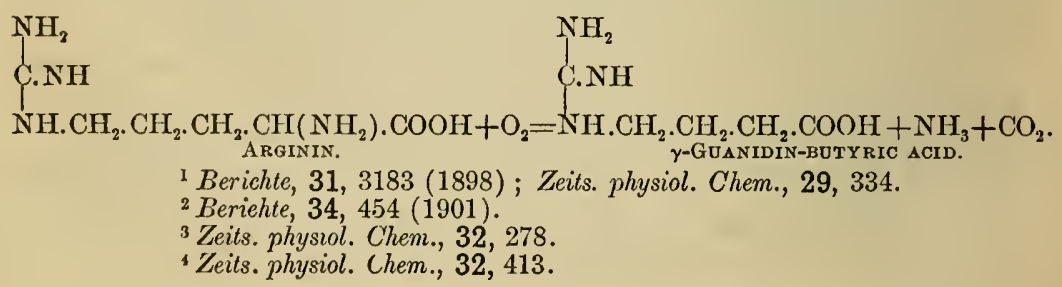




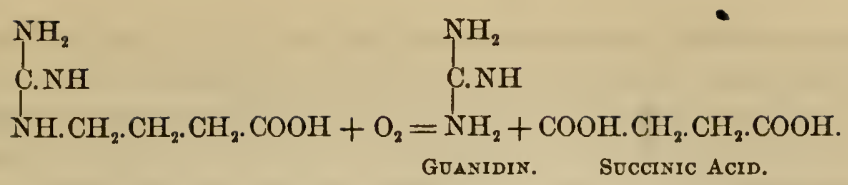

The demonstration of the formation of succinic acid from arginin explains the presence of this acid in fermentation and putrefaction. Indeed it will be seen that the study of the cleavage products of proteids and more directly of arginin have given us an entirely different view of the origin of urea, guanidin, putrescin and of succinic and amido valerianic acids, whether formed within the body, or by the action of bacteria or of chemicals on proteids outside of the body.

Arginin exists in both the active and inactive form. According to Kutscher ${ }^{1}$ all proteids except fibrin yield the dextro-rotatory base. It should be noted in this connection that fibrin is the only proteid examined which has been submitted to tryptic digestion; all others have been hydrolyzed by acids and it is quite probable that if treated with trypsin they will also yield inactive arginin. Moreover, auto-digestion of the pancreas, if short in duration, may yield also the inactive form. From Balke's antipepton obtained by the pancreatic digestion of fibrin he isolated both forms whereas in autodigestion of the pancreas ouly the active arginin was found. It would seem from this as if the fibrin either contained only inactive arginin or else it contains a levo-form which racemizes with the dextro-arginin derived from the pancreas proper. The dextroarginin on heating with sulphuric acid, and its nitrate when exposed to dry-heat of $210^{\circ}$, yields the inactive form. On account of slight differences Gulewitsch believed that the arginin derived from plants was distinct from that of animal proteids but this view has been shown by Schulze ${ }^{2}$ to be incorrect. The active and inactive arginins are the only two kinds known at present.

The free base can be prepared from the acid arginin silver nitrate by changing this to arginin silver which in turn can be decomposed with hydrogen sulphid. It crystallizes in rosette-like masses of plates or thin prisms which melt at $207-207.5^{\circ}$ (corr.). It is odorless but has a slightly bitter taste; is strongly alkaline and takes up carbonic acid. From solutions of the salts of heavy metals it precipitates the oxids and from ammonium salts it liberates ammonia. It is easily soluble in water but is almost insoluble in boiling alcohol. Like ammonium salts and amido compounds it takes up iodin and yields iodic acid. (Schmidt, Z. P. C., 34, 64.) Sodium 
hypobromate sets free about one-third of its nitrogen. The salts of arginin have been especially studied by Gulewitsch. ${ }^{1}$

The neutral chlorid, $\mathrm{C}_{6} \mathrm{H}_{14} \mathrm{~N}_{4} \mathrm{O}_{2} \cdot \mathrm{HCl}+\mathrm{H}_{2} \mathrm{O}$, is easily soluble in water from which it can be crystallized on concentration or on addition of alcohol or ether. It is less soluble in hot than in cold 85 per cent. alcohol. It forms rosette-like masses of plates. The water of crystallization may be driven off at $100^{\circ}$ (Hedin). Moreover, the chlorid may crystallize from water without taking up water of crystallization (Schulze ${ }^{2}$ ). Lawrow obtained a chlorid containing one-half molecule of water. The anhydrous salt sinters at $208^{\circ}$, gives off gas and melts at $209^{\circ}$ or higher. When heated with concentrated hydrochloric acid ammonia is split off, but only at high temperatures $-180^{\circ}-200^{\circ}$. It is strongly dextro-rotatory; the rotation is increased by acid and decreased by alkali. Hedin ${ }^{3}$ was unable to obtain a crystalline acid chlorid.

The neutral nitrate, $\mathrm{C}_{6} \mathrm{H}_{14} \mathrm{~N}_{4} \mathrm{O}_{2} \cdot \mathrm{HNO}_{3}+\frac{1}{2} \mathrm{H}_{2} \mathrm{O}$, results on decomposition of the basic silver or copper nitrate salts. It can be readily crystallized from water or from hot dilute alcohol, forming opaque chalk-like masses composed ot microscopic needles which tend to effloresce. The salt is somewhat hygroscopic; when dry it melts imperfectly at about $175^{\circ}$. At $15^{\circ}$ it is soluble in about two parts of water. It is very easily soluble in hot, difficultly in cold 85 per cent. alcohol. The salt is dextro-rotatory and the rotation is markedly affected by the presence of free acid. It dissolves copper hydrate, forming the double salt.

The neutral nitrate of the inactive arginin which Kutscher isolated from antipepton (pp. 424, 439) forms small, glistening, transparent four-sided prisms or plates, which often unite in groups and do not contain water of crystallization. It is more difficultly soluble than the dextro-salt ( 1 to 17.25 water at $20^{\circ} ; 1$ to 21.7 parts of water at $\left.12^{\circ}\right) .^{4}$ It sinters at $206^{\circ}$ and melts at $211^{\circ}$ with gas formation.

The acid nitrate, $\mathrm{C}_{6} \mathrm{H}_{14} \mathrm{~N}_{4} \mathrm{O}_{2} \cdot 2 \mathrm{HNO}_{3}$, is prepared by evaporating the former salt with excess of acid. It forms long colorless needles or masses of extremely thin elongated plates. The melting point is $144.5-145^{\circ}$.

The sulphate, $\mathrm{C}_{6} \mathrm{H}_{14} \mathrm{~N}_{4} \mathrm{O}_{2} . \mathrm{H}_{2} \mathrm{SO}_{4}$, can be obtained by decomposing the copper sulphate compound. It separates from saturated alcoholic solution as an oil and has not been crystallized (G.). Hedin also was unsuccessful in crystallizing the sulphate. It is hygroscopic and behaves to polarized light the same as the preceding salts.

Zeits. physiol. Chem., 27, 178.

${ }^{2}$ Zeits. physiol. Chem., 29, 331.

${ }^{3}$ Zeits. physiol. Chem., 22, 157.

'Zeits. physiol. Chem., 32, 478. 
The picrate, $\mathrm{C}_{6} \mathrm{H}_{14} \mathrm{~N}_{4} \mathrm{O}_{2} \cdot \mathrm{C}_{6} \mathrm{H}_{3} \mathrm{~N}_{3} \mathrm{O}_{7}$, was prepared by Schulze and Steiger (Z. P. C., 11, 43).

Arginin copper nitrate, $\left(\mathrm{C}_{6} \mathrm{H}_{14} \mathrm{~N}_{4} \mathrm{O}_{2}\right)_{2} \cdot \mathrm{Cu}\left(\mathrm{NO}_{3}\right)_{2}+3 \frac{1}{2} \mathrm{H}_{2} \mathrm{O}$, was obtained by boiling the nitrate with copper carbonate or hydrate. Hedin, ${ }^{1}$ Lawrow, ${ }^{2}$ as well as Schulze ${ }^{3}$ obtain the salt with only three molecules of water, possibly because of loss of same by efflorescence. This takes place slowly when the salt is kept in a desiccator. When crystallized from hot water it forms roundish aggregates of dark blue needles or pointed prisms. Impure solutions are difficult to crystallize. It reacts alkaline and is soluble in 95.5 parts of water at $13^{\circ}$. It melts at $112^{\circ}-114^{\circ}$ but when dehydrated it melts with decomposition at $232^{\circ}-234^{\circ}$.

The copper sulphate, $\left(\mathrm{C}_{6} \mathrm{H}_{14} \mathrm{~N}_{4} \mathrm{O}_{2}\right)_{2} \cdot \mathrm{CuSO}_{4}+5 \frac{1}{2} \mathrm{H}_{2} \mathrm{O}$, was obtained by boiling the sulphate with cupric hydrate. It melts at about $110^{\circ}$ and loses all the water of crystallization at $150^{\circ}$ and decomposes at $170^{\circ}$. The dehydrated salt decomposes at $235^{\circ}-238^{\circ}$.

The acid silver nitrate compound, $\mathrm{C}_{6} \mathrm{H}_{14} \mathrm{~N}_{4} \mathrm{O}_{2} . \mathrm{HNO}_{3}+\mathrm{AgNO}_{3}$, on slow evaporation of its aqueous solution forms long colorless needle-shaped prisms usually grouped in bundles. From hot saturated solution it separates in opaque stellate masses of long, very thin needles. Its solution which is very slightly acid is not reduced on heating. It is soluble in 7.27 parts of water at $16^{\circ}$; is insoluble in alcohol and in ether, and is dextro-rotatory. It melts with decomposition at $176^{\circ}-183^{\circ}$.

The basic silver nitrate compound, ${ }^{4} \mathrm{C}_{6} \mathrm{H}_{14} \mathrm{~N}_{4} \mathrm{O}_{2} \cdot \mathrm{AgNO}_{3}+\frac{1}{2} \mathrm{H}_{2} \mathrm{O}$, forms rosette or wart-like aggregates of short, colorless, transparent prisms. Its solution reacts alkaline; is easily reduced by light and by heating. At $16^{\circ}$ it dissolves in 88.7 parts of water ( 81 parts, Hedin); is easily soluble in hot water. It is insoluble in alcohol and in ether. It decomposes at $164^{\circ}$. This salt has been used as a means of separation (Hedin).

Arginin-silver, ${ }^{5} \mathrm{C}_{6} \mathrm{H}_{12} \mathrm{Ag}_{2} \mathrm{~N}_{4} \mathrm{O}_{2} \cdot \mathrm{H}_{2} \mathrm{O}$, is obtained as a snow-white cheesy precipitate when sodium hydrate is added carefully to the acid silver nitrate compound. It is usually mixed with the similar body containing three atoms of silver. The compound takes up carbonic acid; when powdered is markedly electric and darkens on exposure to light. The moist powder when heated rapidly becomes black. It is very difficultly soluble in water ( 1 in 28,571$)$; easily soluble in acids and in ammonia.

A mercuric chlorid compound forms when this salt is added to a solution of arginin. The voluminous, white, amorphous precipitate is soluble in acids, and in hot water. When purified it becomes very

1 Zeits. physiol. Chem., 20, 191.

2 Zeits. physiol. Chem., 28, 392.

${ }^{3}$ Zeits. physiol. Chem., 11; 52 ; 29, 331 ; 33, 561 ; 34, 138.

- Zeits. physiol. Chem., 20, 188; 21, 156; 27, 200.

${ }^{5}$ Zeits. physiol. Chem., 22, 194 ; 27, 202. 
difficultly soluble in boiling water. It melts and decomposes at $186^{\circ}-189^{\circ}$. Potassium mercuric iodid alone gives no precipitate but on addition of sodium hydrate a heavy white precipitate forms. Potassium bismuth iodid gives a red precipitate. The picrate melts at $205^{\circ}$.

The addition of phosphotungstic acid to solutions of the nitrate produces a pulverulent precipitate which, recrystallized from boiling water, forms very small prisms or needles. It is soluble in large excess of the reagent.

Dibenzoyl arginin, $\mathrm{C}_{6} \mathrm{H}_{12}\left(\mathrm{C}_{6} \mathrm{H}_{5} \cdot \mathrm{CO}\right)_{2} \mathrm{~N}_{4} \mathrm{O}_{2}$, is obtained readily, though the yield is small. On treatment with benzoyl chlorid a slight sticky precipitate forms and from the filtrate the dibenzoyl compound is thrown down on acidulation and is separated from benzoic acid by means of ether. From boiling water it crystallizes in masses of very fine long needles or rhombic plates. The needleshaped crystals are often branched, or stellate and show split ends. It is difficultly soluble in boiling water; more readily in boiling alcohol ; easily in alkali. It melts with slight decomposition at $217^{\circ}-$ $218^{\circ}$. This body is analogous to ornithuric and lysuric acids.

Histidin, $\mathrm{C}_{6} \mathrm{H}_{9} \mathrm{~N}_{3} \mathrm{O}_{2} .-$ As yet nothing definite is known regarding the constitution of this base. From the small amount of hydrogen present it would seem that the structure of histidin must be that of a closed chain. When it is remembered that cadaverin on dry distillation yields piperidin (p. 273), it does not seem improbable but that arginin, the parent substance of cadaverin, may in a similar manner, by closing the chain yield histidin. The change in the case of cadaverin is represented thus :

$$
\mathrm{NH}_{2} \cdot \mathrm{CH}_{2} \cdot \mathrm{CH}_{2} \cdot \mathrm{CH}_{2} \cdot \mathrm{CH}_{2} \mathrm{CH}_{2} \cdot \mathrm{NH}_{2}=\mathrm{NH}_{3} \cdot
$$

The transformation of arginin into histidin would in a similar way be represented by the equation:

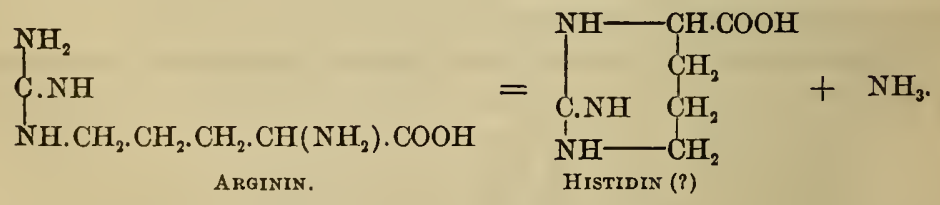

If the above change is correct histidin would possess the formula- 
$\mathrm{C}_{6} \mathrm{H}_{11} \mathrm{~N}_{3} \mathrm{O}_{2}$. The analytical results correspond about as closely with the theoretical percentages required for this formula as with $\mathrm{C}_{6} \mathrm{H}_{9} \mathrm{~N}_{3} \mathrm{O}_{2}{ }^{1}$ According to this interpretation of histidin, like arginin, it should yield on cleavage urea, guanidin and $\delta$ amido valerianic acid. The latter has been repeatedly found among the cleavage products of the protamins. $^{2}$ The conversion of creatin into creatinin is analogous to that shown above with the difference however that water and not ammonia is given off.

If the above view of the origin of histidin be correct it will be seen that the hexon bases acquire a new importance in so far as they would form antecedents of the pyrimidin and even purin bodies. The nucleus of the cell therefore would transform the ready-made protoplasmic bases into the characteristic nuclein bases.

Histidin itself forms well defined rhombic plates or needles. It is not very soluble in water and hence can be crystallized from it direct or by addition of alcohol. The solution shows faint alkaline reaction and does not take up carbonic acid (Hedin). It is insoluble in alcohol and in ether. According to Kossel, ${ }^{3}$ the free base is levowhile its salts are dextro-rotatory; and the rotation is increased by the presence of free acid. Histidin from thymus histon, according to Lawrow, ${ }^{4}$ is dextro-rotatory, which fact considered in connection with the difference observed between the chlorid of histidin from histon and from other sources, may be taken to indicate different histidins.

The free base is usually obtained from the chlorid by decomposition with silver sulphate. Possibly histidin unites with either of the other hexon bases. This is indicated in the isolation of a compound, $\mathrm{C}_{18} \mathrm{H}_{35} \mathrm{~N}_{7} \mathrm{O}_{5}$, by Kossel and Mathews, ${ }^{5}$ from sturin by digestion with trypsin. This compound consists probably of one molecule of histidin and two molecules of lysiu. Sturin itself probably contains the three bases in combination.

The monochlorid, ${ }^{6} \mathrm{C}_{6} \mathrm{H}_{9} \mathrm{~N}_{3} \mathrm{O}_{2} \cdot \mathrm{HCl}+\mathrm{H}_{2} \mathrm{O}$, is obtained on decomposition of the histidin-silver compound with hydrochloric acid (Hedin) and crystallizes in fine, large, glassy plates which do not lose water of crystallization at $120^{\circ}$, but do at $135^{\circ}$ (Hedin), at $105^{\circ}$ (Kossel). It melts at $251^{\circ}-252^{\circ}$; is easily soluble in water, insoluble in alcohol and in ether. The aqueous solution is acid in reaction and is slightly dextro-rotatory, although Hedin first considered it to be inactive. The chlorid from gelatin and from silk melts at $243^{\circ}$ (Wetzel).

The di-chlorid, ${ }^{7} \mathrm{C}_{6} \mathrm{H}_{9} \mathrm{~N}_{3} \mathrm{O}_{2} .2 \mathrm{HCl}$, contains no water of crystalliza-

1 Zeits. physiol. Chem., 22, 184, 194.

2 Zeits. physiol. Chem., 26, 590 ; 31.

3 Zeits. physiol. Chem., 28, 382.

'Zeits. physiol. Chem., 28, 392 ; 31, 193.

5 Zeits. physiol. Chem., 25, 193 ; 31, 185.

6 Zeits. physiol. Chem., 22, 182, 192; 25, 192; 28, 387, 460.

${ }^{7}$ Zeits. physiol. Chem., 28, 383, 392 ; 31, 189, 193. 
tion which in the above appears to be replaced by the second molecule of acid (Schwantke). It can be obtained from the monochlorid by repeated treatment with concentrated hydrochloric acid (Kutscher). On slow crystallization it forms large glassy plates, except when the salt is derived from histon, in which case it crystallizes slowly and with different form. Differences in solubility have also been noted. It sinters at $225^{\circ}$ and finally melts at $231^{\circ}-233^{\circ}$ with evolution of ammonia. The latter is also given off on prolonged heating at $140^{\circ}$.

The nitrate, $\mathrm{C}_{6} \mathrm{H}_{9} \mathrm{~N}_{3} \mathrm{O}_{2} .2 \mathrm{HNO}_{3}$, was obtained by Kossel ${ }^{1}$ in crystalline form, although Hedin was unsuccessful in doing so with the nitrate or sulphate. With platinum chlorid and silver nitrate crystallizable double salts are obtainable. A rather uncertain double salt of barium chlorid has been reported (impure dichlorid ?).

Mercuric chlorid precipitates the alkaline carbonate solution of bistidin and this fact was utilized at one time by Kossel ${ }^{2}$ as a means of separation from arginin.

Silver nitrate when added to an aqueous solution of the base produces no precipitate, but on addition of ammonia a voluminous precipitate forms which is easily soluble in excess (Hedin). ${ }^{3}$ Its formula is $\mathrm{C}_{6} \mathrm{H}_{7} \mathrm{Ag}_{2} \mathrm{~N}_{3} \mathrm{O}_{2}+\mathrm{H}_{2} \mathrm{O}$. Instead of ammonia Kossel employs baryta, which on careful addition throws down the histidin compound first and, subsequently in strong excess, precipitates the arginin compound. These two bases can thus be easily separated (see p. 427).

Lysin, $\mathrm{C}_{6} \mathrm{H}_{14} \mathrm{~N}_{2} \mathrm{O}_{2} \cdot$ - The relation of this base to leucin, $\mathrm{C}_{6} \mathrm{H}_{13} \mathrm{NO}_{2}$, from which it differs by an $\mathrm{NH}_{2}$ group led Drechsel ${ }^{4}$ to regard it as a di-amido caproic acid. He attempted, though unsuccessfully, the conversion of lysin into cadaverin and even pointed out its possible transformation into pyridin. Inasmuch as lysin appeared to be a homologue of ornithin, $\mathrm{C}_{5} \mathrm{H}_{12} \mathrm{~N}_{2} \mathrm{O}_{2}$, Ellinger ${ }^{5}$ reasoned that, like the latter, it should yield on putrefaction the diamin cadaverin, the homologue of putrescin. Experiments with lysin derived from casein and from auto-digested pancreas gave the expected cadaverin and at the same time established the constitution of the former. Lysin, therefore, is $\alpha-\varepsilon$ di-amido caproic acid and is the next homologue of ornithin. The cleavage into cadaverin can be represented by an equation similar to that for ornithin (p. 438).

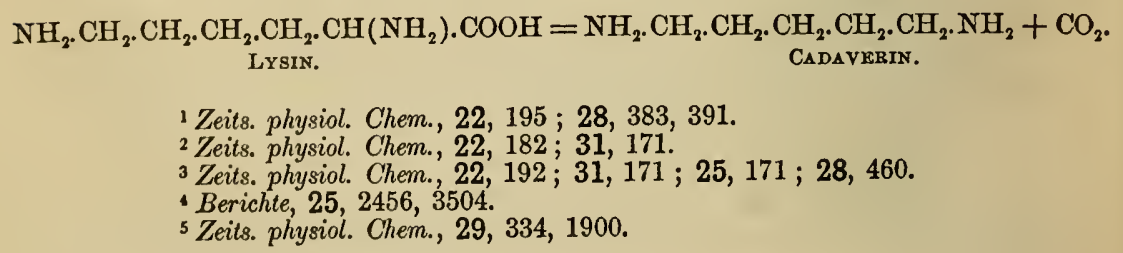
LYSIN.

Cadaverin.

1 Zeits. physiol. Chem., 22, 195 ; 28, 383, 391.

${ }_{2}$ Zeits. physiol. Chem., 22, 182 ; 31, 171.

3 Zeits. physiol. Chem., 22, $192 ; 31,171 ; 25,171 ; 28,460$.

- Berichte, 25, 2456, 3504.

5 Zeits. physiol. Chem., 29, 334, 1900. 
Since lysin is a homologue of ornithin it is possible that like the latter it exists in the proteid molecule in combination with an urea residue. In other words it may have a parent substance which bears to it the same relation that arginin does to ornithin. This theoretical body would possess the formula $\mathrm{C}_{7} \mathrm{H}_{16} \mathrm{~N}_{4} \mathrm{O}_{2}$. On hydration it would yield urea and lysin in the same way that arginin yields urea and ornithin. In this way perhaps may be explained the origin of the ammonia which is formed during the hydrolysis of proteids (see table, p. 426). The existence of the ptomaïn hexamethylene diamin may be taken to indicate a still higher homologue. The relation of these bodies may be indicated thus:

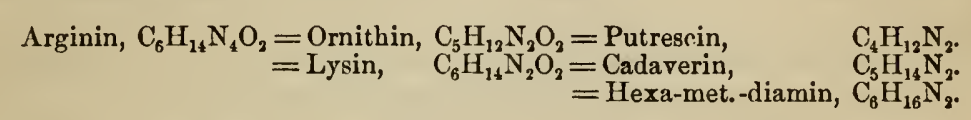

Lysin is easily soluble in water and, like arginin and histidin, it is not precipitated on saturation with ammonium sulphate but is precipitated by alcohol and by phosphotungstic acid. Hence the presence of these bases in antipepton (Kutscher). Unlike the other two bases lysin is not precipitated by silver nitrate and fixed alkali, and this fact Kossel makes use of to effect their separation (p. 428). The free base can be obtained best from the sulphate. On heating it gives off alkaline vapors (Urechsel, Krüger). On fusion with alkali it yields acetic and propionic acids (Henderson).

In the tryptic digestion of sturin Kossel and Mathews obtained a compound, $\mathrm{C}_{18} \mathrm{H}_{35} \mathrm{~N}_{7} \mathrm{O}_{5}$, which crystallized in needles and was evidently a combination of two molecules of lysin with one of histidin. The lysatinin (p. 425) of Drechsel is apparently a similar combination of one molecule each of lysin and of arginin.

The dichlorid, $\mathrm{C}_{6} \mathrm{H}_{14} \mathrm{~N}_{2} \mathrm{O}_{2} .2 \mathrm{HCl}$, can be prepared from the picrate or from the platinum salt. It melts at $217^{\circ}$ and at $261^{\circ}$ it decomposes yielding hydrochloric acid, carbon monoxid, water and a sublimate of ammonium chlorid. Drechsel ${ }^{1}$ interpreted this reaction as leading to amido valeric aldehyde which by loss of water should yield tetrahydropyridin but this transformation, which would be analogous to the change of cadaverin into piperidin, has not as yet been realized. According to Henderson it melts at $192-193^{\circ}$; while Lawrow ${ }^{2}$ states that it begins to melt at $194-195^{\circ}$ and gives off gas at $200-202^{\circ}$. It is not changed to the monochlorid by prolonged heating at $130^{\circ}$. The aqueous solution is strongly acid and on evaporation with the alkali it forms bases possessing a slight coniïn-like odor (Drechsel). The colorless crystals are but slightly soluble in alcohol; readily soluble in methyl alcohol, and readily form from aqueous solution on concentration.

The salt as well as the free base are dextro-rotatory and the devia-

1 Berichte, 25, 2455, 3504.

${ }^{2}$ Zeits. physiol. Chem., 28, 395 ; 29, 321. 
tion of polarized light is increased by acids (Lawrow). On heating with baryta it becomes inactive (Siegfrid, Lawrow). For its behavior to phenyl isocyanate see ornithin. Henderson ${ }^{1}$ examined lysin from six different sources without finding any difference.

The picrate, ${ }^{2} \mathrm{C}_{6} \mathrm{H}_{14} \mathrm{~N}_{2} \mathrm{O}_{2} \cdot \mathrm{C}_{6} \mathrm{H}_{3} \mathrm{~N}_{3} \mathrm{O}_{7}$, is difficultly soluble and is therefore especially useful for the isolation of the base (p. 428). It separates in large crystals, from solutions of the base in alcohol, on the addition of sodium picrate. It is soluble in 185 parts of water (Lawrow).

The carbonate, $2 \mathrm{C}_{6} \mathrm{H}_{14} \mathrm{~N}_{2} \mathrm{O}_{2}+\mathrm{CO}_{2}$, was obtained in crystalline form by Drechsel $^{3}$ and was regarded as analogous to ammonium carbamate.

Phosphotungstic acid gives a precipitate which, when recrystallized from much boiling water, forms needles (Kutscher ${ }^{4}$ ).

Platinum chlorid precipitates lysin from alcoholic solution as $\mathrm{C}_{6} \mathrm{H}_{14} \mathrm{~N}_{2} \mathrm{O}_{2} \cdot 2 \mathrm{HCl} . \mathrm{PtCl}_{4}+\mathrm{C}_{2} \mathrm{H}_{6} \mathrm{O}$ (Drechsel). It forms fine yellowish red prisms from which the alcohol of crystallization can be expelled by drying at $130^{\circ}$. It melts at $219^{\circ}-220^{\circ}$ (Schulze ${ }^{5}$ ). Lysin like arginin, forms two silver salts. The compound $\mathrm{C}_{6} \mathrm{H}_{14} \mathrm{~N}_{2} \mathrm{O}_{2} \cdot \mathrm{AgNO}_{3}$ is more soluble than the corresponding one of arginin and has been used as a means of separation $\left(\right.$ Hedin $\left.^{6}\right)$. The acid compound, $\mathrm{C}_{6} \mathrm{H}_{14} \mathrm{~N}_{2} \mathrm{O}_{2} \cdot \mathrm{HNO}_{3}+\mathrm{AgNO}_{3}$, is easily soluble and crystallizes from water by addition of alcohol and ether in beautiful needles.

Mercuric chlorid ${ }^{7}$ does not precipitate dilute solutions of lysin and arginin. The nitrate by itself does not precipitate lysin or arginin but upon the addition of sodium hydrate these bases are thrown down.

With benzoyl chlorid ${ }^{8}$ lysin forms a di-benzoyl derivativelysuric acid - which corresponds to ornithuric and hippuric acids. This compound forms acid as well as neutral salts which have been studied by Drechsel and by Willdenow. The acid barium salt crystallizes easily and is useful for the separation and purification of lysin. In the presence of acids, lysuric acid is soluble in ether but not in petroleum ether which therefore can be used for its separation (Lawrow ${ }^{9}$ ). A mono-benzoyl derivative has also been prepared.

Ornithin, $\mathrm{C}_{5} \mathrm{H}_{12} \mathrm{~N}_{2} \mathrm{O}_{2}$, was discovered in 1877 by Jaffé who obtained it in the form of ornithuric acid from the urine of chicken

'Zeits. physiol. Chem., 29, 323.

${ }^{2}$ Zeits. physiol. Chem., 25, 180 ; 26, 586 ; 28, 398 ; 33, 553.

${ }^{3}$ Berichte, 25, 2455.

4 Zeils. physiol. Chem., 28, 89.

${ }^{5}$ Zeits. plysiol. Chem., 28, 469 ; 35́, 561.

5 Zeits. phiysiol. Chem., 21, 300, 303.

7 Zeits. physiol. Chem., 21, 303; 25, 176.

${ }^{8}$ Berichte, 28, 3189 ; Zeits. physiol. Chem., 25, 522 ; 26, 398 ; 33, 562.

${ }^{9}$ Zeits. physiol. Chem., 28, 585. 
after administration of benzoic acid. This acid as pointed out on p. 437 is a di-benzoyl compound of ornithin and yields the base on cleavage with concentrated acid. Ornithin therefore can be looked upon as an intermediate waste product (and possibly as an uric acid antecedent) in the chicken just as glycocoll is such in other animals. The base, however, is by no means peculiar to the chicken, inasmuch as it exists preformed in arginin which, as pointed out (p. 426), is present in all proteid matter, whether of animal or plant origin. The partial synthesis of arginin from ornithin and cyanamid has been referred to. Ornithin can be prepared readily from arginin. ${ }^{1}$

The belief of Jaffe that ornithin was a di-amido valerianic acid has been definitely established in the past year or two. The cleavage of arginin into urea and ornithin and the decomposition of the latter by bacteria into putrescin demonstrate that this peculiar base is $\alpha-\delta$ diamino valerianic acid (p. 438).

The synthesis of $\alpha-\hat{o}$ diamino valerianic acid has only recently been effected by Fischer. ${ }^{2}$ It yields with benzoyl chlorid a di-benzoyl derivative, $\mathrm{C}_{19} \mathrm{H}_{20} \mathrm{~N}_{2} \mathrm{O}_{4}$, which is strikingly similar to ornithuric acid. While the latter melts at $182^{\circ}$ (Jaffé), $184^{\circ}$ (Schulze and Winterstein), the synthetic product melts at $184^{\circ}-185^{\circ}$. The synthetic and natural ornithuric acids yield the same characteristic calcium salt, $\left(\mathrm{C}_{19} \mathrm{H}_{19} \mathrm{~N}_{2} \mathrm{O}_{4}\right)_{2} \mathrm{Ca}$. They both, on partial cleavage, form the mono-benzoyl derivative, which in the case of the natural base crystallizes in needles (Jaffe), whereas the synthetic body forms plates.

The synthetic ornithin is strongly alkaline in reaction, gives a flocculent white precipitate with mercuric chlorid and in acid solution gives with phosphotungstic acid a heavy crystalline precipitate which easily dissolves on heating and reappears on cooling in colorless needles. The only real difference between the synthetic and natural ornithin is in the fact that the latter is dextro-rotatory, while the former is inactive. The synthetic ornithin is therefore the racemic form of the natural base as obtained from chicken and from arginin. The properties of the base and of several of its salts have been described by Schulze and Winterstein. ${ }^{3}$ Like lysin it unites with phenyl isocyanate to form an addition product which with hydrochloric acid yields an easily crystallizable hydantoin (Herzog ${ }^{4}$ ).

Ornithin on cleavage by bacteria yields putrescin and it is very probable that this change may be effected by chemical means and also by metabolism within the body. Indeed Lawrow's ${ }^{5}$ studies upon the auto-digestion of pig's stomachs show that pepsin may split up proteids into monamido acids and the diamins-cadaverin and putrescin. The occurrence of these bases in cystinuria may be ac-

1 Zeits. physiol. Chem., 34, 525.

${ }_{2}$ Berichte, 34, 454 (1901).

3 Zeits. physiol. Chem., 34, 128.

4 Zeits. physiol. Chem., 34, 525.

${ }^{5}$ Zeits. physiol. Chem., 33, 312. 
counted for by abnormal metabolism rather than by bacterial activity, and if so, it may be possible to demonstrate the presence of the antecedents ornithin and lysin.

The relation of lysin to cadaverin and of the latter to piperidin (p. 442), suggests a similar relation between ornithin, putrescin and pyrrolidin. The carboxyl derivative of the latter has been obtained from proteids and will be described next.

$\alpha$-Pyrrolidin carbonic acid, $\mathrm{C}_{5} \mathrm{H}_{9} \mathrm{NO}_{2}$, is a product of proteid cleavage which differs from all others in that it has a nitrogen containing ring. It undoubtedly originates from and is related to the hexon bases arginin, histidin and ornithin. This is seen in the fact that Schulze and Winterstein obtained a pyrrolidin-like body on dry distillation of arginin and of ornithin. On the other hand, although this base results, according to Fischer, in the hydrolytic decomposition, with hydrochloric acid, of casein, egg-albumin, fibrin and gelatin (Levene), it does not form when arginin or ornithin are subjected to the same method. For this reason Fischer ${ }^{1}$ is inclined to consider it as a primary cleavage product of proteids. It is conceivable, however, that this base may originate in the cleavage of histidin or its parent substance (p. 442) under conditions similar to the formation of uric acid out of hypoxanthin or out of thymus gland (p. 344), in which case urea would be split off and the chain would then reclose to form the pyrrolidin carbonic acid.

The presumed formation of this base from ornithin can be expressed by the following equation :

$$
\mathrm{NH}_{2} \cdot \mathrm{CH}_{2} \cdot \mathrm{CH}_{2} \cdot \mathrm{CH}_{2} \cdot \mathrm{CH}\left(\mathrm{NH}_{2}\right) \cdot \mathrm{COOH}=\underbrace{\mathrm{CH}_{2}}_{\mathrm{NH}} \mathrm{CH} \cdot \mathrm{COOH}+\mathrm{NH}_{3} \cdot
$$

This equation should be compared with those for piperidin and histidin on page 442 . It is noteworthy that this acid is closely related to the cleavage products of atropin and cocain, namely tropic and hygrinic acids. Moreover, as indicating the possibility of its being a secondary product of proteid cleavage may be mentioned the fact that a methyl 1-4 di-amido valerianic acid on treatment with hydrochloric acid yields a methyl pyrrolidin carbonic acid (Wildstätter and Ettlinger ${ }^{2}$ ).

The synthesis of $\alpha$-pyrrolidin carbonic acid has been effected independently by Wildstätter ${ }^{3}$ and by Fischer. ${ }^{4}$ As in the case of ornithin the synthetic body is the racemic form whereas the natural product is both active and inactive. Thus, from casein Fischer ob-

\footnotetext{
1 Zeits. physiol. Chem., 33, 152, 163, 412.

${ }^{2}$ Berichte, 35, 620 .

${ }^{3}$ Berichte, 33, 1160.

- Berichte, 34, 454 (1901).
} 
tained about 3.2 per cent. yield of the acid, and of this about twothirds was levo-rotatory, the remainder being inactive. On heating with baryta under pressure the active form is changed into the inactive.

The free acid melts at $203-206^{\circ}\left(198^{\circ}\right.$ Wildstätter) with decomposition and gives off an odor of pyrrolidin. This odor is also noted during the evaporation of a solution. It forms flat needles which effloresce. Owing to its marked solubility in water and in alcohol it was overlooked in previous studies of casein. It gives with phosphotungstic acid a crystalline precipitate which easily dissolves on boiling.

The active form unites in alkaline solutions with phenyl isocyanate to form a cyanate, the anhydrid of which readily crystallizes and melts at $143^{\circ}$, whereas that from the inactive form melts at $118^{\circ}$.

On boiling with freshly precipitated copper oxid the acid forms the easily crystallizable salt $\left(\mathrm{C}_{5} \mathrm{H}_{8} \mathrm{NO}_{2}\right)_{2} \mathrm{Cu}+2 \mathrm{H}_{2} \mathrm{O}$, which is especially useful for the isolation of the acid. The salts of both active and inactive forms possess the same properties.

The ethyl ester boils at $75^{\circ}-76^{\circ}$ at $11 \mathrm{~mm}$. pressure. It was by fractional distillation of the esters of the monamino acids which result in the hydrolytic cleavage of proteids that this acid was isolated by Fischer.

Gerontin, $\mathrm{C}_{5} \mathrm{H}_{14} \mathrm{~N}_{2}$, is a base which was isolated by Grandis in 1890. It has been repeatedly observed in the form of peculiar crystals found in the cell nuclei in the liver and kidneys, particularly of old dogs. The free base is an isomer of cadaverin, etc., and resembles it somewhat. It crystallizes in needles which are readily soluble in water and alcohol; possesses a strongly alkaline reaction, and yields the ordinary alkaloidal reactions.

The hydrochlorid forms prismatic crystals, which are deliquescent and easily soluble in alcohol.

The platinochlorid, $\mathrm{C}_{5} \mathrm{H}_{14} \mathrm{~N}_{2} \cdot 2 \mathrm{HCl} \cdot \mathrm{PtCl}_{4}$, is soluble in water and crystallizes in spindle-shaped needles, arranged in rosettes. It decomposes at $115^{\circ}$.

The gold salt forms small needles, and is easily soluble in water and alcohol.

It combines with one molecule of mercuric chlorid to form deliquescent cubes or rectangular prisms containing two molecules of water of crystallization. It decomposes above $100^{\circ}$. This distinguishes it from cadaverin, which combines with three to four molecules of mercuric chlorid. The crystals observed in the liver are probably the phosphate.

The new base also yields a benzoyl compound which melts at $175^{\circ}-176^{\circ}$.

It seems to exert a paralyzing action upon the nerve-centers and 
heart-ganglia, and leaves the nerves and muscles unaffected. $0.5 \mathrm{mg}$. kills $10 \mathrm{~g}$. frogs.

Spermin, $\mathrm{C}_{2} \mathrm{H}_{5} \mathrm{~N}$, or $\mathrm{C}_{5} \mathrm{H}_{14} \mathrm{~N}_{2}$, is the basic substance obtained by Schreiner (1878) from semen, calf's heart, calf's liver, bull's testicles, from the organs of leukemics, and also from the surface of anatomical specimens kept under alcohol. Poehl has found it in the testes, ovaries, prostate, thyroid gland, pancreas and spleen. In $1888 \mathrm{Kunz}$ reported the presence of a non-poisonous base, $\mathrm{C}_{2} \mathrm{H}_{5} \mathrm{~N}$, spermin or ethyleneimid iu cholera cultures. In this case it occurs, then as a ptomaïn. A confirmation of the identity of the two bases is necessary. Previous to this, however, it had been known for a long time under the name of "Charcot-Neumann or Leyden crystals," which are the phosphate of spermin. These peculiarly shaped crystals have been found in the sputa of a case of emphysema with catarrh, in the bronchial discharges in acute bronchitis, as well as in sputa of chronic bronchitis, in the blood, spleen, etc., of leucocythemics and anemics, and in the normal marrow of human bones, as well as in human semen, also in nasal secretions and in feces. Altogether it seems to have a very wide distribution, especially in certain diseases, as in leucocythemia.

Spermatic stains yield with iodin the so-called Florence's crystals which resemble those of Teichmann. These crystals, however, are not characteristic of sperma but may be obtained from any lecithin(cholin)-containing fluid. They have been obtained from liver and brain extracts, and from elder blossoms. According to Bocarius, ${ }^{1}$ the crystals represent a derivative of cholin and not an iodo-spermin as first supposed.

It can be prepared from fresh human semen in the following manner : The semen is washed out of linen by a little warm water, evaporated to dryness, boiled with alcohol, and the insoluble portion is allowed to subside by standing some hours. The precipitate is filtered off, washed, and dried at $100^{\circ}$. This residue, containing the spermin phosphate, is triturated, and then extracted with warm ammoniacal water. From this solution, on slow evaporation, the phosphate crystallizes in its peculiarly shaped crystals.

The free base is obtained, on decomposing the phosphate with baryta and evaporating the filtrate, as a colorless liquid which on cooling crystallizes. From alcohol it crystallizes in wavellite-shaped crystals which readily absorb water and carbonic acid from the atmosphere. They are readily soluble in water and in absolute alcohol, almost insoluble in ether, and possess a strong alkaline reaction. When heated on a platinum foil it gives off thick, white fumes and a weak ammoniacal odor. With potassium bismuth iodid it yields orangecolored crystalline floccules, which under the microscope appear as

${ }^{1}$ Zeits. physiol. Chem., 34, 339. 
long, sharp, plumose needles - distinction from diethylenediamin. The aqueous solution of the base is precipitated by phosphomolybdic, phosphotungstic and tannic acids, gold and platinum chlorids. It cannot be volatilized from alkaline solution by steam without undergoing decomposition (Majert and Schmidt). It is not poisonous.

The hydrochlorid, $\mathrm{C}_{2} \mathrm{H}_{5} \mathrm{~N}$. $\mathrm{HCl}$ (?), crystallizes in six-sided prisms, united in tufts, and is extremely soluble in water, almost insoluble in absolute alcohol and ether.

The aurochlorid, $\mathrm{C}_{2} \mathrm{H}_{5} \mathrm{~N} . \mathrm{HCl} \cdot \mathrm{AuCl}_{3}$ (?), forms shining, goldenyellow, irregular plates, and when freshly precipitated it is easily soluble in water, alcohol, and ether, but the dried salt is incompletely soluble in water. The aqueous solution, treated with magnesia, gives off a sperm-like odor. The platinochlorid crystallizes in prisms.

The phosphate, $\left(\mathrm{C}_{2} \mathrm{H}_{5} \mathrm{~N}\right)_{2} \cdot \mathrm{H}_{3} \mathrm{PO}_{4}+3 \mathrm{H}_{2} \mathrm{O}$ (?), forms prisms and slender double pyramids arranged in rosettes. It is difficultly soluble in hot water, insoluble in alcohol, easily soluble in dilute acids, alkalis and alkali carbonates. It melts with decomposition at about $170^{\circ}$. It is probable that the above formula does not represent the salt as found, and from theoretical considerations Ladenburg is inclined to think that Schreiner's phosphate has the composition $\left(\mathrm{C}_{2} \mathrm{H}_{5} \mathrm{NH}\right)_{4} \mathrm{Ca}\left(\mathrm{PO}_{4}\right)_{2}$.

Ladenburg and Abel prepared in 1888 a compound, ethyleneimin, which was first supposed to be isomeric with spermin. The reaction whereby it is prepared is similar to that by which Ladenburg effected the synthesis of piperidin. Ethylenediamin hydrochlorid is subjected to dry distillation, when it decomposes into ammonium chlorid and the hydrochlorid of the new base. The reaction was supposed to be represented by the equation :

$$
\left.\underset{\mathrm{CH}_{2} \mathrm{NH}_{2} \cdot \mathrm{HCl}}{\mathrm{CH}_{2} \mathrm{NH}_{2} \cdot \mathrm{HCl}}=\underset{\mathrm{CH}_{2}}{\mathrm{CH}_{2}}\right\rangle \mathrm{NH} \cdot \mathrm{HCl}+\mathrm{NH}_{4} \mathrm{Cl} .
$$

Since then Ladenburg showed that the boiling point of this compound did not agree with what it should be theoretically, if represented by the above formula. A determination of the vapor density showed that the molecular weight was twice that corresponding to the formula given, and hence was $\mathrm{C}_{4} \mathrm{H}_{10} \mathrm{~N}_{2}$. Majert and Schmidt assuming spermin to be ethyleneimin, as was apparently shown by Ladenburg and Abel's investigation, attempted to prepare the latter on a manufacturing scale with the expectation that it might be used as a substitute for Brown-Séquard's testicular fluid. They were soon able to show, however, that ethyleneimin did not possess the composition assigned to it, but that it was identical with Hofmann's diethylenediamin (piperazin, p. 418),

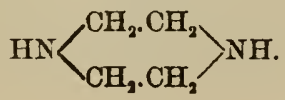


This was soon confirmed by Hofmann and by Ladenburg. Spermin was then assumed to be identical with piperazin, but later (1891) Majert and Schmidt compared some spermin from Schreiner with their own piperazin and found the two bases to be distinct, especially with reference to the phosphate and the potassium bismuth iodid precipitates. Poehl confirmed the difference between spermin and piperazin.

On cleavage of casein with hydrochloric acid Cohn ${ }^{1}$ obtained a substance which at first he regarded as a pyridin derivative, $\mathrm{C}_{5} \mathrm{H}_{7} \mathrm{NO}$. Later (1900), he gave up his view as to its pyridin nature and adopted the formula $\left(\mathrm{C}_{6} \mathrm{H}_{11} \mathrm{NO}\right)_{2}$, that of a leucinimid. This body as well as the basic reduction product $\left(\mathrm{C}_{6} \mathrm{H}_{13} \mathrm{~N}\right)_{2}$ he now regards as derivatives of piperazin or diethylenediamin. The piperazin nature of Cohn's products is supported by the work of Hoyer. ${ }^{2}$ Nevertheless it is true that as yet there is no evidence that piperazin bases exist preformed in the proteid molecule. The related pyrimidin group, however, is present (see p. 418).

In 1891 Poehl announced that the composition of spermin was more complex than was formerly supposed. He ascribed to it the formula $\mathrm{C}_{10} \mathrm{H}_{26} \mathrm{~N}_{4}$. The formula of the platinum salt corresponded to $\mathrm{C}_{10} \mathrm{H}_{26} \mathrm{~N}_{4} .4 \mathrm{HCl} .2 \mathrm{PtCl}_{4}$; and that of the gold salt was represented by $\mathrm{C}_{10} \mathrm{H}_{26} \mathrm{~N}_{4} 4 \mathrm{HCl} .4 \mathrm{AuCl}_{3}$. Later he gave the formula $\mathrm{C}_{5} \mathrm{H}_{14} \mathrm{~N}_{2}$.

From this it would appear that spermin is essentially distinct from piperazin. The composition and structure of this interesting base must therefore be considered as not settled. Undoubtedly bodies of entirely different composition have been included under the head of spermin. It is quite probable that it is related to cadaverin in which case its source might be looked for in the protamin or histon molecule.

According to Poehl, it acts as a tonic on the entire nervous system.

$\alpha$-Methyl quinolin, $\mathrm{C}_{10} \mathrm{H}_{9} \mathrm{~N}$, is present in part or wholly as the free base in the secretion of the anal glands of the skunk (Mephitis mephitica). It was isolated in 1897 by Aldrich and Jones ${ }^{3}$ and is of special interest since, with the exception of the kynurenic acid 4 from the dog's urine, it is the only quinolin compound isolated from the animal body. The structure of these two quinolin derivatives is represented by the formulæ :

${ }_{1}^{1}$ Zeits. physiol. Chem., 22, 153 ; 26, 395 ; 29, 283.

2 Zeits. physiol. Chem., 34, 347.

${ }^{3}$ Journ. Exp. Med., 2, 439, 1897.

1 Zeits. physiol. Chem., 33, 390, 1901. 

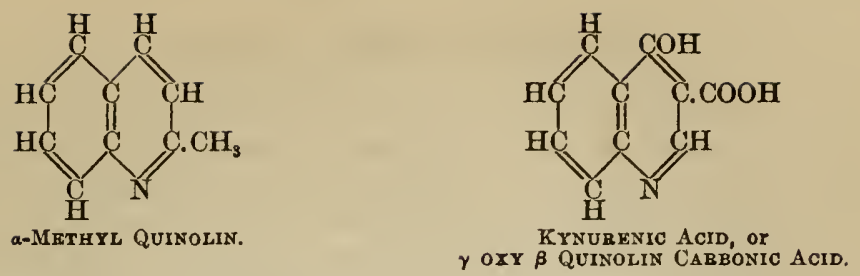

The latter by splitting off the carboxyl group yields kynurin, the homologue of indoxyl. The methyl quinolin may be compared with skatol or $\beta$-methyl indol. The proteid molecule is unquestionably the source of the quinolin as well as of the indol derivatives. The existence of pyrrolidin carbonic acid among the cleavage products of proteids has been noted on p. 448.

The base is a highly refractive, colorless oil possessing a faint quinolin odor which is very pronounced on warming. It is readily volatile with steam and fumes when hydrochloric acid is held near it. It is readily soluble in ether, chloroform, alcohol and in mineral acids; insoluble in alkalis and sparingly soluble in both hot and cold water. It forms addition products with methyl iodid and with bromin. With phthalic anhydrid it yields quinaldin yellow.

The platinochlorid, $\left(\mathrm{C}_{10} \mathrm{H}_{9} \mathrm{~N} . \mathrm{HCl}\right)_{2} \mathrm{PtCl}_{4}$, forms characteristic yellow needles which melt at $226^{\circ}-230^{\circ}$. It is sparingly soluble in cold water and on heating in a test-tube it decomposes, giving rise to a volatile oil which on cooling yields transparent colorless needles.

The aurochlorid is precipitated as fine slender yellow needles which melt at $153^{\circ}$. It is more soluble than the preceding compound. The silver nitrate compound forms colorless needles.

The bichromate, $\left(\mathrm{C}_{10} \mathrm{H}_{9} \mathrm{~N}\right)_{2} \cdot \mathrm{H}_{2} \mathrm{Cr}_{2} \mathrm{O}_{7}$, is extremely characteristic and is precipitated as a yellowish red oil which promptly crystallizes, forming needles which in form and color resemble those of the platinum salt. It melts with decomposition at $130-140^{\circ}$. A ferrocyanid can also be prepared. $177^{\circ}$.

The picrate forms yellow crystals which on rapid heating melt at

The zinc chlorid compound, $\left(\mathrm{C}_{10} \mathrm{H}_{9} \mathrm{~N} . \mathrm{HCl}\right)_{2} \cdot \mathrm{ZnCl}_{2}$, according to Aldrich and Jones is especially useful for the separation and purification of the base. It is readily soluble in hot, insoluble in cold water. It forms gypsum-like crystals which melt at $230^{\circ}-2400^{\circ}$

According to $\mathrm{Cohn}^{1}$ the injection of this base into dogs gives rise to an intense icterus which soon passes away. Rabbits are more susceptible and large doses cause pronounced hemoglobinuria and eventually death. In neither animal does quinolin appear in the urine showing that the group is apparently destroyed in the body.

${ }^{1}$ Zeits. physiol. Chem., 20, 210, 1894. 


\section{THE CREATININ GROUP.}

The knowledge of the formation of basic substances (ptomains) during the putrefaction of nitrogenous organic matter led to a series of investigations having for their object the isolation of alkaloidal bodies, if such existed, from the normal living tissues of the organism. A number of basic compounds, such as the purin bases already described, had been known for a long time, although their physiological relation to the animal economy was little, if at all, understood. Guareschi and Mosso, in the course of their researches on ptomains, were among the first to direct their attention to the possible presence of ptomain-like bodies in fresh tissues. They obtained in those cases where the extraction was carried on without the use of acids only very minute traces of an alkaloidal body (possibly cholin), and an inert substance, methyl hydantoin, which, although it can scarcely be classed as a basic compound, is closely related to creatin, and to methyl guanidin (page 284), and for this reason will be described at the end of this section. Other Italian chemists, as Paterno and Spica and Marino-Zuco, had also shown that the normal fluids and tissues of the body were capable of yielding substances alkaloidal in nature, and these were regarded by them as identical with, or similar to, the ptomaïns of Selmi.

Gautier devoted much time to the study of the leucomainns occurring in fresh muscle tissue, and he succeeded in isolating the several compounds presently to be described.

A number of these substances are credited with possessing an intensely poisonous action, and if this is the case, it is very evident that any undue accumulation of such bases in the system, resulting either from an interference in the elimination or lack of destruction, may give rise to serious disturbances. The amount of these substances present in the urine is said to be very small-so small, indeed, that we must rather look upon this minute quantity as having escaped oxidation in the body. It is well known that the living tissues possess an enormous oxidizing and reducing power, and, according to Gautier, there is constantly going on in the normal tissues of the body a cycle of changes-the formation of leucomaïns and their subsequent destruction by oxidation, before they have accumulated in sufficient quantity to produce poisonous effects. This formation and destruction of bases in the body has been shown to occur in the case of the purin bodies (page 343 ) and obviously intermediate cleavage products in proteid and other metabolisms are being constantly formed to be converted promptly into other and more simple bodies. Creatin and creatinin are examples of this kind, and Gautier's bases show more or less relation to these substances. It is eminently desirable, however, that the results obtained by Gautier and his coworkers be confirmed, if possible, in view of the reasonable doubt 
that may be raised as to the chemical individuality or purity of some of these compounds. The study of the hexon bases and of the monamino- and di-amino acids in general make such a revision all the more necessary.

The following method was employed by Gautier in his study of the leucomains of muscle tissue: The finely divided fresh beef-meat or the Liebig's meat extract is treated with twice its weight of water, containing 0.25 gram of oxalic acid, and one to two c.c. of commercial peroxid of hydrogen per liter. The purpose of these precautions is to prevent fermentation. Inasmuch as peroxid of hydrogen is not without action on organic substances its use should be avoided. At the end of twenty-four hours the liquid is raised to the boiling point, then filtered through linen, and the residue is thoroughly squeezed. The filtrate is again raised to the boiling point in order to coagulate any remaining albumin, and finally filtered through paper. The clear liquid thus obtained is evaporated in a vacuum at a temperature not exceeding $50^{\circ}$, and the acid syrupy residue is extracted with 99 per cent. alcohol ; the alcoholic extract is in turn evaporated in a vacuum, and the residue taken up with warm alcohol of the same strength. The filtered alcoholic solution is set aside for twenty-four hours, and any deposit which forms is removed by filtration; ether $\left(65^{\circ}\right)$ is then added as long as a precipitate continues to form, and the whole is again allowed to stand for twenty-four hours. The ether-alcoholic filtrate from this precipitate is evaporated first on the water-bath, and finally in a vacuum; the slight residue obtained contains a small quantity of basic substances possessing the odor of hawthorn.

The syrupy precipitate produced by the ether partially crystallizes on standing; a little absolute ether is then added, and after standing several days more the liquid is separated by means of an aspirator from the deposit of crystals (A). These are first washed with 99 per cent. alcohol, and then extracted with boiling 95 per cent. alcohol. The alcoholic solution, concentrated by evaporation, gives, on cooling, a deposit of lemon yellow crystals of xantho-creatinin (B), from the mother liquor of which there separates a crop of new crystals (C). The residue of the crystals (A) left after treatment with the boiling 95 per cent. alcohol is extracted with boiling water, which afterward gives a slight deposit of yellowish white crystals of amphicreatin (D). The aqueous mother liquor on concentration yields another deposit of orange colored crystals of cruso-creatinin (E). Gautier has, furthermore, separated three other bases from the mother liquors of the crystals obtained as above. Thus, a base which he named pseudoxanthin is stated to have been obtained by evaporating the alcoholic mother liquors of $\mathrm{B}, \mathrm{D}, \mathrm{E}(?)$ in a vacuum, taking up the residue with water, and precipitating the hot solution with copper acetate. The precipitate is decomposed with hydrogen 
sulphid, and the aqueous solution, filtered while boiling hot, yields a deposit of a sulphur yellow powder of pseudoxanthin. Thus, by the use of alcohol, ether, and water, Gautier, according to his statement, has succeeded in obtaining a sharp separation between these bases. The importance of the subject is such as to require not only confirmation of the results arrived at by Gautier, but also a more detailed and exact study of the chemical and physiological behavior of these bodies.

The following method was employed by Gautier and Landi in 1892 in their study of the changes in meat. The meat extract was concentrated in a vacuum to one-eighth its volume, then cooled, precipitated with neutral lead acetate, filtered, and after washing the precipitate, the filtrate was again concentrated to one-half its volume, and the lead removed by hydrogen sulphid. The filtrate was again concentrated to one-half its bulk and dialyzed. The bases are present in the dialysate. The dialyzed portion therefore was concentrated, acidulated with nitric acid, and precipitated with phosphomolybdic acid. The precipitate is collected and washed at once with very dilute nitric acid, then with water. It is then boiled with neutral lead acetate; the bases and the greater part of the xanthin and carnin pass into solution. After removal of the lead, the filtrate is evaporated in a vacuum, then extracted with alcohol. The residue is examined for:

Bases A.-It is treated with dilute ammonia ; this dissolves xanthin, hypoxanthin, guanin, carnin, etc., whereas creatin, etc., are insoluble. The ammonia is allowed to evaporate, and hence adenin and guanin separate out. Hypoxanthin and xanthin remain in solution.

Bases B.-The alcoholic filtrate from above residue is neutralized, concentrated, and treated with mercuric chlorid. The mercury precipitate is washed, decomposed with hydrogen sulphid, the solution filtered, and the filtrate is precipitated with copper acetate:

1 . In the cold-acids of the carbopyridic series, which are crystalline and give crystalline platinochlorids.

2. In boiling solution-xanthin bases.

3. The portion not precipitated by cold or hot copper acetate is the most important. The copper is removed with hydrogen sulphid, the filtrate evaporated to dryness, and extracted with alcoholguanin, creatin, neurin, chlorin, butylenediamin, etc., neuridin, ethylenediamin; hydropyridin bases and homologues, and bases that give pyrrol on distillation with lime; all are very poisonous.

Bases C:-The mercuric chlorid filtrate is concentrated to drive off the alcohol and the mercury removed with hydrogen sulphid. Lead acctate is added, the liquid filtered, and after removal of the lead is evaporated to dryness and extracted with dilute alcoholthe residue was creatin; the filtrate contained oxy-ethylenamin, 
metbyl guanidin, etc. Almost all of these are poisonous. They are less abundant than the others.

(a) Xanthin bases. Exist in minute amount in meat and are not toxic.

(b) Carboprridic bases. Likewise present only in small amount. They produce stupefaction in animals, but otberwise are not daugerous.

(c) Neurin and hydropyrrolic bases. They are the most abundant leucomaïs in meat, and are the most poisonous. Minute doses in mice produce dyspnœa, spasmodic movements of the extremities, bristling of the hair, paralysis, tetanic convulsions and death. The presence of neurin as a muscle constituent may well be questioned especially since Gulewitsch has been unable to find it in fresh brains.

(d) Creatin bases. These produce in mice vomiting, diarrhœa, tetanic convulsions followed by paralysis of the extremities.

Cruso-creatinin, $\mathrm{C}_{5} \mathrm{H}_{3} \mathrm{~N}_{4} \mathrm{O}$, forms orange vellow crystals which are slightly alkaline in reaction and possess a somewhat bitter taste. It yields a soluble, non-deliquescent hydrochlorid crystallizing in bundles of needles; also a soluble platinochlorid which forms tufts of beautiful, slender prisms. The aurochlorid is obtained as slightly soluble, crystalline grains, and, like the platinum double salt, is partially decomposed on heating. It is not precipitated by acetate of zinc or by mercuric nitrate, but is precipitated in the cold by solutions of alum. Zinc chlorid produces in somewhat concentrated solutions a pulverulent precipitate which dissolves on heating, and recrystallizes again on cooling. Like xantho-creatinin, it is not thrown out of solution by oxalic or nitric acid, and is thus distinguished from urea and guanidin; nor is it precipitated by acetate of copper-a distinction from xanthin derivatives. Mercuric chlorid produces an abundant flocculent precipitate which on heating partially dissolves, decomposing at the same time. Sodium phosphomolybdate gives a heavy yellow precipitate, whereas potassium mercuric chlorid and iodin in potassium iodid have no effect. Potassium ferricyanid is not reduced. This base differs in its composition from creatinin by $\mathrm{HCN}$, the elements of hydrocyanic acid, but in its crystalline form and alkaline reaction, and some other properties, it would seem to be closely related to this latter substance. Because of this apparent relationship and its golden-yellow color, Gautier named it cruso-creatinin.

Xantho-creatinin, $\mathrm{C}_{5} \mathrm{H}_{10} \mathrm{~N}_{4} \mathrm{O}$, is said to be the most abundant of muscle leucomains. It crystallizes in sulphur yellow, thin spangles, consisting of nearly rectangular plates which resemble somewhat those of cholesterin. It is soft and talc-like to the touch; possesses a slightly bitter taste, and when dissolved in boiling alcohol it gives 
off the odor of acetamid, though ordinarily in the cold it has a slight cadaveric odor. When heated, the substance evolves an odor of roast meat, carbonizes in part, and yields ammonia and methylamin. The crystals are amphoteric in reaction, are soluble in cold water, and can be recrystallized from boiling 99 per cent. alcohol.

It forms a hydrochlorid crystallizing in plumose needles, and a very soluble platinochlorid; the aurochlorid crystallizes with diffculty. Like creatinin, it is precipitated by zinc chlorid; the yellowish white precipitate dissolves with partial dissociation on warming, and on cooling separates as isolated or stellate groups of fine needles which possess the composition $\left(\mathrm{C}_{5} \mathrm{H}_{10} \mathrm{~N}_{4} \mathrm{O}\right)_{2} \mathrm{ZnCl}_{2}$. Silver nitrate throws down, in the cold, a flocculent precipitate which likewise dissolves on heating, and recrystallizes in needles. Mercuric chlorid produces a yellowish white precipitate. It is not precipitated by oxalic or nitric acid, nor by potassium mercuric chlorid, or iodin in potassium iodid. Tannin produces in time a slight turbidity, while sodium phosphomolybdate gives a heavy yellowish precipitate. This base is distinguished from the members of the acid group by not giving a precipitate with copper acetate, even on heating.

On gentle oxidation with potassium permanganate it is converted into a black substance insoluble in acids and alkalis, and resembling azulmic acid. By treatment with recently precipitated mercuric oxid it yields a substance which can be recrystallized from boiling 93 per cent. alcohol in needles which possess a slight alkaline reaction, and forms a slightly soluble, crystalline platinochlorid. This new substance is precipitated from alcoholic solution, by the addition of ether, as a mass of beautiful, white silky needles resembling caffein. These crystals melt at $174^{\circ}$; caffein melts at $178^{\circ}$.

Xantho-creatinin, given in fairly large doses, is poisonous, producing in animals depression, somnolence, and extreme fatigue, accompanied by frequent defecation and vomiting. In its general properties this base resembles creatin very much, and it was on account of this resemblance and its yellow color that it was named xantho-creatinin. This relation becomes especially evident since the base appears in the physiologically active muscle at the same time with creatinin, sometimes in about one-tenth of the quantity of the latter. Monari found this base in the aqueous extract of the muscles of an exhausted dog, and also in the urine of soldiers tired by several hours' march. He also claimed to have demonstrated its presence in the urine of a dog after previous injection of creatinin. Stadthagen was not able to isolate this base from his urine after prolonged muscular exercise, and arrived at the conclusion that it does not occur in urine, and that Monari's base was an impure creatinin. Colasanti, in 1884, and again in 1891, isolated from lion's urine by Neubaner's rine chlorid method for creatinin the latter compound and a yellow body which crystallized as canary-yellow, 
small, opaque scales, or as small, orange yellow, granular masses composed of needle-shaped crystals. This he considered to be xanthocreatinin, derived from the large excess of creatinin in the food.

Amphi-creatin, $\mathrm{C}_{9} \mathrm{H}_{19} \mathrm{~N}_{7} \mathrm{O}_{4}$, is slightly soluble and crystallizes from boiling water into yellowish white oblique prisms, which possess, if any, a slightly bitter taste. When heated to $100^{\circ}$ it decrepitates somewhat, and at $110^{\circ}$ it becomes opaque white. Potassium hydrate does not decompose it in the cold. Although a weak base, it combines to form salts just as the preceding members of this group. The hydrochlorid is crystalline, and is not deliquescent; the platinochlorid forms rhombic plates, which are soluble in water, but are insoluble in absolute alcohol; the aurochlorid crystallizes in easily soluble, very small, microscopic crystals, which are tetrahedral to hexahedral in their habit. It is not precipitated by copper acetate or by mercuric chlorid; nor does it give the murexid test, or the xanthin reaction. Sodium phosphomolybdate produces a yellow, pulverulent precipitate. In its properties it resembles creatin, and indeed Gautier thinks it may be possibly a combination of creatin, $\mathrm{C}_{4} \mathrm{H}_{9} \mathrm{~N}_{3} \mathrm{O}_{2}$, and a base, $\mathrm{C}_{5} \mathrm{H}_{10} \mathrm{~N}_{4} \mathrm{O}_{2}$, which, it will be seen, differs from the former only by a HCN group. This second compound, if it really exists, has an analogy in cruso-creatinin, the relation of which to creatinin may be expressed by the equation:

$$
\underset{\text { Cruso-cratimin. }}{\mathrm{C}_{5} \mathrm{H}_{8} \mathrm{~N}_{4} \mathrm{O}}=\underset{\text { Creatinis. }}{\mathrm{C}_{4} \mathrm{H}_{7} \mathrm{~N}_{3} \mathrm{O}}+\mathrm{HCN} \text {. }
$$

In a similar manner, amphi-creatin may be regarded as

$$
\underset{\text { AMPHI-CREATIN. }}{\mathrm{C}_{9} \mathrm{H}_{19} \mathrm{~N}_{7} \mathrm{O}_{4}}=\underset{\text { CREATIN. }}{2 \mathrm{C}_{4} \mathrm{H}_{9} \mathrm{~N}_{3} \mathrm{O}_{2}}+\mathrm{HCN} \text {. }
$$

A Base, $\mathrm{C}_{11} \mathrm{H}_{24} \mathrm{~N}_{10} \mathrm{O}_{5}$, was isolated by Gautier from the mother liquors of xantho-creatinin. It crystallizes in colorless or yellowish, thin, apparently rectangular plates, which are tasteless and possess an amphoteric reaction. The hydrochlorid forms bundles of fine needles; the sulphate yields a confused mass of needles ; the platinochlorid is soluble, non-deliquescent and crystalline. When heated with water in a sealed tube at $180^{\circ}-200^{\circ}$ it gives off ammonia and carbonic acid, and is converted into a new base, which, however, has not been studied. This reaction may be expressed by the equation :

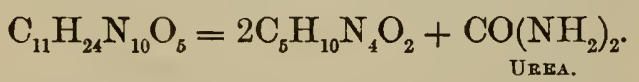

The urea which at first forms is, in turn, decomposed thus :

$$
\mathrm{CO}\left(\mathrm{NH}_{2}\right)_{2}+\mathrm{H}_{2} \mathrm{O}=\mathrm{CO}_{2}+2 \mathrm{NH}_{3} \text {. }
$$


It is to be observed that this base differs in composition from the following one by $\mathrm{HCN}$, the hydrocyanic acid molecule.

A Base, $\mathrm{C}_{12} \mathrm{H}_{25} \mathrm{~N}_{11} \mathrm{O}_{5}$, was obtained from the mother liquors of cruso-creatinin, and forms rectangular silky plates, resembling those of the preceding base and of xantho-creatinin. It forms crystallizable salts.

These complex bases, as already pointed out, require further study in order to elucidate their chemical nature. The equations given above, while they harmonize with Gautier's view as to the rôle of $\mathrm{HCN}$ cannot be looked upon as established facts. On the contrary they are suggestive of the impurity of the substances examined.

$$
\text { Methyl hydantoïn, } \mathrm{C}_{4} \mathrm{H}_{6} \mathrm{~N}_{2} \mathrm{O}_{3}=\mathrm{CO}\left\langle\begin{array}{l}
\mathrm{N}\left(\mathrm{CH}_{3}\right) \cdot \mathrm{CH}_{2} \\
\mathrm{NH}-\mathrm{CO} .
\end{array}\right.
$$

This substance was obtained by Guareschi and Mosso (1883), by extracting fresh meat with $1-1.5$ volumes of water (without addition of acid), for two hours at $50^{\circ}-60^{\circ}$. The aqueous extract was evaporated on a water-bath and the residue was extracted with 95 per cent. alcohol. This alcoholic solution, after the alcohol was driven off, was first taken up in water, filtered, and the aqueous solution was extracted with ether, then rendered alkaline with ammonia, and again extracted with ether. The alkaline ether extract gave on evaporation a white crystalline residue of methyl hydantoïn. The amount of this substance present in flesh appears to be quite variable, since, at times, none whatever can be extracted. Albertoni isolated it from a dog's flesh. Previous to its discovery in flesh by Guareschi and Mosso, it was known for a long time as a decomposition product of various nitrogenous bases of the body. Thus, Neubauer prepared it by heating creatinin with barium hydrate, while Huppert obtained it by fusing together sarcosin with urea. As it occurs in muscle, it is probably derived from the creatin, though under what conditions this splitting up takes place is not definitely known. It may be due to an enzyme or to nitrous acid in which case the change would correspond to the conversion of guanin into xanthin. Acetic and lactic acids are incapable of effecting this change. At all events, it belongs to the ureids, and is intermediate between creatinin, sarcosin, and urea. Compare the above formula with that of creatinin and methyl guanidin (p. 284).

Methyl hydantoïn forms prisms which are easily soluble in water and alcohol, and but slightly soluble in cold ether. It melts at $156^{\circ}$ (Salkowski); at $159^{\circ}-160^{\circ}$ (Guareschi and Mosso). Its aqueons solution is slightly acid in reaction. On strong heating it volatilizes. When fused with potassium hydrate it gives off ammonia; it reduces mercuric nitrate in the cold. Treated with 
mercuric oxid it assumes an alkaline reaction, and the filtrate on heating yields metallic mercury. With silver oxid it forms pearly lanceolate plates having the composition $\mathrm{C}_{4} \mathrm{H}_{5} \mathrm{~N}_{2} \mathrm{O}_{2} \cdot \mathrm{Ag}$. It does not give the alkaloidal reactions.

\section{UNDETERMINED LEUCOMAÏNS.}

\section{Leucomains of Expired Air.}

It was shown at quite an early period that exhalations from animals contain, besides an increased amount of carbonic acid, some organic matter, the nature of which, on account of the exceedingly minute quantity in which it occurs, has never been satisfactorily determined. Ransome, in 1870, estimated the organic matter in expired air by permanganate of potash to be about $0.2 \mathrm{~g}$. per day. Later Uffelmann showed that the amount of the organic matter in occupied closed rooms increased in almost the same ratio as carbonic acid. Herrmanns, however, denied the existence of organic substances in the expired air. Nevertheless, various observers did not hesitate to ascribe to it the ill effects consequent upon breathing impure air, while at the same time the carbonic acid formed during respiration was considered as either entirely inert or as insignificant in its action. Thus, respired air from which moisture and carbonic acid have been removed, but which still contains the organic vapors, was found by some to be highly poisonous. On the other hand, if the respired air is drawn through a red-hot tube, to destroy the organic matter, the air thus purified is capable of sustaining life even in presence of a large percentage of carbonic acid. While it cannot be, therefore, doubted that the organic matter of expired air plays a most important part in producing the well known noxious effects resulting from breathing confined and vitiated air, nevertheless it would seem from experiments made by Angus Smith that the increase of even such small quantities of carbonic acid in the air as from 0.04, the normal amount present, to 0.1 per cent., is capable of producing systemic disturbances characterized by a decrease in the pulse rate and an increase in the rate of respiration.

Smith was consequently of the opinion that the constant lowering of the pulse in impure air, occasioned by the presence of carbonic acid, must have a depressing effect on the vitality. Whatever ill effects the carbonic acid may produce of itself, it remained quite certain that this gas was not the most potent and most injurious constituent of respired air ; and the investigations of Hammond, Nowak, Seegen, and others pointed to the organic matter as the direct and immediate agent which produces those symptoms of sickness and nausea experienced in badly ventilated closed rooms.

Of special importance to the sanitarian and physician is the work on the nature and action of the poisonous principle of expired air 
which was done by Brown-Séquard, d'Arsonval, and R. Wurtz. The first two observers found that the vapors exhaled by dogs, when condensed, and the aqueous liquid (20-44 c.c.) thus obtained was injected into other animals, death was produced, generally within twenty-four hours. The symptoms observed were dilatation of the pupil, increase of heart-beat to 240-280 per minute, which may last for several days or even weeks, while the temperature remains normal; the respiratory movements were generally slowed, and usually paralysis of the posterior members was observed. Choleraic diarrhœa was invariably present. As a rule, larger doses caused labored respiration, violent retching, and contraction of the pupil. A rapid lowering of temperature, $0.5^{\circ}$ to $5^{\circ}$, was sometimes observed. These same symptoms, apparently in aggravated form, were obtained when the liquid had been previously boiled for the purpose of destroying any germs that might be present. The appearances presented on post-mortem were much like those observable in cardiac syncope.

The above work was confirmed in part by $R$. Wurtz, who, by passing expired air through a solution of oxalic acid, obtained, besides ammonia, a volatile organic base which was precipitated by Bouchardat's reagent and by potassio-mercuric iodid. It is said to form a platinum double salt crystallizing in short needles, and a soluble gold salt. When heated to $100^{\circ}$ it gives off a peculiar odor. This basic substance may properly be looked upon as a leucomaïn. The possibility of its being an ammonium compound is not excluded.

Dastre and Loye, Lehmann and Jessen, Geyer, and Merkel have repeated the above experiments with wholly negative results. Similar negative results were obtained by Hoffmann-Wellenhof and by Russo-Giliberti and Alesi, who injected the condensed moisture from expired air without effect. Ben, in 1893, studied the subject of the toxicity of expired air. From about 3000 liters of his expired air (eight hours) he obtained about 100 c.c. of condensed water having a peculiar, not unpleasant odor. It gave a distinct reaction for ammonia with Nessler's reagent, but contained no alkaloids. The organic substance amounted to $5 \mathrm{mg}$., or for twenty-four hours to $15 \mathrm{mg}$. By repeating Wurtz's experiment with 500 and 700 liters of expired air no alkaloidal reactions were obtained nor were any effects produced in animals. From these and other experiments, he concluded that the organic matter of expired air cannot induce acute intoxication. The dyspnœa observed in confined spaces is due to the lack of oxygen. Carbonic acid may give rise to dullness and headache, but the amount may rise considerably and be harmless so long as oxygen is not decreased too much.

Billings, Mitchell and Bergey on examining the water of condensation from expired air obtained traces of ammonia but no reactions for alkaloids. The fatal results met with in Brown-Séquard's ex- 
periments they ascribe to lack of oxygen and to increase of carbonic acid. On the other hand Formanck explains the presence of ammonia as due to external decompositions and the fatal results as due to the combined effects of ammonia and carbonic acid.

The most recent contribution on this subject is that of Sanarelli and Biffi. ${ }^{1}$ These investigators demonstrate that many intestinal products may be absorbed and subsequently be eliminated by the lungs. When rectal injections of ammonia, hydrogen sulphid, butyric acid, aceton, and carbonic acid were made these substances could be detected in the exhaled air.

Sewer air, according to observations made by Odling, contains a basic substance which is probably a compound ammonia. It contains, however, more carbon than methylamin and less than ethylamin.

It should be remarked that Jackson (1887) announced the presence in expired air of quantities of carbon monoxid gas sufficient to produce the ill effects ordinarily attributed to the organic matter. The presence of this poisonous gas must first be fully demonstrated before it can be taken into account in the consideration of the toxicity of air ; certainly, even if present, it cannot explain the results obtained by the French investigators as stated above.

According to Ilosva, expired air contains nitrous acid. This may possibly be derived from that which is constantly being formed in the mouth, either by the reduction of nitrates (Miller) or by surface action.

\section{Leucomains of the Urine.}

A number of basic substances have been isolated at different times from the urine, and on that account they may be properly classed as leucomaïns. Thus, Liebreich (1869) found in the urine a base which apparently was an oxidation product of cholin, and which has since been regarded as identical with betain. In 1866 Dupré and Bence Jones found, among other things in the urine, an alkaloidal body which in sulphuric acid solution possessed a blue fluorescence (see p. 41). Most of the members of the purin group have been detected in the urine and on account of their well defined nature they are described by themselves. It is desirable perhaps in this connection to emphasize the fact already brought out (p. 405) that the greater part of the purin bases found in the urine are derived from the preformed bases in the food. Rachford's view that migraine is due to intoxication with paraxanthin and allied bodies has been referred to on page 403 .

In 1879, Thudichum announced the presence in the urine of four new alkaloids, one of which, urotheobromin, was subsequently rediscovered by Salomon anduamed paraxanthin. Another base which was obtained, namely, reducin, yielded a barium salt which readily re-

${ }^{1}$ Annali d' Igiene sper., 12, 90, 1902. 
duced the salts of silver and mercury. Its formula probably corresponded to $\mathrm{C}_{12} \mathrm{H}_{21} \mathrm{~N}_{6} \mathrm{O}_{9}$ or $\mathrm{C}_{6} \mathrm{H}_{11} \mathrm{~N}_{3} \mathrm{O}_{4}$. A third alkaloid, parareducin, formed a zinc compound having the composition $\mathrm{C}_{6} \mathrm{H}_{9} \mathrm{~N}_{3} \mathrm{O} . \mathrm{ZnO}$. A fourth base is said to give a compound with platinum chlorid and to contain an aromatic nucleus (aromin). Besides these four bases Thudichum described two other substances which he considered to be basic. These were urochrome, the normal pigment of the urine, and creatinin.

In 1880 , Pouchet announced the presence of carnin, $\mathrm{C}_{7} \mathrm{H}_{8} \mathrm{~N}_{4} \mathrm{O}_{3}$. Krüger and Salomon did not meet with carnin in their exhaustive study of the purin bases in urine and, moreover, they question the correctness of Pouchet's finding. The latter also reported another base which he subsequently analyzed and found to have either the composition $\mathrm{C}_{7} \mathrm{H}_{12} \mathrm{~N}_{4} \mathrm{O}_{2}$ or $\mathrm{C}_{7} \mathrm{H}_{14} \mathrm{~N}_{4} \mathrm{O}_{2}$. This substance formed deliquescent fusiform crystals, sometimes grouped in bundles or irregular spheres. It possessed a slight alkaline reaction and combined with acids to form crystallizable salts. It was soluble in dilute alcohol, almost insoluble in strong alcohol, insoluble in ether. The hydrochlorid yielded double salts with gold chlorid, platinum chlorid, and mercuric chlorid. The platinochlorid formed deliquescent golden-yellow rhombic prisms. This base occurred in the dialysate (see page 320). From the non-dialyzable portion, Pouchet obtained another base corresponding to the formula $\mathrm{C}_{3} \mathrm{H}_{5} \mathrm{NO}_{2}$, which he called the "extractive matter of urine." It gave precipitates with the general alkaloidal reagents, was non-crystallizable, altered on exposure to air and was resinified by hydrochloric acid. On the addition of platinum chlorid it rapidly oxidized, but did not yield a platinochlorid. The bases were poisonous to frogs; produced paralysis, loss of reflexes, and stoppage of the heart in systole. The same author regarded the urine as containing very small quantities of some pyridin bases, analogous to or identical with those obtained by Gautier and Etard from decomposing fish.

Baumstark isolated a compound from the urine having the composition $\mathrm{C}_{3} \mathrm{H}_{8} \mathrm{~N}_{2} \mathrm{O}$. In forty liters of urine it could just be detected; was more abundant in one case of icterus. It was not present in dog's urine, except after feeding benzoic acid. It crystallized from water in white prisms resembling hippuric acid. The crystals decrepitate on heating; are unchanged at $250^{\circ}$, but at higher temperature give off dense, white vapors having a peculiar odor; melt and take fire. The odor is that of burned horn. It is rather easily soluble in hot water, difficultly in cold water and in alcohol; not in absolute alcohol or ether. The solutions are neutral. It forms easily soluble salts. The hydrochlorid, $\mathrm{C}_{3} \mathrm{H}_{8} \mathrm{~N}_{2} \mathrm{O} . \mathrm{HCl}$, crystallizes difficultly in dendritic masses; is deliquescent and soluble in alcohol. It does not combine with bases, and is precipitated by mercuric nitrate, thus resembling allantoin and urea. When heated in a glass tube 
or with soda-lime, it gives off a combustible gas having the odor of ethylamin. On boiling with baryta or ammonia, ethylamin and barium carbonate result. With nitrous acid it gives sarcolactic acid. A somewhat similar substance was isolated by Meissner from the urine of the dog.

The distinguished Italian toxicologist Selmi was, perhaps, the first to draw attention to the probable formation of basic substances in the living body during those pathological changes brought on by the presence of pathogenic germs. In a memoir presented to the Academy of Sciences of Bologna, in December, 1880, he announced that infectious diseases, or those in which there occurs an internal disarrangement of some element, either plasmic or histological, must be accompanied or followed by an elimination of more or less characteristic products which would be a sign of the pathological condition of the patient. To support this theory he examined a number of pathological urines, and succeeded in obtaining from them basic substances, some of which were poisonous, others not. Thus, a specimen of urine from a patient with progressive paralysis gave two bases strongly resembling nicotin and coniin; from other pathological urines the bases obtained usually had either an ammoniacal or trimethylamin odor. It is well to note that in normal urine ammonia and trimethylamin are present, while organic bases, as peptotoxin, are absent (Stadhagen). Selmi proposed to designate the basic substances found in disease as pathoamins. The term urotoxin is likewise sometimes employed to designate the urine poison. A strong confirmation of Selmi's theory is seen in the observations made by Bouchard, Villiérs, Lépine, Gautier, and others, all of whom apparently have found basic substances in the urine of various diseases.

Thus, Bouchard asserted the presence in normal urine of two bases, one soluble in ether, the other insoluble in ether, but soluble in chloroform. By the extraction of urine from typhoid fever, pneumonia, pleuritis, and icterus with ether he obtained substances that gave alkaloidal reactions. Lépine and Guérin likewise extracted alkaline urine with ether and obtained a poisonous substance. The extracts from pathological urines were more poisonous than those from normal urine, and the typhoid extract reacted differently from that of pneumonia. Villiérs found the basic substances, as a rule, in pneumonia, tuberculosis, abscesses, but absent in tetanus. In all these cases only extracts were employed, the substance not being isolated in a degree of purity and in amount sufficient for analysis. It is comparatively easy (from the result of the application of the so-called alkaloidal tests) to report upon the presence of alkaloids in so complex a fluid as the urine. It is vastly more difficult, however, to isolate such bodies in a chemically pure condition, thus satisfying the requirements of exact research. 
The criticisms on these older examinations apply with equal force to many of the more recent investigations of the urine in disease. Thus, Chiaruttini applied Spica's method for the extraction of ptomaïns to the urine of various nervous diseases with convulsions. In twelve cases alkaloids were obtained which produced similar toxic effects in animals. Arslan from the urine of two children with ankylostomiasis separated a toxin that induced anæmia in rabbits. Boinet and Silberet isolated three bases from the urine of Basedow's disease that in animals produced effects similar to those observed in the disease. Marino-Zuco believed that the poisonous action of extract of the adrenals, as observed by Foa and Pellacani, was due to cholin (neurin of Marino-Zuco). With Dutto, he found in the urine of Addison's disease a base which behaved with reagents like cholin. They therefore considered the disease as a slow auto-intoxication with this base. It may be mentioned in this connection that eclampsia is considered by Favre as a ptomaïnmia, whereas Bouchard regards it as due to the non-elimination of the normal poisons of the urine. There is more reason, however, in considering it as due to perverted cell metabolism, just as the varnishing of a part or the whole of the skin results, as Kijanitzin has pointed out, in the alteration of the chemical products of the underlying cells. The same author showed that in extensive skin-burns the urine, as well as the blood and organs, contains a basic poisonous substance, presumably peptotoxin (see page 328).

While there may be doubt as to the formation of a basic poison in profound skin-burns, there can be no doubt from the pathological changes as observed by Bardeen and by $\mathrm{McCrae}^{1}$ that toxins, not unlike those of bacterial origin, are produced. The destruction of hemoglobin may lead to the production of histon which as already pointed out possesses marked poisonous properties.

In 1889 Luff examined the urine of infectious diseases for basic products by the following method: A large quantity of the urine was rendered alkaline with sodium carbonate, and agitated with onehalf its volume of ether. After standing for some time the ether was removed, filtered, and then agitated with a solution of tartaric acid, to remove the alkaloids as soluble tartrates. The aqueous acid solution was then rendered alkaline with sodium carbonate, and agitated with one-half its volume of ether. The ether was removed, allowed to evaporate spontaneously, and the residue, after drying over sulphuric acid, was examined for alkaloids.

The urine of typhoid fever, collected during a high fever for four days, gave a small quantity of a white crystalline substance. When dissolved in hydrochloric acid it gave reactions with phosphomolybdic acid, potassium mercuric iodid, iodin solution, tannic and picric acids, and gold chlorid; failed to react with phosphotungstic acid

${ }^{1}$ Trans. Assoc. Am. Phys., 16, 153. 
and platinum chlorid. The examination of a second case was negative.

Scarlet fever urine collected during the height of the fever (four gallons) gave a small amount of a white semi-crystalline alkaloid. The solution in water was faintly alkaline. The hydrochloric acid solution did not react with tannic acid or platinum chlorid, but gave precipitates with the other reagents mentioned above. The amount of substance was insufficient to allow of analysis. In normal urines no such residues were found.

A most prolific supply of alkaloids from the urine of infectious diseases has been furnished by Griffiths. The method employed was identical with that described by Luff. A list of these bases, together with others, is given on page 261 .

Hunter, in 1890, examined the urine of pernicious anæmia by the benzoyl chlorid method, and obtained a very small quantity of a benzoyl compound, which was extremely soluble in alcohol, insoluble in water. It crystallized from alcohol in long, fine needles, grouped in rosettes. The melting point was at $174^{\circ}-175^{\circ}$. The crystalline form and the melting point agreed with putrescin. This compound was usually alone, but sometimes was accompanied by another, forming elongated, rectangular prisms. The crystalline form resembled that of the cadaverin compound. One specimen of urine furnished a dibenzoyl compound, crystallizing in long, rectangular prisms having a melting point between $70^{\circ}$ and $80^{\circ}$. Binet isolated a thermogenic substance from the urine of tuberculosis, and to a less extent from normal urine.

Certain basic substances, as the diamins, cadaverin, and putrescin, have been isolated in a perfectly pure condition. These two basic substances (see page 265) were observed by Baumann and Udránzsky in a case of cystinuria. Later, Brieger and Stadthagen demonstrated the presence of these same bases in two or more cases of that disease. They are absent in normal urine and feces, and exceedingly rare in other diseases. Thus, Roos found diamins (putrescin) in only one case of cholera, and then in the feces, not in the urine ; more frequently in diarrhœa or cholerine; also in one case of dysentery and malaria. As stated above, Hunter apparently succeeded in isolating putrescin from the urine of pernicious anæmia.

Cystin and the diamins are now known to be proteid cleavage products and their presence in the urine is more easily understood. The hexon bases have not been detected in urines but as yet no special studies have been made in that direction. The presence of ornithin in birds, as an intermediate waste product, was recognized by the appearance of ornithuric acid in the urine.

Poehl has proposed the following method for the estimation of the leucomains in the urine. To 100 c.c. of the urine, 25 c.c. of hydrochloric acid (sp. g. 1.134) and 10 c.c. of a 10 per cent. solution of 
phosphotungstic acid are added. Albumin and pepton must first be removed if present. The precipitate is allowed to subside in a graduated tube, and the number of cubic centimeters occupied by the precipitate divided by 8 is to represent the approximate weight of leucomains per liter of urine. The amount thus found in the two cases was 0.6 and 1.69. Cavallero and Olivetti have, with justice, severely attacked this method, and have shown its utter unreliableness.

It is now a well established fact that the urine of certain infectious diseases, as cholera (Bouchard) and septicæmia (Feltz), etc., is far more poisonous than normal urine. That the poisons, basic or otherwise, which are generated within the body by the activity of bacteria can be excreted in the urine is seen in the fact that immunity to the action of bacillus pyocyaneus has been conferred on animals by previous injection of urine taken from animals inoculated with that bacillus (Bouchard) or with filtered cultures of the same (Charrin and Ruffer).

Furthermore the excretion of the tetanus and diphtheria poisons by the urine has been shown to take place. Thus, Brunner demonstrated the tetanus poison in the urine of experimental animals, but failed with the urine of the disease in man. Bruschettini, however, with the urine of a tetanus patient, produced tetanic symptoms in mice by the injection of 3-10 c.c. subcutaneously. In the urine from diphtheria patients Roux and Yersin demonstrated the presence of the diphtheritic poison by inducing paralysis in animals. Although basic substances are not present in the urine of cholera, they are present, but less frequently than was expected, in the discharges (putrescin in only one of four cases, Roos). From cholera-feces Pouchet extracted an oily fluid very poisonous to frogs; whereas, Villiérs obtained a base which produced convulsions in guinea-pigs. Kulneff pointed out the presence of ethylenediamin (?) in the stomach-fluids of gastrectasia, while from the feces of a case of gastroptosis he isolated trimethylamin.

In the consideration of the toxins in the urine of infectious diseases it must not be forgotten, as pointed out by Jawein, that the poison as well as the specific germ may be present in the urine. Thus, in rabbits that died as a result of infection either with anthrax bacilli, erysipelas streptococci, typhoid bacilli, or with pneumonia diplococci, the urine was found to contain these organisms. It, therefore, becomes difficult to decide as to whether the toxin is elaborated within the body or formed subsequently to the secretion of the urine.

The question of the toxicity of normal urine has been the subject of considerable controversy. The early explanations of the cause of uræmia assumed that urine was poisonous, and that uræmic symptoms were the result of the retention of urine. Actual demonstrations of 
the toxicity of urine were made early in the century by Vauquelin and others. On the other hand, disbelievers in the toxicity of urine were not wanting. Thus Frerichs maintained that death, resulting from intravenous injections of urine, was due to the suspended solid elements of the urine; that urea itself was harmless, but that it could by the action of a ferment give rise to the poisonous ammonium carbonate. Voit was among the first to point out that potassium salts, on account of their toxicity, could play an important part in uræmia. It can now be positively stated that normal urine does possess a certain degree of toxicity. It is more difficult to decide upon the nature of this poison. Feltz and Ritter (1881), and independently Astaschewsky, arrived at the opinion that the toxicity was chiefly due to the potassium salts of the urine. Schiffer, while acknowledging the presence and action of the inorganic salts, maintained that the urine contained a definite organic poison, for the reason that the concentrated aqueous solutions from alcoholic extracts of the urine residue, deprived of inorganic salts, killed large rabbits in doses corresponding to $1-1 \frac{1}{2}$ liters of urine.

According to Bouchard, 30-60 c.c. of normal urine, injected intravenously, will kill a rabbit weighing one kilogram. Hence a man weighing 60 kilograms, and excreting per day 1200 c.c., would, if 50 c.c. are necessary to kill one kilogram of living matter, secrete enough poison to kill twenty-four kilograms of animal. Inasmuch as the amount necessary to kill one kilogram of animal is designated as one urotoxy, therefore, in the above case twenty-four urotoxies are formed per day. The urotoxic coefficient is the number of urotoxies which one kilogram of man forms in twenty-four hours. Therefore, $\frac{24}{60}=0.4$, the urotoxic coefficient. The average normal urotoxic coefficient is placed by Bouchard at 0.464. It follows, therefore, that an average man would, if the excretion of urine was stopped, be killed in fifty-two hours. The variations of the urotoxic coefficient in the normal individual is limited. In disease it rarely exceeds 2 , and rarely falls below 0.10 .

According to Bonchard, five kinds of poisons may be met with in the urine, producing narcosis, salivation, mydriasis, paralysis, and convulsions. The day urine, which is chiefly narcotic, is $2-4$ times more toxic than the sleep urine, which induces convulsions and is antagonistic to the former. The toxicity is independent of the density, since night urine is more dense than that secreted during the day.

The greater part of the toxicity of urine is ascribed by Bouchard to organic poisons, especially coloring-matters, whereas potassium salts are regarded as the cause of but a small fraction of the toxicity.

Lépine likewise found that about 60 c.c. of urine sufficed to kill one kg. of animal. The inorganic salts, however, were ascribed by him a much greater importance, inasmuch as he estimated that 85 per cent. of the intoxication was due to this cause. The remainder 
of the toxicity was due to organic matter. Stadthagen arrived at practically the same results, that $80-85$ per cent. of the toxicity was due to the inorganic constituents. A part of the toxicity, 15-20 per cent., is therefore due to organic substances. No one organic substance in the urine, as urea, creatin, etc., possesses this toxicity. Stadthagen has further shown that alkaloidal bodies as peptotoxin, guanidin, methyl guanadin, cholin, neurin, xanthocreatinin are absent from normal urine. 100 liters of urine examined by Brieger's method for bases gave only ammonia, a little trimethylamin, besides creatinin. Dresbach ${ }^{1}(1900)$, employing the same method, obtained poisonous extracts. Gautier has supposed that the urine poison was a proteid analogous to that in the venom of serpents, but Stadthagen showed that proteids were absent. Ferments like pepsin were also excluded from consideration because of their minute amount. His experiments were largely negative, so far as the detection of an organic poison was concerned. Stadthagen disproved the existence of a special urine poison. The poisonous action of normal urine is therefore due to the sum total action of the inorganic salts, chiefly potassium, and the normal organic constituents as urea, creatinin, etc., which by themselves possess but slight action.

Guinard recently tested the action of normal urine from different animals. On an average, the toxicity per kg. rabbit was as follows: Dog, 193 c.c. ; man, 132.7 c.c.; pig, 53 c.c. ; ox, 38.5 c.c. ; guinea-pig, 35 c.c. ; sheep, 33.8 c.c. ; goat, 32 c.c. ; ass, 29.4 c.c. ; horse, 29.2 c.c.; rabbit, 16 c.c.; cat, 13 c.c. The urine of a bear possessed toxicity similar to that of the dog; that of the lion and tiger corresponded to that of the cat. In the case of the horse the urine was less toxic from weak animals, from young animals, and from males than from strong or old animals or females. The urea per liter of urine varied from $15 \mathrm{~g}$. in the dog to $118 \mathrm{~g}$. in the cat. While rabbits are killed, per kilo, by an injection of 45 c.c. of normal urine, dogs are killed by an intravenous injection of 100 c.c. per kilo (Mairet and Bosc). If the thyroid gland is removed, the toxic effects are increased (Godart and Slosse).

While Guinard failed to observe any effect or toxicity following the injection of the urine of pregnancy, Chambrelent and Demont found that the toxicity was diminished in the later months. The average urotoxic coefficient was 0.27. Mairet and Bose examined the toxicity of the urine in nervous disorders and found it to be increased, especially in lypemania and mania. In general, however, the toxic action was the same as that of normal urine, though at times it produced specific nervous symptoms approximating those of the disease.

Increased toxicity of the urine was observed by Surmont in atrophic cirrhosis, tuberculosis, and carcinoma of the liver. On the 
other hand, the toxicity was normal or subnormal in hypertrophic cirrhosis, in hepatic congestion, and in infectious icterus.

Roque and Lemoine showed that there are marked changes in the toxicity of the urine in malaria before and after an attack. Before an attack the urotoxic coefficient was 0.13 and 0.274 , whereas after an attack it rose to 0.684 and 1.276 . It would appear, therefore, that toxic products result from the growth of the malarial plasmodium in the blood, and are largely eliminated by the kidneys. Quinin favors this excretion of poisons.

It does not follow from what has been stated that the urine in disease is always more poisonous than in health. There are diseases, as uræmia, where, as shown by Schiffer and Bouchard, the urine is less toxic than in health. This may be due to a retention of the salts of potassium.

\section{Leucomains of the Saliva.}

According to Gautier (1881), normal human saliva contains divers toxic substances in small quantities which differ very much in their action according to the time of their secretion, and probably according to the individual gland in which they are secreted. The aqueous extract of saliva at $100^{\circ}$ is poisonous or narcotic in its action toward birds. To show the presence of basic substances, the aqueous extract was slightly acidulated with dilute hydrochloric acid, then precipitated by Mayer's reagent; the precipitate was washed, then decomposed by hydrogen sulphid, and the solution filtered. The filtrate on evaporation gave a residue consisting of microscopic slender needles of a soluble hydrochlorid. This salt, purified by extraction with absolute alcohol, formed soluble, crystalline, but easily decomposable double salts with platinum chlorid and with gold chlorid. The solution of the hydrochlorid produced an immediate precipitate of Prussian blue in a mixture of potassium ferricyanid and ferric chlorid, and when injected into birds produced stupor.

\section{Leucomains from Other Tissues of the Body.}

Selmi's work upon the formation of ptomaïns during the process of putrefaction led many investigators to doubt the production of these hases by the decomposition of the proteid or other complex molecules. To substantiate this, a number of chemists, especially Italian, endeavored to show that Selmi's bases, to a large extent at least, exist preformed in the various tissues. Paternò and Spica (1882) succeeded in extracting from fresh blood as well as from fresh albumin of eggs substances identical, or at least similar, to those designated under the name of ptomaïns. Their observations, however, were confined to the detection of alkaloidal reactions in the various extracts obtained by Dragendorff's method, and at no time were they in possession of a definite chemical individual. Marino- 
Zuco (1885) was more successful, inasmuch as he succeeded in obtaining from fresh tissues and organs relevant quantities of a base identical with cholin, and, in addition, he obtained extremely minute traces of other alkaloidal bodies. One of these, obtained by the Stas method from the liver and spleen of an ox, exhibited in hydrochloric acid solution a beautiful violet fluorescence resembling very much that of the salts of quinin. A similar base, probably identical with this one, was obtained by Bence Jones and Dupré (1856) from liver, nerves, tissues, and other organs, and was named by them "animal chinoidin." A greenish-blue fluorescence is frequently observable in the alcoholic extracts of decomposing glue as well as from other putrefying substances, and is undoubtedly due to products formed by some one of the fluorescing bacteria. From a number of very thorough experiments, he concluded that basic substances do not preëxist in fresh organs, but that the acids employed in the process of extraction exert a decomposing action upon the lecithin present in the tissues, resulting in the formation of cholin. He further showed that the method of Dragendorff, on account of the larger quantity of extractives, which forms invariably gave a larger yield of this base than did the Stas-Otto method. Similar observations were made by Guareschi and Mosso, by Coppola and others. At the present time there is no doubt that some basic substances, among these cholin and the hexon bases, can be formed by the action of reagents, and, on the other hand, it is equally well demonstrated that similar bases do preëxist in the physiological condition of the tissues and fluids of the body.

Recently R. Wurtz has obtained from normal blood a number of crystalline products of alkaline reaction, which form well crystallizable double salts with gold, platinum, and mercuric chlorids. These, however, have not been as yet subjected to analysis, because of the minute quantities which were isolated.

Marino-Zuco and Martin in 1894 showed the presence of cholin in fresh blood.

In 1899 Gulewitsch carried out a most painstaking investigation on the leucomains in fresh brains with especial reference to the detection of the poisonous neurin. The possible presence of the latter has been suggested as explaining the auto-intoxications met with in mental disorders. By means of Brieger's method he obtained cholin but no neurin. Moreover, he was unable to obtain the latter from protagon. The aqueous brain extract gave a very small quantity of two bases, probably diamins. Urea was also obtained.

In extensive skin-burns Kijanitzin isolated a peptotoxin-like base from the urine and blood, more abundantly from the organs. A similar base was shown by him to be produced by the action of gastric juice on the blood in the presence of bacteria; also in the early stages of the decomposition of blood. The explanation of the fatal results fol- 
lowing the varnishing of a part or the whole of the body is given on pp. 329,466 . A similar explanation undoubtedly holds true for uræmia.

The presence of specific toxic substances in the blood of infectious diseases is well recognized. Nissen has shown that the blood in suppuration was toxic. In the blood of tetanus in man Kallmeyer and Nissen demonstrated the presence of the tetanic poison. Immerwahr showed the same to be true with the organs of experimental tetanic animals, and that the blood of scarlet fever during uræmia was toxic. Brieger was the first to show the presence of tetanin in the amputated arm of a patient.

Morelle (1886) showed the presence, in the spleen of the ox, of a base, the hydrochlorid of which crystallized in deliquescent needles and likewise formed crystalline platino- and aurochlorids. From experiments made by Laborde, the base would seem to possess decided toxic properties, bringing on a dyspnœic condition with convulsive movements and loss of motion. The post-mortem examinations revealed an extended visceral œdematous infiltration, and stoppage of the heart in systole. For the presence of the xanthin bases, cystin, gerontin, etc., in the organs of the body, see preceding pages.

Viron found a very poisonous albuminoid in a hydrocele fluid from a sheep.

A. W. Blyth claimed to have isolated from milk two alkaloidal substances, namely, galactin, the lead salt of which is said to have the formula $\mathrm{Pb}_{2} \mathrm{O}_{3} \cdot \mathrm{C}_{54} \mathrm{H}_{18} \mathrm{~N}_{4} \mathrm{O}_{25}$, and lactochrome, the mercury salt of which is represented by the formula $\mathrm{HgO} . \mathrm{C}_{6} \mathrm{H}_{18} \mathrm{NO}_{6}$.

The adrenals have been the subject of repeated investigations during the past half a century, but really valuable results have been obtained only within the last three or four years. At an early date the fact was established that these organs perform an extremely important function inasmuch as their extirpation is promptly followed by death. The rapid and severe effects observed have been generally regarded as due to an intoxication of the organism with products which normally were supposed to be destroyed in the adrenals. Thus Marino-Zuco obtained cholin from the adrenals and moreover with Dutto he obtained this base in the urine of Addison's disease. These investigators and also Carbone obtained results showing that cholin was decidedly more toxic to animals deprived of the adrenals than to normal animals. Furthermore, the blood of animals from which the adrenals were removed has been shown to possess a marked toxic action. Only recently, Levin ${ }^{1}$ has shown that the blood of such animals contains something which acts on the blood pressure and which does not exist in normal blood.

The early studies of Oliver and Schäfer and others demonstrated that the adrenals contain a substance which exerts a marked action upon the blood pressure, and thus led to a careful study and search ${ }^{1}$ Amer. Journ. Physiology, 5, 358, 1901. 
for the active principle. According to Mühlmann this substance is pyrocatechin. Fürth ${ }^{1}$ at first also beld that the active principle was a pyrocatechin-like body, but eventually he considered it to be a hydro-dioxypyridin having the formula $\mathrm{C}_{5} \mathrm{H}_{7} \mathrm{NO}_{2}$ or $\mathrm{C}_{5} \mathrm{H}_{9} \mathrm{NO}_{2}$. To this substance be applied the name suprarenin. In this country most painstaking investigations were made by $\mathrm{Abel}^{2}$ who succeeded in isolating a basic product which he designated as epinephrin. The formula which he deduced, $\mathrm{C}_{17} \mathrm{H}_{15} \mathrm{NO}_{4}$, is not that of the free base but rather that of a mono-benzoyl derivative. According to Takamine ${ }^{3}$ the blood pressure raising principle of the gland can be isolated in a pure crystalline form. He has designated this product as adrenalin and to this Aldrich ${ }^{4}$ ascribed the formula $\mathrm{C}_{9} \mathrm{H}_{13} \mathrm{NO}_{3}$. Abel however has shown that the analytical results agree more closely with the formula $\mathrm{C}_{10} \mathrm{H}_{11} \mathrm{NO}_{3}$ which he had proposed. It appears from Abel's recent work that adrenalin does not exist as such in the glands but is rather a modified product of epinephrin, the active base.

It is evident from this that the nature of the active constituent of the suprarenals is as yet undetermined and for this reason it is hardly necessary to enter into a detailed consideration of its properties.

The purin bases in adrenals have been studied by Oberblom (p. 390). The perfectly fresh glands yielded a smaller quantity of these bases than such as were digested for two days at the body temperature in the presence of chloroform. Assuming that the action of bacteria was wholly eliminated it would seem that this increase in bases was probably due to the action of an enzyme. Xanthin, 1-methyl xanthin, hypoxanthin, epiguanin and adenin were detected. The presence of methyl xanthin and methyl guanin is of interest in view of the fact that these derivatives have been looked upon as cleavage products derived from the food. If Oberblom's results can be confirmed it would indicate the existence of a purin antecedent as for example a 1.7-dimethyl guanin which in the body might give rise to paraxanthin or like the latter might be demethylated, forming the 1-methyl and 7-methyl compounds referred to above.

\section{Venoms of Poisonous Serpents.}

The study of the chemistry of the venoms of serpents and of batrachians is fraught with so many difficulties and with so much danger, that we cannot wonder at the present unsatisfactory condition of our knowledge in regard to the poisonous principles which they contain. Much of the early work was not only inaccurate and very contradictory, but was far from meeting the requirements of

${ }^{1}$ Zeit. physiol. Chem., 24, 142; 26, $15 ; 29,105$.

${ }^{2}$ Zeit. plysiol. Chem., 28, 318; Johns Hopkins Hosp. Bull., 1901, 84, 337; 1902, 29.

${ }^{3}$ Journ. Am. Med. Assoc., 38, 150, 153.

4 Amer. Journ. Physiology, 5, 457 (1901). 
exact toxicological research. Recent investigations, however, have made it certain that the most active constituent of the venom of serpents is not alkaloidal in its nature, as was supposed by some. In 1881 Gautier announced the isolation of two alkaloids from the venom of the cobra which gave precipitates with tannin, Mayer's reagent, Nessler's reagent, iodin in potassium iodid, etc. They formed crystallizable platinochlorids and aurochlorids, and also crystalline, neutral, somewhat deliquescent hydrochlorids. The neutral or slightly acid solutions produced an immediate precipitate of Prussian blue in a mixture of potassium ferricyanid and ferric chlorid. The substances possessed a decided physiological action, but Gautier himself did not consider them to be the most dangerous constituents of the venom. This observation of Gautier as to the presence of distinct basic substances in venoms is at variance with that of Wolcott Gibbs, who was unable to obtain an alkaloid from the rattlesnake (Crotalus) venom. S. - Weir Mitchell and E. T. Reichert were likewise unable to substantiate Gautier's statements. Subsequently, Wolfendeu, in an elaborate paper on the nature of cobra venom, confirmed Wolcott Gibbs as to the entire absence of any alkaloidal body.

Mitchell and Reichert made a careful study of the venoms of various serpents, such as cobra, rattlesnake, moccasin, and Indian viper, and succeeded in isolating two proteid constituents, one belon ging to the class of globulins and the other to the peptons. The pepton is said to be non-precipitable by alcohol. According to them, the globulin constituent consisted of at least three distinct globulins. They found that boiling coagulated and destroyed the globulin as a poison, but that the venom pepton was toxically unchanged, so that the solution, though still poisonous, failed to produce the characteristic local lesions due to fresh or unboiled venom. On the other hand, Gautier asserted that the venom was not sensibly altered on being heated to $120^{\circ}-125^{\circ}$ and that the toxic action remained constant even when all the proteid constituents were removed, thus showing that the toxic action cannot be attributed to the albuminoids. Later, Quartier acknowledged that viper venom was destroyed at $100^{\circ}$. Calmette found it to be destroyed at $98^{\circ}$, while still later Phisalix and Bertrand showed that an exposure of five minutes at $80^{\circ}-85^{\circ}$ destroyed the toxicity. The venom pepton from the rattlesnake or the moccasin, however, when injected into animals produced toxic effects which were marked by an œdematous swelling over the site of injection; the tumor was filled with serum, and so also was the subcutaneous cellular tissue. Furthermore, a gradual breaking down of the tissues occurred, accompanied by rapid putrefactive changes and a more or less extensive slough. That peptons may possess intensely poisonous properties has been shown to be the case by a number of authors, among whom may be 
mentioned Schmidt-Mülheim, Hofmeister, Pollitzer, and others. Brieger believed that the formation of peptons in the process of digestion was accompanied by the development of a toxic ptomaïn, which he named peptotoxin. As stated elsewhere, Salkowski has very properly questioned the formation of peptoxin in the ordinary digestion of proteids.

The venom globulins, on the other hand, though present in less quantity than the peptons, induced the same remarkable local effects seen on injection of the pure venom. They caused local bleedings, destroyed the coagulability of the blood, and rapidly corroded the capillaries.

These results of Mitchell and Reichert, which are given somewhat in full, have been questioned by Wolfenden, who, while agreeing in the main that the poisonous property of venom is due to proteid constituents, regarded their pepton not as a true pepton, but rather as one or more bodies of the albumose group of proteids. He likewise regards the globulin of moccasin venom to be some other proteid body. According to him, the cobra venom owed its toxicity to the proteids, globulin, serum albumin, and acid albumin. Occasionally there seem to be present traces of pepton and of hemialbumose.

Brieger was at first apparently inclined to believe that the action of venom was due to animal alkaloids, on the ground that these bases are extremely soluble, and hence always go into solution, along with the likewise very soluble proteid constituents, and that the difficulty in their isolation lies in the elimination of these proteids. Subsequently, however, Brieger and Fränkel pointed out the poisonous nature of some bacterial proteids (toxalbumins) and also showed that cobra poison yields with alcohol a precipitate which gives proteid reactions.

The proteids of serpents' venom should be compared with the toxins formed by the activity of the pathogenic bacteria, and also with the similar compounds, the phytalbumoses of castor beans, jequirity, etc., and with the enzymes. Possibly similar compounds will be found in croton and other species of ricinus, jatropha, locoweed, etc. The poisons secreted by certain spiders and fish may be mentioned in this connection (see p. 193).

Recent researches on the venoms have been productive of very important results, especially from the standpoint of immunity to and cure from the bites of venomous serpents. Although in the higher latitudes poisoning from suake-bites is comparatively rare, it should not be overlooked that in certain portions of the globe, notably India and Australia, the mortality from this cause is exceedingly high, and may well claim the attention of governments. Thus, it is estimated in India that over 20,000 persons die annually from the bites of serpents. 
Phisalix and Bertrand in 1893 confirmed Fontana's previous observation that the garter-snake (couleuvre) was unaffected by repeated bites from vipers or by subcutaneous injection of the venom of the viper. These authors showed that a dose of the venom sufficient to kill 15 to 20 guinea-pigs was without effect on the garter-snake. The natural immunity of the garter-snake to the viper venom is thus firmly established. From previous researches on the natural immunity of the "crepaud" and viper to their own venoms Phisalix and Bertrand showed that the blood or serum of these serpents contained the same poison, echidnin, as was present in the venom. Similarly the blood or serum of the garter-snake when injected in doses of 1.5 c.c. intraperitoneally into guinea-pigs produced death in two hours with the same symptoms as are observed after poisoning with viper venom. Although the several forms of garter-snakes are considered as non-venomous, they nevertheless secrete through the superior maxillary gland (or special glands, Jourdain) toxic products analogous to echidnin, which are excreted into the blood, rendering this, therefore, highly poisonous, and at the same time establishing natural immunity. After the ablation of the venom glands in the viper the blood loses a part of its toxicity, showing that the source of the poison in the blood is the venom gland. It would seem that this immunity is one of tolerance and analogous to that which Sewall obtained with rattlesnake venom.

Later (February, 1894), Phisalix and Bertrand showed that viper venom heated to $75^{\circ}-85^{\circ}$ for five minutes lost its poisonous property with respect to guinea-pigs and acted as a vaccine. The temperature of animals, however, was raised, whereas with unheated venom it is lowered. They were, therefore, led to believe that viper venom contained (1) a phlogogenic substance like the diastases-echidnase, and (2) a general poison-echidnotoxin. Since both are destroyed by heat the vaccine results either from the destruction of these two substances or is preformed in the venom and acts after the toxic principles are destroyed. This behavior of venom to heat and to the production of immunity is analogous to Fränkel's method of producing immunity to diphtheria.

The heated viper venom, or vaccine, does not impart immediate immunity to guinea-pigs, but this condition follows after the lapse of several days - a result of the reaction of the organism. An antitoxin appeared in the blood after the injection of the echidnovaccine, and in less amount in the blood when immunity has been established by tolerance. The amount of antitoxin in the blood could be increased as in the case of tetanus and of diphtheria. A very short time afterward Calmette confirmed the observations that animals could be immunized by repeated injections of the venom, beginning in small doses and gradually increasing. A single non-fatal injection of venom may produce antitoxin in the blood of the animal. 
He furthermore obtained immunity by applying the method employed by Roux and Vaillard in their work on tetanus, that is, by repeated injections of the venom mixed with gold ehlorid, or sodium or calcium hypochlorite. The serum of the immunized animal was found to be antitoxic in the same sense as the serum of animals immunized to diphtheria or tetanus. Furthermore, not only was the blood shown to be antitoxic to the venom employed, but also to the venoms of other serpents. Thus, the serum of a rabbit immunized against the cobra venom is not only antitoxic to this venom, but also to the viper of France, the black snake of Australia, etc.

Immunity, therefore, to venom can be obtained (1) by repeated injections of small doses (Sewall, Phisalix and Bertrand, Calmette); (2) by the use of heated venom or vaccine (Phisalix and Bertrand); (3) by repeated injections of venom mixed with hypochlorite solution (Calmette); (4) by injections of antitoxic serum (Phisalix and Bertrand, Calmette). The immunity according to the first method, by tolerance, has been shown to be due to the presence of antitoxin substances in the blood (Phisalix and Bertrand, Calmette, Fraser). The second and third methods are explainable in the same way.

The application of the latter principle in the treatment of bites from serpents was suggested by Phisalix and Bertrand and carried out by Calmette. The rabbit, dog (?), guinea-pig (Phisalix and Bertrand), horse and ass have been employed to furnish serum antitoxic to venom. Calmette has prepared a serum of a strength of $1: 10,000$; that is, rabbits given a dose of venom sufficient to kill in three or four hours are saved if a quantity of antitoxic serum corresponding to $\frac{1}{10000}$ of their weight is injected not later than one hour after the injection of venom. As stated above, this antitoxic serum protects against all venoms.

Calmette has also shown that the ichneumon of the Antilles is naturally immune to venom, and that it owes this condition to the antitoxic property of its blood. Just as the serum of man or of the horse was sometimes found to be antitoxic to the diphtheria poison, so the serum of dogs was occasionally found to be antitoxic to venom.

The studies of Phisalix ${ }^{2}$ and others show that protection against viper venom may be secured by the injection of serum of diverse origin. The serum of the vipers, garter-snakes, eels, hedge-hog, guinea-pig, frog, toad, dog and horse possesses such action. Moreover, Fraser has found that minute quantities of bile possess a like action. Phisalix has not only confirmed this observation but has demonstrated that the bile acids, cholesterin and tyrosin vaccinate against viper venom. The protecting property however is lost when the material is heated to $120^{\circ}$. The alcoholic precipitates produced

${ }^{1}$ Compt. Rendus, 121, $745 ; 122,1439 ; 123,1305 ; 125,121,977,1053 ; 127$, $1036 ; 126,431$. 
in extracts of dog's liver or pancreas also protect. Phisalix has also found that extracts of mushrooms (Agaricus edulis) and the venom of lornets exert a similar protective action. These various substances are not strictly speaking antitoxins. They, like porcelain, merely modify the venom. This may be due to changes analogous to surface action.

Fraser has independently arrived at substantially the same results as the French investigators. Serum that is antitoxic to venom is designated by Fraser as antivenin. He immunized the horse and cat against the cobra venom. The cat was also rendered immune by administration through the stomach. It is interesting to note that Répin obtained immunity in guinea-pigs to abrin, the poisonous albumose of jequirity, by repeated administrations of small doses by the mouth. Ehrlich has shown that while $\frac{1}{10} \mathrm{mg}$. of abrin is sufficient to kill a guinea-pig in two or three days when injected subcutaneously, one hundred times this amount, $10 \mathrm{mg}$., is necessary to kill by the mouth. Roux and Yersin endeavored to produce immunity to diphtheria by the mouth, but were unsuccessful.

According to Fraser, $0.18 \mathrm{mg}$. of cobra venom constitutes the minimum fatal dose for 1 kilogram of rabbit. The guinea-pig is less susceptible, and the kitten still less so, requiring $2 \mathrm{mg}$. The minimum fatal dose of the rattlesnake venom, per kilogram of rabbit, is placed at $4 \mathrm{mg}$. The cobra venom is, therefore, 16-20 times more powerful than that of the rattlesnake.

Calmette has successfully saved rabbits from intoxication by venom by injecting in a circle, at a distance from the wound, a solution of fresh calcium hypochlorite; in the case of man an injection of 20-30 c.c. of the fresh solution obtained by diluting a $1: 12$ solution (5 c.c.) with boiled water ( 45 c.c.). Mairet and Bosc consider this protection by hypochlorite solution as due to a direct action on the venom poison, and not to the formation of antitoxin. This method of treating venom bites has been tried with success in Australia. It should be noted that a solution of hypochlorite not only destroys the poison of venom, but also the toxin of glanders (Pench), of tetanus, and diphtheria (Roux).

It is therefore evident that a striking similarity exists in the action of venom, of plant albumoses, ${ }^{1}$ of bacterial toxins, and of enzymes. The similarity is strengthened further by the behavior of these poisons to heat and to chemicals, and lastly by development of antitoxic substauces in the blood of animals artificially immunized against these toxins.

Venoms resemble the phytalbumoses and bacterial toxins in their behavior to red blood cells. Mitchell and Stewart, in 1897, showed that these poisons agglutinate and then dissolve the red blood corpuscles. The work of Myers on cobra lysius has been mentioned on

$$
{ }^{1} \text { See Cushny, Archiv. exp. Path. u. Pharm., 41, } 447 \text { (1898). }
$$


page 141. Flexner and Noguchi ${ }^{1}$ have recently made a careful study of the hemolytic action of venoms. Their results briefly stated are as follows: Agglutination of corpuscles occurs rapidly in favorable solutions, while in very weak ones a delay of some minutes up to one hour may be noted. Active agglutination takes place with 0.2 per cent. solutions of venoms but the maximal results are given by 0.5 per cent. solutions. The corpuscles of the rabbit are highly susceptible while those of the guinea-pig, dog, sheep, swine are less and in about the order given. Hemolysis follows agglutination and depends on the strength and kind of venom and on the temperature. The dog's corpuscles are more easily hemolyzed but agglutinate less readily than do corpuscles from other animals. The agglutinating power of venoms is destroyed by heating at $75-80^{\circ}$ for 30 minutes. This temperature has no effect on the hemolytic power which, moreover, is only slightly decreased by heating at $100^{\circ}$ for 15 minutes. The hemolytic power of venoms differs : cobra is most active, watermoccasin, copper-head, and rattle-snake are less in the order named. The different mammalian corpuscles possess a varying degree of susceptibility. The dog's corpuscles are hemolyzed most rapidly while those of sheep, guinea-pig, pig, rabbit and ox are less susceptible in the order given. The hemolytic effects can be brought out usually with 0.2 per cent. solutions of the venoms but in the case of the ox a 0.5 per cent. solution is necessary. Washed corpuscles are agglutinated but not hemolyzed. The addition of serum, however, promptly induces hemolysis. Leucocytes are also agglutinated and hemolyzed by venoms. The agglutinating principle may be common to both the red and white corpuscles while the dissolving principle for leucocytes is distinct from that for red cells. The toxic principle is removed by nerve cells but not by blood cells. Venom destroys the germicidal properties of many blood sera by fixation of the serum complements by the venoms which, however, have no action upon the intermediate bodies of the serum. Antivenin neutralizes venom and removes both the hemolytic and antibacteriolytic actions.

The blood or serum of the common turtle (?) (Bufo vulgaris) is in 1 c.c. dose toxic to frogs. This property of the blood, therefore, is a result, as in the case of the viper and the garter-snake, of the "inner-secretion" of toxic glands (Phisalix and Bertrand).

Cloez and Gratiolet in 1852 examined the poison contained in the cutaneous pustules of some batrachians, and succeeded in extracting a substance which gave a white precipitate with mercuric chlorid and formed a platinum double salt. Beyond this meagre information very little is known in regard to the character of these poisons, though Zalesky, in 1866, announced the isolation of an alkaloid to which he assigned the formula $\mathrm{C}_{34} \mathrm{H}_{60} \mathrm{~N}_{2} \mathrm{O}_{5}$, and which he named samandarin. According to Dutartre (1890), this base is a leuco-

$$
{ }^{1} \text { Journ. Exp. Med., 6, } 277 .
$$


TABLE OF LaUGOMA ÏNs.

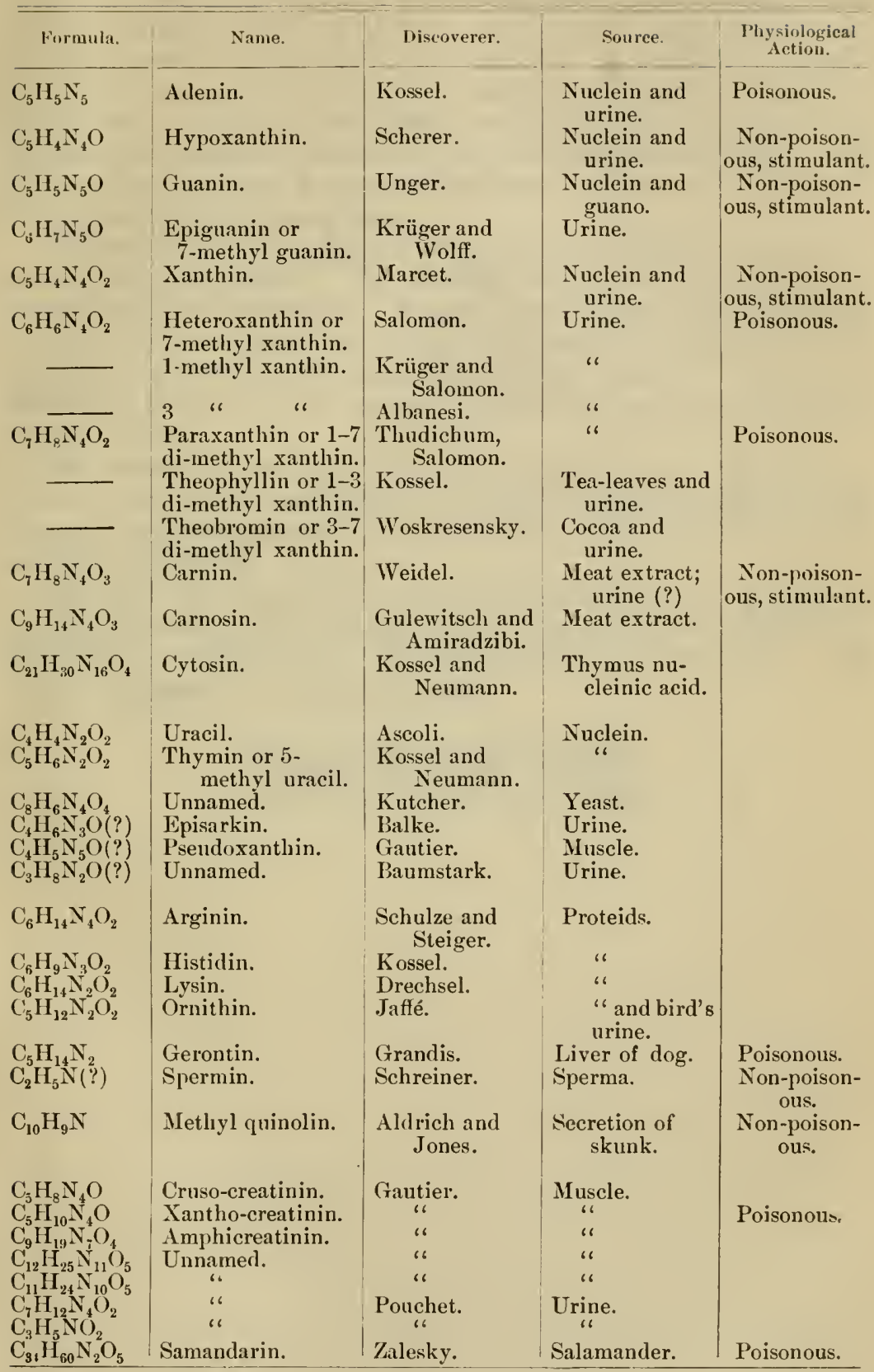



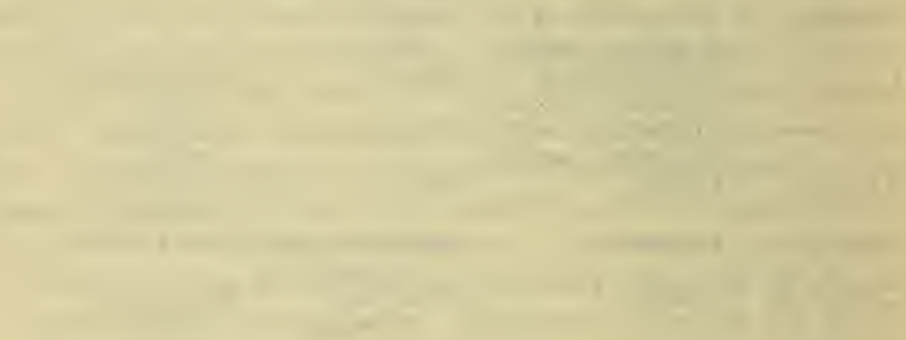

\section{$x^{-2}+x_{n}=$}
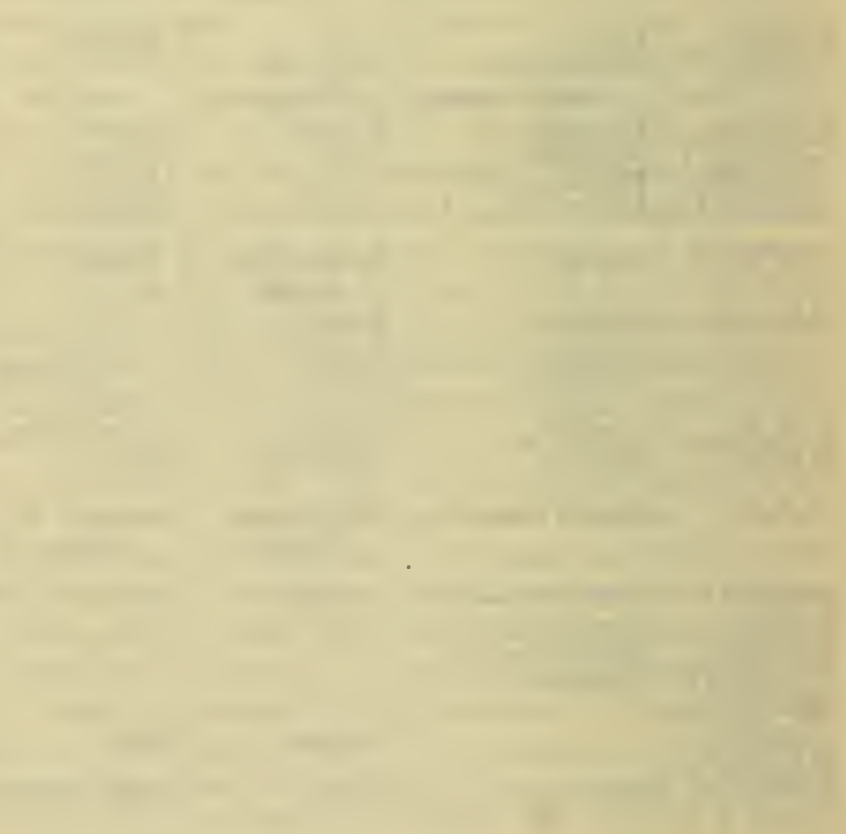
maïn, and similar products, but with different physiological action, are to be found in other batrachians, as the toad, triton (?), green and red frogs, and in the epidermis of some fish. According to Calmeil, the poison from the toad contains methyl carbylamin and isocyanacetic acid. According to Phisalix and Contejean, the blood of the salamander possesses antitoxic action with reference to curare. The salamander, therefore, is naturally immune, and, moreover, its blood will protect frogs against curara.

The recent studies of Faust ${ }^{1}$ show that samandarin acts upon the central nervous system. The chemical nature of the poison, however, remains undetermined. According to Phisalix ${ }^{2}$ the salamander venom loses its toxicity on drying; is promptly destroyed at $100^{\circ}$ and even at $50^{\circ}$; and is also destroyed by alcohol. The venom altered by heating to $60^{\circ}$ acts as a vaccine and hence resembles the serum of eels.

The venom of bees is said to owe its toxicity to an organic base. That of the hornet, according to Phisalix, immunizes against viper venom, is not destroyed at $120^{\circ}$, is soluble in alcohol and does not contain an alkaloid.

Phisalix ${ }^{3}$ has also shown that the ventral glands of myriapods (Iulus terrestris) yield a toxic secretion which is not affected by heating in a sealed tube at $100^{\circ}$ but is destroyed at $120^{\circ}$. The venom is supposed to contain quinone.

1 Archiv. exp. Path. u. Pharm., 41, 229.

${ }^{2}$ Comptes Rendus, 125, 121, 977 (1897).

${ }^{3}$ Comptes Rendus, 131, 955, 1005, 1007. 


\section{CHAPTER XVI.}

\section{THE AUTOGENOUS DISEASES.}

ALL living things are composed of cells. The simplest forms of life are unicellular, and in these all the functions of life devolve upon the single cell ; absorption, secretion, and excretion must be carried on by the same cell. A collection of unicellular organisms may be compared to a community of men with every individual his own tailor, shoemaker, carpenter, cook, farmer, gardener, blacksmith, etc. Nevertheless it is true that in communities of unicellular organisms the accumulation of their own secretions and excretions impair the growth of the individual and finally rob it of its life. But only the lowest forms of life are unicellular; all others are multicellular. In the higher animals there is a differentiation not only in the size and structure of the cells, but in the duties imposed upon them. The body of man may be compared to a community in which labor has been specialized. Certain groups of cells, which we designate by the term "organ," take upon themselves the task of doing some special line of work, the well doing of which is essential to the life, not only of that group, but of other groups as well, or of the body as a whole. There is an interdependence among the various organs. Certain groups of cells supply the fluids or juices which act as digestants, and among these there is again a division of labor. The salivary glands supply a fluid which partially digests the starch of our food ; the peptic glands supply the gastric juice which does the preliminary work in digestion of the proteids, while the pancreatic juice completes the digestion of the starches, begun in the mouth, of the proteids begun in the stomach, and does the special work of emulsifying fats. Harm results to the individual when any part of the digestive processes is not properly carried on, and even some of the products of complete digestion are harmful when permitted to enter the circulation unchanged. The peptons must be converted in part at least into serum albumin by the absorbing mechanism of the walls of the intestines, and while ten per cent. of the fat of the food is split up into glycerin and fatty acids by the action of the pancreatic juice, a much smaller per cent. enters the thoracic duct in this divided form. The food taken may be proper both in quality and in quantity and the digestive juices may do their work promptly and satisfactorily, but if the absorbents fail to perform their functions properly, disease results. Again it may happen that the failure lies in improper or imperfect assimilation, and the result becomes equally dis- 
astrous, and with the effects of non-elimination we are fairly conversant. Of the myriads of cells in the healthy human body there is none which is superfluous. It is true that among these ultimate entities of existence death is constantly occurring, but in health regeneration goes on with equal rapidity and each organ continues to do its daily and hourly task. The microscope has made us familiar with the size and shape of the various cells of the body, and pathologists have described the alterations in form and size characteristic of various diseases; but we must remember that in the study of these ultimate elements of life there are other things besides their morphological history to investigate. They are endowed with life, and they, as well as bacteria, have a physiology and a chemistry which we but slightly know. Their functions are influenced beneficially or harmfully, as the case may be, by their environment. They grow and perform their duties properly when supplied with the needed pabulum, but they are not immune to poisonous agents, and they are injured when the products of their own activity accumulate about them.

It is true, withont exception so far as we know, that the excretions of all living things, plants and animals, contain substances which are poisonous to the organisms which excrete them. A man may drink only chemically pure water, eat only that food which is free from all adulterations, breathe nothing but the purest air, free from all organic matter, both living and dead, and yet that man's excretions would contain poisons. Where do these poisons originate? They are formed within the body. They originate in the metabolic changes by which the complex organic molecule is split up into simpler compounds. We may suppose-indeed we have good reason for believing - that the proteid molecule has certain lines of cleavage along which it breaks when certain forces are applied, and that the resulting fragments have also certain lines of cleavage along which they break under certain influences, and so on until the end products, urea, ammonia, water, and carbon dioxid are reached; also that some of these intermediate products are highly poisonous has been positively demonstrated. The fact that the hydrocyanic acid molecule is a frequent constituent of the leucomaïns, is one to which we have already called attention. We know that chemical composition is an indication of physiological action, and the intensely poisonous character of some of the leucomains conforms to this fact. It matters not whether the proteid molecule be broken up by organized ferments, bacteria, or by the unorganized ferments of the digestive juices, by the cells of the liver or by those still unknown agencies which induce metabolic changes in all the tissues - in all cases poisons may be formed. These poisons will differ in quantity and quality according to the proteid which is acted upon and according to the force which acts.

It is not our intention at this time to write extensively concerning the autogenous diseases. We desire simply to call attention to the 
fact which has been illustrated specifically in the preceding chapter, that among the metabolic products of the animal body there are to be found substances which are more or less toxic in their action. By the word autogenous, as indicating a class of diseases, we understand that in these disorders the materies morbi is a product of some cell of the body, and not, as in the case of the infectious diseases, of cells introduced from without the body. Much has been said about auto-intoxication, and in our opinion a large part of what has been said upon this subject is without sufficient scientific foundation. Auto-intoxication is a word now frequently used to cover up our ignorance, and the time has not yet come for a thorough investigation of this subject. Indeed, it is almost impossible in a given case to exclude external factors which influence diseased conditions. While we must admit that poisons are formed in the animal body, it is not always easy - in fact we may say it is never easy - to be certain that the starting point does not lie outside the body. Some writers have mistaken the secondary effects of ectogenous toxins for auto-intoxications. As an illustration of this we may mention the attempt that has been made to classify alcoholic cirrhosis under the head of anto-intoxications. The explanation which has been offered by those who have advocated this classification is that the changes in the liver are not due to the direct action of alcohol, but that as a result of the abuse of this beverage a diseased stomach results, and on account of its diseased condition permits the absorption of inperfectly digested substances, to the direct action of which the changes in the liver and other organs are to be attributed. According to our way of looking at it, alcoholic cirrhosis is due either directly or indirectly to the action of the toxic substance which is introduced from without, and certainly should not be elassed among autogenous diseases. Auto-infection has also been confounded with auto-intoxication.

These two processes are wholly distinct, and a clear comprehension of each will prevent confusion. The term auto-infection is properly applied to those cases in which the virus of disease is carried locally in some part of the body, and is distributed from this locality to other parts of the body. Illustrations of auto-infection are numerous in certain diseases, and especially in tuberculosis. An individual with pulmonary tuberculosis swallows his sputum and infects his intestinal walls. Another person may carry for years a colony of tubercle bacilli in his lungs, in his bones, or in some other part of his anatomy, and then when this individual is exposed to conditions which materially lower his vitality, the bacilli which have been confined to a narrow locality, pass beyond the bounds to which they have so far heen restricted, and invade every tissue, leading to acute miliary tuberculosis. Post-mortem examinations have shown that one-third or more of all men have latent tuberculosis, and this un- 
doubtedly is the explanation of the frequently observed fact that injury to some part of the body leads to tubercular lesions in that locality. In this way we can account for tuberculosis of the hip joint, of the spine, and of the meninges of the brain. These illustrations must suffice to show the difference between auto-infection and auto-intoxication.

Some writers are inclined to place under the head of auto-intoxications all those diseased conditions in which no lesions can be detected either macroscopically or microscopically, while on the other hand, they exclude from the list of autogenous diseases all those disorders which are accompanied by lesions. This, in our opinion, is plainly unscientific, and, indeed, the error of such a position needs only to be pointed out in order to be plainly understood. The endogenous poisons may induce lesions which are quite as extensive and quite as marked as those which result from the ectogenous toxins. For instance, recent researches have shown that normal, sterile bile when introduced into the pancreatic duct and brought in contact with the tissue of this gland causes most marked necrotic changes; indeed, it is doubtful whether or not we have among the bacterial toxins any substance which will cause more marked morphological changes in any tissue than that induced by normal bile when brought in contact with the pancreas. It must be evident, therefore, that we cannot exclude from the autogenous diseases all of those which show tissue changes. Indeed, it is probable that there is no disease which is unaccompanied by morphological changes in the cells in some part of the body. These alterations may be so slight that they have escaped detection, but it certainly is true that the number of diseases unaccompanied by morphological changes grows less each day.

As has already been stated, we have not as yet sufficient scientific data to enable us to attempt even a provisional classification of the autogenous diseases. However we may point out certain facts connected with this subject.

1. The digestive organs may but imperfectly perform their function, and the products of their incomplete action may be absorbed and may lead to more or less disturbance in certain organs of the body. Moreover in such a case as this every part of the body will suffer more or less from insufficient nutrition due to the fact that properly prepared pabulum is not brought within reach of the cellular elements. That imperfectly digested proteids, and in fact certain proteids wholly undigested, may be absorbed, is a well known fact. To what extent the absorption of undigested proteids may take place in the animal body and how much harm can be wrought in this way, we are not able to say. It is more than probable that the great susceptibility of the infant to bacterial products formed in milk is due to the fact that during this period of life the intestinal 
walls permit the passage of proteid bodies, to which the same structure in the adult is impervious. When peptons and albumoses are injected directly into the blood they act as powerful poisons. They destroy the coagulability of the blood, lower the blood pressure, and in large quantities cause speedy death. The lassitude and depression following a full meal, especially one rich in proteids, is attributed to the absorption of peptons, but so far there is no scientific evidence bearing on this point. Pepton and albumose are frequently found in the urine, but whether the substances found in this excretion have been absorbed from the intestinal wall or have been elaborated elsewhere in the animal body, no one knows.

2. That certain secretions and excretions of the human body are poisonous when brought in contact with tissues with which normally they have no relation, is well known. We have already referred to the action of normal bile when brought in contact with the pancreas, and that the bile acids have a hemolytic action when absorbed into the circulation is a fact which has long been known. Why it is that the bile has a destructive action upon the cells of the pancreas and no such effect upon the cells of the liver, or upon the structures of the gall passages and intestines, we do not know. It is rather strange that with the close relation between the pancreas and the gall bladder that the contents of the latter do not more frequently reach the former. So far as we know this is an accident which very seldom happens, and without the experimental demonstration which has been made on this subject it would be quite impossible to account for the alterations observed in the pancreas in the few instances in which this has happened.

3. It is the function of certain organs of the body to prevent the passage of certain substances in to the general circulation. In other words, it is the duty of certain groups of cells to protect other communities from harmful agents. The rich therapeutical results which have followed experimental investigations of the relation of the thyroid gland to myxedema and cretinism are illustrations under this head. The probabilities are that myxedema is a form of mucinæmia, and that the introduction of an excess of mucus into the other tissues is prevented by the normal action of the thyroid gland. This protective action of certain glands is manifest both in certain internal and external secretions. That the bile consists essentially of excrementitious material is generally believed and in fact has been demonstrated. It is not necessary to multiply illustrations of this kind.

4. That the undue retention of excrementitious substances frequently leads to disturbances of health, is well known. The absorption of effete matter from the intestines and the retention of substances which should be eliminated by the kidneys may lead to disastrous results. We have only to mention as an illustration under this head the retention of urates in the causation of gout, and 
the absorption of bile in cases of obstructive jaundice. Our studies of the leucomaïns have shown that small amounts of substances more or less toxic are constantly being formed in cellular metabolism, and the undue retention of these leads to disease.

5. That certain cells in the body fail to adjust themselves to general alterations taking place in other organs at certain periods of life, is quite evident. So true is this that the physician recognizes the fact that there are certain periods, such as that of puberty and the climacteric, which are accompanied by special dangers to health and even to life. The most plausible explanation of this is on the supposition that in the special disturbances of certain organs other parts of the body fall out of harmony, and the parts no longer work together smoothly.

6. Under conditions but little understood at present certain cells of the body fail to utilize certain food-stuffs. This is true, for instance, in certain forms of diabetes. The cells which are accustomed to absorb and utilize the sugars find themselves unable to accomplish this duty, and the unused sugar acts as a poison to other tissues.

7. Active poisons are sometimes formed by certain cells in the body. In this way we account for the presence of certain of the more highly toxic leucomains and some of the more poisonous acids, such as oxy-butyric, and some of the poisonous gases, such as hydrogen sulphid and methyl mercaptan, and some of the alkaloidal bodies which have been discussed in the chapter on leucomaïns. 



\section{INDEX.}

CCIPENSERIN, 429

Acetrl-adenin, 361 -cholin, 303 -guanin, 383

Addiment, 124

Adenin, 341, 347

-hypoxanthin, 365

-theobromin, 365

Adenylic acid, 347

Adrenals, bases from, 473

Adrenalin, 474

Aërobic bacteria, 30

Agaricin, 293

Agglutinable substance, 154, 158, 161

Agglutinate, 154

Agglutination, 153, 177

and immunity, 179

diagnosis by, 159

differentiation of bacteria by, 160

distinct from bacteriolysis, 123

method of testing, 159

of corpuscles, 115,480

pseudo-, 160

theory of, 161

Agglutinins, 116, 137, 139, 152, 181, 184 properties of, 156 relation of leucocytes to, 157

Albumins, poisonous, 34 precipitins of, 115

Albuminous urine precipitins, 117

Albuminose, 434

Albumoses from anthrax bacillus, 49 tubercle bacillus, 80

hog cholera, 99

immunity from, 479

Alcohol, basic substances in, 231

Alcoholic fermentation, bases in, 280

Aldehyde collidin, 255

Alexins, 109, 151, 167 of Bordet, 135

Alexocrtes, 111

Alkaloids, animal, 29 interference in reactions of, by ptomaïns, 237

Alkapton, 334

Allantiasis, 201

Alloxuric bases, 335 bodies, 335

Amanitin, 293

Amido-ralerianic acid, 28i, 438

Amphi-creatin, 459

Amyladenin, 364 hypoxanthin, 375
Amylamin, 33

Amylic alcohol, impurities in, 231

A naërobic bacteria, 30

Animal chinoidin, 41

coniin, 273

parasites, 18

Anthracen, 330

Anthracin, 49

Anthrax, 48

albumose, 49

bacillus, products of, 48

action of pyocyanase on, 175

action of immune serum on, 179

enzymes, 52

immunity to, 172

by enzymes, 175

proteid, 49,50

ptomaïn, 49, 50

susceptibility to, 166, 167

theories of, 19, 48

toxin, 51,53

Anti-alexins, 136

-enzymes, 150

-hemolytic sera, 137, 141

-infectious sera, 179, 181, 184

-lysin, 65, 130

-spermotoxins, 148

-staphylolysin, 8S

-toxic sera, 179, 181

Antipepton, 424

theory of action of, 181

Antitoxin, 172

artificial, 185, 179

action of, 181, 36, 71

diphtheria, 71

nature, 71,183

production, 130

tetanus, 64,67

venom, 478

Antivenin, 141, 479

Arbacin, 434

Arginin, 423, 436

Aromin, 464

Arrow poison, 68

Asellin, 286

A siatic cholera, 54 see vibrio

Atmid albumoses, 80

Atropin-like substances, 42, 214

Auto-digestion, basic products in, 265, $272,367,416,424$

Autogenous diseases, 18, 482

Auto-infection, 484 
Auto-intoxications, 129, 291，299，334, 484

Autolysins, 129

Auto-spermotoxins, 148 -toxins, 148

Azulmic acid, 352

BACILLUS acidi lactici, 86

$\mathrm{B}^{\mathrm{A}}$ anthracis, 209, 283

botulinus, 211

bovis morbificans, 208

butyricus, 30

coli, $97,153,215,218,220,272$

enteritidis, $153,207,210$

piscicidus agilis, 200

pneumonix, 152, 204

pyocyaneus, $86,141,150,152,166$, 179,330

immunity, 174

subtilis, 86

typhosus, 153

Bacon, poisonous, 206

Bacteria, action of, 19

in summer diarrhoas, 91

relation of, to disease, 24

Bacterial cellular proteids, 32

diseases, 17

intoxications, 24

poisons, definition and classification of, 29

historical sketch of, 38

foods containing, 188

of the infectious diseases, 48

products in toxicology, 237

proteids, 31

toxins, 22

Bacteriolysis, 122,174

Batrachians, poison of, 480

Baumstark's base, 464

Beef, poisonous, 207

Beer, colchicin-like substance in, 247

Bees, poison of, 481

Benzol, impurities in, 231

Benzoyl adenin, 361

chlorid method, 267

cholin, 304

guanin, 383

Benzyl adenin, 362 hypoxanthin, 374

Bergmann and Schmiedeberg's method, 236

Betain, 304, 310

aldehyde, 311

homologues of, 312

Bilineurin, 293

Blood, germicidal properties, 177 hemolysis and agglutination, 123 leucomains in, 472 maternal and fetal, 138 precipitins, 119

Blood-serum, 102 antitoxic and bactericidal, 179 protective action of normal, 180

Bocklisch's base, unnamed, 326

Bois bracelet, 198

Bordet's sensitizer, 161

Botulinic acid, 204

Botulism, 193, 201

Brain, 46, 294, 299, 348 bases in, 472

Bread, poisonous, 220

Brieger's bases, unnamed, 313, 317, 325, 327

methods, 232

Bromatotoxismus, 188

Brom-adenin, 359

-hypoxanthin, 373

-guanin, 383

-xanthin, 392

Brouardel's veratrin, 245

Bujwid's cholera-reaction, 59

Butylamin, 253

Butyric acid, 438

Butyro-cholin, 303

CADAVERIC alkaloids, 29

coniin, 273

Cadaverin, 47, 56, 271

from lysin, 444 urine, 467

Caffein, 340, 405

Caproylamin, 253

Carbon monoxide in expired air, 463

Carbonic acid, 461

Carbopyridic bases, 457

Carnin, 413

Carnosin, 414

Caseic acid, 39, 203, 211

Castor bean, 47

Causation of disease, 17

Charcot Neumann, crystals of, 450

Cheese, poisonous, 39, 211

ptomaïns, $46,47,323$

Chemotaxis, 83, 86, 169

Chicken cholera, 100

immunity to, 172

poisonous, 208

Cholera, 54

bases in, 55, 56, 265

-blue, 60

Bujwid's reaction, 59

enzymes, 54

immunity, 172

-infantum, 61, 90

precipitins, 113

proteids in, 56

-red, 59

serum, 122

-stools, 39, 56, 271

-toxin, 55, 57-59

Cholin, 70, 293

decompositions of, 298

group, 288 constitution of, 310

in Florence's crystals, 450

Chromatin, 430 
Cicuta virosa, 239

Classification of diseases, 18 of bacterial products, 29

Clupein, 429

Codein-like substances, 240

Cod-liver oil, bases from, 285, 286, 319

Colchicin-like substances, 246

Colchicin, Zeisel's test for, 246

Coagulin, 184

Collidin, 255, 256

Collidone, 257

Colon toxin, 215

Complement body, 130, 134, 135, 136

Complementoids, 133

Coniin-like substances, 43, 237

Copper in foods, 191

Copula, 136

Coridin, 261

Corindin, 259

Corn, poisonous, 223

Corn-meal, ptomaïns in, 45, 240

Cornutin, 221

Creatin, 56, 283, 378, 459 relation to arginin, 437

Creatinin, 70

-group, 335, 454

Cruso-creatinin, 457

Cyclopterin, 429

Cystin, 266, 269

Cystinuria, bases in, 265, 268

Cytase, 136

Cytophil group, 131, 182

Cytosin, 415

Cytotoxins, 147, 148

DECONINCK'S bases, 256, 260 Delezinier's base, 263

Delphinin-like substances, 245

Derris, 198

Desmon, 136

Deutero-albumose, 49 toxins, 35,65

Diamins, 263, 271, 272, 472

Di-amido caproic acid, 444 valerianic acid, 438

Diaminuria, 265, 268

Diarrhœa, petromyzon, 197

Diarrhœas of infancy, 90

Diethylamin, 252

Digitalin-like substances, 43, 244

Dihydrolutidin, 254

Dimethylamin, 249

-guanin, 383

-hypoxanthin, 374

-xanthin, 339, 400, 404

Diphtheria, 68

bacillus, frequency of, 25 products of, 47,68

immunity to,

pathological changes in, 75

serum, preparation of, 165

toxalbumin, 70

toxin, 68,72
Diphtheria, Ehrlich's views, 35, 71

Diseases, classification of, 17,18

Dragendorff's method, 232

Drechsel's reaction, 353, 408

CBERTH'S bacillus, products of, 30

1. Echidnase, 477

Echidnin, 477

Echidnotoxin, 477

Eel serum, antitoxic action, 478 hemolytic action, 129, 137 precipitin, 117

Egg albumin precipitin, 115

Ehrlich's view of diphtheria toxin, 35, 71

theory of hemolysis, 129 immunity, 181

Emmerich's theory of immunity, 174

Enzymes, anti, 150

in disease, 21

anthrax, 52

diphtheria, 70

germinating plants, 423

hog cholera, 99

action on agglutinins, 156

bacteriolytic action, 174

relation to immunity, 174

Epiguanin, 386

Epinephrin, 474

Episarkin, 420

Epitheliolysins, 142

Ergot, 221, 250

Ergotismus, 220

Ethyl adenin, 364

guanin, 384

-hypoxanthin, 375

-amin, 251

Ethylenediamin, 47 -imin, 450,451

Ethylidenediamin, 263

Etiology of bacterial diseases, 17

Expired air, leucomains in, 461

Examination of poisonous foods, 226

RATTY acids, 52

Fæces, poisons in, 272

Fish, canned, 199, 200

fermented, 249

poisonous, 193

ptomaïns, 47, 198

Florence's crystals, 450

Food poisoning, 188 examination in, 227

Friedlænder's bacillus, 33

Fugu, 195

Fungous diseases, 17

CADININ, 47, 315

G Gaduin, 320

Galactin, 473

Galactotoxismus, 215

Gautier's leucomaïns, 459, 460

Gautier and Etard's bases, 286

methods, 233 
Gautier and Etard's extraction of leucomaïns, 455

Gelatin, hexon bases from, 426 ptomaïns from, 47

Germicidal action of serum, 102

Gerontin, 449

Glanders, 101, 262

Globin, 435

Globulin, germicidal properties of, 104 Halliburton's, 104 precipitins, 115

Glucosins, 281

Glycogen in tubercle bacillus, 82

Gonococcus, 89

Gonotoxin, 89

Goose grease, poisonous, 209 poisonous, 238

Gram's bases, 326

Griffith's bases, 261

Guanidin, 341, 380 from arginin, 438 butyric acid, 438

Guanin, 340, 376 constitution, 340 preparation of, 384

Guareschi's base, 323 and Mosso's bases, 326

HALICHTHYTOXIN, 198
Ham, poisonous, 210

Haptophorous group, 36, 65, 128, 129, 130,182

Hemitoxin, 35

Hemoglobin, 435

Hemoglobiuuria, 136

Hemolytic action of staphylolysin, 88 of tetanolysin, 63 of venoms, 480

Hemolysis, 123, 134, 137

Hemotropic group, 128

Heteroagglutinins, 140

Heterolysins, 129, 140

Heteroxanthin, 339, 397

Hexamethylenediamin, 279

Hexon bases, 335,422

action of, 433

in proteids, 426

method of separation, 427

Hexylamin, 253

Histidin, 426, 442

Histon, 428, 434 action of, 432

Historical sketch of bacterial poisons, 38

Hog-cholera, 99, 281

Homologous sera, 152

Honey, toxic, 197

Hornet, poison, 481

Hydrocollidin, 258

Hydrocoridin, 261

Hydrogen sulphid, 52, 57

Hydrolutidin, 254

Hydrophobia, 173, 186

Hyoscyamin-like substances, 42, 244
Hypoxanthin, 341, 365

TCE-CREAM, poisonous, 219

Ichneumon, immunity to venom, 170, 478

Ich thyotoxismus, 193

Idio-isolysins, 139

iso-agglutinins, 139

Immune body, 124

Immunity, 163

active, 165

and agglutination, 179

artificial or acquired, 165, 171

bacterial, 179

inherited, 165

methods of inducing, 171

natural, 165

passive, 165

produced by non-bacterial products, 173

rôle of phagocytes, 168

Indol, 59, 241

Indoxyl, 453

Infection, 18

and intoxication, bacterial, 164

Infectious diseases, 18 definition of, 23

how produced, 23

poisons of, 48

Intermediary body, 128, 130, 135, 136 , 151

Intoxications, 18,24

Intracellular toxins, 166

Iso-amylamin, 253 -agglutinins, 139

Isolysins, 129

idio-, 139

Iso-muscarin, 311 -propylamin, 253

JEQURITY, 47

K AKKE, 196

I Koch's rules, 24

Kreotoxismus, 201

Kynurenic acid, 453

I ACTOCHOLIN, 303

1. Lactochrome, 473

Lactoserum, 115

Lafon's reagent, 243

Lathyrismus, 222

Lecithin, decomposition of, 296,305 preparation of, 296

Lepierre's base from cheese, 323

Leucin, 33, 49, 57, 101, 255

homologues of, 317

Leucinimid, 452

Leucocidin, 88, 109

Leucolysin, 149

Leucocythæmia, urine in, 348

Leucocytosis, 87 
Leucomaïns, 29 chemistry of, 332

tables of, 481

Lutidin, 254

Lycin, 305

Lysatinin, 425

Lysin, 425, 444

Lysins, 122, 181

Lysuric acid, 446

MACROPHAGES, 168

M Malignant œdema, 101

Maïdismus, 223

Mallein, 101

Meal and bread, poisonous, 220, 222, 223

Meat, poisonous, 47,201

Mechanical interference, theory of, 21

Meissner's base, 465

Methyladenin, 363

amin, 248

betain, 305

carbylamin, 481

guanin, 383,386

guanidin, $47,56,100,282,284$

hydantoïn, 460

hypoxanthin, 375

quinolin, 452

uramin, 283

xanthin, 339, 394, 395, 397

Milk, agglutinins, 156 bacteria, 94

leucomaïns in, 473

poisonous, 215

precipitins, 114

sera, action of, 143

Monamins, 248

Mongoose, 170,478

Morin's base, 280

Morphin-like substances, 240

Morrhuic acid, 319

Morrhuin, 285

Morvin, 101

Murexid, 392

Muscarin, 47, 307, 311

Mussel, poisonous, 189

Mycoderma aceti, 30

Mycoprotein, 33

Mydatoxin, 47, 311 isomer of, 313

Mydalein, 47, 324

Mydin, 47, 257, 287

Myriapods, poison of, 481

Mytilotoxin, 47, 191, 313

Mytilotoxismus, 189

NARCOTIC substance of Panum, 41 Nencki's base, 255

Nephrotoxin, 89, 143

Neubauer's method, 407

Neuridin, 47, 209, 276

Neurin, 47, 288, 310

Neurotic diseases, 19

Nicotin-like substances, 43, 239
Nicotinic acid, 258

Nuclease, 175, 177

Nuclein bases, 335

Nucleins, 98, 334

germicidal action, 109, 110

metabolism of, 336

Nucleinic acid, 109, 110, 429

in phagocytes, 169

metabolism of, 343

Nucleohiston, 350, 434

Nucleosin, 416

()RITHIN, 437, 446

Ornithuric acid, 437

Oser's base, 286

Orasera, 115

Oxalate plasma, agglutination by, 157

Oxy-betains, 320 -cholin, 308 -neurin, 304

Oxygenated bases, 248

Oysters, poisonous, 192

PACHYRRHIZID, 198

1 Panum's narcotic substance, 41 putrid poison, 40

Paraffin oil, bases in, 281

Parareducin, 464

Paraxanthin, 339, 400

Parvolin, 259

Pathoamins, 465

Pellagra, 223

Pellagroceïn, 240

Pentamethylenediamin, 272

Peptonizing bacteria from milk, 95, 96

Pepton, poisonous nature of, 96 sera, 116

Peptotoxin, 328

Petroleum, bases in, 281

Petromyzon, 197

Pfeiffer's phenomenon, 122

Phagocytes, 168

Phagolysis, 173

Phenol, 33, 243

Phenyl adenin, 364

-alanin, 256

-ethylamin, 256

Phlogosin, 85, 327

Phosphorus-containing substances, 44

Phylocytase, 136

Phytalbumose, 476

Piperazin, 451

Piperidin, synthesis of, 272, 442

Plague precipitins, 113

Plasmolysis, 170

Pneumonia, chemical products in, 100, 283

immunity to, 100

Poisonous arrows, 68

foods, 188

examination of, 226

Pork, 202, 204

Pouchet's bases, 320, 323 
Pouchet's bases in urine, 464

Precipitins, 113

Propylamin, 43, 252

Propionyl guanin, 383

Protalbumose, 49

Protamin, 81, 428 separation of, 430 germicidal action of, 432 physiological action of, 433

Proteids, bacterial, 31 poison, 42 vulgaris, 87, 91, 263

Protons, 429, 431

Prototoxins, 35, 65

Prototoxoid, 65

Protozoal diseases, 18

Pseudo-xanthin, 422

Ptomaïn poisoning, 226

Ptomaïns, chemistry of, 248 definition of, 29 methods of extraction of, 230 table of, 330

Ptomatins, 29

Ptomatropin, 244

Puerperal fever, 101

Purin, 336, 346

bases, separation of, 407

group, 334, 336 action of, 345 metabolism of, $342,344,406$ structure of, 337

Pus organisms, 85 bases from, 349

Putrefactive alkaloids, 29

Putrescin, 47, 56, 265, 438 in urine, 467

Putrid material, effects of, 38 poison of Panum, 40, 42

Pyocyanin, $86,152,330$

Pyocyanase, 175 action on bacteria, 176

Pyogenetic proteids, 83, 86, 90

Pyoxanthose, 330

Pyrazin, 418

Pyridazin, 418

Pyridin, 281, 319

Pyrimidin, 417 group, 334, 415

relation to uric acid, 419

Pyrrolidin carbonic acid, 448

UINOLIN, 453

Quinone, 481

DABBIT septicemia, 100

R Receptors, 129, 130

Reducin, 463

Rennet, anti-, 150

Roussin's test for nicotin, 237

SALAMANDER, 480

$S$ poison, 480

Saliva, leucomaïns in, 471
Salkowski's base, 287

Salmin, 429

Salmon, 199

Samandarin, 480

Saprin, 47, 278

Sarcin, 365

Sardines, poisonous, 200

Sarkin, 365

Sausage, poisonous, 39, 43, 201, 249

Scombrin, 429

Scombron, 434

Sebacic acid, 39, 204, 211

Selmi's method, 43

Sepsin, 41

Septicemia, 24 of rabbits, 100

Septicin, 253

Serum, germicidal action, 102, 169 agglutination by normal, 153, 154 precipitins, 118 -therapy, 47

Siguatera, 193

Silurin, 429

Sinkalin, 289

Sitotoxismus, 220

Skatol, 242, 453

Skin burns, 329, 466

Skunk secretion, 452

Small-pox, 171

Spasmotoxin, 61, 254

Spermatolysins, 145

Spermatoxins, 145

Spermatozoa, agglutination of, 146 composition of, 429,435

Spermin, 450

Sphacelinic acid, 221

Spleen, leucomaïns in, 473

Staphylococcus pyog. aureus, 86,88 bases from, 327

hemolytic action, 88

flavus, 210

Staphylolysin, 88

Staphylotoxin, 88 pathological changes by, 89

Stas-Otto method, 231

Streptococcus, 91, 249, 250, 255

immunity to, 173

Strychnin-like substances, 239

Sturin, 429

Substance sensibilatrice, 135

Succinic acid, 438

Sucholotoxin, 99

Summer diarrhoeas of infancy, 90

Suppuration, 85

Suprarenin, 474

Susotoxin, 99, 280

Swine-plague, immunity to, 180

Symptomatic anthrax, 209

TETANIN, 61, 320

Tetanolysin, 36, 63

Tetanospasmin, 36,63

Tetanotoxin, 61, 254 
Tetanus, 61

antitoxin, 65, 66

bacillus, products of, $34,47,61,254$, 313,320

toxins, 34,61

hemolytic action, 63,64 elimination, 66 action on nerve cells, 66

Tetramethylenediamin, 267

Tetramethyl-putrescin, 269

Tetrodon, 195

Tetrodonin, 196

Thein, see caffein

Theobromin, 339, 404

Theophyllin, 337, 339, 404

Thymin, 416

Toxalbumins, 30, 50, 61, 70, 97

Toxicology of bacterial products, 237

Toxins, 22, 29, 33, 35

conversion into antitoxin, 185

mininum fatal dose, 72

natural immunity to, 170,171

pathological changes by, 73

Toxoid, 35, 65, 133 immunity, 37 of the staphylococcus, 88

Toxons, 35, 65, 72

Toxopeptons, 57

Toxophil chains, 182

Toxophorous group, 36, 65, 130, 182

Traumatic disease, 18

Trichiniasis, 201

Triethylamin, 252

Trigonellin, 305

Trimethylamin, 47, 250

xanthin, 340,405

Trimethylenediamin, 56, 264

Tritotoxins, 35,65

Tubercle bacillus, products of, 77 chemotatic action, 83

fatty products, 79

nucleinic acid, 81 proteid products, 80 protamin, 81 ptomaïns, 82

Tuberculin, 77,83 action of, 84,139

Tuberculinic acid, 81

Tuberculosamin, 81, 429

Tuberculosis, 76

Turtle, 480

Turkey, poisonous, 210

Typhoid bacillus, 97 agglutination of, 113

fever, 97,165 serum agglutination in, 155,159

-like bacilli, 210

Typhotoxin, 47, 97, 316 isomer of, 317

Tyrosin, 49, 57, 101, 255

Tyrotoxicon, 46, 213, 323

in summer diarrhœa, 94

Tyrotoxismus, 211
UNDETERMINED leucomaïns, 461

ptomaïns, 323-330

Uracil, 417, 418

Urea, as cleavage product, 437,459

Uric acid, 333,334

deposits of, 344

group of leucomaïns, 334

metabolism of, 343

purin bases from, 337

reduction of, 337

separation of, from xanthin bases, 412

synthesis of, 419

Urine, agglutination by, 155

diphtheria toxin in, 70,468

leucomaïs in, $250,389,463$

toxicity of, 468

Urochrome, 464

Urotheobromin, 400

Urotoxin, 465

Urotoxy, 469

Urotoxic coefficient, 469

Uschinsky's medium, 34

VALERIANIC acid, 33

Valero-cholin, 303

Veal, poisonous, 205, 209

Vegetable foods, poisoning by, 220

Vetch, poisonous, 222

Venoms, 47,474

agglutinating action, 480

artificial immunity to, 172, 476

hemolytic action, $137,141,479$

natural immunity to, 170

Veratin-like substances, 245, 263

Vernin, 378

Vibrio choleræ asiaticæ, 153, 170, 248, 264

Metchnikovi, 152

Vinylamin, 143

Vitiated air, effects of inhalation of, 461

WEIDEL'S reaction, 352, 370

White liquefying bacterium, products of, 94

Widal's reaction, 154

Y ANTHIN, 339, 388

$\Lambda$ bases, 335

constitution of, 339

isolation of, 410

reaction, 352, 370, 392

Xantho-creatinin, 457

VEAST, 30, 33

auto-digestion of, 348

decomposition of, 326

nuclein, 100

7 EISEL'S test for colchicin, 246 




$$
\begin{array}{lc}
\text { QP801.P7 } & \text { V46 } \\
\text { Vaughan } & \text { 1902. } \\
\text { QP801.P7 } & \text { V46 } \\
& 1902
\end{array}
$$


